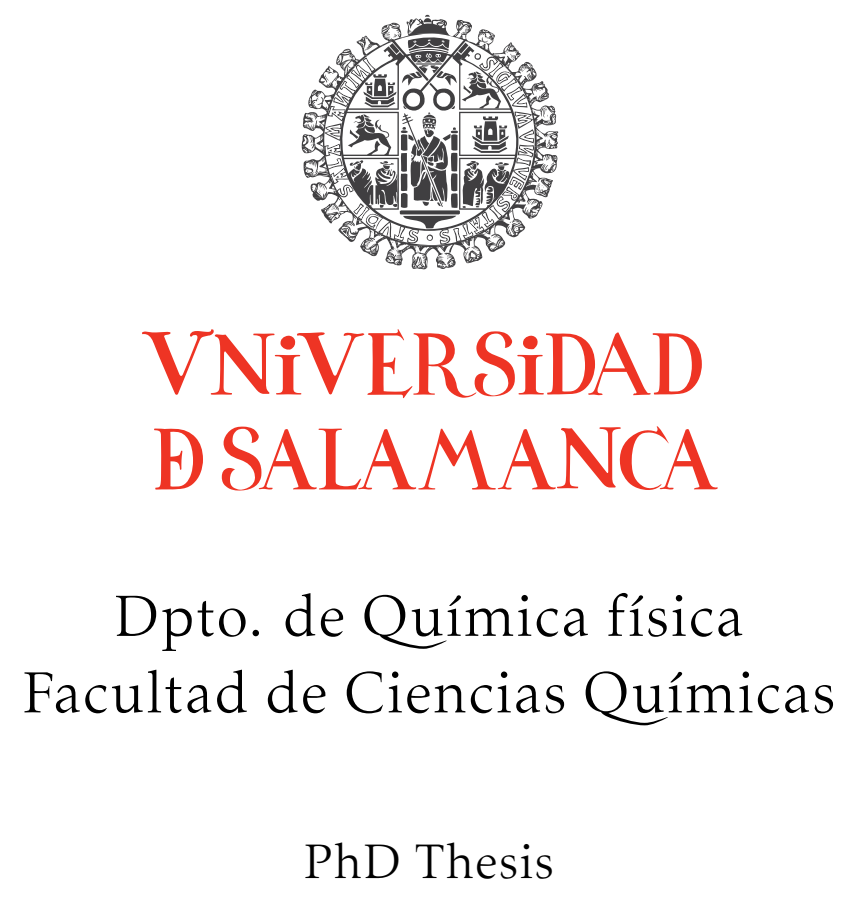

\title{
Chemical processes that can damage cellular DNA: Reactivity and alkylating potential of some $O$-heterocycles
}

Author:

Rafael GómEZ BOMBARELLI
Supervisors:

Prf. Dr. Julio CASADO Prf. Dr. Emilio CALLE 

A Marta, José, Lali y Pepe en ningún orden en particular 

Departamento de Química física

Facultad de Ciencias Químicas

VNiVERSIDAD

DSALAMANCA

The work reported here has been carried out in the Departamento de Química física of the Universidad de Salamanca, under the advice of Prof. Dr. Julio Casado Linarejos and Prof. Dr. Emilio Calle Martín.

Rafael Gómez Bombarelli

Emilio Calle Martín

Julio Casado Linarejos 

It's been emotional.

Big Chris

\section{Acknowledgements}

The author thanks the Spanish Ministerio de Educación for a PhD fellowship (AP2006-01976) and financed research stays at the workgroups of Prof. Dr. José Rueff (Universidade Nova de Lisboa) and Prof. Dr. François Maurel (ITODYS, Paris-VII), who are also thanked for their hospitality.

Financial support of the research reported in this work by the Ministerio de Ciencia e Innovación (projects CTQ2010-18999, CTQ2007-63263, CTQ2004-05048), and the Junta de Castilla y León and European Regional Development Fund (project SA040A08) is also acknowledged.

E. Bombarelli and J. Arenas are also thanked for their generous donation of CPU-time and resources. 



\section{Contents}

List of Figures $v$

List of Tables vii

List of Schemes $\quad i x$

Abbreviations $x i$

Preface xiii

1 Alkylating agents and the NBP Test: a review 1

1.1 Development of the method . . . . . . . . . . . . . . . . . . . . 5

1.1.1 Early examples . . . . . . . . . . . . . . . . . . . . . . . 5

1.1.2 The Epstein test. . . . . . . . . . . . . . . . . . . . . . . . . . 6

1.1.3 Biological samples. . . . . . . . . . . . . . . . . . . . . . 7

1.1.4 Chromatography . . . . . . . . . . . . . . . . . . . . . . 7

$1.2 \mathrm{NBP}$ as a DNA-model. . . . . . . . . . . . . . . . . . . . . . 8

1.2.1 Nucleophilicity of DNA . . . . . . . . . . . . . . . . . . . . . . 8

1.2 .2 Site selectivity . . . . . . . . . . . . . . . . . . . . . . . 10

1.2.3 To what extent is DNA modeled by NBP? . . . . . . . . . . . . . 11

1.2.4 Strengths and weaknesses of the NBP assay. . . . . . . . . . . . . 12

1.3 Uses . . . . . . . . . . . . . . . . . . . . . . . . . . . . . 13

1.3.1 Detection and quantification of alkylating agents . . . . . . . . . . 13

1.3.2 Characterization of alkylating agents . . . . . . . . . . . . . . . 15

References . . . . . . . . . . . . . . . . . . . . 27

2 Reactivity and alkylating potential of diketene 35

2.1 Background . . . . . . . . . . . . . . . . . . . . . . . . . . 39

2.1.1 Genotoxicity of lactones. . . . . . . . . . . . . . . . . . . . 39

2.1.2 Diketene . . . . . . . . . . . . . . . . . . . . . . . . . 41 
2.2 Materials \& methods . . . . . . . . . . . . . . . . . . . . . . . 44

2.2.1 Hydrolysis of diketene . . . . . . . . . . . . . . . . . . . . . . . 44

2.2.2 Alkylating potential of diketene . . . . . . . . . . . . . . . . . . 44

2.2.3 Acidity of lactones and ketones . . . . . . . . . . . . . . . . . . 45

2.3 Results and discussion . . . . . . . . . . . . . . . . . . . . . . 47

2.3.1 Hydrolysis of diketene . . . . . . . . . . . . . . . . . . . . . 47

2.3.2 Alkylating potential of diketene . . . . . . . . . . . . . . . . . . . . 56

2.3.3 Acidity of lactones and cycloketones . . . . . . . . . . . . . . . 63

2.4 Conclusions . . . . . . . . . . . . . . . . . . . . . . . . . . 74

References . . . . . . . . . . . . . . . . . . . . . . . . . 75

3 Theoretical study of lactone hydrolysis 79

3.1 Background . . . . . . . . . . . . . . . . . . . . . . . . . . 83

3.1.1 Hydrolysis of esters . . . . . . . . . . . . . . . . . . . . . . . . . 83

3.1 .2 Hydrolysis of lactones . . . . . . . . . . . . . . . . . . . . . . 108

3.2 Materials \& methods . . . . . . . . . . . . . . . . . . . . . . . 111

3.2.1 Methodology and computational details . . . . . . . . . . . . . . 111

3.2.2 Hydrolysis of 2-5(H)-Furanone . . . . . . . . . . . . . . . . . . . 112

3.3 Results and discussion . . . . . . . . . . . . . . . . . . . . . . . . 113

3.3.1 Neutral hydrolysis. . . . . . . . . . . . . . . . . . . . . . 113

3.3.2 Alkaline hydrolysis . . . . . . . . . . . . . . . . . . . . . 122

3.3.3 Acid hydrolysis . . . . . . . . . . . . . . . . . . . . . . . 128

3.3.4 Conclusions . . . . . . . . . . . . . . . . . . . . . . . . . . 139

References . . . . . . . . . . . . . . . . . . . . . . . . . . . 140

4 Alkylating potential of oxetanes 145

4.1 Background . . . . . . . . . . . . . . . . . . . . . . . . . . . 149

4.1.1 Genotoxicity of ethers . . . . . . . . . . . . . . . . . . . . 149

4.1 .2 Oxetanes . . . . . . . . . . . . . . . . . . . . . . . . . 157

4.2 Materials \& methods . . . . . . . . . . . . . . . . . . . . . . . . . . 161

4.2.1 Alkylating potential of oxetanes . . . . . . . . . . . . . . . . . . . 161

4.2.2 Genotoxicity of oxetanes . . . . . . . . . . . . . . . . . . . . 161

4.3 Results and discussion . . . . . . . . . . . . . . . . . . . . . . . . . . 164

4.3.1 Alkylating potential of oxetanes . . . . . . . . . . . . . . . . . . . . 164

4.3.2 Mutagenicity, genotoxicity and toxicity of oxetanes . . . . . . . . . . . 168

4.4 Conclusions . . . . . . . . . . . . . . . . . . . . . . . . . . 171

References . . . . . . . . . . . . . . . . . . . . . . . . . . . 172 
5 Reactivity and alkylating potential of hydroxyhalofuranones

5.1 Background . . . . . . . . . . . . . . . . . . . . . . . . . . 181

5.1.1 Hydroxyhalofuranones as pollutants . . . . . . . . . . . . . . . 182

5.1.2 Mucohalic acids. . . . . . . . . . . . . . . . . . . . . . 185

5.1 .3 Mutagen X . . . . . . . . . . . . . . . . . . . . . . . . 190

5.2 Materials \& methods . . . . . . . . . . . . . . . . . . . . . . . 199

5.2.1 Reactivity of halohydroxyhalofuranones . . . . . . . . . . . . . . 199

5.2.2 Alkylating potential of halohydroxyfuranones. . . . . . . . . . . . 205

5.3 Results and discussion . . . . . . . . . . . . . . . . . . . . . . 207

5.3.1 Reactivity of halohydroxyhalofuranones . . . . . . . . . . . . . . 207

5.3.2 Alkylating potential of halohydroxyfuranones. . . . . . . . . . . . 234

5.4 Conclusions . . . . . . . . . . . . . . . . . . . . . . . . . 252

References . . . . . . . . . . . . . . . . . . . . 253

A Appendix A1

A.1 DNA-Intercalating photochromic spiropyran . . . . . . . . . . . . . . A5

A.1.1 Methodology and computational details . . . . . . . . . . . . . . A5

A.1.2 Results and discussion . . . . . . . . . . . . . . . . . . . . A6

A.1.3 Conclusions . . . . . . . . . . . . . . . . . . . . . . . . A16

References . . . . . . . . . . . . . . . . . . A17

B Resumen en español C1

Publication list $\quad$ C3 



\section{List of Figures}

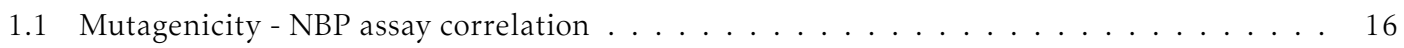

2.1 Typical kinetic run for the neutral hydrolyis of DIK . . . . . . . . . . . . . 48

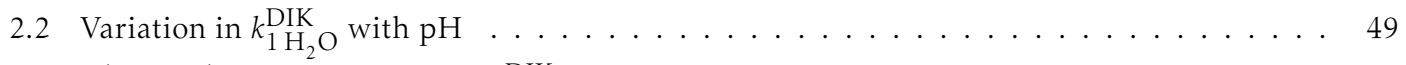

$2.3 \Delta^{\ddagger} H^{\ominus} / \Delta^{\ddagger} S^{\ominus}$ relationship for $k_{\mathrm{H}_{2} \mathrm{O}}^{\mathrm{DIK}}$ in the different $w / d$ media $\ldots \ldots \ldots \ldots \ldots \ldots \ldots$

2.4 Typical kinetic run of alkaline hydrolysis of DIK $\ldots \ldots \ldots \ldots \ldots \ldots \ldots$

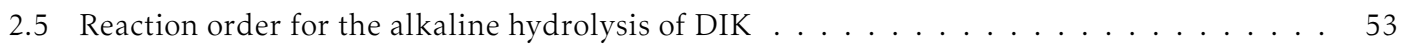

2.6 Variation in $k_{\mathrm{OH}}^{\mathrm{DIK}}$ with $\left[\mathrm{OH}^{-}\right]$and $w / d$ ratio $\ldots \ldots \ldots \ldots \ldots \ldots \ldots \ldots$

$2.7 \Delta^{\ddagger} H^{\ominus} / \Delta^{\ddagger} S^{\ominus}$ relationship for $k_{\mathrm{OH}}^{\mathrm{DIK}}$ in the different $w / d$ media $\ldots \ldots \ldots \ldots \ldots$

2.8 Variation in the UV-Vis spectra of the NBP-DIK alkylation mixture and typical kinetic profile 57

2.9 Formation and decomposition of the NBP-DIK adduct in excess DIK . . . . . . . . . . 58

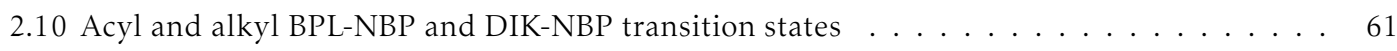

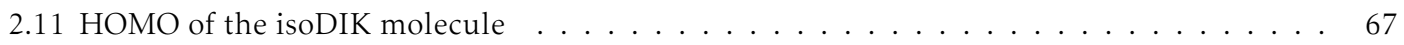

3.1 Hydrolysis rate of some acetates in concentrated sulfuric acid. . . . . . . . . . . 87

3.2 Kinetic order in water for the acid hydrolysis of some acetates. . . . . . . . . . . . . . 95

3.3 Dependence of hydrolysis rate constant on $\mathrm{pH}$ for several esters . . . . . . . . . . . 103

3.4 Hydrolysis rates of some $\beta$-lactones as a function of medium acidity . . . . . . . . . . 109

3.5 Structure of the addition transition state of BIVL in the $\mathrm{B}_{\mathrm{AL}} 1$ mechanism . . . . . . . 115

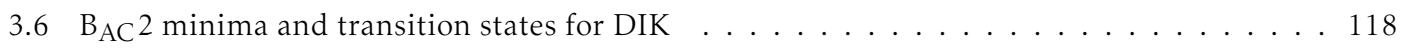

3.7 Structure of the addition and cleavage transition states of GBL in the alkaline $\mathrm{B}_{\mathrm{AC}} 2$ mech-

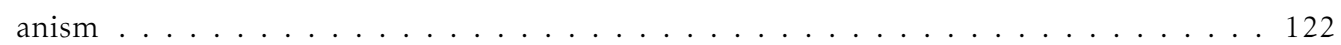

3.8 Transition state for the unimolecular ring-opening reaction of dissociated DIK . . . . . 126

3.9 Equilibrium geometry for the $\mathrm{A}_{\mathrm{AL}} 1$ transition state for BIVL $\ldots \ldots \ldots \ldots$

3.10 Structure of the $\mathrm{A}_{\mathrm{AC}} 1$ cleavage transition state, and its acyl cation product $\ldots \ldots \ldots 133$

3.11 Structure of the intermediate and the transition states in the $\mathrm{A}_{\mathrm{AC}} 2$ mechanism for GBL . . 134

4.1 a) UV-Vis spectrum of NBP-TMO mixtures b) Typical kinetic run of alkylation by oxetanes 164

4.2 a) Variation in $k_{\mathrm{obs}}^{\mathrm{TMO}}$ with $\left.[\mathrm{NBP}] .[\mathrm{TMO}]=5.0 \times 10^{-4} \mathrm{M} . \mathrm{b}\right)$ Variation in $A_{\infty}$ with $[\mathrm{TMO}]_{\mathrm{O}}$,

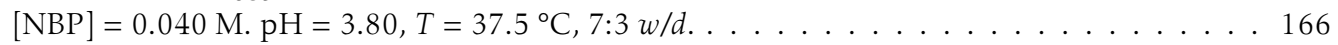

4.3 Revertants per plate in the Salmonella and BTC E. coli tests . . . . . . . . . . . . . . . . . . . . . . . . . . . . . . . . . . . . . .

4.4 Results of the Comet assay . . . . . . . . . . . . . . . . . . 170 
4.5 Relative proliferation as measured in the MTT Assay . . . . . . . . . . . . . . 171

5.1 Carbonyl compounds studied . . . . . . . . . . . . . . . . . 208

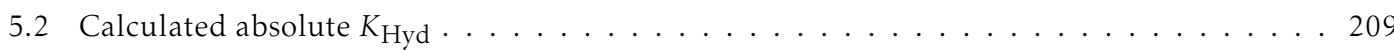

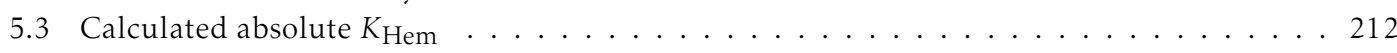

5.4 Variation in the UV-Vis spectra of MXA with $\mathrm{pH} \ldots \ldots \ldots \ldots$

5.5 Spectrophotometric determination of the $\mathrm{p} K_{\mathrm{a}}^{\mathrm{app}}$ of MXA . . . . . . . . . . 216

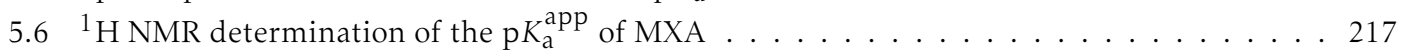

5.7 Calculated equilibrium geometries for mucohalic acids . . . . . . . . . . . . . . . . . . . . . . . . . . . 218

5.8 Energy of $\mathrm{MXA}_{\mathrm{op}}^{-}$as a function of the $\mathrm{C}=\mathrm{C}-\mathrm{C}-\mathrm{O}$ dihedral angle $\ldots \ldots \ldots \ldots$

5.9 Variation with time in the UV-Vis spectra of MXA in the reaction with $\mathrm{OH}^{-} \ldots \ldots 222$

5.10 Kinetic profiles of the reaction of MXA with $\mathrm{OH}^{-} \ldots \ldots \ldots \ldots \ldots \ldots$

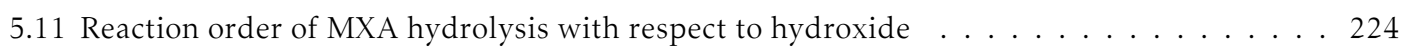

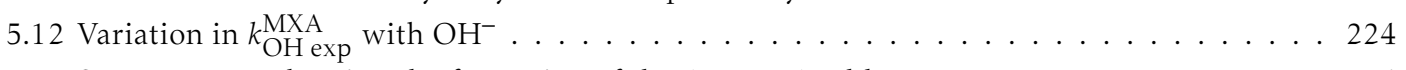

5.13 Spectrograms showing the formation of the AN-MXA adducts . . . . . . . . . 234

5.14 Typical kinetic profile of the alkylation reaction of AN by MXA . . . . . . . . . . . . . . . . 35

5.15 Spectrograms showing the formation of the NBP-MXA adducts . . . . . . . . . 238

5.16 Typical kinetic profile of the alkylation reaction of NBP by MXA . . . . . . . . . . 239

A.1 Calculated geometries for the photochrome $\ldots \ldots \ldots \ldots \ldots \ldots \ldots$

A.2 a) Reported UV-Vis spectra of the open and closed forms of SP, in the absence and presence of DNA. Reproduced from. ${ }^{4}$ b) Calculated UV-Vis spectra of TTC; isolated TTC in its intercalation geometry; $\mathrm{TTC}$ bound to $[\operatorname{tri}(\mathrm{dA}-\mathrm{dT})]_{2} ; \mathrm{CL} \ldots \ldots \ldots \ldots$. . . . . . . . . . . . . . . . . . . . .

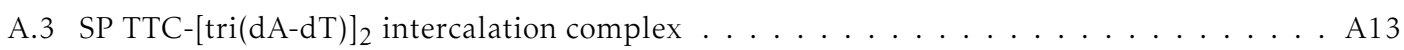




\section{List of Tables}

1.1 Swain-Scott nucleophilicity constants for some common nucleophiles . . . . . . . . . . 9

1.2 Selectivity in DNA-adduct formation in terms of several approaches . . . . . . . . . 10

1.3 Correlation between non-kinetic NBP assay and mutagenicity. . . . . . . . . . 17

1.4 Correlation between non-kinetic NBP assay and carcinogenicty $65 \ldots \ldots \ldots$

1.5 Correlation of Tumorigenicity and NBP Alkylation kinetics for $\beta$-lactones. . . . . . . . 24

1.6 Alkylating Agents that form Unstable Adducts . . . . . . . . . . . . . . . 25

2.1 Rate constants for the hydrolysis of DIK in neutral $w / d \ldots \ldots . \ldots . \ldots . \ldots$

2.2 Activation parameters for the hydrolysis of DIK in neutral $w / d \ldots \ldots \ldots$

2.3 Activation parameters for the neutral hydrolysis of some lactones . . . . . . . . . 50

2.4 Rate constants for the hydrolysis of DIK in alkaline $w / d$ media $\ldots \ldots \ldots \ldots . \ldots . \ldots 54$

2.5 Base dissociation constants for DIK in $w / d$ media $\ldots \ldots \ldots \ldots$

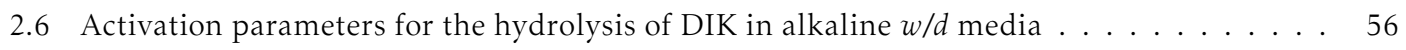

2.7 Rate constants for the alkylation of NBP by DIK . . . . . . . . . . . . . . . . . . 59

2.8 Activation parameters for the alkylation of NBP by DIK in $w / d \ldots \ldots \ldots$

2.9 Activation parameters for the alkylation of NBP by some $\beta$-lactones in $7: 3 w / d \ldots$. . . . . 60

2.10 Computed activation energies for the alkylation of NBP by some $\beta$-lactones . . . . . . . 60

2.11 Rate constants for the decomposition of the NBP-DIK adduct . . . . . . . . . . . . . 62

2.12 NBP catalytic coefficients for the decomposition of the NBP-DIK adduct . . . . . . . . . 62

2.13 Isomerization free energies of some esters and lactones in aqueous solution . . . . . . . 66

2.14 Average effect of cyclization and unsaturation on the $\mathrm{p} K_{\mathrm{a}}$ of esters $\ldots \ldots$. . . . . . 68

2.15 Effect of unsaturation on the $\mathrm{p} K_{\mathrm{a}}$ of esters, with respect to saturated backbone . . . . . . 69

2.16 Isomerization free energies of some cycloketones in aqueous solution $\ldots . . \ldots 72$

2.17 Effect of unsaturation on the $\mathrm{p} K_{\mathrm{a}}$ of esters, with respect to saturated backbone $\ldots \ldots 73$

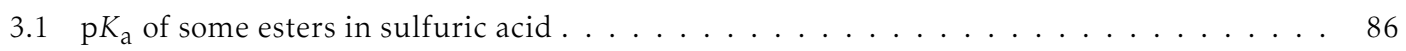

3.2 Effect of solvent on the acid-catalyzed hydrolysis of $t$-butyl acetate . . . . . . . . . . 91

3.3 Activation enthalpy for the acid hydrolysis of $t$-butyl acetate in aqueous acetone . . . . . 92

$3.4 r$-Values for the acid hydrolysis of esters $\ldots \ldots \ldots \ldots$

3.5 Influence of the acyl substituentes on the acid hydrolysis of ethyl esters ${ }^{60-62} \ldots \ldots 100$

3.6 Activation parameters for the alkaline hydrolysis of some lactones . . . . . . . . . . . 111

3.7 Calculated energy barriers for the neutral hydrolysis of some esters . . . . . . . . . . . 114 
3.8 Calculated energy barriers for the neutral $\mathrm{B}_{\mathrm{AC}} 2$ hydrolysis of some lactones $\ldots \ldots$. . . 116

3.9 Calculated energy barriers for the neutral $\mathrm{B}_{\mathrm{AC}} 2$ hydrolysis of some lactones . . . . . 120

3.10 Calculated energy barriers for the alkaline hydrolysis of some lactones. . . . . . . . . 123

3.11 Experimental activation parameters for the alkaline hydrolysis of some lactones in water. . 127

3.12 Energies of dissociation and $\mathrm{pK}_{\mathrm{a}}$ of the studied esters $\ldots \ldots \ldots \ldots$

3.13 Energy barriers for the rate limiting step in the mechanisms acid-catalyzed lactone hydro-

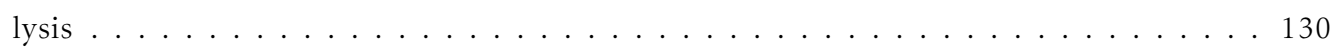

3.14 Energy barriers for the $\mathrm{A}_{\mathrm{AL}} 1$ acid hydrolysis of some lactones $\ldots \ldots \ldots \ldots \ldots 131$

3.15 Energy barriers for the $\mathrm{A}_{\mathrm{AC}} 1$ acid hydrolysis of some lactones . . . . . . . . . . . 132

3.16 Energy barriers for the $\mathrm{A}_{\mathrm{AC}} 2$ hydrolysis of some lactones $\ldots \ldots \ldots \ldots \ldots$

3.17 Experimental activation parameters for the acid hydrolysis of some lactones in water . . . 136

3.18 Energy barriers hydrolysis of some lactones with minimal water molecules . . . . . . . 138

3.19 Energy barriers hydrolysis of some lactones with one additional explicit solvent molecule, plus the one acting as a nucleophile $($ when needed $) \ldots \ldots \ldots \ldots$

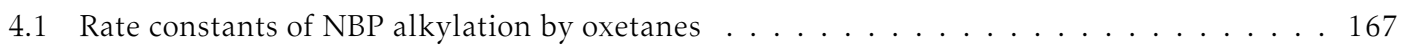

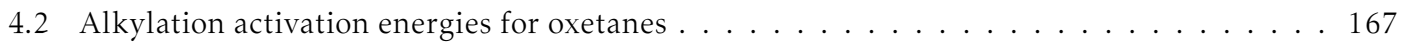

5.1 Concentration of MX in plant effluent and tap waters . . . . . . . . . . . . . . 184

5.2 Maximum concentration of brominated MX in plant effluent . . . . . . . . . . 184

5.3 Genotoxicity of MX in non-mammalian in vitro . . . . . . . . . . . . . . . . . 193

5.4 Genotoxicity of MX in mammalian cells in vitro . . . . . . . . . . . . . . . . . . . . . . . . . . . . . . . .

5.5 Relative genotoxicity of MX to analogue hydroxyhalofuranones label . . . . . . . . . . . 195

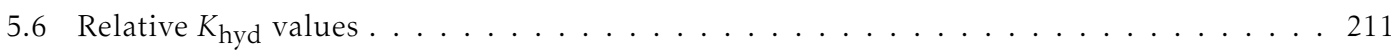

5.7 Mean unsigned errors in relative $K_{\text {hyd }}$ values $\ldots \ldots \ldots \ldots \ldots \ldots \ldots \ldots \ldots \ldots \ldots$

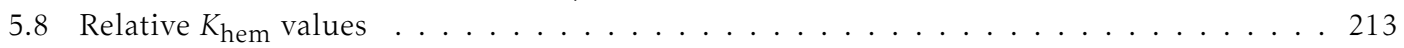

5.9 Mean unsigned errors in relative $K_{\text {hem }}$ values . . . . . . . . . . . . . . . . . . 214

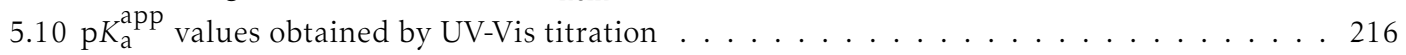

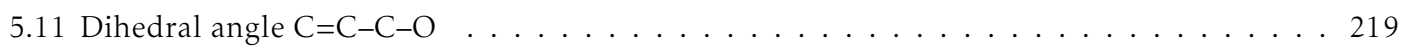

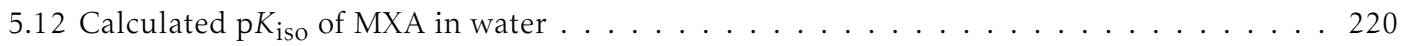

5.13 Calculated $\mathrm{p} K_{\text {iso }}$ of MXA in DMSO and heptane . . . . . . . . . . . . 220

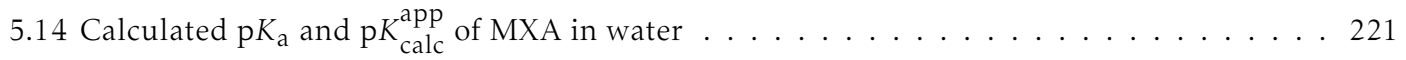

5.15 Rate constants for the reaction of MXA with $\mathrm{OH}^{-} \ldots \ldots \ldots \ldots \ldots \ldots$

5.16 Activation energies for the hydrolysis of MXA . . . . . . . . . . . . 225

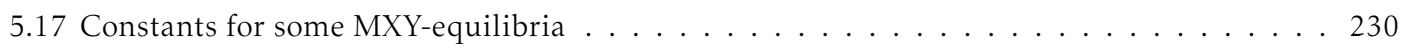

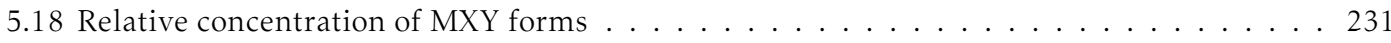

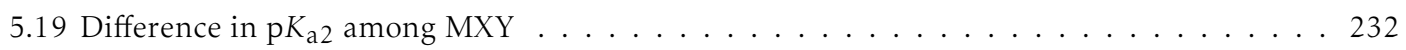

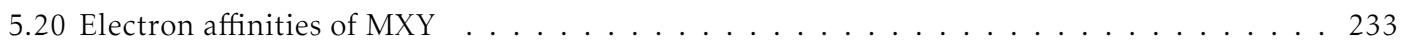

5.21 Rate constants of aniline alkylation by mucohalic acids . . . . . . . . . . . . . . 235

5.22 Activation parameters for the alkylation of aniline by MXA . . . . . . . . . . . . 236

5.23 Rate constants of NBP alkylation by mucohalic acids . . . . . . . . . . . . . . . . . . . . . . 240

5.24 Activation parameters for the alkylation of NBP by MXA . . . . . . . . . . . 240 
5.25 Rate constants of NBP-MXA adduct conversion . . . . . . . . . . . . . . . . 241

5.26 Relative energies along the alkylation path of nucleotides by MXA . . . . . . . . . . . 243

5.27 Relative energies along the alkylation path of nucleotides by MXA . . . . . . . . . . . 244

5.28 Calculated energy barriers for the alkylation of nucleotides by MXY . . . . . . . . . 247

5.29 Energy barriers for further reactions of the nucleotide-MXY adducts . . . . . . . . . . 248

5.30 Electron affinities in aqueous solution for the MXY-Nucleotide adducts . . . . . . . . 250

5.31 Electron affinities in aqueous solution for the closed-chain MX-Nucleotide cationic adducts 251

A.1 Relative energies for the isomers of $\mathrm{SP}$ in gas phase and solution $\ldots \ldots \ldots \ldots$. . . . A7

A.2 energies of the isomers of a SP-analogue lacking the charged substituent $\ldots \ldots \ldots$ A8

A.3 Relative energies of SP-isomers and transition states . . . . . . . . . . . A10

A.4 Wavelength of maximum absorption $(\mathrm{nm})$ for the SP-isomers $\ldots \ldots \ldots \ldots$. . . . . . . . . . . . .

A.5 SP-oligonucleotide interaction energy . . . . . . . . . . . . . . A12

A.6 UV-Vis transitions of the SP-oligonucleotide HL complex . . . . . . . . . . . . . . . . A15 



\section{List of Schemes}

1.1 Development of color after alkylation in the NBP assay . . . . . . . . . . . . . . 6

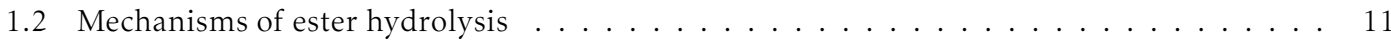

1.3 Reactions involved in the NBP alkylation mechanism . . . . . . . . . . . . . . . . 19

2.1 Absolute approach for the determination of $\mathrm{p} K_{\mathrm{a}}$ values $\ldots \ldots \ldots \ldots$

2.2 Relative approach for the determination of $\mathrm{p} K_{\mathrm{a}}$ values $\ldots \ldots \ldots$. . . . . . . . 46

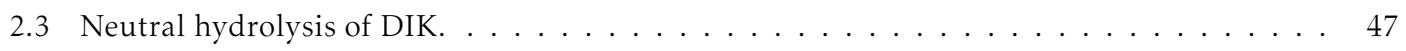

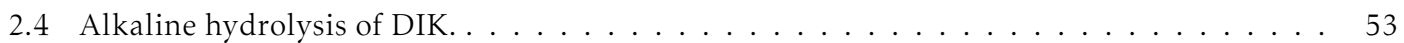

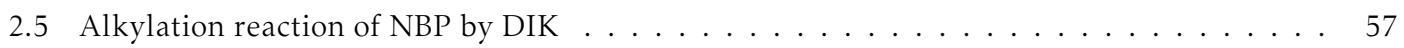

2.6 Calculated $\mathrm{p} K_{\mathrm{a}}$ and isomerization energies of linear esters in aqueous solution. . . . . . . 64

2.7 Calculated $\mathrm{pK}_{\mathrm{a}}$ and isomerization energies of $\beta$-lactones $\ldots \ldots \ldots \ldots \ldots$

2.8 Calculated $\mathrm{p} K_{\mathrm{a}}$ and isomerization energies of $\gamma$-lactones $\ldots \ldots \ldots \ldots$

2.9 Calculated $\mathrm{p} K_{\mathrm{a}}$ and isomerization energies of four-membered cyclic ketones $\ldots \ldots$. . . 70

2.10 Calculated $\mathrm{p} K_{\mathrm{a}}$ and isomerization energies of methyl cyclopentanones and methyl cy-

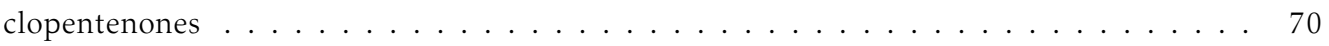

2.11 Calculated $\mathrm{p} K_{\mathrm{a}}$ and isomerization energies of dimethyl cyclopentanones and dimethyl cy-

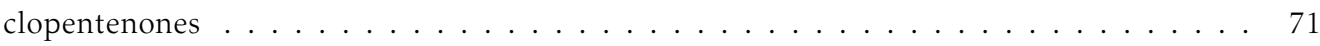

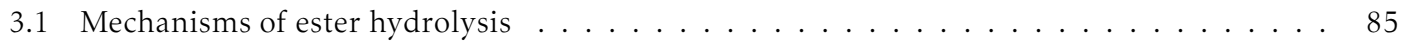

$3.2 \mathrm{~A}_{\mathrm{AC}} 1$ mechanism of ester hydrolysis $\ldots \ldots \ldots \ldots \ldots \ldots$

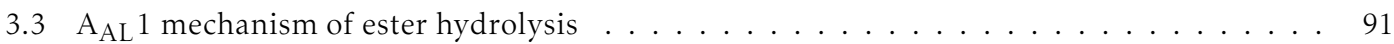

$3.4 \mathrm{~A}_{\mathrm{AL}} 2$ mechanism of ester hydrolysis $\ldots \ldots \ldots \ldots \ldots \ldots \ldots \ldots \ldots$

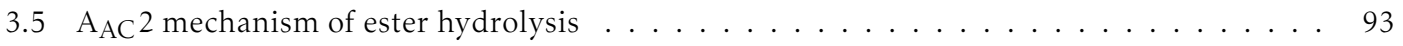

3.6 Concerted mechanism for $\mathrm{A}_{\mathrm{AC}} 2$ ester hydrolysis $\ldots \ldots \ldots \ldots \ldots \ldots$

3.7 Addition of water catalyzed by another water molecule $\ldots \ldots$. . . . . . . . . . 97

3.8 General acid catalysis in the breakdown in the tetrahedral intermediate implies generalbase catalysis in water addition . . . . . . . . . . . . . . . . 98

3.9 Complete accepted mechanism for $\mathrm{A}_{\mathrm{AC}} 2$ ester hydrolysis $\ldots \ldots \ldots$. . . . . . . . . . 99

3.10 Complete accepted mechanism for the neutral $\mathrm{B}_{\mathrm{AC}} 2$ ester hydrolysis . . . . . . . . . 104

$3.11 \mathrm{~B}_{\mathrm{AL}} 2$ mechanism of alkaline ester hydrolysis . . . . . . . . . . . . . 105

3.12 Complete accepted mechanism for the alkaline $\mathrm{B}_{\mathrm{AC}} 2$ ester hydrolysis . . . . . . . . . 105

3.13 Simplified accepted mechanism for the alkaline $\mathrm{B}_{\mathrm{AC}} 2$ ester hydrolysis . . . . . . . . . 106 
$3.14 \mathrm{~B}_{\mathrm{AC}} 1$ (a), $\mathrm{B}_{\mathrm{AL}} 1$ (b), $\mathrm{B}_{\mathrm{AL}} 2$ (c) and simplified $\mathrm{B}_{\mathrm{AC}} 2$ (d) mechanisms of neutral hydrolysis . . 113

3.15 Addition reaction in the $\mathrm{B}_{\mathrm{AC}} 2$ mechanisms of neutral hydrolysis . . . . . . . . . 117

3.16 Acyl oxygen bond cleavage reaction in the $\mathrm{B}_{\mathrm{AC}} 2$ mechanisms of neutral hydrolysis . . . . 119

$3.17 \mathrm{~B}_{\mathrm{AL}} 2$ mechanism of alkaline hydrolysis and $\mathrm{B}_{\mathrm{AC}} 2$ addition step $\ldots \ldots \ldots \ldots \ldots$

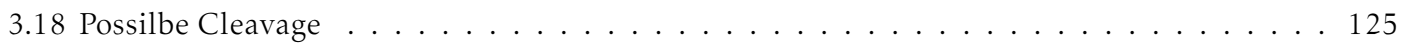

3.19 Elimination-addition hydrolysis mechanism of DIK . . . . . . . . . . . 126

$3.20 \mathrm{~A}_{\mathrm{AL}} 1, \mathrm{~A}_{\mathrm{AC}} 1, \mathrm{~A}_{\mathrm{AL}} 2$ and simplified $\mathrm{A}_{\mathrm{AC}} 2$ mechanisms of hydrolysis $\ldots \ldots \ldots 130$

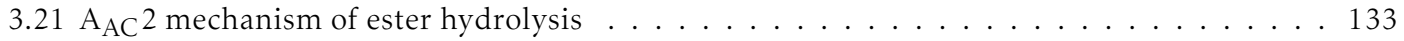

3.22 Possible addition steps in the $\mathrm{A}_{\mathrm{AC}} 2$ hydrolysis mechanism . . . . . . . . . . . . 135

3.23 Observed cleavage steps in the $\mathrm{A}_{\mathrm{AC}} 2$ hydrolysis mechanisms $\ldots \ldots \ldots \ldots$

4.1 Some small heterocyclic alkylating agents. . . . . . . . . . . . . . . . . . . 149

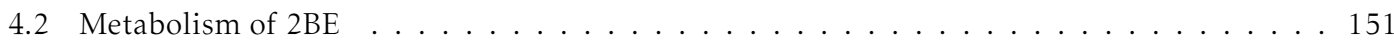

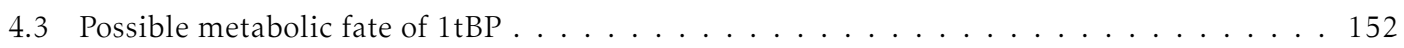

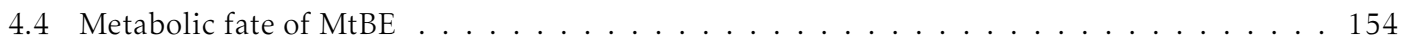

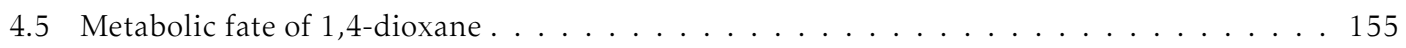

4.6 Metabolic fate of Furan . . . . . . . . . . . . . . . . . . . . 157

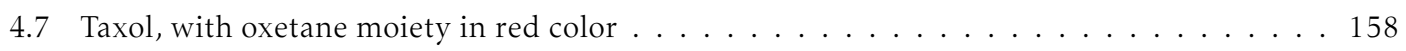

4.8 Oxetane derivatives reported in the literature Reproduced from ${ }^{2} \ldots \ldots$. . . . . . . . 159

4.9 NBP Test: development of color after alkylation . . . . . . . . . . . . . . . . 161

4.10 Alkylation of NBP by oxetanes and concurrent hydrolysis . . . . . . . . . . 165

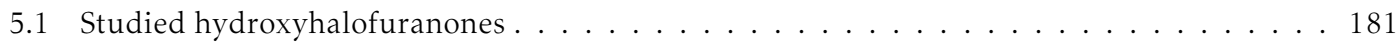

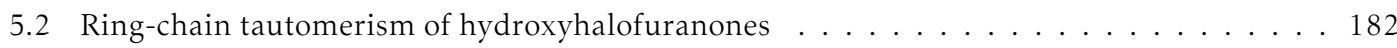

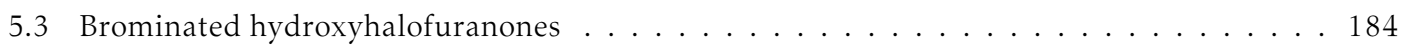

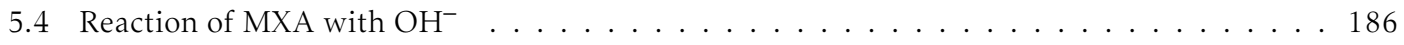

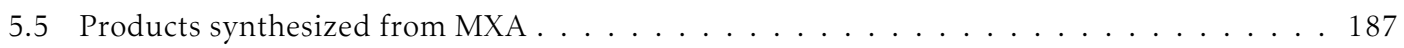

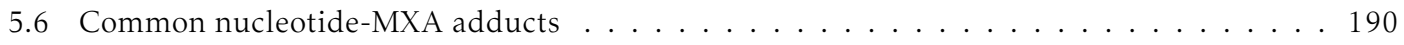

5.7 Rare nucleotide-MXA adducts . . . . . . . . . . . . . . . . . . . . 190

5.8 Mechanism of alkylation by MXA proposed in the literature . . . . . . . . . . . . . 191

5.9 Nucleotide-MXY adducts . . . . . . . . . . . . . . . . . . . . . . . . . . . . . 197

5.10 Proposed mechanisms for the formation of nucleotide-MCF adducts . . . . . . . . 198

5.11 Proposed mechanisms for the formation of nucleotide-CMCF adducts . . . . . . . . 198

5.12 Proposed mechanisms for the formation of nucleotide-MX adducts . . . . . . . . 198

5.13 Thermodynamic cycles for the determination of $K_{\text {hyd }}$ values $\ldots \ldots$. . . . . . . . 199

5.14 Forms of mucohalic acids in equilibrium in aqueous solution. . . . . . . . . . . . 200

5.15 Forms of MX and analogues in equilibrium in aqueous solution. . . . . . . . . . . . 204

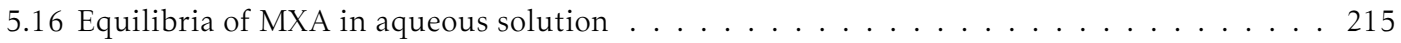

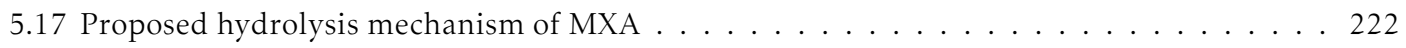

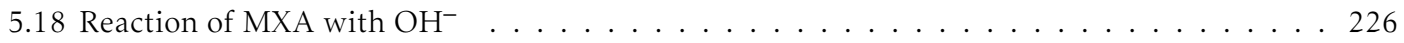

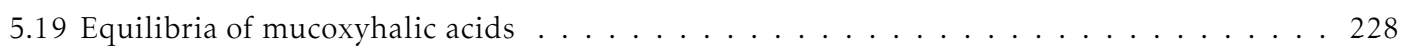

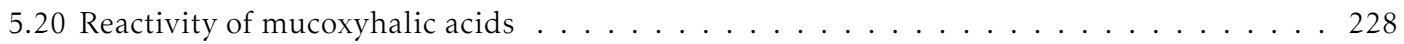


5.21 Forms of MX and analogues in equilibrium in aqueous solution. . . . . . . . . . . . 229

5.22 Reaction of MXA with aniline . . . . . . . . . . . . . . . 237

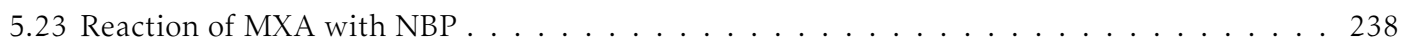

5.24 Reaction of MXA with nucleotides . . . . . . . . . . . . . . . . . . . . . . . . . . . . . . . . .

5.25 Alkylation of nucleotides by $\mathrm{MX}$ and analogues. $\mathrm{X}, \mathrm{Y}=\mathrm{H}, \mathrm{Cl} \ldots \ldots \ldots \ldots$

A.1 Photochromic spiropyran with DNA-intercalating ability $\ldots \ldots \ldots \ldots \ldots$ A5

A.2 Thermal decoloration and isomerization pathways $\ldots \ldots \ldots \ldots \ldots$ A9 

It is worth noting that the notation facilitates discovery. This, in a most wonderful way, reduces the mind's labor.

Gottfried Wilhelm Leibniz

\section{Abbreviations}

1tBP - 1-tert-butoxypropan-2-ol

2BE - 2-butoxyethanol

$\mathrm{cAcH}$ - acetoacetic acid

$\mathrm{BBL}-\beta$-butyrolactone

BIVL - $\beta$-isovalerolactone

BPL - $\beta$-propiolactone

$\mathrm{CHO}$ - Chinese Hamster Ovarian

CHV79 - Chinese Hamster V79

DIK - diketene

DBP - disinfection by-product DVL - $\delta$-valerolactone

GBL $-\gamma$-butyrolactone

GC - Gas Chromatography

IARC - International Agency for Research on Cancer

KIE - Kinetic Isotope Effect

NBP - 4-(p-nitrobenzyl)pyridine

$w / d$ - Water/dioxane

MCA - mucochloric acid (3,4-dichloro-5-hydroxyfuran-2(5H)-one)

MBA - mucobromic acid (3,4-dibromo-5-hydroxyfuran-2(5H)-one)

MS - Mas Spectrometry

MXA - mucohalic acids (3,4-dichalo-5-hydroxyfuran-2(5H)-one)

MX - Mutagen X (3-chloro-4-dichloromethyl-5-hydroxy-2(5H)-furanone)

CMCF - 3-chloro-4-chloromethyl-5-hydroxy-2(5H)-furanone

MCF - 3-chloro-4-methyl-5-hydroxy-2(5H)-furanone

SCE - Sister Chromatide Exchange 

Preface 

Science sans conscience n'est que la ruine de l'âme

Rabelais

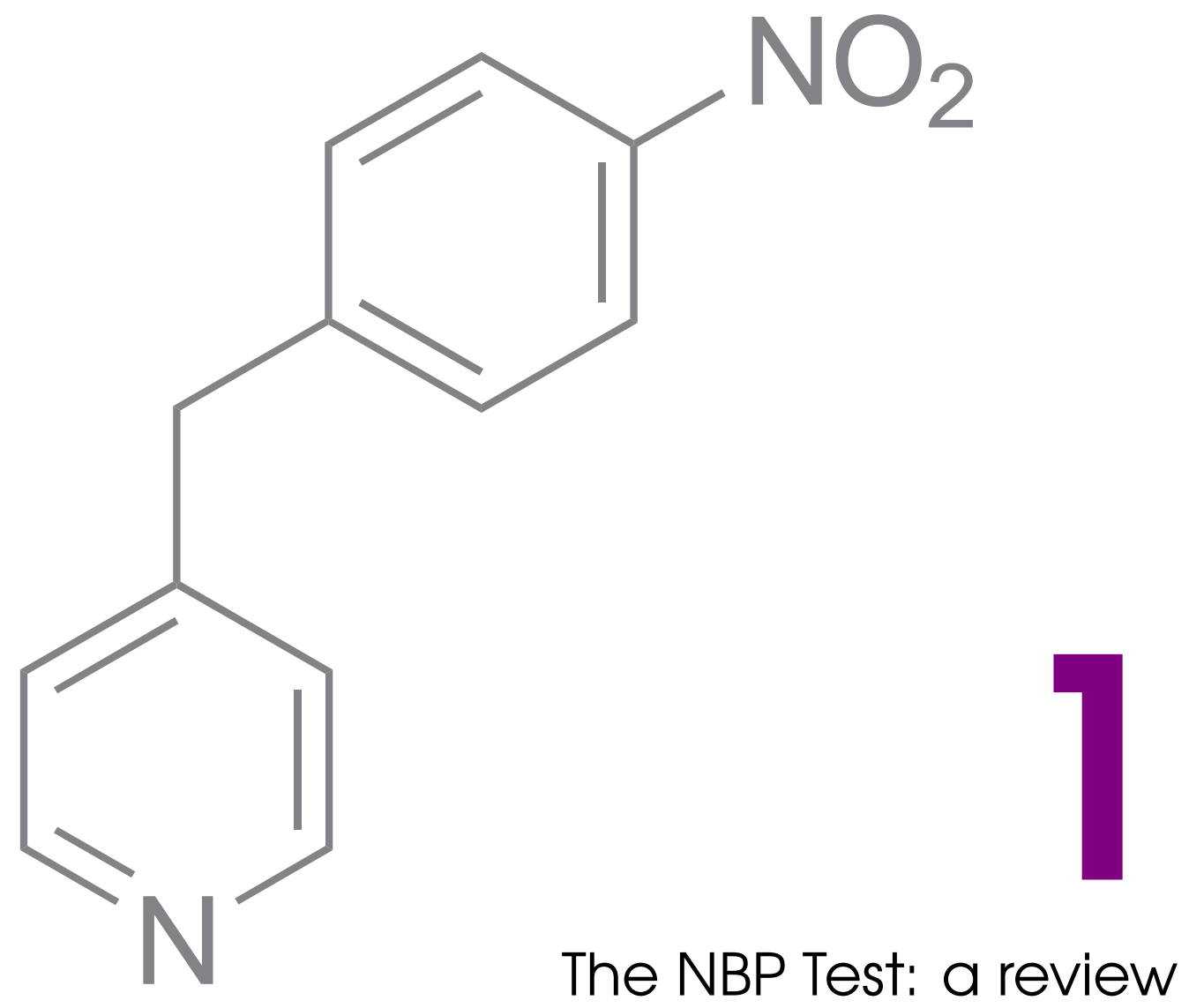





\section{Contents}

1.1 Development of the method .................. 5

1.1.1 Early examples ....................... 5

1.1.2 The Epstein test . . . . . . . . . . . . . . 6

1.1.3 Biological samples . . . . . . . . . . . . . 7

1.1 .4 Chromatography .................... 7

1.2 NBP as a DNA-model . . . . . . . . . . . . . . 8

1.2.1 Nucleophilicity of DNA ................... 8

1.2 .2 Site selectivity . . . . . . . . . . . . . . 10

1.2.3 To what extent is DNA modeled by NBP? . . . . . . . . . . . . . 11

1.2.4 Strengths and weaknesses of the NBP assay . . . . . . . . . . . 12

1.3 Uses . . . . . . . . . . . . . . . . . . 13

1.3.1 Detection and quantification of alkylating agents . . . . . . . 13

1.3.1.1 Detection of Toxic Agents . . . . . . . . . . . . . . . 13

1.3.1.2 Detection of Pharmacological Agents . . . . . . . . . . . . . . . 14

1.3.1.3 Indirect monitoring . . . . . . . . . . . . . . 14

1.3.2 Characterization of alkylating agents . . . . . . . . . 15

1.3.2.1 Non-kinetic applications . . . . . . . . . . . . . . . 15

1.3.2.1.1 Mutagenicity and chemical reactivity . . . . . . . . 16

1.3.2.1.2 Carcinogenicity and chemical reactivity . . . . . . . . 18

1.3.2.1.3 Other attempted correlations . . . . . . . . . . . 18

1.3.2.2 Kinetics of alkylation - alkylation mechanism . . . . . . . . . . . 19

1.3.2.2.1 Only alkylation . . . . . . . . . . . . . . 20

1.3.2.2.2 Competing hydrolysis . . . . . . . . . . . . 22

1.3.2.2.3 Adduct decomposition . . . . . . . . . . . . 24

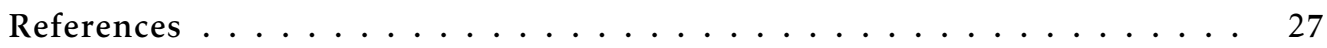



To this date, many chemicals are known or suspected to induce cancer in humans. Institutions like the US Department of Health or International Agency for Research on Cancer (IARC) keep lists in which the available evidence is gathered and conclusions about the carcinogenicity of these compounds are reached. More than 900 chemicals have been tested assessed, and 400 of them have been found to be carcinogenic or possibly carcinogenic, and exert their biological effects throug a wide range of mechanisms. Out of these, the ability to form covalent adducts with DNA is shared by many carcinogens, which are grouped under the label of alkylating agents.

Alkylating agents are archetypical carcinogens, and many works have addressed their characterization and mode of action. Since the early discovery in the 1950's that N-nitrosocompounds (dimethylamine) can induce cancer, large efforts have been devoted to the characterization of alkylating agents.

One of the tools most commonly applied to the study of alkylating agents is the 4- $(p$ nitrobenzyl)pyridine (NBP) test, a chromogenic assay that allows rapid and affordable quantitative detection. Also, since the nucleophilicity of NBP is similar to that of the most reactive sites at DNA, many correlations between biological activity and NBP alkylation (especially alkylation rates) have been reported. Since this assay has been often used by our group, ${ }^{1-16}$ and will be applied several times along this work, this section addresses the history and applicability of the NBP test.

\subsection{Development of the method}

\subsubsection{Early examples}

The first investigations addressing the detection of alkylating agents were made by researchers who worked at medical or warfare agencies in the beginning of the 19 th century. The precursor of the NBP test was developed by Decker et al. who reported that the reaction product of $\alpha$ or $\gamma$ benzylpyridine with methyl iodide yields a colored form in the presence of sodium hydroxide.

The first application of 4-(p-nitrobenzyl)pyridine to detect alkylating agents was in 1925 by Köenigs et al. ${ }^{17}$ and the US military were the first to try to correlate color intensity and reactivity of the alkylating agent, and also to propose a mechanism for the reaction (Scheme $1.1)$.

The early works in the NBP assay are a consequence of the interest in the detection and properties of the vesicant, mutagenic and carcinogenic compounds used in chemical warfare. Among these, Epstein et al. improved the accuracy of method and applied for the quantitative 


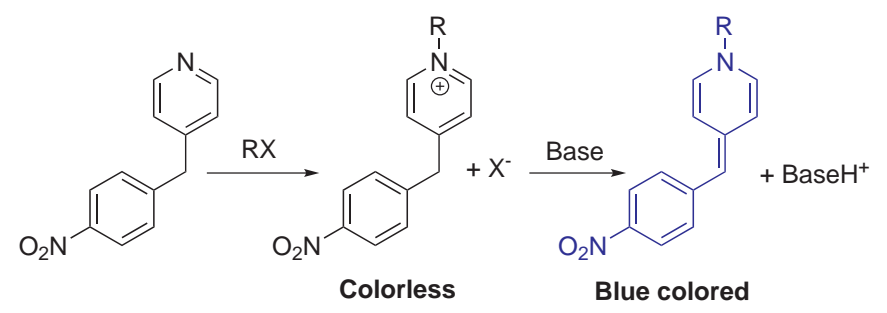

Scheme 1.1. Development of color after alkylation in the NBP assay

determination of alkylating agents. ${ }^{18}$ This seminal work has been quoted profusely and the NBP test called by some authors the Epstein test.

\subsubsection{The Epstein test}

The procedure was based on the spectrophotometric measure of the color intensity of the alkylated NBP after basification. Since they obey the Lambert-Beer law, the measured absorbances can be correlated with the concentration of alkylating agent. Parameters such as $\mathrm{pH}$, buffer, incubation temperature and time were optimized, it was noticed that the color is unstable in the basified medium, and measurements must be taken rapidly.

Depending on the aqueous solubility of the alkylating agent, two modifications of the test have been used:

(i) In the aqueous modification, solutions of the alkylating agent in aqueous phthalate and NBP in acetone are mixed, heated at $100{ }^{\circ} \mathrm{C}$ for 20 minutes and then cooled in an ice-water bath. Potassium carbonate in water:acetone (1:4) is used as the base. Many works have used this form of the assay with minor modifications. ${ }^{19-40}$

(ii) For those compounds that are unsoluble in water, methyl ethyl ketone is used instead of water. Since the alkylation reaction is slower in organic media, it is allowed to run for $45 \mathrm{~min}$. Acetone is added and after cooling, triethylamine is added as a base. Higher temperatures have been used, solving the reactants in less volatile solvents such as acetophenone ${ }^{41}$ and ethylene glycol respectively. ${ }^{42}$

The use of ehtlyene glycol has the additional advantage of avoiding the formation of separate organic and aqueous phases, as often occurs. ${ }^{43-51}$

The method introduced by Preussman uses glycol monomethyl ether as solvent and piperidine as base has been used extensively. ${ }^{52-56}$ 


\subsubsection{Biological samples}

With the advent of alkylating antineoplasic agents, the interest in NBP found as an analytical reagent stirred up. The ability to detect these drugs in biological fluids, however, required some modification to the method in order to:

- improve the sensitivity in these water-rich media, triethylamine was added with a saturated solution of ammonium sulphate. ${ }^{57}$

- improve the reproducibility by solving NBP in a mixture of DMSO, aqueous acetic acid, and 2-butanone, changing the buffer from phthalate to acetate to avoid side reactions. ${ }^{58}$

- separate proteins and other possibly-interfering constituents of biological fluids; e.g. by centrifugation; precipitation with acetone, chemical ${ }^{20,59}$ or thermal denaturation.

Friedman and Boger added more modifications to the original method, such as the extraction of the coloured product with ethyl acetate and use of $\mathrm{NaOH}$ as base. ${ }^{58}$ This approach has been the most commonly used for the estimation of alkylating agents in biological samples. ${ }^{60-71}$

Some variations have been used, which include gassing the sample with either $\mathrm{CO}_{2}, \mathrm{~N}_{2}$, $\mathrm{O}_{2} ;{ }^{35,72-74}$ adding 5\% triethylamine in diethylether and acetone, or replacing ethyl acetate by ethylene dichloride. ${ }^{75-77}$ Skibba et al. also proposed modifications to address the detection of antineoplasic nitrogen mustards in biological fluids. ${ }^{78}$

In order to study the reaction kinetics of a variety of alkylating agents ${ }^{79-81}$ Kawazoe reached several conclusions regarding the influence of temperature and $\mathrm{pH}:{ }^{82}$ attention must be paid to the thermal stability of the reactants, and a $\mathrm{pH}$ above 12.5 is needed for quantitative dissociation of the adduct.

Following the works cite above, only small modifications haven been applied in particular occasions to a base method consisting of: the reaction of excess NBP with the alkylating agent in the reaction media (either aqueous buffer or organic solvent) during a period of time and at fixed temperature (generally either room- or body-temperture, $60{ }^{\circ} \mathrm{C}$ or $100{ }^{\circ} \mathrm{C}$ ); followed by basification (bases used are $\mathrm{NaOH}, \mathrm{K}_{2} \mathrm{CO}_{3}$ in water and $\mathrm{Et}_{3} \mathrm{~N}$ or piperidine) and recording of the UV-Vis absorbance.

\subsubsection{Chromatography}

An additional set of variations exist, as regards the use of NBP as developing reagent in thinlayer chromatography, where NBP is sprayed on the plate and then basified. ${ }^{83-89}$ 


\subsection{NBP as a DNA-model}

NBP is often quoted as model for DNA in general, or for the N-7 position of guanine in particular. ${ }^{59,65,90}$ This assumed relationship is usually taken as a premise and applyed with the aim to establish correlations of the response in the NBP assay with the results in other assays or the effects in vivo. ${ }^{38}$ This section addresses whether NBP is an appropriate model for DNA, and thus the validity of the said premise.

DNA is a very complex polymer, with a variety of $N$ (endo- and exocyclic) and $O$ (carbonyls, phosphates) nucleophilic positions that may react simultaneously affording different reaction products. Even nucleotides, the monomers, have several reactive sites. This, together with other factors such as the low solubility of the nucleobases or the cost of commercial DNA and above all the difficulty of monitoring reactants and products, makes the kinetic study of the DNA-alkylation very difficult and encourages the use of models.

Whereas the multiplicity of reactive sites makes it impossible to condense the reactivity of polymeric DNA into that of one single position, experiments suggest that guanosine (specifically, its N-7 endocyclic nitrogen) is the most nucleophilic position in DNA. ${ }^{91,92}$ In consequence, many of the adducts formed between alkylating agents and DNA are N-7-guanine adducts $^{93}$ and the ability to modify the N-7 site of guanosine bases has been considered evidence of genotoxicity.

The alkylation of N-7 guanine has several possible consequences: (i) Cross-linkage of two adjacent guanine residues in the case of bifunctional agents. (ii) Base mispairing; which produce base-pair substitution (usually GC $\rightarrow$ AT transitions) or strand breaks. (iii) Depurination: N-7 alkylation causes cleavage of the imidazole ring and guanine residue excision. Mutagenesis by depurination may occur by insertion or deletion. ${ }^{94}$ Thus, a reactivity model for the N-7 position of DNA such as NBP is, in fact, a model for the most reactive sites in DNA.

\subsubsection{Nucleophilicity of DNA}

Despite the complexity of DNA, large efforts have been devoted to apply the basic rules of chemical reactivity that govern electrophilicity and nucleophilicity to the reaction of DNA with alkylating agents (AA).

One of the most simple scales of nucleophilicity/electrophilicity, and by far, the one that has been applied most frequently to the reaction of DNA with alkylation agents is the one developed by Swain and Scott, in the equation of the same name. ${ }^{95}$ 
This free-energy relationship relates the pseudo first order reaction rate constant (in water at $25^{\circ} \mathrm{C}$ ), $k$, of a reaction, normalized to the reaction rate, $k_{0}$, of a standard reaction with water as the nucleophile, to a nucleophilic constant $n$ for a given nucleophile and a substrate constant $s$ that depends on the sensitivity of a substrate to nucleophilic attack (defined as 1 for methyl bromide ${ }^{96}$ Since the reaction is assumed to take place in aqueous solution, the influence of solvent is neglected.

More powerful contemporary approaches like the Ritchie equation, ${ }^{97,98}$ or the Mayr-Pratz equation ${ }^{99}$ have been applied less often to the reactions of DNA with electrophiles.

Table 1.1 reports the Swain-Scott nucleophilicity constants for some common nucleophiles.

Table 1.1. Swain-Scott nucleophilicity constants for some common nucleophiles

\begin{tabular}{|c|c|c|}
\hline Nucleophile & Formula & $n$ \\
\hline DNA $^{100}$ & & $2.5-2.6$ \\
\hline Monomeric DNA 101 & & 5.4 \\
\hline N-7-guanine 100 & & $3.5-3.6$ \\
\hline O-6-guanine ${ }^{100}$ & & $0.5-1.5$ \\
\hline DNA phosphate ${ }^{102}$ & & 1 \\
\hline -RS- and -RSH (Avg.) ${ }^{100}$ & & 5 \\
\hline Inorganic phosphate & $\mathrm{H}_{2} \mathrm{PO}_{4}^{-}$ & 2.5 \\
\hline Acetate $^{96}$ & $\mathrm{CH}_{3} \mathrm{COO}^{-}$ & 2.72 \\
\hline Thiosulphate 96 & $\mathrm{~S}_{2} \mathrm{O}_{3}^{2-}$ & 6.36 \\
\hline Water 96 & $\mathrm{H}_{2} \mathrm{O}$ & 0 \\
\hline Azide 96 & $\mathrm{~N}_{3}^{-}$ & 4 \\
\hline Hidroxide $^{96}$ & $\mathrm{HO}^{-}$ & 4.2 \\
\hline Iodide $^{96}$ & $\mathrm{I}^{-}$ & 5.04 \\
\hline Aniline ${ }^{96}$ & $\mathrm{C}_{6} \mathrm{H}_{5} \mathrm{NH}_{2}$ & 4.46 \\
\hline Pyridine ${ }^{103}$ & $\mathrm{C}_{6} \mathrm{H}_{5} \mathrm{~N}$ & 4.27 \\
\hline NBP 101 & & 3.5 \\
\hline
\end{tabular}

Inspection of the reported values rapidly shows that NBP and N-7-guanine have almost identical n values, and thus similar nucleophilicity. Pyridine and aniline have somewhat higher values.

More importantly, these values are very different from those for DNA: double-stranded DNA has much lower nucleophilicity, whereas its monomeric form has much higher values.

However, values for either single-stranded or duplex DNA are difficult to compare to those of monomers and must be taken carefully, since: i) Steric hindrance is not taken into account explicitly in the Swain-Scott equation; ii) DNA has a variety of different nucleophilic sites, with 
very different reactivity; iii) electrophiles like carbocations, which include most $\mathrm{S}_{\mathrm{N}} 1$-acting alkylating agents deviate significantly from ideal Swain-Scott behavior. ${ }^{96}$

\subsubsection{Site selectivity}

An interesting consequence of the multiplicity of reactive sites is that certain alkylating agents show particular preferences for determined nucleophilic positions: for instance, carbocations, or small diazonium ions react preferentially with oxygen atoms, whereas other electrophiles such as oxiranes react mainly with nitrogen nucleophiles. The factors governing this reactivity are a combination of steric and electronic effects. ${ }^{102,104}$

Table 1.2. Selectivity in DNA-adduct formation in terms of several approaches

\begin{tabular}{|c|c|c|c|c|c|}
\hline \multicolumn{2}{|c|}{ Swain-Scott } & \multicolumn{2}{|c|}{ Molecularity } & \multicolumn{2}{|c|}{ Hard-Soft } \\
\hline AA & Site & AA & Site & $\mathrm{AA}$ & Site \\
\hline Low $s$ & $O$ & $\mathrm{~S}_{\mathrm{N}} 1$ & $O$ & Hard & $O$ \\
\hline High $s$ & $N$ & $\mathrm{~S}_{\mathrm{N}} 2$ & $N$ & $\begin{array}{c}\text { Soft } \\
\text { Small \& diffusible }\end{array}$ & $\begin{array}{c}N \\
N-7 \text { Guo }\end{array}$ \\
\hline
\end{tabular}

Several approaches have been proposed to explain this complex behavior (Table 1.2):

- In terms of the Swain-Scott equation: Electrophiles with low s are less sensitive to the greater $n$ value of nitrogen nucleophiles, and thus react preferentially at $O$-sites. Those alkylating agents with high $s$ values are sensitive to the increased nucleophilicity and react with $N$-sites. ${ }^{79,105}$

$$
\log \left(\frac{k_{\mathrm{N}}}{k_{\mathrm{O}}}\right)=\left(n_{\mathrm{N}}-n_{\mathrm{O}}\right) s
$$

- In terms of molecularity: $\mathrm{S}_{\mathrm{N}} 1$ alkylating agents show a greater preference for $O$ nucleophilic positions, whereas $N$ (and especially $N$-7-guanine) adducts are formed via $\mathrm{S}_{\mathrm{N}} 2$ reactions. This view is disputed, and the particular behavior of some alkylating agents has been putatively explained as a yet unexplained deviation from ideal Swain-Scott behavior, rather than a fundamental difference in the alkylation mechanism. ${ }^{106}$

- In terms of the hard-soft reactivity theory, ${ }^{92}$ the observed selectivity has been explained as follows: whereas small, diffusible alkylating agents react with the $N-7$ guanosine position, the reactivities at every other nucleophilic site in DNA can be explained in terms of the soft-soft and hard-hard selectivity. ${ }^{93,106,107}$ 
None of these approaches explains wholly the fact that very different adducts are formed in the reaction of DNA with certain kind of alkylating agents. However, as regards this work, it is perhaps enough to answer the following question.

\subsubsection{To what extent is DNA modeled by NBP?}

The first, and most important, difference between DNA and NBP are their polymeric and monomeric nature, respectively. Large differences in terms of steric hindrance, accessibility and sheer nucleophilicity also exist. Thus, NBP can hardly be an accurate model for DNA as a whole. It is, however a valuable model for one particular position.

The reactive site in NBP is an aromatic amine in the pyridine ring, whose electronic configuration is quite similar to that of the N-7-Guanine (Scheme 1.2), as demonstrated by their almost equal Swain-Scott $s$ factors. ${ }^{108}$ Steric hindrance is also quite similar, both sites being rather unimpeded.

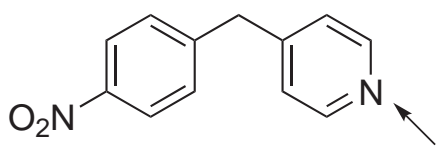

NBP<smiles>Cn1cnc2nc(N)[nH]c(=O)c21</smiles>

\section{Guanosine}

Scheme 1.2. Mechanisms of ester hydrolysis

Thus, NBP is a suitable DNA model insofar as the electrophiles modeled show high affinity for the N-7 position of guanine. That is, for small, diffusible, $\mathrm{S}_{\mathrm{N}} 2$-reacting alkylating agents, the reaction with NBP is very similar to that of DNA, and much insight into the in vivo alkylation mechanism can be obtained. ${ }^{109}$ In this case the observed reaction rate is that of the bimolecular alkylation reaction.

On the contrary, NBP is a poor model when hard, $\mathrm{S}_{\mathrm{N}} 1$-reacting electrophiles, are considered, since these react mostly at oxygen sites in vivo. Also, because the NBP test is unspecific: ${ }^{109}$ when the reaction kinetics is followed, it is the formation of the unimolecular carbocation that is in fact measured.

In conclusion, little insight can be gained from the use of NBP to model the reaction of DNA with alkylating agents that react via unimolecular cleavage, or with great affinity to other DNA centers. In these cases, a test compound with an $O$ alkylation site could be a better model for $\mathrm{O}$ alkylation. For the same reasons, aniline is expected to be a better model compound for the $N$-2 guanine or $N-6$ adenine positions. 


\subsubsection{Strengths and weaknesses of the NBP assay}

Other considerations about the (dis)advantages of the NBP test exist beyond the discussion of its sheer reactivity as a DNA-model. Aside from the ability to model the nucleophilicity of N-7 guanosine, the advantages that have encouraged the use of the NBP assay range from its simplicity (UV-Vis detection), rapidness and low costs 61,110 to its sensitivity, reproducibility ${ }^{41}$ and versatility. ${ }^{41}$ It has also been successfully coupled to in vitro metabolic activation systems.

The fact that the alkylation products can be selectively monitored by UV-Vis spectroscopy has also encouraged the use of NBP in kinetic and mechanistic studies, since the use of DNA is much costlier, and has great experimental difficulties. The NBP assay has several drawbacks that should be kept in mind:

(i) NBP is insoluble in water, and aquo-organic mixtures are required, which are far away from in vivo conditions; (ii) the NBP-AA adducts hydrolyze in basic media, which requires fast measurements, or at least for these to be made at the equal time intervals; ${ }^{25,111}$ (iii) the unspecificity hinders the use of NBP in the case of mixtures of alkylating agents; ${ }^{25,62,75-77}$ (iv) its use in acidic media is complicated by the protonation of the nucleophilic nitrogen, whose $\mathrm{p} K_{\mathrm{a}}$ must be taken into account.

The degree of hindrance -or benefit-introduced by these features depends on the specific application. For instance, in order to correlate kinetic results with in vivo activity, the electrophile under study must fulfill certain requirements (see above), which are not needed for purely analytical applications.

Several, less successful - at least in terms of usage - alternatives to NBP have been proposed, e.g. 8-quinolinol for the detection of bifunctional nitrogen mustards ${ }^{112}$ 2-6-dibromo$N$-chloro- $p$-quinone imine and tricholoro- $p$-quinone imine show selectivity for the detection of compounds containing PO and PS bonds. ${ }^{113}$ Substitute chromogens have also been used, which had lower detection limits, but better color stability. ${ }^{41}$

Fuchsin has been proposed as an alternative for the study of epoxide hydrolases, since it gives superior results for the less reactive oxiranes. ${ }^{114,115}$ When studying the stability of alkylating agents NBP is appropriate only for those with intermediate reactivity. ${ }^{40}$

A colorimetric assay base on nicotinamide has been proposed, which allows use of more biomimetic conditions, and has better color stability. ${ }^{25}$ Rate constants obtained are well correlated with those for NBP. ${ }^{37}$ 


\subsection{Uses}

\subsubsection{Detection and quantification of alkylating agents}

\subsubsection{Detection of Toxic Agents}

Since its conception, the NBP test has been applied to the detection of alkylating agents from a toxicological point of view, that is, to know whether air or water samples contain alkylating agents, independently of their nature.

Some of these uses have been proposed for warfare applications, e.g., Epstein, himself working at the Army Medical Center, quotes previous works, ${ }^{18}$ some of which are purely military. In these works the NBP test is used as a qualitative method for the detection of generic alkylating agents, firstly mustard gas, and later other alkylating agents (diethyl sulfate, butyl thiocyanate, benzene sulfonyl chloride, diphenylchloroarsine, and diethyl phosphorofluoridate).

As regards the formation of alkylating agents in foods, Archer and Eng tested how nitrosodiethylamine reacted to yield an alkylating agent in a non-enzymatic chemical activation system consisting of ascorbic acid, ferrous ions, EDTA and molecular oxygen, which was also applied to other cyclic and acyclic nitrosamines. ${ }^{61}$ Unidentified alkylating nitroso-compounds were detected in the reaction of sauerkraut, certain dairy products, wine and smoked mackerel after incubation with nitrite in simulated gastric conditions. ${ }^{116}$

The formation of mutagenic chlorination by-products was addressed in early studies, ${ }^{34,117}$ before their identification as halogenated hydroxyfuranones.

Several applications have involved organophosphorous pesticides: such as the detection of dichlorvos, trichlorfon and some derivatives have been in water, drinks and urine, ${ }^{118}$ estimating the levels of insecticide on agricultural crop foliage and in the dry mobile soil dust of groves and fields ${ }^{119}$ or validation of an oxidative treatment for alkylating agents present in residual waters. ${ }^{120}$

Very often NBP has been used a TLC-developing agent, often to detect organophosphorous pesticides ${ }^{86,89,113}$ but also aza-heterocycles, as diazinon, ${ }^{121}$ trichothecene mycotoxins, ${ }^{88}$ nitrosoureas, ${ }^{122}$ or drugs like thioTEPA. ${ }^{85,87}$

Addressing the detection of toxic phosgene, an atmospheric pollutant and industrial chemical and more importantly a chemical warfare agent, Nakano et al. developed a porous tape impregnated with a NBP solution able to detect 6 ppb of phosgene in air. ${ }^{110}$ 
The formation of alkylating metabolites of chemicals and drugs has been addressed: Guengerich used NBP to detect 2-cyanoethylene oxide, an alkylating metabolite of acrylonitrile; ${ }^{123}$ Cioli et al. tested the alkylating capacity of a putative metabolite of hycanthone, an antischistosomal drug; ${ }^{83}$ Padgette et al. detected the formation of a epoxide metabolite of 1-phenyl-1(aminomethyl)ethane hydrochloride. ${ }^{30}$

In their characterization of the oxidative activity of methane monooxygenase purified from Methylosinus trichosporium OB3b upon haloalkenes, Fox et al. applied the NBP test for the characterization of the oxidation products. Their alkylating ability helped to confirm that epoxides were formed. ${ }^{124}$ Similarly, Newman et al. used NBP to capture the short-lived epoxide formed in the oxidation of tricholoroethylene by toluene 2-monooxygenase from Burkholderia cepacia G4. ${ }^{125}$ Forkert et al. followed the formation of an epoxide from diallyl sulfone in their study of CYP2E1 inactivation. ${ }^{126,127}$

\subsubsection{Detection of Pharmacological Agents}

The NBP test has been proposed, almost since its conception, to detect antineoplasic alkylating agents present in biological tissues, in order to determine their pharmacokinetics and pharmacodynamics when administered as a treatment.

A myriad of examples exist: phenylalanine mustards in blood; Chlorambucil, Sarcochlorine, mannomustine and others in plasma and urine, ${ }^{124}$ Mannitol Myleran in urine and blood, ${ }^{42}$ bis(2-chloroethyl)methylamine in plasma, lymph, urine or tissue homogenate, ${ }^{128}$ melphalan and mechlorethamine in plasma, ${ }^{78}$ 3-[bis(2-chlorethyl)-carbohydrazide]-2,2,5,5-tetramethylpyroline1-oxyl, a spin-labeled nitrogen mustard, ${ }^{49} \ldots$

NBP has often been the method of choice for the study the metabolic fate of cyclophosphamide; ${ }^{46,129}$ and the activity of its alkylating metabolites; ${ }^{60,62,64,130}$. Also mtomycin $\mathrm{C}^{35,72-74,131}$ thioTEPA (N,N'N"'-triethylenethiophosphoramide) ${ }^{57,66,84,85,87,128,132}$ and ifosfamide ${ }^{75-77,133}$ have received much attention.

As of 2010 work is still being done on updating the NBP test; Dierickx et al. revised the method using some alkylating agents with therapeutical applications (using melphalan, $m$ sarcolysin, chlorambucil, cyclophosphamide and ifosfamide) in human serum and aqueous media. 111

\subsubsection{Indirect monitoring}

The NBP test has also been used as a purely analytical tool to study the solvolysis reactions of alkylating agents by monitoring the concentration of unreacted electrophile. 
Compounds such as bis(2-chloroethyl)methylamine (HN2) and bis(2-chloroethyl)amine (norHN2), ${ }^{58}$ 3-methyl-1-phenyltriazene and some cyclic derivatives ${ }^{24}$ or chloroethylene, ${ }^{134}$ or epoxy-starch derivatives ${ }^{135}$ have been studied.

However, the main application in this field has been the study of epoxide hydrolysis and, especially, the activity of epoxide hydrolases. ${ }^{136}$

One of the first of these NBP-based colorimetric assays was that of Rink et al., who used a microplate variation to assess the concentration of unreacted epoxide, and thus evaluate the catalytic activity. ${ }^{137}$ This method, which was based on the work of Miller and Guengerich (who themselves quote the study of chloroethyle by Barbin ${ }^{19}$ has been improved several times. ${ }^{114,115,138-140}$

These assays have been frequently applied in the study of epoxide hydrolases obtained from a variety of sources, such as Streptomyces, Streptomyces Sphingomonas echinoides, ${ }^{141}$ phanerochaete chrysosporium, ${ }^{142,143}$ Cupriavidus metallidurans-CH34 ${ }^{144}$ and also to study the oxidative activity of cytochromes ${ }^{145}$ or styrene monooxygenase. ${ }^{146}$

Some alternatives to the use of NBP have been proposed, which sometimes are also based on chromogenic reactions, such as that of $p$-nitrothiophenolate. ${ }^{147}$

\subsubsection{Characterization of alkylating agents}

Many mutagenic agents afford positive results in the NBP test (epoxides, lactones, N-nitrosocompounds, ethyleneimines, halogenated compounds, alkyl sulfonic esters...) whereas many non-mutagens or non-carcinogens show no response. ${ }^{43}$

Thus, NBP was proposed as a reagent for the search of quantitative, semi-quantitative or simply qualitative correlations between alkylating activity and biological effects (carcinogenicity, mutagenic potential, toxicity...) or as alert system to assay new compounds whose action in vivo are unknown.

These applications can be divided into two large groups: those that measure the absorbance at a single, fixed time, and those that monitor the alkylation reaction to obtain kinetic parameters.

\subsubsection{Non-kinetic applications}

In non-kinetic applications, the absorbance of an alkylating-agent NBP mixture in an appropriate solvent is measured (after basification) when a certain reaction time has elapsed. The absorbance at the wavelength of measurement is taken as the quantitative response. These 
absorbance values are a measurement of the reactivity of an alkylating agent, and have been correlated, more or less successfully, with biological properties, such as toxicity, mutagenicity, carcinogenicity and antitumoral activity.

1.3.2.1.1. Mutagenicity and chemical reactivity. The most common mutagenicity assays, the bacterial reverse mutation tests (Ames test) use amino-acid-requiring strains of Salmonella typhimurium and Escherichia coli to detect point mutations, which involve substitution (strains TA100, TA1535), addition or deletion of one or several DNA base pairs (TA98, TA1536, TA1537, TA1538). Acceptable qualitative correlation between results in the non-kinetic NBP test and mutagenicity in several test systems have been found (Table 1.3).

Other works have found semiquantitative correlations: mutagenic and alkylating potential followed the same order for: some epoxycyclohexanes ${ }^{33}$ and a series of three 1,2-dibromo alcanes and the compounds resulting from their conjugation with GSH. ${ }^{51}$

More importantly, quantitative correlations between NBP alkylating activity and mutagenicity have also been found (Figure 1.1). These generally hold well within groups of related chemicals, such as haloallyl compounds, 44,45,150 epoxides 23,27,33,37 nitrosocarbamates, nitrosoureas ${ }^{151}$ and other compounds. ${ }^{48}$ In addition, it must be taken into account that usually, equal absorption coefficients are assumed for all the alkylating agents, since it is absorbance - and not concentration of adduct - being measured. This assumption may hold well within classes of compounds, and even then, excluding those that include substituents like carbonyl or diazo groups and conjugated $\pi$ systems like allyl or aryl groups.
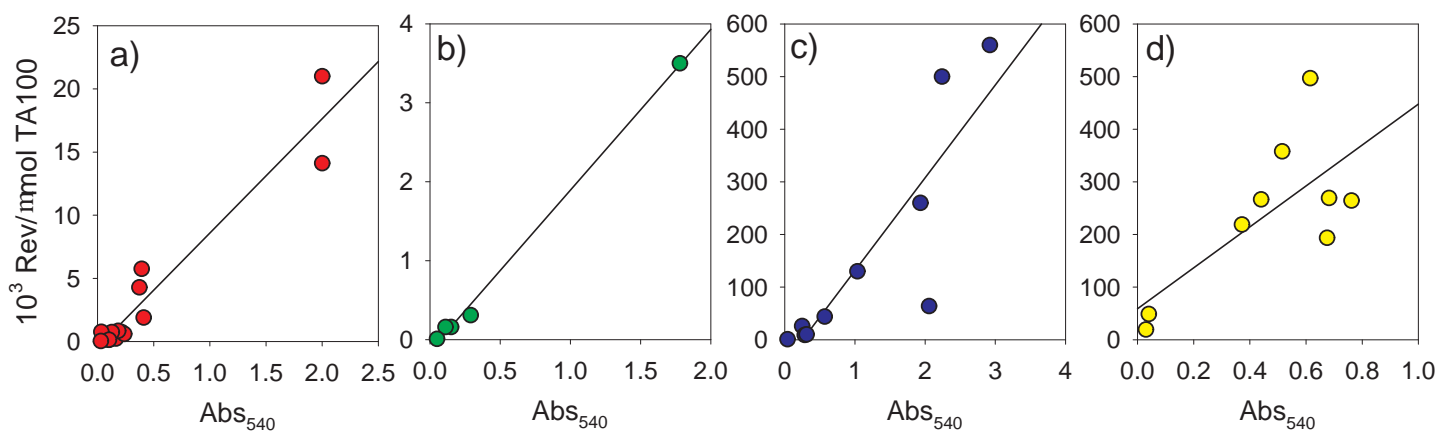

Figure 1.1. Mutagenicity - NBP correlation for some alkylating agents. a) Propyelene oxides ${ }^{37}$ b) Epoxides ${ }^{23}$ c) Haloallyl compounds ${ }^{150}$ d) Nitrosoureas ${ }^{151}$

Inter-group correlation, however, is often poor ${ }^{23,152}$ and discrepancies in response among different compound classes have been obtained. ${ }^{28,32,53,149,153-155}$ Several explanations for this 
Table 1.3. Correlation between non-kinetic NBP assay and mutagenicity.

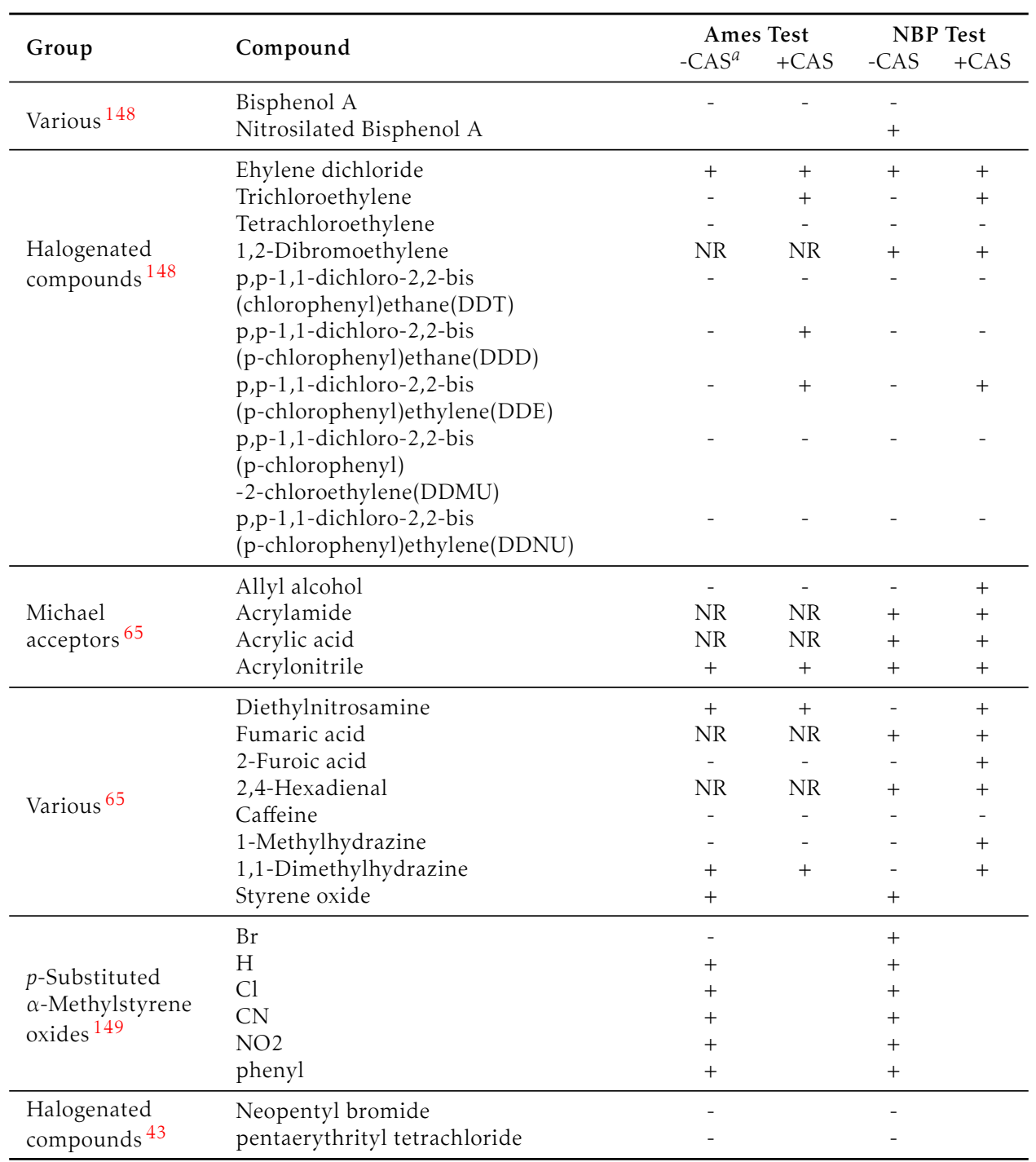

\footnotetext{
${ }^{a}$ Chemical activation system
} 
lack of consistency have been attempted: (i) a higher competition by solvolysis in the case of more reactive compounds. ${ }^{156}$ (ii) Differences in selectivity towards $N$ and $O$ nucleophiles ${ }^{116,157}$ (iii) Differences in the mutagenic events arising from $O-6$ and $N-7$ lesions. ${ }^{156}$ (iv) The mutagenic test system used was not appropriate. ${ }^{53}(\mathrm{v})$ Interference by other reactions, e.g. in the case of $N$-methyl- $N^{\prime}$-alkyl-N-nitrosoureas, the effective alkylating agent is formed in the decomposition of the nitrosouresas. ${ }^{158}$ (vi) Mutagenicity is influenced more intensely than alkylating activity, by properties such as polarity, ${ }^{32}$ molecular volume ${ }^{149}$ or the degree of unsaturation in the case of aryloxides ${ }^{154}$ or glycidyl oxides. ${ }^{153}$

1.3.2.1.2. Carcinogenicity and chemical reactivity. Whereas chemical carcinogens exert their effects by many different mechanisms, the NBP test has also been found to be a good predictor of carcinogenicity (for alkylating agents) as shown in Table 1.4.

Table 1.4. Correlation between non-kinetic NBP assay and carcinogenicty 65

\begin{tabular}{|c|c|c|c|}
\hline \multirow{2}{*}{ Compound } & \multirow{2}{*}{ Carcinogenicity } & \multicolumn{2}{|c|}{ Alkylating activity } \\
\hline & & $+\mathrm{CAS}$ & -CAS \\
\hline Trichloroethylene & + & - & + \\
\hline Tetrachloroethylene & - & - & - \\
\hline 1,2-Dibromoethylene & NR & + & + \\
\hline DDT & + & - & - \\
\hline DDD & + & - & - \\
\hline DDE & + & - & + \\
\hline DDMU & NR & - & - \\
\hline DDNU & NR & - & - \\
\hline Allyl alcohol & NR & - & + \\
\hline Acrylamide & + & + & + \\
\hline Acrylic acid & NR & + & + \\
\hline Acrylonitrile & - & + & + \\
\hline Diethylnitrosamine & + & - & + \\
\hline Fumaric acid & NR & + & + \\
\hline 2-Furoic acid & - & - & + \\
\hline 2,4-Hexadienal & NR & + & + \\
\hline Caffeine & - & - & - \\
\hline 1-Methylhydrazine & + & - & + \\
\hline 1,1-Dimethylhydrazine & + & - & + \\
\hline
\end{tabular}

1.3.2.1.3. Other attempted correlations. Poor correlation was found between toxicity in mice and leukemic L1210 cells for a series of $N$-nitrosoureas, ${ }^{71}$ whereas the agreement between carcinogenicity, mutagenicity and skin sensitization by very different alkylating agents suggested that the three processes share a common mechanism. ${ }^{159}$

Many alkylating agents have found use as antitumoral agents, since cancer cells have a 
high division rate, and thus their DNA is more exposed, as compared to healthy tissue. Thus, it would seem that alkylating ability would be a good predictor for antitumoral properties, which, however is seldom the case. ${ }^{55,69,70,160,161}$

The non-kinetic NBP assay has also been used for the characterization of the in vivo mechanism of action of chemicals like alkylhydrazones, which are autooxidized to alkylating agents; ${ }^{80}$ atmospheric electrophiles; ${ }^{162}$ or [ $\left.{ }^{18} \mathrm{~F}\right]$-labeled benzylfluoride ${ }^{47}$ and drugs like transferrin- and albumin conjugates of the clinical alkylating agent chlorambucil ${ }^{163,164}$ or bifunctional alkylating agents. ${ }^{39}$

\subsubsection{Kinetics of alkylation - alkylation mechanism}

The first efforts to correlate biological effects such antitumor activity, toxicity ${ }^{20}$ or tumor incidence $^{165}$ with kinetic parameters, used common nucleophiles such as water ${ }^{20}$ thiosulfate or azide, ${ }^{166}$ and especially NBP.
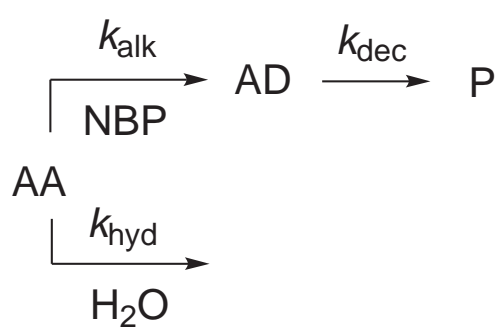

Scheme 1.3. Reactions involved in the NBP alkylation mechanism

A general mechanism for the alkylation of NBP by a generic alkylating agent can be summarized as in Scheme 1.3 the alkylating agent reacts with NBP, affording a labile adduct which, in turn, decomposes. Simultaneously, the alkylating agent undergoes solvolysis (in most cases hydrolysis and also alcoholysis in aquo-acoholic mixtures, ${ }^{103}$ and possibly other deactivation processes like isomerization.

The differential rate equations derived from such a mechanism, when the reaction proceeds through a $\mathrm{S}_{\mathrm{N}} 2$ mechanism are

$$
\begin{aligned}
& \frac{d[\mathrm{AA}]}{d t}=-k_{\mathrm{alk}}[\mathrm{NBP}][\mathrm{AA}]-k_{\mathrm{hyd}}\left[\mathrm{H}_{2} \mathrm{O}\right][\mathrm{AA}] \\
& \frac{d[\mathrm{AD}]}{d t}=k_{\mathrm{alk}}[\mathrm{NBP}][\mathrm{AA}]-k_{\mathrm{dec}}[\mathrm{AD}]
\end{aligned}
$$

And their integrated counterparts are 


$$
\begin{aligned}
& {[\mathrm{AA}]=[\mathrm{AA}]_{\mathrm{o}} e^{-\left(k_{\mathrm{alk}}[\mathrm{NBP}]+k_{\mathrm{hyd}}\left[\mathrm{H}_{2} \mathrm{O}\right]\right) t}} \\
& {[\mathrm{AD}]=[\mathrm{AA}]_{\mathrm{O}} \frac{k_{\mathrm{alk}}[\mathrm{NBP}]}{k_{\mathrm{dec}}-k_{\mathrm{alk}}[\mathrm{NBP}]-k_{\mathrm{hyd}}\left[\mathrm{H}_{2} \mathrm{O}\right]}\left(e^{-\left(k_{\mathrm{alk}}[\mathrm{NBP}]+k_{\mathrm{hyd}}\left[\mathrm{H}_{2} \mathrm{O}\right]\right) t}-e^{-k_{\mathrm{dec}} t}\right)}
\end{aligned}
$$

Depending on the rates of the three processes, three different approaches to the discussion of the kinetic NBP test exist: considering only the alkylation reaction, including also the parallel hydrolysis, or taking into account the three processes simultaneously. The choice of the appropriate approach is of importance, and abundant instances of poor mechanistic perspective in the kinetic application of the NBP test exist.

For instance, the competing hydrolysis and isomerization reactions have been often completely overlooked in the study of epoxides, especially those of polycyclic aromatic hydrocarbons. ${ }^{21,22,54,167-169}$ Such assumptions are not unique to oxiranes, and they have also taken place in the study of nitrogen mustards and aziridines, ${ }^{20}$ chloroethylene, ${ }^{19}$ cyclophosphamide, ${ }^{170}$ 3-methyl-1-phenyltriazenes, ${ }^{24} S$-(2-haloethyl)-1-cysteine analogs, ${ }^{68}$ antineoplasic agents, ${ }^{171}$ haloacetonitriles, ${ }^{172} \mathrm{~N}$-methyl-N'-aryl-N-nitrosoureas, ${ }^{155}$ phosphoraziridines. ${ }^{31}$

The NBP test has obvious limitations, which have been discussed before: lack of selectivity and applicability only to $\mathrm{S}_{\mathrm{N}} 2$ alkylating agents, in addition to the fact that correlating results of the NBP test with in vivo results is sometimes oversimplistic and neglects important processes such as metabolism or DNA-repair systems. However, the results obtained in the kinetic NBP test are often the product of an improper kinetic approach, before anything else. Hence, the presence or lack of in vivo - in vitro - NBP correlations in these faulty kinetic studies must be taken cautiously.

In the next sections, the different mechanistic approximations to the kinetic NBP test will be discussed.

1.3.2.2.1. Only alkylation. If the hydrolysis of the alkylating agent is negligible and the adduct is reasonably stable in the reaction conditions; then, only the rate of alkylation is of importance, and rate equation simplifies to:

$$
[\mathrm{AD}]_{t}=[\mathrm{AA}]_{\mathrm{O}}\left(1-e^{-k_{\mathrm{alk}}[\mathrm{NBP}] t}\right)
$$

In this case, the alkylating potential of the species can be correlated directly with its alkylation rate constant: the faster the alkylation takes place, the less time the organism has to excrete or deactivate the alkylating agent, and the more intense the effects. On the other hand, 
if no alkylation is observed in vitro, or it is extremely slow, it can be concluded that the compound lacks significant alkylating capacity. It should be noted that this conclusion only applies to the tested compound itself; if the effective alkylating agent is formed during the metabolism of the studied compound, the NBP test is useless unless it is carried out in simulated metabolic conditions.

This null reactivity with NBP has been correlated with lack of mutagenicity: neopentyl bromide and pentaerythrityl tetrachloride ${ }^{43}$ or DDNU-oxide, a presumed metabolite of DDT which lacks mutagenic activity. ${ }^{26}$ Also, it has helped understand the small alkylating potential attributed to weak electrophiles as sorbic acid ${ }^{8}$ and sorbates, ${ }^{2}$ and acrylamide ${ }^{13}$ and other $\alpha$ - $\beta$ unsaturated carbonyls. All these compounds are stable in aqueous solution within the length of the experiment, and react very slowly, if at all, with NBP. This low reactivity is agreement with the low -or null-in vivo activity of these compounds.

Occasionally, the assumption of the hydrolysis reaction being negligible is quite arbitrary. For instance Hooberman et al. found good correlation between mutagenicitiy and NBP alkylation rate in a study of seventeen propylene oxides. They attributed some of the deviations to the epoxide decomposition, which they failed to include. ${ }^{37}$ Also failing to acknowledge the hydrolysis reaction, Hemminki et al. compared the alkylation of DNA, desoxyguanosine and NBP by linear epoxides. Better correlation was found for the biological models, which was attributed to the fact that the NBP test was carried in harsh, non-biomimetic conditions. ${ }^{173}$

Styrene oxide derivatives are possibly the most studied oxiranes, for instance the alkylation rate of styrene oxide derivatives - once again, neglecting hydrolysis and variations in the molar absorptivity - has been used to discuss the influence of the site of nucleophilic attack. ${ }^{149}$

Many works have obtained solid relationships between kinetic parameters and biological effects. For instance, a quantitative relationship was found between alkylation rate and ability to induce point mutation, mitotic gene conversion and recombination in a diploid strain (D7)

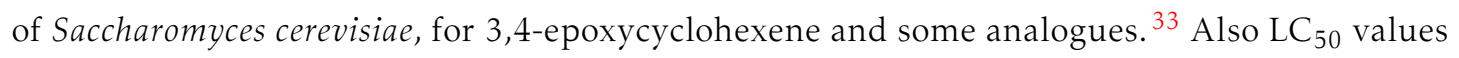
of different epoxy compounds using Quantitative Structure Activity Relationship based on the alkylation rate constant and the hydrophobicity. ${ }^{29} \mathrm{~N}$-alkyl-N-nitrosoureas decompose in water to form diazonium ions, the effective alkylating agents. Benzenediazonium ion reacts with NBP about twenty thousand fold more slowly than methyldiazonium, which correlates with the fact that the methyldiazonium ion shows higher carcinogenicity in animal tests. ${ }^{6}$

Mechanistic conclusions have also been obtained from this approach to the kinetic NBP assay. The relative hydrolysis and alkylation rate of $N$-diazoacetyl derivatives of aminoacids suggest that degradation is a pre-requisite for alkylation. ${ }^{67}$ Peterson et al. concluded, partly from the alkylation and hydrolysis rates of some cysteinyl and homocysteinyl analogues of $S$-(2-haloethyl)glutathione (a carcinogenic metabolic product of ethylene dibromide), that the 
ethylene dibromide-glutathione conjugate forms an episulfonium ion prior to reaction with DNA guanyl residues. ${ }^{174}$ Meier et al. studied the formation of alkylating agents in the nitrosation of some amino acids (aspartic acid, aspartame and glycine ethylester) ${ }^{108}$ and GarcíaSantos et al. demonstrated that these alkylating agents formed in the nitrosation of amino acids are in fact lactones. ${ }^{175}$

The use of the Initial Rate Method ${ }^{176}$ (IRM) allows researchers to neglect both concurring hydrolysis and subsequent adduct decomposition, even in cases where these are quite rapid. Thus, it is a useful approach for the calculation of alkylation rate constants. However, knowledge of the adduct absorption coefficient is essential to calculate the alkylation rate constants.

$$
r_{\mathrm{O}}=\varepsilon l k_{\mathrm{alk}}[\mathrm{AA}]_{\mathrm{O}}[\mathrm{NBP}]_{\mathrm{O}}
$$

As in the non-kinetic approaches, very often the same molar absorption coefficient is used for all the compounds in a study, but this can produce misleading results.

The kinetic NBP test has been most often applied to epoxides, and it is thus unsurprising that most examples of application of the IRM, (and most usually the concomitant approximation regarding the molar extinction coefficients) are found in the study of alkylation by oxiranes. Good correlation has been found between alkylation rate and mutagenicity of trichloropropylene oxide, epichlorohydrin, styrene oxide, glycidol and propylene oxide; ${ }^{23} 4$ vinylcyclohexene metabolites ${ }^{27}$ or oxirane and vinyl oxirane. ${ }^{50}$

Allyl compounds have also received attention: Schiffmann et al. found correlation between unscheduled DNA synthesis, mutagenicity in the Ames test and alkylation of NBP ${ }^{177}$ and Eder et al. found mutagenicity-alkylation rate correlation for six allylic alkylating agents ${ }^{44}$ (allyl bromide, 1,3-dichloropropene, 1-chloro-2-butene, 3-chloro-2-methyl-1-propene, allyl chloride and 2,3-dichloro-1-propene). The authors also concluded that the kinetic variation does not add significantly to the standard NBP test. Indeed, the use of a kinetic approach without a mechanistic interpretation of the results makes the kinetic data of little use.

Other systems for which reasonable rate-activity relationships have been found include organophosphate impurities of commercial malathion; ${ }^{53}$ pyrrolizidine alkaloid pyrroles ${ }^{178}$ and their metabolites; ${ }^{179}$ acridine-linked aniline mustards, ${ }^{36}$ whose alkylation rate constant also correlates well with the ${ }^{15} \mathrm{~N}-\mathrm{NMR}$ shifts; ${ }^{134}$ or quaternary ammonium derivatives of chlorambucil and melphalan. ${ }^{180}$

1.3.2.2.2. Competing hydrolysis. Since alkylating agents are potent electrophiles, they often undergo hydrolysis in aqueous medium, and thus a parallel reaction must be considered. The rate equation in this case is 


$$
[\mathrm{AD}]_{t}=[\mathrm{AA}]_{\mathrm{o}} \frac{k_{\mathrm{alk}}[\mathrm{NBP}]}{k_{\mathrm{alk}}[\mathrm{NBP}]+k_{\mathrm{hyd}}\left[\mathrm{H}_{2} \mathrm{O}\right]}\left(1-e^{-\left(k_{\mathrm{alk}}[\mathrm{NBP}]+k_{\mathrm{hyd}}\left[\mathrm{H}_{2} \mathrm{O}\right]\right) t}\right)
$$

Therefore, the experimentally observed rate constant is the sum of both the pseudo-firstorder hydrolysis and alkylation rate constants.

$$
k_{\mathrm{obs}}=k_{\mathrm{alk}}[\mathrm{NBP}]+k_{\mathrm{hyd}}\left[\mathrm{H}_{2} \mathrm{O}\right]
$$

From a kinetic point of view, the selectivity towards NBP, or alkylating efficiency can be expressed as the quotient between the alkylation rate constant and the hydrolysis constant:

$$
S_{\mathrm{NBP}}=\frac{k_{\mathrm{alk}}}{k_{\mathrm{hyd}}}
$$

The ratio between the alkylation product and the hydrolysis product, even for a $\mathrm{S}_{\mathrm{N}} 1$ reacting agent, can be considered a selectivity factor not necessarily derived from measurable kinetic rate constants. Another useful parameter, which depends on the concentration of NBP, is the fraction of the alkylating agent that finally forms adduct $(f)$ :

$$
f=\frac{[\mathrm{AD}]}{[\mathrm{AA}]_{\mathrm{O}}}=\frac{k_{\mathrm{alk}}[\mathrm{NBP}]}{k_{\mathrm{alk}}[\mathrm{NBP}]+k_{\text {hyd }}\left[\mathrm{H}_{2} \mathrm{O}\right]}
$$

$S_{\mathrm{NBP}}$ and $f$ are closely related since:

$$
\frac{1}{f}=1+\frac{1}{S_{\mathrm{NBP}}} \frac{\left[\mathrm{H}_{2} \mathrm{O}\right]}{[\mathrm{NBP}]}
$$

Kawazoe et al. synthesized some N-Alkyl-4-( $p$-nitrobenzyl) pyridonium salts and making used of their absorption coefficients calculated the $S_{\mathrm{NBP}}$ values for a number of $S_{N} 1$ and $S_{N} 2$ alkylating agents. The found that $\log \left(S_{\mathrm{NBP}}\right)$ ranged from 4.3 to $0.7(f=0.99-0.05)$ for some alkyl halides and were as low as $-1\left(f=10^{-3}\right)$ for some nitrosoureas and nitrosoguanidines. ${ }^{82}$ The intermediate-low values indicate a large amount of competing hydrolysis (diethylsulfate, ethyl methanesulfonate, pentyl methanesulfonate...), whereas the very low values obtained for some compounds suggest that they act through $\mathrm{S}_{\mathrm{N}} 1$ mechanisms.

Ninomiya et al. also calculated the $S_{\text {NBP }}$ values for a number of alkyl sulfonates $(1.0<$ $\left.\log \left(S_{\mathrm{NBP}}\right)<4.3\right)$. They observed that chemoselectivity depends strongly on the nature of alkoxy group: the bulkier the substituent (and thus the larger the $\mathrm{S}_{\mathrm{N}} 1$-character), the smaller the selectivity. The order of selectivity towards NBP for the compounds studied is then methyl $>$ ethyl $>$ isopropyl. The nature of the leaving group $\left(\mathrm{R}_{1} \mathrm{SO}_{3}^{-}\right)$is of less importance, although 
electron deficient sulfonic groups tends to decrease NBP selectivity. ${ }^{81}$ In terms of rate constant the order of reactivity is methyl > isopropyl > ethyl, the increase of reactivity of the isopropyl group with respect to the ethyl group can be attributed to the $S_{N} 1$ reaction path.

Kang and Spears characterized the reactivity of some selenium mustards by studying their alkylation rate and competitive hydrolysis, finding a very similar reactivity to nitrogen mustards, and pointed out their possible use as drugs. ${ }^{79}$

Values of either $S_{\text {NBP }}$ or $f$ have been used to correlate reactivity against NBP and carcinogenicity/mutagenicity: It was observed that the doses needed to equate the carcinogenic effects of $\beta$-propiolactone (BPL) and $\beta$-butyrolactone (BBL) - which are considered as possibly carcinogenic to humans (IARC 2A) - are well correlated with both their alkylation rate constants and their $S_{\mathrm{NBP}}$ values. ${ }^{1}$ Since the competing hydrolysis reaction is significant, but not major, similar results were obtained by Hemminki, who neglected the parallel hydrolysis reaction. ${ }^{152}$

Table 1.5. Correlation of Tumorigenicity and NBP Alkylation kinetics for $\beta$-lactones.

\begin{tabular}{|c|c|c|c|c|c|c|}
\hline \multirow{3}{*}{ Lactone } & \multirow{2}{*}{\multicolumn{2}{|c|}{ NBP assay }} & \multicolumn{4}{|c|}{ Tumorigenicity } \\
\hline & & & \multicolumn{2}{|c|}{ Mice } & \multicolumn{2}{|c|}{ Rats } \\
\hline & $\begin{array}{c}10 k_{\mathrm{alk}} \\
\left(\mathrm{M}^{-1} \mathrm{~min}^{-1}\right)\end{array}$ & $S_{\mathrm{NBP}}$ & $\begin{array}{l}\text { dose } \\
(\mathrm{mg})\end{array}$ & $\begin{array}{l}\text { tumors / } \\
\text { animals }\end{array}$ & $\begin{array}{l}\text { dose } \\
(\mathrm{mg})\end{array}$ & $\begin{array}{l}\text { tumors / } \\
\text { animals }\end{array}$ \\
\hline $\mathrm{BPL}$ & 8.2 & 5000 & 4 & $13 / 20$ & 0.7 & $18 / 30$ \\
\hline $\mathrm{BBL}$ & 0.8 & 3200 & 100 & $9 / 20$ & 10 & $18 / 30$ \\
\hline
\end{tabular}

As regards $N$-nitrosoureas, which decompose to form alkylating diazonium ions, it has been observed that their $f$ and $S_{\mathrm{NBP}}$ values correlate with their tumorigenicity in animal tests. ${ }^{6}$

1,4-Dinitro-2-methylpyrrole (NMP), a product of the reaction between nitrite and sorbic acid, both of which are common food additives, is a proven mutagen. An experimental study of the NBP alkylation mechanism, together with a theoretical study of its reactivity have shown that the effective alkylating agent is 5-methyl-3-nitro-2H-pyrrol-2-ol, a decomposition product formed after nucleophilic attack of a hydroxide ion, loss of nitrite and subsequent isomerization. The rather low $f$ value obtained $\left(0.07 ; S_{\mathrm{NBP}}=155\right.$ at $37.5^{\circ} \mathrm{C}$ in $7: 3$ water:dioxane medium. $\left.[\mathrm{NBP}]_{\mathrm{o}}=0.02 \mathrm{M}\right)$ is coherent with the low mutagenicity of the compound. ${ }^{14}$

1.3.2.2.3. Adduct decomposition. In the more complex cases, the adducts formed are unstable and undergo hydrolysis in the reaction medium. This reactivity explains the disappearance of the color for some cromophores and their increased stability in non-aqueous media. In general, this decomposition reaction has been observed to undergo general base 
catalysis by hydroxide, acetate, phosphate, and even NBP itself. ${ }^{111}$ In these cases, the alkylation efficiency does not include all possible effects and a new parameter can be introduced to take into account the decomposition of the NBP-AA adduct: the adduct life (AL).

$$
\begin{gathered}
\mathrm{AL}=\frac{\int_{0}^{\infty}[\mathrm{AD}] d t}{[\mathrm{AA}]_{\mathrm{O}}}=\int_{0}^{\infty} \frac{k_{\mathrm{alk}}[\mathrm{NBP}]}{k_{\mathrm{alk}}[\mathrm{NBP}]+k_{\mathrm{hyd}}\left[\mathrm{H}_{2} \mathrm{O}\right]-k_{\mathrm{dec}}}\left(e^{-\left(k_{\mathrm{alk}}[\mathrm{NBP}]+k_{\mathrm{hyd}}\left[\mathrm{H}_{2} \mathrm{O}\right]\right) t}-e^{-k_{\mathrm{dec}} t}\right) \\
\mathrm{AL}=\frac{k_{\mathrm{alk}}[\mathrm{NBP}]}{\left(k_{\mathrm{alk}}[\mathrm{NBP}]+k_{\mathrm{hyd}}\left[\mathrm{H}_{2} \mathrm{O}\right]\right) k_{\mathrm{dec}}}
\end{gathered}
$$

$\mathrm{AL}$ is defined as the area under the kinetic profile of the reaction, per unit of alkylating agent.

Since it includes all possible effectors (the alkylation rate, the rate of the competing hydrolysis and the adduct stability) this parameter gives an idea of the cumulative effect of an alkylating agent, combining the concentration and time factors. A higher AL implies a combination of more adduct being formed, and this having a longer life, and thus more time to exert its biological effect. AL is related to $f$, since

$$
\mathrm{AL}=\frac{f}{k_{\mathrm{dec}}}
$$

Quite intuitively, AL depends directly on $f$ : a greater selectivity for NBP implies a larger concentration of adduct, and thus a larger cumulative effect. In addition, the faster the adduct

\begin{tabular}{|c|c|c|c|}
\hline Molecule & $\log \left(\mathrm{S}_{\mathrm{NBP}}\right)$ & $f$ & $\mathrm{AL}(\min )$ \\
\hline Ethylnitrolic acid ${ }^{11}$ & 2.5 & 0.13 & 7.3 \\
\hline$p$-Nitrostyrene oxide ${ }^{15}$ & 2.4 & 0.82 & 3300 \\
\hline
\end{tabular}
decomposition, the lower AL.

Table 1.6. Alkylating Agents that form Unstable Adducts

Ethylnitrolic acid ${ }^{7,12}$ (ENA), also a product of the reaction between nitrite and sorbic acid, is a proven mutagen. ENA decomposes in aqueous medium, which, together with the presence of an induction period in the reaction between ENA and NBP suggests that the active alkylating agent is a decomposition product of ENA. Ethylnitrolic acid is transformed in aqueous media into acetonitrile oxide, the active species that alkylates NBP. The adduct formed is rather shortlived and both the low $f$ and AL values are consistent with its low mutagenicity (Table 1.6). ${ }^{11}$ 
The alkylating potential of $\alpha$-angelicalactone (AAL), a $\gamma$-lactone with chemopreventive activity has also been discussed on the basis of its alkylation rate and adduct life. ${ }^{3}$ The extremely high value of $k_{\text {alk }}$ (the alkylation reaction lasting less than a second) suggests that $\alpha$-angelicalactone might react rapidly with the most exposed positions, protecting them from other alkylating agents. The short life of the AAL-DNA adducts would then prevent $\alpha$-angelicalactone from causing permanent DNA damage.

p-Nitrostyrene oxide (pNSO), a mutagenic compound used as a model for epoxide hydrolase activity due to its slow spontaneous hydrolysis, was also investigated using the NBP test. Although styrene oxides react generally through both $\alpha$ and $\beta$ carbons of the oxirane ring, $\alpha$ adduct formation is negligible for pNSO. The hydrolysis reaction of the epoxide competes with the formation of the unstable $\beta$ adduct, whose decomposition is also relevant. ${ }^{15}$ The high $f(0.82)$ and the low AL (3300 min) values indicate that it is a strong alkylating agent with a low alkylating effectiveness. This is in accordance with the lower mutagenicity of pNSO in comparison with BPL, which has a similar $f$ value but it forms a stable adduct. 


\section{References}

[1] Manso, J. A.; Pérez-Prior, M. T.; García-Santos, M. P.; Calle, E.; Casado, J. Chem. Res. Toxicol. 2005, $18,1161-1166$.

[2] Pérez-Prior, M. T.; Manso, J. A.; García-Santos, M. D.; Calle, E.; Casado, J. J. Agric. Food Chem. 2005, $53,10244-10247$.

[3] Fernández-Rodríguez, E.; Manso, J. A.; Pérez-Prior, M. T.; García-Santos, M. D. P.; Calle, E.; Casado, J. Int. J. Chem. Kinet. 2007, 39, 591-594.

[4] Manso, J. A. Estudio cinético del potencial alquilante de lactonas y N-alquil-N-nitrosoureas. Ph.D. thesis, Universidad de Salamanca, Dpto Química física, 2007.

[5] Manso, J. A.; Pérez-Prior, M. T.; García-Santos, M. d. P.; Calle, E.; Casado, J. J. Solution Chem. 2008, $37,451-457$.

[6] Manso, J. A.; Pérez-Prior, M. T.; García-Santos, M. P.; Calle, E.; Casado, J. J. Phys. Org. Chem. 2008, $21,932-938$.

[7] Pérez-Prior, M. T., M Trez-Prior; Manso, J. A.; Gómez-Bombarelli, R.; González-Pérez, M.; GarcíaSantos, M. P.; Calle, E.; Caballero, M. C.; Caballero, M. C.; Casado, J. J. Agric. Food Chem. 2008, 56, 11824-11829.

[8] Pérez-Prior, M. T.; Manso, J. A.; García-Santos, M. P.; Calle, E.; Casado, J. J. Solution Chem. 2008, $37,459-466$.

[9] Manso, J. A.; Pérez-Prior, M. T.; Gómez-Bombarelli, R.; González-Pérez, M.; Céspedes, I. F.; GarcíaSantos, M. P.; Calle, E.; Casado, J. J. Phys. Org. Chem. 2009, 22, 386-389.

[10] Pérez-Prior, M. Estudio cinético del potencial alquilante del ácido sórbico y de los productos de su reacción con el nitrito. Ph.D. thesis, Dpto Química física; Facultad de Ciencias Químicas, Universidad de Salamanca, 2009.

[11] Pérez-Prior, M. T.; Gómez-Bombarelli, R.; González-Pérez, M.; Manso, J. A.; García-Santos, M. P.; Calle, E.; Casado, J. Chem. Res. Toxicol. 2009, 22, 1320-1324.

[12] Pérez-Prior, M. T.; Manso, J. A.; Gómez-Bombarelli, R.; González-Pérez, M.; Céspedes, I. F.; GarcíaSantos, M. P.; Calle, E.; Casado, J. J. Phys. Org. Chem. 2009, 22, 418-424.

[13] Céspedes, I. F.; Manso, J. A.; Pérez-Prior, M. T.; Gómez-Bombarelli, R.; González-Pérez, M.; Calle, E.; Casado, J. J. Phys. Org. Chem. 2010, 23, 171-175.

[14] Pérez-Prior, M. T.; Gómez-Bombarelli, R.; González-Pérez, M.; Manso, J. A.; García-Santos, M. P.; Calle, E.; Casado, J. J. Org. Chem. 2010, 75, 1444-1449.

[15] González-Pérez, M.; Gómez-Bombarelli, R.; Pérez-Prior, M. T.; Manso, J. A.; CespedesCamacho, I. F.; Calle, E.; Casado, J. Org. Biomol. Chem. 2011, 9, 7016-7022.

[16] Manso, J. A.; Cespedes Camacho, I. F.; Calle, E.; Casado, J. Org. Biomol. Chem. 2011, 9, 6226-6233.

[17] Koenigs, E.; Köhler, K.; Blindow, K. Berichte der deutschen chemischen Gesellschaft ( $A$ and B Series) $1925,58,933-940$.

[18] Epstein, J.; Rosenthal, R. W.; Ess, R. J. Anal. Chem. 1955, 27, 1435-1439. 
[19] Barbin, A.; Bresil, H.; Croisy, A.; Jacquignon, P.; Malaveille, C.; Montesano, R.; Bartsch, H. Biochem. Biophys. Res. Commun. 1975, 67, 596-603.

[20] Bardos, T. J.; Datta-Gupts, N.; Hebborn, P.; Triggle, D. J. J. Med. Chem. 1965, 8, 167-174.

[21] Sims, P. Biochem. J. 1973, 131, 405-413.

[22] Swaisland, A. J.; Grover, P. L.; Sims, P. Biochem. Pharmacol. 1973, 22, 1547-1556.

[23] Hemminki, K.; Falck, K. Toxicol. Lett. 1979, 4, 103-106.

[24] Malaveille, C.; Brun, G.; Kolar, G.; Bartsch, H. Cancer Res. 1982, 42, 1446-1453.

[25] Nelis, H. J. C. F.; Airy, S. C.; Sinsheimer, J. E. Anal. Chem. 1982, 54, 213-216.

[26] Planche, G.; Croisy, A.; Malaveille, C.; Tomatis, L.; Bartsch, H. Chem.-Biol. Interact. 1979, 25, 157175.

[27] Turchi, G.; Bonatti, S.; Citti, L.; Gervasi, P. G.; Abbondandolo, A.; Presciuttini, S. Mutat. Res. 1981, 83, 419-430.

[28] Bartsch, H.; Terracini, B.; Malaveille, C.; Tomatis, L.; Wahrendorf, J.; Brun, G.; Dodet, B. Mutat. Res.-Fund. Mol. M. 1983, 110, 181-219.

[29] Deneer, J. W.; Sinnige, T. L.; Seinen, W.; Hermens, J. L. M. Aquat. Toxicol. 1988, 13, 195-204.

[30] Padgette, S. R.; Wimalasena, K.; Herman, H. H.; Sirimanne, S. R.; May, S. W. Biochemistry 1985, 24, 5826-5839.

[31] Perlman, M. E.; Bardos, T. J. J. Org. Chem. 1988, 53, 1761-1767.

[32] Sinsheimer, J. E.; Chakraborty, P. K.; Messerly, E. A.; Gaddamidi, V. Mutat. Res.-Genet. Tox. 1989, $224,171-175$.

[33] Turchi, G.; Bauer, C.; Bronzetti, G.; Citti, L.; Corsi, C.; Fassina, G. F.; Gervasi, P. G.; Lippi, A.; Nieri, R.; Abbondandolo, A.; Berti, G.; Mastrorilli, E. Mutat. Res. 1983, 117, 213-224.

[34] Van Duuren, B. L.; Melchionne, S.; Seidman, I.; Pereira, M. A. Environ. Health Perspect. 1986, 69, 109-117.

[35] Chakrabarty, S.; Danels, Y. J.; Long, B. H.; Willson, J. K. V.; Brattain, M. G. Cancer Res. 1986, 46, 3456-3458.

[36] Gourdie, T. A.; Valu, K. K.; Gravatt, G. L.; Boritzki, T. J.; Baguley, B. C.; Wakelin, L. P. G.; Wilson, W. R.; Woodgate, P. D.; Denny, W. A. J. Med. Chem. 1990, 33, 1177-1186.

[37] Hooberman, B. H.; Chakraborty, P. K.; Sinsheimer, J. E. Mutat. Res.-Genet. Tox. 1993, 299, 85-93.

[38] Sobol, Z.; Engel, M. E.; Rubitski, E.; Ku, W. W.; Aubrecht, J.; Schiestl, R. H. Mut. Res. 2007, 633, $80-94$.

[39] Thier, R.; Muller, M.; Taylor, J. B.; Pemble, S. E.; Ketterer, B.; Guengerich, F. P. Chem. Res. Toxicol. 1995, 8, 465-472.

[40] Wilman, D. E. V.; Palmer, B. D.; Denny, W. A. J. Med. Chem. 1995, 38, 2256-2258.

[41] Sawicki, E.; Bender, D. F.; Houser, T. R.; Wilson, R. M.; Meeker, J. E. Anal. Chem. 1963, 35, 14791486. 
[42] Connors, T. A.; Elson, L. A.; Leese, C. L. Biochem. Pharmacol. 1964, 13, 963-968.

[43] Ashby, J.; Callander, R. D.; Gilman, D. Mutat. Res. Lett. 1984, 140, 71-74.

[44] Eder, E.; Neudecker, T.; Lutz, D.; Henschler, D. 1982, 38, 303-315.

[45] Eder, E.; Neudecker, T.; Lutz, D.; Henschler, D. Biochem. Pharmacol. 1980, 29, 993-998.

[46] Hill, B. T.; Harrap, K. R. Chem.-Biol. Interact. 1972, 5, 117-125.

[47] Magata, Y.; Lang, L.; Kiesewetter, D. O.; Jagoda, E. M.; Channing, M. A.; Eckelman, W. C. Nucl. Med. Biol. 2000, 27, 163-168.

[48] Neudecker, T.; Lutz, D.; Eder, E.; Henschler, D. Biochem. Pharmacol. 1980, 29, 2611-2617.

[49] Raïkova, E.; Grueva, D.; Golovinsky, E. Biochem. Pharmacol. 1981, 30, 289-291.

[50] Schmiedel, G.; Filser, J. G.; Bolt, H. M. Toxicol. Lett. 1983, 19, 293-297.

[51] Zoetemelk, C. E. M.; Mohn, G. R.; Vandergen, A.; Breimer, D. D. Biochem. Pharmacol. 1987, 36, $1829-1835$.

[52] Budzisz, E.; Nawrot, E.; Malecka, M. Archiv Der Pharmazie 2001, 334, 381-387.

[53] Imamura, T.; Talcott, R. E. Mutat. Res.-Genet. Tox. 1985, 155, 1-6.

[54] Katzenellenbogen, J. A.; McGorrin, R. J.; Tatee, T.; Kempton, R. J.; Carlson, K. E.; Kinder, D. H. J. Med. Chem. 1981, 24, 435-450.

[55] Miyahara, M.; Miyahara, M.; Kamiya, S.; Maekawa, A. Chem. Pharm. Bull. 1981, 29, 2366-2369.

[56] Nawrot-Modranka, J.; Nawrot, E.; Graczyk, J. Eur. J. Med. Chem. 2006, 41, 1301-1309.

[57] Truhaut, R.; Delacoux, E.; Brule, G.; Bohuon, C. Clin. Chim. Acta 1963, 8, 235-245.

[58] Friedman, O. M.; Boger, E. Anal. Chem. 1961, 33, 906-910.

[59] Chmielewicz, F. Z.; Fiel, R. J.; Bardos, T. J.; Ambrus, J. L. Cancer Res. 1967, 27, 1248-1257.

[60] Acosta, D.; Mitchell, D. B. Biochem. Pharmacol. 1981, 30, 3225-3230.

[61] Archer, M. C.; Eng, V. W. S. Chem.-Biol. Interact. 1981, 33, 207-214.

[62] Chan, K. K.; Hong, P. S.; Tutsch, K.; Trump, D. L. Cancer Res. 1994, 54, 6421-9.

[63] Fisher, G. R.; Donis, J.; Gutierrez, P. L. Biochem. Pharmacol. 1992, 44, 1625-1635.

[64] Hipkens, J. H.; Struck, R. F.; Gurtoo, H. L. Cancer Res. 1981, 41, 3571-3583.

[65] Kim, J. H.; Thomas, J. J. Bull. Environ. Contam. Toxicol. 1992, 49, 879-885.

[66] van Maanen, M. J.; Tijhof, I. M.; Damen, J. M. A.; Versluis, C.; van den Bosch, J. J. K.; Heck, A. J. R.; Rodenhuis, S.; Beijnen, J. H. Cancer Res. 1999, 59, 4720-4724.

[67] Parodi, S.; Picca, M.; Bolognesi, C.; Cavanna, M.; Carlo, P.; Finollo, R.; Brambilla, G. Pharmacol. Res. Comm. 1977, 9, 621-631.

[68] Schasteen, C. S.; Reed, D. J. Toxicol. Appl. Pharmacol. 1983, 70, 423-432.

[69] Schmid, F. A.; Otter, G. M.; Mehta, B. M. Cancer Res. 1985, 45, 40-44. 
[70] Shyam, K.; Cosby, L. A.; Sartorelli, A. C. J. Med. Chem. 1986, 29, 1323-1325.

[71] Wheeler, G. P.; Bowdon, B. J.; Grimsley, J. A.; Lloyd, H. H. Cancer Res. 1974, 34, 194-200.

[72] Kennedy, K. A.; Mimnaugh, E. G.; Trush, M. A.; Sinha, B. K. Cancer Res. 1985, 45, 4071-4076.

[73] Kennedy, K. A.; Sligar, S. G.; Polomski, L.; Sartorelli, A. C. Biochem. Pharmacol. 1982, 31, $2011-$ 2016.

[74] Marshall, R. S.; Paterson, M. C.; Rauth, A. M. Biochem. Pharmacol. 1991, 41, 1351-1360.

[75] Lewis, L. D.; Burton, L. C.; Harper, P. G.; Rogers, H. J. Eur. J. Cancer 1992, 28, 1976-1981.

[76] Lewis, L. D.; Fitzgerald, D. L.; Harper, P. G.; Rogers, H. J. Brit. J. Clin. Pharmacol. 1990, 30, 725-732.

[77] Lewis, L. D.; Fitzgerald, D. L.; Mohan, P.; Thatcher, N.; Harper, P. G.; Rogers, H. J. Brit. J. Clin. Pharmacol. 1991, 31, 77-82.

[78] Skibba, J. L.; Collins, F. G. Journal of Pharmacological Methods 1980, 4, 155-163.

[79] Kang, S. I.; Spears, C. P. J. Med. Chem. 1987, 30, 597-602.

[80] Mochizuki, M.; Michihiro, K.; Shiomi, K.; Itoh, K.; Tange, Y.; Hizatate, S. Biol. Farm. Bull. 1993, 16, 96-98.

[81] Ninomiya, S.; Kohda, K.; Kawazoe, Y. Chem. Pharm. Bull. 1984, 32, 1326-1332.

[82] Kawazoe, Y.; Tamura, N.; Yoshimura, T. Chem. Pharm. Bull. 1982, 30, 2077-2086.

[83] Cioli, D.; Picamattoccia, L.; Rosenberg, S.; Archer, S. Life Sci. 1985, 37, 161-167.

[84] Cohen, B. E.; Egorin, M. J.; Nayar, M. S. B.; Gutierrez, P. L. Cancer Res. 1984, 44, 4312-4316.

[85] Egorin, M. J.; Snyder, S. W. Cancer Res. 1990, 50, 4044-4049.

[86] Mori, H.; Sato, T.; Nagase, H.; Sakai, Y.; Yamaguchi, S.; Iwata, Y.; Hashimoto, R.; Yamazaki, F.; Hayata, M. Japanese Journal of Toxicology and Environmental Health 1994, 40, 101-110.

[87] Struck, R. F.; Alberts, D. S.; Horne, K.; Phillips, J. G.; Peng, Y.-M.; Roe, D. J. Cancer Res. 1987, 47, $2723-2726$.

[88] Takitani, S.; Asabe, Y.; Kato, T.; Suzuki, M.; Ueno, Y. Journal of Chromatography 1979, 172, 335-342.

[89] Tsunoda, N. Japanese Journal of Toxicology and Environmental Health 1986, 32, 447-454.

[90] Van Duuren, B. L. Ann. NY Acad. Sci. 1969, 163, 633-650.

[91] Lawley, P. D.; Orr, D. J.; Jarman, M. Biochem. J. 1975, 145, 73-84.

[92] Lo, T. Chem. Rev. 1975, 75, 1-20.

[93] Gates, K. S. Chem. Res. Toxicol. 2009, 22, 1747-1760.

[94] Offermanns, S. Encyclopedia of molecular pharmacology; Vol. 1.

[95] Pratihar, S.; Roy, S. J. Org. Chem. 2010, 75, 4957-4963.

[96] Swain, C. G.; Scott, C. B. J. Am. Chem. Soc. 1953, 75, 141-147.

[97] Ritchie, C. D. Acc. Chem. Res. 1972, 5, 348-354. 
[98] Ritchie, C. D. J. Am. Chem. Soc. 1975, 97, 1170-1179.

[99] Mayr, H.; Patz, M. Angew. Chem., Int. Ed. 1994, 33, 938-957.

[100] Vogel, E. W.; Nivard, M. J. M. Mutat. Res.-Fund. Mol. M. 1994, 305, 13-32.

[101] Spears, C. P. Mol. Pharmacol. 1981, 19, 496-504.

[102] Swenson, D. H.; Lawley, P. D. Biochem. J. 1978, 171, 575-587.

[103] Walles, S. A. S. Toxicol. Lett. 1980, 5, 161-167.

[104] Lawley, P. D.; Thatcher, C. J. Biochem. J. 1970, 116, 693-\&.

[105] Singer, B.; Grunberger, D. Molecular biology of mutagens and carcinogens; Plenum Press: New York, 1983.

[106] Loechler, E. L. Chem. Res. Toxicol. 1994, 7, 277-280.

[107] Beranek, D. T. Mutat. Res.-Fund. Mol. M. 1990, 231, 11-30.

[108] Meier, I.; Shephard, S. E.; Lutz, W. K. Mutat. Res. 1990, 238, 193-201.

[109] Boerth, D. W.; Eder, E.; Rasul, G.; Morais, J. Chem. Res. Toxicol. 1991, 4, 368-372.

[110] Nakano, N.; Yamamoto, A.; Kobayashi, Y.; Nagashima, K. Talanta 1995, 42, 641-645.

[111] Dierickx, K. M. E.; Journé, F.; Gerbaux, P.; Morandini, R.; Kauffmann, J.-M.; Ghanem, G. E. Talanta $2009,77,1370-1375$.

[112] Trams, E. G. Anal. Chem. 1958, 30, 256-259.

[113] Mason, W. A.; Meloan, C. E. J. Agric. Food Chem. 1976, 24, 299-304.

[114] Doderer, K.; Lutz-Wahl, S.; Hauer, B.; Schmid, R. D. Anal. Biochem. 2003, 321, 131-134.

[115] Zocher, F.; Enzelberger, M. M.; Bornscheuer, U. T.; Hauer, B.; Schmid, R. D. Anal. Chim. Acta 1999, $391,345-351$.

[116] Groenen, P. J.; Busink, E. Food Chem. Toxicol. 1988, 26, 215-225.

[117] Agarwal, S. C.; Neton, J. Sci. Total Environ. 1989, 79, 69-83.

[118] Katagi, M.; Tsuchihashi, H.; Hanada, S.; Jinmori, H.; Otsuki, K. Japanese Journal of Toxicology and Environmental Health 1993, 39, 459-468.

[119] Berck, B.; Iwata, Y.; Gunther, F. A. J. Agric. Food Chem. 1981, 29, 209-216.

[120] Tingfa, D.; Shiguang, Z.; Mousheng, T. Water, Air, E Soil Pollution 1990, 49, 63-67.

[121] Guth, J. A.; Manner, L. P. Journal of Chromatography 1967, 30, 514-521.

[122] Asami, M.; Nakamura, K.-I.; Kawada, K.; Tanaka, M. Journal of Chromatography A 1979, 174, $216-$ 220.

[123] Guengerich, F. P.; Geiger, L. E.; Hogy, L. L.; Wright, P. L. Cancer Res. 1981, 41, 4925-4933.

[124] Fox, B. G.; Borneman, J. G.; Wackett, L. P.; Lipscomb, J. D. Biochemistry 1990, 29, 6419-6427.

[125] Newman, L. M.; Wackett, L. P. J. Bacteriol. 1997, 179, 90-96. 
[126] Forkert, P. G.; Premdas, P. D.; Bowers, R. J. Am. J. Respir. Cell Mol. Biol. 2000, 23, 687-695.

[127] Premdas, P. D.; Bowers, R. J.; Forkert, P. G. J. Pharmacol. Exp. Ther. 2000, 293, 1112-1120.

[128] Tan, Y. L.; Cole, D. R. Clinical Chemistry 1965, 11, 58-62.

[129] Cox, P. J.; Farmer, P. B. Chem.-Biol. Interact. 1975, 10, 103-114.

[130] Christian, R. A.; Chaffee, S. K.; Hovick, C. J.; Steele, W. J. Life Sci. 1980, 27, 2595-2599.

[131] Jiang, H. B.; Ichikawa, M.; Furukawa, A.; Tomita, S.; Ichikawa, Y. Biochem. Pharmacol. 2000, 60, 571-579.

[132] Mellett, L. B.; Woods, L. A. Cancer Res. 1960, 20, 524-532.

[133] Aeschlimann, C.; Kupfer, A.; Schefer, H.; Cerny, T. Drug Metab. Dispos. 1998, 26, 883-890.

[134] Barbin, A.; Bereziat, J. C.; Croisy, A.; Oneill, I. K.; Bartsch, H. Chem.-Biol. Interact. 1990, 73, $261-$ 277.

[135] Huijbrechts, A. M. L.; Haar, R. t.; Schols, H. A.; Franssen, M. C. R.; Boeriu, C. G.; Sudhölter, E. J. R. Carbohydr. Polym. 2011, 79, 858-866.

[136] Magdalou, J.; Hammock, B. D. Biochem. Pharmacol. 1988, 37, 2717-2722.

[137] Rink, R.; Fennema, M.; Smids, M.; Dehmel, U.; Janssen, D. B. J. Biol. Chem. 1997, 272, 14650-14657.

[138] Alcalde, M.; Farinas, E. T.; Arnold, F. H. J. Biomol. Screen. 2004, 9, 141-146.

[139] Bhatnagar, T.; Manoj, K. M.; Baratti, J. C. J. Biochem. Biophys. Methods 2001, 50, 1-13.

[140] Cedrone, F.; Bhatnagar, T.; Baratti, J. C. Biotechnol. Lett. 2005, 27, 1921-1927.

[141] Kim, H. S.; Lee, O. K.; Lee, S. J.; Hwang, S.; Kim, S. J.; Yang, S. H.; Park, S.; Lee, E. Y. J. Mol. Catal. B: Enzym. 2006, 41, 130-135.

[142] Li, N.; Zhang, Y.; Feng, H. Acta Biochimica et Biophysica Sinica 2009, 41, 638-647.

[143] Zhang, L. F.; Wu, J. M.; Feng, H. J. Biochem. 2011, 149, 673-684.

[144] Kumar, R.; Wani, S. I.; Chauhan, N. S.; Sharma, R.; Sareen, D. Protein Expression Purif. 2011, 79, 49-59.

[145] Kubo, T.; Peters, M. W.; Meinhold, P.; Arnold, F. H. Chem. Eur. J. 2006, 12, 1216-1220.

[146] Qaed, A. A.; Lin, H.; Tang, D. F.; Wu, Z. L. Biotechnol. Lett. 2011, 33, 611-616.

[147] Tee, K. L.; Schwaneberg, U. Angew. Chem., Int. Ed. 2006, 45, 5380-5383.

[148] Schrader, T. J.; Langlois, I.; Soper, K.; Cherry, W. Teratogenesis Carcinogenesis and Mutagenesis 2002, $22,425-441$.

[149] Rosman, L. B.; Beylin, V. G.; Gaddamidi, V.; Hooberman, B. H.; Sinsheimer, J. E. Mutat. Res. 1986, $171,63-70$.

[150] Eder, E.; Henschler, D.; Neudecker, T. Xenobiotica 1982, 12, 831-48.

[151] Yano, K.; Isobe, M. Cancer Res. 1979, 39, 5147-5149.

[152] Hemminki, K. Chem.-Biol. Interact. 1981, 34, 323-331. 
[153] Rosman, L. B.; Chakraborty, P. K.; Messerly, E. A.; Sinsheimer, J. E. Mutat. Res.-Genet. Tox. 1988, $206,115-125$.

[154] Rosman, L. B.; Gaddamidi, V.; Sinsheimer, J. E. Mutat. Res.-Genet. Tox. 1987, 189, 189-204.

[155] Yano, K.; Katayama, H.; Takemoto, K. Cancer Res. 1984, 44, 1027-1030.

[156] Eder, E.; Kutt, W. Chem.-Biol. Interact. 1989, 69, 45-59.

[157] Lawley, P. In Carcinogenesis by alkylating agents; Searle, C., Ed.; American Chemical Society,: Washington, 1976; Vol. American Chemical Society Monograph 173; pp 83-244.

[158] Isobe, M.; Yano, K. Mutat. Res. 1982, 93, 57-66.

[159] Ashby, J.; Hilton, J.; Dearman, R. J.; Callander, R. D.; Kimber, I. Environ. Health Perspect. 1993, 101, 62-67.

[160] Jesson, M. I.; Johnston, J. B.; Robotham, E.; Begleiter, A. Cancer Res. 1989, 49, 7031-7036.

[161] Shyam, K.; Penketh, P. G.; Divo, A. A.; Loomis, R. H.; Rose, W. C.; Sartorelli, A. C. J. Med. Chem. 1993, 36, 3496-3502.

[162] Freeman, J. A.; Johnson, J. V.; Yost, R. A.; Kuehl, D. W. Anal. Chem. 1994, 66, 1902-1910.

[163] Beyer, U.; Roth, T.; Schumacher, P.; Maier, G.; Unold, A.; Frahm, A. W.; Fiebig, H. H.; Unger, C.; Kratz, F. J. Med. Chem. 1998, 41, 2701-2708.

[164] Kratz, F.; Beyer, U.; Roth, T.; Schütte, M. T.; Unold, A.; Fiebig, H. H.; Unger, C. Archiv Der Pharmazie 1998, 331, 47-53.

[165] Dipple, A.; Slade, T. A. Eur. J. Cancer 1970, 6, 417-423.

[166] Van Duuren, B. L.; Goldschmidt, B. M. J. Med. Chem. 1966, 9, 77-79.

[167] Peterson, A. R.; Peterson, H.; Spears, C. P.; Trosko, J. E.; Sevanian, A. Mutat. Res.-Envir. Muta. 1988, 203, 355-366.

[168] Phillips, D. H.; Grover, P. L.; Sims, P. Chem.-Biol. Interact. 1978, 20, 63-75.

[169] Sims, P. Biochem. J. 1972, 130, 27-35.

[170] Lalka, D.; Bardos, T. J. Biochem. Pharmacol. 1975, 24, 455-462.

[171] Anderson, W. K.; Heider, A. R. J. Med. Chem. 1986, 29, 2392-2395.

[172] Lin, E. L. C.; Daniel, F. B.; Herrenfreund, S. L.; Pereira, M. A. Environ. Health Perspect. 1986, 69, $67-71$.

[173] Hemminki, A.; Vayrynen, T.; Hemminki, K. Chem.-Biol. Interact. 1994, 93, 51-58.

[174] Peterson, L. A.; Harris, T. M.; Guengerich, F. P. J. Am. Chem. Soc. 1988, 110, 3284-3291.

[175] García-Santos, M. P.; Calle, E.; Casado, J. J. Am. Chem. Soc. 2001, 123, 7506-7510.

[176] Casado, J.; López-Quintela, M. A.; Lorenzo-Barral, F. M. J. Chem. Ed. 1986, 63, 450.

[177] Schiffmann, D.; Eder, E.; Neudecker, T.; Henschler, D. Cancer Lett. 1983, 20, 263-269.

[178] Karchesy, J. J.; Arbogast, B.; Deinzer, M. L. J. Org. Chem. 1987, 52, 3867-3872.

[179] Cooper, R. A.; Huxtable, R. J. In The relationship between reactivity of metabolites of pyrrolizidine alkaloids and extrahepatic toxicity; Huxtable, R., Ed.; 1999; Vol. 42; pp 13-16.

[180] Giraud, I.; Rapp, M.; Maurizis, J.-C.; Madelmont, J.-C. J. Med. Chem. 2002, 45, 2116-2119. 

<smiles>[Z6]OC1=CC=C1</smiles> 



\section{Contents}

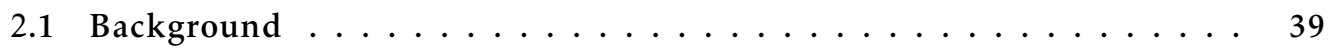

2.1.1 Genotoxicity of lactones .................... 39

$2.1 .1 .1 \quad \beta$-Lactones . . . . . . . . . . . . . . . . . . 39

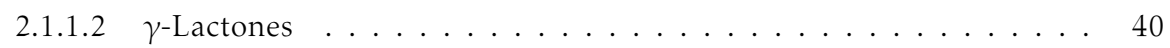

2.1.1.3 $\delta$-Lactones . . . . . . . . . . . . . . . . . 40

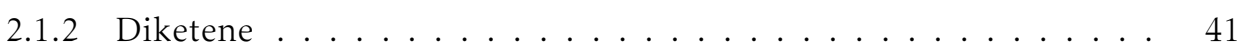

2.1.2.1 Chemical reactivity . . . . . . . . . . . . . . 41

2.1.2.1.1 Hydrolysis . . . . . . . . . . . . . . . 41

2.1.2.1.2 Acetoacetylation . . . . . . . . . . . . . 42

2.1.2.2 Carcinogenicity . . . . . . . . . . . . . 42

2.2 Materials \& methods . . . . . . . . . . . . . . . . 44

2.2.1 Hydrolysis of diketene . . . . . . . . . . . . . . . . . . . . . 44

2.2.1.1 Neutral hydrolysis of diketene . . . . . . . . . . . . . . . 44

2.2.1.2 Base hydrolysis of diketene . . . . . . . . . . . . . . . . . . . . . . . . . . . .

2.2.2 Alkylating potential of diketene . . . . . . . . . . . . . 44

2.2.3 Acidity of lactones and ketones . . . . . . . . . . . . . . 45

2.2.3.1 Absolute approach . . . . . . . . . . . . . . . 45

2.2.3.2 Relative approach . . . . . . . . . . . . . . . 46

2.2.3.3 Nucleus-Independent Chemical Shift . . . . . . . . . . . . . . . . . . . . . . . . . . . . . . .

2.2.3.4 Computational details . . . . . . . . . . . . . . . . 47

2.3 Results and discussion . . . . . . . . . . . . . . . 47

2.3.1 Hydrolysis of diketene . . . . . . . . . . . . . . . . 47

2.3.1.1 Neutral hydrolysis . . . . . . . . . . . . . . . . . . . 47

2.3.1.2 Base hydrolysis . . . . . . . . . . . . . . . 52

2.3.2 Alkylating potential of diketene . . . . . . . . . . . . 56

2.3.2.1 Formation of the DIK-NBP adduct . . . . . . . . . . . . . . . . 59

2.3.2.2 Decomposition of the NBP-DIK adduct . . . . . . . . . . . . . 61

2.3.3 Acidity of lactones and cycloketones ............. 63

2.3.3.1 Esters and lactones . . . . . . . . . . . . . . . 63

2.3.3.1.1 Isomerization energies . . . . . . . . . . . . . . . . . . . . . . 64

2.3.3.1.2 Effect of structure upon $\mathrm{p}_{\mathrm{a}} \ldots \ldots \ldots . \ldots 67$

2.3.3.2 Ketones . . . . . . . . . . . . . . . . . . . . . . 69

2.3.3.2.1 Isomerization energies . . . . . . . . . . 72 
2.3.3.2.2 Effect of structure upon $\mathrm{p}_{\mathrm{a}} \ldots \ldots \ldots \ldots \ldots$

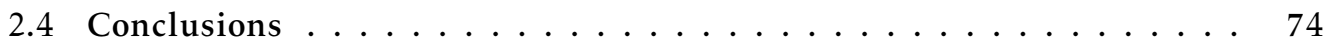

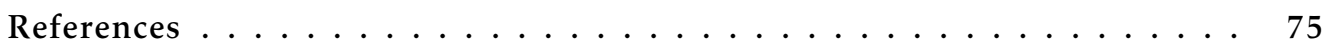




\subsection{Background}

Small, highly strained heterocycles are archetypical alkylating agents, for instance, $\beta$-lactones were among the first alkylating agents discovered that did not require metabolic activation to exert their genotoxic effects. In recent years, the finding that nitrosation of amino acids yields lactones as a product has attracted renewed interest in the alkylating potential of lactones.

\subsubsection{Genotoxicity of lactones}

The first studies dealing with the tumorigenicity of lactones in test animals date back to the 1950's, when most of the classic studies about the carcinogenicity of direct alkylating agents upon mice and rats were carried out. Later works have addressed the genotoxicity of lactones and their ability to modify DNA, using contemporary assays.

\subsubsection{1. $\beta$-Lactones}

Among all the four-membered lactones, $\beta$-propiolactone is the most studied as regards its ability to damage DNA. One of the very first works in which BPL is detected to be a powerful mutagen dates back to $1951^{1}$ with later works addressed the tumorigenicity of BPL in mice and rats. ${ }^{2,3}$ In 1961 Dickens and Jones determined that weekly doses of 0.1 and $1.0 \mathrm{mg}$ of BPL are carcinogenic in rats treated for 25 weeks or more. Time elapsed until the appearance of tumors was the same for all doses; however, dosage during four weeks did not induce tumors, nor did the hydrolysis product, $\beta$-hydroxypropionic acid. ${ }^{4,5}$ BPL was also observed to be carcinogenic at similar doses and time lapses in golden hamsters and guinea pigs. ${ }^{6,7}$

Other works also focused on the correlation of the reactivity of BPL with its carcinogenicity, ${ }^{8}$ especially as regards its reaction with nucleotides ${ }^{9,10}$ and both DNA and RNA. ${ }^{11-15}$ Since it is a powerful electrophile, BPL also binds to proteins and other cellular structures like membranes in vivo. ${ }^{16-18}$

Dickens and Jones also found that $\alpha$-carboxy- $\beta$-phenyl- $\beta$-propiolactone and $\alpha, \alpha$-diphenyl- $\beta$-propiolactone give rise to the formation of tumors in rats. ${ }^{19}$ Results obtained from the study of other lactones suggest that substitution reduces the activity of $\beta$-lactones as alkylating agents, mostly by steric hindrance of their alkylation reaction.

Van Duuren et al., in some of their works about chemical carcinogens, studied some $\beta$-lactones; three out of the eight studied lactones tested positive: BPL, BBL and 2,2,4-trimethyl-3-hydroxy-3-pentenoic- $\beta$ lactone. This suggests, too, that substitution in the $\alpha$ and $\beta$ positions blocks, sometimes completely, the carcinogenicity of four-membered lactones. ${ }^{20-25}$

More modern assays have also proved BPL a powerful genotoxin: ${ }^{26}$ it produces hepatomas after a single application, ${ }^{27}$ is mutagenic to bacteriophages and Salmonella typhimurium, ${ }^{28,29}$ and also to Saccharomyces cerevisiae. ${ }^{30}$ It induces sex-linked recessive lethals and autosomal translocations in Drosophila ${ }^{31}$ and is clastogenic after a single application. ${ }^{32}$ 
BPL-DNA adducts have been identified as carboxyethyl derivatives of adenine, ${ }^{33,34}$ guanosine, ${ }^{34}$ thymine and cytosine, ${ }^{35}$ arising from the alkyl-oxygen cleavage of the lactone ring.

The case of diketene (4-methylenoxetan-2-one, DIK) is surprising: given its similarities with carcinogenic lactones, it would be likely to be carcinogenic. Nevertheless, no carcinogenicity has been observed in animal tests (vide infra).

\subsubsection{2. $\gamma$-Lactones}

As a rule, $\gamma$-lactones are less reactive than $\beta$-lactones due to the smaller ring strain, as can be observed in their hydrolysis rates, for instance. Neither $\gamma$ - nor $\delta$-lactones undergo uncatalyzed hydrolysis, which suggests that, in the absence of activating groups, both are unreactive against nucleophiles like DNA bases.

As occurs with their antibiotic properties, carcinogenicity of $\gamma$-lactones is almost exclusive of $\alpha, \beta$ unsaturated ones. This is possibly due to these compounds being Michael acceptors, and not so much to the ring-opening reactions, as is the case with $\beta$-lactones.

For instance, biweekly doses of $2 \mathrm{mg}$ methylprotoanemonine produced lethal sarcomes in three out of five rats. ${ }^{4} 2$-hexenoic- $\gamma$-lactone, 4 -hexenoic- $\gamma$-lactone and penicillinic acid, which suggests that $\alpha$ - $\beta$ unsaturation is a required condition for carcinogenicty. Although Dickens found that $\beta$-angelicalactone was carcinogenic in one out of six mice, ${ }^{5}$ van Duuren et al. found no proof for carcinogenicity in a assay lasting 546 days.

A very important group of genotoxic lactones discovered in the late 80's are those arising from the chlorination of humic substances. These compounds will be discussed in depth somewhere else (vide infra) and will not be discussed here.

\subsubsection{3. $\delta$-Lactones}

$\delta$-Lactones are more reactive than five-membered lactones, as regards their hydrolysis reaction. Nevertheless, simple $\delta$-lactones do not react with biological nucleophiles.

Parasorbic acid has been proven to be carcinogenic in repeated doses of $2 \mathrm{mg}$ and $0.2 \mathrm{mg}$ during 32 weeks, giving rise to tumor in eight out of 11 rats. $^{5}$

Aflatoxines are naturally occurring mycotoxins that are produced by many species of Aspergillus, a fungus, most notably Aspergillus flavus and Aspergillus parasiticus. Aflatoxins are toxic and among the most carcinogenic substances known and can infect crops like cereals (maize, sorghum, pearl millet, rice, wheat), oilseeds (peanut, soybean, sunflower, cotton), spices (chilli peppers, black pepper, coriander, turmeric, ginger), and tree nuts (almond, pistachio, walnut, coconut, Brazil nut). They possess a $\delta$-lactone moiety in their structure, but their alkylating ability arises from the presence of an epoxide group that is formed during metabolization, and, as proposed by Dickens, their carcinogenicity is unrelated to the lactone function. ${ }^{19}$ 


\subsubsection{Diketene}

Diketene (4-methylene-2-oxetanone, DIK) consists of a four-membered lactone ring adjacent to a methylene function and it can be considered the anhydride of acetoacetic acid, that is obtained from the dimerization of ketene, which is in turn obtained from the pyrolysis of acetic acid, acetone or acetic anhydride. Diketene is an industrial chemical, and its derivatives have versatile applications, including the manufacture of agrochemicals, dyes, pigments, pharmaceuticals (including vitamins), and stabilizers for PVC and polyester. Its reactivity and applications have been reviewed by Clemens. ${ }^{36}$

\subsubsection{Chemical reactivity}

DIK is highly reactive towards nucleophilic and electrophilic reagents such as alcohols, aldehydes, amines, and ketones. A significant part of this reactivity arises from the highly tensioned heterocycle, whose strain energy has been estimated in $90 \mathrm{~kJ} \mathrm{~mol}^{-1} .37$

When compared to BPL, DIK shows an additional $s p^{2}$ hybridized atom in its cycle. Since the $120^{\circ}$ bonding angles required by $s p^{2}$ hybridazation are far from the $90^{\circ}$ bonding angles in square lactones, the extra $s p^{2}$ hybridized atom is expected to add significant strain. The structure of diketene, ${ }^{38}$ and the product selectiviy of the cycloadditive dimerization of ketene have been studied computationally and experimentally. ${ }^{39,40}$

The reactions of DIK with nucleophiles usually imply ring opening, mainly through acyl-oxygen cleavage and conduct to acetoacetic acid derivatives. However, cases are known in which DIK undergoes alkyl-oxygen cleavage. Electrophiles react mostly with the exocyclic double bond and are followed by nucleophilic attack on the lactone carbonyl group.

2.1.2.1.1. Hydrolysis. As other $\beta$-lactones diketene undergoes spontaneous hydrolysis in aqueous medium. The initial product is acetoacetic acid, which in turn undergoes decarboxylation - specially in acidic medium - affording acetone and $\mathrm{CO}_{2}$. Sodium acetoacetate from the alkaline hydrolysis of DIK has been used in situ - as an equivalent to acetone enolate - in Knoevenagel reactions or for the preparation of allylacetone.

The hydrolysis of diketene was studied by Briody and Satchell, 41,42 who obtained the following conclusions:

- The neutral hydrolysis of diketene takes place with acyl-oxygen cleavage. Anions do not attack the $\beta$ carbon of DIK.

- In strongly acidic medium DIK follows $H_{0}$, rather than hydronium ion concentration. This has been interpreted as evidence that the acid catalyzed hydrolysis of DIK occurs through the $\mathrm{A}_{\mathrm{AC}} 1$ mechanism, as occurs with other $\beta$ lactones

- Diketene undergoes mild general base catalysis: acetate, choloroacetate and acetoacetate were seen to catalyze the hydrolysis reaction. 
- Reaction with hydroxide ions is very fast.

- Pyridine, and other amines, are nucleophilic catalysts of the reaction.

The hydrolysis of diketene was also studied in the context of the catalytic activity of sodium tetrachloropalladate. ${ }^{43}$ Also Van Duuren et al. tackled some aspects of diketene spontaenous hydrolysis in their study of lactone carcinogenicty. ${ }^{44}$

Decarboxylation of acetoacetate has also been subject of kinetic investigations by both spectrophotometric and manometric methods. It was found that the acid for of the carboxylic acid decomposes about 50 times faster than the basic form, which was attributed to an entropic effect. ${ }^{45}$

2.1.2.1.2. Acetoacetylation. Whereas the number of nucleophilic reactants susceptible of being acetoacetylated with DIK is very high (aliphatic alcohols, phenols, amines, carbon nucleophiles, ...) the reactivity of diketene with nucleophilic aromatic nitrogen heteroycles deserves special attention, since these include, in a broad sense both NBP and DNA bases.

Diketene reacts easily with nitrogen heterocycles yielding polycyclic products. Pyridine reacts with ketene or diketene affording a tricyclic system known as the Wollenberg compound. ${ }^{46}$ The reactivity of diketene with quinoline or isoquinoline has also been studied in excess DIK both in reflux and at room temperature. ${ }^{47}$

Thus, excess diketene reacts with cyclic aromatic nitrogen atoms, first through acetoacetylation of the nitrogen atom, followed by cyclization through the formed enolate, and finally continues with acetoacetylation of the formed cycle. The life of the intermediate enolate in a protic solvent such as water, must be greatly reduced, which would end the reaction at the initial amide.

\subsubsection{Carcinogenicity}

Diketene, as a $\beta$-lactone, would be expected to be a carcinogenic alkylating agent. However, despite its high chemical reactivity, diketene is inactive as a carcinogen in a variety of assays. ${ }^{20,23,25}$ No tumorigenic effects were observed when $1 \mathrm{ml}$ 10\% diketene was applied to mouse skin three times per week either in tricarpylin or acetone, ${ }^{22,48}$ nor when injected in $1.1 \mathrm{mg}$ doses, once a week, ${ }^{21}$ nor when it was subcutaneously implanted in a gelatin capsule containin $1.1 \mathrm{mg} .{ }^{24}$ This lack of activity has been attributed to the fast hydrolysis of diketene and its lack of solubility in water:

Van Duuren and Goldschmidt reported that "in the $\beta$-lactone series, $\beta$-propiolactone and $\beta$-butyrolactone are carcinogenic and water-soluble, whereas diketene is inactive [as a carcinogen] and water-insoluble. This correlation suggests that solubility plays some role in facilitating the expression of biological activity by these materials". ${ }^{44}$ Van Duuren et al. also referred to the rapid hydrolysis of diketene as a possible cause for its lack of carcinogenicity, since it may preclude its in vivo reactivity. ${ }^{24}$

Nevertheless, the solubility of diketene in water is around $0.1 \mathrm{M}$ and the values found in the literature for its hydrolysis rate constant in water at $25^{\circ} \mathrm{C}$ are close to those of the carcinogenic $\beta$-lactones BPL 
and BBL. These observations suggest that the reason for the lack of carcinogenicity of diketene must lie somewhere else. 


\subsection{Materials \& methods}

\subsubsection{Hydrolysis of diketene}

\subsubsection{Neutral hydrolysis of diketene}

Since the product of lactone hydrolysis are hidroxyacids, (acetoacetic acid in the case of diketene) the formation of the product was followed kinetically by means of and acid-base titration. Kinetic runs were carried out in a automated titrator (Metrohm 718 Stat Titrino).

$210 \mu \mathrm{L}$ diketene (Panreac, Barcelona) were solved in $100 \mathrm{~cm}^{3}$ of the appropriate water/dioxane (w/d) mixture, giving a final concentration of $2.5 \times 10^{-3} \mathrm{M}$ and the auto-titrator was set off. In order to keep $\mathrm{pH}$ constant, the device added previously normalized sodium hydroxide $\left(9.38 \times 10^{-2} \mathrm{M}\right)$, to compensate for the formation of the hydroxiacid. By the end of the reaction approximately $2.5 \mathrm{~cm}^{3}$ of sodium hydroxide had been added. Thus, the effect of dilution of the reaction mixture was negligible (less than 2.5\%).

In the working conditions acetoacetic acid $\left(\mathrm{p} K_{\mathrm{a}}=3.77\right)$ is completely dissociated, which allowed us to link quantitatively the hydroxide consumed in the reaction with the formed acetoacteic acid. No influence of acetoacetic acid decarboxylation during the length of the experiment was observed. The reaction temperature was fixed using a Lauda Ecoline RE120 cryostat between 15 and $35^{\circ} \mathrm{C}$.

\subsubsection{Base hydrolysis of diketene}

Due to its high rate, the base-catalyzed hydrolysis of diketene was studied using the stopped-flow technique (Biologic Sequential Mixing Stopped Flow SFM300). NaOH and diketene were prepared in the desired solvent mixture $\left(0.2-1.0 \mathrm{M}\right.$ and $5 \times 10^{-3} \mathrm{M}$, respectively) and loaded onto the syringes. Diketene was renewed frequently enough, so that its neutral hydrolysis could be neglected. $\mathrm{NaCl}$ was used to adjust the ionic intensity to $1.0 \mathrm{M}$.

The contents of the syringes were mixed in the cuvette in different volumetric proportions (1:1.6 to $1.6: 1)$ up to a total volume of $300 \mu \mathrm{L}$. That way, varying concentrations of hydroxide were obtained ( $\mathrm{NaOH}$ was in great excess with respect to diketene in all runs).

Since the $\alpha$ hydrogen in acetoacetate is highly acidic $\left(\mathrm{p} K_{\mathrm{a}} \simeq 11\right)$, almost all acetoacetate is in the dianion form, and thus, the reaction was followed at $\lambda=400 \mathrm{~nm}$, where the AcAc ${ }^{2-}$ shows maximum absorption. Temperature control was achieved via a thermostatic circulating bath (Lauda Ecoline RE120).

\subsubsection{Alkylating potential of diketene}

Since the NBP-DIK adduct is rather labile, the usual NBP test, in which basification induces a change in color, could not be used. Instead the adduct was followed spectrophotometrically at $\lambda=450 \mathrm{~nm}$, 
where it shows maximum absorption. Measurements were carried out as follows: $3 \mathrm{~cm}^{3}$ of the reaction mixture (NBP $0.020 \mathrm{M}$ ) were kept at constant temperature in a quartz cuvette $(10.0 \mathrm{~mm}$ optic path) and the alkylation reaction was started by addition of $210 \mu \mathrm{L}$ diketene $0.238 \mathrm{M}$, giving a final concentration of $0.010 \mathrm{M}$. Immediately after the addition of diketene, recording of the absorption was started using a Shimadzu UV-2401 PC spectrophotometer, equipped with a CPS 240A thermostatted six-cell holder.

\subsubsection{Acidity of lactones and ketones}

In recent years, computational methods have reached a level of accuracy that allows calculation of equilibrium constants in solution, using the thermodynamic definition of equilibrium constant.

$$
\mathrm{p} K_{\mathrm{a}}=-\log K_{\mathrm{a}}=\frac{\Delta G_{\mathrm{aq}}^{\ominus}}{\ln 10 R T}
$$

Gas-phase free energies can be computed within less than $1 \mathrm{kcal}$ and the largest cause of error lies in solvation free energies. Computational determination of $\mathrm{p} K_{\mathrm{a}}$ values is particularly challenging, since the solvation energy of the proton is hard to determine computationally and even experimental values have enough indetermination to make results vary in some log units. Also, solvation patterns of the acid and its conjugated base are very different, and systematic errors are likely to appear.

Therefore, the use of a direct approach is error-prone, and several solutions have been proposed. These normally include use of a relative method and/or explicit solvent molecules, to better model specific solvent-solute interactions such as hydrogen bonding. In this work we have opted for a relative approach.

\subsubsection{Absolute approach}

In the absolute approach the gas-phase free energy of reaction is calculated using high-level theoretical methods, and then a polarizable continuum method is used to calculate solvation energies. From the thermodynamic cycle depicted in Scheme 2.1, $\Delta G_{\text {aq }}^{\ominus}$ can be computed as:

$$
\Delta G_{\mathrm{aq}}^{\ominus}=\Delta G_{\mathrm{g}}^{\ominus}-\Delta G_{\text {solv }}^{\ominus}(\mathrm{AH})+\Delta G_{\text {solv }}^{\ominus}\left(\mathrm{A}^{-}\right)+-\Delta G_{\text {solv }}^{\ominus}\left(\mathrm{H}^{+}\right)
$$

A thermodynamic correction of $-\delta n R T \ln V$ has to be considered because the gas-phase calculations are referred to a standard state of $1 \mathrm{~atm}$ and the solvation calculations are referred to a standard state of $1 \mathrm{~mol} \mathrm{dm}^{-3}$. There is a broad range of proposed values for the solvation free energy of the proton (from -254 to $-261 \mathrm{kcal} \mathrm{mol}^{-1}$ ); here, the most accepted value of $\mathrm{G}_{\mathrm{H}}^{\mathrm{aq}}=1129.8 \mathrm{~kJ} \mathrm{~mol}^{-1}$ was used for the free energy of the proton in aqueous solution. 


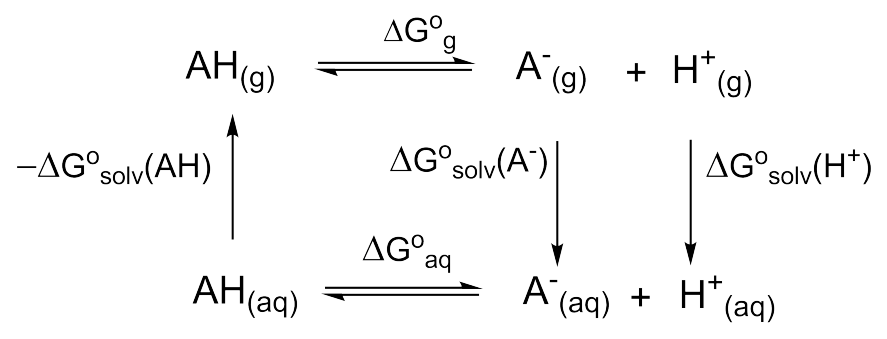

Scheme 2.1. Absolute approach for the determination of $\mathrm{p} K_{\mathrm{a}}$ values

\subsubsection{Relative approach}

In the relative approach (Scheme 2.2), a reference molecule, whose $\mathrm{p} K_{\mathrm{a}}$ is well-known experimentally. The use of a proton-exchange reaction with $\mathrm{BH}$, a species similar to $\mathrm{AH}$, makes the use of the solvation free energy of the proton unnecessary.

$$
\begin{gathered}
\mathrm{B}_{(\mathrm{g})}^{-}+\mathrm{AH}_{(\mathrm{g})} \stackrel{\Delta \mathrm{G}_{\mathrm{g}}^{\circ}}{\rightleftharpoons} \mathrm{A}_{(\mathrm{g})}^{-}+\mathrm{BH}_{(\mathrm{g})} \\
-\Delta \mathrm{G}_{\text {solv }}^{\circ}\left(\mathrm{B}^{-}\right) \mid \\
\mathrm{B}_{(\mathrm{aq})}^{-}+\mathrm{AH}_{(\mathrm{aq})} \stackrel{\Delta \mathrm{G}_{\text {solv }}^{\circ}(\mathrm{AH})}{\stackrel{\Delta \mathrm{G}_{\text {solv }}^{\circ}\left(\mathrm{A}^{-}\right)}{\Delta \mathrm{G}_{\mathrm{aq}}}} \mathrm{A}_{(\mathrm{aq})}^{-}+\mathrm{BH}_{(\mathrm{aq})}
\end{gathered}
$$

Scheme 2.2. Relative approach for the determination of $\mathrm{p} K_{\mathrm{a}}$ values

Since the method relies on the use of an experimental reference, the values obtained are termed relative.

The use of a homodesmic reaction allows (partial) compensation of the effects that could be due to the method, systematically evaluating differently gas-phase energies and solvation energies for ions or neutral molecules, since there is both an ion and a neutral molecule on each side of the chemical equation. The effect of solvent hydrogen bonding, which is not taken into account in SCRF models, is also diminished, because the species on both sides of the chemical equation should show very similar degrees of hydrogen bonding with the solvent.

\subsubsection{Nucleus-Independent Chemical Shift}

The nucleus-independent chemical shift (NICS) is a computational method that calculates the absolute magnetic shieldings at the center of the ring taken with reversed sign. In this method negative NICS values indicate aromaticity and positive values antiaromaticity. ${ }^{49,50}$ An improved method (NICS(1)), measures the chemical shift $1 \AA$ above the central point of the ring, in order to above spurious effects. 


\subsubsection{Computational details}

Gas-phase equilibrium geometries and free energies were computed at the DFT-B3LYP/6-31++G(2df,2pd), CBS-Q, and G2 levels, and all structures were checked to be true minima (zero imaginary frequencies).

Solvation free energies were computed as single point calculations via the SCFVAC keyword on the gas-phase geometries using the Gaussian 03 default Integral Equation Formalism IEF-PCM.

Two hydration radii were used: UAKS, in combination with the DFT-B3LYP/6-31+G(d) level of theory and UAHF at the HF/6-31+G(d) level of theory. Use of other solvation methods (CPCM or DPCM) has negligible influence on the solvation energies.

Once diffuse functions are added, so that anions are properly modeled, the choice of the basis set has little influence in the solvation energies, thus, relatively small basis sets were chosen, in order to speed up the calculations. Since solvation radii have been parametrized using specific levels of theory (Hartree-Fock for UAHF and DFT-B3LYP for UAKS) any changes in the method produce inferior results.

NICS was determined as predicted NMR shielding tensors, computed with the Gauge-Independent Atomic Orbital (GIAO) method at the DFT-B3LYP/6-311+G(d,p) level of theory on the equilibrium geometries obtained at the same level. ${ }^{51-55}$

\subsection{Results and discussion}

\subsubsection{Hydrolysis of diketene}

\subsubsection{Neutral hydrolysis}

Diketene hydrolyzes following the reaction depicted in the Scheme 2.3, yielding acetoacetic acid (AcACH).

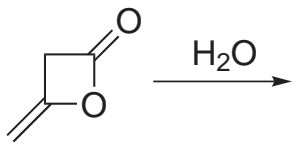

DIK<smiles>CC(=O)CC(=O)O</smiles>

$\mathrm{AcAcH}$

Scheme 2.3. Neutral hydrolysis of DIK.

The differential rate equation for the process is eq. 2.3. Since water is in a large excess, the pseudoorder approximation was applied.

$$
\begin{aligned}
r & =\frac{d[\mathrm{AcAcH}]}{d t}=-\frac{d[\mathrm{DIK}]}{d t}=k_{\mathrm{H}_{2} \mathrm{O}}^{\mathrm{DIK}}\left[\mathrm{H}_{2} \mathrm{O}\right][\mathrm{DIK}] \\
& =k_{1 \mathrm{H}_{2} \mathrm{O}}^{\mathrm{DIK}}[\mathrm{DIK}]=k_{1 \mathrm{H}_{2} \mathrm{O}}^{\mathrm{DIK}}\left([\mathrm{DIK}]_{\circ}-[\mathrm{AcAcH}]\right)
\end{aligned}
$$


[AcAcH] stands for the concentration of acetoacetic acid at a time $t, k_{\mathrm{H}_{2} \mathrm{O}}$ is the bimolecular hidrolysis rate constant and $[\mathrm{DIK}]_{\circ}$ represents the initial concentration of diketene. $k_{1 \mathrm{H}_{2} \mathrm{O}}^{\mathrm{DIK}}$ stands for the product of the bimolecular rate constant by water concentration:

$$
k_{1 \mathrm{H}_{2} \mathrm{O}}^{\mathrm{DIK}}=k_{\mathrm{H}_{2} \mathrm{O}}^{\mathrm{DIK}}\left[\mathrm{H}_{2} \mathrm{O}\right]
$$

Integration of eq. 2.3 yields eq. 2.5 and 2.6.

$$
\begin{aligned}
{[\mathrm{AcAcH}] } & =[\mathrm{DIK}]_{\circ}\left(1-e^{-k_{1 \mathrm{H}_{2} \mathrm{O}}^{\mathrm{DIK}} t}\right) \\
{[\mathrm{DIK}] } & =[\mathrm{DIK}]_{\circ} e^{-k_{1 \mathrm{H}_{2} \mathrm{O}}^{\mathrm{DIK}} t}
\end{aligned}
$$

The concetration of formed AcAcH expressed as the amount of consumed $\mathrm{NaOH}$ in the titrator is

$$
V_{\mathrm{NaOH}}=V_{\mathrm{NaOH}}^{\max }\left(1-e^{-k_{1 \mathrm{H}_{2} \mathrm{O}}^{\mathrm{DIK}}}\right)
$$

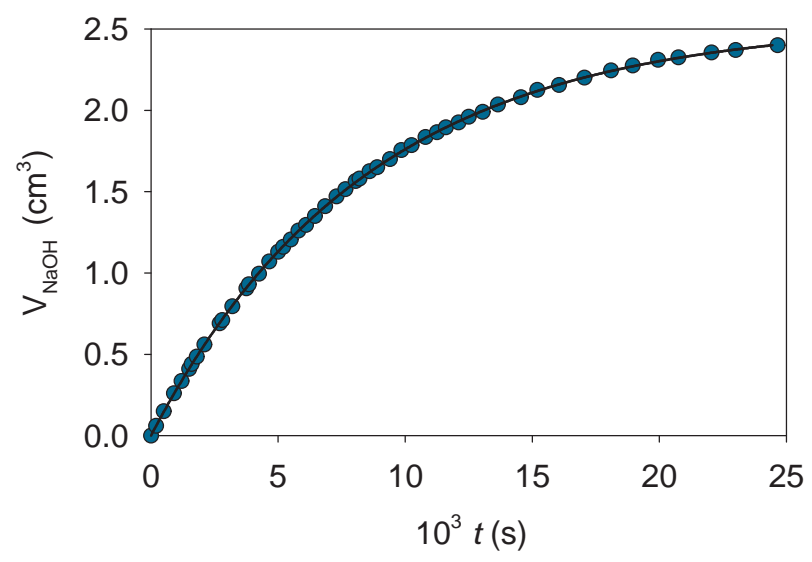

Figure 2.1. Fit of experimental data to eq. 2.5

Figure 2.1 shows the fit of experimental titration data to eq. 2.7

As shown in Figure 2.2, no acid-catalyzed hydrolysis of diketene was observed above $\mathrm{pH}=2$. The reaction is more sensitive to base catalysis, and catalysis by hydroxide ions is observed at $\mathrm{pH}$ values higher than 7. In order to obtain maximum signal and avoid the base-catalyzed region, experimental measurements were carried out at $\mathrm{pH}=6$.

Results obtained in a variety of $w / d$ media at different temperatures are gathered in Table 2.1.

It can be seen that, the rate constant of diketene hydrolysis increases with the fraction of water in the reaction medium. When compared to values for other $\beta$-lactones, diketene hydrolyzes about two-fold faster than BPL and $\sim 20$ times faster than BBL. $\left(k_{\mathrm{H}_{2} \mathrm{O}}^{\mathrm{DIK}}\right.$ being respectively $8.1 \times 10^{-7}$ and $1.3 \times 10^{-7} \mathrm{M}^{-1} \mathrm{~s}^{-1}$ in $7: 3 \mathrm{w} / \mathrm{d}$ at $25^{\circ} \mathrm{C}$ ) 


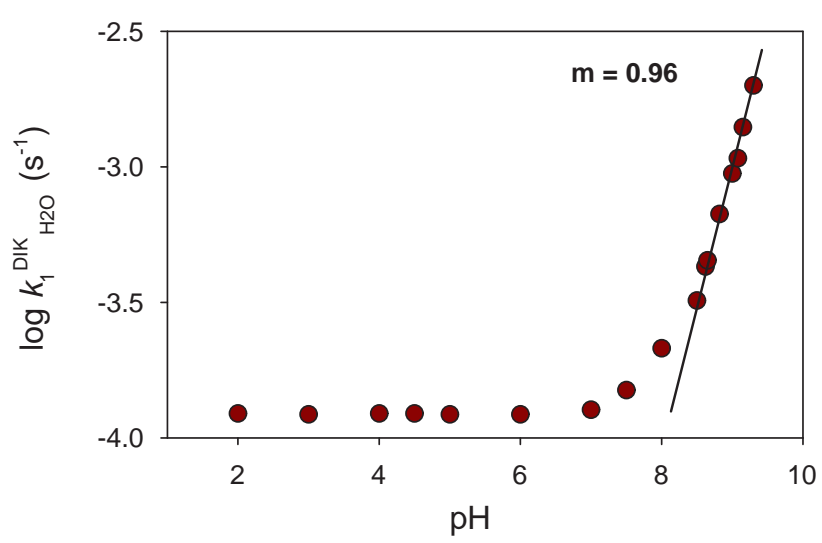

Figure 2.2. Variation in $k_{1}^{\mathrm{DIK}} \mathrm{H} \mathrm{O}^{\mathrm{O}}$ with $\mathrm{pH}$

Table 2.1. Rate constants for the hydrolysis of DIK in neutral $w / d$ media

\begin{tabular}{|c|c|c|c|c|c|}
\hline \multirow{2}{*}{$w / d$} & \multicolumn{5}{|c|}{$10^{6} \times k_{\mathrm{H}_{2} \mathrm{O}}^{\mathrm{DIK}}\left(\mathrm{M}^{-1} \mathrm{~s}^{-1}\right)^{a}$} \\
\hline & $15^{\circ} \mathrm{C}$ & $20^{\circ} \mathrm{C}$ & $25^{\circ} \mathrm{C}$ & $30^{\circ} \mathrm{C}$ & $35^{\circ} \mathrm{C}$ \\
\hline $4: 6$ & 0.292 & 0.495 & 0.810 & 1.35 & 2.21 \\
\hline $5: 5$ & 0.396 & 0.749 & 1.21 & 2.03 & 3.24 \\
\hline $6: 4$ & 0.575 & 0.904 & 1.43 & 2.08 & 3.30 \\
\hline $7: 3$ & 0.856 & 1.30 & 2.03 & 3.06 & 4.65 \\
\hline $8: 2$ & 1.17 & 1.84 & 2.77 & 4.18 & 6.19 \\
\hline $9: 1$ & 1.52 & 2.25 & 3.54 & 5.16 & 7.49 \\
\hline 10:0 & 1.86 & 2.95 & 4.50 & 6.15 & 9.37 \\
\hline
\end{tabular}

${ }^{a}$ Values are reproducible to $2 \%$

In their 1965 work, Briody and Satchell studied the neutral hydrolysis of diketene in $6: 4 \mathrm{w} / \mathrm{d}$, and found a $k_{\mathrm{H}_{2} \mathrm{O}}^{\mathrm{DIK}}$ value at $25^{\circ} \mathrm{C}$ of $1.0 \times 10^{-6} \mathrm{M}^{-1} \mathrm{~s}^{-1}$, which is about two thirds of the one here determined. ${ }^{42}$ The authors froze the hydrolysis reaction with the competitive reaction of DIK with aniline. Since this reaction is only moderately faster, they waited one hour for completion before spectrophotometric analysis. This freezing process must imply an appreciable imprecision in their results.

In 1984 Okeya and Kawaguchi studied the sodium tetracholoropalladate-catalyzed hydrolysis of diketene. ${ }^{43}$ In aqueous media at $20{ }^{\circ} \mathrm{C}$ and an ionic strength of $I=1.0 \mathrm{M}$ they found the value $k_{\mathrm{H}_{2} \mathrm{O}}^{\text {DIK }}=$ $1.5 \times 10^{-6} \mathrm{M}^{-1} \mathrm{~s}^{-1}$. This is about $50 \%$ of the value hereby reported. The authors point the rate constant decreases to approximately one half when the ionic strength is increased from 0 to 1 , so this likely to be the cause for the discrepancy.

The difference between our value and that of Van Duuren et al. is larger and of the opposite sign. At $25^{\circ} \mathrm{C}$ in neutral aqueous medium, they found $k_{\mathrm{H}_{2} \mathrm{O}}^{\mathrm{DIK}}=3.6 \times 10^{-5} \mathrm{M}^{-1} \mathrm{~s}^{-1}$, which is in strong disagreement 
with both results in the literature and those hereby presented. ${ }^{44}$

Table 2.2 shows the values of the activation parameters obtained from fitting rate constant values (Table 2.1) to the Eyring-Wynne-Jones equation:

$$
k=\frac{\mathbf{k} T}{h} K_{e}^{\ddagger}=\frac{\mathbf{k} T}{h} e^{-\frac{\Delta^{\ddagger} G^{\ominus}}{R T}}=\frac{\mathbf{k} T}{h} \exp -\frac{\Delta^{\ddagger} H^{\ominus}}{R T} \exp -\frac{\Delta^{\ddagger} S^{\ominus}}{R}
$$

Table 2.2. Activation parameters for the hydrolysis of DIK in neutral water/dioxane media

\begin{tabular}{|c|c|c|c|}
\hline$w / d$ & $\begin{array}{c}E_{\mathrm{a}} \\
\left(\mathrm{kJ} \mathrm{mol}^{-1}\right)\end{array}$ & $\begin{array}{c}\Delta^{\ddagger} H^{\ominus} \\
\left(\mathrm{kJ} \mathrm{mol}{ }^{-1}\right)\end{array}$ & $\begin{array}{c}-\Delta^{\ddagger} S^{\ominus} \\
\left(\mathrm{J} \mathrm{mol}^{-1} \mathrm{~K}^{-1}\right)\end{array}$ \\
\hline $4: 6$ & $75 \pm 2$ & $72 \pm 2$ & $119 \pm 4$ \\
\hline $5: 5$ & $77 \pm 2$ & $74 \pm 2$ & $108 \pm 6$ \\
\hline $6: 4$ & $62 \pm 2$ & $61 \pm 2$ & $151 \pm 3$ \\
\hline $7: 3$ & $61 \pm 1$ & $60 \pm 1$ & $152 \pm 2$ \\
\hline $8: 2$ & $58 \pm 1$ & $59 \pm 1$ & $154 \pm 1$ \\
\hline $9: 1$ & $59 \pm 1$ & $57 \pm 1$ & $158 \pm 3$ \\
\hline $10: 0$ & $59 \pm 2$ & $56 \pm 2$ & $159 \pm 6$ \\
\hline
\end{tabular}

Our results are in reasonable agreement with those in the literature: Briody and Satchell obtained a value of $\Delta^{\ddagger} H^{\ominus}=53 \mathrm{~kJ} / \mathrm{mol}$ in 6:4 $\mathrm{w} / \mathrm{d}$ medium, although they worked only at two different temperatures. The large negative activation entropies suggest that the transition state is highly ordered, and thus, that the lactone ring is not open at that point. The little variation in $\Delta^{\ddagger} S^{\ominus}$ with the $w / d$ ratio suggests that solvation has little influence on this effect.

Table 2.3. Activation parameters for the neutral hydrolysis of some $\beta$-lactones in water ${ }^{56}$

\begin{tabular}{|c|c|c|c|}
\hline Lactone & $\begin{array}{c}\Delta^{\ddagger} H^{\ominus} \\
\left(\mathrm{kJ} \mathrm{mol}{ }^{-1}\right)\end{array}$ & $\begin{array}{c}-\Delta^{\ddagger} S^{\ominus} \\
\left(\mathrm{J} \mathrm{mol}^{-1} \mathrm{~K}^{-1}\right)\end{array}$ & $\begin{array}{c}\Delta^{\ddagger} G^{\ominus}\left(35^{\circ} \mathrm{C}\right) \\
\left(\mathrm{kJ} \mathrm{mol}^{-1}\right)\end{array}$ \\
\hline BBL & $83 \pm 1$ & $98 \pm 3$ & $113 \pm 1$ \\
\hline BPL & $79 \pm 1$ & $95 \pm 3$ & $108 \pm 1$ \\
\hline DIK & $56 \pm 1$ & $159 \pm 5$ & $105 \pm 1$ \\
\hline
\end{tabular}

On comparing the activation parameters to those of the carcinogenic $\beta$-lactones BPL and BBL (Table 2.3) it may be seen that the activation free energy for the hydrolysis of diketene is slightly smaller than that of BPL, and thus, that DIK is slightly more reactive. The hydrolysis of diketene is only slightly faster than that of BPL and about one order of magnitude faster than that of BBL ( $k_{\mathrm{H} 2 \mathrm{O}}$ being $1.1 \times 10^{-6}$ and $0.2 \times 10^{-6} \mathrm{M}^{-1} \mathrm{~s}^{-1}$, respectively as compared to $4.5 \times 10^{-6} \mathrm{M}^{-1} \mathrm{~s}^{-1}$ for diketene in pure water at $\left.25^{\circ} \mathrm{C}\right) .{ }^{56}$ Interestingly, there are large differences in $\Delta^{\ddagger} H^{\ominus}$ and $\Delta^{\ddagger} S^{\ominus}$ between DIK and BPL: the activation enthalpy is much lower in the case of DIK, suggesting a lower energy barrier and the activation entropy is 
more negative, suggesting a more ordered transition state. These two effects are somewhat compensated, yielding very similar activation free energies for the two lactones.

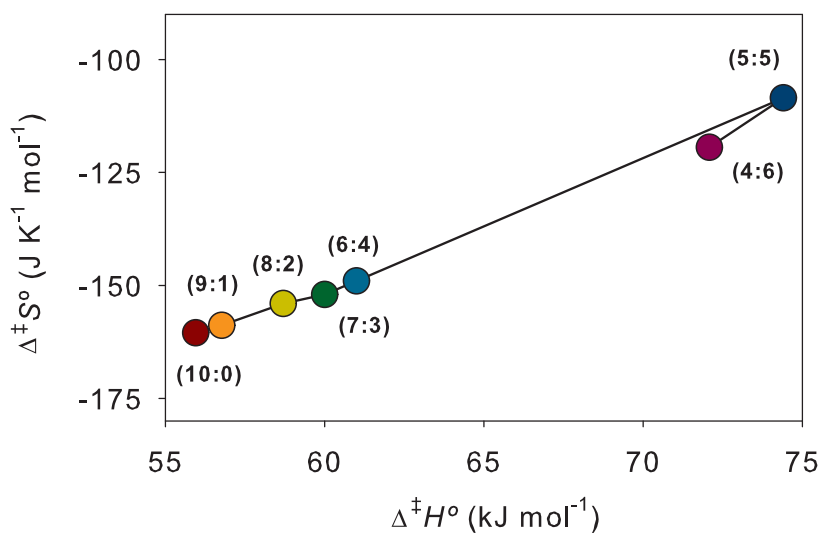

Figure 2.3. $\Delta^{\ddagger} H^{\ominus} / \Delta^{\ddagger} S^{\ominus}$ relationship for $k_{\mathrm{H}_{2} \mathrm{O}}^{\mathrm{DIK}}$ in the different $w / d$ media

Figure 2.3 shows the existence of an isokinetic $\Delta^{\ddagger} H^{\ominus} / \Delta^{\ddagger} S^{\ominus}$ relationship for $k_{\mathrm{H}_{2} \mathrm{O}}^{\mathrm{DIK}}$ in the different media. The existence of the isokinetic relationship has been reported for many reaction series, in particular those that serve to support the common theoretical conceptions. It can serve as an argument - not a proof - that the reactions in question show some similarity, or a common feature; in this case a common mechanism in all the water/dioxane media hereby investigated .

It can be seen that the $\Delta^{\ddagger} H^{\ominus} / \Delta^{\ddagger} S^{\ominus}$ correlation shows a large difference for the activation parameters for 6:4 and 5:5 $w / d$ media. This correlates with abrupt changes in the properties of the water-dioxane mixtures, taking place around a ratio of $6: 4$ vol. or $\sim 0.1$ dioxane molar fraction $\left(X_{\text {Diox }}\right)$; for instance, the melting points and the heats of mixing of the mixtures show minima at approximately $6: 4 .{ }^{57}$

These sudden changes correlate with the structure of the mixtures; the number of hydrogen bonds, per water and dioxane oxygen atom, estimated from X-Ray data, show an inflection point at about $X_{\text {Diox }} \simeq 0.1$. At $X_{\text {Diox }}<0.1$, the structure of the solution is that of water, while at $0.1<X_{\text {Diox }}<0.2$ the formation of small binary clusters consisting of one or two dioxane molecules and several water molecules is observed. ${ }^{58}$

The parameters for the 4:6 medium show an inversion of the observed tendencies, suggesting that the $\Delta^{\ddagger} H^{\ominus} / \Delta^{\ddagger} S^{\ominus}$ relationships become complex for media with a higher dioxane ratio. A similar behavior has been observed in the case of BPL and BBL, both of which have N-shaped $\Delta^{\ddagger} H^{\ominus} / \Delta^{\ddagger} S^{\ominus}$ plots for neutral hydrolysis in water-dioxane mixtures. This curve appears at a $w / d$ ratio of approximately 5:5, and has been classified in terms of the shape of the $\Delta^{\ddagger} H^{\ominus} / \Delta^{\ddagger} S^{\ominus}$ plot as ${ }_{20} \mathrm{~A}_{35} \mathrm{C}_{40} \mathrm{~A}_{100} .{ }^{59}$ 


\subsubsection{Base hydrolysis}

If the base hydrolysis of diketene is supposed to be first order with respect to hydroxide ions, the differential rate equation for the process can be written to be eq. 2.9. Since hydroxide is in a large excess, the pseudo-order approximation was applied.

$$
\begin{aligned}
r & =\frac{d\left[\mathrm{AcAc}^{2-}\right]}{d t}=-\frac{d[\mathrm{DIK}]}{d t}=k_{\mathrm{OH}}^{\mathrm{DIK}}\left[\mathrm{OH}^{-}\right][\mathrm{DIK}] \\
& =k_{1 \mathrm{OH}}^{\mathrm{DIK}}[\mathrm{DIK}]=k_{1 \mathrm{OH}}^{\mathrm{DIK}}\left([\mathrm{DIK}]_{0}-\left[\mathrm{AcAc}^{2-}\right]\right)
\end{aligned}
$$

$\left[\mathrm{AcAc}^{2-}\right]$ stands for the concentration of acetoacetate dianion at a time $t, k_{\mathrm{OH}}{ }^{\mathrm{DIK}}$ is the bimolecular hydrolysis rate constant and $[\mathrm{DIK}]_{0}$ represents the initial concentration of diketene. $k_{1 \mathrm{OH}}^{\mathrm{DIK}}$ stands for the product of the bimolecular rate constant by hydroxide concentration, and $k_{\text {expOH }}^{\mathrm{DIK}}$ is the experimental rate constant for the base hydrolysis of diketene:

$$
k_{\operatorname{expOH}}^{\mathrm{DIK}}=k_{1 \mathrm{OH}}^{\mathrm{DIK}}=k_{\mathrm{OH}}^{\mathrm{DIK}}\left[\mathrm{OH}^{-}\right]
$$

Integration of eq. 2.9 yields eq. 2.11 and 2.12 .

$$
\begin{aligned}
{\left[\operatorname{AcAc}^{2-}\right] } & =[\mathrm{DIK}]_{\circ}\left(1-e^{-k_{\operatorname{expOH}}^{\mathrm{DIK}} t}\right) \\
{[\mathrm{DIK}] } & =[\mathrm{DIK}]_{0} e^{-k_{\operatorname{expOH}}^{\mathrm{DIK}} t}
\end{aligned}
$$

Figure 2.4 shows a typical kinetic run, and the excellent fit of experimental data to eq. 2.11

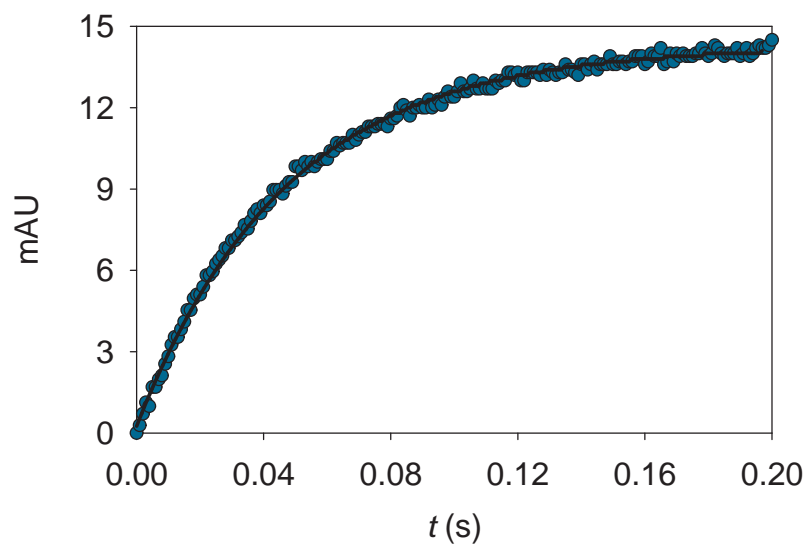

Figure 2.4. Typical kinetic run of alkaline hydrolysis of DIK: Fit of experimental absorption data to eq. $2.11(\lambda=400 \mathrm{~nm})$

However, when $\log k_{\text {expOH }}^{\mathrm{DIK}}$ values are plotted against the logarithm of hydroxide concentration (Figure 2.5), somewhat curved plots are obtained and the obtained reaction orders are less than unity.

This is hardly a consequence of the ionic intensity, since its value is fixed; and the curvature is apparent at low concentrations of hydroxide, which discards an specific ion effect. We propose that, since 
<smiles>C=C1CC(=O)OC1=C[CH-]C(C)=O</smiles>

Scheme 2.4. Alkaline hydrolysis of DIK.

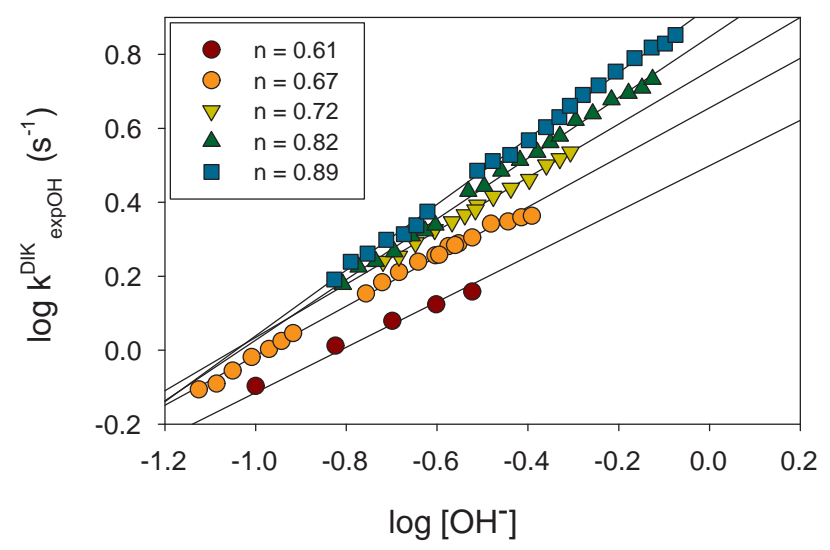

Figure 2.5. Reaction order for $k_{\text {expOH }}^{\mathrm{DIK}} . w / d 4: 6(\bullet) ; 5: 5(\bullet) ; 6: 4(\nabla) ; 7: 3(\boldsymbol{\Delta}) ; 8: 2(\boldsymbol{\square})$

diketene is a $\beta, \gamma$-unsaturated carbonyl, its $\alpha$ hydrogen is highly acidic and dissociates at low $\mathrm{pH}$ values. This reaction mechanism is shown in the Scheme 2.4. Since the dissociated form of diketene is negatively charged, it must show very limited reactivity towards hydroxide ions. This is in keeping with the observation that the increased lability of the $\alpha$ hydrogen of lactones is not accompanied by a change in the addition-elimination to the elimination-addition mechanism. ${ }^{60}$ If we assume that $\mathrm{DIK}^{-}$does not react significantly with $\mathrm{OH}^{-}$and we define $K_{\mathrm{b}}^{\mathrm{DIK}}$ as in eq. 2.13

$$
K_{\mathrm{b}}^{\mathrm{DIK}}=\frac{\left[\mathrm{DIK}^{-}\right]}{[\mathrm{DIK}]\left[\mathrm{OH}^{-}\right]}
$$

we have that, when the dissociated fraction of diketene is accounted for, the differential rate equation is:

$$
r=k_{\mathrm{OH}}^{\mathrm{DIK}}\left[\mathrm{OH}^{-}\right] \frac{[\mathrm{DIK}]_{\mathrm{Tot}}}{1+K_{\mathrm{b}}^{\mathrm{DIK}}\left[\mathrm{OH}^{-}\right]}=\frac{k_{1 \mathrm{OH}}^{\mathrm{DIK}}[\mathrm{DIK}]_{T o t}}{1+K_{\mathrm{b}}^{\mathrm{DIK}}\left[\mathrm{OH}^{-}\right]}
$$

eq. 2.14 can be integrated to yield eq. 2.15

$$
\left[\mathrm{AcAc}^{2-}\right]=[\mathrm{DIK}]_{\circ}\left(1-\exp \left(-\frac{k_{1 \mathrm{OH}}^{\mathrm{DIK}}}{1+K_{\mathrm{b}}^{\mathrm{DIK}}\left[\mathrm{OH}^{-}\right]} t\right)\right)
$$

And thus,

$$
k_{\operatorname{expOH}}^{\mathrm{DIK}}=\frac{k_{1 \mathrm{OH}}^{\mathrm{DIK}}}{1+K_{\mathrm{b}}^{\mathrm{DIK}}\left[\mathrm{OH}^{-}\right]}=k_{\mathrm{OH}}^{\mathrm{DIK}} \frac{\left[\mathrm{OH}^{-}\right]}{1+K_{\mathrm{b}}^{\mathrm{DIK}}\left[\mathrm{OH}^{-}\right]}
$$


Fitting $k_{\operatorname{expOH}}^{\mathrm{DIK}}$ values to eq. 2.16 (Figure 2.6) affords both $k_{\mathrm{OH}}^{\mathrm{DIK}}$ (Table 2.4) and $K_{\mathrm{b}}^{\mathrm{DIK}}$ (Table 2.5)

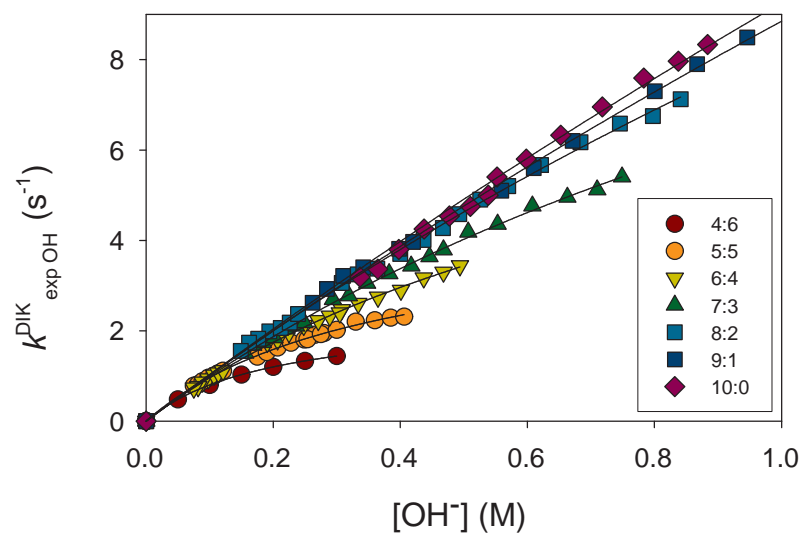

Figure 2.6. Variation in $k_{\mathrm{OH}}^{\mathrm{DIK}}$ with $\left[\mathrm{OH}^{-}\right]$and $w / d$ ratio

Table 2.4. Rate constants for the hydrolysis of DIK in alkaline $\mathrm{w} / \mathrm{d}$ media

\begin{tabular}{|c|c|c|c|c|c|}
\hline \multirow{2}{*}{$w / d$} & \multicolumn{5}{|c|}{$k_{\mathrm{OH}}^{\mathrm{DIK}}\left(\mathrm{M}^{-1} \mathrm{~s}^{-1}\right)^{a}$} \\
\hline & $15^{\circ} \mathrm{C}$ & $20^{\circ} \mathrm{C}$ & $25^{\circ} \mathrm{C}$ & $30^{\circ} \mathrm{C}$ & $35^{\circ} \mathrm{C}$ \\
\hline $4: 6$ & 7.5 & 12.0 & 21.0 & 32.1 & 50.5 \\
\hline $5: 5$ & 7.4 & 11.6 & 18.7 & 29.8 & 43.4 \\
\hline $6: 4$ & 7.8 & 10.9 & 15.8 & 22.9 & 32.3 \\
\hline $7: 3$ & 7.7 & 11.1 & 15.5 & 23.2 & 31.1 \\
\hline $8: 2$ & 7.9 & 10.5 & 15.0 & 23.0 & 31.6 \\
\hline $9: 1$ & 7.7 & 10.4 & 15.1 & 22.1 & 29.6 \\
\hline 10:0 & 7.5 & 10.4 & 15.0 & 21.6 & 28.7 \\
\hline
\end{tabular}

${ }^{a}$ Values are reproducible to $1 \%$

Table 2.4 shows that reaction medium has little influence on the base hydrolysis rate. At low temperatures, the variation lies within the experimental error and, at higher temperatures, the values of the base hydrolysis rate constants increase as the polarity of the reaction medium decreases. A larger water content implies more intense solvation of both reactant and transition state, and thus, a possible interpretation is that the increase in polarity would stabilize the transition state less than the reactants, since the charge is more delocalized in the transition state. This would imply an increase in the activation free energy, and thus, a decrease in reaction rate.

Literature dealing with the base hydrolysis of $\beta$-lactones is scarce. In the particular case of diketene, no references were found, aside from the bald information that above $\mathrm{pH}=9$ the hydroxide-catalyzed reaction takes place, and that it was too fast to be measured. ${ }^{42}$

In their review of lactone hydrolysis, Kaiser and Kezdy pointed a value of $k_{\mathrm{OH}} \simeq 1.2 \mathrm{M}^{-1} \mathrm{~s}^{-1}$ at 
$0{ }^{\circ} \mathrm{C}$ which could be compared with the extrapolated value of $2.5 \mathrm{M}^{-1} \mathrm{~s}^{-1}$ in this work. ${ }^{61}$ Blackburn and Dodds found $k_{\mathrm{OH}}^{\mathrm{BPL}}=8.8 \mathrm{M}^{-1} \mathrm{~s}^{-1}$ at $46{ }^{\circ} \mathrm{C}$, quite less that the extrapolated value of $57 \mathrm{M}^{-1} \mathrm{~s}^{-1}$ for diketene, which is coherent with the greater reactivity of the latter. ${ }^{62}$

Long and Purchase found $k_{\mathrm{OH}}^{\mathrm{BPL}}=0.28 \mathrm{M}^{-1} \mathrm{~s}^{-1}$ at $0^{\circ} \mathrm{C}$ and 0.49 at $\mathrm{M}^{-1} \mathrm{~s}^{-1}$ at $6.9^{\circ} \mathrm{C}$, also inferior to the extrapolated values for diketene (2.5 and 3.7). ${ }^{63}$ Barlett and Small give $\left(1.2 \mathrm{M}^{-1} \mathrm{~s}^{-1} \text { for BPL at } 25^{\circ} \mathrm{C}\right)^{64}$

Olson and Miller published $k_{\mathrm{OH}}^{\mathrm{BBL}}=0.8 \mathrm{M}^{-1} \mathrm{~s}^{-1}$ at $25^{\circ} \mathrm{C}$, which is about 20 -fold smaller that the value here obtained $\left(15 \mathrm{M}^{-1} \mathrm{~s}^{-1}\right)$, once again in agreement with the enhanced reactivity of diketene when compared to other $\beta$-lactones. ${ }^{65}$

Table 2.5. Base dissociation constants for DIK in w/d media

\begin{tabular}{ccc}
\cline { 2 - 3 }$\frac{w / d}{4: 6}$ & & $\frac{K_{\mathrm{b}}^{\mathrm{DIK}}\left(\mathrm{M}^{-1}\right)^{a}}{5.0}$ \\
$5: 5$ & & 2.6 \\
$6: 4$ & 1.1 \\
$7: 3$ & 0.64 \\
$8: 2$ & 0.36 \\
$9: 1$ & 0.13 \\
10:0 & 0.07 \\
\hline Values are reproducible to $20 \%$
\end{tabular}

$K_{\mathrm{b}}^{\text {DIK }}$ was found to show no variation with temperature, within experimental error. Table 2.5 shows that the equilibrium constant, and thus the acidity of diketene, increases with the dioxane ratio. This result can be explained by the loss of solvation that hydroxide ions undergo as the polarity of the medium decreases. Their charges are then more stable in diketene, delocalized in the conjugated $\pi$ sistem of the methylene and carbonyl groups. This $K_{\mathrm{b}}^{\mathrm{DIK}}$ implies that $\mathrm{p} K_{\mathrm{a}}^{\mathrm{DIK}} \simeq 15$ in water, which is unexpectedly acidic for the $\alpha$-hydrogen of a lactone. This particularity of diketene will be studied in depth in following sections (vide infra).

When $k_{\mathrm{OH}}^{\mathrm{DIK}}$ are fitted to eq. 2.8, the following activation parameters are obtained (Table 2.6)

The observed trend in the activation enthalpy is in keeping with the general results for the alkaline hydrolysis of esters (vide supra): $\Delta^{\ddagger} H^{\ominus}$ increases with the amount of organic co-solvent. An increase is seen in $-\Delta^{\ddagger} S^{\ominus}$, also in keeping with the effects observed in the hydrolysis of other esters, such as, ethyl benzoates.

Blackburn and Dodds found $\Delta^{\ddagger} H^{\ominus}=50 \mathrm{~kJ} \mathrm{~mol}^{-1}$ and $\Delta^{\ddagger} S^{\ominus}=-70.5 \mathrm{~J} \mathrm{~mol}^{-1} \mathrm{~K}^{-1}$ for BPL, which are consistent with the slightly more favorable values for more reactive diketene $\left(\Delta^{\ddagger} H^{\ominus}=47.4 \mathrm{~kJ} \mathrm{~mol}^{-1}\right.$ and $\left.\Delta^{\ddagger} S^{\ominus}=-63 \mathrm{~J} \mathrm{~mol}^{-1} \mathrm{~K}^{-1}\right) .{ }^{62}$ Previous $\Delta^{\ddagger} H^{\ominus}$ values for BPL and BBL are 45.2 and $58.5 \mathrm{~kJ} \mathrm{~mol}^{-1}$, and are also consistent with a similar mechanism involving acyl-oxygen cleavage for DIK. 
Table 2.6. Activation parameters for the hydrolysis of DIK in alkaline $w / d$ media

\begin{tabular}{|c|c|c|c|}
\hline$w / d$ & $\begin{array}{c}E_{\mathrm{a}} \\
\left(\mathrm{kJ} \mathrm{mol}^{-1}\right)\end{array}$ & $\begin{array}{c}\Delta^{\ddagger} H^{\ominus} \\
\left(\mathrm{kJ} \mathrm{mol}{ }^{-1}\right)\end{array}$ & $\begin{array}{c}-\Delta^{\ddagger} S^{\ominus} \\
\left(\mathrm{J} \mathrm{mol}^{-1} \mathrm{~K}^{-1}\right)\end{array}$ \\
\hline $4: 6$ & $69.1 \pm 1.0$ & $66.6 \pm 1.0$ & $-4 \pm 1$ \\
\hline $5: 5$ & $63.4 \pm 0.2$ & $60.9 \pm 0.2$ & $16 \pm 1$ \\
\hline $6: 4$ & $52.8 \pm 0.3$ & $50.3 \pm 0.3$ & $53 \pm 1$ \\
\hline $7: 3$ & $52.6 \pm 0.8$ & $50.2 \pm 0.8$ & $54 \pm 3$ \\
\hline $8: 2$ & $51.8 \pm 1.4$ & $49.4 \pm 1.4$ & $56 \pm 5$ \\
\hline $9: 1$ & $50.6 \pm 0.8$ & $48.1 \pm 0.8$ & $61 \pm 3$ \\
\hline 10:0 & $49.9 \pm 0.6$ & $47.4 \pm 0.6$ & $63 \pm 2$ \\
\hline
\end{tabular}

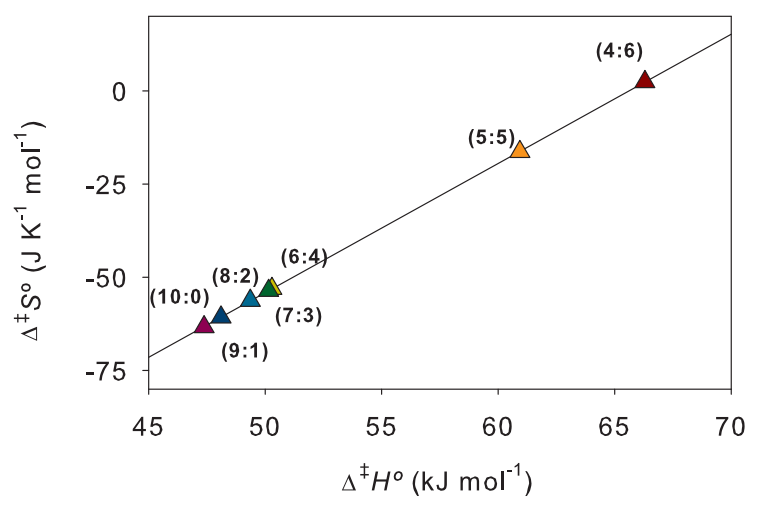

Figure 2.7. $\Delta^{\ddagger} H^{\ominus} / \Delta^{\ddagger} S^{\ominus}$ relationship for $k_{\mathrm{OH}}^{\mathrm{DIK}}$ in the different $w / d$ media

As shown in Figure $2.7(\boldsymbol{\Delta})$, a $\Delta^{\ddagger} H^{\ominus} / \Delta^{\ddagger} S^{\ominus}$ relationship exists for $k_{\mathrm{OH}}^{\mathrm{DIK}}$ in the different media, suggesting that base-hydrolysis mechanism is the same in all the water-dioxane media. As was the case with the neutral hydrolysis, large differences in the activation parameters are observed at $w / d$ ratios 4:6 and 5:5, but not the inversion of the observed tendencies.

\subsubsection{Alkylating potential of diketene}

The aduct formed in the reaction of NBP and DIK was detected using negative-mode mass spectrometry, both in aquo-organic mixtures and in pure dioxane. The experimental value $m / z=297.1$ was obtained. Comparison with the calculated value $(\mathrm{M}-\mathrm{H}=297.10)$ is coherent with adduct depicted in the Scheme 2.5 .

Figure 2.8 a) shows the variation in the UV-Vis spectrum of the reaction mixture along time, and in Figure $2.8 \mathrm{~b}$ ) a typical kinetic run of the alkylation reaction is compared to that of hydrolysis in the same conditions. Since the alkylation reaction is much faster, the competing hydrolysis reaction of DIK during 
<smiles>C=C1CC(=O)OC1=CCc1ccc([N+](=O)[O-])cc1</smiles>

Scheme 2.5. Alkylation reaction of NBP by DIK
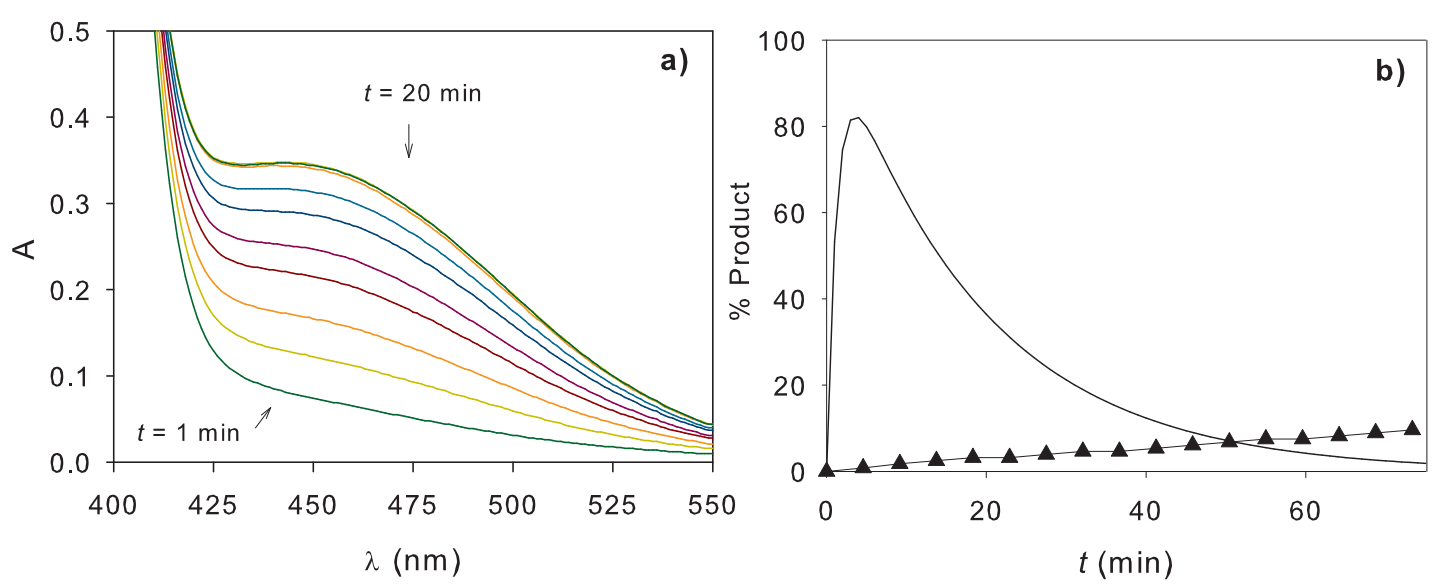

Figure 2.8. a) Variation in the UV-Vis spectra of the NBP-DIK alkylation mixture.

b) Typical kinetic profile at $\lambda=450 \mathrm{~nm}(-)$ and comparison to DIK hydrolysis in the same conditions $(\boldsymbol{\Lambda})$

alkylation was neglected. It can be also observed that the NBP-DIK adduct is unstable. Since its decomposition yields AcAcH and NBP, (Scheme 2.5) we can write the following differential rate equations.

$$
\begin{aligned}
\frac{d[\mathrm{DIK}]}{d t} & =-k_{\mathrm{alk}}^{\mathrm{DIK}}[\mathrm{DIK}][\mathrm{NBP}] \\
\frac{d[\mathrm{NBP}]}{d t} & =-k_{\mathrm{alk}}^{\mathrm{DIK}}[\mathrm{DIK}][\mathrm{NBP}]+k_{\mathrm{dec}}^{\mathrm{AD}_{\mathrm{DIK}}}\left[\mathrm{AD}_{\mathrm{NBP}}^{\mathrm{DIK}}\right] \\
\frac{d\left[\mathrm{AD}_{\mathrm{NBP}}^{\mathrm{DIK}}\right]}{d t} & =k_{\mathrm{alk}}^{\mathrm{DIK}}[\mathrm{DIK}][\mathrm{NBP}]-k_{\mathrm{dec}}^{\mathrm{AD}_{\mathrm{DIK}}}\left[\mathrm{AD}_{\mathrm{NBP}}^{\mathrm{DIK}}\right]
\end{aligned}
$$

Since NBP was present in a large excess, the pseudo-order approximation was applied and its concentration was assumed to remain constant along the reaction; hence eqs. $2.17-2.19$ can be converted to eqs. 2.20 and 2.21, where $k_{1 \mathrm{alk}}^{\mathrm{DIK}}=k_{\mathrm{alk}}^{\mathrm{DIK}}[\mathrm{NBP}]$ 


$$
\begin{gathered}
\frac{d[\mathrm{DIK}]}{d t}=-k_{1 \mathrm{alk}}^{\mathrm{DIK}}[\mathrm{DIK}] \\
\frac{d\left[\mathrm{AD}_{\mathrm{NBP}}^{\mathrm{DIK}}\right]}{d t}=k_{1 \mathrm{alk}}^{\mathrm{DIK}}[\mathrm{DIK}]-k_{\mathrm{dec}}^{\mathrm{AD}_{\mathrm{DIK}}}\left[\mathrm{AD}_{\mathrm{NBP}}^{\mathrm{DIK}}\right]
\end{gathered}
$$

Integration of eqs 2.20 and 2.21 and expression in terms of absorbance yield

$$
A_{\mathrm{AD}}^{\mathrm{DIK}}=\epsilon l \frac{k_{1 \mathrm{alk}}^{\mathrm{DIK}}}{k_{\mathrm{dec}}^{\mathrm{AD} \text { DIK }}-k_{1 \mathrm{alk}}^{\mathrm{DIK}}}[\mathrm{DIK}]_{\circ}\left(e^{-k_{\text {alk }}^{\mathrm{DIK}} t}-e^{-k_{\mathrm{dec}}^{\mathrm{AD} \mathrm{DIK}_{t}} t}\right)
$$

By fitting experimental data to eq. 2.22, $k_{1 \mathrm{alk}}^{\mathrm{DIK}}, k_{\mathrm{dec}}^{\mathrm{AD} \mathrm{D}_{\mathrm{DIK}}}$ and $\epsilon$ values were obtained.

The proposed reaction mechanism holds that NBP is regenerated along the reactions. Thus, once the reaction is over, all DIK has consumed and is present as AcAcH, and NBP remains unreacted. To confirm that no NBP is consumed along the reaction, we performed the alkyation in excess DIK. In these conditions, when the reaction is finished, addition of more DIK to the exhausted reaction mixtured started the reaction again with a similar kinetic profile (see Figure 2.9), which shows that NBP was still present in the reaction mixture. The large increase in absorption at the end of the reaction is due to the low, but non-zero molar absorption coefficient of AcAcH. Whereas this contribution can be neglected in the usual conditions, in excess DIK, a large increase in absorption is observed at the end of the reaction.

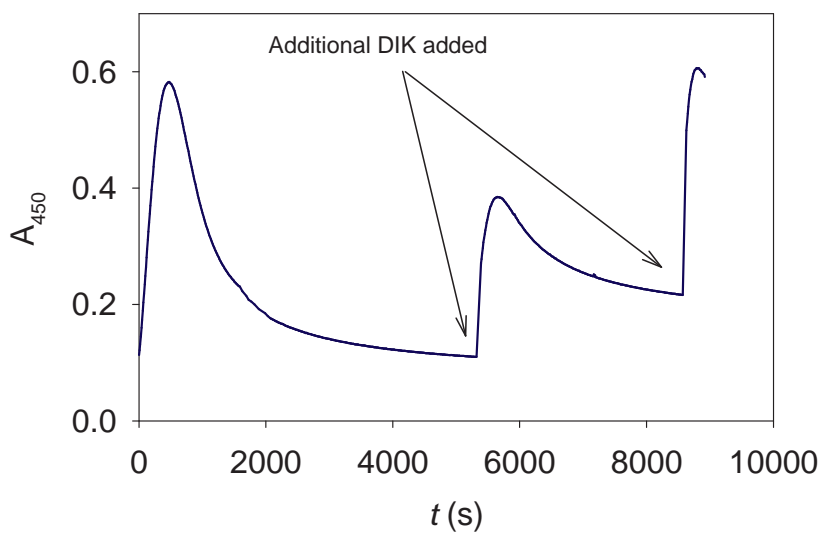

Figure 2.9. Formation and decomposition of the NBP-DIK adduct in excess DIK. Additional DIK was inserted at $t=$ $5500 \mathrm{~s}$ and $t=8500 \mathrm{~s}$

The proposed reaction mechanism is also supported by the fact that, when the reaction is carried out in excess DIK, the reaction profiles do not fit the rate equations derived from either of these reaction mechanisms:

$$
\begin{aligned}
& \mathrm{NBP} \rightarrow \mathrm{AD} \rightarrow \text { Products } \\
& \mathrm{NBP} \rightleftharpoons \mathrm{AD} \rightarrow \text { Products }
\end{aligned}
$$




$$
\mathrm{NBP} \rightarrow \mathrm{AD} \rightleftharpoons \text { Products }
$$

However, reaction profiles do fit to the numerical solution of 2.19 , which lacks an analytical solution in those conditions.

From the standpoint of net converstion of DIK to AcAcH, the whole alkylation and adduct decomposition process, can be considered as the nucleophile-catalyzed hydrolysis of DIK. Briody and Satchell have reported that pyridine is a nucleophilic catalyst for the hydrolysis of DIK, ${ }^{41,42}$ and NBP, as a pyridine derivative, is likely to have a similar effect. That the final product is AcAcH has been confirmed by UV-Vis spectra and by the decrease in $\mathrm{pH}$ after adduct hydrolysis.

\subsubsection{Formation of the DIK-NBP adduct}

The alkylation rate constants $\left(k_{\mathrm{alk}}^{\mathrm{DIK}}\right)$ in various $w / d$ at several temperatures are given in Table 2.7.

Table 2.7. $k_{\text {alk }}^{\mathrm{DIK}}$ rate constants for the alkylation of NBP by DIK

\begin{tabular}{|c|c|c|c|c|c|}
\hline \multirow[t]{2}{*}{$w / d$} & \multicolumn{5}{|c|}{$k_{\mathrm{alk}}^{\mathrm{DIK}}\left(\mathrm{M}^{-1} \mathrm{~s}^{-1}\right)^{a}$} \\
\hline & $15^{\circ} \mathrm{C}$ & $20^{\circ} \mathrm{C}$ & $25^{\circ} \mathrm{C}$ & $30^{\circ} \mathrm{C}$ & $35^{\circ} \mathrm{C}$ \\
\hline $3: 7$ & 0.035 & 0.047 & 0.061 & 0.073 & 0.094 \\
\hline $4: 6$ & 0.080 & 0.102 & 0.141 & 0.182 & 0.220 \\
\hline $5: 5$ & 0.156 & 0.195 & 0.260 & 0.321 & 0.410 \\
\hline $6: 4$ & 0.292 & 0.336 & 0.438 & 0.517 & 0.602 \\
\hline $7: 3$ & 1.05 & 1.32 & 1.51 & 1.95 & 2.30 \\
\hline
\end{tabular}

Alkylation rate constants for DIK are much higher than those of other $\beta$-lactones with higher alkylating potentials, e.g., in 7:3 (vol.) $w / d$ at $25^{\circ} \mathrm{C} k_{\text {alk }}^{\text {DIK }}$ values for BPL and BBL are $7.8 \times 10^{-3}$ and $6.7 \times 10^{-4} \mathrm{M}^{-1} \mathrm{~s}^{-1}$ respectively, as compared with $1.5 \mathrm{M}^{-1} \mathrm{~s}^{-1}$, i.e., a 200 -fold difference in the case of BPL and two thousandfold in the case of BBL. 66

The hydrolysis of DIK is very slow when compared to its alkylation reaction, and thus, $f$ (the percentage of alkylating agent that reacts with NBP and is not lost through the competing hydrolysis) is very close to unity for DIK. Carcinogenic lactones BPL and BBL have $f$ values around 0.8 , which suggests that, contrarily to the hypothesis of Van Duuren et al., 24,44 a very fast competing hydrolysis is not the cause for the lack of carcinogenicity of DIK.

The activation parameters for the alkylation of NBP by DIK are reported in Table 2.8. When compared to those of carcinogenic BPL and BBL (Table 2.9), the activation parameters suggest that DIK is a stronger alkylating agent (expressed as the alkylation rate constant), since the activation free energy is about 15 
Table 2.8. Activation parameters for the alkylation of NBP by DIK in water/dioxane media

\begin{tabular}{|c|c|c|c|}
\hline$w / d$ & $\begin{array}{c}E_{\mathrm{a}} \\
\left(\mathrm{kJ} \mathrm{mol}^{-1}\right)\end{array}$ & $\begin{array}{c}\Delta^{\ddagger} H^{\ominus} \\
\left(\mathrm{kJ} \mathrm{mol}^{-1}\right)\end{array}$ & $\begin{array}{c}-\Delta^{\ddagger} S^{\ominus} \\
\left(\mathrm{J} \mathrm{mol}^{-1} \mathrm{~K}^{-1}\right)\end{array}$ \\
\hline $3: 7$ & $36 \pm 1$ & $33 \pm 1$ & $158 \pm 4$ \\
\hline $4: 6$ & $39 \pm 2$ & $36 \pm 2$ & $140 \pm 5$ \\
\hline $5: 5$ & $36 \pm 1$ & $33 \pm 1$ & $144 \pm 3$ \\
\hline $6: 4$ & $28 \pm 2$ & $26 \pm 2$ & $167 \pm 5$ \\
\hline $7: 3$ & $29 \pm 2$ & $26 \pm 2$ & $152 \pm 5$ \\
\hline
\end{tabular}

Table 2.9. Activation parameters for the alkylation of NBP by some $\beta$-lactones in 7:3 water/dioxane media

\begin{tabular}{|c|c|c|c|}
\hline Lactone & $\begin{array}{c}\Delta^{\ddagger} H^{\ominus} \\
\left(\mathrm{kJ} \mathrm{mol}{ }^{-1}\right)\end{array}$ & $\begin{array}{c}-\Delta^{\ddagger} S^{\ominus} \\
\left(\mathrm{J} \mathrm{mol}^{-1} \mathrm{~K}^{-1}\right)\end{array}$ & $\begin{array}{c}\Delta^{\ddagger} G^{\ominus}\left(35^{\circ} \mathrm{C}\right) \\
\left(\mathrm{kJ} \mathrm{mol}{ }^{-1}\right)\end{array}$ \\
\hline $\mathrm{BPL}$ & $41 \pm 2$ & $148 \pm 6$ & $87 \pm 2$ \\
\hline BBL & $47 \pm 2$ & $148 \pm 6$ & $93 \pm 2$ \\
\hline DIK & $26 \pm 2$ & $156 \pm 5$ & $71 \pm 2$ \\
\hline
\end{tabular}

$\mathrm{kJ} \mathrm{mol}^{-1}$ less, due mainly to an enthalpic effect. However, we cannot talk about enthalpic control in the strict sense, since the alkylation reactions take place by different mechanisms: alkyl-oxygen fission for BPL and BBL and acyl-oxygen fission for DIK. The hydrolysis of DIK is slightly faster than that of BPL (vide supra), but its alkylation reaction is some hundred of times fasters. The comparatively slow hydrolysis of DIK gives this compound a higher alkylation efficiency (expressed as $f$ ) than BPL or BBL. Thus, it is reasonable to assume that the lack of genotoxicity of DIK is not due to its fast hydrolysis, but possibly to the lack of stability of the formed adducts, which decompose into non-toxic acetoacetic acid and the unreacted nucleotide.

We have computed the activation free energies for the acyl- and alkyl-oxygen cleavage alkylation of NBP by three $\beta$-lactones BPL, BBL and DIK. Results at the MP2/6-31G++(d,p)//RB3LYP/6-31G++(d,p) level of theory using IEF-PCM for solvation are resumed in Table 2.10.

Table 2.10. Computed activation energies for the alkylation of NBP by some $\beta$-lactones

\begin{tabular}{|c|c|c|c|}
\hline \multirow{2}{*}{ Lactone } & \multicolumn{3}{|c|}{$E_{a}\left(\mathrm{~kJ} \mathrm{~mol}^{-1}\right)$} \\
\hline & Experimental $^{a}$ & Calcd Alkyl $^{b}$ & Calcd Acyl $^{b}$ \\
\hline $\mathrm{BPL}^{66}$ & 44 & 56 & 117 \\
\hline $\mathrm{BBL}^{66}$ & 50 & 75 & 125 \\
\hline DIK & 29 & 104 & 33 \\
\hline
\end{tabular}

${ }^{a}$ In $7: 3 w / d$ medium

${ }^{b}$ ZPE energy corrections are included 
Figure 2.10 shows the calculated transition state geometries for the acyl and alkyl attacks of pyridine on BPL and DIK, respectively. The structure of the alkyl-cleavage transition state for BPL is straightforward: the formation of the $\mathrm{N}-\mathrm{C}$ bond and breaking of the $\mathrm{C}-\mathrm{O}$ bond are concerted. The corresponding structures for BBL and DIK are also very similar. The transition states for the acyl attack of pyridine onto $\beta$-lactones are also concerted. However, they are characterized by an important asymmetry: bond formation is practically barrierless and the energy barrier corresponds almost exclusively to the bond breaking, which corresponds with the structure seen in Figure 2.10.
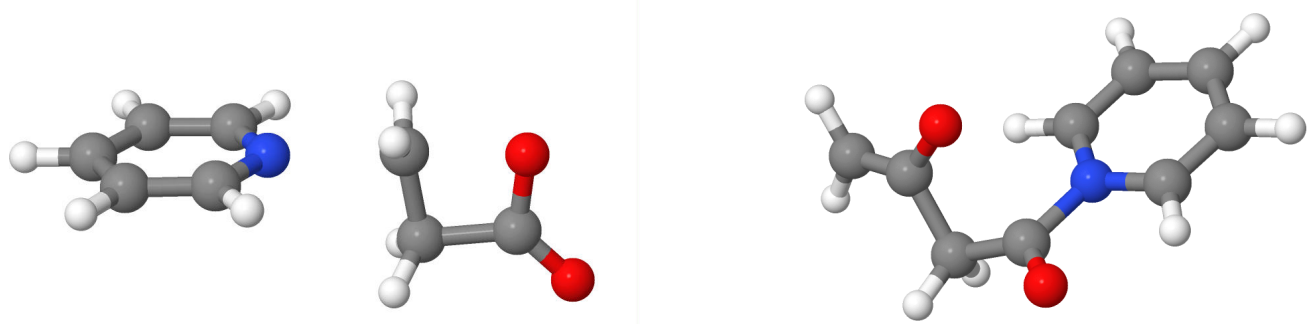

Figure 2.10. Calculated TS geometries for the acyl and alkyl attacks of pyridine on BPL and DIK

\subsubsection{Decomposition of the NBP-DIK adduct}

Contrarily to what occurs with BPL, BBL and other alkylating agents, the adduct formed by NBP and DIK readily undergoes hydrolysis in the reaction conditions.

When the decomposition rate constant of the adduct $\left(k_{\text {dec }}^{\mathrm{AD} D_{\text {DIK }}}\right)$ is plotted against the concentration of NBP, a straight line with a significant $y$-intercept is obtained. This was attributed to general base catalysis (or perhaps nucleophilic catalysis) since NBP, as a pyridine derivative, is rather basic ( $\mathrm{p} K_{\mathrm{a}}=$ 4.30). In order to test the catalysis hypothesis, alkylation reactions were performed in the presence of various concentrations of acetate, with respect to which $k_{\mathrm{dec}}^{\mathrm{AD} \text { DIK }}$ was observed to be linear. Thus, we propose eq. 2.23, in which $k_{\mathrm{B}}^{\mathrm{AD}}$ DIK stands for the catalytic coefficient for each catalyst.

$$
k_{\mathrm{dec}}^{\mathrm{AD}}=\left(k_{\mathrm{H}_{2} \mathrm{O}}^{\mathrm{AD}}+k_{\mathrm{B}-\mathrm{NBP}}^{\mathrm{AD}} \mathrm{D}_{\text {DIK }}[\mathrm{NBP}]+\ldots\right)\left[\mathrm{H}_{2} \mathrm{O}\right]
$$

$k_{\mathrm{H}_{2} \mathrm{O}}^{\mathrm{AD}} \mathrm{D}$ and $k_{\mathrm{B}-\mathrm{NBP}}^{\mathrm{AD}}$ values are reported in Tables 2.11 and 2.12

Contrary to what occurs with BPL and BBL and other alkylating agents, the adduct formed by DIK readily undergoes hydrolysis in the reaction conditions used here. The cause could lie in the fact that the 
Table 2.11. Rate constants for the decomposition of the NBP-DIK adduct

\begin{tabular}{|c|c|c|c|c|c|}
\hline \multirow{2}{*}{$w / d$} & \multicolumn{5}{|c|}{$10^{5} \times k_{\mathrm{H}_{2} \mathrm{O}}^{\mathrm{AD}_{\mathrm{DIK}}}\left(\mathrm{M}^{-1} \mathrm{~s}^{-1}\right)$} \\
\hline & $15^{\circ} \mathrm{C}$ & $20^{\circ} \mathrm{C}$ & $25^{\circ} \mathrm{C}$ & $30^{\circ} \mathrm{C}$ & $35^{\circ} \mathrm{C}$ \\
\hline $3: 7$ & 0.593 & 0.834 & 1.13 & 1.48 & 1.94 \\
\hline $4: 6$ & 1.06 & 1.40 & 1.75 & 2.47 & 3.11 \\
\hline $5: 5$ & 1.85 & 2.41 & 3.34 & 4.54 & 6.23 \\
\hline $6: 4$ & 3.24 & 4.68 & 6.54 & 8.13 & 11.1 \\
\hline $7: 3$ & 10.5 & 14.8 & 19.3 & 26.1 & 34.6 \\
\hline
\end{tabular}

Table 2.12. NBP catalytic coefficients for the decomposition of the NBP-DIK adduct

\begin{tabular}{|c|c|c|c|c|c|}
\hline \multirow{2}{*}{$w / d$} & \multicolumn{5}{|c|}{$10^{4} \times k_{\mathrm{B}-\mathrm{NBP}}^{\mathrm{AD}}\left(\mathrm{M}^{-2} \mathrm{~s}^{-1}\right)$} \\
\hline & $15^{\circ} \mathrm{C}$ & $20^{\circ} \mathrm{C}$ & $25^{\circ} \mathrm{C}$ & $30^{\circ} \mathrm{C}$ & $35^{\circ} \mathrm{C}$ \\
\hline $3: 7$ & 0.49 & 0.68 & 0.97 & 1.32 & 1.86 \\
\hline $4: 6$ & 0.96 & 1.27 & 1.78 & 2.45 & 3.54 \\
\hline $5: 5$ & 1.29 & 1.94 & 2.50 & 3.23 & 4.47 \\
\hline $6: 4$ & 2.00 & 2.64 & 3.48 & 4.74 & 6.25 \\
\hline $7: 3$ & 4.66 & 6.33 & 7.64 & 9.29 & 12.3 \\
\hline
\end{tabular}

$\beta$-ketoamide bond formed by the acyl fission on DIK is more easily cleaved than the amine bond formed by alkyl fission on BPL and BBL.

If we compute the adduct life (AL, vide supra) for diketene in $7: 3$ (vol.) $w / d$ at $35.0{ }^{\circ} \mathrm{C}$, considering only the non-catalyzed hydrolysis of the adduct, $\mathrm{AL} \simeq 1$ minute. This very low value suggests that the putative adducts formed between DNA nucleophilic sites and DIK are very short-lived, and thus, incapable of having enduring genotoxic effects.

In view of the observed dependency of the rate constants on the medium composition, an increase in $k_{\mathrm{dec}}^{\mathrm{AD} \mathrm{D}_{\mathrm{DIK}}}$ is expected when moving from the aquo-organic mixtures to pure water. Also, the phosphate buffer present in the cellular environment can act as a general base catalyst, and thus accelerate the decomposition of adducts in the biological medium. This increase in $k_{\mathrm{dec}}^{\mathrm{AD} \mathrm{D}_{\mathrm{DK}}}$ translates in a lower $\mathrm{AL}$, i.e. a shorter adduct life.

Our results suggest that DIK is water-soluble and its hydrolysis is slow enough not to compete with its alkylating ability, which in vitro is much higher than that of BPL and BBL. Thus, its lack of carcinogenicity could be explained in terms of the instability of the adducts that it may form with DNA. DIK could react rapidly with the nucleophilic sites in DNA (such as nitrogen atoms of purine and pyrimidine nucleotides), but because the adducts would have a very short life $(<1 \mathrm{~min})$, they would decompose into the free nucleotide and $\mathrm{AcAcH}$, the latter of which is in turn metabolized in the organism. 


\subsubsection{Acidity of lactones and cycloketones}

While the structure of diketene was still being discussed, the acidity of its $\alpha$-hydrogen was considered. An extremely low value of $\mathrm{p} K_{\mathrm{a}} \sim 7$ was proposed but rapidly discarded. The value obtained in this work (vide supra) $\left(\mathrm{p} K_{\mathrm{a}} \simeq 15.2\right)$ is unexpectedly low in comparison to saturated non-functionalized open-chain esters, which are generally assumed to have $\mathrm{p} K_{\mathrm{a}}$ values close to that of ethyl acetate $\left(\mathrm{p} K_{\mathrm{a}} \simeq 25-26\right.$ ).

Since esters are weak acids that rapidly hydrolyze in basic aqueous media, the experimental determination of their acid dissociation constants is challenging, and generally $\mathrm{p} K_{\mathrm{a}}$ values are accurately known only for esters with acidifying substituents, such as $\beta$-keto esters (e.g. acetylacetate derivatives), with $\mathrm{p} K_{\mathrm{a}}$ around 11. The scarceness of experimental values for comparison imposes the need to search for additional ways to confirm the rather low value obtained for the $\mathrm{p} K_{\mathrm{a}}$ of diketene.

In recent years, accurate computational calculation of $\mathrm{p} K_{\mathrm{a}}$ values has attracted a great deal of attention as a plausible alternative to experiment in the cases of intermediates and very weak or very strong acids. Several approaches have been developed ${ }^{67,68}$ which have almost the so called chemical accuracy, that is $\pm 1 \mathrm{p} K_{\mathrm{a}}$ units. This have been applied successfully to a variety of compounds. ${ }^{69-73}$

In order to confirm the unexpectedly low value obtained for the acid dissociation constant of diketene, a first-principle study of the acidity of some alkylic esters and ketones (Schemes 2.6 - 2.8) has been carried out. Also aiming to test contemporary methodologies for theoretical $\mathrm{p} K_{\mathrm{a}}$ calculation, the influence of some structural parameters (presence and position of a double bond and cyclization) on the stability and the acid dissociation constant of these compounds has been studied.

\subsubsection{Esters and lactones}

$\mathrm{pK}_{\mathrm{a}}$ values were computed using the relative method at the level of theory shown in the figures (DFTB3LYP/6-31G++(2df,2pd), CBS-Q and G2). Solvation free energies were computed as DFT-B3LYP/6$31 \mathrm{G}+(\mathrm{d})$ single point calculations, using the IEF-PCM formalism with default parameters and UAKSradii. Results in which solvation energies were computed at the HF/6-31G+(d) level of theory using UAHF radii are similar to $\pm 0.3 \log$ units and are not shown.

Gas-phase proton affinities, where available, are in excellent agreement with computational results, e.g. the tabulated $\Delta G_{g}^{\ominus}$ for ethyl acetate is $1527 \pm 17$ and the value obtained here are $1524.4,1533.1$ and 1535.4 for the DFT, CBS-Q and G2 methods respectively. Values for the two compound methods are in good accord and suggest that DFT values are poorer. This accuracy is in agreement with the reach of compound methods used to calculate gas-phase free energies (CBS-Q and G2), which are accurate to within $1 \mathrm{kcal} \mathrm{mol}^{-1}$.

In the first place, acid dissociation constants and isomerization free energies for $\alpha-\beta$ and $\beta-\gamma$ unsaturated compounds were computed for linear esters. (Scheme 2.6).

It can be observed that alkyl esters are about as acidic as ethyl acetate, whereas unsaturated esters are stronger acids, since the extended conjugation that stabilizes the anion, as would be expected. 


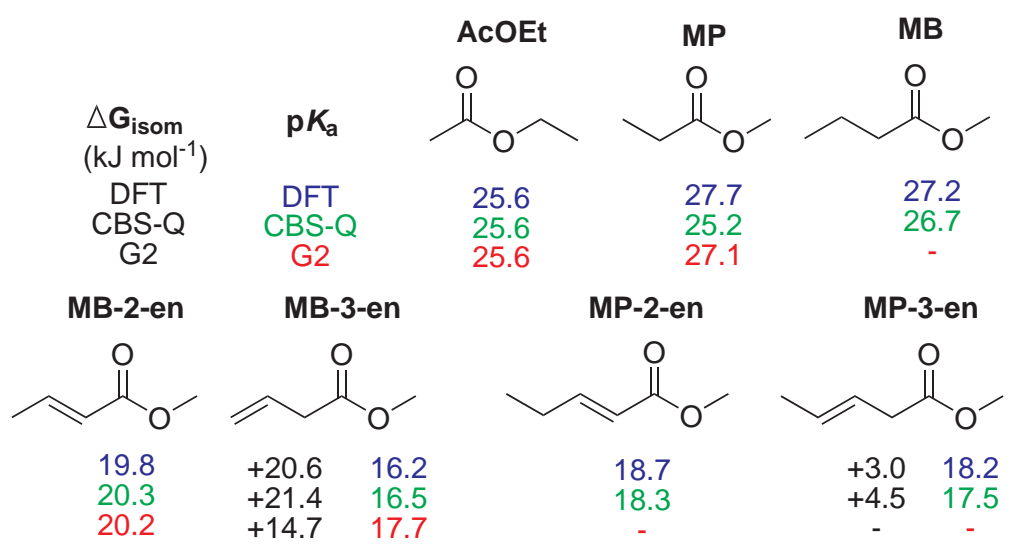

Scheme 2.6. Calculated $\mathrm{p} \mathrm{K}_{\mathrm{a}}$ and isomerization energies of linear esters in aqueous solution.

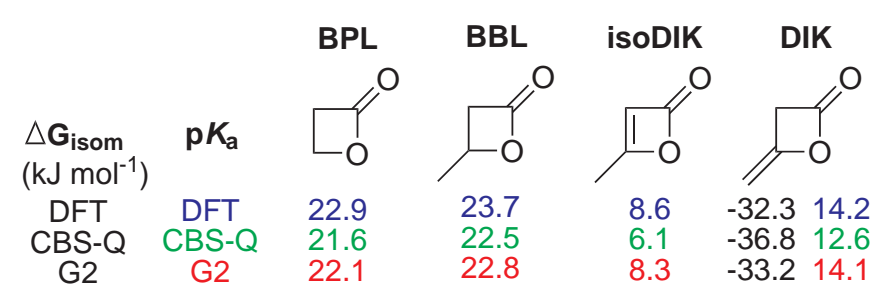

Scheme 2.7. Calculated $\mathrm{pK} a$ and isomerization energies of $\beta$-lactones

$\mathrm{p} K_{\mathrm{a}}$ values and isomerization free energies were computed for $\beta$ - and $\gamma$-lactones and are shown in Schemes 2.7 and 2.8 These values suggest that small lactones are more acidic than linear esters, and, as seen before, that unsaturated compounds are stronger acids. Agreement among the three levels of theory, including the lower level DFT, is usually very good (results are within \pm 1 log unit for all methods) and speaks in favor of the accuracy of our calculations.

From a close inspection of the values reported, some trends can be noticed, which will be discussed in the the following sections.

2.3.3.1.1. Isomerization energies. Table 2.13 shows the free energies of isomerization for $\alpha-\beta$ and $\beta-\gamma$ unsaturated esters, referred to the $\alpha-\beta$-isomer with the same alkyl backbone.

In all the cases be them linear or cyclic, but that of diketene, the most stable isomer is the $\alpha-\beta$ unsaturated one. All $\beta-\gamma$-unsaturated esters are between from $\sim 4$ to $\sim 50 \mathrm{~kJ} \mathrm{~mol}^{-1}$ less stable than their $\alpha-\beta$-unsaturated counterparts. Exocyclic methylene groups are very usually unstable, whereas the $\beta-\gamma$ unsaturation is more favored in long alkyl chains and certain $\gamma$-butyrolactones: AAL is only $\sim 8 \mathrm{~kJ} \mathrm{~mol}^{-1}$ higher in energy than BAL and the difference in the case of MP-3-en decreases to only $\sim 4 \mathrm{~kJ} \mathrm{~mol}^{-1}$.

A possible explanation for the fact that isoDIK is less stable than DIK is that the $s p^{2}$ hybridization would create additional tension in yet another cycle atom, since the square structure of $\beta$-lactones en- 


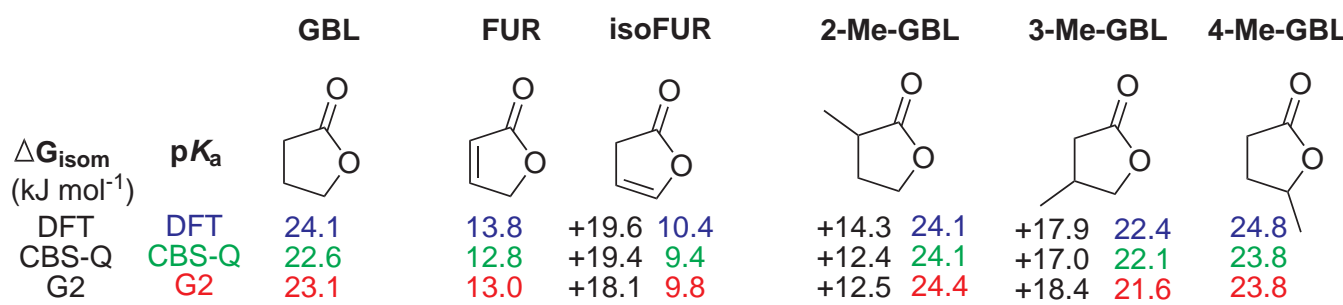

2-Me-FUR 2-Me-isoFUR 3-Me-FUR 3-Me-isoFUR 3-MET-GBL<smiles>CC1=CCOC1=O</smiles>

$+7.216 .7$ $+2.416 .2$ $+6.016 .2$<smiles>CC1C=COC1=O</smiles>

$+46.29 .9$

$+39.09 .8$

+38.7 10.5<smiles>CC1=CC(=O)OC1</smiles>

16.6

15.3 15.6<smiles>CC1=COC(=O)C1</smiles>

$+28.211 .6$ $+27.010 .5$ $+26.111 .0$<smiles>C=C1COC(=O)C1</smiles>

$+40.313 .1$

$+38.613 .3$ $+36.813 .8$
BAL<smiles>O=C1C=CC([131I])O1</smiles>

$+13.613 .2$

$\begin{array}{lll}+9.0 & 13.4 & +16.212 .2\end{array}$

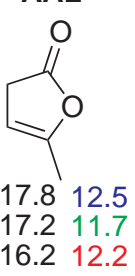

$c-2,3-M e-G B L \quad t-2,3-M e-G B L \quad c-2,4-M e-G B L \quad t-2,4-M e-G B L \quad c-3,4-M e-G B L \quad t-3,4-M e-G B L \quad 3,3-M e-G B L$

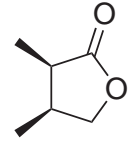

+27.922.1 $+31.121 .5$ $+22.722 .7$<smiles>C[C@@H]1COC(=O)[C@H]1C</smiles>

+25.922.6

$+30.722 .2$ $+20.523 .2$<smiles>CC1CC(C)C(=O)O1</smiles>

25.8 26.8 25.8<smiles>C[C@H]1C[C@@H](C)OC1=O</smiles>

$+5.424 .8+12724.4$

+13.3 24.5 +18.5 22.6

$+3.525 .1+8.2 \quad 23.9$<smiles>CC1CC(=O)O[C@@H]1C</smiles>

$+5.023 .5$

$+11.622 .3$ $+4.022 .7$

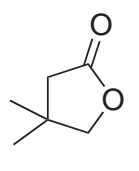

$+26.822 .0$ $+30.720 .9$ +19.421.3

2,3-Me-FUR 2,3-Me-isoFUR 2,3-MET-GBL 2,4-Me-FUR 2,4-Me-isoFUR<smiles>CC1=C(C)C(=O)OC1</smiles>

19.5

18.8

18.6<smiles>CC1=COC(=O)C1C</smiles>

$+47.211 .2$ $+42.911 .3$ $+39.311 .7$

\section{3,4-Me-FUR}<smiles>CC1=CC(=O)OC1C</smiles>

$+6.416 .3$ $+4.915 .4$ $+1.716 .4$<smiles>C=C1COC(=O)C1C</smiles>

$+55.812 .4$ $+44.510 .6$ $+47.613 .6$<smiles>CC1=CC(C)OC1=O</smiles><smiles>CC1=CC(C)C(=O)O1</smiles>

+10.7 16.7 +34.512.5

$+0.816 .7+29.211 .7$

$\begin{array}{lll}+7.0 & 17.0+29.213 .1\end{array}$ 
Table 2.13. Isomerization free energies of some esters and lactones in aqueous solution

\begin{tabular}{|c|c|c|c|c|}
\hline & \multicolumn{4}{|c|}{$\Delta G_{\text {isom }}(\alpha-\beta \rightarrow \beta-\gamma)\left(\mathrm{kJ} \mathrm{mol}^{-1}\right)$} \\
\hline & $\begin{array}{c}\text { DFT-B3LYP } \\
6-31++(2 \mathrm{df}, 2 \mathrm{pd})\end{array}$ & CBS-Q & G2 & Mean \\
\hline MB-3-en & 20.6 & 21.4 & 14.7 & 18.9 \\
\hline MP-3-en & 3.0 & 4.5 & - & 3.8 \\
\hline DIK & -32.3 & -36.8 & -33.2 & -34.1 \\
\hline isoFUR & 19.6 & 19.4 & 18.1 & 19.0 \\
\hline 2-Me-isoFUR & 38.9 & 36.6 & 32.7 & 36.1 \\
\hline 3-Me-isoFUR & 28.2 & 27.0 & 26.1 & 27.1 \\
\hline 3-MET-GBL & 40.3 & 38.6 & 36.8 & 38.6 \\
\hline AAL & 4.3 & 7.7 & 7.3 & 6.4 \\
\hline 2,3-Me-isoFUR & 47.2 & 42.9 & 39.3 & 43.1 \\
\hline 2,3-MET-GBL & 55.8 & 44.5 & 47.6 & 49.3 \\
\hline 2,4-Me-isoFUR & 23.8 & 28.4 & 22.2 & 24.8 \\
\hline 3,4-Me-isoFUR & 14.7 & 15.5 & 17.7 & 16.0 \\
\hline 3,4-MET-GBL & 37.1 & 31.2 & 34.8 & 34.4 \\
\hline
\end{tabular}

forces $90^{\circ}$ angles, instead of the $120^{\circ}$ favored by said hybridization. Thus, isoDIK has three highly closed angles, as opposed to the two present in DIK. This, however, is disproved by the fact that, 3ethylenecyclobutanone, the analogous ketone (vide infra) favors the $\alpha-\beta$ unsaturated isomer.

The instability of isoDIK as compared to DIK is unexpected, and a possible explanation was sought in a putative antiaromaticity that would arise from in the structure of isoDIK. Nucleus Independent Chemical Shift calculations (a measure of the aromatic ring current), however, disprove this idea.

An aromatic ring current is an effect observed in aromatic molecules such as benzene and naphthalene. If a magnetic field is directed perpendicular to the plane of the aromatic system, a ring current is induced in the delocalized $\pi$ electrons of the aromatic ring. This is a direct consequence of Ampère's law; since the electrons involved are free to circulate, rather than being localized in bonds as they would be in most non-aromatic molecules, they respond much more strongly to the magnetic field.

The nucleus-independent chemical shift (NICS) is a computational method that calculates the absolute magnetic shieldings at the center of the ring taken with reversed sign. In this method negative NICS values indicate aromaticity and positive values antiaromaticity. ${ }^{49,50}$ Common values are around -10 - 15 for aromats like pyrrole, naphthalene or benzene and 20-25 for antiaromats like pentalene, heptalene or cyclobutadiene. Non-aromatic molecules have low negative values, e.g. -3.2 for cyclopentadiene or -2.2 for cyclohexane.

The calculated chemical shift for a non-existent atom located in the center of the ring of the ring of isoDIK (NICS(0)) or $1 \AA$ above the ring $(\operatorname{NICS}(1))$ is very far from the values normally observed for antiaromats. We obtained the following values $\operatorname{NICS}(0)_{\text {isoDIK }}=0.15$ and $\operatorname{NICS}(1)_{\text {isoDIK }}=0.2$, when 
values for the clearly non-aromatic diketene are $\operatorname{NICS}(0)_{\text {DIK }}=-0.3$ and NICS(1) DIK $=-2.1$ (analogue methylenecyclobutanone shows NICS $(0)_{\text {MET-CB }}=12.6$ and NICS $(1)_{\text {MET-CB }}=-0.5$, and its $\alpha-\beta$ unsaturated isomer has NICS $(0)_{\text {MET-CB }}=5.1$ and NICS $\left.(1)_{\text {MET-CB }}=-2.5\right)$.

Figure 2.11 shows the HOMO of isoDIK, which clearly has several nodes that cut through the ring, making it impossible for the molecule to show aromaticity effects.

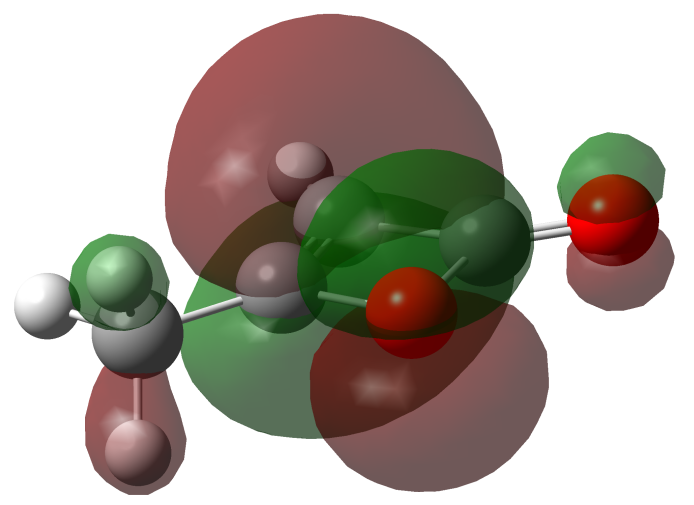

Figure 2.11. HOMO of the isoDIK molecule

2.3.3.1.2. Effect of structure upon $\mathbf{p} \boldsymbol{K}_{\mathbf{a}}$. Some structural parameters were observed to affect the acid dissociation constants:

2.3.3.1.2.1. Cyclization. Lactones are observed to be about $\sim 2 \log$ units more acidic than linear esters, both saturated and unsaturated compounds (Table 2.14). In addition, no differences in acidity are observed between $\beta$ and $\gamma$ lactones, including DIK. One single exception is observed: isoDIK.

2.3.3.1.2.2. Unsaturation. Inspection of Table 2.14 also shows that unsaturated esters are more acidic than the saturated molecules with the same carbon backbone. The average difference is approximately 6-7 $\mathrm{p} K_{\mathrm{a}}$ units for $\alpha, \beta$ unsaturated esters and $\beta, \gamma$ unsaturated compounds are a further 2-3 $\log$ units more acidic. This is not unexpected since the $\alpha, \beta$ and $\beta, \gamma$ isomers share the same anion and the former are $\sim 4$ to $\sim 50 \mathrm{~kJ} \mathrm{~mol}^{-1}$ more stable and thus, less prone to dissociate. Detailed values are given in Table 2.15.

Results so far indicate that DIK is as acidic as would be expected for an average $\beta, \gamma$ unsaturated lactone. isoDIK, however is exceptionally acidic for a $\alpha, \beta$ unsaturated compound, which suggests that isoDIK is exceptionally unstable, rather than DIK being exceptionally stable. Thus, the cause for the 
Table 2.14. Average effect of cyclization and unsaturation on the $\mathrm{p} K_{\mathrm{a}}$ of esters

\begin{tabular}{|c|c|c|c|}
\hline & \multicolumn{3}{|c|}{ Average $\mathrm{p} K_{\mathrm{a}}$} \\
\hline & $\begin{array}{c}\text { DFT-B3LYP } \\
6-31++(2 \mathrm{df}, 2 \mathrm{pd})\end{array}$ & CBS-Q & G2 \\
\hline \multicolumn{4}{|c|}{ Saturated } \\
\hline Linear esters & 26.8 & 25.8 & 26.3 \\
\hline$\beta$-lactones & 23.3 & 22.0 & 22.5 \\
\hline$\gamma$-lactones & 23.7 & 23.0 & 23.4 \\
\hline \multicolumn{4}{|c|}{$\alpha-\beta$ Unsaturated } \\
\hline Linear esters & 19.3 & 19.3 & 20.2 \\
\hline$\beta$-lactones (isoDIK) & 8.6 & 6.1 & 8.3 \\
\hline$\gamma$-Lactones & 16.1 & 15.5 & 15.7 \\
\hline \multicolumn{4}{|c|}{$\beta-\gamma$ Unsaturated } \\
\hline Linear esters & 17.2 & 17.0 & 17.7 \\
\hline$\beta$-lactones (DIK) & 14.2 & 12.6 & 14.1 \\
\hline$\gamma$-lactones & 14.0 & 13.5 & 12.4 \\
\hline
\end{tabular}

exceptionally low $\mathrm{p} K_{\mathrm{a}}$ of DIK lies in not having a more stable isomer to convert to. Whereas equally acidic $\beta, \gamma$ unsaturated $\gamma$ lactones rapidly isomerize to their more stable and less acid $\alpha, \beta$ unsaturated counterparts, making it impossible to measure their $\mathrm{p} K_{\mathrm{a}}$, DIK is already the most stable form and its $\mathrm{p} K_{\mathrm{a}}$ can be measured. 
Table 2.15. Effect of unsaturation on the $\mathrm{p} K_{\mathrm{a}}$ of esters, with respect to saturated backbone

\begin{tabular}{|c|c|c|c|c|c|c|c|}
\hline & \multicolumn{7}{|c|}{$\Delta \mathrm{p} K_{\mathrm{a}}$} \\
\hline & \multicolumn{3}{|c|}{$\alpha-\beta$-unsaturated } & & \multicolumn{3}{|c|}{$\beta-\gamma$-unsaturated } \\
\hline & DFT & CBS-Q & G2 & & DFT & CBS-Q & G2 \\
\hline MB-2-en & -7.4 & -6.4 & - & MB-3-en & -11.0 & -10.2 & - \\
\hline isoDIK & -15.2 & -16.4 & -14.5 & DIK & -9.5 & -9.9 & -8.7 \\
\hline FUR & -10.2 & -9.8 & -10.1 & isoFUR & -13.7 & -13.2 & -13.2 \\
\hline 2-Me-FUR & -7.4 & -7.9 & -8.2 & 2-Me-isoFUR & -14.2 & -14.3 & -14.0 \\
\hline \multirow[t]{2}{*}{ 3-Me-FUR } & -5.8 & -6.8 & -6.0 & 3-Me-isoFUR & -10.8 & -11.5 & -10.6 \\
\hline & & & & 3-METGBL & -9.3 & -8.8 & -7.9 \\
\hline BAL & -11.6 & -10.7 & -10.3 & AAL & -12.3 & -12.1 & -11.6 \\
\hline \multirow[t]{2}{*}{ 2,3-Me-FUR } & -3.1 & -3.3 & -4.6 & 2,3-Me-isoFUR & -11.4 & -10.9 & -11.5 \\
\hline & & & & 2,3-METGBL & -10.2 & -11.5 & -9.6 \\
\hline 2,4-Me-FUR & -9.1 & -10.1 & -8.8 & 2,4-Me-isoFUR & -13.3 & -15.1 & -12.7 \\
\hline \multirow[t]{2}{*}{ 3,4-Me-FUR } & -8.2 & -7.2 & -7.5 & 3,4-Me-isoFUR & -10.7 & -9.9 & -10.6 \\
\hline & & & & 3,4-METGBL & -10.8 & -9.8 & -9.9 \\
\hline
\end{tabular}

\subsubsection{Ketones}

In order to check whether the trends in $\mathrm{p} K_{\mathrm{a}}$ observed for esters also hold for ketones, and to search for exceptional cases such as that of diketene, the acid dissociation constants of a number of ketones, mostly cyclopentanones, were computed.

Unlike esters, ketones have two $\alpha$-hydrogen atoms able to dissociate. The presence of unsaturations and methyl groups alters which of the two possible positions is more acidic.

In the first place, the acid dissociation constants of some cyclobutanones were calculated (Scheme 2.9). The results suggest that the case of diketene is indeed exceptional, since the analogous cyclobutanones behave in the same general terms as most $\beta-\gamma$ unsaturated compounds. The $\beta-\gamma$ isomer of the cyclobutanone analogue of diketene is $15-20 \mathrm{~kJ} \mathrm{~mol}^{-1}$ higher in energy than its $\alpha$ - $\beta$ unsaturated counterpart.

Schemes 2.10 and 2.11 report the computed acid dissociation constants of methyl and dimethyl cyclopentanones, respectively. 


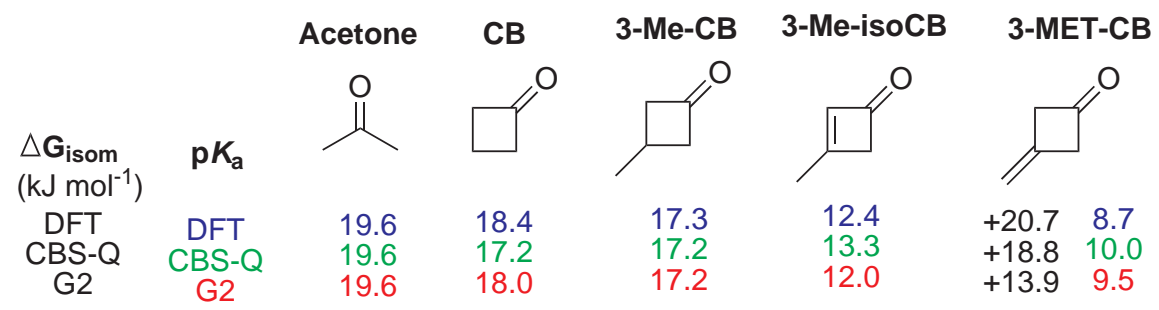

Scheme 2.9. Calculated $\mathrm{pK} \mathrm{K}_{\mathrm{a}}$ and isomerization energies of four-membered cyclic ketones

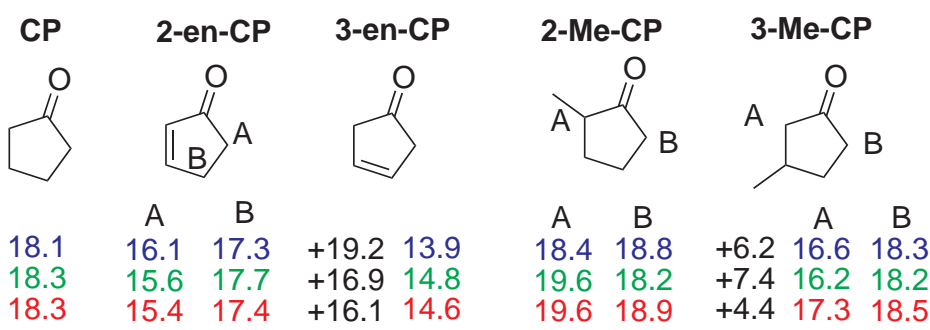

2-en-2-Me-CP<smiles>CC1=C[B]CC1=O</smiles>

A B

$\begin{array}{lll}+8.7 \quad 17.2 \quad 20.1 \\ -3.5 & 18.4 & 22.6\end{array}$

$\begin{array}{lll}-3.5 & 18.4 & 22.6\end{array}$

$+7.516 .4 \quad 20.5$

2-en-3-Me-CP<smiles>CC1=CC(=O)CC1</smiles>

A $B$

$18.1 \quad 19.4$

$17.5 \quad 19.6$

17.319 .4
3-en-2-Me-CP<smiles>CC1C=C[B]C1=O</smiles>

A B

$+45.4 \quad 14.3 \quad 15.5$

$+35.915 .7 \quad 14.5$

$+36.8 \quad 15.4 \quad 15.0$

3-en-3-Me-CP

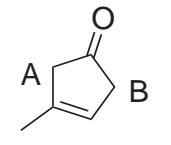

A B
4-en-2-Me-CP<smiles>[B]C1C=CCC1=O</smiles>

A B

$+27.015 .8 \quad 17.7$

$+19.216 .317 .4$

$\begin{array}{lll}+21.1 & 16.4 \quad 17.8\end{array}$

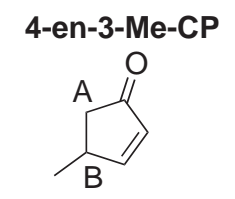

A B

Scheme 2.10. Calculated $\mathrm{p} K_{\mathrm{a}}$ and isomerization energies of methyl cyclopentanones and methyl cyclopentenones 
$c-2,3-M e-C P \quad t-2,3-M e-C P \quad c-2,4-M e-C P \quad t-2,4-M e-C P$<smiles>[B]C1CC[C@@H](C)[C]1C</smiles>

A B

$+10.017 .019 .9$

$+15.317 .418 .8$

$+5.818 .620 .0$

c-3,4-Me-CP<smiles>C[C@H]1CC(=O)C[C@H]1C</smiles>

$+11.918 .5$ +20.617.4<smiles>[B]C1C[C@@H](C)[C@@H](C)C1=O</smiles>

A B $+0.618 .219 .2$ $\begin{array}{ll}-0.5 & 19.318 .7\end{array}$ 19.319 .3

$t-3,4-\mathrm{Me}-\mathrm{CP}$<smiles>C[C@H]1CC(=O)C[C@H]1C</smiles>

+3.3 17.8 $+10.017 .5$<smiles>[B]C1C[C@H](C)C[C]1C</smiles><smiles>[13CH3]</smiles>
$18.818 .2+6.217 .717 .7$

$20.316 .8+12.118 .216 .3$

2,2-Me-CP<smiles>CC1(C)CCCC1=O</smiles>

+5.418.6 +9.5 17.2<smiles>C[C@@H]1CCCC1=O</smiles>

$\mathrm{A}^{\doteqdot} \mathrm{B}$
3,3-Me-CP

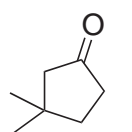

A B

$+7.616 .918 .9$ $+14.316 .619 .2$

\section{2-en-2,3-Me-CP}

3-en-2,3-Me-CP

c-4-en-2,3-Me-CP<smiles>CC1=C(C)C(=O)C[B]1</smiles>

A $B$<smiles>C=C1C(=O)[B]C=C1C</smiles>

A B<smiles>C[C@@H]1C(=O)C=C[C@H]1C</smiles>

A B

$+10.019 .821 .3$

$+15.318 .923 .1$

$+10.014 .216 .4$

$+15.315 .917 .4$

$+10.015 .115 .5$

$+15.315 .516 .9$

$+5.815 .716 .7$

t-4-en-2,3-Me-CP

+5.8 18.522 .0

3-en-2,4-Me-CP

4-en-2,4-Me-CP

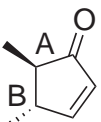

A B $+10.016 .416 .8$ +15.316.5 17.8 $+5.816 .717 .7$

2-en-2,4-Me-CP<smiles>CC1=CC(C)C(C)C1=O</smiles>

A B

$+10.017 .519 .0$

$+15.315 .718 .8$ $+5.816 .920 .7$<smiles>CC1=CC(C)=C(C)C1=O</smiles>

A B $+10.015 .615 .2$

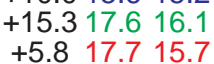<smiles>CC1=C2C=C(C(=O)C2)C1=O</smiles>

A B
$+10.018 .019 .9$

$+15.318 .322 .0$ $+5.818 .519 .7$
4-en-3,3-Me-CP<smiles>CC1(C)C=CC(=O)C1</smiles>

+36.617.2 $+34.716 .8$

2-en-3,4-Me-CP 3-en-3,4-Me-CP

3-en-2,2-Me-CP

4-en-2,2-Me-CP<smiles>CC1=CC(=O)C(C)[C+]1C</smiles>

A B

+10.018.2 18.4

$+15.317 .619 .4$

+5.817.619.6<smiles>CC1=C(C)CC(=O)C1</smiles>

$\begin{array}{ll}+10.0 & 16.4 \\ +15.3 & 17.5 \\ +5.8 & 17.3\end{array}$<smiles>CC1(C)C=CC2CC1C2</smiles>

+58.914 .8
+26.417 .0 $+44.415 .1$<smiles>CC1(C)CC=CC1=O</smiles>

$+40.018 .1$ $+19.818 .1$ +26.918.1

Scheme 2.11. Calculated $\mathrm{p} K_{\mathrm{a}}$ and isomerization energies of dimethyl cyclopentanones and dimethyl cyclopentenones 
2.3.3.2.1. Isomerization energies. Free energy differences between (the most stable, when more than one) $\alpha-\beta$ and $\beta-\gamma$ unsaturated ketones, are reported in Table 2.16. Results suggest that, with no exceptions within the studied compounds, $\alpha-\beta$ unsaturated cycloketones are more stable than their $\beta-\gamma$ counterparts.

Table 2.16. Isomerization free energies of some cycloketones in aqueous solution

\begin{tabular}{|c|c|c|c|c|}
\hline & \multicolumn{4}{|c|}{$\Delta G_{\text {isom }}(\alpha-\beta \rightarrow \beta-\gamma)\left(\mathrm{kJ} \mathrm{mol}^{-1}\right)$} \\
\hline & $\begin{array}{c}\text { DFT-B3LYP } \\
6-31++(2 \mathrm{df}, 2 \mathrm{pd})\end{array}$ & CBS-Q & G2 & Mean \\
\hline 3-MET-СB & 20.7 & 18.8 & 13.9 & 17.8 \\
\hline 3-en-CP & 19.2 & 16.9 & 16.1 & 17.4 \\
\hline 2-Me-3-en-CP & 36.7 & 39.4 & 29.3 & 35.1 \\
\hline 3-Me-3-en-CP & 26.9 & 23.3 & 23.1 & 24.4 \\
\hline 3-MET-CP & 48.2 & 45.7 & 42.3 & 45.4 \\
\hline 2,3-Me-3-en-CP & 40.8 & 42.0 & 33.9 & 38.9 \\
\hline 2,4-Me-3-en-CP & 19.5 & 35.2 & 16.9 & 23.9 \\
\hline 3,4-Me-3-en-CP & 11.3 & 10.6 & 13.4 & 11.7 \\
\hline 2,2-Me-3-en-CP & 18.9 & 6.6 & 17.5 & 14.3 \\
\hline
\end{tabular}

2.3.3.2.2. Effect of structure upon $\mathbf{p} \boldsymbol{K}_{\mathrm{a}}$. Some structural parameters were observed to affect the acid dissociation constants. As was the case with lactones, the presence of the ring decreases the $\mathrm{p} K_{\mathrm{a}}$ in about 1 or $2 \log$ units.

2.3.3.2.2.1. Unsaturation. As expected, conjugation of the keto group with a double bond generally increases the acidity of the carbon atoms. However this effect is not as strong as the one detected in esters. (Table 2.17). $\beta-\gamma$ Unsaturation largely increases the acidity of the $\alpha$-hydrogen, since the extended conjugation stabilizes the negative charge. Additionally, $\alpha-\beta$ unsaturation does not always imply an increase in acidity of the $\gamma$ position, in contrast with esters. 
Table 2.17. Effect of unsaturation on the $\mathrm{p} K_{\mathrm{a}}$ of esters, with respect to saturated backbone

\begin{tabular}{|c|c|c|c|c|c|c|c|c|}
\hline \multicolumn{5}{|c|}{$\alpha-\beta$-Unsaturated } & \multicolumn{4}{|c|}{$\beta-\gamma$-unsaturated } \\
\hline & & DFT & CBS-Q & G2 & & DFT & CBS-Q & G2 \\
\hline 3-Me-isoCB & & -4.9 & -3.9 & -5.2 & 3-MET-CB & -9.7 & -7.3 & -8.5 \\
\hline 2-en-CP & $\gamma$ & -0.8 & -0.5 & -0.8 & 3-en-CP & -4.2 & -3.5 & -3.6 \\
\hline$"$ & $\alpha$ & -2.0 & -2.7 & -0.8 & & & & \\
\hline 2-Me-2-en-CP & $\gamma$ & 1.7 & 3.0 & 0.9 & 2-Me-3-en-CP & -4.1 & -3.9 & -4.3 \\
\hline$"$ & $\alpha$ & -1.6 & 0.2 & -2.5 & $"$ & -4.3 & -3.8 & -3.9 \\
\hline 2-Me-4-en-CP & $\gamma$ & -1.0 & -0.9 & -1.2 & & & & \\
\hline$"$ & $\alpha$ & -2.5 & -3.3 & -3.3 & & & & \\
\hline 3-Me-2-en-CP & $\gamma$ & 2.8 & 3.4 & 2.1 & 3-Me-3-en-CP & -1.9 & -0.7 & -2.0 \\
\hline$"$ & $\alpha$ & -0.2 & -0.7 & -1.2 & $"$ & -2.5 & -1.6 & -1.7 \\
\hline 3-Me-4-en-CP & $\gamma$ & -2.1 & -0.8 & -1.1 & 3-MET-CP & -7.7 & -7.4 & -7.3 \\
\hline$"$ & $\alpha$ & 0.0 & -1.6 & -1.3 & & 0.7 & 1.5 & 0.1 \\
\hline 2,3-Me-2-en-CP & $\gamma$ & 3.1 & 3.8 & 2.6 & 2,3-Me-3-en-CP & -4.0 & -3.4 & -3.3 \\
\hline$"$ & $\alpha$ & 0.6 & 0.3 & -0.8 & " & -3.0 & -1.3 & -2.3 \\
\hline c-2,3-Me-4-en-CP & $\gamma$ & -4.3 & -2.0 & -3.3 & & & & \\
\hline$"$ & $\alpha$ & -1.9 & -1.9 & -2.9 & & & & \\
\hline t-2,3-Me-4-en-CP & $\gamma$ & -2.4 & -0.8 & -1.6 & & & & \\
\hline$"$ & $\alpha$ & -1.8 & -2.8 & -2.7 & & & & \\
\hline 2,4-Me-2-en-CP & $\gamma$ & 0.3 & -1.5 & 0.7 & 2,4-Me-3-en-CP & -2.9 & -0.7 & 2.7 \\
\hline$"$ & $\alpha$ & -0.6 & -1.1 & 1.5 & $"$ & -3.2 & -2.7 & -2.3 \\
\hline 2,4-Me-4-en-CP & $\gamma$ & 1.7 & 5.1 & 1.2 & & & & \\
\hline$"$ & $\alpha$ & -0.8 & -2.0 & -1.5 & & & & \\
\hline 3,4-Me-2-en-CP & $\gamma$ & 0.6 & 1.9 & 1.6 & 3,4-Me-3-en-CP & -1.4 & 0.1 & -0.8 \\
\hline$"$ & $\alpha$ & 0.5 & 0.2 & -0.4 & & & & \\
\hline 2,2-Me-4-en-CP & & -0.5 & 1.0 & -0.4 & 2,2-Me-3-en-CP & -3.8 & -0.2 & -3.5 \\
\hline
\end{tabular}




\subsection{Conclusions}

1. Diketene was found to hydrolyze rapidly in water in both neutral and alkaline media, with activation parameters $\Delta^{\ddagger} H^{\ominus}=56 \pm 2 \mathrm{~kJ} \mathrm{~mol}^{-1}, \Delta^{\ddagger} S^{\ominus}=-159 \pm 6 \mathrm{~J} \mathrm{~K}^{-1} \mathrm{~mol}^{-1}$ and $\Delta^{\ddagger} H^{\ominus}=-47.4 \pm 0.6$ $\mathrm{kJ} \mathrm{mol}^{-1}, \Delta^{\ddagger} S^{\ominus}=63 \pm 2 \mathrm{~J} \mathrm{~K}^{-1} \mathrm{~mol}^{-1}$, respectively.

2. The influence of the reaction medium in the activation parameters is in keeping with that observed for other $\beta$-lactones, and with the properties of the water-dioxane mixtures.

3. Diketene is a strong electrophile that reacts with NBP at faster rate than other $\beta$-lactones.

4. Since the alkylation reaction of DIK is much faster than its hydrolysis reaction, the lack of biological effects of diketene must be due to the short life of the amide alkylation adducts.

5. The $\alpha$-hydrogen in DIK was found to have a $\mathrm{p} K_{\mathrm{a}} \sim 15.0$, a result in keeping with theoretical values for this and similar compounds.

6. The fact that this high dissociation constant can be measured is a consequence of the DIK being a unique case in which the $\beta-\gamma$ unsaturated isomer is favored over its $\alpha-\beta$ unsaturated counterpart. 


\section{References}

[1] Smith, H. H.; Srb, A. M. Science 1951, 114, 490-492.

[2] Roe, F. J.; Salaman, M. H. Br. J. Cancer 1955, 9, 1772-1803.

[3] Glendenning, O. M.; Roe, F. J. Br. J. Cancer 1956, 10, 357362.

[4] Dickens, F.; Jones, H. E. H. Br. J. Cancer 1961, 15, 85-\&.

[5] Dickens, F.; Jones, H. E. H. Br. J. Cancer 1963, 17, 100-108.

[6] Parish, D. J.; Searle, C. E. Br. J. Cancer 1966, 20, 200-205.

[7] Parish, D. J.; Searle, C. E. Br. J. Cancer 1966, 20, 206-209.

[8] Searle, C. E. Br. J. Cancer 1961, 15, 804-811.

[9] Roberts, J. J.; Warwick, G. P. Biochem. Pharmacol. 1963, 12, 1441-1442.

[10] Colburn, N. H.; Richardson, R. G.; Boutwell, R. K. Biochem. Pharmacol. 1965, 14, 1113-1118.

[11] Colburn, N. H.; Boutwell, R. K. Cancer Res. 1966, 26, 1701-1706.

[12] Colburn, N. H.; Boutwell, R. K. Cancer Res. 1968, 28, 642-652.

[13] Colburn, N. H.; Boutwell, R. K. Cancer Res. 1968, 28, 653-660.

[14] Kubinski, H.; Szybalski, E. H. Chem.-Biol. Interact. 1975, 10, 4155.

[15] Hemminki, K. Chem.-Biol. Interact. 1981, 34, 323331.

[16] Nietert, W. C.; Kellicutt, L. M.; Kubinski, H. Cancer Res. 1974, 34, 859-864.

[17] Kubinski, H.; Andersen, P. R.; Kellicutt, L. M. Chem.-Biol. Interact. 1972, 5, 279283.

[18] Segal, A.; Maté, U.; Wortman, M. Chem.-Biol. Interact. 1978, 21, 249-261.

[19] Dickens, F. Br. Med. Bull. 1964, 20, 96-\&.

[20] Van Duuren, B. L.; Orris, L.; Nelson, N. J. Natl. Cancer Inst. 1965, 35, 707-717.

[21] Van Duuren, B. L.; Langseth, L.; Orris, L.; Teebor, G.; Nelson, N.; Kuschner, M. J. Natl. Cancer Inst. $1966,37,825-838$.

[22] Van Duuren, B. L.; Langseth, L.; Goldschmidt, B. M.; Orris, L. J. Natl. Cancer Inst. 1967, 39, 12171227.

[23] Van Duuren, B. L.; Langseth, L.; Orris, L.; Baden, M.; Kuschner, M. J. Natl. Cancer Inst. 1967, 39, 1213-1216.

[24] Van Duuren, B. L. Ann. NY Acad. Sci. 1969, 163, 633-650.

[25] Van Duuren, B.; Goldschmidt, B.; Katz, C.; Seidman, I.; Paul, J. J. Natl. Cancer Inst. 1974, 53, 695700.

[26] Brusick, D. J. Mutat. Res. 1977, 39, 241255. 
[27] Chernozemski, I. N.; Warwick, G. P. J. Natl. Cancer Inst. 1970, 45, 709717.

[28] Fukuda, S.; Yamamoto, N. Cancer Res. 1970, 30, 830-833.

[29] Penman, B. W.; Hoppe, H.; Thilly, W. G. J. Natl. Cancer Inst. 1979, 63, 903-907.

[30] Brusick, D. J. Mutat. Res. 1972, 15, 425434.

[31] Kortselius, M. J. Mutat. Res. 1979, 66, 5563.

[32] Rees, E. D.; Shuck, A. E.; Lowry, J. Q.; Smith, T. M.; Lipscomb, H. J. Environ. Pathol. Toxicol. 1979, 2, 1475-1485.

[33] Maté, U.; Solomon, J. J.; Segal, A. Chem.-Biol. Interact. 1977, 18, 327-336.

[34] Chen, R.; Mieyal, J. J.; Goldthwait, D. A. Carcinogenesis 1981, 2, 7380.

[35] Segal, A.; Solomon, J. J.; Mignano, J.; Dino, J. Chem.-Biol. Interact. 1981, 35, 349-361.

[36] Clemens, R. J. Chem. Rev. 1986, 86, 241-318.

[37] Mansson M, S. S., Nakase Y Acta Chem. Scand. 1968, 22, 171-\&.

[38] Seidl, E. T.; Schaefer, H. F. J. Phys. Chem. 1992, 96, 657-661.

[39] Seidl, E. T.; Schaefer, H. F. J. Am. Chem. Soc. 1990, 112, 1493-1499.

[40] Morales, G.; Martinez, R. J. Phys. Chem. A 2009, 113, 8683-8703.

[41] Briody, J. M.; Satchell, D. P. N. Chem. Ind. 1964, 893-893.

[42] Briody, J. M.; Satchell, D. P. J. Chem. Soc., Chem. Comm. 1965, 3778-3785.

[43] Okeya, S.; Kawaguchi, S. Bull. Chem. Soc. Jpn. 1984, 57, 1217-1221.

[44] Van Duuren, B. L.; Goldschmidt, B. M. J. Med. Chem. 1966, 9, 77-79.

[45] Hay, R. W.; Bond, M. A. Aust. J. Chem. 1967, 20, 1823-\&.

[46] Wollenberg, O. Ber. Dtsch. Chem. Ges. 1934, 67, 1675-1679.

[47] Kato, T.; Yamanaka, H.; Wagatsum.K,; Oizumi, M.; Niitsuma, T. Chem. Pharm. Bull. 1964, 12, 910-\&.

[48] Van Duuren, B. L.; Nelson, N.; Orris, L.; Palmes, E. D. J. Natl. Cancer Inst. 1963, 31, 41-55.

[49] Chen, Z.; Wannere, C.; Corminboeuf, C.; Puchta, R.; Schleyer, P. Chem. Rev. 2005, 105, 3842-3888.

[50] Schleyer, P.; Maerker, C.; Dransfeld, A.; Jiao, H.; Hommes, N. J. Am. Chem. Soc. 1996, 118, 63176318.

[51] Cheeseman, J. R.; Trucks, G. W.; Keith, T. A.; Frisch, M. J. J. Chem. Phys. 1996, 104, 5497-5509.

[52] Ditchfield, R. Mol. Phys. 1974, 27, 789-807.

[53] London, F. J. Phys. Radium 1937, 8, 397-409.

[54] McWeeny, R. Phys. Rev. 1962, 126, 1028.

[55] Wolinski, K.; Hilton, J. F.; Pulay, P. J. Am. Chem. Soc. 1990, 1990, 8251-8260. 
[56] Pérez-Prior, M. T.; Manso, J. A.; García-Santos, M. P.; Calle, E.; Casado, J. J. Org. Chem. 2005, 70, $420-426$.

[57] Goates, R.; Sullivan, R. J. J. Phys. Chem. 1958, 62, 188-190.

[58] Takamuku, T.; Yamaguchi, A.; Tabata, M.; Nishi, N.; Yoshida, K.; Wakita, H.; Yamaguchi, T. J. Mol. Liq. 1999, 83, 163-177.

[59] Manso, J. A.; Pérez-Prior, M. T.; García-Santos, M. P.; Calle, E.; Casado, J. J. Solution Chem. 2008, 37, $451-457$.

[60] Tobias, P. S.; Kezdy, F. J. J. Am. Chem. Soc. 1969, 91, 5171-5173.

[61] Kaiser, E. T.; Kézdy, F. J. Prog. Bioorg. Chem. 1976, 4, 239.

[62] Blackburn, G. M.; Dodds, H. L. H. J. Chem. Soc., Perkin Trans. 2 1974, 377-382.

[63] Long, F. A.; Purchase, M. J. Am. Chem. Soc. 1950, 72, 3267-3273.

[64] Bartlett, P. D.; Small, G. J. Am. Chem. Soc. 1950, 72, 4867-4869.

[65] Olson, A. R.; Miller, R. J. J. Am. Chem. Soc. 1938, 60, 2687-2692.

[66] Manso, J. A.; Pérez-Prior, M. T.; García-Santos, M. P.; Calle, E.; Casado, J. Chem. Res. Toxicol. 2005, $18,1161-1166$.

[67] Ho, J.; Coote, M. L. J. Chem. Theory Comput. 2009, 5, 295-306.

[68] Ho, J. M.; Coote, M. L. Theor. Chem. Acc. 2010, 125, 3-21.

[69] Brown, T. N.; Mora-Díez, N. J. Phys. Chem. B 2006, 110, 9270-9279.

[70] Ding, F.; Smith, J. M.; Wang, H. J. Org. Chem. 2009, 74, 2679-2691.

[71] Li, J. N.; Fu, Y.; Liu, L.; Guo, Q. X. Tetrahedron 2006, 62, 11801-11813.

[72] Shen, K.; Fu, Y.; Li, J. N.; Liu, L.; Guo, Q. X. Tetrahedron 2007, 63, 1568-1576.

[73] Wang, B.; Cao, Z. J. Phys. Chem. A 2010, 114, 12918-12927. 

Hydrolysis of lactones: a theoretical study 



\section{Contents}

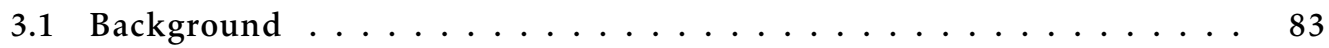

3.1 .1 Hydrolysis of esters . . . . . . . . . . . . . . 83

3.1.1.1 Acid hydrolysis . . . . . . . . . . . . . . . . . . 84

3.1.1.1.1 General considerations . . . . . . . . . . . . 84

$3.1 .1 .1 .2 \quad \mathrm{~A}_{\mathrm{AC}} 1 \ldots \ldots \ldots \ldots \ldots \ldots \ldots$

3.1.1.1.3 $\mathrm{A}_{\mathrm{AL}} 1 \ldots \ldots \ldots \ldots \ldots \ldots$

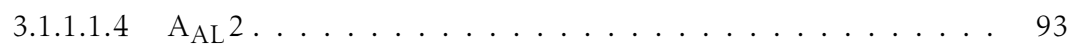

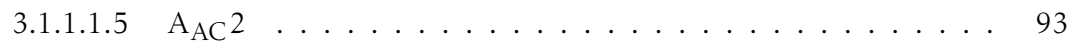

3.1.1.2 Neutral hydrolysis . . . . . . . . . . . . . . . . . . . . . . 101

3.1.1.2.1 $\mathrm{B}_{\mathrm{AL}} 2$ mechanism . . . . . . . . . . . . 101

3.1.1.2.2 $\mathrm{B}_{\mathrm{AL}} 1$ mechanism . . . . . . . . . . . . . . . . . . . . . . . . . . 102

3.1.1.2.3 $\mathrm{B}_{\mathrm{AC}} 2$ mechanism .................. 102

3.1.1.3 Base hydrolysis . . . . . . . . . . . . . . . . . . . . 104

3.1.1.3.1 $\mathrm{B}_{\mathrm{AL}} 2$ mechanism . . . . . . . . . . . . 105

3.1.1.3.2 $\mathrm{B}_{\mathrm{AC}} 2$ mechanism . . . . . . . . . . . . 105

3.1 .2 Hydrolysis of lactones . . . . . . . . . . . . . . . 108

3.1.2.1 Acid hydrolysis . . . . . . . . . . . . . . . . . . 108

3.1.2.2 Neutral hydrolysis . . . . . . . . . . . . . . . . . . . 110

3.1.2.3 Base hydrolysis . . . . . . . . . . . . . . . . . 110

3.2 Materials \& methods . . . . . . . . . . . . . . . . 111

3.2.1 Methodology and computational details . . . . . . . . . . . . 111

3.2.2 Hydrolysis of 2-5(H)-Furanone . . . . . . . . . . . . . 112

3.3 Results and discussion . . . . . . . . . . . . . . . . 113

3.3 .1 Neutral hydrolysis . . . . . . . . . . . . . . . . 113

3.3.1.1 $\mathrm{B}_{\mathrm{AL}} 2$ and $\mathrm{B}_{\mathrm{AL}} 1$ mechanisms .................. 114

$3.3 .1 .2 \mathrm{~B}_{\mathrm{AC}} 2$ mechanism .......................... 116

3.3.1.2.1 Addition . . . . . . . . . . . . . . . . 116

3.3.1.2.2 Intermediate $\ldots \ldots \ldots \ldots \ldots \ldots \ldots$

3.3.1.2.3 Breakdown . . . . . . . . . . . . . . . . 119

3.3.1.2.4 Concerted Reaction _. . . . . . . . . . . . . . 120

3.3.1.3 Comparison with experimental data . . . . . . . . . . . . . 120

3.3.1.3.1 Favored pathway . . . . . . . . . . . . . . . 120

3.3.1.3.2 Accuracy ..................... 121 
3.3.2 Alkaline hydrolysis . . . . . . . . . . . . . . . 122

3.3.2.1 $\mathrm{B}_{\mathrm{AC}} 2$ mechanism . . . . . . . . . . . . . . . 123

3.3.2.1.1 Addition . . . . . . . . . . . . . . . . . . . 123

3.3.2.1.2 Intermediate . . . . . . . . . . . . . . . . . 124

3.3.2.1.3 Breakdown . . . . . . . . . . . . . . . 124

3.3.2.2 Hydrolysis of diketene . . . . . . . . . . . . . . . . 125

3.3.2.3 Comparison with experimental data . . . . . . . . . . . . . 127

3.3.2.3.1 Preferred pathway . . . . . . . . . . . . . . . 127

3.3 .2 .3 .2 Accuracy . . . . . . . . . . . . . . 127

3.3 .3 Acid hydrolysis . . . . . . . . . . . . . . . . . . 128

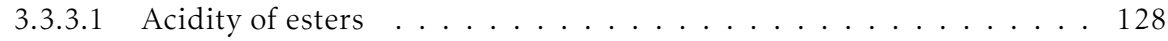

3.3.3.2 $\mathrm{A}_{\mathrm{AL}} 1, \mathrm{~A}_{\mathrm{AC}} 1$ and $\mathrm{A}_{\mathrm{AL}} 2$ mechanisms . . . . . . . . . . 129

3.3.3.2.1 $\mathrm{A}_{\mathrm{AL}} 1$ mechanism . . . . . . . . . . . . . . . 131

3.3.3.2.2 $\mathrm{A}_{\mathrm{AC}} 1$ mechanism . . . . . . . . . . . 132

$3.3 .3 .3 \mathrm{~A}_{\mathrm{AC}} 2$ mechanism . . . . . . . . . . . . . . 133

3.3.3.3.1 Addition . . . . . . . . . . . . . . . . . . . 134

3.3.3.3.2 Intermediate . . . . . . . . . . . . . . 135

3.3.3.3.3 Cleavage. . . . . . . . . . . . . . . 135

3.3.3.4 Comparison to experimental results . . . . . . . . . . . 136

3.3.3.4.1 Preferred mechanism . . . . . . . . . . . . . 136

3.3.3.4.2 Accuracy . . . . . . . . . . . . . . 137

3.3.3.5 Acid hydrolysis at low water concentrations . . . . . . . . . . . 137

3.3 .4 Conclusions . . . . . . . . . . . . . . . . . . . . . 139

References ......................... 140 


\section{Introduction}

Lactones have a variety of applications as intermediates in organic synthesis or in the chemical industry, and even unsubstituted ones have wide applications: e. g. $\beta$-propiolactone has been used as a disinfectant, viral inactivating agent in vaccines, in the manufacture of acrylic acid and its esters, and as a monomer; $\gamma$ butyrolactone is used as a solvent and is also a prodrug of $\gamma$-hydroxybutyric acid (GHB or liquid ecstasy), an illegal recreational drug analogue of neurotransmitter GABA; $\delta$-valerolactone is used in the synthesis of polyesters; etc.. Many drugs and natural products have the lactone functional group:

- Macrolides: Antibiotics like azithromycin, clarithromycin, dirithromycin, erythromycin, roxithromycin or telithromycin or immunosupresants like tacrolimus, pimecrolimus and sirolimus.

- Sesquiterpene lactones artemisinin and analogues artesunate, artemether, dihydroartemisinin, artelinic acid, artenimol and artemotil are used against falciparum malaria.

- Camptothecin and its analogues topotecan, irinotecan, exatecan or lurtotecan are anticancer topoisomerase I inhibitors in which the lactone moiety directly interacts with the enzyme-DNA complex.

- 4-Hydroxycoumarins have anticoagulant properties. First generation compounds like acenocoumarol, dicumarol, ethyl biscoumacetate, phenprocoumon and warfarin are used as anticoagulants, whereas coumatetralyl, difenacoum, flocoumafen, bromadiolone, coumatetralyl, tioclomarol and brodifacoum are used as rodenticides.

As regards this work, the most important aspect of lactone reactivity is their electrophilicity, which is responsible for their hydrolysis and alkylation reactions.

\subsection{Background}

Esters undergo hydrolysis in a variety of experimental conditions that, most usually, include some catalyst: acidic or basic medium, metal ions, cyclodextrins, enzymes or nucleophiles. Being somewhat more electrophilic than their open-chain counterparts, non-catalyzed reaction pathways exist in the case of lactone hydrolysis. In the first place, the general aspects of ester hydrolysis will be reviewed.

\subsubsection{Hydrolysis of esters}

The hydrolysis of esters has been the subject of a myriad of experimental and theoretical works. From the earliest kinetic works from which many of the general concepts and relationships in chemical kinetics have been derived (such as the Hammett equation and the Taft equation), to modern theoretical works in which the subtleties of the mechanisms are explored and the accuracy of computational methods is tested against a very solid body of work that includes thousands of experimental rate constants and 
activation parameters. The catalyzed and uncatalyzed hydrolysis of esters have been reviewed in depth in an excellent work by Kirby. ${ }^{1}$

The most used classification of ester hydrolysis was proposed by Ingold. ${ }^{2,3}$ Eight possible mechanisms exist depending on the following criteria: (1) acid- or base-catalyzed; (2) unimolecular or bimolecular; (3) acyl or alkyl cleavage. These possible mechanisms $\left(\mathrm{B}_{\mathrm{AC}} 1, \mathrm{~B}_{\mathrm{AC}} 2, \mathrm{~B}_{\mathrm{AL}} 1, \mathrm{~B}_{\mathrm{AL}} 2, \mathrm{~A}_{\mathrm{AC}} 1, \mathrm{~A}_{\mathrm{AC}} 2\right.$, $A_{A L} 1, A_{A L}$ 2) are shown in Scheme 3.1. Internal proton transfers may occur directly, as depicted, or through the solvent.

Acid catalyzed mechanism are reversible and thus, shown with double harpoons; on the contrary, base-catalyzed pathways are only reversible in theory and thus, shown with simple arrows. Mechanisms involving water as a nucleophile are also described as B and it can be observed that, despite their names, $\mathrm{B}_{\mathrm{AC}} 1$ and $\mathrm{B}_{\mathrm{AL}} 1$ are non-catalyzed.

Whereas it is known that esters favor protonation of the acyl over the alkyl oxygen, mechanism $\mathrm{A}_{\mathrm{AC}} 1$ is shown as proceeding through the ether-protonated intermediate, since it is always possible for a reaction to proceed through a minor species and it is difficult to envision this mechanism as occurring through the carbonyl-protonated form.

Seven of these mechanisms have been observed experimentally in the hydrolysis of carboxylic acid esters; only $\mathrm{B}_{\mathrm{AC}} 1$ has not been ever observed. The catalyzed pathways proceeding through tetrahedral intermediates $\mathrm{B}_{\mathrm{AC}} 2$ and $\mathrm{A}_{\mathrm{AC}} 2$ are the most common mechanisms, both of them involving acyl-oxygen cleavage.

\subsubsection{Acid hydrolysis}

Although acidic aqueous solution is not usually the medium of choice for preparative ester hydrolysis, it is where the mechanisms of ester hydrolysis are more varied and complex. Aside from the particularities of each mechanism type, some general aspects of acid-catalyzed ester hydrolysis exist.

3.1.1.1.1. General considerations. In what conditions each particular carboxylic acid ester follows a given mechanism depends on a combination of internal and external parameters: the acyl and alkyl substituents of the ester that determine, and the concentrations - or rather activities - of protons and water.

3.1.1.1.1.1. Basicity of esters. Since all the mechanisms of acid-catalyzed hydrolysis involve protonation of the ester, some attention should be addressed to the basicity of esters. Whereas the basicities of their parents carboxylic acids are better known, there have been very few studies of the basicity of carboxylic esters, mainly because they are generally rapidly hydrolyzed in the region of acidity in which ionization takes place. $\mathrm{p} K_{\mathrm{a}}$ values for such strong acids are commonly determined by NMR and UV-Vis spectroscopy, and in the case of the esters measurements have to be extrapolated to zero time to take account of hydrolysis. Rather expectedly, NMR studies suggest that protonation takes place on the carbonyl oxygen atom over the ether oxygen. Table 3.1 reports the acid dissociation constants of some alkyl esters. 


\section{$\mathrm{A}_{\mathrm{AC}} \mathbf{1}$}

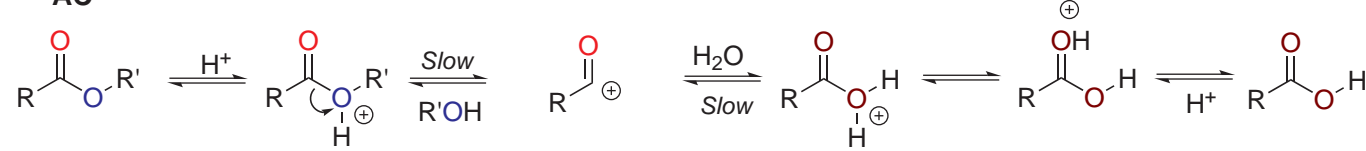

\section{$\mathrm{A}_{\mathrm{AC}} \mathbf{2}$}

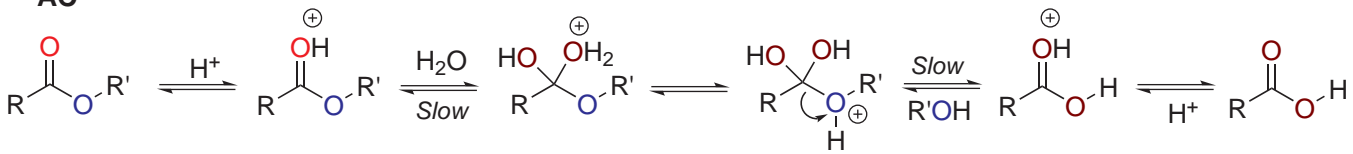

\section{$A_{A L} 1$}

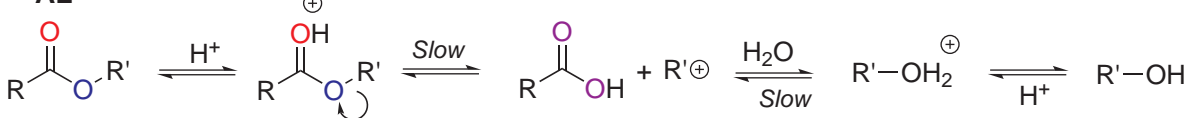

\section{$\mathrm{A}_{\mathrm{AL}} \mathbf{2}$}

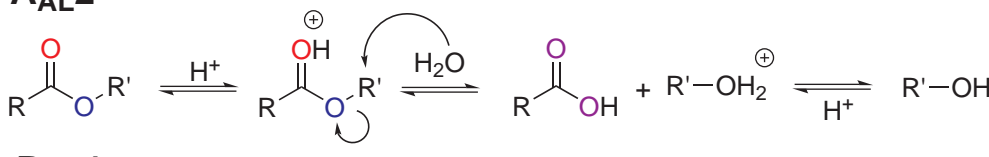

\section{$\mathrm{B}_{\mathrm{AC}} \mathbf{1}$}

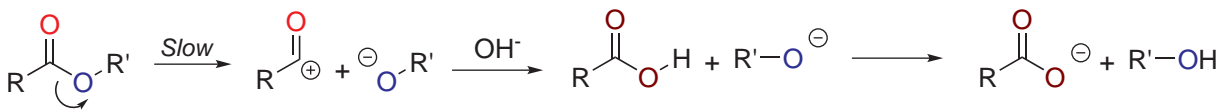

\section{$\mathrm{B}_{\mathrm{AC}} \mathbf{2}$}

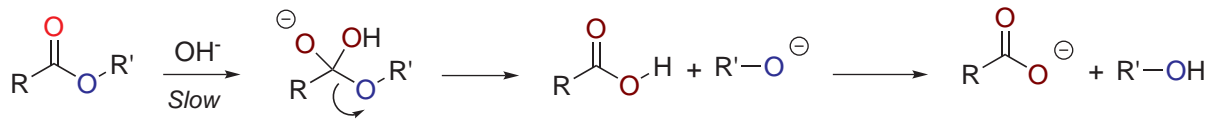

\section{$\mathrm{B}_{\mathrm{AL}} \mathbf{1}$}

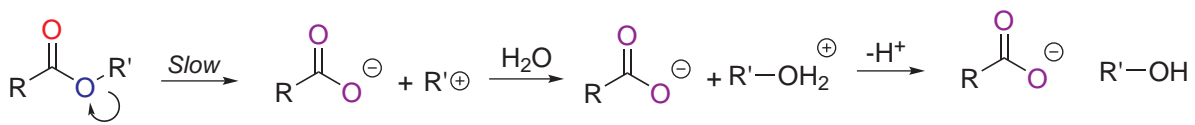

\section{$\mathrm{B}_{\mathrm{AL}}$ 2}

$\overbrace{\mathrm{O}^{-}}^{\mathrm{O}} \stackrel{\mathrm{OH}^{-}}{\longrightarrow} \stackrel{\mathrm{O}}{\mathrm{O}} \odot \mathrm{R}$ 
Table 3.1. $\mathrm{p} K_{\mathrm{a}}$ of some esters in sulfuric acid

\begin{tabular}{|c|c|c|c|}
\hline Acid & $\mathrm{p} K_{\mathrm{a}}$ & Method & Ref. \\
\hline Methyl acetate & -7.25 & UV & 4 \\
\hline Ethyl acetate & -6.93 & NMR, UV & 5,6 \\
\hline Propyl acetate & -7.18 & UV & 4 \\
\hline iso-Propyl acetate ${ }^{a}$ & -7.2 & UV & 4 \\
\hline Ethyl benzoate & -7.7 & UV & 7 \\
\hline
\end{tabular}

Since the few aliphatic esters for which $\mathrm{p} K_{\mathrm{a}}$ values are only known are very similar (methyl, $n$-propyl and iso-propyl acetate esters), all of the measured values lie in the same range, and the alkyl group seems to have little effect. Benzoate esters seem to have $\mathrm{p} K_{\mathrm{a}}$ values close to those of benzoic acid; one log unit less basic than aliphatic esters. The protonation constants of benzoic acids are correlated by the Hammet equation with $\rho \sim-1.09$, but esters hardly behave like Hammet bases at all.

3.1.1.1.1.2. Kinetics of reaction in strongly acidic media. The rate of hydrolysis of a carboxylic ester in strong sulfuric acid $\left(k_{\mathrm{H}}\right)$ generally shows one of the three types of dependence on acid concentration illustrated in Figure 3.1

- An increase in hydrolysis rate with increasing acid concentration is observed in the hydrolysis of tertiary alcohols esters. This reaction is very fast even in mildly acidic conditions. It is also shown by phenol esters, both substituted and unsubstituted, which are somewhat less reactive. This corresponds to the $\mathrm{A}_{\mathrm{AL}} 1$ mechanism.

- Esters of primary aliphatic alcohols show an initial increase in rate with increasing acid concentration. Around $50-60 \% \mathrm{H}_{2} \mathrm{SO}_{4}$ the curve reaches a maximum, after which the rate coefficient subsequently falls almost to zero in the region of $80 \%$ acid. Finally, the rate increases very slightly between $80 \%$ and $100 \% \mathrm{H}_{2} \mathrm{SO}_{4}$. Methyl acetate is rather more reactive than the ethyl ester in above about $50 \% \mathrm{H}_{2} \mathrm{SO}_{4}$, and $n$-propyl acetate shows closely similar reactivity. This has been interpreted as a transition from $\mathrm{A}_{\mathrm{AC}} 2$ to $\mathrm{A}_{\mathrm{AC}} 1$.

- Secondary aliphatic alcohols and benzyl acetate show an intermediate behavior: a very sharp increase in rate at moderate acid concentrations, typical of the first type of ester, followed with a rate maximum at intermediate acidities typical of the second and a final continuous increase at very high acid concentrations.

3.1.1.1.1.3. Acyl vs. Alkyl cleavage. In the regions of concentrated acid (above $50 \% \mathrm{H}_{2} \mathrm{SO}_{4}$ ), two types of cleavage are possible: alkyl-oxygen cleavage is a very fast reaction for the conjugate acids of tertiary alcohols and also occurs at a measurable rate at low temperatures for the protonated esters of secondary alcohols, but is not important for primary alkyl esters. Acyl-oxygen cleavage, on the other 

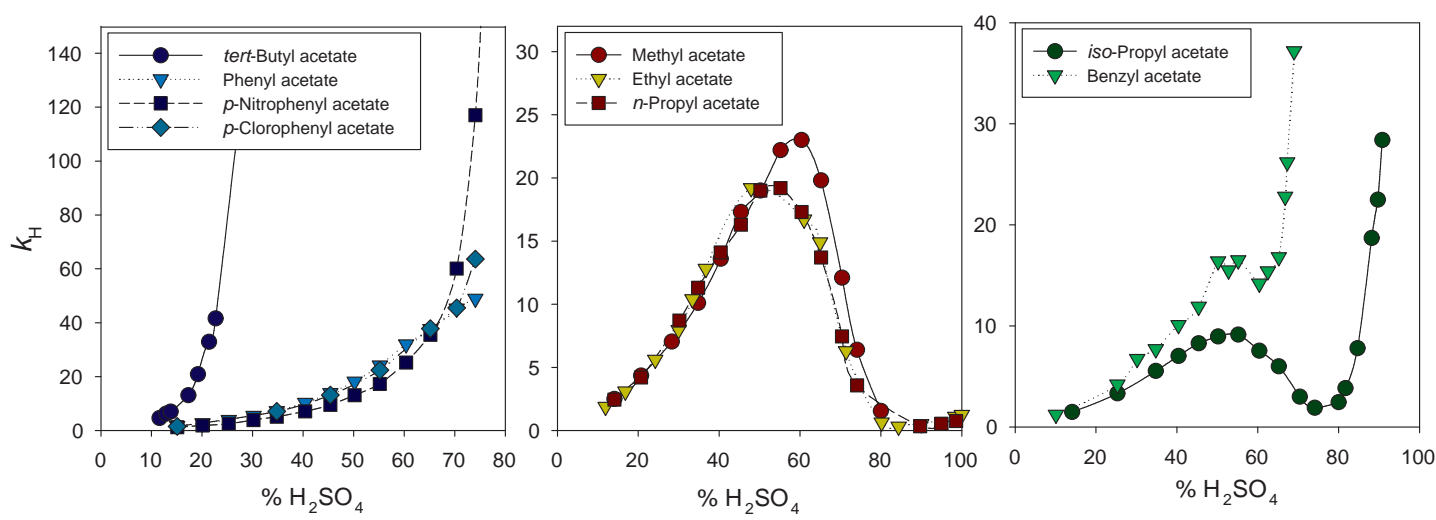

Figure 3.1. Hydrolysis rate of some acetates in concentrated sulfuric acid. Recalculated from. ${ }^{1}$ Methyl, $n$-propyl, phenyl, benzyl,p-nitrophenyl and $p$-chlorophenyl from; ${ }^{4}$ ethyl acetate from ${ }^{8,9}$ and $t$-butyl acetate from ${ }^{1}$

hand, becomes significant only in very concentrated media, where the activity of water is very low, like concentrated sulfuric acid, and thus is important only with esters of primary alcohols.

In the case of some particular esters, these reactions can be very fast even in less concentrated conditions: for example, $t$-butyl acetate shows very rapid alkyl oxygen cleavage, to the point that the formation of only a small proportion of the protonated form leads to a rapid hydrolysis. The rapid increase in the rate of hydrolysis of secondary esters like iso-propyl acetate above about $80 \% \mathrm{H}_{2} \mathrm{SO}_{4}$ is probably due to this process. However, the reaction does not become too fast for convenient measurement until the ester is nearly completely protonated. The curve for iso-propyl acetate could be expected to reach a plateau, if it could be measured in the $100 \% \mathrm{H}_{2} \mathrm{SO}_{4}$ region.

The hydrolysis of methyl acetate is faster than that of ethyl acetate. Since this is the opposite of the expected behavior if alkyl-oxygen cleavage takes place, the final increase in the rate of hydrolysis of the esters of primary aliphatic alcohols, in $85-100 \% \mathrm{H}_{2} \mathrm{SO}_{4}$ is presumably due to acyl-oxygen cleavage. Since aryl-oxygen cleavage is not expected to be so fast, the hydrolysis of aryl acetates at moderate acidity is possibly due to acyl-cleavage.

This dichotomy can explain qualitatively why the hydrolysis of some esters becomes faster as the acidity of the medium is increased. However, it does not explain the observed decreases in hydrolysis rate with increasing acidity.

3.1.1.1.1.4. Unimolecular vs. bimolecular. This decline can be explained if another reaction of the protonated ester is considered, i.e. a bimolecular reaction with a molecule or molecules of water.

Two opposing processes take place as the concentration of acid increases: the activity of water declines and the concentration of the highly reactive protonated ester rises. However, since the ester is fully protonated around $60-70 \% \mathrm{H}_{2} \mathrm{SO}_{4}$, a situation is reached in which increasing the concentration of acid decreases the activity of one reactant (water), but does not increase the concentration of the other 
(the protonated ester). Thus, bimolecular -or higher molecularity- hydrolysis slows down as the acidity increases. Similar behavior is observed in the hydrolysis of amides in strong acid solutions, but the rate maximum occurs at lower acid concentration, since amides are more basic than esters.

Most esters are, in fact, hydrolyzed by the $\mathrm{A}_{\mathrm{AC}} 2$ mechanism in less concentrated solutions of strong acids: all the esters in Figure 3.1, for example, with the exception of $t$-butyl acetate, are thought to be hydrolyzed by the $\mathrm{A}_{\mathrm{AC}} 2$ mechanism below $50 \% \mathrm{H}_{2} \mathrm{SO}_{4}$.

3.1.1.1.1.5. Kinetic order in water: higher molecularity?. The Zucker-Hammett hypothesis, which has been applied often to the study of ester hydrolysis, states that reactions for which $\log k_{\mathrm{obs}}$ is proportional to the Hammett acidity function $\left(H_{0}\right.$, eq. 3.1) are generally those thought for other reasons to involve a rate-determining unimolecular reaction of the protonated substrate. On the other hand, reactions for which $\log k_{\text {obs }}$ is proportional to $\log \left[\mathrm{H}_{3} \mathrm{O}^{+}\right]$appeared to be those in which a molecule of solvent is involved in the transition state, as well as the protonated substrate, e.g. the acid-catalyzed hydrolysis of simple carboxylic esters. ${ }^{10}$

$$
H_{0}=\mathrm{pK}_{\mathrm{BH}^{+}}+\log \frac{[\mathrm{B}]}{\left[\mathrm{BH}^{+}\right]}=-\log \left(\mathrm{a}_{\mathrm{H}^{+}} \frac{\gamma_{\mathrm{B}}}{\gamma_{\mathrm{BH}^{+}}}\right)
$$

Although the Zucker-Hammett hypothesis works remarkably well in some cases, it also leads to incorrect conclusions sometimes. This is because one of its main underlying assumptions, that the protonation behavior of all neutral substrates is quantitatively similar, is incorrect. More powerful approaches have been proposed such as Bunnett's $w$ or that by Yates and McClelland.

Bunnett proposed a new mechanistic parameters on the divergences between experimental results and ideal Zucker-Hammett behavior. He found that $\log k_{\mathrm{obs}}+H_{0}$, or $\log k_{\mathrm{obs}}-\log \left[\mathrm{H}_{3} \mathrm{O}^{+}\right]$plotted against the logarithm the activity of water, give straights. The slope of that line defines $w$ (or $\left.w^{*}\right)^{11-14}$

$$
\log k_{\mathrm{obs}}+H_{0}=w \log a_{\mathrm{H}_{2} \mathrm{O}}+\text { constant }
$$

The $w$-values range between -3 and +7 and are used by Bunnett as a new criterion of mechanism for acid-catalyzed reactions, e.g. from the value of $w \sim 5$, it can be inferred that two molecules of water take part in the transition state both as a proton donor and a nucleophile in the $\mathrm{A}_{\mathrm{AC}} 2$ mechanism. This treatment includes the influence of the activity, to which it relates divergences from ideal Zucker-Hammett.

The $w$ parameter has been improved. ${ }^{15}$ However, $H_{0}$ does not describe the protonation behavior of all neutral substrates and it can hardly be extrapolated among different compounds, even very similar ones. Thus, the minimum requirements for a valid treatment of the data for the hydrolysis of any given substrate are both an accurate knowledge of its protonation behavior in the medium used, and due allowance for the variation in the activity of water

Yates and McClelland extended proposed a hydration number treatment, based in a definition of an acidity function, $H_{S}=m H_{0}$, for the ionization of protonated acetate esters. ${ }^{4}$ Since all aliphatic acetates measured behave similarly, a common equation is proposed for all acetate esters: 


$$
\log \frac{\left[\mathrm{BH}^{+}\right]}{[\mathrm{BH}]}=0.62\left(-\mathrm{H}_{0}-7.2\right)
$$

The use of $H_{S}$ requires less of approximations that those implicit in the use of $H_{0}$, while the treatment remains more general than the experimentally unattainable ideal of measuring the acidity function directly for every substrate. Despite its accuracy in the study of aliphatic ester hydrolysis, $H_{S}$ fares less well in the description of the protonation behavior of substituted phenyl acetates.

From the proposed hydrolysis mechanism, involving fast pre-equilibrium protonation of the substrate, followed by rate-determining attack of water on the conjugate acid of the ester, they deduce that

$$
\log k_{\mathrm{obs}}-\log \left(\frac{h_{\mathrm{S}}}{h_{\mathrm{S}}+K_{\mathrm{SH}}}\right)=r \log a_{\mathrm{H}_{2} \mathrm{O}}+\text { constant }
$$

Where $K_{\mathrm{SH}}$ is the acid dissociation constant of the ester, $h_{\mathrm{S}}$ is the acidity function for the ester $a_{\mathrm{H}_{2} \mathrm{O}}$ is the activity of water and $r$ the kinetic order of the reaction in water. If it is assumed that only a fraction of the ester is protonated, then $K_{\mathrm{SH}} \gg h_{\mathrm{S}}$

$$
\log k_{\mathrm{obs}}+H_{\mathrm{S}}=r \log a_{\mathrm{H}_{2} \mathrm{O}}+\text { constant }
$$

which is similar as the equation derived by Bunnett, that defines the $w$-parameter (eq. 3.2).

3.1.1.1.2. AAC 1. Acyl (or acylium) cations are well-established chemical species. ${ }^{3}$ In the absence of more favorable processes, acids and esters are generally cleaved to acyl cations in sufficiently strong acid, which is followed by reaction with water in the $\mathrm{A}_{\mathrm{AC}} 1$ mechanism (3.2).

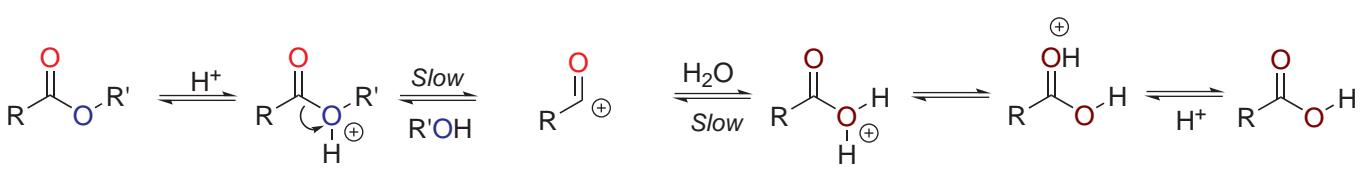

Scheme 3.2. $A_{A C} 1$ mechanism of ester hydrolysis

The existence of the $\mathrm{A}_{\mathrm{AC}} 1$ mechanism was first inferred from the cryoscopic behavior of mesitoic acid (2,4,6-trilmethylbenzoic acid). ${ }^{16}$ The primary alkyl derivatives of mesitoic acid have been frequently used in the study of the $\mathrm{A}_{\mathrm{AC}} 1$ mechanism since the bimolecular acylic attack is subject to severe steric hindrance and unimolecular alkyl cleavage can be ruled out. ${ }^{17}$ Aside from the hindrance of alternative mechanisms, the $\mathrm{A}_{\mathrm{AC}} 1$ mechanism seems to be particularly favorable for derivatives of mesitoic acid, presumably because of steric assistance. ${ }^{17}$

Activation parameters in are agreement with the unimolecular mechanism: positive activation entropies and high activation enthalpies: $\Delta^{\ddagger} S^{\ominus}=82 \pm 21$ and $25 \pm 21 \mathrm{~J} \mathrm{~mol}^{-1} \mathrm{~K}^{-1}, \Delta^{\ddagger} H^{\ominus}=119 \pm 4$ and $90 \pm 4 \mathrm{~kJ} \mathrm{~mol}^{-1}$, in 9.8 and $11.5 \mathrm{M}$ sulfuric acid, respectively. ${ }^{17}$ 
It has been found that the logarithms of the rate coefficients for hydrolysis at $90{ }^{\circ} \mathrm{C}$ in aqueous solutions of perchloric and sulfuric acids (1-6 M) are linear functions of $H_{0}$ (at $25^{\circ} \mathrm{C}$ ), with slopes of about 1.2 , but do not vary linearly with concentration of $\mathrm{H}_{3} \mathrm{O}^{+}$. This is the expected result for a unimolecular reaction according to the Zucker-Hammett hypothesis. ${ }^{18}$

However, results using different mineral acids suggest that the (un)fulfillment of the Zucker-Hammett hypothesis depends, quite randomly, on the acid used. ${ }^{19}$ The value of Bunnett's $w$ parameter for the reaction in sulfuric acid is calculated as -1.1 , and in perchloric acid as -2.5 , both values falling in the region $(-2.5<w<0)$ characteristic of reactions not involving a molecule of solvent in the transition state. ${ }^{11,12}$

The ${ }^{18} \mathrm{O}$ exchange reaction of methyl mesitoate in $\mathrm{H}_{2} \mathrm{SO}_{4}$ behaves similarly to the hydrolysis, both as regards the activation parameters, and the fulfillment of the Zucker-Hammet hypothesis. ${ }^{20}$

Since the activity of water decreases significantly with increasing acid concentration, the kinetic order of the $\mathrm{A}_{\mathrm{AC}} 1$ mechanism with respect to water can be estimated. The hydrolysis of methyl benzoate in sulfuric acid at $20^{\circ} \mathrm{C}$ has been observed to be first-order with respect to ester concentration, but zerothorder with respect to water in concentrations up to $1 \mathrm{M}^{1}{ }^{1}$ Additional evidence of the first order for the $\mathrm{A}_{\mathrm{AC}} 1$ can be found in the analysis developed by Lane, ${ }^{5,6}$ and also Jaques, ${ }^{9}$ in their works about the $\mathrm{A}_{\mathrm{AC}}{ }^{2}$ hydrolysis of ethyl acetate. Yates and McClelland expanded those conclusions using a similar method, derived from Bunnett's hydration parameter. ${ }^{4}$

Acetates of phenols or primary alcohols in concentrated sulfuric acid (>90\%) also hydrolyze following the $\mathrm{A}_{\mathrm{AC}} 1$ pathway, whereas in more dilute conditions they follow the more common $\mathrm{A}_{\mathrm{AC}} 2$ pathway. Phenols are less basic than alcohols, and thus better leaving groups. ${ }^{4}$ Therefore, they could be expected to hydrolyze faster than the primary alkyl esters by the $\mathrm{A}_{\mathrm{AC}} 1$ mechanism. Although this effect may be partially weakened by the less favorable protonation constant, the rate coefficients for the hydrolysis of aryl acetates at high acid concentrations are in the order $p$-nitrophenyl $>$-chlorophenyl $>$ phenyl (although the reverse order is found in the $\mathrm{A}_{\mathrm{AC}} 2$ region).

$\beta$-Lactones, due to their special structural features, also react in strong acid by the $\mathrm{A}_{\mathrm{AC}} 1$ mechanism (vide infra).

The assertion that esters of primary aliphatic alcohols hydrolyze by the $\mathrm{A}_{\mathrm{AC}} 1$ mechanism in very strongly acidic media requires a qualification: sufficiently strongly electron-withdrawing substituents on the acyl group (as in ethyl 4-nitro-4-chloro-3-nitro, and 3,5-dinitro-benzoates ${ }^{21}$ ) can make the formation of the acyl cation even less favorable than that of a primary carbonium ion; under these circumstances even a primary alkyl ester may be hydrolyzed by the $\mathrm{A}_{\mathrm{AL}} 1$ mechanism.

3.1.1.1.3. A A $\mathbf{A}$ 1. The study of ester hydrolysis involving alkyl-oxygen cleavage requires much less concentrated solutions of the strong acids than those required for significant rates of reaction by the $\mathrm{A}_{\mathrm{AC}} 1$ mechanism. The alkyl-oxygen cleavage of the protonated forms of tertiary alkyl esters is a very fast reaction in concentrated and dilute aqueous acid, e.g. the hydrolysis of $t$-butyl acetate becomes too fast for convenient measurement in $20-30 \% \mathrm{H}_{2} \mathrm{SO}_{4}$ where only a very small proportion of the protonated species is present. ${ }^{4,19}$ However, it is not faster than the proton transfers involved (Scheme 3.3) 
A variety of tests have been used to prove that alkyl-oxygen cleavage takes place ${ }^{22}$ such as the use of $\mathrm{H}_{2}{ }^{18} \mathrm{O}$ as a tracer ${ }^{23}$ or use of chiral alcohol groups, which undergo racemization upon hydrolysis. ${ }^{24}$

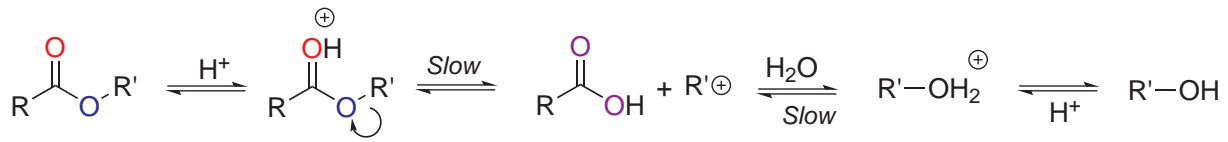

Scheme 3.3. $A_{A L} 1$ mechanism of ester hydrolysis

As regards the unimolecular nature of the reaction, the high activation enthalpies (typically 100$\left.120 \mathrm{~kJ} \mathrm{~mol}^{-1}\right)$ and positive activation entropies $\left(\sim 50 \mathrm{~J} \mathrm{~mol}^{-1} \mathrm{~K}^{-1}\right)^{25-32}$ are consistent with the unimolecular nature of $\mathrm{A}_{\mathrm{AL}} 1$, as is the null volume of activation. ${ }^{33}$

Bunnett's $w$-parameter is -1.2 for the hydrolysis of $t$-butyl acetate catalyzed by $\mathrm{HCl}$ at $25^{\circ} \mathrm{C}$ falls in the region expected for a reaction not involving a molecule of water in the transition state. ${ }^{11}$ Yates and McClelland, using their hydration number treatment (vide supra) provided evidence that the transition state for rate-determining step does not include water molecules, even more, they suggest that a water molecule is released. ${ }^{4}$

Very often a combination of the major $\mathrm{A}_{\mathrm{AL}} 1$ and the minor $\mathrm{A}_{\mathrm{AC}} 2$ mechanisms is observed. Using ${ }^{18} \mathrm{O}$, it is possible to determine the relative contribution of the two pathways, and even, to determine the activation parameters for the two processes. ${ }^{34}$ The activation parameters for the acid-catalyzed hydrolysis of $t$-butyl acetate in water are $\Delta^{\ddagger} H^{\ominus}=112 \mathrm{~kJ} \mathrm{~mol}^{-1}, \Delta^{\ddagger} S^{\ominus}=+55 \mathrm{~J} \mathrm{~mol}^{-1} \mathrm{~K}^{-1}$ for the $\mathrm{A}_{\mathrm{AL}} 1$ pathway and $\Delta^{\ddagger} H^{\ominus}=69 \mathrm{~kJ} \mathrm{~mol}^{-1}, \Delta^{\ddagger} S^{\ominus}=-102 \mathrm{~J} \mathrm{~mol}^{-1} \mathrm{~K}^{-1}$ for the $\mathrm{A}_{\mathrm{AC}} 2$ pathway. ${ }^{32}$

The higher activation enthalpy is responsible for the increase of alkyl-oxygen cleavage with temperature, for instance, in the hydrolysis of $t$-butyl acetate the percentage of alkyl-oxygen cleavage goes from $84.8 \%$ at $25{ }^{\circ} \mathrm{C}$ to $95.5 \%$ at $70{ }^{\circ} \mathrm{C}$. This effect is also responsible for the non-fulfillment of the Arrhenius equation, since at higher temperatures it is the activation parameters of the major $\mathrm{A}_{\mathrm{AL}} 1$ reaction that are measured (see Table 3.2). ${ }^{32}$

Table 3.2. Effect of solvent on the acid-catalyzed hydrolysis of $t$-butyl acetate

\begin{tabular}{|c|c|c|c|}
\hline \multirow{2}{*}{ Solvent } & \multicolumn{3}{|c|}{$10^{6} \times \mathrm{k}_{\mathrm{H}}\left(\mathrm{M}^{-1} \mathrm{~s}^{-1}\right)$ at $25^{\circ} \mathrm{C}$} \\
\hline & Overall & $\mathrm{A}_{\mathrm{AL}} 1$ & $A_{A C}{ }^{2}$ \\
\hline Water & 128 & $\sim 124$ & $\sim 4$ \\
\hline $70 \%$ water/dioxane & 6.8 & 2.4 & 4.3 \\
\hline
\end{tabular}

An interesting feature of the $\mathrm{A}_{\mathrm{AL}} 1$ mechanism is that it is very sensitive to the water content of the reaction medium. ${ }^{34}$ Whereas the $\mathrm{A}_{\mathrm{AC}} 2$ contribution remains almost constant when going from pure water to a medium with a significant proportion of organic cosolvent, the reaction rate of the formerly major $\mathrm{A}_{\mathrm{AL}} 1$ pathway is decreased, sometimes to the point it may become minor, or event non-existent. ${ }^{35}$ For instance, the contribution of $\mathrm{A}_{\mathrm{AL}} 1$ to the hydrolysis of $t$-butyl acetate at $25{ }^{\circ} \mathrm{C}$ is $85 \%$ in water, $70 \%$ in 
$50 \%$ aqueous dioxane and $39 \%$ in $75 \%$. This occurs because the transition state for the $\mathrm{A}_{\mathrm{AL}} 1$ mechanism is highly polarized, and thus, very sensitive to the dielectric constant of the reaction medium.

Table 3.3. Activation enthalpy for the acid hydrolysis of $t$-butyl acetate in aqueous acetone. $[\mathrm{HCl}]=0.005-0.02$ $\mathrm{M}$

\begin{tabular}{|c|c|c|}
\hline$\%$ Acetone & $T\left({ }^{\circ} \mathrm{C}\right)$ & $\Delta^{\ddagger} H^{\ominus}\left(\mathrm{kJ} \mathrm{mol}^{-132}\right)$ \\
\hline 80 & $85.3-97.1$ & 126 \\
\hline 80 & $72.4-85.3$ & 121 \\
\hline 60 & $85.3-97.1$ & 120 \\
\hline 60 & $72.4-85.3$ & 114 \\
\hline 60 & $48.7-72.4$ & 106 \\
\hline 40 & $85.3-97.1$ & 109 \\
\hline 40 & $72.4-85.3$ & 107 \\
\hline 40 & $48.7-72.4$ & 106 \\
\hline
\end{tabular}

For the same reasons, when the reaction is carried out in increasingly non-aqueous medium, the activation parameters vary from those typical of the bimolecular $\mathrm{A}_{\mathrm{AC}} 2$ reaction (lower activation enthalpies and negative activation entropies) for to those of the unimolecular $\mathrm{A}_{\mathrm{AL}} 1$ (high activation enthalpies and positive activation entropies) mechanism (Table 3.2).

Despite the bulky substituent, the hydrolysis of $t$-butyl formate occurs through the $\mathrm{A}_{\mathrm{AC}} 2$ mechanism, being one of the very few tertiary esters hydrolyzed by this mechanism. Because of the high activation enthalpy of the $\mathrm{A}_{\mathrm{AL}} 1$ mechanism, the alkyl contribution becomes significant at very high temperatures. ${ }^{29}$ Triphenylmethyl acetate and benzoate are solvolyzed with alkyl-oxygen fission even under neutral conditions in a strongly acid-catalyzed reaction. ${ }^{35-37}$ The hydrolysis of triphenylmethyl acetate involves almost quantitative alkyl-oxygen fission in both acidic and initially neutral solution, as shown by the incorporation of ${ }^{18} \mathrm{O}$ from enriched $\mathrm{H}_{2} \mathrm{O}$ into the triphenylmethyl alcohol produced, and by the lack of incorporation into the acetic acid. ${ }^{35}$

Diphenylmethyl esters hydrolyze in a slower reaction and diphenylmethyl acetate has been shown to follow the $\mathrm{A}_{\mathrm{AL}} 1$ mechanism at high temperature and low ratios of organic cosolvent; on the other hand, the contribution of the $\mathrm{A}_{\mathrm{AC}} 2$ mechanism is enhanced as temperature decreases. Diphenylmethyl formate has a stronger $\mathrm{A}_{\mathrm{AC}} 2$ contribution and the acid-catalyzed hydrolysis proceeds with exclusive acyloxygen cleavage, in $70 \%$ aqueous dioxan $(0.078$ and $0.141 \mathrm{M}$ in $\mathrm{HCl})$ at $35^{\circ} \mathrm{C}$. The activation parameters, $\Delta^{\ddagger} H^{\ominus}=65 \mathrm{~kJ} \mathrm{~mol}^{-1}$ and $\Delta^{\ddagger} S^{\ominus}=-97 \mathrm{~J} \mathrm{~mol}^{-1} \mathrm{~K}^{-1} \cdot 31,36$

The introduction of one $p$-methoxyl substituent into diphenylmethy acetate increases the rate of the $\mathrm{A}_{\mathrm{AL}} 1$, so that only akyl-oxygen cleavage is observed, as evidenced by the second-order rate coefficients following $\mathrm{H}_{0}$ rather than concentration of $\mathrm{H}_{3} \mathrm{O}^{+}$; the observed deuterium isotope effect, $k_{\mathrm{D}} / k_{\mathrm{H}}$ is $\sim 2$ and the activation parameters are $\Delta^{\ddagger} H^{\ominus}=100 \mathrm{~kJ} \mathrm{~mol}^{-1}$ and $\Delta^{\ddagger} S^{\ominus}=-2.5 \mathrm{~J} \mathrm{~mol}^{-1} \mathrm{~K}^{-1} \cdot{ }^{38}$ In diphenylmethyl trifluoroacetate both the $\mathrm{A}_{\mathrm{AC}} 2$ and $\mathrm{A}_{\mathrm{AL}} 1$ pathways are favored by the strong electron-withdrawing substituents and which one of them dominates is again a matter of temperature and organic cosolvent ra- 
tio. $^{39}$

Two other class of esters hydrolyze by the $\mathrm{A}_{\mathrm{AL}} 1$ mechanism: alkoxymethyl acetates and $\beta$-isovalerolactone. In alkoxymethyl acetates the ether is probably more basic than the ester group, so these substrates react as acetals. As esters, alkyl-oxygen cleavage is not unexpected, since the methoxyl group helps stabilize the carbonium ion. The hydrolysis mechanism of BIVL is discussed somewhere else (vida infra) The hydrolysis of secondary alkyl esters, such as isopropyl acetate, and that of benzyl acetate are also thought to follow the $\mathrm{A}_{\mathrm{AL}} 1$ mechanism, but they require much higher acid concentrations. 4,19

3.1.1.1.4. $\mathbf{A}_{\mathbf{A L}}$ 2. $\quad \mathrm{A}_{\mathrm{AL}} 2$ has only been reported in the hydrolysis of some $\gamma$-lactones, and thus, its existence is somewhat disputed. Moore et al. observed that (2RS,3S, $4 \mathrm{~S})-\mathrm{N}$-benzoyl- $\left[3,4-{ }^{2}-\mathrm{H}_{2}\right]$ homoserine lactone undergoes partial epimerization at C-4 (but not C-3) when heated at reflux in $6 \mathrm{~N} \mathrm{HCl} \mathrm{H}{ }_{2}^{18} \mathrm{O} .{ }^{40,41}$ From the possible reaction mechanisms, based on the ${ }^{18} \mathrm{O}$ incorporation and the epimerization, it was concluded that the reaction proceeded partially through an $\mathrm{A}_{\mathrm{AL}} 2$ mechanism.

Since the $\mathrm{A}_{\mathrm{AC}} 2$ lactone hydrolysis is a reversible process, it can occur many times without epimerization or incoporation of the ${ }^{18} \mathrm{O}$ tracer. Thus, under the working conditions, the very minor $\mathrm{A}_{\mathrm{AL}} 2$ contribution could be isolated even if the attack at the carbonyl group was many orders of magnitude faster. However, the harsh conditions and prolonged reaction times suggest that the $\mathrm{A}_{\mathrm{AL}} 2$ contribution is minute, and unobservable in direct competence with $\mathrm{A}_{\mathrm{AC}} 2$ hydrolysis.

Although they are not esters stricto sensu, $\mathrm{A}_{\mathrm{AL}} 2$ hydroylsis has been reported for benzimidate esters. ${ }^{42}$

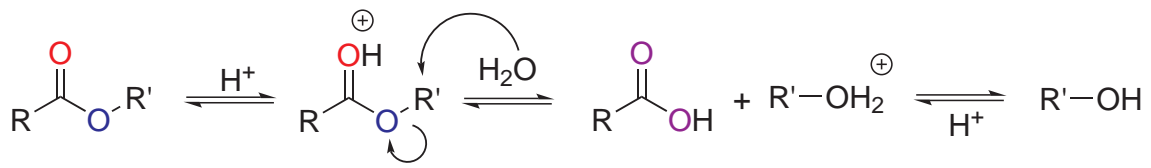

Scheme 3.4. $A_{A L} 2$ mechanism of ester hydrolysis

3.1.1.1.5. A AC2. The mechanisms for acid-catalyzed ester hydrolysis described so far represent exceptional behavior; they are generally observed only with compounds with narrowly defined structural characteristics, or under extreme conditions. The vast majority of esters are hydrolyzed in most acidic conditions by the $\mathrm{A}_{\mathrm{AC}} 2$ mechanism. Whereas some particulars of this mechanism are disputed, many of its features are well known and must be taken into account when proposing a detailed reaction pathway (Scheme 3.5), namely that it proceeds through acyl-oxygen cleavage, its two-step nature and the observed reaction order.

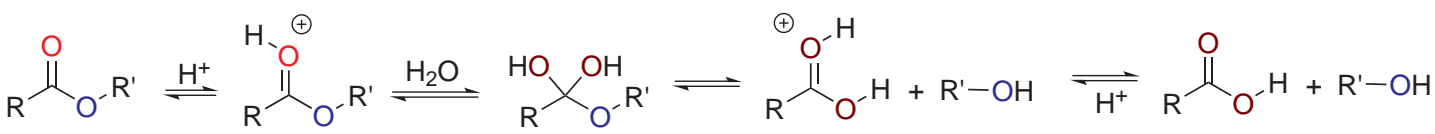


3.1.1.1.5.1. Acyl-oxygen cleavage. The fact that acyl-oxygen cleavage takes place, is supported in a combination of evidence suggesting that acyl-cleavage takes place, or that alkyl cleavage does not:

1. Hydrolysis in $\mathrm{H}_{2}^{18} \mathrm{O}$ results in the marked ${ }^{18} \mathrm{O}$ atom appearing in the acid, not in the alcohol. ${ }^{36,43-45}$

2. Alcohols formed retain the configuration of chiral R' groups. ${ }^{46}$

3. No allylic rearrangement is observed when $R^{\prime}$ is allylic. ${ }^{47}$

4. Neopently R' gives no rearrangement. ${ }^{48}$

3.1.1.1.5.2. Two-step nature. Whereas convincing evidence supports a two-step mechanism involving formation and cleavage of a tetrahedral intermediate, this addition product is very labile, and has never been observed. ${ }^{49}$ The proof for the two-step nature of this mechanism has also been obtained by means of ${ }^{18} \mathrm{O}$ labeling. ${ }^{50}$

Hydrolysis of isotopically labeled ethyl benzoate in non-marked aqueous $1 \mathrm{M}$ acid at $99^{\circ} \mathrm{C}$ shows that oxygen exchange between the unreacted ester and the solvent takes place, and the enrichment of the remaining ester decreases steadily as hydrolysis proceeds. This is in keeping with a mechanism involving a full intermediate. The addition elimination mechanism receives further support from the observation that hydrolysis and exchange proceed at similar rates, with a constant ratio, $k_{\text {hyd }} / k_{\text {ex }}=5.2$ for ethylbenzoate.

Whereas this does not necessarily imply that the tetrahedral species through which the exchange takes place is an intermediate, plots of $k_{o b s} /\left[\mathrm{BH}^{+}\right]$against $\log a_{\mathrm{H}_{2} \mathrm{O}}$ have a slope of 2.07 for hydrolysis and 1.84 for ${ }^{18} \mathrm{O}$ exchange. ${ }^{5,6}$ This is interpreted as evidence of two water molecules being involved in the respective transition states, suggesting that they share the rate-determining step. Finally, no exchange is observed in the acid hydrolysis of methyl 2,4,6-trimethylbenzoate, ${ }^{17}$ a typical $\mathrm{A}_{\mathrm{AC}} 1$-reacting species. Additional evidence exists for the two-step nature of the alkaline hydrolysis mechanism $\mathrm{B}_{\mathrm{AC}} 2$ (vide infra).

3.1.1.1.5.3. Kinetic order. The kinetic order of the hydrolysis reaction is easily determined to be unity for both ester concentration and the hydronium ions. However, determination of the reaction order with respect to solvent is more difficult and many different approaches have been attempted; from the earlier and less conclusive works of Friedman and Elmore ${ }^{51}$ or Sadek et al., ${ }^{1}$ which already suggest that the reaction is second order in water, to more conclusive studies, which have often applied the Zucker-Hammett hypothesis. ${ }^{10}$

An archetypal case is that of $\gamma$-butyrolactone, which was found to undergo acyl fission using ${ }^{18} \mathrm{O}$ labelling. ${ }^{44}$ Since its hydrolysis is not quantitative and an equilibrium exists between the open and closed forms, it is possible to determine both hydrolysis and cyclization rate constants. The plots of $\log k$, against $\log \left[\mathrm{H}_{3} \mathrm{O}^{+}\right]$give straight lines with slope close to unity. Similar plots of the cyclization rate constant deviate highly from a straight line, but the data are now linear with respect to $H_{0}$, the slope being also close to unity. ${ }^{52,53}$ This is what the Zucker-Hammett hypothesis predicts: one water molecule is necessary for the hydrolysis, but none for the lactone formation. 
By plotting $\log k_{\mathrm{obs}} /\left[\mathrm{BH}^{+}\right]$against $\log a_{\mathrm{H}_{2} \mathrm{O}}$, Lane obtained order two for both the hydrolysis and the ${ }^{18} \mathrm{O}$ exchange in the hydrolysis of ethyl acetate in $11-79 \% \mathrm{H}_{2} \mathrm{SO}_{4}$ at $25{ }^{\circ} \mathrm{C} .{ }^{5,6} \mathrm{In}$ an extension of this work to $100 \% \mathrm{H}_{2} \mathrm{SO}_{4}$ it was observed that, above about $80 \%$ sulfuric acid, there is a sudden change of slope from close to 2.0 to about -0.16 , this is attributed to a change of mechanism to $\mathrm{A}_{\mathrm{AC}} 1$ at very low water activity. ${ }^{9}$
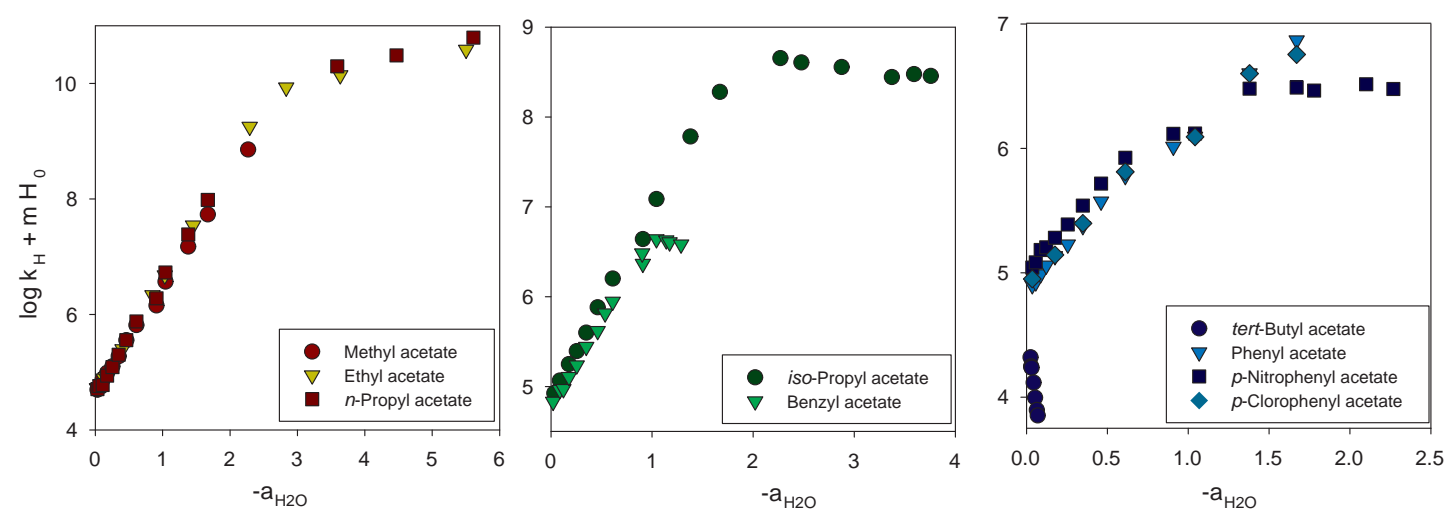

Figure 3.2. Kinetic order in water for the acid hydrolysis of some acetates.

When the method by Yates and McClelland is applied, ${ }^{4}$ similar results are obtained for methyl, ethyl and $n$-propyl acetates (Figure 3.2): excellent straight lines of almost identical slope, $r,(r \simeq 2)$ in the region $0-80 \% \mathrm{H}_{2} \mathrm{SO}_{4}$ and a break at $80-85 \%$ acid, followed by a second linear region, where the slope has a much smaller, negative value $(r \simeq-0.2)$. The plot for $p$-nitrophenyl acetate also levels out to a small negative slope above about $70 \% \mathrm{H}_{2} \mathrm{SO}_{4}$. This is almost certainly a result of a change from $\mathrm{A}_{\mathrm{AC}} 2$ to the $\mathrm{A}_{\mathrm{AC}} 1$ mechanism. This weak dependence of the rate constant on water activity is evidence that water is not involved as a reactant in the transition state for hydrolysis, which is a unimolecular process involving acyl-oxygen fission. Even more, the negative value is interpreted as water being expelled in the rate determining step of the reaction.

As has been said, the acetate esters of secondary aliphatic alcohols and benzyl alcohol are hydrolyzed by the $\mathrm{A}_{\mathrm{AL}} 1$ mechanism at high acid concentration. The same type of behavior as before is observed (Figure 3.2): a good straight line $(r \simeq 2)$ at lower acid concentrations is succeeded for each ester by a break at an intermediate acidity region, and a different linear dependence, with a smaller, negative, slope $(r \simeq-0.6)$ in the high acidity region.

The method, however, fails completely for $t$-butyl acetate, which is known to follow the $\mathrm{A}_{\mathrm{AL}} 1$ mechanism: the plot shows a very strongly negative slope, which can be shown to be in the region of $\sim-10$. The rate coefficient for the hydrolysis of the protonated form of $t$-butyl acetate increases very rapidly with increasing acid concentration, or with decreasing water activity. This unexpected result could be explained by the Yates and McClelland method not being applicable to $t$-butyl acetate, which seems unlikely, since all alkyl esters seem to obey $H_{S}$ or by some factor not being allowed for. This deviation has been explained as an effect of increasing ionic strength, since it is well-known that increasing ionic strength strongly favors ionization reactions such as $\mathrm{S}_{\mathrm{N}} 1$. 
The results for three aryl esters are also plotted in Figure 3.2: an initial linear region with a slope in the region of $1.5<r<2$ gives way in more concentrated acid to a region where the slope appears to settle down to a small, negative value.

Less deactivated esters undergo sulfonation at high acid concentrations, and thus, data are limited at the higher acidities. It can be observed that the linear region is much smaller in the case of aryl esters, which suggests that $H_{S}$ fails partially in this case.

Table 3.4 summarizes the $r$ values for the hydrolysis of some esters.

Table 3.4. $r$-Values for the acid hydrolysis of esters ${ }^{4}$

\begin{tabular}{|c|c|c|c|c|}
\hline Acetate & $r$ & $\% \mathrm{H}_{2} \mathrm{SO}_{4}$ & $r$ & $\% \mathrm{H}_{2} \mathrm{SO}_{4}$ \\
\hline Methyl & 1.92 & $0-80$ & -0.2 & $>80$ \\
\hline Ethyl & 2.10 & $0-80$ & -0.18 & $>85$ \\
\hline n-Propyl & 2.06 & $0-80$ & -0.2 & $>85$ \\
\hline iso-Propyl & 2.11 & $0-75$ & -0.57 & $>80$ \\
\hline sec-Butyl & 2.18 & $0-70$ & -0.66 & $>75$ \\
\hline Benyl & 1.9 & $0-60$ & -0.5 & 60 \\
\hline Phenyl & 1.6 & $0-50$ & $<0$ & $>70$ \\
\hline p-Nitrophenyl & 1.6 & $0-50$ & -0.2 & $>70$ \\
\hline p-Chlorophenyl & 1.5 & $0-55$ & & \\
\hline$t$-Butyl & $\sim-10$ & $0-30$ & & \\
\hline
\end{tabular}

The corresponding treatment for data for amide hydrolysis gives a $r$ values approaching 3 , and the fact that low integral values are obtained in each case is in agreement with the interpretation of $r$ as the kinetic order of the reaction with respect to water. The results for ester hydrolysis, where an $r$ value close to 2.0 is found for all but the most reactive (t-butyl) ester, at low to medium acid concentrations, are encouragingly consistent with a common mechanism in this acidity region. Evidence suggests that this is the so called $\mathrm{A}_{\mathrm{AC}} 2$ mechanism, and that it is order two in water.

3.1.1.1.5.4. Pathway. The $\mathrm{A}_{\mathrm{AC}} 2$ hydrolysis mechanism has been concluded to be first order in the ester and the acid concentration, and second order in water, which is consistent with the proposition of five exchangeable protons are involved in the reaction ${ }^{54}$ and Burnett's $w$ parameter, that suggests that water is involved as a proton transfer agent. ${ }^{14}$

Therefore, the structure of the transition state includes an ester molecule, a proton and two water molecules; whether the proton is involved in a hydronium ion or as the conjugate acid of the ester is somewhat unclear. Also, a termolecular process could, in principle, be discarded, since activation entropies are in a range of -80 to $-120 \mathrm{~J} \mathrm{~K}^{-1} \mathrm{~mol}^{-1}$ and processes suspected to be termolecular have entropies of activation around -160 to $-200 \mathrm{~J} \mathrm{~K}^{-1} \mathrm{~mol}^{-1}$. Enthalpies of activation are usually around $\sim 65$ $\mathrm{kJ} \mathrm{mol}{ }^{-1} \cdot 20,55$ 
Both two-step and concerted pathways have been proposed for this mechanism. The concerted mechanism proposed by Lane ${ }^{5,6}$ (Scheme 3.6) justifies the observed correlation between hydrolysis and ${ }^{18} \mathrm{O}$ exchange, since both oxygen atoms are in similar chemical environments. Since a neutral water molecule can hardly expel a hydroxide/alkoxide leaving group, the leaving group must be protonated. Given that the proton from the acid catalyst is present in the substrate, this second hydrogen must come from the attacking water molecule, and be transferred through the second water molecule. An argument against this mechanism is that it is termolecular.

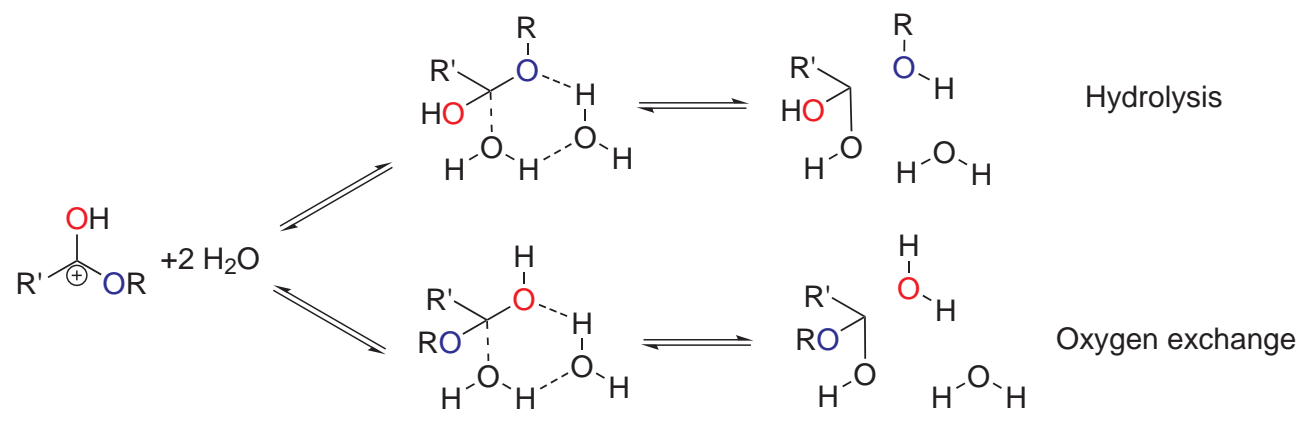

Scheme 3.6. Concerted mechanism for $A_{A C} 2$ ester hydrolysis

Mechanisms involving general species catalysis of the formation of a tetrahedral intermediate seem unlikely, since they involve termolecular collisions. General acid catalysis of the addition of water by the hydronium ion can be ruled out, since it involves the addition of water to what can be considered as a partially protonated carbonyl group, a process which must be slower than the addition to the fully protonated group.

An addition mechanism is possible, in which one water molecule acts as a general base in the addition of another water molecule to the protonated ester (Figure 3.7). Water is a very weak base as regards general base catalysis, and the fact that the process is termolecular plays against this mechanism. However, the products formed in the base-catalyzed pathway are hydronium ion and the ester gem-diol, which are presumably more stable than the protonated orthoester formed in the direct addition, which is expected to be highly acidic. ${ }^{1}$

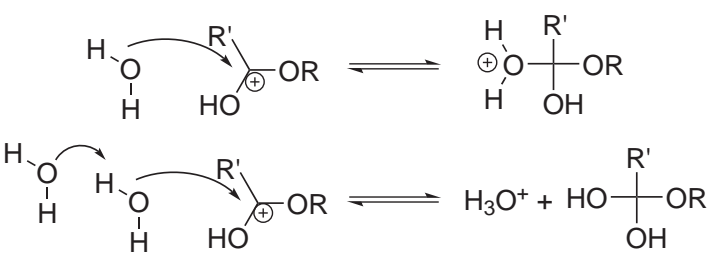

Scheme 3.7. Addition of water catalyzed by another water molecule

The fact that $O$-exchange and hydrolysis rates are very similar suggests that the forward and back reactions of the tetrahedral intermediate take place at similar rates, and thus neither formation or cleavage of the tetrahedral intermediate are rate-limiting. Since the tetrahedral intermediate in the $\mathrm{A}_{\mathrm{AC}} 2$ is a 
monoalkyl orthoester, comparison to the hydrolysis of trialkyl orthoesters can be useful. The hydrolysis of fully esterified orthoesters shows general acid catalysis, even by weak acids, and both the proton transfer and the carbon-oxygen bond cleavage are thought to occur in a concerted step. The transition state is thought to have the proton transfer well advanced, but a relatively small amount of carbon-oxygen bond breaking.

The hydrolysis of esters is many times slower than that of trialkyl orthoesters (e.g. ethyl orthoacetate hydrolyzes $\sim 10^{8}$ times faster than ethyl acetate) and monoalkyl orthoesters must hydrolyze even faster. If breakdown of the tetrahedral intermediate is partially rate-limiting, the concentration of the tetrahedral intermediate must be very low.

Given the similarities between the nucleophile and the leaving group, the mechanism can be considered quite symmetrical. Thus, if the breakdown of the tetrahedral intermediate is subject to general acid catalysis by the hydronium ion, as is the case with fully sterified orthoesters, then the addition of water must be subject to general-base catalysis by another water molecule, as suggested above (Figure 3.8).

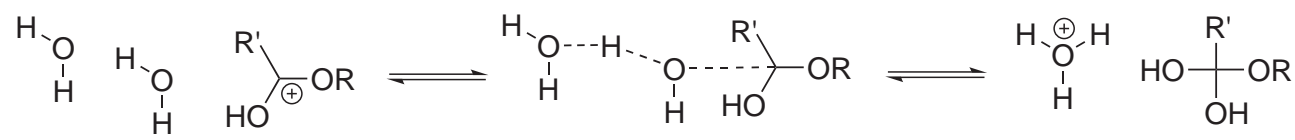

Scheme 3.8. General acid catalysis in the breakdown in the tetrahedral intermediate implies general-base catalysis in water addition

Although, as said before, water is a weak base, the initial product of the addition of a molecule of water to a protonated ester would be the protonated orthoester. In the hydrolysis of orthoesters carbonoxygen bond-cleavage is concerted with the transfer of the proton to the orthoester, which suggests that a protonated orthoester is too energetic to exist in aqueous solution. This implies that formation of a neutral orthoester, by a general base catalyzed process, even by such a weak base, will be more favorable than formation of the protonated orthoester. Thus, the full mechanism can be written as shown in Scheme 3.9. Although $k_{4}$ and $k_{5}$ are labelled as fast, they are partially rate-determining, since the concentration of tetrahedral intermediate is very low.

The rate equation for such a process is complex, even though the possible role of $\mathrm{ROH}$ and $\mathrm{ROH}_{2}^{+}$as general catalysts is not included. If the steady-state approximation is applied to the tetrahedral intermediate, the rate of disappearance of the ester is:

$$
-\frac{d[\mathrm{E}]}{d t}=\frac{k_{3} k_{5}\left[\mathrm{EH}^{+}\right]\left[\mathrm{H}_{2} \mathrm{O}\right]^{2}}{k_{4}+k_{5}}=\frac{K_{\mathrm{w}} k_{3} k_{5}[\mathrm{E}]\left[\mathrm{H}_{3} \mathrm{O}^{+}\right]\left[\mathrm{H}_{2} \mathrm{O}\right]}{K_{\mathrm{a}}\left(k_{4}+k_{5}\right)}
$$

3.1.1.1.5.5. Polar effects. The protonation reaction of the ester has a negative Hammett sensitivity constant $(\rho \sim-1)$, and so does the breakdown of the intermediate; however, the attack of water at the carbonyl is expected to have a large positive $\rho$ value. In other words, substituents that increase the acidity of the ester, decrease its electrophilicity, and thus polar effects for the global reaction, which includes the pre-equilibrium, the addition and the cleavage steps, are small. 


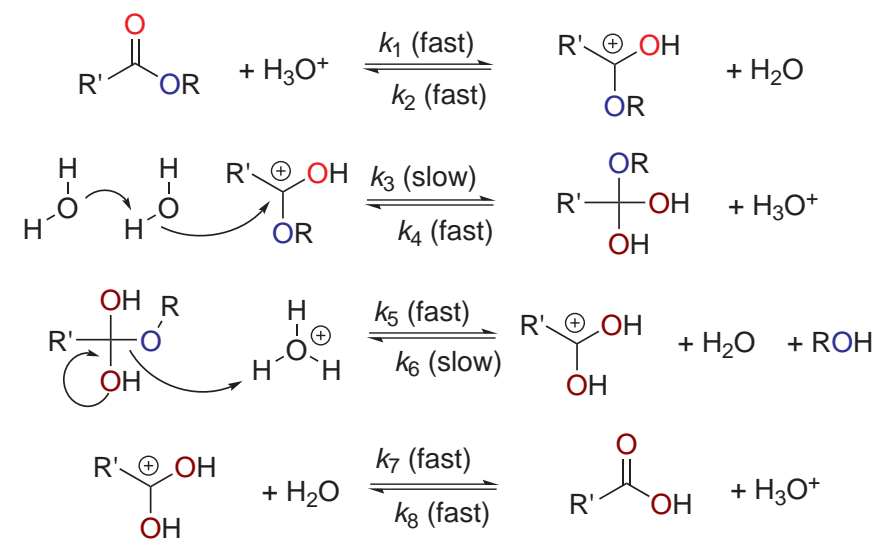

Scheme 3.9. Complete accepted mechanism for $A_{A C} 2$ ester hydrolysis

Thus, it is no unexpected that the acid-catalyzed hydrolysis of benzilic and aliphatic esters have $\rho$ values close to zero. ${ }^{55-57}$

For instance, chlorine and methyl substituents have similar effects in the hydrolysis rates of ethyl acetate $^{58}$ and the hydrolysis of $p-\mathrm{NO}_{2}$ ethyl benzoate is only two-fold faster than that of $p$-OHethyl benzoate, but the hydrolysis of the $o-\mathrm{NO}_{2}$ benzoate compound is 20 times slower. ${ }^{58}$ Therefore, only steric effect are of importance. Based on this argument, and on the hydrolysis rate constants of methyl esters, Taft developed the well-known free energy relationship that carries his name.

As regards the leaving group, electron-withdrawing groups reduce the hydrolysis rate of alkyl and phenyl acetates, albeit slightly. ${ }^{59}$

3.1.1.1.5.6. Steric effects. In the hydrolysis of benzylic esters, the substituents in ortho have the strongest steric effect, since they block the ester group. Rate constants and activation parameters for the hydrolysis of different ethyl esters are given in Table 3.5. The values show the great influence of steric effects on the rate.

Since the alkyl group is one bond further from the electrophilic carbon, steric effects in the leaving group are also very mild; e.g. the rate coefficients for the hydrolysis of ethyl, iso-propyl and tert-butyl formates in dilute aqueous $\mathrm{HCl}$ at $25^{\circ} \mathrm{C}$ are 3.36, 2.92 and $5.98 \times 10^{-3} \mathrm{M}^{-1} \mathrm{~s}^{-1}$, respectively (the last figure possibly includes a contribution from the $\mathrm{A}_{\mathrm{AL}} 1$ mechanism). However, this effect is increased in the hydrolysis of esters with very bulky acids. ${ }^{63}$

The contribution of the steric effects to the activation parameters are usually compensating. ${ }^{55,60-62}$ The presence of bulky substituents increases the compression energy of all the groups, and thus, increments the activation enthalpy. The effect upon solvation is as follows: a rise in $\Delta^{\ddagger} H^{\ominus}$, since the solvent molecules cannot reach the ester easily, and a simultaneous relaxation of the solvation shell, and thus, a partially compensating increase $\Delta^{\ddagger} S^{\ominus}$. Very bulky groups can block the rotation or vibration of sub- 
Table 3.5. Influence of the acyl substituentes on the acid hydrolysis of ethyl esters ${ }^{60-62}$

\begin{tabular}{|c|c|c|c|c|}
\hline R-COOEt & $\begin{array}{c}k_{\mathrm{H}^{+}} \\
\mathrm{M}^{-1} \mathrm{~s}^{-1}\end{array}$ & $\begin{array}{c}\Delta^{\ddagger} H^{\ominus} \\
\left(\mathrm{kJ} \mathrm{mol}{ }^{-1}\right)\end{array}$ & $\begin{array}{c}\Delta^{\ddagger} S^{\ominus} \\
\left(\mathrm{J} \mathrm{mol}^{-1} \mathrm{~K}^{-1}\right)\end{array}$ & $\begin{array}{c}\Delta^{\ddagger} G^{\ominus} \\
\left(\mathrm{kJ} \mathrm{mol}^{-1}\right)\end{array}$ \\
\hline $\mathrm{H}$ & 3360 & 61.4 & -85.7 & 87.0 \\
\hline $\mathrm{CH}_{3}$ & 44.7 & 65.2 & -109.1 & 97.8 \\
\hline $\mathrm{CH}_{3} \mathrm{CH}_{2}$ & 37 & 64.8 & -110.4 & 98.0 \\
\hline $\mathrm{CH}_{3}\left(\mathrm{CH}_{2}\right)_{2}$ & 19.6 & - & - & 99.8 \\
\hline $\mathrm{CH}_{3}\left(\mathrm{CH}_{2}\right)_{3}$ & 17.9 & - & - & 100.1 \\
\hline $\mathrm{CH}_{3}\left(\mathrm{CH}_{2}\right)_{4}$ & 17.7 & 65.2 & -116.6 & 100.0 \\
\hline $\mathrm{CH}_{3}\left(\mathrm{CH}_{2}\right)_{5}$ & 16.4 & - & - & 100.3 \\
\hline $\mathrm{CH}_{3}\left(\mathrm{CH}_{2}\right)_{6}$ & 15.5 & - & - & 100.4 \\
\hline $\mathrm{C}\left(\mathrm{CH}_{3}\right)_{2} \mathrm{CH}$ & 13.5 & 66.0 & -116.6 & 100.8 \\
\hline $\mathrm{C}\left(\mathrm{CH}_{3}\right)_{3} \mathrm{C}$ & 1.3 & 77.3 & -98.6 & 106.7 \\
\hline $\mathrm{C}_{2} \mathrm{H}_{5}\left(\mathrm{CH}_{3}\right) \mathrm{CH}$ & 5.7 & - & - & 102.9 \\
\hline$\left(\mathrm{C}_{2} \mathrm{H}_{5}\right)_{2} \mathrm{CH}$ & 1.2 & - & - & 106.8 \\
\hline $\mathrm{C}_{6} \mathrm{H}_{11}$ & 7.4 & 68.1 & -118.3 & 102.8 \\
\hline $\mathrm{C}_{6} \mathrm{H}_{5}$ & - & 79.0 & -110.4 & 111.9 \\
\hline $\mathrm{C}_{6} \mathrm{H}_{5} \mathrm{CH}_{2}$ & 9.3 & 64.8 & -120.4 & 101.2 \\
\hline $\mathrm{C}_{6} \mathrm{H}_{5}\left(\mathrm{CH}_{2}\right)_{2}$ & 8.1 & 65.6 & -118.7 & 101.5 \\
\hline $\mathrm{C}_{6} \mathrm{H}_{5}\left(\mathrm{CH}_{2}\right)_{3}$ & 8 & - & - & 101.1 \\
\hline $\mathrm{C}_{6} \mathrm{H}_{5}\left(\mathrm{CH}_{2}\right)_{4}$ & 8.6 & 66.9 & -113.7 & 101.3 \\
\hline $\mathrm{C}_{6} \mathrm{H}_{5} \mathrm{CH}\left(\mathrm{CH}_{3}\right)$ & 0.171 & 68.1 & -122.9 & 104.3 \\
\hline $\mathrm{C}_{6} \mathrm{H}_{5} \mathrm{CH}\left(\mathrm{C}_{2} \mathrm{H}_{5}\right)$ & 0.067 & 69.0 & -127.9 & 106.7 \\
\hline $\mathrm{C}\left(\mathrm{C}_{6} \mathrm{H}_{5}\right)_{2} \mathrm{CH}$ & 0.030 & 71.5 & -126.2 & 108.7 \\
\hline $\mathrm{C}\left(\mathrm{CH}_{3}\right)_{3} \mathrm{NCH}_{2} \mathrm{CH}_{2}$ & - & 66.9 & -106.2 & 98.5 \\
\hline $\mathrm{ClCH}_{2}$ & - & 61.4 & -121.6 & 97.7 \\
\hline $\mathrm{Cl}_{2} \mathrm{CH}$ & - & 56.8 & -141.7 & 99.1 \\
\hline $\mathrm{Cl}_{3} \mathrm{C}$ & - & 48.1 & -158.4 & 95.3 \\
\hline $\mathrm{FCH}_{2}$ & 23.5 & - & - & 91.7 \\
\hline $\mathrm{F}_{2} \mathrm{CH}$ & 95 & - & - & 88.3 \\
\hline
\end{tabular}


stituents, and thus decrease $\Delta^{\ddagger} S^{\ominus} .^{1}$

An interesting feature is that formates hydrolyze 10- to 100-fold faster than acetates. Since polar effects reflect mainly in $\Delta^{\ddagger} H^{\ominus}$, this effect must arise from steric contributions. In general, hydrolysis rates decrease with increasing chain length of primary alkyl groups, until this decrease levels out, in a mainly entropic effect. The fact that cyclohexane substituents, which are already rather rigid, have a normal entropic term confirms that the slowing down is due to the reduction of degrees of freedom in the transition state.

3.1.1.1.5.7. Weakly acidic esters. Acids with very strongly electron-withdrawing substituents, such as haloesters have very low $\mathrm{p} K_{\mathrm{a}}$ values, and are not significantly protonated even in $100 \%$ sulfuric acid. As a consequence, these ester show very weak acid catalysis, and a very fast neutral hydrolysis rate. $^{1}$

Interestingly, in the esters that do show some catalysis, the reaction rate shows a moderate increase until it reaches a maximum at about 1-2 $\mathrm{M}$ catalyst concentration, then, it decreases slowly. This decline is attributed to the salt effect, which decreases the activity of water. ${ }^{38,39}$ A Bunnett plot for the hydrolysis of $o$-nitrophenyl oxalates or phenyl trifluoroacetate gives $w$ vales around 10 , which is explained as water acting a proton transfer agent in the rate-limiting step. ${ }^{64}$ Experiments at constant ionic strength make it unclear whether the catalysis at 1-2 $\mathrm{M}$ acid is a specific salt effect on the neutral hydrolysis reaction, or represents a genuine, though very weakly, acid-catalyzed reaction.

The order of sensitivity to acid catalysis is o-nitrophenyl oxalates $<$ phenyl trifluoroacetate $<$ methyl trifluoroacetate. The fact that this also is the inverse order of leaving group ability (catalysis is observed for the poorer leaving groups), suggests that breakdown of the intermediate is both (co)rate-limiting and sensitive to acid catalysis. Since the reverse addition reaction and the breakdown are acid catalyzed, the breakdown reaction is rate-limiting for the poorer leaving groups, and thus, is accelerated by acid. For better leaving groups, the breakdown reaction is faster and no catalysis is observed.

\subsubsection{Neutral hydrolysis}

The reactions discussed in this section are those of ester hydrolysis under neutral conditions, designated together with the base catalyzed ones by the letter B in Ingold's classification. As stated above, mechanism $\mathrm{B}_{\mathrm{AC}} 1$ involves a $\mathrm{S}_{\mathrm{N}} 1$ reaction with $\mathrm{OR}^{\prime}$ as a leaving group, which does not occur.

3.1.1.2.1. B $\mathbf{B}_{\mathbf{A L}} \mathbf{2}$ mechanism. Bimolecular mechanisms involving alkyl-oxygen cleavage are rare, since oxygen nucleophiles are normally more reactive towards $s p^{2}$ - than $s p^{3}$-hybridized carbon. As rule, less hindered methyl esters would be expected to be more susceptible to these reactions. Bimolecular alkyloxygen fission, is not normally observed in ester solvolysis reactions. The single exception is the neutral hydrolysis or solvolysis of $\beta$-lactones (vide infra). Also, in the alcoholysis of esters, when the nucleophile is the same as the leaving group, the attack at the carbonyl gives no net reaction, and thus, the minor attack at the alkyl-oxygen carbon is the observed reaction. ${ }^{65,66}$ 
3.1.1.2.2. B $_{\mathbf{A L}} 1$ mechanism. As stated above, the unimolecular mechanism involving alkyl-oxygen cleavage, $\mathrm{A}_{\mathrm{AL}} 1$ occurs mainly when $\mathrm{R}^{\prime}$ comes of as a stable carbocation, that is, when $\mathrm{R}^{\prime}$ is tertiary alkyl, allylic, benzylic and so on. ${ }^{22,25,30,35-38}$ This is also the case with $\mathrm{B}_{\mathrm{AL}} 1$. Even more so, $\mathrm{B}_{\mathrm{AL}} 1$ only occurs in neutral solution, where the (relatively) faster attack by hydroxide ions is slowed. Since the reaction is an unimolecular cleavage, the reaction has high activation enthalpies $\left(\Delta^{\ddagger} H^{\ominus} \sim 95 \mathrm{~kJ} \mathrm{~mol}^{-1}\right)$ and null or slightly positive activation entropies. ${ }^{67}$

It has been observed that a significant part of the carbocation yields the elimination olefin, instead of the alcohol. Both the activation parameters and the percentage of elimination are very similar for $t$-alkyl trifluoroacetates and their $t$-alkyl halide counterparts. ${ }^{67}$

3.1.1.2.3. $\mathrm{B}_{\mathrm{AC}} 2$ mechanism. Whereas, the category $\mathrm{B}_{\mathrm{AC}} 2$ referred originally to alkaline hydrolysis, and little attention was drawn by reactions in neutral medium, a multitude of catalytic processes can assist hydrolysis in neutral solutions. Alkaline hydrolysis itself can be regarded as just one example, albeit a special case, of a large class of reactions which involve nucleophilic attack on esters. Thus, the un-catalyzed neutral hydrolysis of esters and the general base and nucleophilic catalysis will be discussed separately.

3.1.1.2.3.1. Non-catalyzed hydrolysis. At constant $\mathrm{pH}$, and discarding the aforementioned catalytic processes, the experimental hydrolysis rate constant of an ester is:

$$
k_{\mathrm{obs}}=k_{0}+k_{\mathrm{H}^{+}}\left[\mathrm{H}_{3} \mathrm{O}^{+}\right]+k_{\mathrm{OH}^{-}}\left[\mathrm{OH}^{-}\right]
$$

Depending on the type of ester, and thus the relative values of $k_{0}, k_{\mathrm{H}^{+}}$and $k_{\mathrm{OH}^{-}}$, three different plots of $k_{\text {obs }}$ can be obtained, as shown in Figure 3.3:

- The acid and base catalysis lines intercept at a minimum. This is e.g. the kinetic profile shown by ethyl acetate at $25{ }^{\circ} \mathrm{C}$ and similarly unreactive esters. Since at the minimum the sum of the acid- and base-catalyzed reactions is the same, we have that, $k_{\min }=k_{0}+2\left(k_{\mathrm{H}^{+}} \times k_{\mathrm{OH}^{-}} \times K_{\mathrm{W}}\right)^{1 / 2}$. Thus, the actual presence of spontaneous hydrolysis will only be detected depending on the relative values of the two summands.

- More reactive esters, like phenyl acetate and other with electron-withdrawing substituents in either the acyl or the leaving group of the ester, give plots with a central plateau or even lack an observable acid-catalyzed reaction, in the case of very reactive esters.

The three reactions are intrinsically different, and thus have different sensitivities towards electronic effects: $\rho$ for the acid-catalyzed hydrolysis is close to zero (vide supra). The alkaline hydrolysis of substituted alkyl benzoates has $\rho$ values around 2-2.5 and a plot of $\log k_{\mathrm{OH}^{-}}$against the $\mathrm{pK}$ of the leaving group for a large number of acetate esters has a slope of -0.26 . Thus, the acid-catalyzed reaction is almost insensitive to the presence of electron withdrawing substituents - in fact, electron-withdrawing substituents 


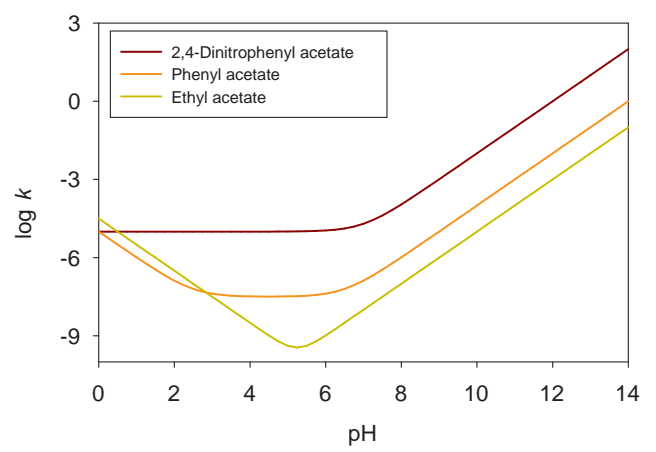

Figure 3.3. Approximate dependence of hydrolysis rate constant on $\mathrm{pH}$ for several esters. ${ }^{56,59,68}$

slow down the reaction -, and the relative importance of alkaline hydrolysis increases with the reactivity of the ester.

Of the three mechanisms, the neutral hydrolysis is the most sensitive, and a plot of log $k_{0}$ against the $\mathrm{pK}$ of the leaving group for a large number of acetate esters has a slope of -0.38 , which would predict $t_{1 / 2}=75$ years for ethyl acetate.

This sensitivity to substitution of neutral hydrolysis means that, as the reactivity increases, the $\mathrm{pH}$ independent reaction becomes more important than the hydroxide reaction at the high $\mathrm{pH}$ end of the region, and becomes much more rapidly more important than acid-catalyzed hydrolysis at low $\mathrm{pH}$.

Aside from small lactones, only very activated esters exhibit neutral hydrolysis, and this has not attracted much attention. Most studies have dealt with the hydrolysis of alkyl and aryl haloacetates, in which electron-withdrawing substituents activate the carbonyl group, in a similar way as protonation does. ${ }^{69-72}$ The results can be summarized in the activation parameters: $\Delta^{\ddagger} S^{\ominus}=-160$ to $-200 \mathrm{~J} \mathrm{~mol}^{-1}$ $\mathrm{K}^{-1}$, and the increases in reactivity due to both polar and steric factors are mainly an enthalpic effect. $\left(\Delta^{\ddagger} H^{\ominus}=25-55 \mathrm{~kJ} \mathrm{~mol}^{-1}\right.$.

High positive kinetic isotope effects of about $\sim 2$ and as high as 5 have been measured when the reaction is carried out in deuterated water. The rate of hydrolysis is reduced by organic solvents and high ionic intensities. ${ }^{73}$ This evidence points out in the direction of two water molecules (four exchangeable hydrogen atoms) being involved in the transition state. This is usually interpreted as the general base catalysis by a water molecule of the addition of another water molecule (the indistinguishable general acid catalysis by hydronium on the addition of hydroxide is regarded as improbable), in a close parallel of the $\mathrm{A}_{\mathrm{AC}} 2$ mechanism described above. ${ }^{1}$

The breakdown of the tetrahedral intermediate is expected to be partially rate-determining in the hydrolysis of alkyl esters, since the leaving group and the nucleophile are very similar, and thus the reaction is quite symmetrical. Since aryl esters have better leaving groups, the breakdown of the intermediate is probably faster, and thus, non rate-limiting. 


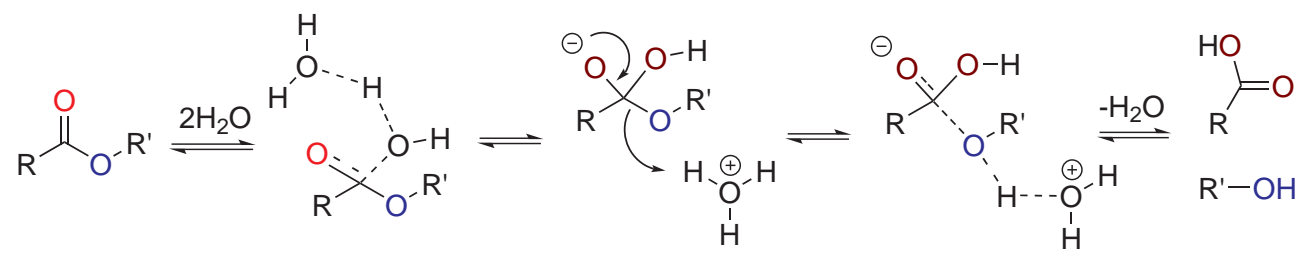

Scheme 3.10. Complete accepted mechanism for the neutral $\mathrm{B}_{\mathrm{AC}} 2$ ester hydrolysis

3.1.1.2.3.2. Nucleophilic and general base catalysis. In general, for nucleophilic catalysis of hydrolysis to be observed two conditions must concur: (i) reaction of the ester with the nucleophile is faster than its hydrolysis and (ii) the initial product is more reactive than the ester and undergoes rapid hydrolysis.

The rate-limiting step is often the initial nucleophilic displacement, and the alkaline hydrolysis can be seen as an extreme case that includes only one displacement, the step concerned is the same as the rate determining step for the reaction with many other nucleophiles, and thus, the reactions are comparable from a kinetic point of view.

A second important type of catalysis by nucleophilic reagents does not involve direct attack by the nucleophile on the ester, but rather that it acts as a general base. Two kinetically indistinguishable alternatives exist for this reaction: either the nucleophile assists the attack of a water molecule on the carbonyl group, by removing a proton from it in the rate-determining step of the reaction or the protonated nucleophile catalyzes of the attack of hydroxide ion on the carbonyl group. The first option is regarded as much more probable. This is a termolecular process and would be expected to be entropically unfavourable. Intermolecular reactions thought for other reasons to involve general base catalysis commonly have $\Delta^{\ddagger} S^{\ominus}$ around -150 to $-200 \mathrm{~J} \mathrm{~mol}^{-1} \mathrm{~K}^{-1}$.

In those cases where the breakdown of the tetrahedral intermediate is the slow step of the reaction, two kinetically equivalent mechanisms are also possible. The catalyst may be involved as a general base, catalyzing the elimination of the leaving group or it may be involved as its conjugate acid, assisting the breakdown of the anion of the tetrahedral intermediate.

The addition of water, catalyzed by general acids and bases, is most readily observed under conditions where the uncatalyzed reaction is significant. This water reaction is important only for reactive esters, which are those with electron-withdrawing substituents in either the acyl group or the leaving group. Since general base catalysis is expected to be most important for esters with leaving groups much more strongly basic than the nucleophile, it is thus observed most readily for esters with poor leaving groups that are activated by electron-withdrawing substituents on the acyl group.

\subsubsection{Base hydrolysis}

Except for extremely rare exceptions, alkaline hydrolysis of esters takes place by the $\mathrm{B}_{\mathrm{AC}} 2$ mechanism. 
3.1.1.3.1. $\mathbf{B}_{\mathrm{AL}} \mathbf{2}$ mechanism. Alkaline $\mathrm{B}_{\mathrm{AL}} 2$ has only been observed in the hydrolysis of extremely hindered esters, and its existence beyond those outmost cases is disputed. $\mathrm{B}_{\mathrm{AL}} 2$ is the only hydroylsis pathway for 2,4,6-tri-tert-butyl benzoate. ${ }^{74}$ Methyl 2-methyl-4,6-di-tert-butylbenzoate is hydrolyzed by a mixture of $\mathrm{B}_{\mathrm{AL}} 2$ and $\mathrm{B}_{\mathrm{AC}}{ }^{2}$ mechanisms, and other hindered esters (such as 2,4,6-triphenylbenzoate) exclusively by $\mathrm{B}_{\mathrm{AC}} 2 .{ }^{30}$ The contribution of the $\mathrm{B}_{\mathrm{AL}} 2$ mechanism to the hydrolysis of methyl 2,2-dimethylpropionate and methyl triphenylacetate, potential candidates for alkyl-oxygen cleavage, is negligible for the former and about $5 \%$ for the latter. ${ }^{75}$ These evidence suggests that alkaline $\mathrm{B}_{\mathrm{AL}} 2$ hydrolysis is an extremely rare event.

As regards the alkaline alcoholysis of esters, the $S_{N} 2$ reaction of alkyl carboxylates can be isolated under conditions where the attack at the carbonyl group is the faster reaction, if the nucleophile concerned is identical with the leaving group. Under these conditions displacement at the carbonyl carbon leads to no net reaction (unless the alcohol is labelled).

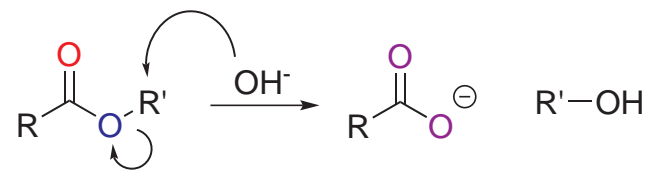

Scheme 3.11. $B_{A L} 2$ mechanism of alkaline ester hydrolysis

3.1.1.3.2. BAC2 mechanism. The full mechanism of $B_{A C} 2$ hydrolysis is though to occur as depicted in Scheme 3.12. ${ }^{1}$ Although formally reversible, the acidity of the carboxylic group is so high that ester formation from the carboxylate is not possible under basic conditions.

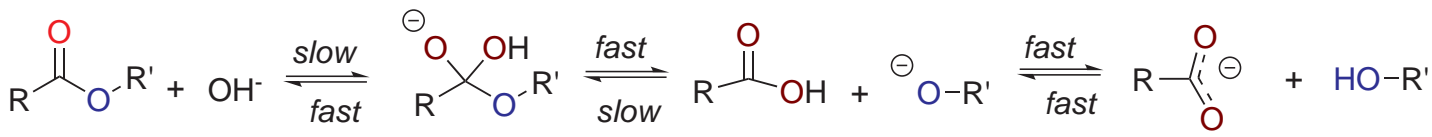

Scheme 3.12. Complete accepted mechanism for the alkaline $\mathrm{B}_{\mathrm{AC}} 2$ ester hydrolysis

The fact that the reaction takes place by acyl cleavage is well known and has been proved by similar methods as those used in the acid-catalyzed reactions: chiral alcohols retain their configuration, and when the hydrolysis is carried out in isotopically-labelled water, the ${ }^{18} \mathrm{O}$ atom ends up in the acid moiety. $36,38,39,44,45,50,76,77$ Use of the isotopic tracer has also helped prove that the reaction takes place in two steps: exchange of ${ }^{18} \mathrm{O}$ takes place at a rate comparable to that of hydrolysis. ${ }^{50}$ The same argument was used in the discussion of the $\mathrm{A}_{\mathrm{AC}} 2$ mechanism, and it is even stronger now: the protonated carbonyl could lose $\mathrm{H}_{2} \mathrm{O}$ or $\mathrm{ROH}$ by direct displacement, but an unprotonated carbonyl oxygen can only become a leaving group by the formation of the tetrahedral intermediate.

Although the existence of the tetrahedral intermediate can be hardly disputed, this does not ensure its role in the hydrolysis mechanism. The ratio of hydrolysis and O-exchange is small and independent of the rate of hydrolysis, and its values are higher for esters with good leaving groups. This suggests that the tetrahedral species is indeed an intermediate and not just a dead end in the reaction pathway. Although 
broadly accepted, the existence of the tetrahedral intermediate has not been detected experimentally, and other possible concerted reaction pathways have been proposed.

The $\mathrm{B}_{\mathrm{AC}} 2$ mechanism has been studied on many occasions, and there is little controversy about the nature and properties of the mechanism and it is the effect of structure upon the reactivity that has attracted most attention. it is not possible to discuss the structure-activity relationships on for the addition and oxygen-exchange reaction separately, since they have only been measured for few compounds.

The kinetically important steps of the hydrolysis reaction can be summarized by the simplified reaction path shown in Scheme 3.13. Although the breakdown of the tetrahedral intermediate $\left(k_{3}\right)$ takes place in a series of steps, the proton transfers involved are expected to be very fast, and thus, not rate-limiting.

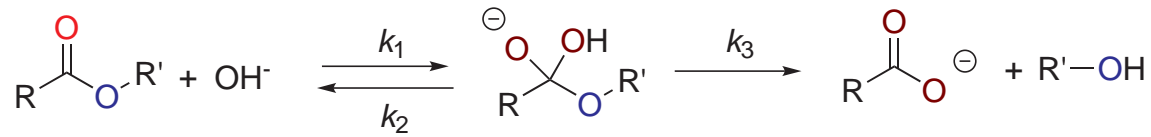

Scheme 3.13. Simplified accepted mechanism for the alkaline $B_{A C} 2$ ester hydrolysis

Using the steady-state approximation, the rate constant for the overall reaction is:

$$
k_{\text {hyd }}=\frac{k_{1} k_{3}}{k_{2}+k_{3}}
$$

In the cases when $k_{3} \gg k_{2}$, that is, for good leaving groups, the global reaction constant equals the addition constant $k_{1}$. For alkyl esters, the alkoxide and hydroxide leaving groups are very similar and thus $k_{3} \simeq k_{2}$, which forces the use of the full expression. Since only one of the equivalent oxygen atoms in the tetrahedral intermediate is istopically marked, the observed rate coefficient for oxygen exchange is just half that for the breakdown of the tetrahedral intermediate to reactants.

$$
k_{\text {exch }}=\frac{k_{1} k_{2}}{2\left(k_{2}+k_{3}\right)}
$$

Thus, knowledge of $k_{\text {hyd }}$ and $k_{\text {hyd }} / k_{\text {exch }}$ allows calculation of $k_{1}$ and $k_{3} / k_{2} .{ }^{77}$ Bender et al. studied the alkaline hydrolysis of ethyl benzoate, and they obtained very similar activation parameters for $k_{\text {hyd }}$ and $k_{1}$, which suggests that this is the main step, and that the rate decrease due to the breakdown step is an entropy effect. The hydrolysis of methyl and substituted benzyl benzoates, phthalide and $\gamma$-butyrolactone, no concurrent oxygen exchange could be detected on alkaline hydrolysis, so that $k_{\text {hyd }} \gg k_{\text {exch. }}{ }^{78}$

Since $k_{3}$ is lower for the less acidic esters and $k_{2}$ should not show much dependence on the nature of the alcohol, $k_{\text {hyd }} / k_{\text {exch }}$ decreases for worse, that is, more basic, leaving groups. Therefore, only rarely will the addition of hydroxide not be rate-determining. As a consequence, structure-activity relationships will arise from this first step.

Since the minimum in the $\mathrm{pH}$-rate plot (or the center of the $\mathrm{pH}$-independent region) falls within in the acidic region, acid catalysis can be considered less efficient than base catalysis. Also, alkaline hydrolysis is much more sensitive to polar effects (although possibly less than neutral hydrolysis), which sometimes 
screens the steric effects, to which base hydrolysis is equally sensitive, as was assumed in the development of the Taft equation. ${ }^{58}$

3.1.1.3.2.1. Polar effects. The polar effects of substituents are easily explained: since addition is most usually the limiting step, electron-withdrawing substituents will increase the electrophilicity of the carbonyl carbon, increasing the reaction rate. Values or $\rho \sim 2.4$ have been found for the alkaline hydrolysis of methyl and ethyl benzoates. ${ }^{79,80}$ In more polar media, the solvent can stabilize the charges better and smaller values are obtained: $\rho \sim 2.0$ was obtained in the hydrolysis benzoates of phenyl derivatives in $33 \%$ aqueous acetonitrile. ${ }^{81}$

ortho-Substituted benzoates have lower polar sensitivity factors, which is explained by the loss of coplanarity between the phenyl ring and the carbonyl, thus disrupting the transmission of the electronic effects. ${ }^{82}$ Substituents placed further away give lower $\rho$ (e.g. 1.21 for ortho-substituted ethyl cinnamates ${ }^{83}$ ), whereas trans allyl substituents have stronger effects (the ones in cis force the said loss of conjugation), and $\rho=3.74$ in acrylates. ${ }^{84}$

Polar effects on the leaving group are smaller, ( $\rho=1.24$ for substituted phenyl benzoates), as expected by the screening effect of the interposed oxygen atom. ${ }^{81}$ From the study of thirty five alkyl benzoates, substituted in the ring, or the alkyl group, or both, eq. 3.10 was obtained. The 2.22/1.53=0.69 ratio is similar to the difference between acyl and alkyl substituents described before and suggests that addition is mostly rate-limiting, since the bond-breaking would be more sensitive to polar effects. ${ }^{1}$

$$
\log k=0.174+2.22 \sigma_{\mathrm{AC}}+1.53 \sigma_{\mathrm{ALK}}+0.668 E_{\mathrm{S}}^{c}
$$

3.1.1.3.2.2. Steric effects. The transition states for the addition reaction (oftehn the ratelimiting step) of acid and base hydrolysis are very similar, and only differ in two protons. Therefore, steric effects are very similar for these two reactions, as was acknowledged by Taft, who assumed them to be identical in the development of the free energy relationship that carries his name. ${ }^{58}$ The steric effects on the $\mathrm{A}_{\mathrm{AC}} 2$ mechanism have been described before (vide supra)

3.1.1.3.2.3. Solvent effects. Since only one of the reactants is charged, little influence of the ionic strength on the $\mathrm{B}_{\mathrm{AC}} 2$ hydrolysis rate is observed. In general, the mild influence of the ionic strength is negative: rates decrease as the ionic strength increases, and this effect is larger for less reactive esters. ${ }^{85}$

Usually, added organic solvents also decrease the reaction rate, in what is mainly, an enthalpic effect. ${ }^{86}$ If the charge was dispersed in the transition state, the opposite effect would be expected: increasing the ratio of organic co-solvent, would destabilize the reactants more than the transition state, lowering the barrier. That is the behavior observed in many $\mathrm{S}_{\mathrm{N}} 2$ reactions, but addition to the carbonyl is complex: the more symmetric transition state for the $S_{N} 2$ reaction is expected to have better solvation and distribution of the charge that the compact transition state for the addition of hydroxide. Whereas, some authors have attributed this decrease in the reaction rate to the presence of an additional water 
molecule in the transition state, bridging the nucleophile and the leaving group; efforts to measure the kinetic order of basic hydrolysis in water have proved unsuccessful. ${ }^{87}$

Due to its particular effect as a dipolar aprotic solvent on the activity of hydroxide, moderate concentrations of dimehtyl sulfoxide bring on an increase in reaction rates. ${ }^{1}$

\subsubsection{Hydrolysis of lactones}

In the case of lactones, the presence of a cycle conditions the reactivity, with respect to that of open-chain esters: restrictions are imposed on the relative movements of the acyl and alkyl groups, which alters the entropy changes. The strain created by the ring size can also modify the enthalpy changes in the process. Acyl and alkyl groups are in cis position, contrarily to open chain esters or large lactones, and the fact that in the hydrolysis product the hydroxyl group is physically linked to the carboxylic acid makes the effective concentration of the reactants very high, favoring esterification the reaction. The closed structure also imposes restrictions on the stereochemistry of the substituents linked to the carbonyl carbon in the moment when the tetrahedral dihydroxyeter intermediate is formed.

For the reason explained above, lactones have a very particular behavior as regards their hydrolysis reactions, especially $\beta$-lactones. Cyclic esters of this type containing the highly strained four-membered ring, are very reactive towards hydrolysis by all mechanisms. Addition to the carbonyl group reduces the strain as the $s p^{2}$-hybridized carbonyl carbon is converted to the $s p^{3}$ - hybrid, with bond angles that are naturally more acute, while mechanisms involving cleavage of either the acyl-oxygen or the alkyloxygen bonds in the rate-determining step are assisted directly by the release of strain consequent upon the partial opening of the ring in the transition state.

It was stated above, that acid hydrolysis shows little dependence on substitution, and thus, highly reactive esters such show very ample $\mathrm{pH}$-independent regions. $\beta$-Lactones are no exception. Since their cyclic structure is very activated, the $\mathrm{pH}$ independent region is wide; acid catalysis is only observed below $H_{0} \sim 0$ and base catalysis only above $\mathrm{pH}>9$ (Figure 3.4). Among the three lactones depicted, DIK is the most activated, followed by BBL and BPL, and the observed plots are consistent with that order.

\subsubsection{Acid hydrolysis}

As a rule, lactone hydrolysis in moderately acidic medium, like that of linear esters, takes place by the usual $\mathrm{A}_{\mathrm{AC}} 2$ mechanism in most cases, or through the $\mathrm{A}_{\mathrm{AL}} 1$ pathway for lactones that give off particularly stable carbocations. Nevertheless, acid hydrolysis is no exception to the particular character of $\beta$-lactones: primary and secondary $\beta$-lactones in strongly acidic medium follow the less common $\mathrm{A}_{\mathrm{AC}} 1$ mechanism:

Olson and Miller, using optically active $\beta$-butyrolactone, showed that the hydroxybutyric acid produced on acid hydrolysis has the opposite configuration to that produced by the reaction in neutral water. ${ }^{88}$ Later work using ${ }^{18} \mathrm{O}$-labelled water, showed that the hydrolysis in concentrated solutions of strong acids proceeds with acyl-oxygen fission. ${ }^{89}$ 


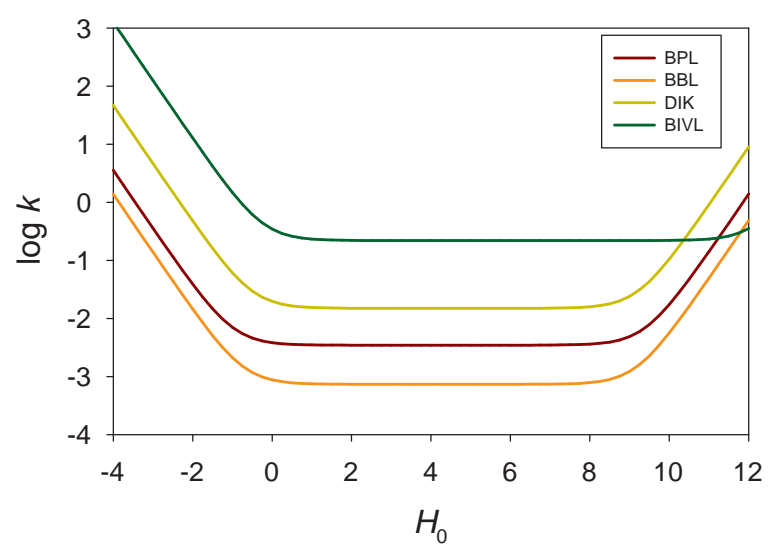

Figure 3.4. Hydrolysis rates of some $\beta$-lactones as a function of medium acidity.

A later and more complete work of the similar reaction of $\beta$-propiolactone showed that the rate of hydrolysis at $25^{\circ} \mathrm{C}$ in up to 6-7 M perchloric, sulfuric and nitric acids, is not directly correlated with acid concentration, as would be expected for a normal ester hydrolysis reaction. ${ }^{90}$ The reaction is also characterized by a very large positive salt effect. Long and Purchase show that their data are, in fact, correlated; a plot of $\log k_{\mathrm{H}} v s$. $H_{0}$, is a good straight line, of slope close to unity. It seems clear that this is a case where the Zucker-Hammett hypothesis leads to a correct conclusion: the hydrolysis of $\beta$ lactones involves a unimolecular reaction of the conjugate acid. This proves that primary and secondary $\beta$-lactones undergo acyl-oxygen fission, and thus, follow the $\mathrm{A}_{\mathrm{AC}} 1$ mechanism.

However, $\beta$-isovalerolactone (BIVL) follows a $\mathrm{A}_{\mathrm{AL}} 1$ mechanism. Whereas the addition of a single methyl group to $\beta$-propiolactone reduces the rate of hydrolysis slightly, as expected on the basis of the $\mathrm{A}_{\mathrm{AC}} 1$ mechanism, $\beta$-isovalerolactone (with a second methyl group) is hydrolyzed about a thousand times faster than $\beta$-butyrolactone. This indicates a change in the mechanism to a rate-limiting unimolecular step: $\mathrm{A}_{\mathrm{AL}} 1 .{ }^{91}$

Unlike $\beta$-lactones, the hydrolysis of five-membered lactones follow the standard $\mathrm{A}_{\mathrm{AC}} 2$ reaction mechanism, of which $\gamma$-butyrolactone (GBL) is a prototypical example. ${ }^{44,52,53}$ Also, the hydrolysis of simple $\gamma$-lactones is not quantitative, the equilibrium constants for the hydrolysis of methyl and dimethyl $\gamma$ lactones follow the sequence: $\mathrm{H} \gg \gamma-\mathrm{CH}_{3}>\beta-\mathrm{CH}_{3}>\alpha-\mathrm{CH}_{3} \simeq \gamma, \gamma-\mathrm{CH}_{3} \gg \beta, \beta-\mathrm{CH}_{3} \simeq \alpha, \alpha-\mathrm{CH}_{3}$, the last two undergoing almost no hydrolysis. ${ }^{92}$

Pérez-Prior et al. studied the acid hydrolysis of $\gamma$-butyrolactone and $\delta$-valerolactone in acidic aqueous solution $1.0<\mathrm{pH}<3.0$. They concluded that the hydrolysis reaction of GBL is an endothermic equilibrium $\left(\Delta H^{\ominus}=3.6 \mathrm{~kJ} \mathrm{~mol}^{-1}\right)$ whereas that of DVL is quantitative. The fact that the hydrolysis of DVL is two orders of magnitude faster was explained in terms of its greater basicity. ${ }^{93}$ 


\subsubsection{Neutral hydrolysis}

Unsubstituted $\beta$-lactones constitute a rather specific case, since, unlike linear esters, a non-catalyzed hydrolysis mechanism exists at low temperatures.

Oxygen nucleophiles are normally more reactive towards $s p^{2}$ than $s p^{3}$ hybridized carbon, and consequently bimolecular alkyl-oxygen fission, identified (for example) by using optically active alkyl carboxylates, is not normally observed in ester solvolysis reactions. The single exception is the neutral hydrolysis or solvolysis of $\beta$-lactones

Olson and Miller, ${ }^{88,89}$ using its optical activity and ${ }^{18} \mathrm{O}$ as a tracer, proved that $\beta$-butyrolactone (BBL) hydrolyzes in neutral medium affording the alkyl-cleavage product whereas the reaction products in basic and acidic media are those of acyl-oxgen fission, an observation similar to that made earlier by lngold et al. using $\beta$-malolactonate. Bartlett and Rylander showed that the methanolysis of $\beta$-propiolactone leads to $\beta$-methoxypropionic acid ${ }^{94}$ and Long and Purchase concluded that also $\beta$-propiolactone (BPL) hydrolyzes in neutral medium through $\mathrm{B}_{\mathrm{AL}} 2$ mechanism. ${ }^{90}$

The neutral hydrolysis of $\beta$-propiolactone is four times faster than that of $\beta$-butyrolactone, as expected for the $\mathrm{S}_{\mathrm{N}} 2$ mechanism (but not the $\mathrm{S}_{\mathrm{N}} 1$ ). The activation parameters for the neutral hydrolysis of are $\Delta^{\ddagger} H^{\ominus}=83 \pm 2 \mathrm{~kJ} \mathrm{~mol}^{-1}$ and $\Delta^{\ddagger} S^{\ominus}=-80 \pm 8 \mathrm{~J} \mathrm{~mol}^{-1} \mathrm{~K}^{-1}$ for BPL (previously obtained values are $\Delta^{\ddagger} H^{\ominus}=79 \mathrm{~kJ} \mathrm{~mol}^{-1}$ and $\left.\Delta^{\ddagger} S^{\ominus}=-62 \mathrm{~J} \mathrm{~mol}^{-1} \mathrm{~K}^{-190}\right)$ and $\Delta^{\ddagger} H^{\ominus}=88 \pm 2 \mathrm{~kJ} \mathrm{~mol}^{-1}$ and $\Delta^{\ddagger} S^{\ominus}=-78 \pm 10 \mathrm{~J} \mathrm{~mol}^{-1} \mathrm{~K}^{-1}$ for BBL. ${ }^{93}$

This evidence is consistent with the neutral hydrolysis of $\beta$-propiolactone and $\beta$-butyrolactone being unique cases of the $\mathrm{B}_{\mathrm{AL}} 2$ reaction.

Pérez-Prior et al. studied the neutral hydrolysis of BPL and BBL in water-dioxane mixtures, ${ }^{93}$ and concluded that the reactivity of these lactones is a good measure of their electrophilicity, as regards their reactivity with nucleotides in vivo. Manso et al. observed that the hydrolysis of BPL and BBL is essentially enthalpy controlled and also that an increase in the dioxane percentage reduces the enthalpy of activation and simultaneously decreases the entropy of activation for solvent compositions up to $60 \%$ dioxane. A complex enthalphy-entropy correlation was observed, as a consequence of the quadratic and cubic terms appearing in the expressions of $\Delta^{\ddagger} H^{\ominus}$ and $\Delta^{\ddagger} H^{\ominus}$ as functions of the solvent composition. ${ }^{95}$

Interestingly, as was the case in the acid hydrolysis of $\beta$-lactones, $\beta$-isovalerolactone behaves differently. The base hydrolysis of $\beta$-isovalerolactone is slower than that of BBL, but its neutral hydrolysis is about 100 times faster (at $25^{\circ} \mathrm{C}$ ). ${ }^{91}$ This is a consequence of a change in the mechanism to a rate-determining ionization. The major product of neutral solvolysis of BIVL is isobutene, resulting from its decarboxylation, which confirms the unimolecular.

\subsubsection{Base hydrolysis}

One of the first studies dealing with the influence of ring size on the rate of lactone hydrolysis dates back to $1959 .{ }^{96}$ Results can be summarized in the hydrolysis of five, seven and eight membered rings 
being about three hundred-fold faster than those of the open chain counterparts. Larger rings have little effect on hydrolysis rate. Four- and six- membered lactones hydrolyze thousands of times faster than the corresponding open-chain compounds. ${ }^{97}$

Such acceleration is due, possibly to the a combination of ring-strain, restriction of the ester group to cis configuration, lack of planarity of the ester group and changes in the rate-limiting step or in the whole mechanism. ${ }^{97}$ Some works suggest that the contribution of the cis configuration is only partially responsible for the acceleration, even in the case of lactones with less strained cycles. For instance, the hydrolysis of linear 2,2,2-trifluorethyl formiate is not particularly fast, although it has a cis configuration. ${ }^{98}$

Brown ${ }^{99}$ et al. concluded that for $\delta$-lactones, formation of the tetrahedral intermediate cancels part of the ring strain, since it allows the lactone to adopt the chair conformation. On the contrary, on $\gamma$-lactones, formation of the tetrahedral intermediate adds extra steric repulsion without easing the adoption of more stable conformations. For these same reasons, exocyclic double bonds (carbonyl or methylene) stabilize five-membered rings and destabilize six-membered cycles. Therefore, the increase in hydrolysis rate in $\gamma$-lactones $(10-15$ fold) is mainly due to the cis effect, whereas the extra increase seen in $\delta$-lactones (15 40 fold) is due to the extra loss of ring strain.

In their investigation of the effect of ring strain of acyl-transfer reactions, ${ }^{100}$ Blackburn and Dodds studied the alkaline hydrolysis of a series of lactones and esters. From the analysis of the activation parameters (Table 3.6), they reached the conclusion that BPL owns its enhanced reactivity to a favorable increase in the activation entropy with respect to other lactones. They also concluded that the high hydrolysis rate of $\gamma$-butyrolactone (GBL) was due to a low activation enthalpy.

Table 3.6. Activation parameters for the alkaline hydrolysis of some lactones. Reproduced from ${ }^{100}$

\begin{tabular}{|c|c|c|c|}
\hline Substrate & $\begin{array}{c}\Delta^{\ddagger} H^{\ominus} \\
\left(\mathrm{kJ} \mathrm{mol}{ }^{-1}\right)\end{array}$ & $\begin{array}{c}-\Delta^{\ddagger} S^{\ominus} \\
\left(\mathrm{J} \mathrm{mol}^{-1} \mathrm{~K}^{-1}\right)\end{array}$ & $\begin{array}{c}\Delta^{\ddagger} G^{\ominus} 25^{\circ} \mathrm{C} \\
\left(\mathrm{kJ} \mathrm{mol}{ }^{-1}\right)\end{array}$ \\
\hline $\mathrm{BPL}$ & $50.1 \pm 0.9$ & $70.5 \pm 20$ & 71.1 \\
\hline GBL & $44.6 \pm 1.3$ & $94 \pm 5$ & 72.8 \\
\hline DVL & $30.1 \pm 1.7$ & $117 \pm 6$ & 65.0 \\
\hline$\epsilon$-Caprolactone & $38.9 \pm 2.1$ & $114 \pm 7$ & 72.9 \\
\hline Methyl formate & $38.7 \pm 2.5$ & $83 \pm 8$ & 63.4 \\
\hline Methyl acetate & $46.4 \pm 1.7$ & $101 \pm 7$ & 76.5 \\
\hline Ethyl acetate & 45.1 & 112 & 78.5 \\
\hline
\end{tabular}

\subsection{Materials \& methods}

\subsubsection{Methodology and computational details}


3.2.2. Hydrolysis of 2-5(H)-Furanone 


\subsection{Results and discussion}

\subsubsection{Neutral hydrolysis}

As a rule, only activated esters show significant hydrolysis in neutral conditions, mostly by the $\mathrm{B}_{\mathrm{AC}}{ }^{2}$ mechanism. This enhanced reactivity usually arises from ring-strain, in lactones, or from the presence of electron-withdrawing substituents that increase the electrophilicity of the carbonyl carbon (haloesters, benzoates...). Formates are also very reactive in comparison to alkyl esters, due to steric and electronic reasons, since they lack electron-donating, bulky alkyl substituents. Hence, linear unactivated alkyl esters and large lactones do not hydrolyze in neutral medium.

Alkyl $\beta$-lactones have long been known to undergo neutral hydrolysis: BPL and BBL are two of the few examples of the $\mathrm{B}_{\mathrm{AL}} 2$ mechanism, and $\mathrm{BIVL}$ and DIK follow the $\mathrm{B}_{\mathrm{AL}} 1$ and $\mathrm{B}_{\mathrm{AC}} 2$ mechanisms respectively, confirming that very little structural variation can completely change the hydrolysis mechanism of lactones.

We have modeled the reaction pathways for all four possible neutral hydrolysis mechanisms. Three of them ( $\mathrm{B}_{\mathrm{AL}} 1, \mathrm{~B}_{\mathrm{AC}} 1$ and $\left.\mathrm{B}_{\mathrm{AL}} 2\right)$ are rather simple and take place in a single rate-limiting step (labeled $\mathrm{RL}$ ), followed by rapid addition of water to carbocations (or elimination) and proton transfers. Scheme 3.14 a) to c) shows the reaction pathways proposed in the literature for these three neutral hydrolysis mechanisms.

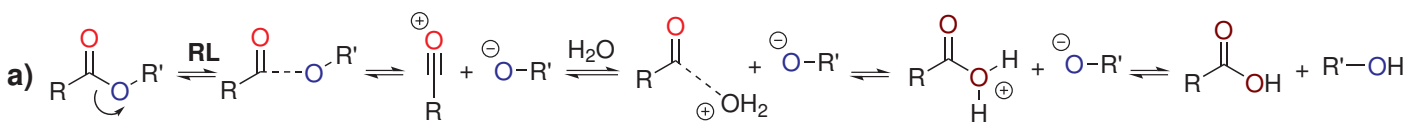

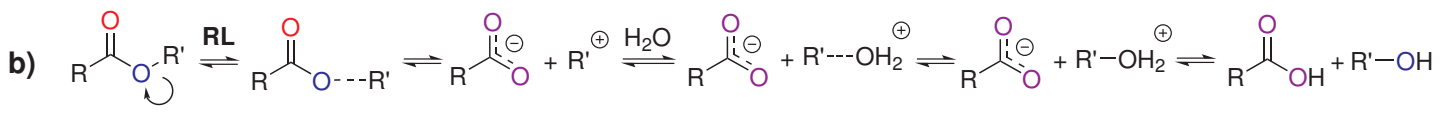

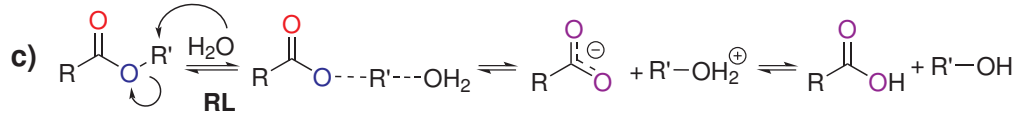

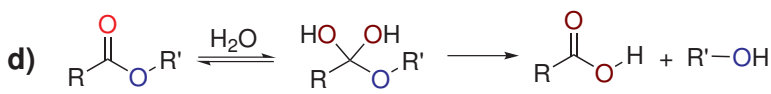

Scheme 3.14. $\mathrm{B}_{A C^{1}}{ }^{(a)}, \mathrm{B}_{\mathrm{AL}} 1$ (b), $\mathrm{B}_{\mathrm{AL}} 2$ (c) and simplified $\mathrm{B}_{\mathrm{AC}} 2$ (d) mechanisms of neutral hydrolysis

The $\mathrm{B}_{\mathrm{AC}} 2$ hydrolysis mechanism is more complex and occurs in two steps (Scheme $3.14 \mathrm{~d}$ ). In the first one, a water molecule attacks the carbonyl carbon in an addition reaction that yields a tetrahedral intermediate. This intermediate is unstable, and decomposes in the second step.

Table 3.7 reports the calculated activation parameters for each of these esters in the neutral hydrolysis mechanisms. Since the addition step in the $\mathrm{B}_{\mathrm{AC}} 2$ mechanism precludes any further reaction, a high barrier blocks the entire pathway, and thus, only this step was included in this comparison. 
Table 3.7. Calculated energy barriers for the neutral hydrolysis of some esters

\begin{tabular}{|c|c|c|c|c|c|c|c|c|}
\hline & \multicolumn{4}{|c|}{$\Delta H\left(\mathrm{~kJ} \mathrm{~mol}^{-1}\right)$} & \multicolumn{4}{|c|}{$\Delta G\left(25^{\circ} \mathrm{C}\right)\left(\mathrm{kJ} \mathrm{mol}^{-1}\right)$} \\
\hline & $\mathrm{TS}_{\mathrm{BAL} 2}$ & $\mathrm{TS}_{\mathrm{BAL} 1}$ & $\mathrm{TS}_{\mathrm{BAC} 1}$ & $\mathrm{TS}_{\mathrm{BAC} 2}$ & $\mathrm{TS}_{\mathrm{BAL} 2}$ & $\mathrm{TS}_{\mathrm{BAL} 1}$ & $\mathrm{TS}_{\mathrm{BAC} 1}$ & $\mathrm{TS}_{\mathrm{BAC} 2}$ \\
\hline COOMe & 160.3 & 289.7 & & 69.3 & 142.2 & 270.1 & & 109.1 \\
\hline AcOEt & 182.5 & 225.0 & 265.9 & 89.5 & 151.4 & 235.5 & 271.1 & 128.8 \\
\hline BPL & 84.8 & 146.2 & 221.3 & 81.3 & 91.8 & 146.2 & 201.0 & 111.3 \\
\hline BBL & 99.1 & 107.5 & 189.4 & 83.8 & 95.9 & 109.1 & 203.5 & 110.4 \\
\hline BIVL & - & 75.5 & 239.9 & 87.6 & - & 79.7 & 226.0 & 118.7 \\
\hline DIK & - & 140.2 & 158.3 & 56.6 & - & 148.1 & 152.5 & 90.9 \\
\hline GBL & 145.4 & 233.5 & 198.3 & 100.2 & 138.8 & 215.1 & 216.6 & 134.4 \\
\hline FUR & 181.9 & 236.0 & 210.0 & & 166.1 & 212.5 & 227.2 & \\
\hline DVL & 184.1 & 220.7 & 198.5 & 92.6 & 198.1 & 234.5 & 216.0 & 135.3 \\
\hline
\end{tabular}

Calculated free energies suggest that $\beta$-lactones are the most susceptible to neutral hydrolysis: BPL and $\mathrm{BBL}$ show very low barriers in the $\mathrm{B}_{\mathrm{AL}} 2$ pathways; tertiary BIVL follows the $\mathrm{B}_{\mathrm{AL}} 1$ pathway and the $\mathrm{B}_{\mathrm{AC}} 2$ addition transition state is the lowest energy path for DIK (the steps following the nucleophilic attack are discussed below). Since formates are quite reactive, COOMe is also prone to neutral hydrolysis by the $\mathrm{B}_{\mathrm{AC}} 2$ mechanism.

Larger $\gamma$ and $\delta$-lactones and AcOEt show very high energy barriers for all the mechanisms and would not be expected to hydrolyze in neutral aqueous solution. In general, the larger the ring, the lower the reactivity.

The $\mathrm{B}_{\mathrm{AC}} 1$ barriers are extremely high in energy for all the compounds, and everyone of them has more favored hydrolysis pathways. This is not unexpected, since this mechanism has never been observed experimentally.

\subsubsection{1. $B_{A L} 2$ and $B_{A L} 1$ mechanisms}

No transition states could be located for the $\mathrm{B}_{\mathrm{AL}} 2$ hydrolysis of BIVL and DIK, since the steric hindrance in the case of BIVL and a combination of steric repulsion and the $s p^{2}$ hybridization of the alkyl-oxygen carbon in DIK disfavor this mechanism. BPL and BBL do favor this pathway: the barriers are quite low and also much lower than those for the $\mathrm{B}_{\mathrm{AC}} 2$ standard hydrolysis mechanism. The rest of the esters show very high barriers for the bimolecular alky-cleavage.

As regards the hydrolysis of $\mathrm{BBL}$, it is interesting to notice that, being a secondary ester, $\mathrm{B}_{\mathrm{AL}} 1$ and $\mathrm{B}_{\mathrm{AL}} 2$ transition states are not very far in terms of free energy. The extra methyl group hinders the nucleophilic attack of water in the $\mathrm{B}_{\mathrm{AL}} 2$ pathway, and, at the same time, stabilizes the partial positive charge that develops on the alkyl-oxygen carbon in the $\mathrm{B}_{\mathrm{AL}} 1$ mechanism.

Due to its particular structure, BIVL is the only among the studied compounds to clearly favor the $\mathrm{B}_{\mathrm{AL}} 1$ mechanism. It could be thought that this occurs because a stable tertiary carbocation is formed as a 
product (Scheme 3.14). However, in the located transition state, both alkyl- and acyl-oxygen cleavage take place almost simultaneously (Scheme 3.5), and thus, rather than hydrolysis, a decarboxylation reaction takes place. Since the reaction is concerted, the carbocation does not develop fully. Nevertheless, C-O bond cleavage is much more advanced than $\mathrm{C}$-C cleavage in the transition state (bond lengths are $219 \mathrm{vs}$ $160 \mathrm{pm}$ ) and a large partial positive charge concentrates in the alkyl carbon (Mulliken and APT atomic charges are 0.773 and 1.225 , respectively), even if it is not a wholly developed cation.

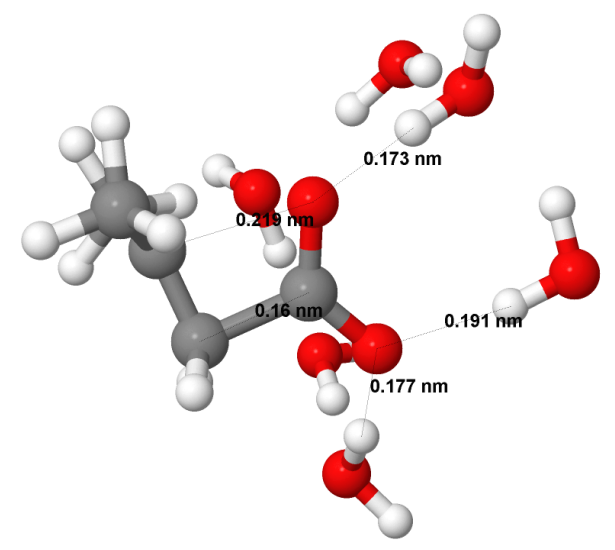

Figure 3.5. Structure of the addition transition state of $B I V L$ in the $B_{A L} 1$ mechanism

The reaction products for all the instances of $\mathrm{B}_{\mathrm{AL}} 1$ hydrolysis in lactones were found to be carbon dioxide and the elimination product, through IRC calculations. However, since these reactions have very high barriers, these products have only been observed experimentally in the decomposition of BIVL. On the contrary, the virtually inexistent $\mathrm{B}_{\mathrm{AL}} 1$ hydrolysis of AcOEt is not decarboxylative and would yield the secondary carbocation from a $\sigma$-rearrangement.

A close inspection of the reported values shows that, both for $\mathrm{B}_{\mathrm{AL}} 2$ and $\mathrm{B}_{\mathrm{AL}} 1$, activation enthalpies and free energies are very similar, which implies that activation entropies are null, or in some cases slightly positive. This is the expected result for an unimolecular ring-opening reaction. However, in the case of the bimolecular reaction, a negative value would be expected (experimental activation entropies for the few known cases of bimolecular alkyl-cleavage have low negative values: $\sim-50 \mathrm{~J} \mathrm{~mol}^{-1} \mathrm{~K}^{-1}$, as shown in Table 3.9). This suggests that the entropic contributions are somewhat misestimated in the $\mathrm{B}_{\mathrm{AL}} 2$ mechanism. A possible cause could be a decrease in the rigidity of the hydration shell. Since the atoms upon which partial charge develops (the attacking water molecule and the carboxylate leaving group) are quite far from each other, the two solvation subshells are less tightly connected than in the reactants. The loss of inter-solvent hydrogen bonds would be expected to produce a increase in the activation enthalpy and also an increase in activation entropy, since the molecules are less tightly bound and hence, freer. 


\subsubsection{2. $B_{A C} 2$ mechanism}

Among the studied lactones, only DIK and COOMe have low barriers for the $\mathrm{B}_{\mathrm{AC}} 2$ addition and lack an alternative preferred hydrolysis mechanism. However, the $\mathrm{B}_{\mathrm{AC}} 2$ mechanism is the more common pathway of neutral hydroylsis for activated linear esters, and both its alkaline and acid counterparts $\left(\mathrm{B}_{\mathrm{AC}} 2\right.$ and $\left.\mathrm{A}_{\mathrm{AC}} 2\right)$ are the usual mechanisms of catalyzed hydrolysis (see below). Thus, it is interesting to study the full two-step reaction pathway in detail.

The calculated differences in free energy and enthalpy for the full mechanism are reported in Table 3.8. Since two different transition states were found for the addition step (catalyzed and concerted, see below), these were labeled $\mathrm{TS}_{\mathrm{AD}} \mathrm{CAT}$ and $\mathrm{TS}_{\mathrm{AD}} \mathrm{CON}$; we have labeled the tetrahedral intermediate $\mathrm{TD}$, and the cleavage transition state is $\mathrm{TS}_{\mathrm{CLV}}$.

Also, a concerted mechanism exists ( $\mathrm{TS}_{\mathrm{UNI}}$ ), in which the leaving group is expelled synchronously with the attack of water at the carbonyl group, and thus, bypassing the intermediate.

Table 3.8. Calculated energy barriers for the neutral $\mathrm{B}_{\mathrm{AC}} 2$ hydrolysis of some lactones

\begin{tabular}{|c|c|c|c|c|c|c|c|c|c|c|}
\hline & \multicolumn{5}{|c|}{$\Delta H\left(\mathrm{~kJ} \mathrm{~mol}^{-1}\right)$} & \multicolumn{5}{|c|}{$\Delta G\left(25^{\circ} \mathrm{C}\right)\left(\mathrm{kJ} \mathrm{mol}^{-1}\right)$} \\
\hline & \multicolumn{2}{|c|}{$\mathrm{TS}_{\mathrm{AD}}$} & \multirow{2}{*}{$\mathrm{TD}$} & \multirow{2}{*}{$\mathrm{TS}_{\mathrm{CLV}}$} & \multirow{2}{*}{$\mathrm{TS}_{\mathrm{UNI}}$} & \multicolumn{2}{|c|}{$\mathrm{TS}_{\mathrm{AD}}$} & \multirow{2}{*}{$\mathrm{TD}$} & \multirow{2}{*}{$\mathrm{TS}_{\mathrm{CLV}}$} & \multirow{2}{*}{$\mathrm{TS}_{\mathrm{UNI}}$} \\
\hline & CAT & $\mathrm{CON}$ & & & & CAT & $\mathrm{CON}$ & & & \\
\hline COOMe & 69.3 & 108.4 & 53.1 & 62.2 & & 109.1 & 130.6 & 71.3 & 99.2 & \\
\hline $\mathrm{AcOEt}$ & 89.5 & 125.3 & 53.1 & 88.0 & 197.2 & 128.8 & 138.4 & 80.5 & 129.0 & 221.3 \\
\hline BPL & 81.3 & 124.5 & 79.9 & 67.9 & 171.5 & 111.3 & 136.3 & 67.4 & 106.4 & 189.6 \\
\hline $\mathrm{BBL}$ & 83.8 & 129.8 & 82.3 & 65.1 & 181.2 & 110.4 & 140.6 & 73.3 & 100.8 & 190.9 \\
\hline BIVL & 89.7 & 126.3 & 84.5 & 72.3 & 182.7 & 118.7 & 147.5 & 78.3 & 107.6 & 191.1 \\
\hline DIK & 56.6 & 88.3 & 52.1 & 41.2 & 125.5 & 90.9 & 106.1 & 49.9 & 79.1 & 123.0 \\
\hline GBL & 100.2 & 134.7 & 71.0 & 87.1 & 192.3 & 134.4 & 143.5 & 84.9 & 126.4 & 207.2 \\
\hline FUR & & 144.6 & 85.2 & 101.1 & 204.6 & & 150.9 & 92.4 & 131.8 & 214.2 \\
\hline DVL & 92.6 & 147.7 & 73.5 & 75.7 & 185.5 & 135.3 & 154.9 & 83.3 & 117.4 & 211.9 \\
\hline
\end{tabular}

3.3.1.2.1. Addition. The addition reaction in the neutral $\mathrm{B}_{\mathrm{AC}} 2$ mechanism pathway has been proposed to occur through a variety of pathways (Scheme 3.15):

(a) Direct addition. (b) Some works have suggested that the base-catalyzed addition is concerted with a direct or water-mediated proton transfer from the water molecule acting as a catalyst to the carbonyl oxygen. However, the availability of this pathway has sometimes been attributed to the lack of sufficient explicit solvent molecules in the modelling of the system. (c) A base-catalyzed mechanism, which affords a negatively charged intermediate, and a hydronium ion, which rapidly transfers a proton to the intermediate anion. 
Gunaydin et al. propose an addition mechanism catalyzed simultaneously by a hydroxide and hydronium ion. ${ }^{101}$ These are formed in the autoionization of water, which is the limiting step at $\Delta^{\ddagger} G=$ $99 \mathrm{~kJ} \mathrm{~mol}^{-1}$. Whereas such a mechanism can hardly explain the observed dependence of hydrolysis rate on the nature of the ester, its study in a cluster-continuum approach would require significantly more explicit water molecules and is beyond the reach of this work.

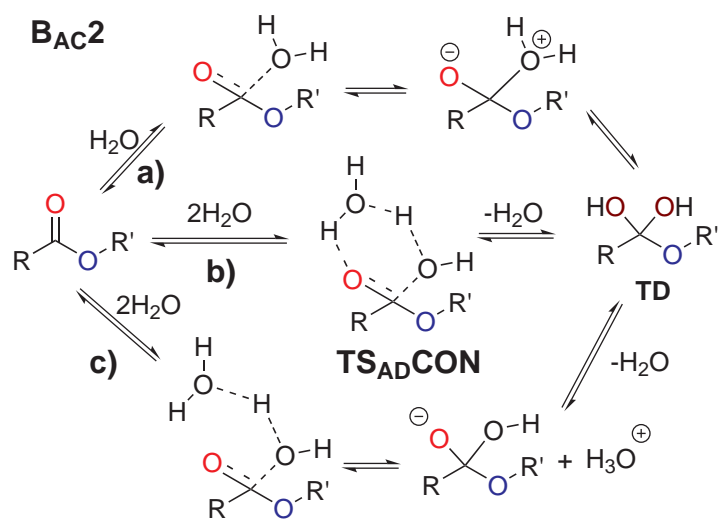

$\mathrm{TS}_{\mathrm{AD}} \mathrm{CAT}$

Scheme 3.15. Addition reaction in the $\mathrm{B}_{\mathrm{AC}} 2$ mechanisms of neutral hydrolysis

Our observations agree with those of Wang and Cao and suggest that a base-catalyzed multi-step mechanism takes place (Scheme 3.15, c; TS $\mathrm{AD}_{\mathrm{CAT}}$ ). ${ }^{102}$ In this, the water-ester encounter is the limiting step $\left(\mathrm{TS}_{\mathrm{AD}} \mathrm{CAT}\right)$ and the subsequent proton transfers are almost isoenergetic and barrierless.

In addition, two less favored transition states were found for the concerted water-mediated addition reaction (Scheme 3.15, b). In these, either one or two water molecules assist the proton transfer from the nucleophilic water to the carbonyl. The transition state involving two solvent molecules is lower in energy, since the bond angles for the six-membered ring transition states are more favorable, and thus it is the one reported in Table 3.8 as $\mathrm{TS}_{\mathrm{AD}} \mathrm{CON}$.

Excluding the $\beta$-lactones, the ring strain seems to play little role in the addition step, the effect being mainly entropic.

3.3.1.2.2. Intermediate. The tetrahedral intermediates are quite high in energy, since they are the product of the ester hydration reaction, which is very displaced to the reactants side.

Thermoentropic contributions are more favorable for the more reactive esters. Within $\beta$-lactones, the order of free energy is also $D I K<B P L<B B L<B I V L$, because of the electron-donating nature of the methyl substituents.

The tetrahedral intermediate of DIK is exceptionally stable when compared to other $\beta$-lactones (also in the base- and acid-catalyzed mechanisms, see below), since they favorable enthalpic and thermoentropic contributions are combined. This is probably due to the effect of the methylene group: the square 

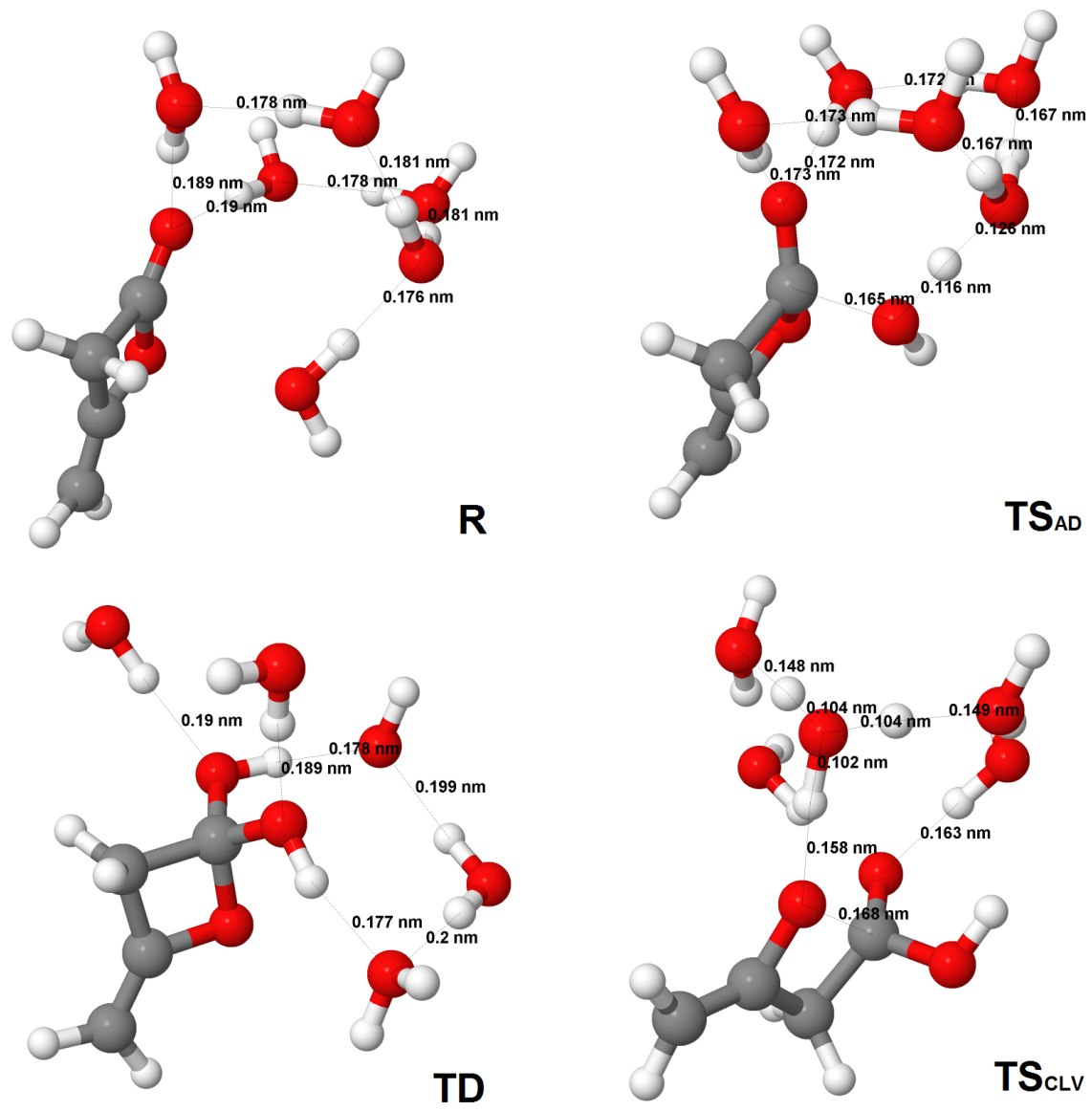

Figure 3.6. $\mathrm{B}_{\mathrm{AC}} 2$ minima and transition states for DIK 
structure of $\beta$-lactones enforces $90^{\circ}$ angles, and DIK has two $s p^{2}$ carbons, whose preferred geometry is $120^{\circ}$. Transition of the $s p^{2}$ carbonyl carbon the $s p^{3}$ hybridization is, thus, especially favored in DIK.

The transition states for the reverse addition reaction (the reaction from TD back to reactants through $\mathrm{TS}_{\mathrm{AD}}$ ) are around 40-50 $\mathrm{kJ} \mathrm{mol}^{-1}$ higher than the intermediates, and the breakdown reactions have similar or even lower barriers (see below, $\mathrm{TS}_{\mathrm{CLV}}$ ). This suggests that intermediates are short-lived, with half-lives of microseconds or below.

3.3.1.2.3. Breakdown. The ester breakdown step involves both cleavage of the ester bond and transfer of a hydrogen atom from the acyl to the alkyl oxygen as shown in Scheme 3.14. Several possible transition states were located for this step (Scheme 3.16):

(a) cleavage of the acyl-oxygen bond and direct concerted proton transfer from the carbonyl oxygen to the leaving group; (b) a breakdown occurring in one step, symmetrical to the unfavored concerted addition mechanism $\left(\mathrm{TS}_{\mathrm{AD}} \mathrm{CON}\right)$, in which proton transfer and bond cleavage occur simultaneously ; and (c) a transition state symmetrical to the one observed for the addition reaction ( $\mathrm{TS}_{\mathrm{AD}} \mathrm{CAT}$ ); after the dissociation of the intermediate in a fast proton transfer to the surrounding solvation shell, the formed hydronium ion catalyzes the cleavage step $\left(\mathrm{TS}_{\mathrm{CLV}}\right)$.

Of these breakdown pathways, the one symmetrical to $\left(\mathrm{TS}_{\mathrm{AD}} \mathrm{CAT}\right)$ is the most favored. Since the addition step is base-catalyzed, the symmetrical cleavage is catalyzed by a hydronium ion, which proceeds from the dissociation of the intermediate. This behavior has been observed experimentally in the hydrolysis of activated esters with poor leaving groups, whose breakdown is catalyzed in acidic media. Thus, the results reported in Table 3.8 correspond to the hydronium-catalyzed cleavage of the dissociated intermediate.

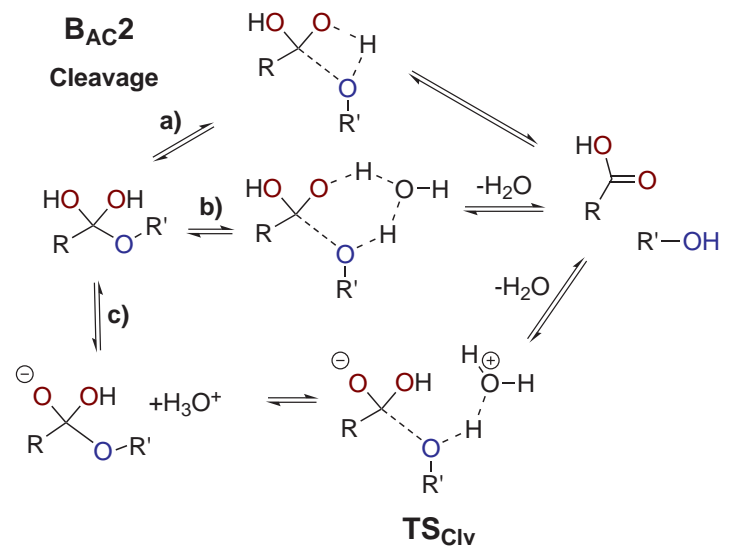

Scheme 3.16. Acyl oxygen bond cleavage reaction in the $\mathrm{B}_{\mathrm{AC}} 2$ mechanisms of neutral hydrolysis

The reasons for the symmetry between addition and cleavage steps lie in the similarity between the nucleophile and the leaving group. Notwithstanding ring strain, the leaving groups in all the compounds are (mostly primary) alkyl alcohols, which behave similarly to water in terms of nucleophilicity and acidbase behavior. 
Ring strain seems to play a moderate role in the breakdown free energies (Table 3.8), and, in general, the breakdown transition states are about $\sim 35 \mathrm{~kJ} \mathrm{~mol}^{-1}$ higher in free energy than the tetrahedral intermediates.

For the larger lactones and AcOEt, the energy barriers for the addition and cleavage step are very similar, which is in keeping with the usual assumption that, in principle, neither the addition or breakdown step is, by itself, rate-limiting in the $\mathrm{B}_{\mathrm{AC}} 2$ mechanism, especially in the absence of acids that may catalyze the cleavage step. As a matter of fact, it has been suggested that breakdown of the intermediate is the limiting step for activated esters with poor leaving groups.

3.3.1.2.4. Concerted Reaction. As regards the concerted mechanism found ( $T S_{U N I}$ ), the energy barrier is higher than that of the addition-breakdown mechanism due mostly to an enthalpic effect (Table 3.8).

\subsubsection{Comparison with experimental data}

Table 3.9 compares calculated and experimental (when available) activation parameters for the studied compounds. Although the activation parameters for the hydrolysis/decomposition of BIVL are not known, the neutral reaction rate constant is $2.7 \times 10^{-4} \mathrm{~min}^{-1}$, which allows its $\Delta^{\ddagger} G^{\ominus}$ to be estimated. The case of AcOEt is similar, since the reaction is very slow (half-life is $\sim 75$ years), and the $\Delta^{\ddagger} G^{\ominus}$ value is extrapolated.

Whereas the calculated activation free energies for the hydrolysis of the $\gamma$ - and $\delta$-lactones are similar to that of AcOEt, no experimental values are available for comparison.

Table 3.9. Calculated energy barriers for the neutral $\mathrm{B}_{\mathrm{AC}} 2$ hydrolysis of some lactones

\begin{tabular}{|c|c|c|c|c|c|c|c|c|}
\hline & \multicolumn{2}{|c|}{$\begin{array}{c}\Delta^{\ddagger} H^{\ominus} \\
\left(\mathrm{kJ} \mathrm{mol}{ }^{-1}\right)\end{array}$} & \multicolumn{2}{|c|}{$\begin{array}{c}\Delta^{\ddagger} S^{\ominus} \\
\left(\mathrm{J} \mathrm{mol}^{-1} \mathrm{~K}^{-1}\right)\end{array}$} & \multicolumn{2}{|c|}{$\begin{array}{c}\Delta^{\ddagger} G^{\ominus}\left(25^{\circ} \mathrm{C}\right) \\
\left(\mathrm{kJ} \mathrm{mol}{ }^{-1}\right)\end{array}$} & \multicolumn{2}{|c|}{ Mechanism } \\
\hline & Exp & Calc & Exp & Calc & Exp & Calc & Exp & Calc \\
\hline $\mathrm{COOMe}^{103}$ & 76 & 69 & -115 & -134 & 110 & 109 & $\mathrm{~B}_{\mathrm{AC}}{ }^{2}$ & $\mathrm{~B}_{\mathrm{AC}}{ }^{2}$ \\
\hline $\mathrm{AcOEt}^{1}$ & - & 90 & - & -131 & $\sim 128$ & 129 & $\mathrm{~B}_{\mathrm{AC}}{ }^{2}$ & $\mathrm{~B}_{\mathrm{AC}}{ }^{2}$ \\
\hline$B P{ }^{93} \mathrm{~L}$ & $83 \pm 2$ & 85 & $-46 \pm 8$ & -24 & 97 & 92 & $\mathrm{~B}_{\mathrm{AL}} 2$ & $\mathrm{~B}_{\mathrm{AL}}{ }^{2}$ \\
\hline $\mathrm{BBL}^{93}$ & $88 \pm 3$ & 99 & $-44 \pm 9$ & +10 & 101 & 96 & $\mathrm{~B}_{\mathrm{AL}} 2$ & $\mathrm{~B}_{\mathrm{AL}} 2$ \\
\hline BIVL $^{91}$ & - & 76 & - & -13 & $\sim 87$ & 80 & $\mathrm{~B}_{\mathrm{AL}} 1$ & $\mathrm{~B}_{\mathrm{AL}} 1$ \\
\hline $\operatorname{DIK}^{104}$ & $56 \pm 2$ & 57 & $-126 \pm 6$ & -115 & 94 & 91 & $\mathrm{~B}_{\mathrm{AC}}{ }^{2}$ & $\mathrm{~B}_{\mathrm{AC}}{ }^{2}$ \\
\hline
\end{tabular}

3.3.1.3.1. Favored pathway. The free energy differences (Table 3.7) are in excellent qualitative agreement with the experimental observations: $\mathrm{B}_{\mathrm{AC}} 1$ barriers are very high, and the mechanism is greatly unfavored. BPL and $\mathrm{BBL}$, which are indeed the two only known examples of $\mathrm{B}_{\mathrm{AL}} 2$ hydrolysis are correctly 
predicted to follow the bimolecular alkyl-cleavage mechanism. The least energetic pathways for AcOEt, COOMe and DIK are $\mathrm{B}_{\mathrm{AC}} 2$, whereas the only tertiary alkyl lactone studied, BIVL, favors the $\mathrm{B}_{\mathrm{AL}} 1$ mechanism, in keeping with experiments. Energy barriers for all pathways are high in the cases of the esters that do not to hydrolyze in neutral medium (GBL, FUR, DVL and AcOEt).

The reaction products found in the $\mathrm{B}_{\mathrm{AL}} 1$ mechanism by IRC calculations are consistent with the experimental observation that the major decomposition products of $\mathrm{BIVL}$ in water are $\mathrm{CO}_{2}$, isobutene and also isobutanol. In these experiments, the corresponding addition product is minor, even in the presence of strong nucleophiles that should be able to scavenge the putative carbocation.

Also, the mechanism involving several simultaneous proton transfers is in keeping with the primary kinetic isotope effect (KIE) observed in experiments: values of $k_{H} / k_{D} \sim 2$ are common (and KIE as high as 5 have been reported in the hydrolysis of ethyl dichloroacetate), which is indicative of the involvement of proton transfers in the limiting step.

3.3.1.3.2. Accuracy. The activation parameters are generally in good accord with empirical results. Activation free energies show errors below $5 \mathrm{~kJ} \mathrm{~mol}^{-1}$, and activation enthalpies are also in reasonable agreement with experimental values.

The trends in the activation entropies are fairly correct: the $\mathrm{B}_{\mathrm{AC}} 2$ additions show smaller $\Delta^{\ddagger} S$ than the $\mathrm{B}_{\mathrm{AL}} 2$ reactions. This is coherent with the fact that in the $\mathrm{B}_{\mathrm{AC}} 2$ mechanism, the bond cleavage is delayed, whereas in $\mathrm{B}_{\mathrm{AL}}$ 2, the positive contribution of bond-breaking to the entropy is simultaneous to the nucleophilic attack.

Experimentally, the neutral hydrolysis of linear alkyl haloesters is enthalpy-controlled, and activation entropies are very much constant for most compounds $\left(-\Delta^{\ddagger} S^{\ominus} \sim 160-200 \mathrm{~J} \mathrm{~mol}^{-1} \mathrm{~K}^{-1}\right)$. Whereas only one of the studied compounds is susceptible to the $\mathrm{B}_{\mathrm{AC}} 2$ mechanism in neutral medium, calculated activation entropies for all compounds are close to that value, especially in the case of the larger ester, AcOEt.

The results at the higher levels of theory are quite inferior. Whereas the activation energies at the DFTB3LYP/6-31++G(d,p) level are in reasonably good agreement with experimental values, $\Delta^{\ddagger} G^{\ominus}$ values at the post-HF levels of theory used are overestimated. If the DFT values are to be trusted, for which we have no indication against, $\Delta G^{\ominus}$ is understimated for the diol intermediates.

This errors can be understood in terms of the loose hydrogen bonds that connect the water molecules among them and to the lactones. The energy and the bond distance of these weak bonds are highly dependent on the method used, and hence geometries frozen at the DFT-B3LYP/6-31++G(d,p) are quite far from the equilibrium geometries at the MP-n or QCISD levels of theory, both in term of energy and distance. The size of this error varies for each particular step in the pathway, and thus, high random errors are encountered. Deviations of this kind and size have been reported in similar systems. ${ }^{102}$ Determination of the equilibrium geometries at these higher levels of theory is computationally costly. Moreover, since the hydration shell is so loosely bound, force constants should be calculated the higher levels of theory for the optimization step to reach the equilibrium geometry. The computational cost of this approach is beyond our reach, and, at the sight of the results, possibly not necessary. 


\subsubsection{Alkaline hydrolysis}

Since the unimolecular $\mathrm{B}_{\mathrm{AC}} 1$ and $\mathrm{B}_{\mathrm{AL}} 1$ mechanisms are uncatalyzed, only two base-catalyzed mechanisms of ester hydrolysis exist (Scheme 3.17): the controverted $\mathrm{B}_{\mathrm{AL}} 2^{74,75}$ and the almost universal $\mathrm{B}_{\mathrm{AC}} 2$.

\section{$\mathrm{B}_{\mathrm{AL}} 2$}

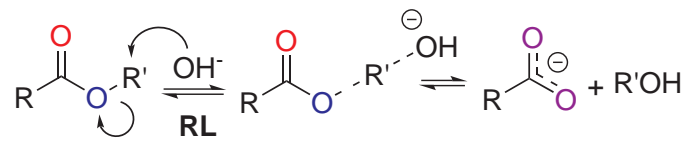

\section{$\mathrm{B}_{\mathrm{AC}} 2$ - Addition}

AD.1)

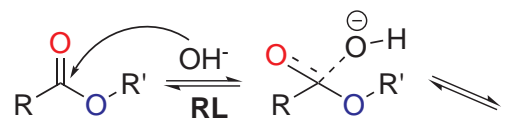

AD.2)

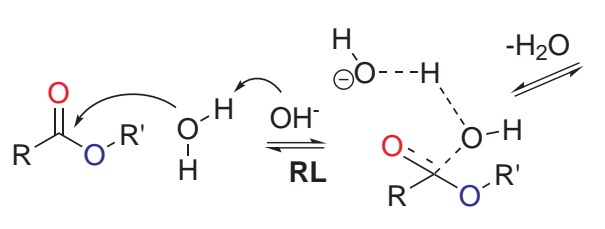<smiles>[O-]C(O)O</smiles><smiles>[R]C1(O)CCCC1</smiles>

Scheme 3.17. $\mathrm{B}_{\mathrm{AL}} 2$ mechanism of alkaline hydrolysis and $\mathrm{B}_{\mathrm{AC}} 2$ addition step

The theoretical $\mathrm{B}_{\mathrm{AL}} 2$ and $\mathrm{B}_{\mathrm{AC}} 2$ energy barriers for the studied compounds are summarized in Table 3.10, and show that acyl-oxygen cleavage is favored over the alkyl-oxygen cleavage in all the cases, including the $\beta$-lactones, which are known to react in neutral medium with water and alcohols with alkyl-oxygen cleavage. For BPL, the less hindered $\beta$-lactone, the $\mathrm{B}_{\mathrm{AL}} 2$ transition state is $7 \mathrm{~kJ} \mathrm{~mol}^{-1}$ higher in energy than the $\mathrm{B}_{\mathrm{AC}} 2$ addition, which would mean about $10 \%$ of alkyl-cleavage products in the basic hydrolysis of BPL. Such a minor contribution could have gone undetected in the experiments from which it was concluded that BPL undergoes acyl-oxygen cleavage.
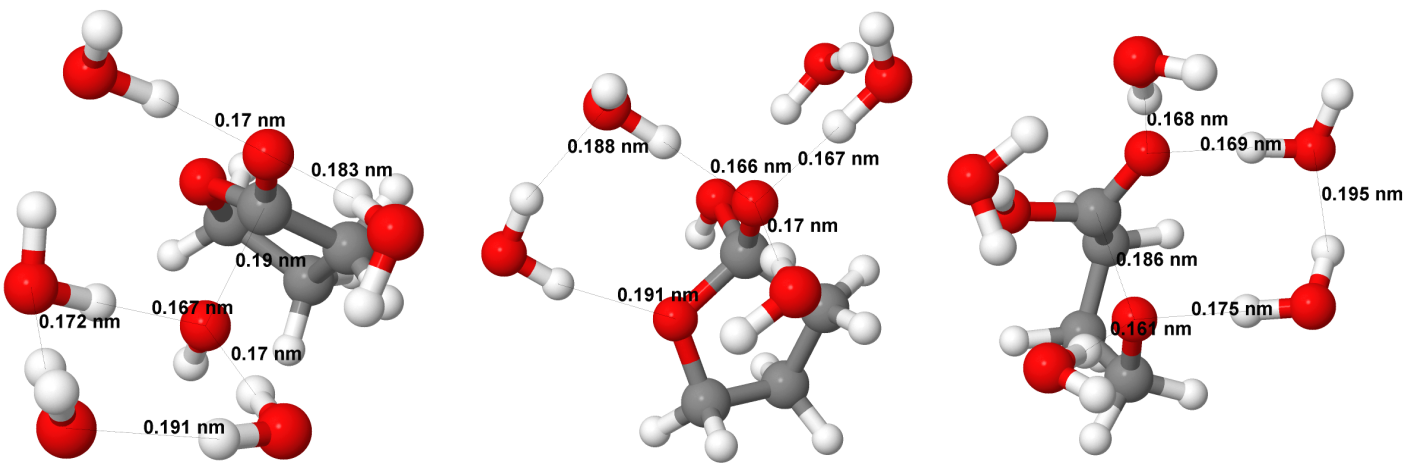

Figure 3.7. Structure of the addition and cleavage transition states of $G B L$ in the alkaline $B_{A C} 2$ mechanism 
Table 3.10. Calculated energy barriers for the alkaline hydrolysis of some lactones.

\begin{tabular}{|c|c|c|c|c|c|c|c|c|}
\hline & \multicolumn{4}{|c|}{$\Delta H\left(\mathrm{~kJ} \mathrm{~mol}^{-1}\right)$} & \multicolumn{4}{|c|}{$\Delta G\left(25^{\ominus} \mathrm{C}\right)\left(\mathrm{kJ} \mathrm{mol}^{-1}\right)$} \\
\hline & \multirow{2}{*}{$\mathrm{B}_{\mathrm{AL}}{ }^{2}$} & \multicolumn{3}{|c|}{$\mathrm{B}_{\mathrm{AC}}{ }^{2}$} & \multirow{2}{*}{$\mathrm{B}_{\mathrm{AL}}{ }^{2}$} & \multicolumn{3}{|c|}{$\mathrm{B}_{\mathrm{AC}}{ }^{2}$} \\
\hline & & $\mathrm{TS}_{\mathrm{AD}}$ & $\mathrm{TD}$ & $\mathrm{TS}_{\mathrm{CLV}}$ & & $\mathrm{TS}_{\mathrm{AD}}$ & $\mathrm{TD}$ & $\mathrm{TS}_{\mathrm{CLV}}$ \\
\hline COOMe & 135.6 & 35.4 & 26.6 & 26.7 & 132.5 & 67.2 & 46.6 & 59.2 \\
\hline $\mathrm{AcOEt}$ & 160.1 & 50.7 & 47.2 & 54.0 & 144.6 & 85.9 & 72.9 & 89.1 \\
\hline $\mathrm{BPL}$ & 88.0 & 46.5 & 23.1 & 28.0 & 88.1 & 78.8 & 49.8 & 56.9 \\
\hline $\mathrm{BBL}$ & 102.1 & 49.1 & 29.0 & 29.3 & 102.1 & 81.6 & 54.0 & 69.1 \\
\hline BIVL & 138.4 & 55.5 & 32.6 & 31.8 & 141.4 & 87.7 & 58.7 & 72.8 \\
\hline DIK & - & 27.0 & -15.3 & -18.2 & - & 60.8 & 10.5 & 20.3 \\
\hline GBL & 135.5 & 56.3 & 38.0 & 40.3 & 130.9 & 86.8 & 60.1 & 73.2 \\
\hline FUR & 140.1 & 63.8 & 51.1 & 54.2 & 133.5 & 95.3 & 74.1 & 89.7 \\
\hline DVL & 150.8 & 45.5 & 33.9 & 29.7 & 141.8 & 80.4 & 51.7 & 68.2 \\
\hline
\end{tabular}

\subsubsection{1. $B_{A C} 2$ mechanism}

The $\mathrm{B}_{\mathrm{AC}} 2$ mechanism has been proposed to occur through several pathways, depending on the nature of the leaving group: concerted or featuring a tetrahedral intermediate. There is also doubt about whether the hydroxide ion acts as a nucleophile or as a base catalyst in the addition step.

Figure 3.7 shows the equilibrium geometries for the addition transition state, the intermediate and the cleavage transition state for GBL as were calculated in this work.

3.3.2.1.1. Addition. Some authors have proposed that the hydroxide-catalyzed hydrolysis of esters does not imply the attack of hydroxide at the carbonyl (Scheme 3.17 AD.1), but rather, that hydroxide catalyzes the addition of neutral water (Scheme 3.17 AD.2). ${ }^{105,106}$ This water-mediated mechanism has been observed in computational studies using few explicit water molecules, possibly because in these, the negative charge is poorly solvated, and hence it is unrealistically stabilized in the proton-transfer mechanism.

In this work, the transitions states located correspond to the direct addition (Scheme 3.17 AD.1), as shown by the fact that all the hydrogen atoms are tightly bound to the corresponding oxygen atoms in 3.7. If proton transfers were to take place, $\mathrm{O}-\mathrm{H}$ bond distances between 100 and $160 \mathrm{pm}$ would be expected

The structure of the addition transition states has two water molecules hydrogen-bonded to the nucleophile, and two solvating the incipient charge in the carbonyl group, the fifth one is bound to those solvating the hydroxyde ion.

As regards the effect of structure upon reactivity, all lactones show quite similar reaction free energies, DVL and $\beta$-lactones being more reactive. The activation free energies for the lactones lie amidst those for the linear esters: COOMe is very reactive and AcOEt is least reactive, together with BIVL, whose methyl 
groups have an electron-donating effect that decreases the electrophilicity of the carbonyl. The high reactivity of COOMe and DVL is due to steric factors: COOMe lacks acyl and alkyl substituents, and thus, the addition of hydroxide is unhindered. In the case of DVL, the chair conformation and the fact that the hydrogen atom facing hydroxide is in equatorial position favor the attack.

Interestingly, the double bond in FUR has a significant effect in the addition barrier with respect to GBL, about $8 \mathrm{~kJ} \mathrm{~mol}^{-1}$ on $\Delta G$ (experimentally the reaction is about hundred-fold slower). This effect is a consequence of the ring geometry, since alkaline hydrolysis rate constants of their open-chain counterparts (ethyl propanoate and ethyl propenoate) are only $20 \%$ different (less than $0.5 \mathrm{~kJ} \mathrm{~mol}^{-1}$ in $\Delta G$ ).

3.3.2.1.2. Intermediate. The intermediates are quite high in energy, with free energies around 40$50 \mathrm{~kJ} \mathrm{~mol}^{-1}$ above the reactants, and thus their equilibrium ratio with respect to the reactants would be around $10^{-7}-10^{-10}$ to 1 . A single exception exists: the tetrahedral intermediate of DIK is calculated to be exceptionally stable, possibly for the reasons given above. Within $\beta$-lactones, the order of free energy of the intermediates is DIK $<\mathrm{BPL}<\mathrm{BBL}<\mathrm{BIVL}$ as in the addition reaction?, because of the electrondonating nature of the methyl substituents.

The transition states for the reverse addition reaction (from $\mathrm{TD}$ to the reactants through $\mathrm{TS}_{\mathrm{AD}}$ ) are around $20 \mathrm{~kJ} \mathrm{~mol}^{-1}$ higher than the intermediates, and the breakdown reactions have similar or even lower barriers (see below, $\mathrm{TS}_{\mathrm{CLV}}$ ). This suggests that intermediates are very short-lived, with half-lives below nanoseconds.

3.3.2.1.3. Breakdown. In close analogy to those observed for the neutral $\mathrm{B}_{\mathrm{AC}} 2$ hydrolysis, several transition states were located for the cleavage step, which, as before, includes acyl-bond cleavage and a proton transfer from the acyl to the alkyl oxygen.

(i) One-step cleavage pathways were observed, which correspond to the breakdown of the intermediate and concerted proton transfer, either direct (Scheme 3.18 a) or mediated by a water molecule (Scheme $3.18 \mathrm{~b})$.

Two step-breakdown reactions were also observed, in which the cleavage and the proton transfer occur sequentially. Two possibilities were found: (ii) a cleavage reaction with water acting as an acid catalyst, which yields hydroxide and the hydroxyacid as products, and would be the symmetrical counterpart of the unobserved hydroxide-catalyzed and (iii) the symmetrical counterpart of the addition reaction (Scheme $3.18 \mathrm{~d}$ ), with the alkoxide (instead of hydroxide) as a leaving group (or nucleophile in the reverse reaction); addition of water.

The breakdown transition states are about $\sim 15 \mathrm{~kJ} \mathrm{~mol}^{-1}$ higher in free energy than the tetrahedral intermediates and little influence of ring-strain upon this difference is observed.

In all the cases, the lowest-energy path was the one symmetrical to the addition. Since it yields the alkoxide and the neutral carboxylic acid, subsequent fast proton transfers must occur before the final products are formed. This pathway is preferred over the acid-catalyzed alternatives because the negative charge does not increase much the basicity of the alkyl oxygen, since it sits mostly on the unprotonated 


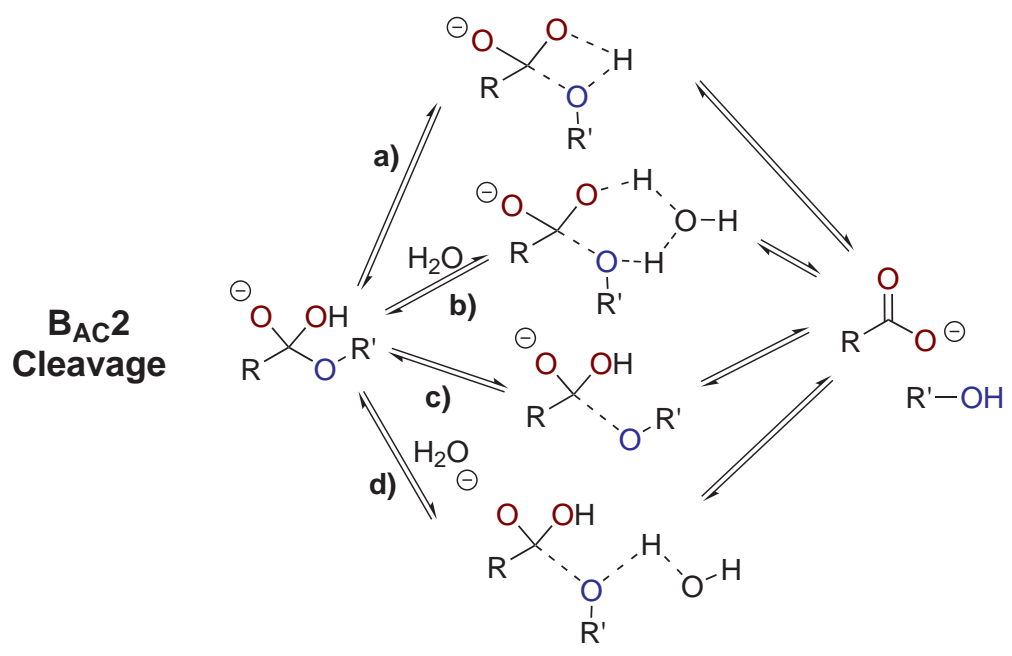

Scheme 3.18. Possilbe Cleavage

acyl oxygen (APT atomic charges are -1.56 and -1.20 for the acyl and alkyl oxygen atoms, respectively, in the alkaline tetrahedral intermediate, compared to -1.15 and -1.10 for the neutral diol). Thus, water is too a poor acid catalyst to assist the reaction, either directly or indirectly. The symmetry of the mechanism arises from the fact that the alkoxide leaving group and the hydroxide nucleophile are very similar, especially as regards their ability to stabilize their negative charge, acting as hydrogen bond acceptors with two water molecules in the transition state.

Table 3.10 reports the values for the lowest path, (Scheme $3.18 \mathrm{~d}$ and 3.7).

For the less reactive species, AcOEt, the breakdown reaction has a similar activation free energy to that of the addition step, which suggests that the cleavage step is not rapid enough to be neglected. On the contrary, COOMe and lactones show much lower energy barriers for their cleavage reactions, because of their ring-strain, and the addition step can be considered fully rate-limiting.

\subsubsection{Hydrolysis of diketene}

Whereas the theoretical and experimental activation free energies for the $\mathrm{B}_{\mathrm{AC}} 2$ hydrolysis of DIK are in reasonable agreement (see Table 3.11), the calculated activation enthalpy for diketene is very low, when compared to any other ester, and especially $\beta$-lactones.

The $\alpha$ hydrogen in diketene is known to be highly acidic, with an experimental $p K_{\mathrm{a}}$ value of 15 , a result that has also been replicated theoretically. In the experimental study of the alkaline hydrolysis of DIK, it was assumed that the dissociated form was unreactive, as regards hydrolysis and constituted a kinetic dead alley. However, the large deviation between experimental and theoretical activation enthalpies has motivated us to search for an additional parallel reaction: and elimination-addition pathway (3.19).

Such reaction has been observed in the hydrolysis of esters ${ }^{107,108}$ and amides ${ }^{97}$ carrying very labile 
<smiles>C=C1CC(=O)O1</smiles>

Scheme 3.19. Elimination-addition hydrolysis mechanism of DIK

$\alpha$ hydrogens. Since the addition-elimination and the elimination-addition pathway are kinetically indistinguishable, the preference for one or the other must be based on additional evidence, such as solvent isotopic effects, based on which it has been concluded that the addition-elimination mechanism does not to take place in the case of lactones such as the very acidic 2-coumaranones. However, diketene is intrinsically more strained than the five-membered cycle in coumaranones and could perhaps react by this mechanism. The equilibrium geometry for the corresponding transition state is shown in Figure 3.8.

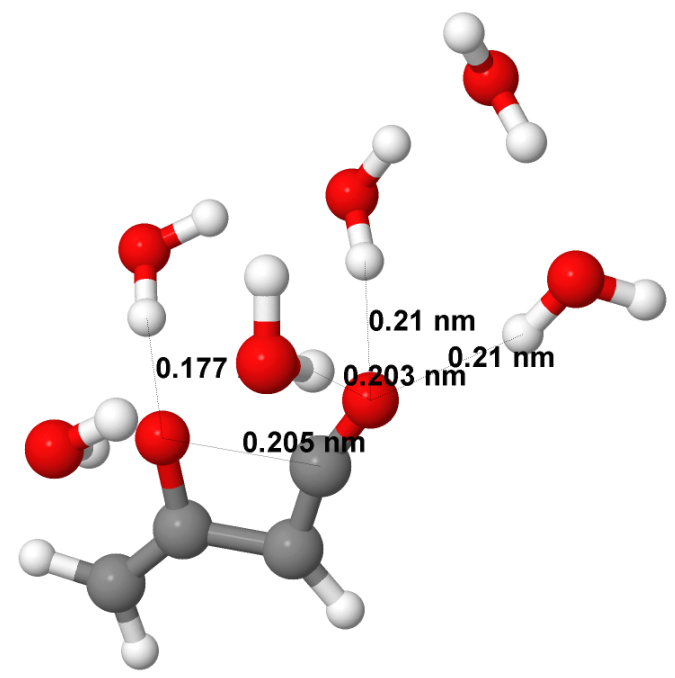

Figure 3.8. Transition state for the unimolecular ring-opening reaction of dissociated DIK

The theoretical barrier for the unimolecular acyl cleavage of the dissociated diketene is $\Delta^{\ddagger} H^{\ominus}=$ $58.0 \mathrm{~kJ} \mathrm{~mol}^{-1}$ and $\Delta^{\ddagger} G^{\ominus}=63.3 \mathrm{~kJ} \mathrm{~mol}^{-1}$, with respect to the lactone+ $\mathrm{OH}^{-}+5 \mathrm{H}_{2} \mathrm{O}$ cluster. The higher activation enthalpy and entropy are in keeping with a unimolecular reaction.

The addition-elimination and the elimination-addition pathways have very similar activation free energies (see Table 3.10), and thus, the reaction could be expected to proceed by a mixture of both. In this case, the measured rate coefficient would be the sum of the rate constants for the two processes, and thus, the activation parameters would have intermediate values between those for the two reactions. Interestingly, this is what occurs: the experimental $\Delta^{\ddagger} H^{\ominus}$ is halfway between the theoretical values for the two pathways (Table 3.11). 


\subsubsection{Comparison with experimental data}

The experimental activation parameters for the alkaline hydrolysis reaction of the studied compounds in water are given in Table 3.11 .

Table 3.1 1. Experimental activation parameters for the alkaline hydrolysis of some lactones in water.

\begin{tabular}{|c|c|c|c|c|c|c|c|c|}
\hline & \multicolumn{2}{|c|}{$\begin{array}{c}\Delta^{\ddagger} H^{\ominus} \\
\left(\mathrm{kJ} \mathrm{mol}^{-1}\right)\end{array}$} & \multicolumn{2}{|c|}{$\begin{array}{c}\Delta^{\ddagger} S^{\ominus} \\
\left(\mathrm{J} \mathrm{mol}^{-1} \mathrm{~K}^{-1}\right)\end{array}$} & \multicolumn{2}{|c|}{$\begin{array}{c}\Delta^{\ddagger} G^{\ominus}\left(25^{\ominus} \mathrm{C}\right) \\
\left(\mathrm{kJ} \mathrm{mol}{ }^{-1}\right)\end{array}$} & \multicolumn{2}{|c|}{ Mechanism } \\
\hline & Exp & Calc & Exp & Calc & Exp & Calc & Exp & Calc \\
\hline $\mathrm{COOMe}^{100}$ & $38.7 \pm 2.5$ & 35.4 & $-83 \pm 8$ & -77 & $63.4 \pm 4.9$ & 67.2 & $\mathrm{~B}_{\mathrm{AC}}{ }^{2}$ & $\mathrm{~B}_{\mathrm{AC}}{ }^{2}$ \\
\hline AcOEt 109 & $45.3 \pm$ & 50.7 & $-111 \pm$ & -112 & $78.5 \pm$ & 85.9 & $\mathrm{~B}_{\mathrm{AC}}{ }^{2}$ & $\mathrm{~B}_{\mathrm{AC}}{ }^{2}$ \\
\hline $\mathrm{BPL}^{100}$ & $50.1 \pm 0.9$ & 46.5 & $-71 \pm 3$ & -61 & $71.3 \pm 1.3$ & 78.8 & $\mathrm{~B}_{\mathrm{AC}}{ }^{2}$ & $\mathrm{~B}_{\mathrm{AC}}{ }^{2}$ \\
\hline $\mathrm{BBL}^{110}$ & $51.3 \pm 2.1$ & 49.1 & $-74 \pm 6$ & -76 & $73.4 \pm 2.7$ & 81.6 & $\mathrm{~B}_{\mathrm{AC}}{ }^{2}$ & $\mathrm{~B}_{\mathrm{AC}}{ }^{2}$ \\
\hline BIVL & - & 55.5 & - & -63 & $\sim 77$ & 87.7 & $\mathrm{~B}_{\mathrm{AC}}{ }^{2}$ & $\mathrm{~B}_{\mathrm{AC}}{ }^{2}$ \\
\hline $\operatorname{DIK}^{104}$ & $47.4 \pm 0.6$ & 27.0 & $-63 \pm 2$ & -89 & $66.1 \pm 0.8$ & 60.8 & $\mathrm{~B}_{\mathrm{AC}}{ }^{2}$ & $\mathrm{~B}_{\mathrm{AC}}{ }^{2}$ \\
\hline GBL $^{100}$ & $44.6 \pm 1.3$ & 56.3 & $-94 \pm 5$ & -97 & $72.6 \pm 2.0$ & 86.8 & $\mathrm{~B}_{\mathrm{AC}}{ }^{2}$ & $\mathrm{~B}_{\mathrm{AC}}{ }^{2}$ \\
\hline FUR & $49.8 \pm 0.8$ & 63.8 & $-104 \pm 3$ & -102 & $80.8 \pm 1.8$ & 95.3 & $\mathrm{~B}_{\mathrm{AC}}{ }^{2}$ & $\mathrm{~B}_{\mathrm{AC}}{ }^{2}$ \\
\hline DVL $^{100}$ & $30.1 \pm 1.7$ & 45.5 & $-117 \pm 6$ & -127 & $65.0 \pm 2.5$ & 80.4 & $\mathrm{~B}_{\mathrm{AC}}{ }^{2}$ & $\mathrm{~B}_{\mathrm{AC}}{ }^{2}$ \\
\hline
\end{tabular}

3.3.2.3.1. Preferred pathway. The results are in good qualitative agreement with experimental values: all molecules clearly favor the $\mathrm{B}_{\mathrm{AC}} 2$ mechanism over the alkyl alternative, with the exception of the elimination-addition mechanism for the hydrolysis of DIK.

Due to the symmetry of the hydroxide nucleophile and the alkoxide leaving group, it has been proposed that nor the addition or breakdown reactions are fully rate-limiting except for esters with very good leaving groups, for which the addition is indeed a kinetic bottleneck. This is in excellent agreement with our results: as the ring-strain increases, so does the difference between addition and cleavage activation free energies. The obvious example are $\beta$-lactones, whose high ring strain lowers the energetic barrier for the cleavage step by almost $20 \mathrm{~kJ} \mathrm{~mol}^{-1}$ (Table 3.11 ).

GBL has been observed not to undergo any ${ }^{18} \mathrm{O}$ isotopic exchange during alkaline hydrolysis, which suggests that cleavage of the intermediate $\left(k_{\mathrm{clv}}\right)$ is much faster than the inverse addition reaction $\left(k_{\text {exch }}\right)$. In this work, based on the activation free energies a $k_{\mathrm{clv}} / k_{\mathrm{exch}}$ ratio of about one hundred would be expected, and thus, negligible O-exchange.

3.3.2.3.2. Accuracy. Moreover, reasonable quantitative agreement is also obtained. For the linear esters and the $\beta$-lactones, activation free energies are somewhat overestimated by more than $5 \mathrm{~kJ} \mathrm{~mol}^{-1}$, whereas activation enthalpies are underestimated by less than $5 \mathrm{~kJ} \mathrm{~mol}^{-1}$. The errors are much higher for the larger lactones which exhibit overestimated enthalpies and free energies, by $\sim 15 \mathrm{~kJ} \mathrm{~mol}^{-1}$. 
The divergence between the experimental and theoretical $\mathrm{B}_{\mathrm{AC}} 2$ activation enthalpy for DIK has been discussed on the basis of the base-catalyzed elimination-addition mechanism.

The errors in the activation entropy observed for the neutral $B_{A C} 2$ mechanism are not present in the basic counterpart.

As regards the results using the postHF ab initio methods, the results are inferior. As occurred in the neutral mechanisms and for the same reasons, $\Delta G^{\ominus}$ at the post-HF levels of theory shows large deviations from the experimental values, when available, and from DFT-B3LYP results. Both free energies differences for the transition states and the tetrahedral intermediates are underestimated, even to the point that, for some lactones, the intermediate is more stable than the reactants.

\subsubsection{Acid hydrolysis}

The existing mechanisms of acid-catalyzed ester hydrolysis can be seen as the counterparts of those described in Schemes 3.14, 3.15 and 3.16, albeit involving the protonated ester.

Non-activated esters favor the $\mathrm{A}_{\mathrm{AC}} 2$ mechanism, whereas those species prone to give off stable carbocations, that is, tertiary alkyl esters, hydrolyze rapidly at low acid concentrations according to the $\mathrm{A}_{\mathrm{AL}} 1$ mechanism.

Once again, $\beta$-lactones are an example of particular behavior and variation in the mechanism with small changes in structure: $\mathrm{BPL}, \mathrm{BBL}$ and DIK are known to follow the $\mathrm{A}_{\mathrm{AC}} 1$ mechanism even in conditions where water is relatively abundant. The tertiary alkyl nature of the alkyl-oxygen carbon in BIVL rules its reactivity in acidic media, and thus it is an example of the $\mathrm{A}_{\mathrm{AL}} 1$ mechanism. Larger primary lactones (GBL, FUR and DVL) and linear esters (AcOEt and COOMe) follow the $\mathrm{A}_{\mathrm{AC}} 2$ pathway.

Since the ester group is a first step common to all acid-catalyzed mechanisms, the determination of the acid dissociation constants of the protonated esters is of importance and will be addressed first.

\subsubsection{Acidity of esters}

The protonation pre-equilibrium is of great importance; for instance, esters with electron-withdrawing substituents, such as haloesters, are weakly basic and show little acid catalysis. Thus, variations in reactivity can sometimes be explained by differences in the protonation free energy. Also, the contribution of this initial step to the overall entropy and enthalpy of activation is not known, since experimental activation parameters are measured for the global reaction.

Esters are weak bases that only undergo protonation in strongly acidic media, and hence their $\mathrm{p} K_{\mathrm{a}}$ values have been defined in terms of Hammett's acidity function. In general, akyl esters have very similar acid dissociation constants, around a standard value of $\mathrm{p} K_{\mathrm{a}}(\mathrm{AcOEt})=-7.0$, which is also quite similar to the equilibrium constant for the protonation of carboxylic acids. Esters, however, are not well behaved Hammet bases. 
The values obtained for the protonation reaction of esters using the absolute (??) and proton transfer (??) methods are reported in Table 3.12.

Table 3.12. Energies of dissociation and $\mathrm{p} K_{\mathrm{a}}$ of the studied esters

\begin{tabular}{cccccccc}
\hline & \multicolumn{3}{c}{ Absolute } & & \multicolumn{3}{c}{ Proton Transfer } \\
\cline { 2 - 3 } \cline { 6 - 8 } & $\begin{array}{c}-\Delta H \\
\left(\mathrm{~kJ} \mathrm{~mol}^{-1}\right)\end{array}$ & $\begin{array}{c}-\Delta G \\
\left(\mathrm{~kJ} \mathrm{~mol}^{-1}\right)\end{array}$ & $-\mathrm{p} K_{\mathrm{a}}$ & & $\begin{array}{c}-\Delta H \\
\left(\mathrm{~kJ} \mathrm{~mol}^{-1}\right)\end{array}$ & $\begin{array}{c}-\Delta G \\
\left(\mathrm{~kJ} \mathrm{~mol}^{-1}\right)\end{array}$ & $-\mathrm{p} K_{\mathrm{a}}$ \\
\hline COOMe & 64.2 & 61.2 & 10.7 & & 40.0 & 53.1 & 9.3 \\
AcOEt & 57.1 & 42.9 & 7.5 & & 33.2 & 41.2 & 7.2 \\
BPL & 67.9 & 56.5 & 10.5 & & 32.3 & 41.9 & 7.3 \\
BBL & 64.3 & 54.0 & 9.9 & & 29.1 & 41.9 & 7.3 \\
BIVL & 60.5 & 54.0 & 9.5 & & 26.7 & 37.8 & 6.6 \\
DIK & 78.9 & 65.5 & 11.5 & & 40.0 & 49.6 & 8.7 \\
GBL & 52.5 & 49.2 & 8.6 & & 16.9 & 24.9 & 4.4 \\
FUR & 55.8 & 43.4 & 7.6 & & 20.0 & 27.1 & 4.7 \\
DVL & 42.8 & 42.9 & 7.5 & & 8.4 & 20.5 & 3.6 \\
\hline
\end{tabular}

The determination of the absolute enthalpy of the proton in solution has received significantly less attention than its free energy, and this is observed in the large differences observed in the absolute and proton-transfer dissociation enthalpies. Free energy and $\mathrm{p} K_{\mathrm{a}}$ values, however, are in reasonable agreement between the two methods and in close agreement with available experimental result for AcOEt ( $\mathrm{p} K_{\mathrm{a}}$ $\simeq-7.0$ ).

It has been discussed whether the pre-protonation step is entropically favorable. The two methods are quite contradictory in this regard: the absolute method predicts somewhat negative entropies, whereas the proton transfer method predicts similar entropies but of the opposite sign. Since the value for the enthalpy of the proton in aqueous solution is quite dubious, the values of the proton-transfer method are probably more realistic.

Results suggest that $\beta$ lactones, especially DIK, are significantly less basic (more acidic) than linear esters. This effect has been studied both theoretically and empirically in the gas-phase and is attributed to the lower $s p^{2}$ character of the ester carbon in four-membered lactone. ${ }^{111}$ The electron-donating effect increases the basicity of the $\beta$-lactones, as observed in the BPL - BBL - BIVL series. The $\gamma$ and $\delta$ lactones are approximately as acidic as the open chain esters.

\subsubsection{2. $A_{A L} 1, A_{A C} 1$ and $A_{A L} 2$ mechanisms}

The $\mathrm{A}_{\mathrm{AL}} 1, \mathrm{~A}_{\mathrm{AC}} 1$ and $\mathrm{A}_{\mathrm{AL}} 2$ mechanisms are simple and take place in a single, rate-limiting step; excluding rapid addition of water to carbocations (or elimination) and proton transfers (Scheme 3.20). $\mathrm{A}_{\mathrm{AC}} 2$ is more complex and is discussed separately (see below). 


\section{$\mathrm{A}_{\mathrm{AL}} \mathbf{1}$}

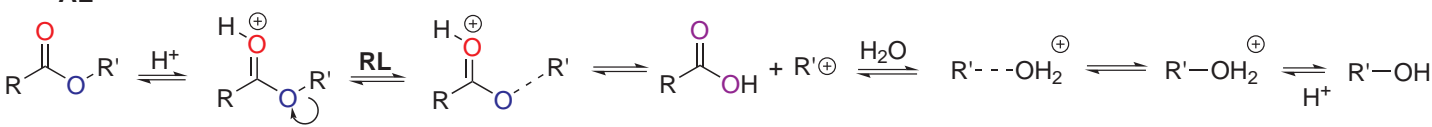

$\mathrm{A}_{\mathrm{AC}} 1$

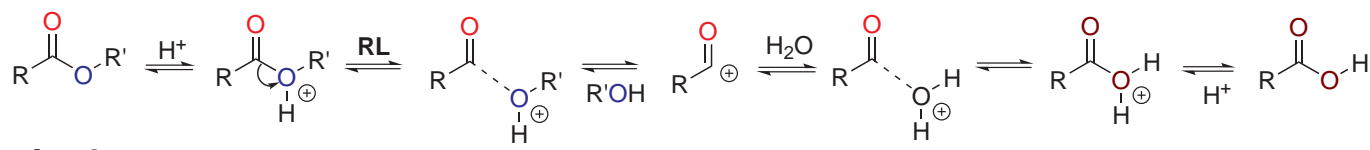

\section{$\mathrm{A}_{\mathrm{AL}} \mathbf{2}$}

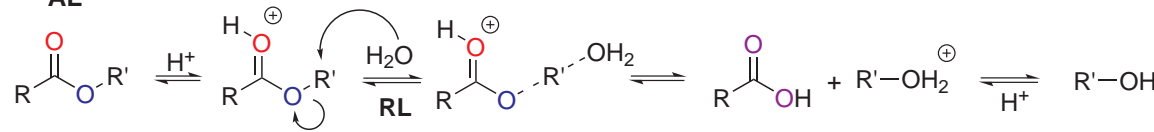

\section{$\mathrm{A}_{\mathrm{AC}} \mathbf{2}$}

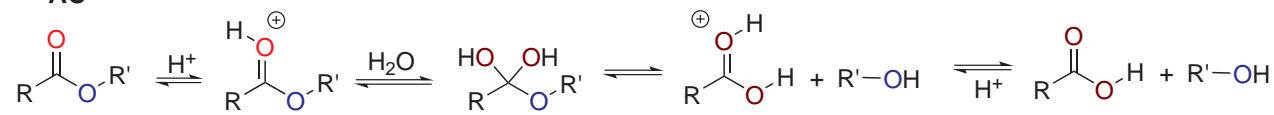

Scheme 3.20. $A_{A L} 1, A_{A C} 1, A_{A L} 2$ and simplified $A_{A C} 2$ mechanisms of hydrolysis

For ease of comparison, Table 3.13 reports the enthalpy and free energy differences between the reactants and the transition state for the rate-limiting step for all the studied compounds. In analogy to the experimental situation, the water+hydronium+ester cluster has been chose as reactants, rather than the protonated ester.

Table 3.13. Energy barriers for the rate limiting step in the mechanisms acid-catalyzed lactone hydrolysis

\begin{tabular}{|c|c|c|c|c|c|c|c|c|}
\hline & \multicolumn{4}{|c|}{$\Delta H\left(\mathrm{~kJ} \mathrm{~mol}^{-1}\right)$} & \multicolumn{4}{|c|}{$\Delta G\left(25^{\circ} \mathrm{C}\right)\left(\mathrm{kJ} \mathrm{mol}^{-1}\right)$} \\
\hline & $\mathrm{A}_{\mathrm{AL}} 2$ & $\mathrm{~A}_{\mathrm{AL}} 1$ & $\mathrm{~A}_{\mathrm{AC}} 1$ & $\mathrm{~A}_{\mathrm{AC}}{ }^{2}$ & $\mathrm{~A}_{\mathrm{AL}} 2$ & $\mathrm{~A}_{\mathrm{AL}} 1$ & $\mathrm{~A}_{\mathrm{AC}} 1$ & $\mathrm{~A}_{\mathrm{AC}}{ }^{2}$ \\
\hline COOMe & 185.8 & & 234.4 & 86.9 & 164.3 & & 228.9 & 93.6 \\
\hline $\mathrm{AcOEt}$ & 188.8 & 228.8 & 184.9 & 96.8 & 171.3 & 204.3 & 177.8 & 114.3 \\
\hline BPL & 120.3 & 193.9 & 101.7 & 94.2 & 108.8 & 192.1 & 103.0 & 98.1 \\
\hline BBL & 108.9 & 130.2 & 105.1 & 93.6 & 119.4 & 113.6 & 111.2 & 98.5 \\
\hline BIVL & - & 90.8 & 112.3 & 96.5 & - & 70.7 & 123.8 & 101.1 \\
\hline DIK & - & 168.4 & 84.6 & 87.5 & - & 145.6 & 92.2 & 93.0 \\
\hline GBL & 128.1 & 208.1 & 191.8 & 86.1 & 123.6 & 179.2 & 173.6 & 86.6 \\
\hline FUR & 142.5 & 224.3 & 183.3 & 104.1 & 145.9 & 200.4 & 167.0 & 112.1 \\
\hline DVL & 151.7 & 173.3 & 171.4 & 72.7 & 145.0 & 171.8 & 182.2 & 83.6 \\
\hline
\end{tabular}

As occurred in the neutral hydrolysis, no transition states could be located for the bimolecular alkylcleavage of BIVL and DIK. Also in a similar fashion as occurred with the neutral $\mathrm{B}_{\mathrm{AL}} 2$ mechanism, activation entropies for $\mathrm{A}_{\mathrm{AL}} 2$ are quite high, even positive in some cases, whereas, in analogy to the neutral counterpart, (Table 3.9), small negative values would be expected. 
These uncommon mechanisms are very unfavored for the larger lactones and the linear esters, which are known to hydrolyze through the $\mathrm{A}_{\mathrm{AC}} 2$ mechanism (see below).

Only $\beta$-lactones favor (at least in part) these three less common hydrolysis mechanisms: the only tertiary alkyl lactone, BIVL is hydrolyzed by the $\mathrm{A}_{\mathrm{AL}} 1$ mechanism, which is quite clearly the most favored path. As regards the $\mathrm{A}_{\mathrm{AC}} 1$ cleavage, it is very close in activation free energy to the $\mathrm{A}_{\mathrm{AC}} 2$ addition step for $\beta$-lactones (Table 3.16).

Since $\mathrm{A}_{\mathrm{AL}} 2$ is disfavored for all the compounds - in keeping with the fact that it has never been observed experimentally to be major - only the unimolecular mechanisms will be discussed in this section.

3.3.3.2.1. $\mathbf{A}_{\mathbf{A L}} \mathbf{1}$ mechanism. Table 3.14 reports the enthalpy and free energy differences between the reactants and the significant species in the reaction path for the $\mathrm{A}_{\mathrm{AL}} 1$ mechanism. Since the reaction products following the unimolecular cleavage $\left(\mathrm{TS}_{\mathrm{CLV}}\right)$ are carbocations that either undergo elimination or add a water molecule in a non rate-limiting step, we have included the carbocation (CAT) and the transition state corresponding to the attack of water $\left(\mathrm{TS}_{\mathrm{AD}}\right)$ in cases for which this mechanism seem preferred.

As stated above, $\mathrm{A}_{\mathrm{AL}} 1$ is observed in the hydrolysis of esters whose leaving group is capable of yielding stable carbocations, and thus mainly for esters of tertiary alcohols, such as tert-butyl acetates. Among the compounds of choice, BIVL is the only one that clearly (more than $30 \mathrm{KJ} \mathrm{mol}^{-1}$ ) favors this $\mathrm{A}_{\mathrm{AL}} 1$ over the alternatives (Table 3.13).

Table 3.14. Energy barriers for the $\mathrm{A}_{\mathrm{AL}} 1$ acid hydrolysis of some lactones

\begin{tabular}{|c|c|c|c|c|c|c|}
\hline & \multicolumn{3}{|c|}{$\Delta H\left(\mathrm{~kJ} \mathrm{~mol}^{-1}\right)$} & \multicolumn{3}{|c|}{$\Delta G\left(25^{\circ} \mathrm{C}\right)\left(\mathrm{kJ} \mathrm{mol}^{-1}\right)$} \\
\hline & $\mathrm{TS}_{\mathrm{CLV}}$ & CAT & $\mathrm{TS}_{\mathrm{AD}}$ & $\mathrm{TS}_{\mathrm{CLV}}$ & CAT & $\mathrm{TS}_{\mathrm{AD}}$ \\
\hline COOMe & & - & - & & - & - \\
\hline AcOEt & 228.8 & - & - & 204.3 & - & - \\
\hline $\mathrm{BPL}$ & 193.9 & - & - & 192.1 & - & - \\
\hline BBL & 130.2 & & 128.2 & 113.6 & & 126.0 \\
\hline BIVL & 90.8 & 85.5 & 68.9 & 70.7 & 56.9 & 84.0 \\
\hline DIK & 168.4 & - & - & 145.6 & - & - \\
\hline GBL & 208.1 & - & - & 179.2 & - & - \\
\hline FUR & 224.3 & - & - & 200.4 & - & - \\
\hline DVL & 173.3 & - & - & 171.8 & - & - \\
\hline
\end{tabular}

The stabilizing effect of the substituent on the alkyl carbon from primary to tertiary is evident in the BPL - BBL - BIVL series.

The $\mathrm{A}_{\mathrm{AL}} 1$ reaction has positive activation entropies, in keeping with the fact that a bond is broken and none are formed. Interestingly, the addition of water to the carbocation has a barrier (referred to the 
reactants as starting point) quite similar for that of unimolecular cleavage itself, which makes the step kinetically significant.

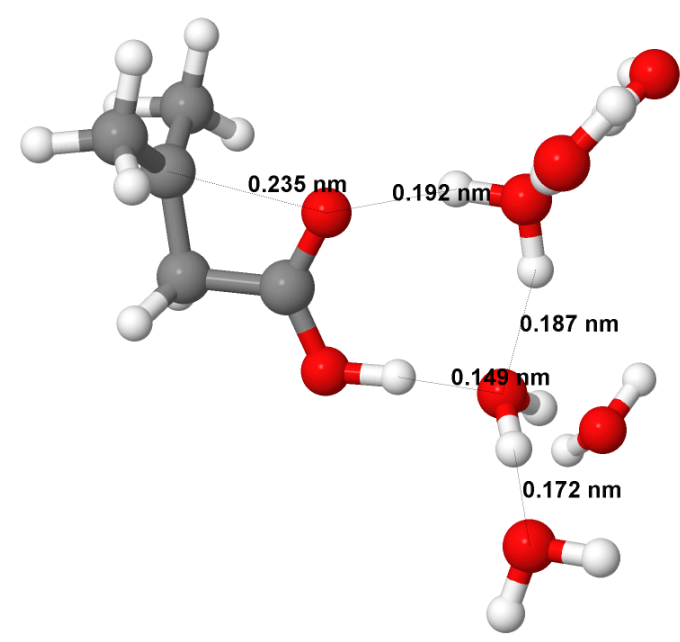

Figure 3.9. Equilibrium geometry for the $A_{A L} 1$ transition state for BIVL

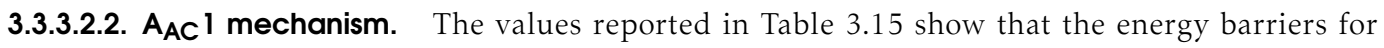
the $A_{A C} 1$ cleavage are very high, except in the case of three $\beta$-lactones. Activation enthalpies are very high and the thermoentropic contributions are close to null, in keeping with the unimolecular nature of the mechanism.

Table 3.15. Energy barriers for the $A_{A C} 1$ acid hydrolysis of some lactones

\begin{tabular}{|c|c|c|c|c|c|c|}
\hline & \multicolumn{3}{|c|}{$\Delta H\left(\mathrm{~kJ} \mathrm{~mol}^{-1}\right)$} & \multicolumn{3}{|c|}{$\Delta G\left(25^{\circ} \mathrm{C}\right)\left(\mathrm{kJ} \mathrm{mol}^{-1}\right)$} \\
\hline & $\mathrm{TS}_{\mathrm{CLV}}$ & CAT & $\mathrm{TS}_{\mathrm{AD}}$ & $\mathrm{TS}_{\mathrm{CLV}}$ & CAT & $\mathrm{TS}_{\mathrm{AD}}$ \\
\hline COOMe & 234.4 & - & - & 228.9 & - & - \\
\hline $\mathrm{AcOEt}$ & 184.9 & - & - & 177.8 & - & - \\
\hline $\mathrm{BPL}$ & 101.7 & 99.1 & 116.4 & 103.0 & 99.6 & 111.6 \\
\hline BBL & 105.1 & 103.1 & 115.5 & 111.2 & 113.8 & 118.5 \\
\hline BIVL & 112.3 & 111.5 & & 123.8 & 126.4 & \\
\hline DIK & 84.6 & 80.2 & 94.6 & 92.2 & 82.6 & 100.3 \\
\hline GBL & 191.8 & - & - & 173.6 & - & - \\
\hline FUR & 183.3 & - & - & 167.0 & - & - \\
\hline DVL & 171.4 & - & 166.1 & 182.2 & - & - \\
\hline
\end{tabular}

Since the reaction products following the unimolecular cleavage $\left(\mathrm{TS}_{\mathrm{CLV}}\right)$ are carbocations that either 
undergo elimination or add a water molecule in a non rate-limiting step, we have included the carbocation $(\mathrm{CAT})$ and the transition state corresponding to the attack of water $\left(\mathrm{TS}_{\mathrm{AD}}\right)$ in cases for which this mechanism seem preferred.

The species formed following the carbon-oxygen bond cleavage in the $\mathrm{A}_{\mathrm{AC}} 1$ mechanism are the corresponding acyl cations. These are quite high in energy for the larger lactones, but not so much in the case of BPL, BBL and DIK. The acyl cation moiety is not hydrogen-bonded to its water hydration shell, and a lack of specific interactions with the solvent can be deduced from the large distance to the solvent molecules (Figure 3.10). Since they lie so high in energy, their hydration step is predicted to be partially rate-limiting, as regards the global progress of the reaction.

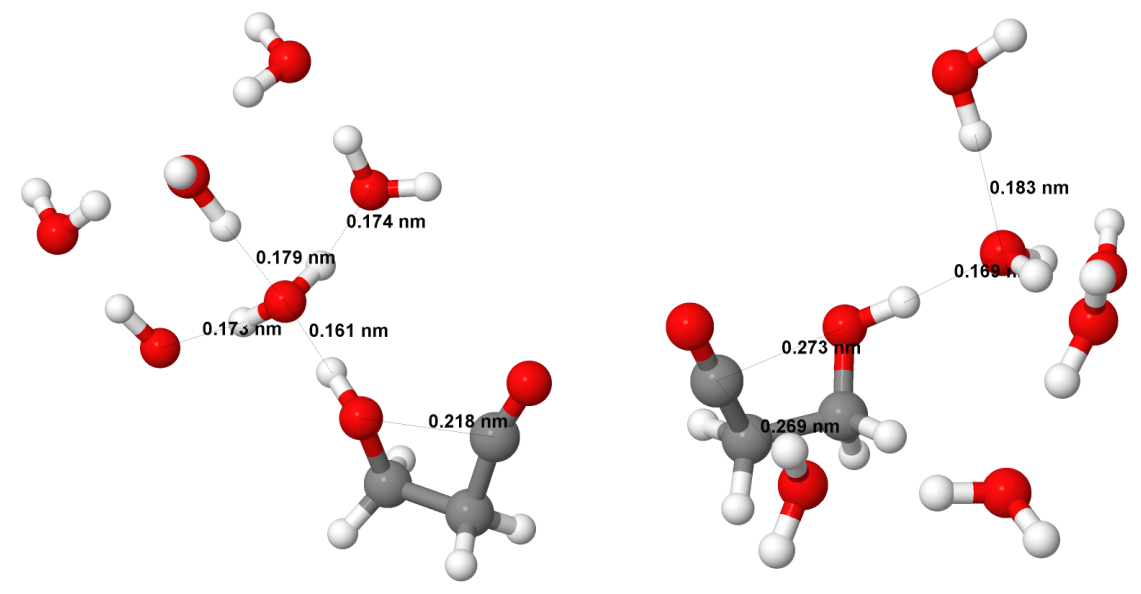

Figure 3.10. Structure of the $A_{A C} l$ cleavage transition state, and its acyl cation product

\subsubsection{3. $A_{A C} 2$ mechanism}

Like its neutral and base-catalyzed analogues, the $\mathrm{A}_{\mathrm{AC}} 2$ mechanism takes place in two steps (Scheme 3.21): an addition reaction leads to the tetrahedral intermediate, which in turn decomposes. Whereas the breakdown kinetics of the intermediate has received less attention, the addition step, and the nature of the intermediate have attracted much discussion. Especially, as regards whether the reaction takes place by the base catalyzed addition of water on the protonated carbonyl (which yields a neutral tetrahedral intermediate) or the direct attack of water, affording a charged intermediate.

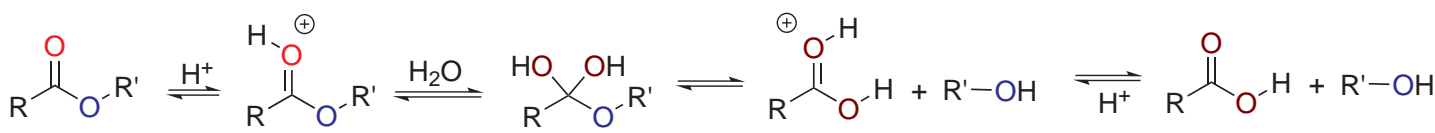

Scheme 3.21. $A_{A C} 2$ mechanism of ester hydrolysis

The calculated differences in free energy and enthalpy for the addition step $\left(\mathrm{TS}_{\mathrm{AD}}\right)$ the intermediate (DIOL), and the cleavage reaction $\left(\mathrm{TS}_{\mathrm{CLV}}\right)$ are reported in Table 3.16. 
Table 3.16. Energy barriers for the $\mathrm{A}_{\mathrm{AC}} 2$ hydrolysis of some lactones

\begin{tabular}{cccccccc}
\hline & \multicolumn{3}{c}{$\Delta H\left(\mathrm{~kJ} \mathrm{~mol}^{-1}\right)$} & & \multicolumn{3}{c}{$\Delta G\left(25^{\circ} \mathrm{C}\right)\left(\mathrm{kJ} \mathrm{mol}^{-1}\right)$} \\
\cline { 2 - 3 } \cline { 7 - 9 } & $\mathrm{TS}_{\mathrm{AD}}$ & DIOL & $\mathrm{TS}_{\mathrm{CLV}}$ & & $\mathrm{TS}_{\mathrm{AD}}$ & $\mathrm{DIOL}$ & $\mathrm{TS}_{\mathrm{CLV}}$ \\
\hline COOMe & 86.9 & 63.9 & 77.7 & & 93.6 & 80.3 & 88.1 \\
AcOEt & 96.8 & 80.4 & 104.6 & & 114.3 & 107.7 & 106.7 \\
BPL & 94.2 & 61.2 & 60.6 & & 98.1 & 75.0 & 77.3 \\
BBL & 93.6 & 64.9 & 62.3 & & 98.5 & 78.2 & 84.7 \\
BIVL & 96.5 & 65.7 & 68.7 & & 101.1 & 77.1 & 88.8 \\
DIK & 87.5 & 37.5 & 42.1 & & 93.0 & 50.7 & 63.8 \\
GBL & 86.1 & 69.0 & 81.7 & & 86.6 & 73.3 & 89.0 \\
FUR & 104.1 & 87.2 & 100.3 & & 112.1 & 92.9 & 114.5 \\
DVL & 72.7 & 62.8 & 68.5 & & 83.6 & 79.7 & 89.9 \\
\hline
\end{tabular}
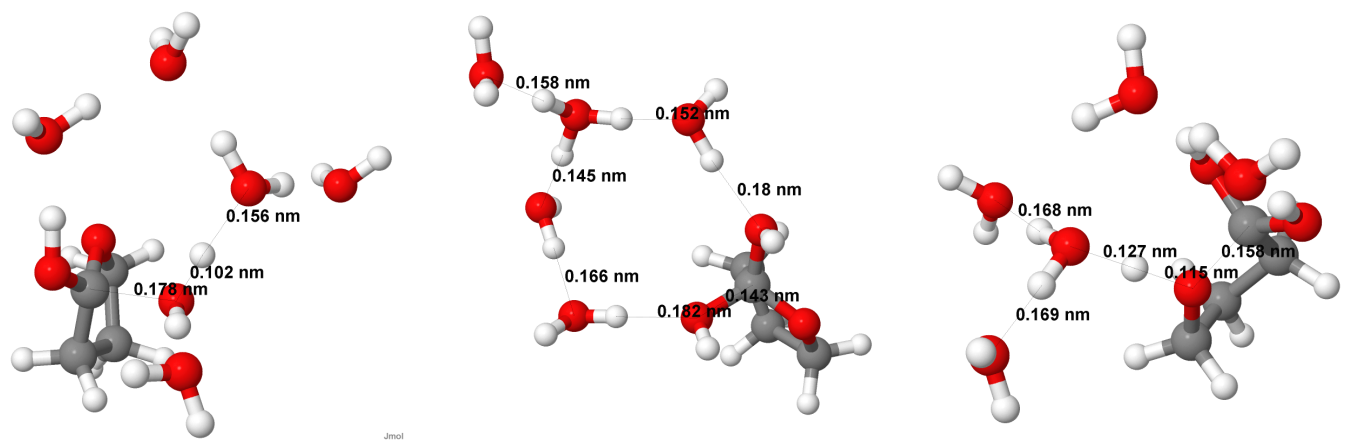

Figure 3.1 1. Structure of the intermediate and the transition states in the $A_{A C} 2$ mechanism for $G B L$

3.3.3.3.1. Addition. It has been suggested that the addition reaction of a water molecule to the protonated carbonyl is base-catalyzed by an additional water molecule. Thus, the reaction would yield a unprotonated partially sterified orthoacid as the tetrahedral intermediate, and a hydronium ion (Scheme 3.22). The reasons for this proposed mechanism range from reaction symmetry (if the breakdown in the tetrahedral intermediate is acid-catalyzed, then the opposite must occur in the addition reaction) to the very high acidity of the protonated diol that is the tetrahedral intermediate. The transition states found in this work correspond to such a base-catalyzed mechanism (AD.2 in Scheme 3.22). Two molecules are involved the rate-limiting step, in keeping with the known fact that the acid hydrolysis of most esters in acidic conditions is second order in water.

$\beta$-Lactones, excluding the very reactive DIK, show high barriers for this mechanism, which is most possibly related to their low basicity. The barriers are quite high for FUR and AcOEt, whereas COOMe and especially GBL and DVL are very reactive. 


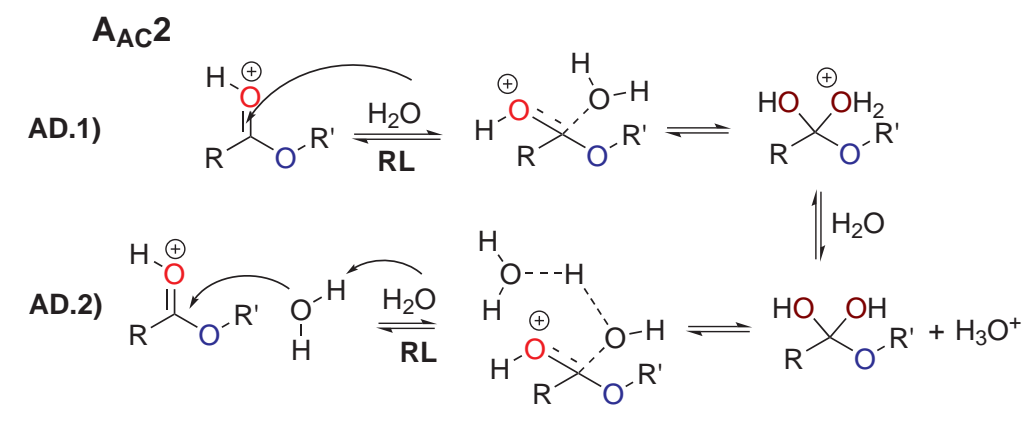

Scheme 3.22. Possible addition steps in the $A_{A C} 2$ hydrolysis mechanism

3.3.3.3.2. Intermediate. The tetrahedral intermediates formed are partially esterified orthoacids, and, as occurred in the neutral and alkaline hydrolysis, these species lie rather high in energy, with the exception of DIK, for the geometrical reasons given above.

3.3.3.3.3. Cleavage. The breakdown transition states are symmetrical to those for the addition reaction, and feature a hydronium-catalyzed cleavage step (Scheme 3.23 CLV.2), which is in agreement with results in the literature. ${ }^{112}$

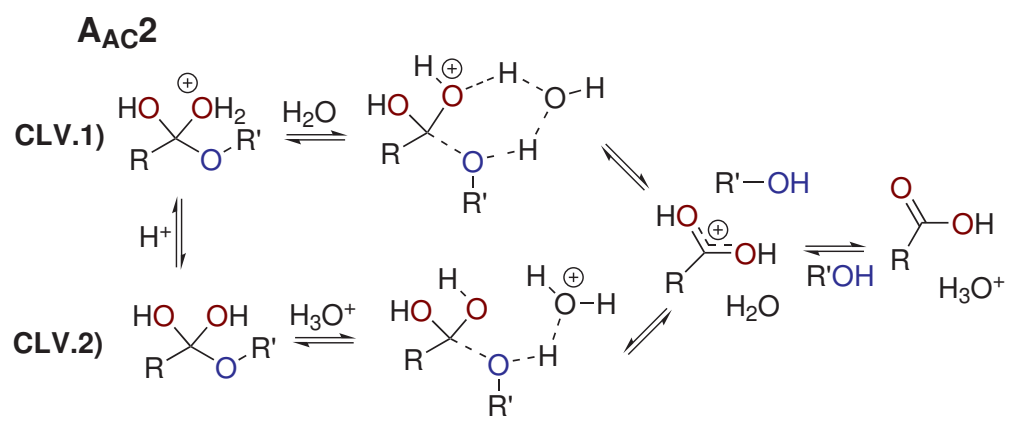

Scheme 3.23. Observed cleavage steps in the $A_{A C} 2$ hydrolysis mechanisms

For the larger lactones and the linear compounds the cleavage step is kinetically significant, since the barrier for the breakdown is very similar to that for the addition.

Weakly basic esters are known to show very limited acid-catalysis, and even then, only those with poor leaving groups. These esters are not protonated even in 100\% sulfuric acid, and hence this has been attributed to the cleavage step being (co)rate-limiting. Since the acid catalyzed hydrolysis shares intermediate uncharged diol with the neutral mechanism, the $\mathrm{H}_{3} \mathrm{O}^{+}$-catalyzed cleavage is also available for weakly acidic esters, whose addition reaction follows the already described neutral $\mathrm{B}_{\mathrm{AC}} 2$ pathway. The breakdown reaction is rate-limiting for the poorer leaving groups, and thus is accelerated by acid. For better leaving groups, the breakdown reaction is non the limiting step and no catalysis is observed. 


\subsubsection{Comparison to experimental results}

The experimental and theoretical activation parameters for the acid hydrolysis reaction of the studied compounds in water are given in Table 3.17. Experimental activation parameters for acid catalyzed hydrolysis reactions are computed from the catalytic term, $k_{\mathrm{H}} / K_{\mathrm{a}}$, and thus include the contribution of both the protonation pre-equilibrium and the rate-limiting step. For consistency, values reported in Table 3.17 are referred to the unprotonated reactants (hydronium+water cluster+ester).

Table 3.17. Experimental activation parameters for the acid hydrolysis of some lactones in water

\begin{tabular}{|c|c|c|c|c|c|c|c|c|}
\hline & \multicolumn{2}{|c|}{$\begin{array}{c}\Delta^{\ddagger} H^{\ominus} \\
\left(\mathrm{kJ} \mathrm{mol}{ }^{-1}\right)\end{array}$} & \multicolumn{2}{|c|}{$\begin{array}{c}\Delta^{\ddagger} S^{\ominus} \\
\left(\mathrm{J} \mathrm{mol}^{-1} \mathrm{~K}^{-1}\right)\end{array}$} & \multicolumn{2}{|c|}{$\begin{array}{c}\Delta^{\ddagger} G^{\ominus}\left(25^{\circ} \mathrm{C}\right) \\
\left(\mathrm{kJ} \mathrm{mol}{ }^{-1}\right)\end{array}$} & \multicolumn{2}{|c|}{ Mechanism } \\
\hline & Exp & Calc & Exp & Calc & Exp & Calc & Exp & Calc \\
\hline COOMe & $60 \pm 2$ & 87 & $-89 \pm 7$ & -22 & $87 \pm 3$ & 94 & $\mathrm{~A}_{\mathrm{AC}}{ }^{2}$ & $A_{A C}{ }^{2}$ \\
\hline $\mathrm{AcOEt}^{113}$ & $68 \pm 1$ & 97 & $-94 \pm 1$ & -59 & $96 \pm 1$ & 114 & $\mathrm{~A}_{\mathrm{AC}}{ }^{2}$ & $\mathrm{~A}_{\mathrm{AC}}{ }^{2}$ \\
\hline BPL $^{90}$ & - & $94 / 97$ & - & $-4 /$ & $\sim 103$ & $98 / 103$ & $\mathrm{~A}_{\mathrm{AC}} 1$ & $\mathrm{~A}_{\mathrm{AC}} 2 / \mathrm{A}_{\mathrm{AC}} 1$ \\
\hline $\mathrm{BBL}^{88}$ & - & $94 / 102$ & - & $-20 /$ & $\sim 105$ & 99 / 111 & $\mathrm{~A}_{\mathrm{AC}} 1$ & $\mathrm{~A}_{\mathrm{AC}} 2 / \mathrm{A}_{\mathrm{AC}} 1$ \\
\hline BIVL $^{91}$ & - & 91 & - & 33 & $\sim 88$ & 81 & $\mathrm{~A}_{\mathrm{AL}} 1$ & $\mathrm{~A}_{\mathrm{AL}} 1$ \\
\hline $\operatorname{DIK}^{114}$ & - & $88 / 85$ & - & $-25 /$ & $\sim 96$ & $93 / 92$ & $\mathrm{~A}_{\mathrm{AC}} 1$ & $\mathrm{~A}_{\mathrm{AC}} 2 / \mathrm{A}_{\mathrm{AC}} 1$ \\
\hline $\mathrm{GBL}^{93}$ & $65 \pm 1$ & 86 & $-96 \pm 3$ & -2 & $94 \pm 2$ & 87 & $\mathrm{~A}_{\mathrm{AC}}{ }^{2}$ & $\mathrm{~A}_{\mathrm{AC}} 2$ \\
\hline FUR & - & 104 & - & -27 & - & 112 & $\mathrm{~A}_{\mathrm{AC}}{ }^{2}$ & $\mathrm{~A}_{\mathrm{AC}}{ }^{2}$ \\
\hline DVL $^{93}$ & $47 \pm 1$ & 73 & $-113 \pm 5$ & -46 & $81 \pm 2$ & 84 & $\mathrm{~A}_{\mathrm{AC}}{ }^{2}$ & $\mathrm{~A}_{\mathrm{AC}}{ }^{2}$ \\
\hline
\end{tabular}

3.3.3.4.1. Preferred mechanism. The results are in good qualitative agreement with the experimental mechanisms: no esters are known to follow the $\mathrm{A}_{\mathrm{AL}} 2$ mechanism, which is disfavored in all the studied cases; linear esters and larger lactones are correctly predicted to follow the $\mathrm{A}_{\mathrm{AC}} 2$ mechanism, and BIVL is hydrolyzed by the $\mathrm{A}_{\mathrm{AL}} 1$ pathway.

The behavior of the other $\beta$-lactones, which experimentally follow the $\mathrm{A}_{\mathrm{AC}} 1$ pathway, is more complex. $A_{A C} 1$ and $A_{A C} 2$ hydrolyses are predicted to have very similar barriers (BBL favors the bimolecular pathway by almost $10 \mathrm{~kJ} \mathrm{~mol}^{-1}$ ). This can be reconciled with the fact the most kinetic evidence points in the direction of (mostly) pure $\mathrm{A}_{\mathrm{AC}} 1$.

Usually, the increased reactivity of activated esters is more evident in the neutral hydrolysis, and the acid hydrolysis is the least sensitive to increases in reactivity. Thus, high concentrations of acid are needed in experimental conditions, in order to accelerate the acid-catalyzed reaction to a point where it is faster than the enhanced neutral reaction.

This is exactly the case with BPL, BBL and DIK: their acid-catalyzed reaction is unfavored by their low basicity and ocluded by their fast neutral hydrolysis. Catalysis by hydronium ions cannot be observed at acid concentrations with $H_{0}<-1$, which is equivalent to $20 \%$ sulfuric acid. At these high acid concentra- 
tions the activity of water is significantly lower $\left(a_{\mathrm{H}_{2} \mathrm{O}}<0.75\right)$. This decrease in water activity disfavors the bimolecular mechanism (especially since the reaction is order two in water) and also, hinders the formation of the highly solvated $\mathrm{A}_{\mathrm{AC}} 2$ intermediate.

It is not unexpected then, for the $\mathrm{A}_{\mathrm{AC}} 2 / \mathrm{A}_{\mathrm{AC}} 1$ ratio to go down as the concentration of acid increases and water becomes more scarce: at low acidities, in which the reaction cannot be observed experimentally $\mathrm{A}_{\mathrm{AC}} 2$ is possibly equally rapid or even faster than $\mathrm{A}_{\mathrm{AC}} 1$, as predicted by our results. However, in the working conditions (concentrated mineral acid) the barrier for $\mathrm{A}_{\mathrm{AC}} 2$ is increased, which means that the measured kinetic parameters correspond to the now major $\mathrm{A}_{\mathrm{AC}} 1$ pathway.

Experiments show that the $\mathrm{A}_{\mathrm{AL}} 1$ reaction is very sensitive to the dielectric constant of the medium, and is decreased in media with organic co-solvent, sometimes, to such a point that the mechanism switches to the standard $A_{A C} 2$. This is consistent with the observed dipolar moment of $A_{A L} 1$ transition states, which is around 20 Debye, and much higher than those of the alternative $\mathrm{A}_{\mathrm{AC}} 2$ addition, around 5-6 Debye. For this reason, together with the proximity of the $\mathrm{A}_{\mathrm{AC}} 1$ and $\mathrm{A}_{\mathrm{AL}} 1$ barriers, $\mathrm{BBL}$ could perhaps show a minor alkyl contribution to its hydroylsis mechanism that has gone undetected.

The structures of the acyl cations are in good agreement with crystallographic values: $\mathrm{C}-\mathrm{C}$ bond around $140 \mathrm{pm}$ and $\mathrm{C} \equiv \mathrm{O}$ about $110 \mathrm{pm}$. (Figure 3.10)

3.3.3.4.2. Accuracy. The calculated $\mathrm{p} K_{\mathrm{a}}$ value for AcOEt is in excellent agreement with the experimental value available in the literature. In addition, the values obtained for the lactones can help understand the reactivity of these compounds. Of the three ring sizes studied, $\beta$-lactones are the least sensitive to acid catalysis, and usually very high acid concentrations are needed to observe an increase in reaction rate. On the opposite extreme are $\gamma$ and $\delta$-lactones, which react readily in mildly acidic media. This can be explained partially in terms of their acid dissociation constants: the higher the acidity, the lower the concentration of the highly reactive protonated ester, and thus the lower the catalytic effect of hydronium ions.

As regards the activation parameters, free energies show errors from -7 to $+15 \mathrm{~kJ} \mathrm{~mol}^{-1}$, and thus ours results are insufficient to predict reaction rates within the so-called chemical accuracy $\left( \pm 1 \mathrm{kcal} \mathrm{mol}^{-1}\right)$. Activation enthalpies, in the few cases where experimental values are available for comparison, show large positive errors.

\subsubsection{Acid hydrolysis at low water concentrations}

The $\mathrm{A}_{\mathrm{AC}} 2$ reaction requires the simultaneous availability of several water molecules: the nucleophile, the base catalyst, and those necessary for solvating the formed hydronium ion. As a result, the $\mathrm{A}_{\mathrm{AC}} 2$ pathway is expected to be disfavored in situations where few water molecules are present. On the contrary, a decrease in the availability of water molecules has little effect on the $\mathrm{A}_{\mathrm{AC}} 1$ mechanism, since water does not play a significant role in the limiting steps of the other than generic hydration

This is precisely what occurs in experiments: the hydrolysis of primary and secondary alkyl esters in very concentrated acid solutions switches from a the standard $\mathrm{A}_{\mathrm{AC}} 2$ mechanism to $\mathrm{A}_{\mathrm{AC}} 1$ when the 
concentration of mineral acid reaches $70-80 \%$ and few water molecules are available to simultaneously hydrate the ester. This is a more extreme version of the behavior of $\beta$-lactones, whose high ring-strain increases the tendency to undergo unimolecular cleavage even at moderate water concentration.

In order to emulate this empirical observation, the energy barriers for the pertinent mechanisms in the absence of solvent molecules were calculated. The activation parameters when only the absolutely essential water molecules are included (one for bimolecular mechanisms, none for unimolecular ones) are reported in Table 3.18. Table 3.19 shows the calculated activation parameters when one extra water molecule is included, for a total of one water molecule for unimolecular reactions and two water molecules (nucleophile plus catalyst) for bimolecular pathways.

Table 3.18. Energy barriers hydrolysis of some lactones with minimal water molecules

\begin{tabular}{|c|c|c|c|c|c|c|c|c|}
\hline & \multicolumn{4}{|c|}{$\Delta H\left(\mathrm{~kJ} \mathrm{~mol}^{-1}\right)$} & \multicolumn{4}{|c|}{$\Delta G\left(25^{\circ} \mathrm{C}\right)\left(\mathrm{kJ} \mathrm{mol}^{-1}\right)$} \\
\hline & $\mathrm{A}_{\mathrm{AL}} 1$ & $\mathrm{~A}_{\mathrm{AC}} 1$ & $\mathrm{~A}_{\mathrm{AL}} 2$ & $\mathrm{~A}_{\mathrm{AC}}{ }^{2}$ & $\mathrm{~A}_{\mathrm{AL}} 1$ & $\mathrm{~A}_{\mathrm{AC}} 1$ & $\mathrm{~A}_{\mathrm{AL}} 2$ & $\mathrm{~A}_{\mathrm{AC}}{ }^{2}$ \\
\hline COOMe & 239.5 & 165.8 & 117.5 & 101.0 & 221.6 & 159.0 & 117.7 & 108.7 \\
\hline AcOEt & 125.2 & 87.9 & 118.0 & 109.5 & 112.7 & 88.1 & 115.9 & 126.4 \\
\hline $\mathrm{BPL}$ & 91.7 & 44.7 & 84.8 & 196.2 & 88.3 & 42.6 & 87.6 & 207.4 \\
\hline BBL & 42.9 & 40.5 & 84.3 & 199.8 & 39.0 & 38.3 & 80.3 & 210.3 \\
\hline BIVL & 11.5 & 50.7 & 63.7 & 200.7 & 7.2 & 49.0 & 51.0 & 211.7 \\
\hline DIK & 68.9 & 38.8 & - & 198.3 & 61.8 & 35.4 & - & 209.3 \\
\hline GBL & 162.6 & 85.4 & 115.7 & 203.3 & 154.1 & 77.4 & 119.0 & 215.8 \\
\hline FUR & 230.3 & 117.7 & 140.5 & 229.8 & 222.4 & 103.5 & 141.6 & 241.0 \\
\hline DVL & 129.4 & 95.3 & 116.4 & 206.0 & 123.0 & 85.4 & 118.3 & 218.2 \\
\hline
\end{tabular}

When only the strictly necessary water molecules are present, the situation changes significantly. The acidic tetrahedral intermediate in the $\mathrm{A}_{\mathrm{AC}} 2$ mechanism is so high in energy, and behind such a towering energy barrier, that, for all the studied molecules (with the obvious exception of tertiary BIVL), the $\mathrm{A}_{\mathrm{AC}}{ }^{1}$ mechanism is the lowest-energy reaction pathway. The energy barrier for this pathway is higher than that of standard well-solvated $\mathrm{A}_{\mathrm{AC}} 2$ hydrolysis, in accord with the experimentally observed lower reaction rate once the change in the mechanism occurs. The barrier for the $\mathrm{A}_{\mathrm{AC}} 2$ addition in the case of AcOEt is exceptionally favored, in comparison with the lactones - although still higher in energy than the $\mathrm{A}_{\mathrm{AC}} 1$ transition state. This effect could be related to the favored trans configuration of the ester bond.

In an intermediate situation, the presence of one explicit solvent molecule favors the $\mathrm{A}_{\mathrm{AC}} 2$ contribution: the linear esters are now predicted to follow the $\mathrm{A}_{\mathrm{AC}} 2$ mechanism; the less strained lactones show similar barriers for $\mathrm{A}_{\mathrm{AC}} 2$ and $\mathrm{A}_{\mathrm{AC}} 1$; and the primary and secondary $\beta$-lactones still hydrolyse by the $\mathrm{A}_{\mathrm{AC}} 1$ mechanism.

Therefore, the change from the bimolecular to the unimolecular acyl cleavage mechanisms can be interpreted as being influenced by exogenous and endogenous parameters: solvation and ring strain, respectively. If the activity of water is lowered, the availability the $\mathrm{A}_{\mathrm{AC}} 2$ mechanism decreases, since it is 
Table 3.19. Energy barriers hydrolysis of some lactones with one additional explicit solvent molecule, plus the one acting as a nucleophile (when needed)

\begin{tabular}{|c|c|c|c|c|c|c|c|c|}
\hline & \multicolumn{4}{|c|}{$\Delta H\left(\mathrm{~kJ} \mathrm{~mol}^{-1}\right)$} & \multicolumn{4}{|c|}{$\Delta G\left(25^{\circ} \mathrm{C}\right)\left(\mathrm{kJ} \mathrm{mol}^{-1}\right)$} \\
\hline & $\mathrm{A}_{\mathrm{AL}} 1$ & $\mathrm{~A}_{\mathrm{AC}}{ }^{1}$ & $\mathrm{~A}_{\mathrm{AL}} 2$ & $\mathrm{~A}_{\mathrm{AC}}{ }^{2}$ & $\mathrm{~A}_{\mathrm{AL}} 1$ & $\mathrm{~A}_{\mathrm{AC}}{ }^{1}$ & $\mathrm{~A}_{\mathrm{AL}} 2$ & $\mathrm{~A}_{\mathrm{AC}}{ }^{2}$ \\
\hline COOMe & 222.3 & 194.2 & 118.8 & 93.8 & 210.6 & 184.1 & 112.5 & 99.1 \\
\hline AcOEt & 170.5 & 145.1 & 121.5 & 98.2 & 113.7 & 142.4 & 117.1 & 117.2 \\
\hline $\mathrm{BPL}$ & 105.5 & 67.5 & 68.8 & 86.5 & 101.9 & 51.4 & 68.0 & 92.8 \\
\hline $\mathrm{BBL}$ & 58.9 & 50.2 & 48.0 & 64.4 & 60.2 & 45.7 & 50.9 & 79.8 \\
\hline BIVL & 23.2 & 62.3 & & 72.8 & 22.6 & 58.2 & & 89.0 \\
\hline DIK & 90.2 & 21.2 & - & 54.9 & 88.0 & 17.4 & - & 68.3 \\
\hline GBL & 170.1 & 130.9 & 117.5 & 99.2 & 158.6 & 107.0 & 112.1 & 105.9 \\
\hline FUR & 191.5 & 123.6 & 134.6 & & 180.8 & 113.4 & 130.0 & \\
\hline DVL & 148.6 & 126.4 & 135.0 & 103.7 & 149.0 & 107.8 & 132.4 & 109.6 \\
\hline
\end{tabular}

order two in water. Also, the presence of a strained ring increases the tendency of the lactones to undergo unimolecular ring-cleavage.

Thus, the acid-catalyzed hydrolysis of BPL, BBL and DIK can be seen as an extreme version of this behavior, in which the decrease in water activity necessary for the change in the mechanism takes place at rather low acid concentrations. Since DIK is the most reactive $\beta$-lactone, it is also the one showing the larger unimolecular contribution. On the opposite side sits AcOEt, which requires very high concentrations of acid before the $\mathrm{A}_{\mathrm{AC}} 1$ mechanism can be observed.

The only exception to this behavior is the formate, for which the formation of the $\mathrm{A}_{\mathrm{AC}} 1$ transition state is very unfavored in any conditions. This is so because of the lack of substituents than can stabilize the acyl cation.

\subsubsection{Conclusions}

1. The neutral, alkaline and acid hydrolysis of lactones have been modeled in silico.

2. The predicted mechanisms are in good qualitative agreement with the experimental results reported in the literature.

3. Theoretical activation parameters are in good quantitative agreement with experimental values, the errors usually below $5 \mathrm{~kJ} \mathrm{~mol}^{-1}$.

4. Diketene was found to hydrolyze by the uncommon elimination-addition mechanism in alkaline media. 


\section{References}

[1] Kirby, A. J. In Ester formation and hydrolysis and related reactions; Bamford, C. H., Tipper, C. F. H., Eds.; Comprehensive Chemical Kinetics; Elsevier, Amsterdam The Netherlands, 1972; Vol. 10; pp $57-201$.

[2] Ingold, C. K. Structure and Mechanism in Organic Chemistry; Bell. London, 1953.

[3] March, J. Advanced Organic Chemistry. Reactions, Mechanisms and Structure, 4th ed.; John Wiley: New York, 1992.

[4] Yates, K.; McClelland, R. A. J. Am. Chem. Soc. 1967, 89, 2686-2692.

[5] Lane, C. A. J. Am. Chem. Soc. 1964, 86, 2521-2523.

[6] Lane, C. A.; Cheung, M. F.; Dorsey, G. F. J. Am. Chem. Soc. 1968, 90, 6492-6494.

[7] Hine, J.; Bayer, R. P. J. Am. Chem. Soc. 1962, 84, 1989-1993.

[8] Bell, R.; Dowding, A.; Noble, J. J. Chem. Soc. 1955, 3106-3110.

[9] Jaques, D. J. Chem. Soc. 1965, 3874-\&.

[10] Zucker, L.; Hammett, L. P. J. Am. Chem. Soc. 1939, 61, 2791-2798.

[11] Bunnett, J. F. J. Am. Chem. Soc. 1961, 83, 4956-4967.

[12] Bunnett, J. F. J. Am. Chem. Soc. 1961, 83, 4968-4973.

[13] Bunnett, J. F. J. Am. Chem. Soc. 1961, 83, 4973-4977.

[14] Bunnett, J. F. J. Am. Chem. Soc. 1961, 83, 4978-4983.

[15] Bunnett, J. F.; Olsen, F. P. Can. J. Chem. 1966, 44, 1899-1916.

[16] Treffers, H. P.; Hammett, L. P. J. Am. Chem. Soc. 1937, 59, 1708-1712.

[17] Bender, M. L.; Ladenheim, H.; Chen, M. C. J. Am. Chem. Soc. 1961, 83, 123-127.

[18] Chmiel, C. T.; Long, F. A. J. Am. Chem. Soc. 1956, 78, 3326-3330.

[19] Bunton, C. A.; Crabtree, J. H.; Robinson, L. J. Am. Chem. Soc. 1968, 90, 1258-1265.

[20] Bunton, C.; James, D.; Senior, J. J. Chem. Soc. 1960, 3364-3367.

[21] Kernshaw, D. N.; Lesten, J. A. Proc. Chem. Soc. 1960, 84.

[22] Davies, A. G.; Kenyon, J. Q. Rev. Chem. Soc. 1955, 9, 203-228.

[23] Cohen, S. G.; Schneider, A. J. Am. Chem. Soc. 1941, 63, 3382-3388.

[24] Bunton, C. A.; Hughes, E. D.; Ingold, C. K.; Meigh, D. F. Nature 1950, 166, 680680.

[25] Hammond, G. S.; Rudesill, J. T. J. Am. Chem. Soc. 1950, 72, 2769-2770.

[26] Stimson, V. R.; Watson, E. J. J. Chem. Soc. 1954, 28482852.

[27] Stimson, V. R. J. Chem. Soc. 1955, 26732675. 
[28] Stimson, V. R. J. Chem. Soc. 1955, 20102013.

[29] Stimson, V. R. J. Chem. Soc. 1955, 40204028.

[30] Bunton, C.; Comyns, A.; Graham, J.; Quayle, J. J. Chem. Soc. 1955, 3817-3824.

[31] Harvey, G. J.; Stimson, V. R. J. Chem. Soc. 1956, 36293631.

[32] Adam, K.; Lauder, I.; Stimson, V. Aust. J. Chem. 1962, 15, 467479.

[33] Osborn, A.; Whalley, E. Can. J. Chem. 1961, 39, 1094-\&.

[34] Bunton, C. A.; Wood, J. L. J. Chem. Soc. 1955, 1522-1525.

[35] Bunton, C. A.; Konasiewicz, A. J. Chem. Soc. 1955, 1354-1359.

[36] Bunton, C. A.; Day, J. N. E.; Flowers, R. H.; Sheel, P.; Wood, J. L. J. Chem. Soc. 1957, 963-967.

[37] Swain, C. G.; Knee, T. E. C.; MacLachlan, A. J. Am. Chem. Soc. 1960, 82, 6101-6104.

[38] Bunton, C. A.; Hadwick, T. J. Chem. Soc. 1957, 3043-3047.

[39] Bunton, C. A.; Hadwick, T. J. Chem. Soc. 1961, 943-953.

[40] Schwab, J. M.; Ray, T.; Ho, C.-K. J. Am. Chem. Soc. 1988, 111, 1057.

[41] Moore, J. A.; Schwab, J. M. Tetrahedron Lett. 1991, 32, 2331-2334.

[42] McClelland, R. A. J. Am. Chem. Soc. 1974, 97, 3177-3181.

[43] Datta, S. C.; Day, J. N. E.; Ingold, C. K. J. Chem. Soc. 1939, 838-840.

[44] Long, F. A.; Friedman, L. J. Am. Chem. Soc. 1950, 72, 3692-3695.

[45] Bunton, C. A.; Hadwick, T. J. Chem. Soc. 1958, 3248-3256.

[46] Holmberg, B. Chem. Ber. 1912, 45, 2997.

[47] Ingold, E. H.; Ingold, C. K. J. Chem. Soc. 1932, 756-760.

[48] Quayle, O. R.; Norton, H. M. J. Am. Chem. Soc. 1940, 62, 1170-1171.

[49] Johnson, S. In be; Gold, V., Ed.; Advances in Physical Organic Chemistry; Academic Press, 1967; Vol. 5; pp $237-330$.

[50] Bender, M. L. J. Am. Chem. Soc. 1951, 73, 1626-1629.

[51] Friedman, H. B.; Elmore, G. V. J. Am. Chem. Soc. 1941, 63, 864-867.

[52] Long, F. A.; Dunkle, F. B.; McDevit, W. F. J. Phys. Chem. 1951, 55, 829-842.

[53] Long, F. A.; McDevit, W. F.; Dunkle, F. B. J. Phys. Chem. 1951, 55, 813-829.

[54] Salomaa, P.; Schaleger, L. L.; Long, F. A. J. Am. Chem. Soc. 1964, 86, 1-7.

[55] Timm, E. W.; Hinshelwood, C. N. J. Chem. Soc. 1938, 862-869.

[56] Jaffé, H. H. Chem. Rev. 1953, 53, 191-261.

[57] Chapman, N. B.; Shorter, J.; Utley, J. H. P. J. Chem. Soc. 1963, 1291-1299. 
[58] Taft, R. In Steric effects in organic chemistry; Newman, M., Ed.; Wiley, New York, 1956.

[59] Tommila, E.; Hinshelwood, C. N. J. Chem. Soc. 1938, 1801-1810.

[60] Davies, G.; Evans, D. P. 1940, 339-345.

[61] Smith, H. A.; Steele, J. H. J. Am. Chem. Soc. 1941, 63, 3466-3469.

[62] Smith, H. A.; Myers, R. R. J. Am. Chem. Soc. 1942, 64, 2362-2365.

[63] Salomaa, P. Acta Chem. Scand. 1960, 14, 577-585.

[64] Bruice, T. C.; Holmquist, B. J. Am. Chem. Soc. 1967, 89, 4028-4034.

[65] Bunnett, J. F.; Robison, M. M.; Pennington, F. C. J. Am. Chem. Soc. 1950, 72, 2378-2381.

[66] Johnson, S. L. J. Am. Chem. Soc. 1964, 86, 3819-3824.

[67] Moffat, A.; Hunt, H. J. Am. Chem. Soc. 1958, 80, 2985-2986.

[68] Kirsch, J. F.; Jencks, W. P. J. Am. Chem. Soc. 1964, 86, 837-846.

[69] Palomaa, M. H.; Salmi, E. J.; Korte, R. Chem. Ber. 1939, 72, 790797.

[70] Salmi, E. J.; Suonpää, T. Chem. Ber. 1940, 73, 11261131.

[71] Moffat, A.; Hunt, H. J. Am. Chem. Soc. 1959, 81, 2082-2086.

[72] Euranto, E.; Cleve, N. Acta Chem. Scand. 1963, 17, 1584.

[73] Jencks, W. P.; Carriuolo, J. J. Am. Chem. Soc. 1961, 83, 1743-1750.

[74] Barclay, L. R. C.; Hall, N. D.; Cooke, G. A. Can. J. Chem. 1962, 40, 1981-1985.

[75] Douglas, J. E.; Campbell, G.; Wigfield, D. C. Can. J. Chem. 1993, 1841-1844.

[76] Bender, M. L.; Kemp, K. C. J. Am. Chem. Soc. 1957, 79, 111-116.

[77] Bender, M. L.; Ginger, R. D.; Unik, J. P. J. Am. Chem. Soc. 1958, 80, 1044-1048.

[78] Bender, M. L.; Matsui, H.; Thomas, R. J.; Tobey, S. W. J. Am. Chem. Soc. 1961, 83, 4193-4196.

[79] Taft, R. W.; Newman, M. S.; Verhoek, F. H. J. Am. Chem. Soc. 1950, 72, 4511-4519.

[80] Jones, L. B.; Sloane, T. M. Tetrahedron Lett. 1966, 7, 831 - 837.

[81] Kirsch, J. F.; Clewell, W.; Simon, A. J. Org. Chem. 1968, 33, 127-132.

[82] Goering, H. L.; Rubin, T.; Newman, M. S. J. Am. Chem. Soc. 1954, 76, 787-791.

[83] Charton, M. Can. J. Chem. 1960, 38, 2493.

[84] Bowden, K. Can. J. Chem. 1966, 44, 661kris.

[85] Hay, R. W.; Porter, L. J. J. Chem. Soc. B 1967, 1261-1264.

[86] Nolan, G. J.; Amis, E. S. J. Phys. Chem. 1961, 65, 1556-1560.

[87] Parker, A. In Rates of bimolecular substitution reactions in protic and dipolar aprotic solvents; Gold, V., Ed.; Advances in Physical Organic Chemistry; Academic Press, 1967; Vol. 5; pp 173 - 235. 
[88] Olson, A. R.; Miller, R. J. J. Am. Chem. Soc. 1938, 60, 2687-2692.

[89] Olson, A. R.; Hyde, J. L. J. Am. Chem. Soc. 1941, 63, 2459-2461.

[90] Long, F. A.; Purchase, M. J. Am. Chem. Soc. 1950, 72, 3267-3273.

[91] Liang, H. T.; Bartlett, P. D. J. Am. Chem. Soc. 1958, 80, 3585-3590.

[92] Wheeler, O. H.; Derodriguez, E. E. J. Org. Chem. 1964, 29, 1227.

[93] Pérez-Prior, M. T.; Manso, J. A.; García-Santos, M. P.; Calle, E.; Casado, J. J. Org. Chem. 2005, 70, $420-426$.

[94] Bartlett, P. D.; Rylander, P. N. J. Am. Chem. Soc. 1951, 73, 4273-4274.

[95] Manso, J. A.; Pérez-Prior, M. T.; García-Santos, M. P.; Calle, E.; Casado, J. J. Solution Chem. 2008, $37,451-457$.

[96] Huisgen, R.; Ott, H. Tetrahedron 1959, 6, 253-267.

[97] Kaiser, E. T.; Kézdy, F. J. Prog. Bioorg. Chem. 1976, 4, 239.

[98] Blackburn, G. M.; Dodds, H. L. H. J. Chem. Soc. B 1971, 826831.

[99] Brown, H. C.; Brewster, J. H.; Shechter, H. J. Am. Chem. Soc. 1954, 76, 467-474.

[100] Blackburn, G. M.; Dodds, H. L. H. J. Chem. Soc., Perkin Trans. 2 1974, 377-382.

[101] Gunaydin, H.; Houk, K. N. J. Am. Chem. Soc. 2008, 130, 15232-15233.

[102] Wang, B.; Cao, Z. Angew. Chem., Int. Ed. 2011, 50, 32663270.

[103] Begum, S.; Zeb, M. A.; Pirzada, N. J. Chem. Soc. Pak. 2000, 22, 255-259.

[104] Gómez-Bombarelli, R.; González-Pérez, M.; Pérez-Prior, M. T.; Manso, J. A.; Calle, E.; Casado, J. J. Phys. Org. Chem. 2008, manso.

[105] Mata-Segreda, J. F. J. Am. Chem. Soc. 2002, 124, 2259-2262.

[106] Marlier, J. F. J. Am. Chem. Soc. 1993, 115, 5953-5956.

[107] Holmquist, B.; Bruice, T. C. J. Am. Chem. Soc. 1969, 91, 2993-3002.

[108] Holmquist, B.; Bruice, T. C. J. Am. Chem. Soc. 1969, 91, 3003-3009.

[109] Tommila, E.; Murto, M. Acta Chem. Scand. 1963, 17, 1947-\&.

[110] Olson, A. R.; Youle, P. V. J. Am. Chem. Soc. 1951, 73, 2468-2471.

[111] Bordeje, M. C.; Mo, O.; Yanez, M.; Herreros, M.; Abboud, J. L. M. Journal of the American Chemical Society 1993, 115, 7389-7396.

[112] Yamabe, S.; Fukuda, T.; Ishii, M. Theor. Chem. Acc. 2011, DOI 10.1007/s00214-011-1019-4, 1-10.

[113] Tommila, E.; Murto, M. Acta Chem. Scand. 1963, 17, 1957-\&.

[114] Briody, J. M.; Satchell, D. P. J. Chem. Soc., Chem. Comm. 1965, 3778-3785. 


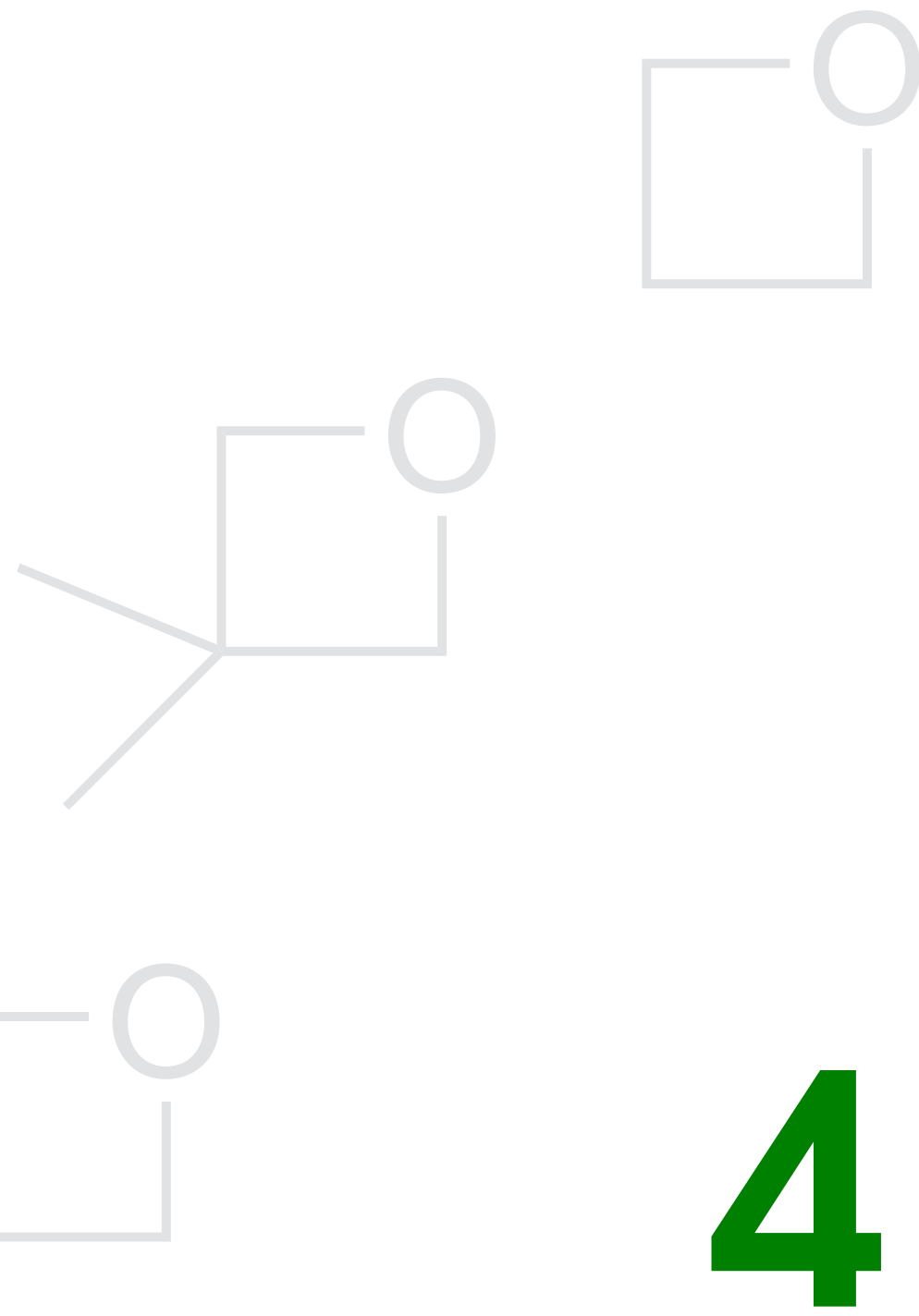

Oxetanes 



\section{Contents}

4.1 Background . . . . . . . . . . . . . . . . . . . . . 149

4.1 .1 Genotoxicity of ethers . . . . . . . . . . . . . . . . 149

4.1 .1 .1 Glycol ethers . . . . . . . . . . . . . . . . 150

4.1.1.1.1 Ethoxyethanol . . . . . . . . . . . . . . 150

4.1.1.1.2 2-Butoxyethanol . . . . . . . . . . . . 150

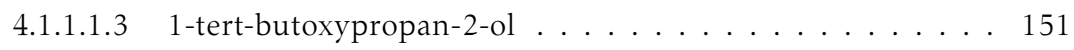

4.1.1.2 Methyl-tert-butyl ether . . . . . . . . . . . . . 153

4.1.1.3 1,4-Dioxane . . . . . . . . . . . . . . . . . . 155

4.1.1.4 Furan . . . . . . . . . . . . . . . . . . . . 156

4.1 .2 Oxetanes . . . . . . . . . . . . . . . . . . . . . 157

4.1.2.1 Applications of oxetanes . . . . . . . . . . . . . . 158

4.1.2.2 Carcinogenicity of oxetanes . . . . . . . . . . . . . . 160

4.2 Materials \& methods . . . . . . . . . . . . . . . . . 161

4.2.1 Alkylating potential of oxetanes . . . . . . . . . . . . 161

4.2.2 Genotoxicity of oxetanes . . . . . . . . . . . . . . . . 161

4.2.2.1 Ames Salmonella/Microsome Assay _. . . . . . . . . . . 161

4.2.2.2 BTC E. coli Test . . . . . . . . . . . . . . . . . . . . . 162

4.2.2.3 Alkaline Comet Assay . . . . . . . . . . . . . . . . 162

4.2.2.4 Cell viability: MTT reduction assay $\ldots \ldots \ldots \ldots \ldots \ldots$

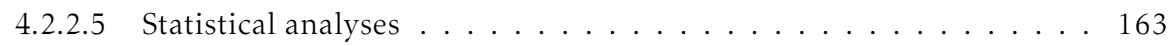

4.3 Results and discussion . . . . . . . . . . . . . . . . . . . . . . 164

4.3.1 Alkylating potential of oxetanes . . . . . . . . . . . . . . 164

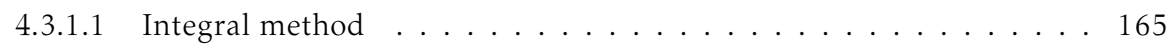

4.3.1.2 Initial Rate Method . . . . . . . . . . . . . . . . . . 166

4.3.2 Mutagenicity, genotoxicity and toxicity of oxetanes . . . . . . 168

4.3.2.1 Ames Salmonella/Microsome Assay _. . . . . . . . . . . 168

4.3.2.2 BTC E. coli Test . . . . . . . . . . . . . . . . . . . . 168

4.3.2.3 Alkaline Comet Assay . . . . . . . . . . . . . . . . 168

4.3.2.4 Cell viability: MTT reduction assay $\ldots \ldots \ldots \ldots \ldots \ldots$

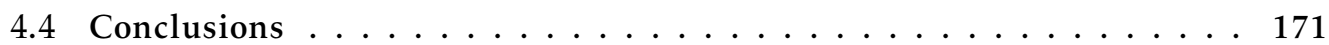

References . . . . . . . . . . . . . . . . . . . 172 



\section{Introduction}

From a structural point of view, oxetanes - tetragonal ethers - lie in between of two groups of powerful genotoxins. Oxetanes are higher homologues of oxiranes (epoxides), their rings being one link larger and thus less strained, and also have a structure similar to that of more oxidized and more electrophilic $\beta$-lactones. These two groups of compounds have long been known to be direct alkylating agents and powerful carcinogens without metabolic activation as so are other small strained heterocycles, such as, aziridinium rings from nitrogen mustards and thiirinium rings from sulfur mustards (Scheme 4.1).

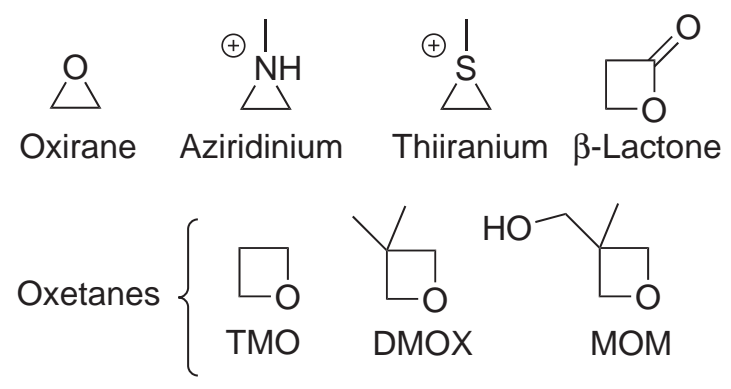

Scheme 4.1. Some small heterocyclic alkylating agents.

Oxetanes are fairly stable molecules like most aliphatic ethers, ${ }^{1}$ and contrarily to the effect of the three-membered ring in oxiranes or the four-membered cycle in $\beta$-lactones, the ring strain has little effect upon their reactivity. Like simple linear ethers and unlike other genotoxic heterocycles, oxetanes are reactive only in the presence of an acid catalyst. Trimethylene oxide (TMO), for instance, does not undergo hydrolysis in neutral aqueous medium and its acid-catalyzed hydrolysis is rather slow. ${ }^{2}$ Its reaction with amines to form 3-hydroxypropylamines is also negligible at room temperature in neutral medium. ${ }^{3}$

\subsection{Background}

\subsubsection{Genotoxicity of ethers}

Whereas very little or no information exists about the biological effects of oxetanes, the metabolism and genotoxicity of linear and cyclic ethers has attracted widespread attention in recent years. The genotoxic effects of chemicals such as glycol ethers, gasoline additives such as methyl-tert-butyl ether, ether solvents such as 1,4-dioxane, or reagents such as furan have been addressed in several studies. Thus, the following section reviews the genotoxicity of several ethers, with the aim of setting a background for the likely behavior of oxetanes. 


\subsubsection{Glycol ethers}

Glycol ethers have numerous industrial (paints, pharmaceutical products, inks) and consumer (cosmetics, detergents) applications. They have the ability to dissolve in both water and oil and a relatively slow rate of evaporation, which makes them useful as solvents and coalescing agents in paints. Other uses include inks, cleaning products, chemical intermediates, process solvents, brake fluids and de-icers. More than 30 different ethylene and propylene glycol ethers, (E- and P-series, respectively) are in use since the 1930s.

Since they are present in such a large number of consumer products, the metabolism, ${ }^{4}$ and health effects $^{5,6}$ of glycol ethers are an important matter of discussion.

4.1.1.1.1. Ethoxyethanol. Although it has tested negatively in many in vitro assays, ethoxyethanol has been found to induce chromosomal aberrations and sister-chromatid exchanges. ${ }^{7}$

4.1.1.1.2. 2-Butoxyethanol. Since the early 1980s, 2-butoxyethanol has been the most widely produced and consumed glycol ether in the USA, with a yearly production of some 226 thousand tonnes in 1999. Of this, $80 \%$ finds use in the industry, mostly in paints and coatings and also in cleaners, inks, synthesis and the electronic, rubber and oil industries, agricultural products, adhesives, oil spill dispersants, leather finishing, fire foams, pharmaceuticals and construction materials. Consumer use is mostly in paints and cleaners.

2-Butoxyethanol (2BE) is present in the environment as pollutant. It has been measure in air $(\leq 1$ $\left.\mu \mathrm{g} / \mathrm{m}^{38}\right)$ in remote and forested areas, and $\sim 3 \mu \mathrm{g} / \mathrm{m}^{3}$ in French households. ${ }^{9}$ It has been listed as a contaminant in drinking water and was found in samples collected in Canada with with a mean concentration of $0.21 \mu \mathrm{g} / \mathrm{L} .{ }^{10}$ Samples from landfill water were $<0.4$ to $84 \mathrm{mg} / \mathrm{L}^{11}$ and as high as $1.3-5.7$ $\mathrm{mg} / \mathrm{L}$ in a polluted river. ${ }^{12}$ Its presence in consumer products ranges from a few percent to almost half the weight of paints, cleaners, polishes, degreasers... and occupational exposure limits of about 10-75 $\mu \mathrm{g} / \mathrm{m}^{3}$ in workplace air exist. ${ }^{11}$

2-Butoxyacetic acid is a principal metabolic end-point for 2-butoxyethanol (Scheme 4.2). ${ }^{13}$ Although it mainly occurs in the liver, there is potential for the formation of 2-butoxyacetic acid in tissues at or near the site of contact that contain alcohol dehydrogenase ${ }^{14}$ and aldehyde dehydrogenase, ${ }^{15}$ e.g. the skin, forestomach or glandular stomach. ${ }^{16}$

Significantly positive results were afforded in inhalation carcinogenicity tests in $\mathrm{B} 6 \mathrm{C} 3 \mathrm{~F}_{1}$ mice at doses $0,302,604$ or $1208 \mathrm{mg} / \mathrm{m}^{3}$. However, none were observed in female Fischer 344/N rats. ${ }^{17}$ However, no induction of gene mutations by 2-butoxyethanol was observed in Escherichia coli strain WP2uvrA or in Salmonella typhimurium strains TA100, TA102, TA1535, TA1537, TA98 or TA97, although the results in TA97 have been disputed. ${ }^{18-20}$ Negative results have been obtained in the presence and absence of exogenous metabolic system in a number of in vitro assays: Oxidative DNA damage (8-hydroxyguanosine) ${ }^{21} \mathrm{SCE},{ }^{22,23}$ micronucleus, ${ }^{17}$ cell transformation, ${ }^{23,24}$ chromosomal aberrations, DNA adduct 


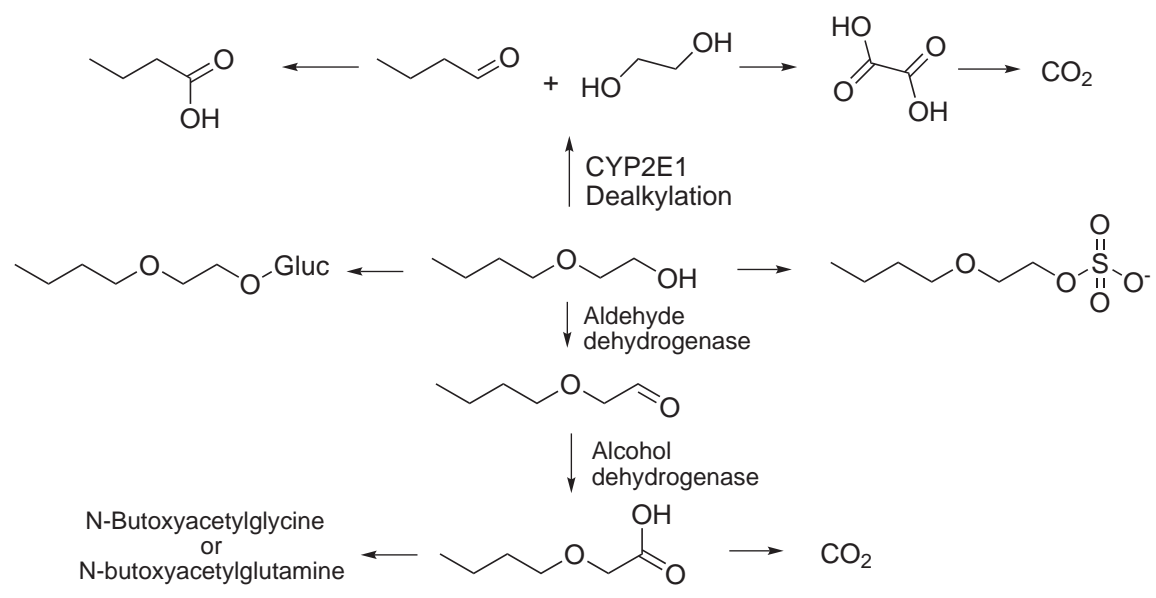

Scheme 4.2. Metabolism of 2BE

formation) for 2BE, and also for metabolites 2-butoxyethanal and 2-butoxyacetic acid. Recently, it has been reported that chronic exposure to 2-butoxyethanol increased liver hemangiosarcomas in male mice. ${ }^{25}$

Results in the literature suggest that $2 \mathrm{BE}$ lacks genotoxic effects. ${ }^{26}$ It does not induce gene mutations in bacteria or structural chromosomal aberrations in mammalian cells in vitro. Weakly positive results have been reported for high concentrations of 2-butoxyethanol in some in vitro studies of cultured mammalian cells. These genotoxic effects may be caused by the metabolite, 2-butoxyacetaldehyde, which may be formed in small amounts and has a weak capacity to cause genotoxic effects in vitro, largely at the chromosomal level. However, the genotoxic activity of this aldehyde appears to be lower than that of other glycol ether-derived aldehydes with shorter alkyl groups. The product of further oxidation, 2butoxyacetic acid, does not appear to be genotoxic. Thus, it has been proposed that tumour induction is the consequence of a sustained exposure of this organ to high concentrations of 2-butoxyethanol and its toxic metabolites, primarily 2-butoxyacetic acid, which results in tissue damage, reparative regeneration and hyperplasia. ${ }^{27,28}$

Based on this evidence, 2-butoxyethanol has been included in Group 3 by the IARC: not classifiable as to its carcinogenicity to humans. ${ }^{11}$

4.1.1.1.3. 1-tert-butoxypropan-2-ol. This compound is also known as propylene glycol mono-tertbutyl ether. However, two species - known as $\alpha$ - and $\beta$-isomers - share that name, and thus it must be stated that the acohol is secondary and the ether is primary.

Concerns about the toxic effects of the E-series glycol ethers have stimulated the development of P-series products as potential substitutes. 1-tert-Butoxypropan-2-ol (1tBP) is one of several monoalkyl ethers of propylene glycol that is finding increasing use as a replacement for the E-series glycol ethers. ${ }^{29}$

Due to their non-polar nature of the bulky tert-butyl group, $t$-butyl ethers have limited solubility in water but are miscible with most organic solvents, which favors their use as coupling, coalescing and 
dispersing agents.

Butyl glycol ethers have been used as solvents for surface coatings, inks, lacquers, paints, resins, dyes, agricultural chemicals and other oils and greases. 1-tert-Butoxypropan-2-ol is a better coupling agent and has higher electrolyte solubility than 2-butoxyethanol and it is used commercially as a solvent in water-reducible coatings and in a variety of commercial cleaners. ${ }^{29}$

Exposure to $1 \mathrm{tBP}$ during manufacture is unlikely, since it is enclosed; however, since it is present in a large, growing number of consumer products, exposure may occur. This, nevertheless has not been quantified.

Only two studies have addressed the kinetics and metabolic fate of 1tBP: single oral, intravenous or dermal administrations ${ }^{30}$ or chronic intravenous and inhalation. ${ }^{31,32} 1 \mathrm{tBP}$ was observed to be rapidly absorbed and eliminated in rats and mice, and eliminated through a concentration-dependent non-linear kinetics with a half-life of approximately 16 and $10 \mathrm{~min}$ in rats and mice, respectively. Elimination occurred mostly in the urine (48-67\%) in the form of glucuronide (23-52\%) and sulfate (7-13\%) conjugates. Expired carbon dioxide accounted for up to $26 \%$. Metabolites resulting from other potential pathways of metabolism have not been investigated experimentally. The metabolism of $1 \mathrm{tBP}$ has been proposed to occur as depicted in Scheme 4.3 .<smiles>CC(O)COC(C)(C)C</smiles>

$\underset{\text { Conjugation }}{\longrightarrow}$ P450?<smiles>CC(O)C(O)OC(C)(C)C</smiles><smiles>CC(O)C=O</smiles><smiles>[OH2+][CoH]</smiles><smiles>COC(C)(C)C</smiles><smiles>CC(=O)CO</smiles>

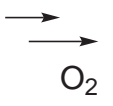
$3 \mathrm{CO}_{2}$ $2 \mathrm{H}_{2} \mathrm{O}$

Scheme 4.3. Possible metabolic fate of 1tBP

1tBP has been tested for carcinogenicity in $\mathrm{B}_{6} \mathrm{C} 3 \mathrm{~F}_{1}$ mice in doses ranging from 405 to $6480 \mathrm{mg}$ $\mathrm{m}^{-3}$ during two years, and increases in the incidences of neoplastic and non-neoplastic lesions in the livers of both male and female mice were observed. Positive trends were observed in the incidences of hepatoblastoma, hepatocellular adenoma and adenoma or carcinoma (combined); and the incidences in the high-dose groups were significantly increased. ${ }^{31,33}$ However, in a study using Fisher 344/N rats no increase in the incidence of tumors was observed in females and only marginal increases in the incidences of kidney and liver tumors were observed in males. ${ }^{31,33}$ 
As regards the genotoxicity of $1 \mathrm{tBP}$ in vitro, negative results were obtained in the S. typhimurium assay in strain TA100, TA1535 or TA98 in the presence and absence of $S 9$ mix. However, in strain TA97, 1tBP caused a concentration-related increase in mutant frequency in in the absence of an exogenous metabolic activation. No mutagenicity was observed in strain TA97 in the presence of metabolic activation. Negative results were also obtained in tests for induction of SCE and chromosomal aberrations in CHO cells, both in the presence and absence of an exogenous metabolic activation system.

The renal lesions observed in male rats were characteristic of $\alpha 2 u$-globulin-associated nephropathy, which suggests a possible mechanistic link between renal toxicity and renal tumor response. However, neither the binding of $1 \mathrm{tBP}$ to $\alpha 2 \mathrm{u}$-globulin nor the formation of tert-butanol, which does bind to $\alpha 2 \mathrm{u}$ globulin, ${ }^{34,35}$ have been investigated. ${ }^{32}$

No mechanistic information related to the occurrence of increased incidences of liver neoplasms in male rats and both male and female mice exposed to 1 tBP was available. ${ }^{31,33}$

In the light of the scarce and somewhat contradictory evidence, 1-tert-butoxy-propan-2-ol has been included in Group 3 by the IARC: not classifiable as to its carcinogenicity to humans. ${ }^{29}$

\subsubsection{Methyl-tert-butyl ether}

Methyl-tert-butyl (MtBE) ether was found to be a high-octane fuel component during the Second World War; however, it was not until the reduction in the lead content of gasoline in the mid-1970s that its commercial production and use as an octane enhancer began. ${ }^{36}$

The 1990 Clean Air Act Amendments impose a minimum oxygenate content in gasolines to improve the combustion, and thus the tailpipe gas emissions. Since it is cheaper than other alternatives such as ethanol, MtBE is also used as a gasoline oxygenate, usually in higher concentrations than as a antiknocking agent. More than $95 \%$ of the methyl tert-butyl ether produced is used as a component of gasoline. After 1992, it represented approximately 12\% of the content of reformulated gasolines sold year-round in 37 areas with ozone pollution associated with emissions of volatile organic compounds. It was present in 1997 in more than 30\% of gasoline sold in the United States, representing about 15\% of the content of oxygenated fuels sold during winter months to reduce carbon monoxide in areas where there is heavy pollution by carbon monoxide. 37,38

World production reached 7.3 million tonnes in 54 plants worldwide in $1990^{36}$ and it was 8.0 million tonnes in the US in 1995. ${ }^{39}$

As a pollutant, MeBE enters the environment (principally air and water) during all phases of the petroleum fuel cycle, which includes production refinery stack releases, storage tank releases, pipeline leaks and significant releases from underground storage tanks, evaporative losses from gasoline stations and vehicles, .... Industrial releases of methyl tert-butyl ether reported in 1993 totalled about 1.7 million $\mathrm{kg}$, about $84 \%$ of which were from refineries. ${ }^{40}$ Because of dispersion, mixing and the relatively short half-life of MtBE in the atmosphere (1-11 days) it is found near the areas of release. Since MtBE ether 
sorbs only weakly to subsurface solids, sorption does not substantially retard its transport by groundwater. $^{41}$

MtBE has been reported in air, rainwater, surface water, groundwater, drinking-water and human tissues. ${ }^{40-45}$ Excluding the areas surrounding refineries and gasoline processing and storage plants, MtBE has been measured at low concentrations ranging from below the detection limit, to less than $100 \mu \mathrm{g}$ $\mathrm{m}^{-3}$. Consumers are exposed especially at pumping stations, where concentrations of MtBE can reach the hundreds, and even thousands $\mu \mathrm{g} \mathrm{\textrm {m } ^ { - 3 }}$

MtBE is highly soluble in water, and it can partition readily from gasoline into water, resulting in high aqueous concentrations. It has been found in storm-water, groundwater, reservoir water and drinkingwater, especially in areas where it is used extensively in gasoline. In shallow groundwater below underground storage tanks, concentrations up to $200 \mathrm{mg} / \mathrm{L}$ have been found. ${ }^{46}$ Methyl tert-butyl ether is a potentially important groundwater contaminant because of its mobility and persistence. It persists in groundwater under both aerobic and anaerobic conditions because it resists physical, chemical and microbial degradation. ${ }^{40,41,44}$

Maximum concentrations of MtBE in workspaces are regulated in some countries, with 8-h timeweighted average maximum concentration around 100-180 $\mathrm{mg} \mathrm{m}^{-3}$ and term exposure limit of 100-200 $\mathrm{mg} \mathrm{m}^{-3}$

Several metabolic products of MtBE have been identified in urine (Scheme 4.4, compounds between brackets have not been found in urine). The main path is oxidative, conjugation routes being minor. ${ }^{44}$

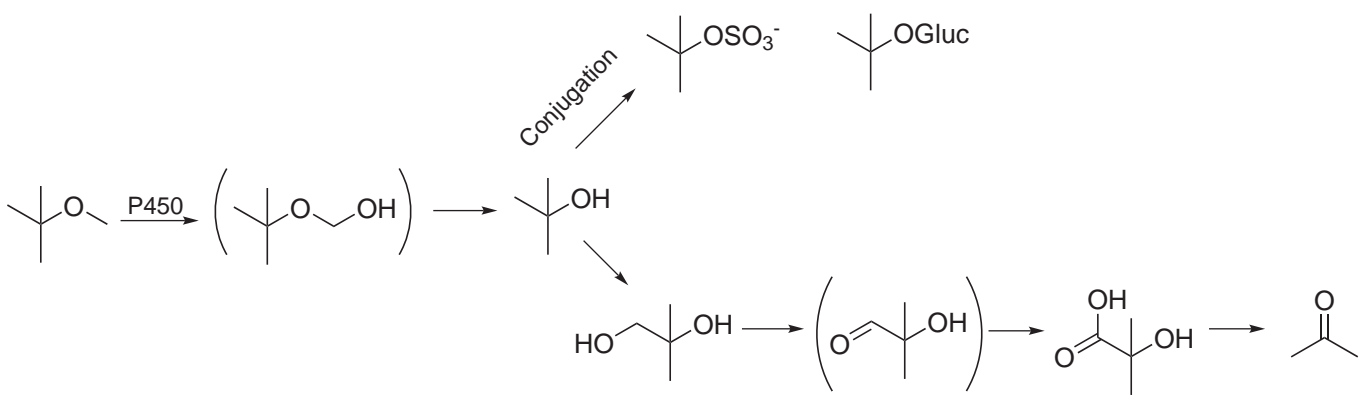

Scheme 4.4. Metabolic fate of MtBE

The tumorigenicity of MtBE has been addressed in oral administration and inhalation studies. Some of them, however, suffered from some deficiencies, such as lack of mortality-adjusted analysis. SpragueDawley rats, were given gastric instillations of 0,250 or $1000 \mathrm{mg} / \mathrm{kg}$ bw methyl tert-butyl ether. Increases in the incidence of Leydig-cell testicular adenomas was found in males at the high dose and lymphomas and leukemias was seen in female rats but not in males. ${ }^{47,48} \mathrm{CD}-1$ mice were exposed to $0-29 \times 10^{3} \mathrm{mg}$ $\mathrm{m}^{-3} \mathrm{MtBE}$. The incidence of hepatocellular adenomas (but not carcinomas) was increased in females at the high dose. At the same doses an increased incidence of renal tubular-cell tumours was observed in male Fischer 344 rats, but not in females. ${ }^{49,50}$

The tumorigenicity of the metabolites of $\mathrm{MtBE}$ has also been adressed: $\mathrm{B} 6 \mathrm{C}_{3} \mathrm{~F}_{1}$ mice were given 
0-20 $\mathrm{g} \mathrm{dm}^{-3}$ tert-butyl alcohol in their drinking water. A significantly-increased incidence of thyroid gland follicular-cell hyperplasia was observed for both males and females. The incidence of follicularcell adenoma was also increased at the high dose, but this was only significant for females. In Fischer 344 rats, kidney tumor incidences were, and so was the incidence of focal renal tubule hyperplasia was also increased in males. ${ }^{51}$

No mutagenicity to Salmonella typhimurium strains TA98, TA100, TA104 or TA1535 in the presence and absence of an exogenous activation system has been measured. Whereas, MtBE did induce gene mutation in mouse lymphoma L $5178 \mathrm{Y} t k+/-$ cells in the presence of exogenous activation, the mutagenicity was eliminated when formaldehyde dehydrogenase was added to the assay system. ${ }^{52,53}$

In vivo, methyl tert-butyl ether did not induce sex-linked recessive lethal mutation in Drosophila melanogaster. Neither did it induce unscheduled DNA synthesis in the liver of mice exposed by inhalation nor micronuclei in mouse bone marrow or chromosomal aberrations in rat bone marrow. Negative results were also obtained for micronucleus formation in mice treated with high doses by intraperitoneal injection. ${ }^{54}$ However, it induced DNA damage in isolated human lymphocytes which was identified as combination of single-strand breaks, double-strand breaks, and oxidative base modification. ${ }^{55}$

In the light of the scarce and somewhat contradictory evidence, methyl tert-butyl ether has been included in Group 3 by the IARC: not classifiable as to its carcinogenicity to humans. ${ }^{45}$

\subsubsection{1,4-Dioxane}

1,4-Dioxane is used as a solvent in a wide range of organic products: lacquers, paints, varnishes, paint and varnish removers, cleaning and detergent preparations, cements, cosmetics, deodorants, fumigants, emulsions, and polishing compositions. It is also used as a stabilizer for chlorinated solvents. In workspaces maximum values for dioxane in air of $\sim 100 \mathrm{mg} \mathrm{m}^{-3}$. Like other ethers, metabolism of 1,4-dioxane consists mainly in oxidation by cytochrome P450 affording a hemiacetal, which hydrolyzes to the alcohol and the aldehyde, which are subject to further oxidation.

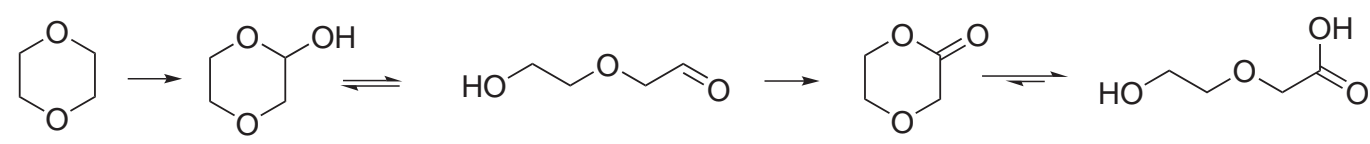

Scheme 4.5. Metabolic fate of 1,4-dioxane

1,4-Dioxane was tested in rats and guinea-pigs by oral administration: it produced malignant tumors of the nasal cavity and liver in rats and tumors of the liver and gallbladder in guinea-pigs. It was also active as a promoter in a two-stage skin carcinogenesis study in mice. No carcinogenic effect was observed in one inhalation study in rats. 56

Crj:BDF ${ }_{1}$ mice given $0-8000 \mathrm{mg} / \mathrm{L}$ dioxane in their drinking water experienced an increase in the incidence of hepatocellular adenomas and carcinomas combined. Also in F344/DuCrj rats a significant 
increase in the incidence of hepatocellular adenoma and carcinoma occurred. Mesotheliomas of the peritoneum, subcutaneous fibromas and mammary fibroadenoma in males and nasal cavity tumors and mammary adenomes in females were also increased. ${ }^{57}$ Intraperitoneal injection of dioxane also increased the incidence of lung tumors. ${ }^{58,59}$ In a recent study increases in nasal squamous cell carcinomas, hepatocellular adenomas and carcinomas, peritoneal mesotheliomas and mammary gland adenomas were observed in F344/DuCrj. In Crj:BDF1 mice significant induction of hepatocellular tumors was observed. ${ }^{60}$

Dioxane has also been observed to enhance the tumorigenicity of $N$-nitrosodiethylamine. ${ }^{61}$

Genotoxicity results both in vivo and in vitro are mostly negative: no increase in chromosomal aberrations was found in six workers employed in 1,4-dioxane production. ${ }^{57}$ 1,4-Dioxane with or without metabolic activation did not induce differential DNA repair in E. coli K-12 uvrB/rec A, was not mutagenic in Salmonella typhimurium or in L5178Y mouse lymphoma cells. ${ }^{62-65}$ and did not induce sex-linked recessive lethal mutations in Drosophila melanogaster. ${ }^{66}$

In Chinese hamster ovary $\mathrm{CHO}$ cells, it did not cause chromosomal aberrations, although it did cause a slight increase in sister chromatid exchange in the absence of metabolic activation. ${ }^{67}$ It has also been reported to cause morphological transformation of BALB/c 3T3 mouse cells. ${ }^{68}$ Oral administration of 1,4-dioxane to rats caused DNA strand breaks in liver cells. ${ }^{69}$ However, no covalent DNA binding was detected in rat liver. ${ }^{62}$ No induction of unscheduled DNA synthesis was observed in rat hepatocytes after either in vivo treatment or in vitro cell treatment. ${ }^{70}$

Of several studies on the induction of bone-marrow micronuclei it was concluded that a weak, strainspecific clastogenic activity possibly exists. ${ }^{71-73}$

Based on the sufficient evidence in experimental animals for the carcinogenicity of 1,4-dioxane, it has been concluded to be possibly carcinogenic to humans (Group 2B). ${ }^{57}$ However, this conclusion has been disputed, based on the high doses used in animal tests and a different slope factor for the risk. ${ }^{74}$

\subsubsection{Furan}

Furan is a relatively common industrial chemical that is used as an intermediate in the production of tetrahydrofuran, pyrrole and thiophene and also in the in the manufacture of lacquers and solvent for resins. It is also used in the production of pharmaceuticals, agricultural chemicals and stabilizers. ${ }^{75}$

Furan is released into the air as a gas-phase component of cigarette smoke and exhaust gas from diesel and gasoline engines. It has been detected in the expired air of both smokers, at $0-98 \mu \mathrm{g} / \mathrm{h}$, and non-smokers, at $0-28 \mu \mathrm{g} / \mathrm{h}$. Furan has identified in river waters ${ }^{76,77}$ and also in industrial effluents $(7 \pm 4$ $\mu \mathrm{g} / \mathrm{L})$ (Perry et aL., 1979).

As regards its biological action, furan requires metabolism by cytochrome P450 2E1 to elicit its toxic effects (Scheme 4.6). The initial product of furan oxidation is cis-2-butene-1,4-dial which further reacts with cellular nucleophiles such as glutathione (GSH), cysteine, and lysine. Glutation-conjugated butenedial is an electrophile that reacts with cellular amines like putrescine, spermidine and ornithine. ${ }^{78}$ 


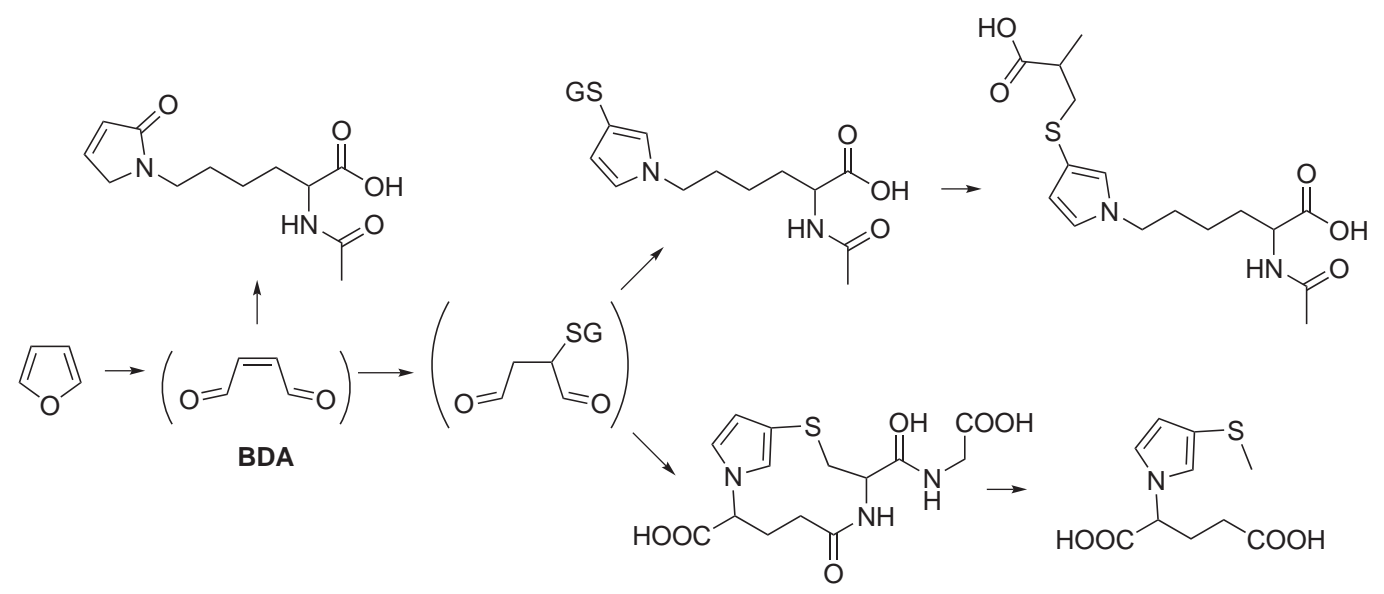

Scheme 4.6. Metabolic fate of Furan

Furan has been submitted to oral-administration tumorigenicity tests. In these, B6C3F 1 mice were fed 0,8 or $15 \mathrm{mg} / \mathrm{kg}$ bw furan. It was observed that the incidence of both hepatocellular adenomas and carcinomas. Also, high incidences of biliary hyperplasia, cholangiofibrosis, hepatocellular necrosis and focal hyperplasia were observed. ${ }^{79}$ In Fischer $344 / \mathrm{N}$ rats given $0,2,4$ or $8 \mathrm{mg} / \mathrm{kg}$ bw the incidence of hepatocellular adenomas was increased, and many rats had multiple adenomas. Hepatocellular carcinomas occurred only in males. Cholangiocarcinomas in practically all treated animals, as well as biliary hyperplasia, cholangiofibrosis, hepatocellular necrosis and hyperplasia were observed. An increase in mononuclear-cell leukaemia was also measured. ${ }^{79}$

However, in vitro furan was not mutagenic to Salmonella typhimurium after preincubation in the presence or absence of an exogenous metabolic activation system. ${ }^{75,79}$ nor did it induce sex-linked recessive lethal mutations in Drosophila melanogaster when administered to adult flies by feeding or abdominal injection. ${ }^{80}$ It was reported in an abstract that furan did not induce unscheduled DNA synthesis in rat or mouse hepatocytes after treatment in vitro or in vivo. ${ }^{75}$

Furan did afford positive results in mutation assays at the thymidine kinase locus of L5 178Y mouse lymphoma cells in the absence of metabolic activation. McGregor et al. (1988) and it also induced sister chromatid exchange and chromosomal aberrations in $\mathrm{CHO}$ cells both in the presence and absence of metabolic activation. (United States National Toxicology Program, 1993).

Given the sufficient evidence in experimental animals for the carcinogenicity of furan, it is considered to be possibly carcinogenic to humans (Croup 2B). ${ }^{75}$

\subsubsection{Oxetanes}

All marketed drugs containing the oxetane ring are derived from one family of natural products: Taxol was isolated from the bark of the western yew (Taxus brevifolia) and is, together with the structurally 
related Docetaxel currently used in cancer chemotherapy, since they interfere with the breakdown of mictrotubules during mitosis. Oxetanes are found only in a few other natural products, many of them terpenoids: ${ }^{81}$ oxetanocin, thromboxane $\mathrm{A}_{2}$, merrilactone $\mathrm{A}$, mitrephorone $\mathrm{A}$, oxetin, aoyecrystal I, dictyoxetane, bradyoxetin are all examples of natural products including oxetane moities that have attracted interest in synthetic and medicinal chemistry. ${ }^{81}$

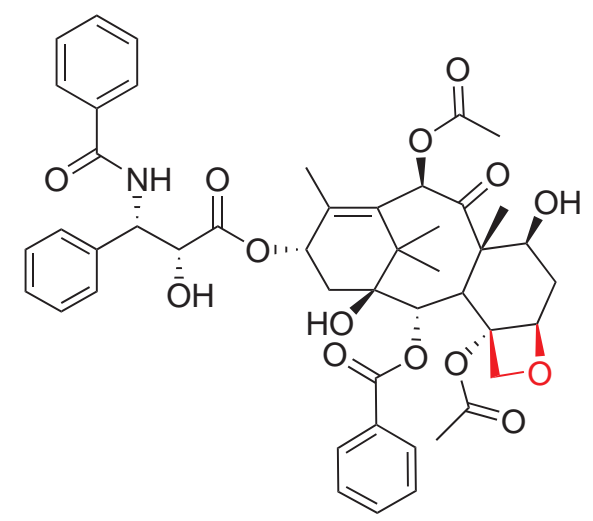

Scheme 4.7. Taxol, with oxetane moiety in red color

Whereas some of the properties of the oxetane group are a consequence of its cyclic structure, whose strain energy has been determined to be $106 \mathrm{~kJ} \mathrm{~mol}^{-1}$ (only $6 \mathrm{~kJ} \mathrm{~mol}^{-1}$ less than in oxirane and $81 \mathrm{~kJ}$ $\mathrm{mol}^{-1}$ more than in tetrahydrofuran, ${ }^{82}$ the ring-opening reactions of oxetanes usually require powerful acid catalysts. The alkaline hydrolysis of oxetane is three orders of magnitude slower than ethylene oxide. $^{2}$

This difference in reaction rate has been attributed to ring strain being released in the transition state in the case of the oxirane but not the oxetane ${ }^{83}$ and also to the transition states being aromatic and antiaromatic for the three- and four-membered rings, respectively. ${ }^{84}$

Oxetane is the strongest hydrogen-bond acceptor of all the common alicyclic ethers. This is due to the balance of two opposing effects: the smaller COC angle means that the oxygen atom is more exposed, and, at the same time, the non-bonding orbitals show less $s$ character than in the three-membered oxirane. It is also a stronger acceptor than most carbonyl compounds except amides. ${ }^{81}$

\subsubsection{Applications of oxetanes}

Oxetanes have largely remained a neglected unit in synthetic organic chemistry. For more than 130 years since the first preparation of the parent structure oxetanes have been used almost exclusively as monomers in the manufacture of polymers, ${ }^{85,86}$ such as organic light-emitting diodes (OLEDs) ${ }^{87-89}$ or support for enzymatic and solid-phase methods. ${ }^{90}$

In recent years the application of oxetanes in drug discovery has attracted increased attention. One of the earliest examples of this renewed interest is the use of oxetane moieties instead of gem-dimethyl units 
to protect metabolically exposed sites in drugs. Since the oxetane group is quite polar, this avoids the increase in lipophilicity associated with the gem-dimethyl unit. It was found that introduction of oxetane moieties - or substitution of tert-butyl groups by the more hydrophilic gem-dimethyl unit- resulted in a clear improvement of the physico chemical properties of $N, N$-dimethyl-4-( $p$-tert-butylphenyl)butylamine, a chemical chosen for its poor properties in a medicinal chemistry context. ${ }^{91}$

Oxetanes have also been found to be good surrogate groups for the carbonyl moiety, which is sometimes undesired in biologically active compounds, since it can act as en electrophile, and is susceptible to enzymatic modification and to the epimerization of adjacent stereogenic centers. It has been found that spirocyclic oxetanes, analogues of of piperidones, pyrrolidones, and azetidinone has very favorable physico-chemical properties. ${ }^{92,93}$

Given their ability to graft bulky substituents onto a scaffold of interest without increasing the lipophilicity (that is, liponeutral bulk increase) and other useful properties, like the ability to modify the basicity of proximal amines or their metabolic and chemical stability in physiological conditions, the interest in oxetane chemistry has grown rapidly $(4.8) .{ }^{81}$

Recent applications include catalytic asymmetric synthesis of 2,2-disubstituted oxetanes from ketones, ${ }^{94}$ synthesis of azaspiro[3.3] heptanes, ${ }^{95} \mathrm{Rh}$-catalyzed addition of arylboroxines to activated $\mathrm{N}$ tert-butanesulfinyl ketimines ${ }^{96}$ or synthesis of isoxazoles by base-catalyzed rearrangement. ${ }^{97}$ Most of these have oxetan-3-one 98 as their starting material.

\subsubsection{Carcinogenicity of oxetanes}

Due to their structural analogy with oxiranes and $\beta$-lactones, oxetanes were tested for their carcinogenicity in the late 1960's. Animal tests, in which the compound was delivered by subcutaneous injection, revealed that trimethylene oxide (TMO), the parent compound, $(80 \mathrm{mg} / \mathrm{kg} / \mathrm{week}, \mathrm{BD}-\mathrm{rats}),{ }^{99}$ and its dimethyl derivative, $\beta, \beta$-dimethyloxetane (DMOX) ${ }^{100}$ induce tumors at the site of injection. Nevertheless, these results have been quoted as equivocal (Registry of Toxic Effects of Chemical Substances: RQ6825000).

This shows that, although some evidence in favor of the carcinogenicity of oxetanes exists, it is very limited. No works have studied the genotoxicity or mutagenicity of oxetanes, and no contemporary studies have confirmed the results previously obtained in test animals. Thus, a re-examination of oxetanes under new light would be advisable, in order to confirm whether the behavior of these compounds is closer to their highly strained electrophilic analogues or to unreactive esters.

In this part of the work, a mixed approach was applied to study three model oxetanes (OX, in general), namely, trimethylene oxide (TMO), 3,3-dimethyloxetane (DMOX), and methyloxetanemethanol(MOM) (Scheme 4.1). The first approach involved a battery of biological tests, the Ames Salmonella assay, the BTC E. coli test, the MTT cell viability assay, and the comet assay, to search for mutagenic or toxic effects of these three compounds in both the absence and presence of a microsomal activation system or the human cytchrome P450s CYP2A6 and CYP2E1. 


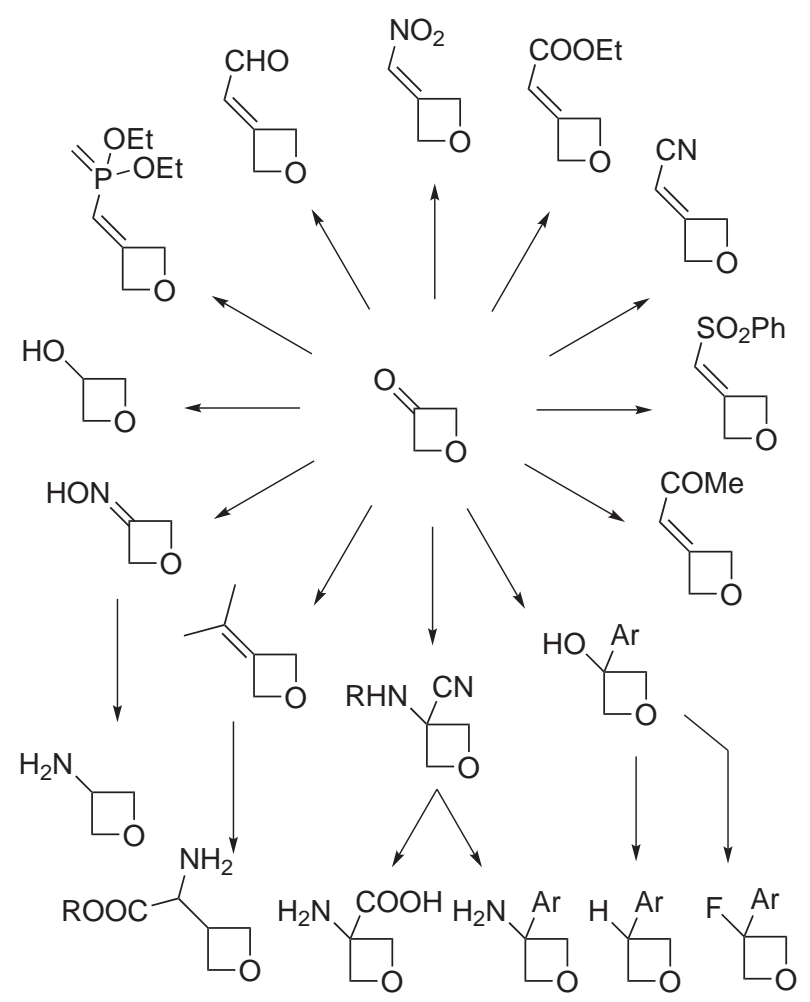

Scheme 4.8. Oxetane derivatives reported in the literature. Reproduced from ${ }^{81}$

The second approach involved the study of their reactivity with NBP. 


\subsection{Materials \& methods}

\subsubsection{Alkylating potential of oxetanes}

Alkylation reactions were performed in the $2-5 \mathrm{pH}$ range. $\mathrm{NBP}\left(\mathrm{p} K_{\mathrm{a}}=4.40\right)$ was used as a buffer solution to maintain constant $\mathrm{pH}$, and $\mathrm{HCl}$ was added to fix the $\mathrm{pH}$ value. To render NBP soluble, the reaction mixtures were prepared in 7:3 (v/v) water/dioxane medium. To monitor the alkylation reactions, $2.4 \mathrm{~cm}^{3}$-aliquots of the alkylation mixture were removed at different times and added to a cuvette containing $0.6 \mathrm{~cm}^{3}$ of $99 \%$ triethylamine $\left(\mathrm{Et}_{3} \mathrm{~N}\right)$ to form the colored compounds (Scheme 4.9), after which absorbance was measured at the wavelength of maximum absorption $(\lambda=570 \mathrm{~nm})$. The NBP concentration was in the $5-50 \times 10^{-3} \mathrm{M}$ range, and oxetane concentrations were in the $0.1-5 \times 10^{-3} \mathrm{M}$ range. Detailed reaction conditions are given in the figure and table legends. NBP was from Aldrich (98\%), and dioxane (99\%) was from Panreac.

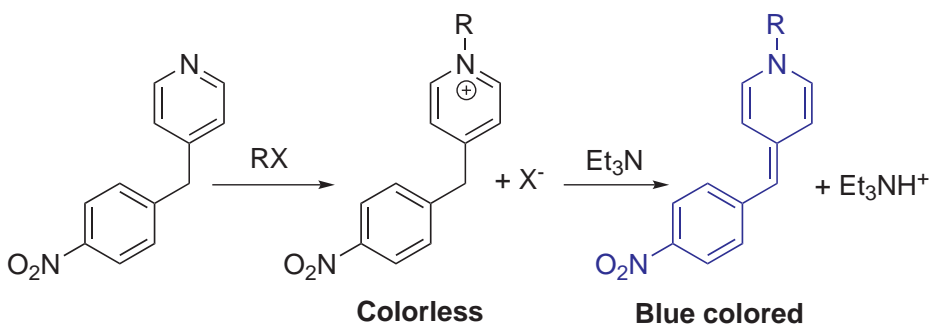

Scheme 4.9. NBP Test: development of color after alkylation

\subsubsection{Genotoxicity of oxetanes}

\subsubsection{Ames Salmonella/Microsome Assay}

The oxetanes were tested for mutagenic activity using the preincubation version of Salmonella Mutagenicity Test as described in Mortelmans and Zeiger. ${ }^{101}$

Briefly, 1.5-500 $\mu \mathrm{g}$ of each compound was combined with the Salmonella tester strain, a metabolic activation mixture derived from Aroclor 1254-induced rat liver (as required), and incubated for $20 \mathrm{~min}$ at $37^{\circ} \mathrm{C}$. The contents were then mixed with molten agar and poured onto glucose minimal medium agar plates. The plates were inverted and incubated at $37^{\circ} \mathrm{C}$ for $48 \mathrm{~h}$. Following incubation, the plates were photographed digitally, and the number of revertant colonies per plate was determined using Labworks Software (version 4.6) (UVP. Inc. USA). Two bacterial test strains were used: TA98 and TA100. Suitable positive controls were used, as suggested by Mortelmans and Zeiger: ${ }^{101} 2.5 \mu \mathrm{g} /$ plate 4-nitro-ophenylendiamine for TA98 without activation, $5.0 \mu \mathrm{g} /$ plate sodium azide for TA100 without activation, and $2.5 \mu \mathrm{g} /$ plate 2 -aminoanthracene for both TA98 and TA100 with metabolic activation. Samples were 
tested both with and without a metabolic activation mixture. The S9 metabolic activation mixture (10\%) was prepared as suggested by Mortelmans and Zeiger, ${ }^{101}$ using Aroclor 1254 induced rat liver S9 (Moltox Inc.).

\subsubsection{BTC E. coli Test}

This assay uses E. coli strains that are capable of expressing human biotransformation enzymes, in particular cytochrome P450, cytochrome P450 NADPH oxidoreductase, and, optionally, cytochrome b5. ${ }^{102}$ These strains have been used to study the role of specific human CYPs in the bioactivation of several premutagens, in particular alkylating agents, ${ }^{103}$ and the importance of human cytochrome b5 in this bioactivation. ${ }^{104}$ Briefly, an aliquot of $1.5-500 \mu \mathrm{g}$ of each compound was combined with the BTC-CYP tester strain and preincubated for $45 \mathrm{~min}$ at $37^{\circ} \mathrm{C}$. The contents were then mixed with molten agar and poured onto glucose minimal media agar plates. The plates were then inverted and incubated at $37^{\circ} \mathrm{C}$ for $48 \mathrm{~h}$. Following incubation, the plates were photographed digitally, and the number of revertant colonies per plate was determined using Labworks Software (version 4.6) (UVP Inc., USA). Suitable positive controls were used: $60 \mu \mathrm{g} /$ plate N-nitrosodiethylamine for BTC-2E1-POR and BTC-2E1-b5-POR, and 100 $\mu \mathrm{g} /$ plate 4-(methylnitrosamino)-1-(3-pyridyl)-1-butanone for BTC2A6-POR and BTC2A6-b5-POR.

\subsubsection{Alkaline Comet Assay}

Conventional microscope slides were dipped in a solution of $1 \%$ agarose in water and allowed to dry on a flat surface. Chinese hamster cells (MZ) (kindly provided by Professor H. R. Glatt, German Institute of Human Nutrition, Nuthetal, Germany) were cultured in $1 \mathrm{~cm}^{3}$ of culture medium per well (Ham's F-10 medium, supplemented with 10\% newborn calf serum and 1\% antibiotic solution [penicillinstreptomycin]) in 12-well plates, and incubated at $37{ }^{\circ} \mathrm{C}$ under a $5 \% \mathrm{CO}_{2}$ atmosphere. The cells were grown for $24 \mathrm{~h}$ and then exposed to different concentrations of TMO, DMOX, and MOM (dissolved in 10 $\mu \mathrm{L}$ of DMSO), ranging from 1 to $30 \mathrm{mM}$, for a $1 \mathrm{~h}$ period; $250 \mu \mathrm{M}$ hydrogen peroxide was used as a positive control. After trypsinization and a rinse with PBS, cells were centrifuged at $250 \mathrm{~g}$ for $5 \mathrm{~min}$ at $4{ }^{\circ} \mathrm{C}$ and were mixed with $500 \mu \mathrm{L}$ of $1 \%$ low-melting-point agarose (LMPA). $100 \mu \mathrm{L}$ of the mixture was applied to each precoated slide and coverslipped. The slides were maintained at $4{ }^{\circ} \mathrm{C}$ for $20 \mathrm{~min}$, the coverslip was removed, and the cells were immersed in a freshly made lysis solution $\left(2.5 \mathrm{M} \mathrm{NaCl}, 100 \mathrm{mM} \mathrm{Na}{ }_{2} \mathrm{EDTA}\right.$, $10 \mathrm{mM}$ Tris, and $1(v / v)$ Triton $\mathrm{X}-100$ at $\mathrm{pH} 10)$ for $1 \mathrm{~h}$ at $4{ }^{\circ} \mathrm{C}$. The slides were then incubated in alkaline buffer $\left(0.3 \mathrm{M} \mathrm{NaOH}\right.$ and $1 \mathrm{mM} \mathrm{Na}{ }_{2}$ EDTA) for $20 \mathrm{~min}$ at $4{ }^{\circ} \mathrm{C}$ in an electrophoresis tank. Electrophoresis was conducted at $0.8 \mathrm{~V} / \mathrm{cm}(20 \mathrm{~V})$ and $300 \mathrm{~mA}$ for $20 \mathrm{~min}$ at $4{ }^{\circ} \mathrm{C}$, after which the slides were washed three times in neutralizing buffer $(400 \mathrm{mM}$ Tris, $\mathrm{pH} 7.50)$ for $5 \mathrm{~min}$ at $4{ }^{\circ} \mathrm{C}$. The slides were subsequently dried for $5 \mathrm{~min}$ in water-ethanol mixtures with increasing ethanol contents, $50 \%, 70 \%$, and $100 \%$, and stained with $100 \mu \mathrm{L}$ of ethidium bromide $\left(20 \mu \mathrm{g} / \mathrm{cm}^{3}\right)$. Fifty comets per slide, two slides per treatment, were analyzed at 200× magnification by fluorescence microscopy (Leica DMLB, equipped with a short-arc mercury lamp, HBO 103 W/2) attached to a digital camera (Applied Imaging Corp., now Genetix) and connected to a personal computer, and images of randomly selected cells were captured from each slide 
using Cytovision (v3.0) capture software (Genetix). The cells were then photographed, and the images were analyzed; tail moment and DNA percentage in tail were scored using CometScore software (Tritek CometScore freeware, v1.5; www.autocomet.com).

\subsubsection{Cell viability: MTT reduction assay}

The MTT assay is based on the reduction of the yellow MTT tetrazolium salt (3-(4,5-dimethylthiazol-2yl)-2,5-diphenyltetrazolium bromide) by mitochondrial dehydrogenases affording a blue MTT formazan 105,106 and was performed according to Martins et al. ${ }^{107}$ Approximately, $7.5 \times 10^{3}$ V79 Chinese hamster lung fibroblast cells were cultured in $200 \mu \mathrm{L}$ of culture medium per well (Ham's F-10 medium, supplemented with $10 \%$ newborn calf serum and $1 \%$ antibiotic solution [penicillin-streptomycin]) in 96-well plates and incubated at $37^{\circ} \mathrm{Cunder}$ a $5 \% \mathrm{CO}_{2}$ atmosphere. The cells were grown for $16 \mathrm{~h}$ and then exposed to different concentrations of the oxetanes (dissolved in DMSO), ranging from 0.10 to $30 \mathrm{mM}$, for a $24 \mathrm{~h}$ period. Hydrogen peroxide $(1 \mathrm{mM})$ was used as a positive control and the appropriate volume of DMSO $(10 \mu \mathrm{L})$ as a negative control. The cells were washed with culture medium, incubated with MTT $\left(500 \mu \mathrm{g} / \mathrm{cm}^{3}\right)$ for a further $4 \mathrm{~h}$, and then carefully rinsed with PBS. At the end of the incubation period, the medium was discarded and DMSO $(200 \mu \mathrm{L})$ was added to each well. Absorbance was read at $595 \mathrm{~nm}$ in a Zenyth 3100 microplate reader. Four independent experiments were performed with two replicates per dose, and four individual cultures were used for each oxetane concentration in each independent experiment.

\subsubsection{Statistical analyses}

Dose-related effects were assessed using linear regression analysis. One-way ANOVA and Student's ttest were used to compare treatment levels with the control group. Kinetic profiles were fitted using SigmaPlot 10.0. 


\subsection{Results and discussion}

\subsubsection{Alkylating potential of oxetanes}

The alkylation reaction of NBP by the selected oxetanes was investigated kinetically. NBP-oxetane adducts were formed in the reaction mixture, and showed maximum absorption (after addition of triethylamine) at $\lambda=570 \mathrm{~nm}$ (Figure $4.1 \mathrm{a}$ ).
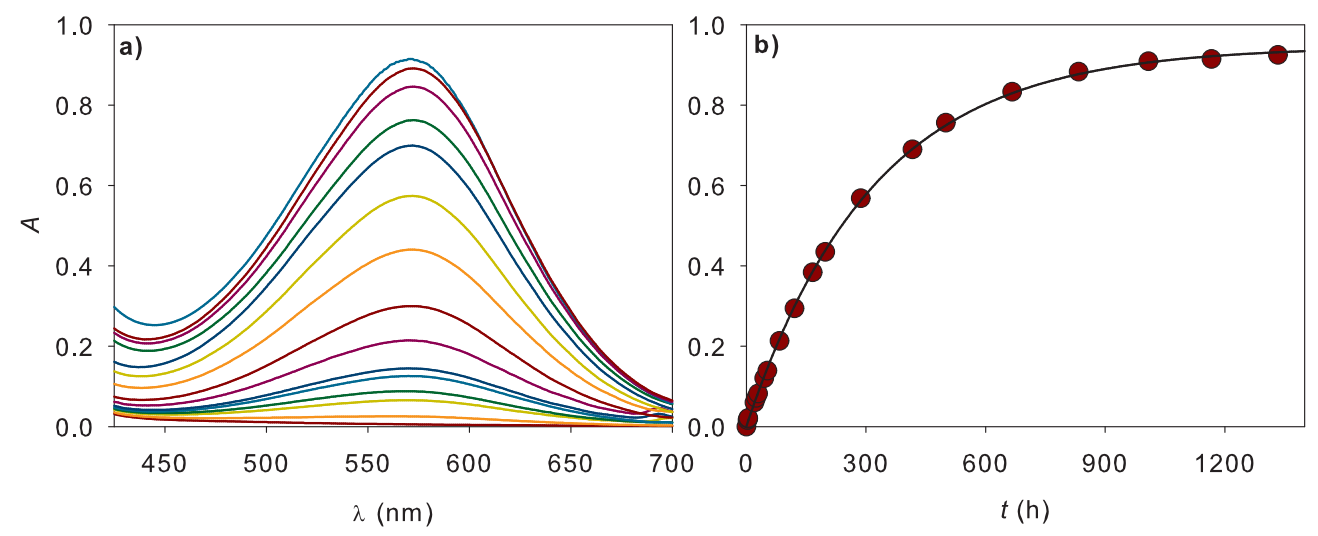

Figure 4.1. a) Variation in the UV-Vis spectrum of NBP-TMO mixtures. b) Typical kinetic run of alkylation by TMO and fit of data to eq. 4.5. $\mathrm{pH}=4.00, T=37.5^{\circ} \mathrm{C}, 7: 3 \mathrm{w} / \mathrm{d}$, (NBP) $=0.040 \mathrm{M},(\mathrm{OX})=5.0 \times 10^{-4} \mathrm{M}$.

No reaction was observed at neutral $\mathrm{pH}$ and adduct formation was only observed in acidic medium, which suggests that the alkylation reaction is acid-catalyzed. The oxetane-NBP adducts were detected by mass-spectrometry as showing $m / z$ values of 273.1, 301.1 and 317.2 for TMO, DMOX and MOM respectively, which corresponds to the $\mathrm{M}+\mathrm{H}^{+}$ions (calc $\mathrm{m} / z=273.12,301.16$ and 317.15). Since oxetanes undergo hydrolysis in acidic media, their hydrolysis reaction must also be included in reaction mechanism. Considering that NBP, as a pyridine derivative, has a mildly basic nitrogen atom, the reaction mechanism depicted in Scheme 4.10 is proposed.

From Scheme 4.10, the following rate equations are derived:

$$
\begin{gathered}
\frac{d[\mathrm{OX}]}{d t}=-k_{\mathrm{alk}}^{\mathrm{OX}}[\mathrm{OX}][\mathrm{NBP}]_{f}\left[\mathrm{H}^{+}\right]-k_{\mathrm{hyd}}^{\mathrm{OX}}[\mathrm{OX}]\left[\mathrm{H}^{+}\right] \\
\frac{d\left[\mathrm{AD}_{\mathrm{NBP}}^{\mathrm{OX}}\right]}{d t}=k_{\mathrm{alk}}^{\mathrm{OX}}[\mathrm{OX}][\mathrm{NBP}]_{f}\left[\mathrm{H}^{+}\right]
\end{gathered}
$$

$[\mathrm{NBP}]_{f}$ stands for the concentration of free, unprotonated, NBP. If $[\mathrm{NBP}]_{t}$ is the total concentration of NBP and $K_{\mathrm{a}}^{\mathrm{NBP}}$ is the acid dissociation constant of NBP $\left(10^{-4.40}\right.$ in 7:3 $\left.w / d\right)$ we have that: 


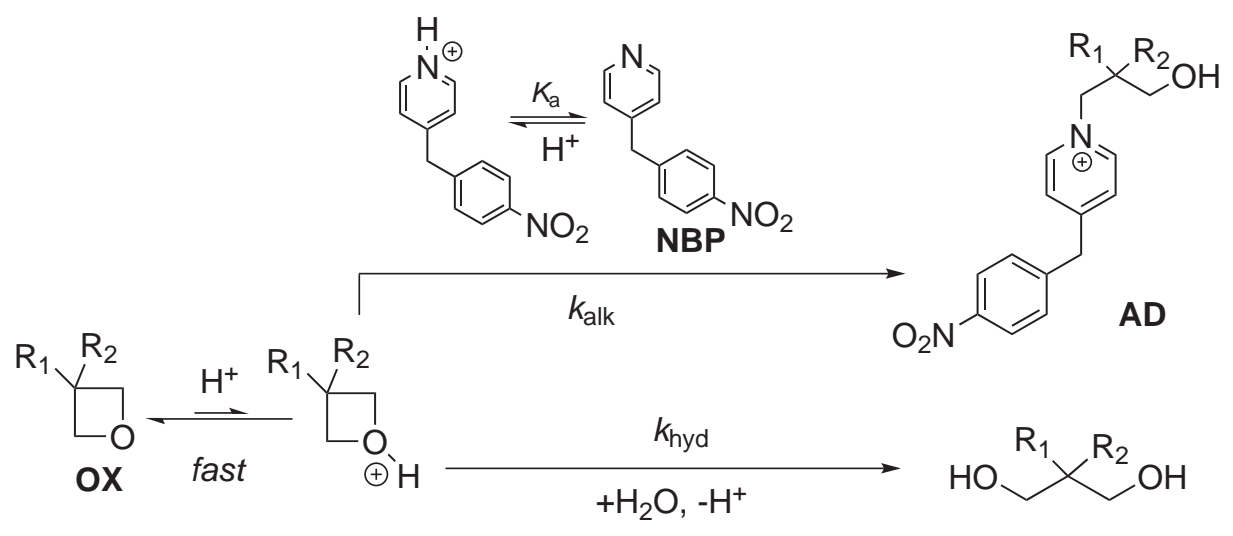

Scheme 4.10. Alkylation of NBP by oxetanes and concurrent hydrolysis

$$
[\mathrm{NBP}]_{f}=\frac{K_{\mathrm{a}}^{\mathrm{NBP}}}{\left[\mathrm{H}^{+}\right]+K_{\mathrm{a}}^{\mathrm{NBP}}}[\mathrm{NBP}]_{t}
$$

NBP was in a large excess with respect to the oxetanes, so that its concentration was assumed to remain constant during the reaction. Applying the pseudofirst-order approximation, we can define the experimental alkylation rate consntat $\left(k_{\text {alkexp }}^{\mathrm{OX}}\right)$

$$
k_{\text {alkexp }}^{\mathrm{OX}}=k_{\mathrm{alk}}^{\mathrm{OX}} \frac{K_{\mathrm{a}}^{\mathrm{NBP}}}{\left[\mathrm{H}^{+}\right]+K_{\mathrm{a}}^{\mathrm{NBP}}}[\mathrm{NBP}]_{t}\left[\mathrm{H}^{+}\right]
$$

Integration of eqs 4.1 and 4.2 , and use of the definition in eq. 4.4 afford the concentration of adduct along the reaction, which can be expresed in terms of absorbance.

$$
A=\epsilon_{\mathrm{AD}}^{\mathrm{OX}} l[\mathrm{OX}]_{\mathrm{NBP}} \frac{k_{\text {alkexp }}^{\mathrm{OX}}}{k_{\text {alkexp }}^{\mathrm{OX}}+k_{\text {hyd }}^{\mathrm{OX}}\left[\mathrm{H}^{+}\right]}\left[1-\exp \left(\left(-k_{\text {alkexp }}^{\mathrm{OX}}-k_{\mathrm{hyd}}^{\mathrm{OX}}\left[\mathrm{H}^{+}\right]\right) t\right)\right]
$$

$\epsilon_{\mathrm{AD}}^{\mathrm{OX}}$ is is the molar absorption coefficient of the NBP-OX adduct at the wavelength of measure, and $l$ is the optic path of the cuvette $(1.00 \mathrm{~cm})$.

Whereas the alkylation of TMO is moderately slow (with reaction times up to a month in optimal conditions), DMOX and MOM react with NBP very slowly (with reaction times of several months). Accordingly, the integral method could only be applied to TMO. DMOX and MOM were studied using the initial rate method ${ }^{108}$ (IRM) (vide infra).

\subsubsection{Integral method}

Figure $4.1 \mathrm{~b}$ shows the excellent fit of the experimental measurements of absorbance to eq 4.5. From such fits, the global rate constant $k_{\mathrm{obs}}^{\mathrm{TMO}}$, which is the sum of the alkylation and hydrolysis experimental rate 
constants, was obtained (4.6).

$$
k_{\mathrm{obs}}^{\mathrm{TMO}}=k_{\mathrm{alkexp}}^{\mathrm{TMO}}+k_{\text {hyd }}^{\mathrm{TMO}}\left[\mathrm{H}^{+}\right]
$$

If $k_{\mathrm{obs}}^{\mathrm{TMO}}$ values obtained at constant $\mathrm{pH}$ are plotted against their respective NBP concentrations (Figure $4.2 \mathrm{a}), k_{\text {hyd }}^{\mathrm{TMO}}$ and $k_{\text {alkexp }}^{\mathrm{TMO}}$ values can be obtained from the intercept and slope, respectively.
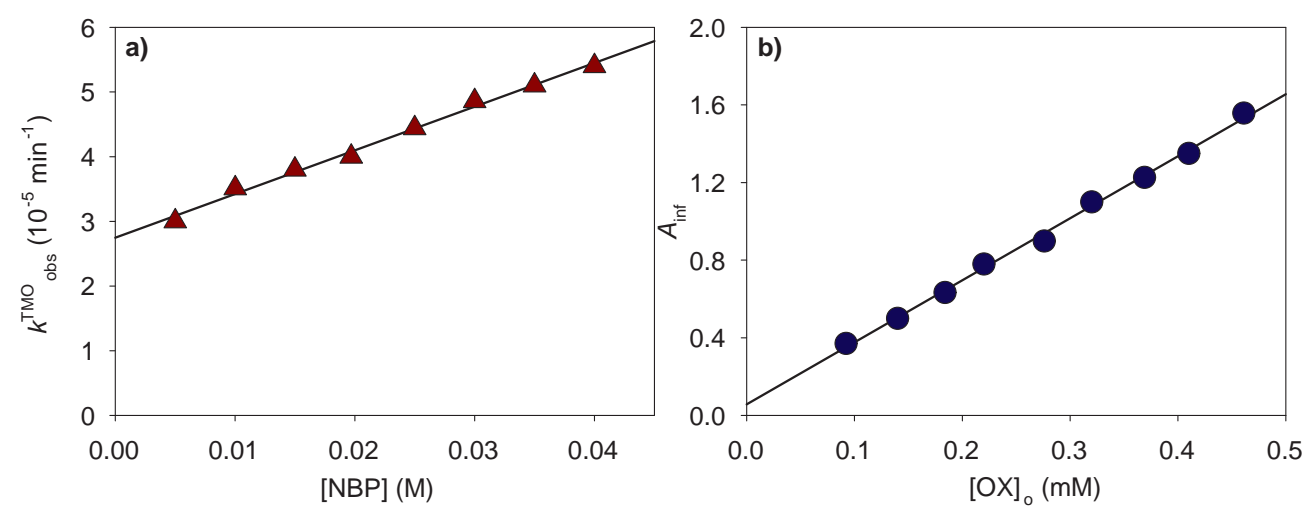

Figure 4.2. a) Variation in $k_{\mathrm{Obs}}^{\mathrm{TMO}}$ with $\left.[\mathrm{NBP}] .(\mathrm{TMO})=5.0 \times 10^{-4} \mathrm{M} . \mathrm{b}\right)$ Variation in $A_{\infty}$ with $[\mathrm{TMO}]_{0},(\mathrm{NBP})=0.040 \mathrm{M}$. $\mathrm{pH}=3.80, T=37.5^{\circ} \mathrm{C}, 7: 3 \mathrm{w} / \mathrm{d}$.

The value obtained at $37.5^{\circ} \mathrm{C}$ in $7: 3 w / d$ medium is: $k_{\text {hyd }}^{\mathrm{TMO}}=2.7 \times 10^{-3} \mathrm{M}^{-1} \mathrm{~s}^{-1} \cdot k_{\text {alk }}^{\mathrm{TMO}}$ values are reported below together with values obtained with the Initial Rate Method ${ }^{108}$ (Table 4.1).

Knowledge of the hydrolysis and alkylation constants allow calculation of the molar absorption coefficient of the adduct, by plotting the absorbance value once the plateau is reached $\left(A_{\infty}\right)$ against TMO concentration (Figure $4.2 \mathrm{~b}$ ), and setting $t=\infty$ in eq. 4.5 to correct for the fraction of hydrolyzed TMO (eq. 4.7). The obtained value is $\epsilon_{\mathrm{AD}_{\mathrm{NBP}}^{\mathrm{TMO}}}^{\mathrm{TMO}}=4.02 \times 10^{-3} \mathrm{M}^{-1} \mathrm{~cm}^{-1}$.

$$
A_{\infty}=\epsilon_{\mathrm{AD}}^{\mathrm{TMO}} l \frac{k_{\mathrm{N} P}^{\mathrm{TMO}} l}{k_{\text {alkexp }}^{\mathrm{TMO}}+k_{\text {hyd }}^{\mathrm{TMO}}\left[\mathrm{H}^{+}\right]}[\mathrm{TMO}]_{\circ}
$$

\subsubsection{Initial Rate Method}

The alkylation reactions of NBP by DMOX and MOM are extremely slow, and could only be monitored by making use of the Initial Rate Method ${ }^{108}$ (IRM) (eq. 4.8)

$$
v_{\circ}=\epsilon_{\mathrm{AD}}^{\mathrm{OX}} l k_{\mathrm{NBP}}^{\mathrm{OX}}[\mathrm{OX}]
$$

Since the alkylation reactions by DMOX and TMO could not reach a plateau in feasible times, the molar absorption coefficients for the NBP-DMOX and NBP-TMO adducts could not be calculated. Lack- 
ing these experimental values, $\epsilon_{\mathrm{AD}}^{\mathrm{TMO}}$ used for all three compounds. This assumption is based on the fact that substituents without significant electronic or conjugative effects have little influence on the molar absorption coefficient. For instance, increasingly substituted 1,3-butadiene, isoprene, and 2,4hexadiene show molar absorption values differing by less than $10 \% .{ }^{109}$ It is known that alkyl 4 - $(p$ nitrobenzyl)pyridonium adducts lacking conjugated double bonds show very similar absorption coefficients, such as the adducts formed by acrylamide ${ }^{110}$ or $\beta$-lactones. ${ }^{111}$

Comparison of the rate constants obtained from the IRM and from the integral method for TMO showed that the two were equal within experimental uncertainty. TMO, DMOX and MOM were tested to fulfill eq. 4.8, that is to be order one in the oxetane and in NBP, and to depend on $\mathrm{pH}$ as described by eq 4.4 .

Table 4.1. Rate constants of NBP alkylation by TMO, DMOX and MOM as a function of temperature

\begin{tabular}{cccc}
\hline & \multicolumn{3}{c}{$k_{\text {alk }}^{\mathrm{OX}}\left(\mathrm{M}^{-2} \mathrm{~min}^{-1}\right)$} \\
\cline { 2 - 4 } $\mathrm{T}\left({ }^{\circ} \mathrm{C}\right)$ & \multicolumn{1}{c}{$\mathrm{TMO}$} & $\mathrm{DMOX}$ & $\mathrm{MOM}$ \\
\hline 20.0 & $3.4 \pm 0.1$ & $0.52 \pm 0.02$ & - \\
22.5 & $4.3 \pm 0.1$ & $0.68 \pm 0.02$ & - \\
25.0 & $5.8 \pm 0.2$ & $0.92 \pm 0.03$ & $0.16 \pm 0.02$ \\
27.5 & $7.6 \pm 0.3$ & $1.23 \pm 0.05$ & $0.21 \pm 0.03$ \\
30.0 & $10.8 \pm 0.5$ & $1.62 \pm 0.07$ & $0.29 \pm 0.03$ \\
32.5 & $14.9 \pm 0.5$ & $2.12 \pm 0.09$ & $0.37 \pm 0.05$ \\
35.0 & $17.8 \pm 0.8$ & $3.18 \pm 0.12$ & $0.46 \pm 0.04$ \\
37.5 & $23.1 \pm 0.9$ & $4.07 \pm 0.15$ & $0.76 \pm 0.06$ \\
\hline
\end{tabular}

The obtained values for $k_{\text {alk }}^{\mathrm{OX}}$ are reported in Table 4.1, and activation energies obtained from the fit of the rate constants to the Arrhenius equation are given in Table 4.2.

Table 4.2. Activation energies for the alkylation of NBP by some oxetanes

\begin{tabular}{ccccc}
\hline Oxetane & & TMO & DMOX & MOM \\
\cline { 1 - 3 } \cline { 5 - 6 }$E_{\mathrm{a}}\left(\mathrm{kJ} \mathrm{mol}^{-1}\right)$ & & $85 \pm 2$ & $90 \pm 2$ & $93 \pm 4$ \\
\hline
\end{tabular}

The fact that the alkylation reaction takes place only at acidic $\mathrm{pH}$ values is consistent with the reactivity of ethers in general and oxetanes in particular. For example, trimethylene oxide undergoes hydrolysis only in strongly acidic media with a rate constant of $1.6 \times 10^{-3} \mathrm{M}^{-1} \mathrm{~s}^{-1}$ at $25^{\circ} \mathrm{C}$ in $6: 4 \mathrm{w} / \mathrm{d}^{2}$, which is in reasonable agreement with our value $\left(2.7 \times 10^{-3} \mathrm{M}^{-1} \mathrm{~s}^{-1}\right.$ at $37.5^{\circ} \mathrm{C}$ in $\left.7: 3 \mathrm{w} / \mathrm{d}\right)$. Some discrepancy arises because our value was obtained at a higher temperature in a more polar reaction medium.

The half-reaction times for the alkylation reaction, even under the most favorable conditions, extend up to one month in the case of TMO and are 10- to 40-fold longer in the cases of DMOX and MOM 
(and were therefore prohibitively time-consuming for study by the integral method). The high values of the activation energies obtained, especially in the case of DMOX and MOM, indicate that a significant energy barrier exists in the alkylation reaction, which is coherent with the generally low reactivity of these ethers. ${ }^{1}$ These lower rate constants and activation energies are consistent with the steric effect of the substituents at position 3; indeed, both compounds are neopenyl-like and thus show very limited reactivity in $S_{N} 2$ reactions

At the neutral $\mathrm{pH}$ of the cellular medium, these alkylation reactions can be considered to be nonexistent or, at least, insignificant.

It would still be possible that the metabolism of oxetanes ultimately yields the effective alkylating agents, as occurs with many compounds. The mutagenicity and genotoxicity of oxetanes under simulated hepatic activation, as measured in a series of biological tests, will be addressed in the next section.

\subsubsection{Mutagenicity, genotoxicity and toxicity of oxetanes}

\subsubsection{Ames Salmonella/Microsome Assay}

The three oxetanes were tested at concentrations ranging from 1.5 to $500 \mu \mathrm{g} /$ plate spaced at half-logarithm intervals. The results using strains TA98 and TA100 with and without S9 mix are shown in Figure 4.3. At all doses tested, the number of revertants in the two strains was not significantly different from the negative control, and no dose-response relationship was observed. This was the case both when using microsomal activation and when not.

\subsubsection{BTC E. coli Test}

The results obtained with the BTC assay are shown in Figure 4.3. At all doses tested, the number of revertants in the two strains was not significantly different from the negative control. Also, there was a lack of any dose-response relationship. This suggests that neither TMO, DMOX, nor MOM were activated by CYP2E1 or CYP2A6 (in the presence or absence of cytochrome $b_{5}$ ) to form mutagens.

The three compounds also tested negative in the BTC-CYP E. coli test, using strains expressing human cytochromes CYP2E1 and CYP2A6, ${ }^{103}$ both of which are known to be involved in the activation of xenobiotics. Since oxetanes are small and rather polar molecules, CYP2E1 would be a likely candidate involved in their biotransformation. ${ }^{112}$ While the coexpression of cytochrome b5 increases the bioactivation of some alkylating compounds by CYP2A6 and 2E1, 104,113 the results were equally negative when the strains BTC-2A6-b5-POR or BTC-2E1-b5-POR were applied. 

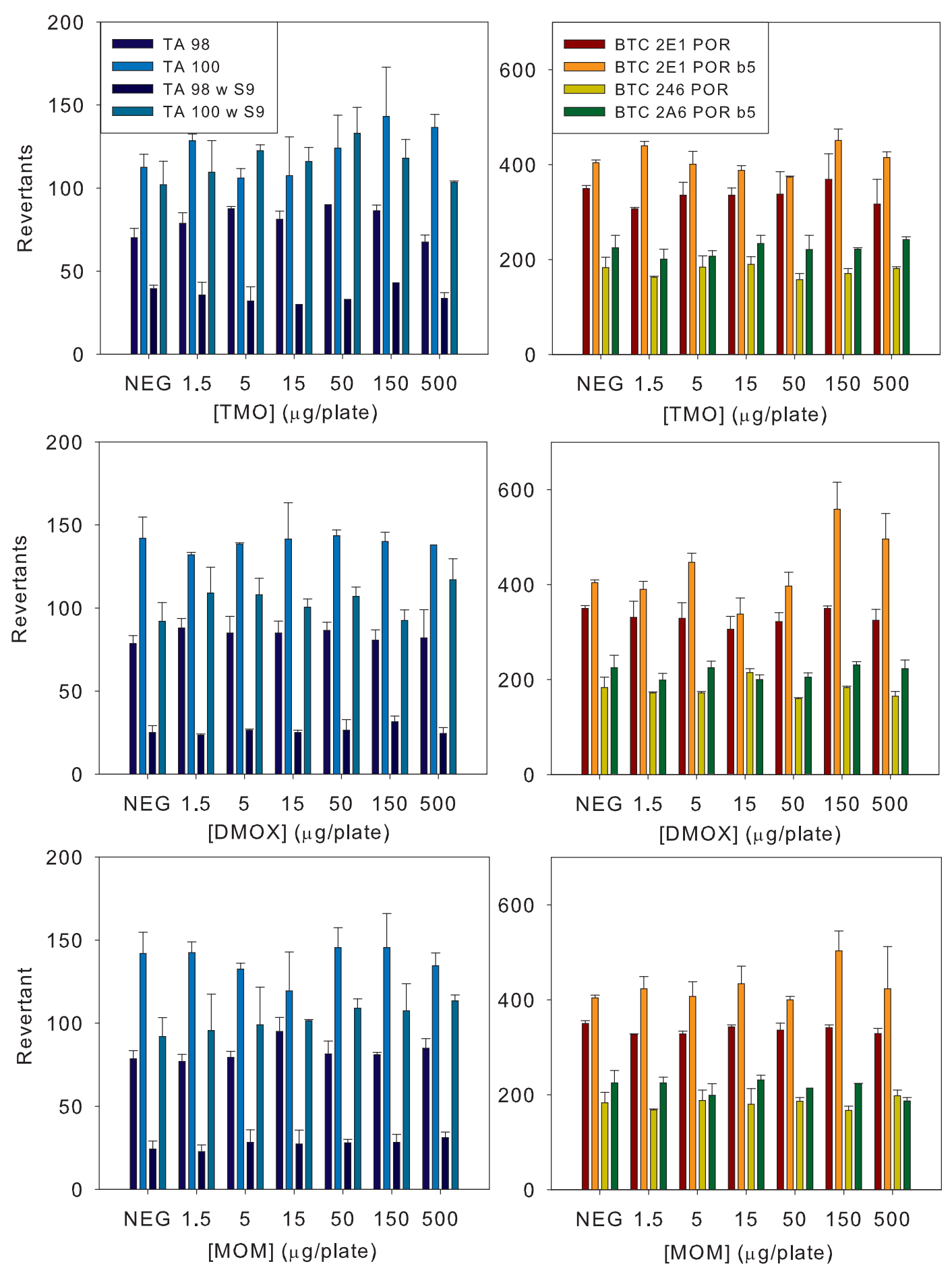

Figure 4.3. Revertants per plate in the Salmonella and BTC E. coli tests 


\subsubsection{Alkaline Comet Assay}

The results, measured as percentage of DNA in tail or as tail moment, are shown in Figure 4.4. For the three compounds, the results were not significantly different from the negative control or the S9 activation mix. This suggests that TMO, DMOX, and MOM lack the potential to produce DNA strand breaks.

The three model oxetanes demonstrated no DNA strand breaks in the comet assay, using Chinese hamster cells, either in the presence or absence of the S9 mix. This inability to form strand breaks points further in the direction of oxetanes and their oxidation metabolites having very limited or no genotoxic effects.
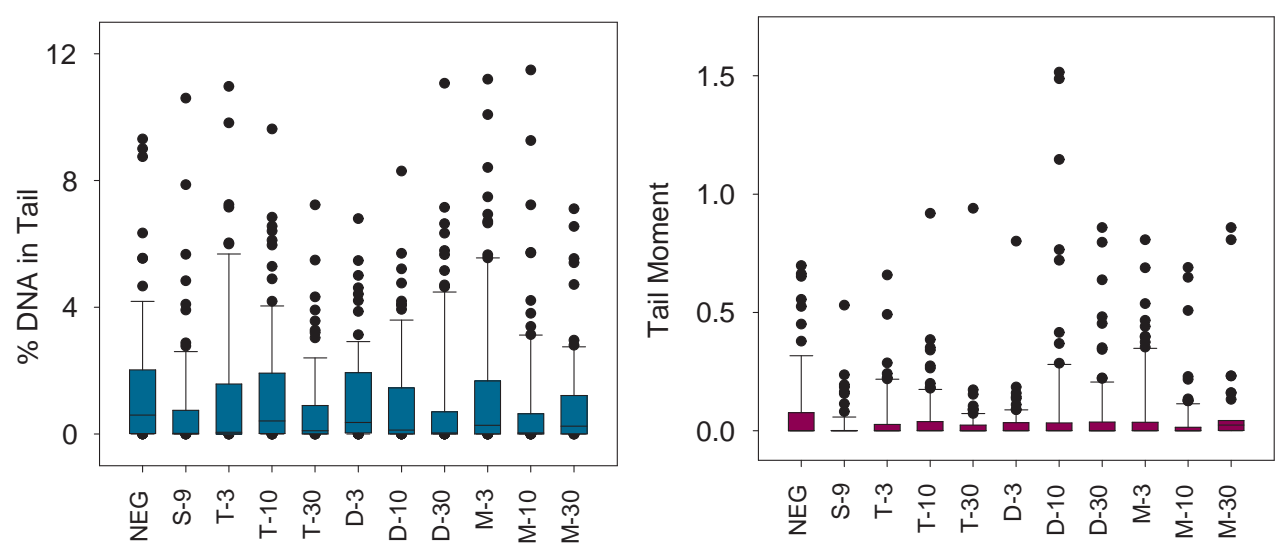

Figure 4.4. Results of the Comet assay (T, TMO; D, DMOX; M, MOM) $[\mathrm{OX}]=10$ and $30 \mathrm{mM}$ with and w/O the S9 activation mix.

These negative results could cast certain doubts on previous positive results obtained in animal tests, which have been called equivocal.3 For instance, those results may have been influenced by the presence of minor impurities (the boiling point referred to by Druckrey et al. ${ }^{99}$ differs significatively from the one given in the literature) that became significant at high dose usage or perhaps may have been due to the acute toxicity of oxetanes, which, followed by compensatory cell proliferation, could increase the likelihood of tumor production (mitogenesis).

\subsubsection{Cell viability: MTT reduction assay}

The three oxetanes were tested at concentrations ranging from 0.1 to $30 \mathrm{mM}$, spaced at half-logarithm concentration intervals; the results are shown in Figure 4.5. At all doses tested, the ratio of survival was not significantly different from that of the negative control, either in the presence or in the absence of microsomal activation. In addition, there was a complete lack of any dose-response relationship, which suggests a very low toxicity of the three oxetanes or their metabolites at below the highest concentrations tested. 
This negative result seems to indicate that oxetanes and their oxidation metabolites are very sparingly toxic for mammalian cells, which is consistent with the $\mathrm{LD}_{50}$ value reported for DB-rats: $500 \mathrm{mg} / \mathrm{kg} .{ }^{99}$ This suggests that previous positive results in animal test systems were possibly not due to the toxicity of the compounds and subsequent cell proliferation but rather to some other factor, such as the presence of impurities.
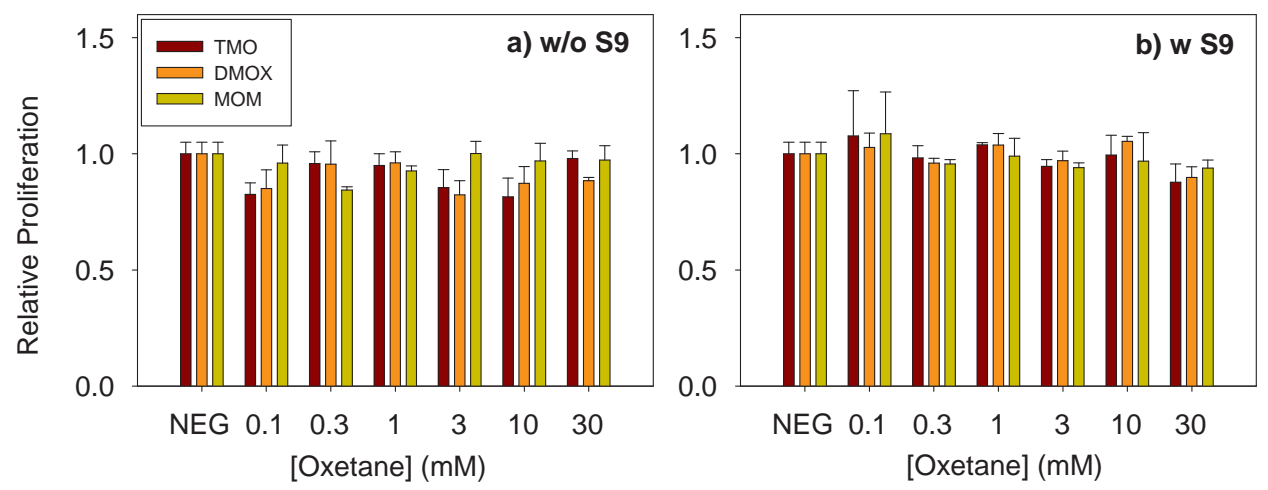

Figure 4.5. Relative proliferation as measured in the MTT Assay, in absence (a) or presence (b) of the S9 mixture

\subsection{Conclusions}

1. From the combined kinetic data and the biological results obtained in the absence of microsomal activaction, it can be safely assumed that oxetanes are not direct alkylating agents at physiological $\mathrm{pH}$.

2. The lack of mutagenicity in Salmonella and E. coli; genotoxicity as measured in the alkaline comet assay, and cytotoxicity to mammalian cells in the presence of the S9 mix and human cytochromes CYP2E1 and CYP2A6, with or without coexpression of cytochrome $b_{5}$, may also indicate that oxetanes are not activated to alkylating agents or other direct-acting mutagens in vivo.

3. The apparent contradiction between our results and previous animal tests suggests either that (i) oxetanes are extremely weak genotoxins or nongenotoxic and that previous positive results would have been due to spurious factors such as the use of unrealistically high doses or the presence of impurities or that (ii) their carcinogenic effect is exerted by mechanisms completely different from those of similar compounds, such as oxiranes and $\beta$-lactones, which do cause direct DNA damage. 


\section{References}

[1] March, J. Advanced Organic Chemistry. Reactions, Mechanisms and Structure, 4th ed.; John Wiley: New York, 1992.

[2] Pritchard, J. G.; Long, F. A. J. Am. Chem. Soc. 1958, 80, 4162-4165.

[3] Searles, S.; Gregory, V. P. J. Am. Chem. Soc. 1954, 76, 2789-2790.

[4] Miller, R. R. Drug Metab. Rev. 1987, 18, 1-22.

[5] Foster, P. M. D.; Creasy, D. M.; Foster, J. R.; Thomas, L. V.; Cook, M. W.; Gangolli, S. D. Toxicol. Appl. Pharmacol. 1983, 69, 385-399.

[6] Correa, A.; Gray, R. H.; Cohen, R.; Rothman, N.; Shah, F.; Seacat, H.; Corn, M. Am. J. Epidemiol. 1996, 143, 707-717.

[7] Johnson, W.; Cosmetic Ingredient Review, E. Int. J. Toxicol. 2002, 21, 9-62.

[8] Ciccioli, P.; Brancaleoni, E.; Cecinato, A.; Sparapani, R.; Frattoni, M. J. Chromatogr. 1993, 643, 5569.

[9] Union, E. European Union Risk Assessment Report - 2-Butoxyethanol (Draft Report),; 2004.

[10] Canada, E. C. Priority Substances List Assessment Report: 2-Butoxyethanol, Quebec/Montreal; 2002.

[11] IARC, In Formaldehyde, 2-Butoxyethanol and 1-tert-Butoxypropan-2-ol; Lyon,, Ed.; IARC Monographs on the Evaluation of Carcinogenic Risks to Human; IARC Monograph 88, IARC, 2006; Vol. 88; pp 328-414.

[12] Yasuhara, A.; Shiraishi, H.; Tsuji, M.; Okuno, T. Environ. Sci. Technol. 1981, 15, 570-573.

[13] Ghanayem, B. I.; Burka, L. T.; Matthews, H. B. J. Pharmacol. Exp. Ther. 1987, 242, 222-231.

[14] Aasmoe, L.; Winberg, J.-O.; Aarbakke, J. Toxicol. Appl. Pharmacol. 1998, 150, 86-90.

[15] Gross, A.; Ong, T. R.; Grant, R.; Hoffmann, T.; Gregory, D. D.; Sreerama, L. Chem.-Biol. Interact. 2008, In Press, doi 10.1016/j.cbi.2008.09.025.

[16] Sabourin, P. J.; Medinsky, M. A.; Birnbaum, L. S.; Griffith, W. C.; Henderson, R. F. Toxicol. Appl. Pharmacol. 1992, 114, 232238.

[17] Program, N. T. Toxicology and Carcinogenesis Studies of 2-Butoxyethanol (CAS No. 111-76-2) in F344/N Rats and B6C3F1 Mice (Inhalation Studies); 2000.

[18] Zeiger, E.; Anderson, B.; Haworth, S.; Lawlor, T.; Mortelmans, K. Environ. Mol. Mutagen. 1992, 19 Suppl 21, 2141.

[19] Hoflack, J. C.; Lambolez, L.; Elias, Z.; Vasseur, P. Mutat. Res. 1995, 341, 281287.

[20] Gollapudi, B. B.; Barber, E. D.; Lawlor, T. E.; Lewis, S. A. Mutat. Res. 1996, 370, 6164.

[21] Park, J.; Kamendulis, L.; Klaunig, J. Toxicol. Lett. 2002, 126, 19-29.

[22] Program, N. T. Toxicity Studies of Ethylene Glycol Ethers 2-Methoxyethanol,2-Ethoxyethanol, 2Butoxyethanol (CAS Nos. 109-86-4, 110-80-5, 111-76-2) Administered in Drinking Water to F344/N Rats and B6C3F1 Mice; 1993. 
[23] Elias, Z.; Danière, M.; Marande, A.; Poirot, O.; Terzetti, F.; Schneider, O. Occup. Hyg. 1996, 2, $187-212$.

[24] Park, J.; Kamendulis, L.; Klaunig, J. Toxicol. Sci. 2002, 68, 43-50.

[25] Corthals, S. M.; Kamendulis, L. M.; Klaunig, J. E. Toxicol. Sci. 2006, 92, 378-386.

[26] Elliott, B. M.; Ashby, J. Mutat. Res.-Rev. Mutat. 1997, 387, 89 - 96.

[27] Green, T.; Toghill, A.; Lee, R.; Moore, R.; Foster, J. Toxicology 2002, 180, 257273.

[28] Poet, T. S.; Soelberg, J. J.; Weitz, K. K.; Mast, T. J.; Miller, R. A.; Thrall, B. D.; Corley, R. A. Toxicol. Sci. 2003, 71, 176189.

[29] IARC, In Formaldehyde, 2-Butoxyethanol and 1-tert-Butoxypropan-2-ol; Lyon,, Ed.; IARC Monographs on the Evaluation of Carcinogenic Risks to Human; IARC Monograph 88, IARC, 2006; Vol. 88; pp 416-438.

[30] Program, N. T. Chemical Disposition in Mammals: The Metabolism and Disposition of Propylene Glycol t-Butyl Ether in the Male Fischer 344 Rat; 1994.

[31] Program, N. T. NTP Technical Report on the Toxicology and Carcinogenesis Studies of Propylene Glycol Mono-t-Butyl Ether (CAS No. 57018-52-7) in F344/N Rats and B6C3F1 Mice and a Toxicology Study of Propylene Glycol Mono-t-Butyl Ether in Male NBR Rats (Inhalation Studies); 2004.

[32] Dill, J.; Fuciarelli, A.; Lee, K.; Mellinger, K.; Burka, L.; Roycroft, J. Inhal. Toxicol. 2004, 16, 271-290.

[33] Doi, A. M.; Roycroft, J. H.; Herbert, R. A.; Haseman, J. K.; Hailey, J. R.; Chou, B. J.; Dill, J. A.; Grumbein, S. L.; Miller, R. A.; Renne, R. A.; Bucher, J. R. Toxicology 2004, 199, 122.

[34] Borghoff, S. J.; Prescott, J. S.; Janszen, D. B.; Wong, B. A.; Everitt, J. I. Toxicol. Sci. 2001, 61, 176186.

[35] Williams, T. M.; Borghoff, S. J. Toxicol. Sci. 2001, 62, 228235.

[36] Scholz, B.; Butzert, H.; Neumeister, J.; Nierlich, F. In Elvers, B., Ullmann's Encyclopedia of Industrial Chemistry, 5th rev .Ed. Vol. A16; Hawkins, S., Schulz, G., Eds.; New York, VCH Publishers, 1990; Chapter Methyl tert-butyl ether, p 543-550.

[37] Piel, W. Historical perspective on the use of ethers in fuels. 1995.

[38] Stern, B. R.; Kneiss, J. J. J. Appl. Toxicol. 1997, 17 Suppl 1, S1S2.

[39] Kirschner, E. Chem. Eng. News 1996, 74, 16-20.

[40] Zogorski, J.; Morduchowitz, A.; Baehr, A.; Bauman, B.; Dwayne, L.; Drew, R.; Korte, N.; Lapham, W.; Pankow, J.; Washington, E. Fuel Oxygenates and Water Quality: Current Understanding of Sources, Occurrence in Natural Waters, Environmental Behavior, Fate and Significance; 1996.

[41] Squillace, P.; Pankow, J.; Korte, N.; Zogorski, J. Environ. Toxicol. Chem. 1997, 16, 1836-1844.

[42] Agency, E. P. Chemical Summary for Methyl-tert-Butyl Ether (EPA-749/F-94-017a); 1994.

[43] Brown, S. L. Regul Toxicol Pharmacol 1997, 25, 256276.

[44] WHO, Methyl tertiary-Butyl Ether (Environmental Health Criteria 206); 1998.

[45] IARC, In Some Chemicals that Cause Tumours of the Kidney or Urinary Bladder in Rodents and Some Other Substances; Lyon,, Ed.; IARC Monographs on the Evaluation of Carcinogenic Risks to Human; IARC Monograph 73, IARC, 1999; Vol. 88; pp 328-414. 
[46] Davidson, J. Fate and transport of MTBE: The latest data. 1996.

[47] Belpoggi, F.; Soffritti, M.; Filippini, F.; Maltoni, C. Ann. NY Acad. Sci. 1997, 837, 7795.

[48] Belpoggi, F.; Soffritti, M.; Maltoni, C. Toxicol Ind Health 1995, 11, 119149.

[49] Bird, M. G.; Burleigh-Flayer, H. D.; Chun, J. S.; Douglas, J. F.; Kneiss, J. J.; Andrews, L. S. J. Appl. Toxicol. 1997, 17 Suppl 1, S45S55.

[50] Mennear, J. H. Risk Anal 1997, 17, 673681.

[51] Cirvello, J. D.; Radovsky, A.; Heath, J. E.; Farnell, D. R.; Lindamood, C., 3rd Toxicol Ind Health 1995, $11,151165$.

[52] Mackerer, C. R.; Angelosanto, F. A.; Blackburn, G. R.; Schreiner, C. A. Proc Soc Exp Biol Med 1996, 212,338341 .

[53] Kado, N. Y.; Kuzmicky, P. A.; Loarca-Piña, G.; Moiz Mumtaz, M. Mutat. Res. 1998, 412, 131138.

[54] McKee, R. H.; Vergnes, J. S.; Galvin, J. B.; Douglas, J. F.; Kneiss, J. J.; Andrews, L. S. J. Appl. Toxicol. 1997, 17 Suppl 1, S31S36.

[55] Chen, C. S.; Hseu, Y. C.; Liang, S. H.; Kuo, J. Y.; Chen, S. C. J. Hazard. Mater. 2008, 153, 351-356.

[56] IARC, In IARC Monographs on the Evaluation of the Carcinogenic Risk of Chemicals to Man, Vol. 11, Cadmium, Nickel, Some Epoxides, Miscellaneous Industrial Chemicals and General Considerations on Volatile Anaesthetics; Lyon,, Ed.; IARC Monograph 11, IARC, 1976.

[57] IARC, Re-Evaluation of Some Organic Chemicals, Hydrazine and Hydrogen Peroxide; IARC Monograph 71, IARC: Lyon, France, 1999; pp 591-601.

[58] Maronpot, R. R.; Shimkin, M. B.; Witschi, H. P.; Smith, L. H.; Cline, J. M. J. Natl. Cancer Inst. 1986, 76,11011112 .

[59] Stoner, G. D.; Conran, P. B.; Greisiger, E. A.; Stober, J.; Morgan, M.; Pereira, M. A. Toxicol. Appl. Pharmacol. 1986, 82, 1931.

[60] Kano, H.; Umeda, Y.; Kasai, T.; Sasaki, T.; Matsumoto, M.; Yamazaki, K.; Nagano, K.; Arito, H.; Fukushima, S. Food Chem. Toxicol. 2009, 47, 2776-2784.

[61] Lundberg, I.; Högberg, J.; Kronevi, T.; Holmberg, B. Cancer Lett. 1987, 36, 2933.

[62] Stott, W. T.; Quast, J. F.; Watanabe, P. G. Toxicol. Appl. Pharmacol. 1981, 60, 287300.

[63] Haworth, S.; Lawlor, T.; Mortelmans, K.; Speck, W.; Zeiger, E. Environ. Mutagen. 1983, 5 Suppl 1, $11-42$.

[64] Khudoley, V. V.; Mizgireuv, I.; Pliss, G. B. Arch. Geschwulstforsch. 1987, 57, 453462.

[65] Hellmér, L.; Bolcsfoldi, G. Mutat. Res. 1992, 272, 145160.

[66] Yoon, J. S.; Mason, J. M.; Valencia, R.; Woodruff, R. C.; Zimmering, S. Environ. Mutagen. 1985, 7, 349-367.

[67] Galloway, S. M.; Armstrong, M. J.; Reuben, C.; Colman, S.; Brown, B.; Cannon, C.; Bloom, A. D.; Nakamura, F.; Ahmed, M.; Duk, S. Environ. Mol. Mutagen. 1987, 10 Suppl 10, 1175.

[68] Sheu, C. W.; Moreland, F. M.; Lee, J. K.; Dunkel, V. C. Environ. Mol. Mutagen. 1988, 11, 4148. 
[69] Sina, J. F.; Bean, C. L.; Dysart, G. R.; Taylor, V. I.; Bradley, M. O. Mutat. Res. 1983, 113, 357391.

[70] Goldsworthy, T. L.; Monticello, T. M.; Morgan, K. T.; Bermudez, E.; Wilson, D. M.; Jäckh, R.; Butterworth, B. E. Arch. Toxicol. 1991, 65, 19.

[71] McFee, A. F.; Abbott, M. G.; Gulati, D. K.; Shelby, M. D. Mutat. Res. 1994, 322, 145148.

[72] Mirkova, E. T. Mutat. Res. 1994, 322, 142144.

[73] Tinwell, H.; Ashby, J. Mutat. Res. 1994, 322, 148150.

[74] Stickney, J. A.; Sager, S. L.; Clarkson, J. R.; Smith, L. A.; Locey, B. J.; Bock, M. J.; Hartung, R.; Olp, S. F. regulatory Toxicology and Pharmacology 2003, 38, 183-195.

[75] IARC, In Dry Cleaning, Some Chlorinated Solvents and Other Industrial Chemicals; Lyon,, Ed.; IARC Monograph 63, IARC, 1995.

[76] Elder, V. A.; Proctor, B. L.; Hites, R. A. Biomed. Mass. Spectrom. 1981, 8, 409-415.

[77] Howard, P., Sage, G., Jarvis, W., Gray, D., Eds. Handbook of Environmental Fate andExposure Datafor Organic Chemicals, Vol. Il, Solvents; Chelsea, MI, Lewis Publishers, 1990.

[78] Peterson, L. A.; Phillips, M. B.; Lu, D.; Sullivan, M. M. Chem. Res. Toxicol. 2011,

[79] Program, U. S. N. T. Toxicology and Carcinogenesis Studies of Furan (CAS No. 110-00-9) in F344/N Rats and B6C3FI Mice (Gavage Studies); 1993.

[80] Foureman, P.; Mason, J. M.; Valencia, R.; Zimmering, S. Environ. Mol. Mutagen. 1994, 23, 51-63.

[81] Burkhard, J. A.; Wuitschik, G.; Rogers-Evans, M.; Müller, K.; Carreira, E. M. Angew. Chem., Int. Ed. 2010, 49, 90529067.

[82] Eigenmann, H. K.; Golden, D. M.; Benson, S. W. J. Phys. Chem. 1973, 77, 1687-1691.

[83] Wolk, J. L.; Sprecher, M.; Basch, H.; Hoz, S. Org. Biomol. Chem. 2004, 2, 1065-1069.

[84] Sawicka, D.; Houk, K. N. Journal of Molecular Modeling 2000, 6, 158-165.

[85] Moussa, K.; Decker, C. Journal of Polymer Science Part A: Polymer Chemistry 1993, 31, 2197-2203.

[86] Yan, D.; Hou, J.; Zhu, X.; Kosman, J.; Wu, H. Macromol. Rapid Commun. 2000, 21, 557-561.

[87] Bayerl, M.; Braig, T.; Nuyken, O.; Muller, D.; Gross, M.; Meerholz, K. Macromol. Rapid Commun. 1999, 20, 224-228.

[88] Muller, C.; Falcou, A.; Reckefuss, N.; Rojahn, M.; Wiederhirn, V.; Rudati, P.; Frohne, H.; Nuyken, O.; Becker, H.; Meerholz, K. Nature 2003, 421, 829-833.

[89] Yang, X.; Muller, D.; Neher, D.; Meerholz, K. Adv. Mater. 2006, 18, 948-954.

[90] Rademann, J.; Grotli, M.; Meldal, M.; Bock, K. J. Am. Chem. Soc. 1999, 121, 5459-5466.

[91] Wuitschik, G.; Rogers-Evans, M.; Müller, K.; Fischer, H.; Wagner, B.; Schuler, F.; Polonchuk, L.; Carreira, E. M. Angew. Chem., Int. Ed. 2006, 45, 77367739.

[92] Wuitschik, G.; Rogers-Evans, M.; Buckl, A.; Bernasconi, M.; Märki, M.; Godel, T.; Fischer, H.; Wagner, B.; Parrilla, I.; Schuler, F.; Schneider, J.; Alker, A.; Schweizer, W. B.; Müller, K.; Carreira, E. M. Angew. Chem., Int. Ed. 2008, 47, 45124515. 
[93] Wuitschik, G.; Carreira, E. M.; Wagner, B.; Fischer, H.; Parrilla, I.; Schuler, F.; Rogers-Evans, M.; MuÃàller, K. J. Med. Chem. 2010, 53, 3227-3246.

[94] Sone, T.; Lu, G.; Matsunaga, S.; Shibasaki, M. Angew. Chem., Int. Ed. 2009, 48, 16771680.

[95] Burkhard, J. A.; GueÃÅrot, C.; Knust, H.; Rogers-Evans, M.; Carreira, E. M. Org. Lett. 2010, 12, 1944-1947.

[96] Jung, H. H.; Buesking, A. W.; Ellman, J. A. Org. Lett. 2011, 13, 3912-3915.

[97] Burkhard, J. A.; Tchitchanov, B. H.; Carreira, E. M. Angew. Chem., Int. Ed. 2011, 50, 53795382.

[98] Dejaegher, Y.; Kuz'menok, N. M.; Zvonok, A. M.; De Kimpe, N. Chem. Rev. 2002, 102, 29-60.

[99] Druckrey, H.; Kruse, H.; Preussman, R.; Ivankovic, S.; Landschütz, C. Z. Krebsforsch. 1970, 74, 241-270.

[100] Dickens, F.; Jones, H. E. H. Br. J. Cancer 1963, 17, 100-108.

[101] Mortelmans, K.; Zeiger, E. Mutat. Res. 2000, 455, 29-60.

[102] Kranendonk, M.; Mesquita, P.; Laires, A.; Vermeulen, N. P. E.; Rueff, J. Mutagenesis 1998, 3, $263-$ 269.

[103] Duarte, M. P.; Palma, B. B.; Laires, A.; Oliveira, J. S.; Rueff, J.; Kranendonk, M. Mutagenesis 2005, 20, 199-208.

[104] Duarte, M. P.; Palma, B. B.; Gilep, A. A.; Laires, A.; Oliveira, J. S.; Usanov, S. A.; Rueff, J.; Kranendonk, M. Mutagenesis 2007, 22, 75-81.

[105] Carmichael, J.; DeGraff, W.; Gazdar, A.; Minna, J.; Mitchell, J. Cancer Res. 1987, 47, 936-941.

[106] Wilson, A. P. Animal Cell Culture: A Practical Approach; Oxford University Press: Oxford, 2000; Vol. 3rd.

[107] Martins, C.; Oliveira, N. G.; Pingarilho, M.; da Costa, G. G.; Martins, V.; Marques, M. M.; Beland, F. A.; Churchwell, M. I.; Doerge, D. R.; Rueff, J.; Gaspar, J. F. Toxicol. Sci. 2007, 95, 383-390.

[108] Casado, J.; López-Quintela, M. A.; Lorenzo-Barral, F. M. J. Chem. Ed. 1986, 63, 450.

[109] Rao, C. Ultra-Violet and Visible Spectroscopy; Butterworth, London., 1967; Chapter 4.

[110] Céspedes, I. F.; Manso, J. A.; Pérez-Prior, M. T.; Gómez-Bombarelli, R.; González-Pérez, M.; Calle, E.; Casado, J. J. Phys. Org. Chem. 2010, 23, 171-175.

[111] Manso, J. A.; Pérez-Prior, M. T.; García-Santos, M. P.; Calle, E.; Casado, J. Chem. Res. Toxicol. 2005, $18,1161-1166$.

[112] Guegenrich, F. Cytochrome P450 Structure, Mechanism and Biochemistry; Kluwer, Academic/Plenum Publishers: New York, 2005.

[113] Duarte, M. P.; Palma, B. B.; Gilep, A. A.; Laires, A.; Oliveira, J. S.; Usanov, S. A.; Rueff, J.; Kranendonk, M. Mutagenesis 2005, 20, 93-100. 

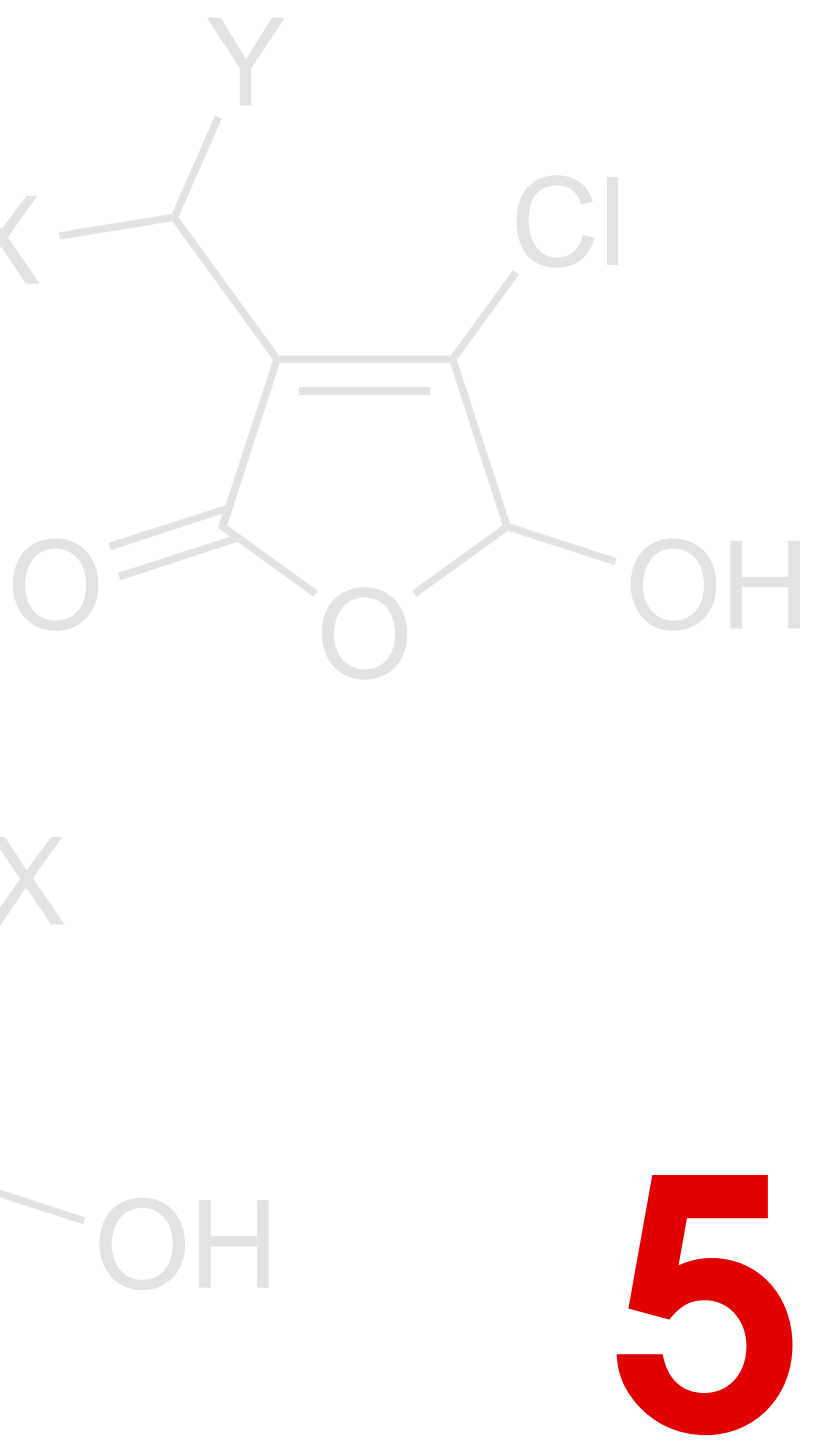

Hydroxyhalofuranones 



\section{Contents}

5.1 Background ........................... 181

5.1.1 Hydroxyhalofuranones as pollutants . . . . . . . . . . . 182

5.1.1.1 Formation . . . . . . . . . . . . . . . . . . 182

5.1 .1 .2 Analysis . . . . . . . . . . . . . . . 183

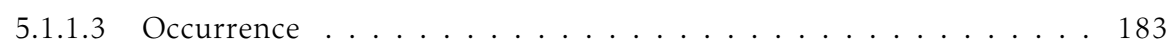

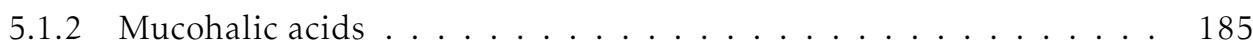

5.1 .2 .1 Reactivity . . . . . . . . . . . . . . . . 186

5.1.2.1.1 Applications in organic synthesis . . . . . . . . . . 187

5.1 .2 .2 Genotoxicity . . . . . . . . . . . . . . . . 188

5.1.2.2.1 Biological assays . . . . . . . . . . . . . 188

5.1.2.2.2 DNA adduct formation . . . . . . . . . . . . 189

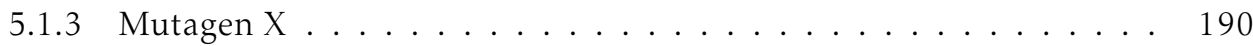

5.1.3.1 Genotoxicity of MXY . . . . . . . . . . . . . . . 191

5.1.3.1.1 Tumorigenicity in animal models . . . . . . . . . . . 192

5.1.3.1.2 Genotoxicity in vitro . . . . . . . . . . . . . . . . . . 192

5.1.3.1.3 Genotoxicity in vivo . . . . . . . . . . . . . . . . . . 195

5.1.3.1.4 DNA adduct formation . . . . . . . . . . . . 196

5.2 Materials \& methods . . . . . . . . . . . . . . . . . . . . . . . 199

5.2.1 Reactivity of halohydroxyhalofuranones . . . . . . . . . . . . 199

5.2.1.1 Calculation of hydration and hemiacetalization constants . . . . . . 199

5.2.1.2 Equilibria of mucohalic acids . . . . . . . . . . . . . 200

5.2.1.2.1 Experimental study . . . . . . . . . . . . . 200

5.2.1.2.2 Theoretical study . . . . . . . . . . . . . . 202

5.2.1.3 Hydrolysis of mucohalic acids . . . . . . . . . . . . . . 203

5.2.1.3.1 Experimental study . . . . . . . . . . . . . 203

5.2.1.4 Reactivity of MXY _. . . . . . . . . . . . . . . . 203

5.2.1.4.1 Equilibrium constants ... . . . . . . . . . 204

5.2.1.4.2 Electron affinities ................ . 205

5.2.1.4.3 Computational details ............... 205

5.2.2 Alkylating potential of halohydroxyfuranones . . . . . . . . 205

5.2.2.1 Alkylating potential of mucohalic acids . . . . . . . . . . . . 205

5.2.2.1.1 Reaction with aniline ... . . . . . . . . 205

5.2.2.1.2 Reaction with NBP . . . . . . . . . . . . . . 206 
5.2.2.1.3 Computational study of nucleobase alkylation . . . . . . . 206

5.2.2.2 Alkylating potential of MXY . . . . . . . . . . . . . 206

5.2.2.2.1 Reaction pathway . . . . . . . . . . . . 206

5.2.2.2.2 Computational details . . . . . . . . . . . . . 206

5.3 Results and discussion . . . . . . . . . . . . . . 207

5.3.1 Reactivity of halohydroxyhalofuranones . . . . . . . . . . . . 207

5.3.1.1 Calculation of hydration and hemiacetalization constants . . . . . . 207

5.3.1.1.1 Hydration constants . . . . . . . . . . . . . . . 207

5.3.1.1.2 Hemiacetalization constants . . . . . . . . . . . . 210

5.3.1.2 Equilibria of mucohalic acids . . . . . . . . . . . . 215

5.3.1.2.1 Experimental study . . . . . . . . . . . . . . . 215

5.3.1.2.2 Theoretical study . . . . . . . . . . . . . . . . 218

5.3.1.3 Hydrolysis of mucohalic acids . . . . . . . . . . . . . . 222

5.3.1.3.1 Kinetics of MXA decomposition ............ 222

5.3.1.3.2 Mechanism of MXA decomposition . . . . . . . . . 226

5.3.1.3.3 Mucoxyhalic acids . . . . . . . . . . . . . . 227

5.3.1.4 Reactivity of MXY . . . . . . . . . . . . . . . . . . . . . 229

5.3.1.4.1 Equilibrium constants . . . . . . . . . . . . . . 229

5.3.1.4.2 Electron affinities . . . . . . . . . . . . . 232

5.3.2 Alkylating potential of halohydroxyfuranones . . . . . . . . . 234

5.3.2.1 Alkylating potential of mucohalic acids . . . . . . . . . . . 234

5.3.2.1.1 Reaction with aniline . . . . . . . . . . . . . 234

5.3.2.1.2 Reaction with NBP . . . . . . . . . . . . . . . . 237

5.3.2.1.3 Computational study of nucleobase alkylation . . . . . . . 241

5.3.2.2 Alkylating potential of MXY . . . . . . . . . . . . . . . . . 245

5.3.2.2.1 Adduct formation . . . . . . . . . . . . . . . . 245

5.3.2.2.2 Unimolecular cleavage . . . . . . . . . . . . . . 249

5.3 .2 .2 .3 Reduction . . . . . . . . . . . . . . . . 250

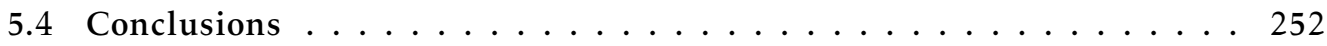

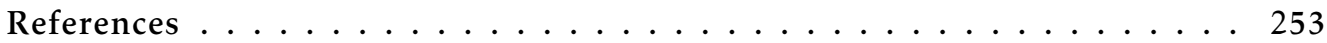




\section{Introduction}

Several studies have shown that chlorinated water is associated with an increased incidence of bladder ${ }^{1}$ and also rectal cancer. ${ }^{2}$ This effect is attributed to the presence of genotoxic Disinfection By-Products (DBPs). These pollutants are formed when disinfectants such as chlorine, ozone, chlorine dioxide or chloramines react with naturally occurring organic matter, anthropogenic contaminants, bromide, or iodide during the production of drinking water. ${ }^{3}$ DBPs include well known toxic contaminants such as halometanes or haloacetic acids.

Give their negative health effects, DBPs have become an important research topic in the last decades and constant work is made to identify new compounds and to assess their potential hazards. ${ }^{4-8}$ Regulation exists regarding the presence of many DBPs in drinking water; however the more recently discovered compounds may take time before the make it into legislation.

A group of DBPs of increasing interest and yet to be regulated are halohydroxyfuranones, which are formed in the chlorination of humic substances (organic matter present in soils), and whose genotoxic and carcinogenic properties are well known. ${ }^{9,10}$ These DBPs are responsible for most of the observed mutagenicity of chlorinated tap water. ${ }^{11-13}$

In this work, the reactivity of two classes of halohydroxyfuranones was addressed (Scheme 5.1): 3,4dihalo-5-hydroxy-2(5H)-furanones, also known as mucohalic acids (MCA and MBA, MXA in general) and three compounds bearing (halo)methylgroups in their 4-position (MCF, CMCF and MX, MXY in general).

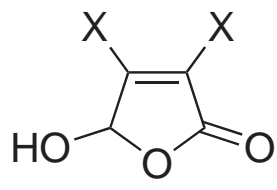

$\begin{array}{ll}\mathrm{X} X \mathrm{Cl} & \mathrm{MCA} \\ \mathrm{X}=\mathrm{Br}: \text { MBA }\end{array}$

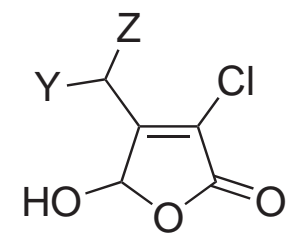

$Y=H, Z=H: M C F$

MXY $Y=\mathrm{Cl}, \mathrm{Z}=\mathrm{H}: \mathbf{C M C F}$

$\mathrm{Y}=\mathrm{Cl}, \mathrm{Z}=\mathrm{Cl}: \mathbf{M X}$

Scheme 5.1. Studied hydroxyhalofuranones

\subsection{Background}

Despite their name, hydroxyhalofuranones only exist as the furanone structure in organic or acidic aqueous medium, where a minor amount of open-chain aldehyde-acid coexists in equilibrium with the closedchain form. In neutral or basic medium hydroxyhalofuranones exist mostly as open chain aldehydecarboxylates. ${ }^{14-18}$ 


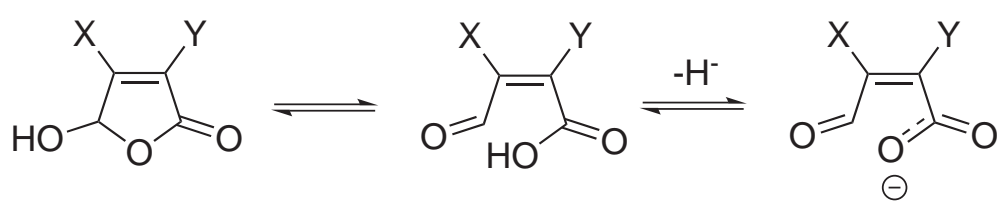

Scheme 5.2. Ring-chain tautomerism of hydroxyhalofuranones

\subsubsection{Hydroxyhalofuranones as pollutants}

\subsubsection{Formation of hydroxyhalofuranones}

Mutagenic hydroxyhalofuranones were first detected in fractions from pulp chlorination process for paper production, ${ }^{19-21}$ which were detected to be mutagenic, and eventually in drinking water. ${ }^{22}$ One single compounds was found to be responsible for the most part of the measured mutagenicity: 3-chloro4-dichloromethyl-5-hydroxy-2(5H)-furanone. Since its detection as a powerful mutagen until the final determination of its structure, this compound was known as "Mutagen X", which in its abbreviated form (MX) has become the common name of 3-chloro-4-dichloromethyl-5-hydroxy-2(5H)-furanone.

As many disinfection by-products, MX is formed at the first stage of the treatment process (prechlorination), then decreases in subsequent treatment stages, is totally removed in the carbon filters and finally may be formed again in the post-chlorination step. Of a variety of disinfectants (chlorine, chlorine dioxide-chlorine mixtures and chloramines) the highest concentrations of MX were found when chlorine was used. An increased proportion of chlorine dioxide in the combined chlorine/chlorine dioxide treatment resulted in a decrease in the mutagenic activity, and so did preozonization. Water chloramination produced considerably lower mutagenicity and lower concentrations of MX. ${ }^{23,24}$ The precursors to MX have been shown to be destroyed by both ozone and the combination of ozone and UV irradiation, in a dose-dependent manner. ${ }^{25}$

As regards the role of $\mathrm{pH}$ and chlorine dose, the formation of MX is favoured by acidic reaction conditions and high chlorine doses. No MX was detected when chlorination was carried out at pH 9. ${ }^{26,27}$ Acidic conditions also favored formation of MX in the chlorination of chlorinated phenolic compounds with structures related to aromatic units in aquatic humus. ${ }^{28}$

Zhuo et al. found that the influences of $\mathrm{pH}$, chlorine dose, reaction time, and temperature are quite complex, the optimal chlorination conditions for the formation of $\mathrm{MX}$ being $\mathrm{pH}=2, \mathrm{~T}=45^{\circ} \mathrm{C}, \mathrm{C} / \mathrm{Cl}_{2}=$ $1 / 4$, and $\mathrm{t}=12 \mathrm{~h} .{ }^{29}$ Most MX was formed in the first two hours and MX yield increased with chlorine dose until a maximum was reached and then decreased, this is possibly possibly due to the reaction of MX with chlorine. ${ }^{24}$ At higher temperature, the stability of MX declined dramatically. As would be expected, MX formation was linear in the total organic carbon concentration.

In a study of MX formation at water treatement plants, concentrations of MX increased with multiple chlorine applications, chlorine dose and total organic carbon and decreased with an increase in $\mathrm{pH}$. Levels 
were lower in chloraminated systems and showed seasonal variation with higher levels occurring in the spring compared with the autumn ${ }^{30}$

The presence of bromide in water, results in the formation of hydroxybromofuranones, which are equally (or more) genotoxic than their chlorine-bearing counterparts.

The synthesis of halofuranones for research purposes has also been addressed. ${ }^{31}$

\subsubsection{Analysis of hydroxyhalofuranones}

Since MX is usually present at concentrations below $\mu \mathrm{g} \mathrm{L}^{-1}$, which quite low in comparison with other DBP, and is also thermo-labile, its analysis is difficult. ${ }^{9}$ It requires pre-concentration of several liters of water, a clean-up of the water extract (adsorption on XAD resins, desorption with ethyl acetate, evaporation of the solvent), its derivatization (methylation with an acidic methanol solution) and high-resolution gas chromatography (GC) coupled to low- or high-resolution mass spectrometry (MS) or an ion-trap mass spectrometer with MS capabilities as an alternative. Electron capture detection is usually only suitable to detect MX present in clean matrices; chlorinated tap-water cannot be analysed for MX by this method due to the presence of many interfering contaminants. ${ }^{32-36}$

The second approach, an ion-trap detector with electron ionization and MS-MS fragmentation has been used for the selective determination of MX and its chlorinated and brominated analogues. ${ }^{37-40}$ Liquid-liquid extraction followed by methylation and GC/ $\mu$-ECD detection was used to measure halogenated furanones in a U.S. Nationwide Occurrence Study. ${ }^{41}$

In routine analysis of MX it is usually derivatized with methanol, although derivatization with other alcohols, such as propanol ${ }^{42}$ and sec-butanol, ${ }^{43}$ can improve the detection of MX. New methods using $N$ methyl-bis-trifluoroacetamide (MBTFA) derivatization and GC/ion trap-MS/MS have been developed. ${ }^{44}$ These reduce analysis time and improved detection limits (7.7 ng/l). Solid Phase MicroExtraction (SPME) with online bis(trimethylsilyl)trifluoroacetamide derivatization-GC/MS has also been applied ${ }^{45}$ with a detection limit of $30 \mathrm{ng} / \mathrm{l}$ were achieved.

\subsubsection{Occurrence of hydroxyhalofuranones}

Given its extreme biological effects (vide infra), MX is the halofuranone that has attracted most attention. Other analogues of MX have also been identified in chlorinated drinking water, including its geometric isomer (EMX), ${ }^{11,12}$ oxidized and reduced forms of MX. ${ }^{46}$

Brominated analogues (Scheme 5.3) and their respective $E$ isomers (the so-called B(E)MXs) have also been detected. ${ }^{47}$

All halohydroxyfuranones, including MCA, together with bromonitromethanes, iodo-trihalomethanes, haloamides, as well as other priority DBPs were incorporated by scientists of the U.S. Environmental Protection Agency in the list of priority DBPs for a Nationwide Occurrence Study. ${ }^{48,49}$ 
<smiles>O=C1OC(O)C(C(Cl)Br)=C1Cl</smiles>

BMX-1<smiles>O=C1OC(O)C(C(Br)Br)=C1Cl</smiles>

BMX-2<smiles>O=C1OC(O)C(C(Br)Br)=C1Br</smiles>

BMX-3

Scheme 5.3. Brominated hydroxyhalofuranones

The concentration of MX in drinking water has been measured in Canada, ${ }^{50}$ China, ${ }^{51}$ Finland, ${ }^{12,21,22,52,53}$ Japan, ${ }^{47,54,55}$ Rusia, ${ }^{52,56}$ Spain, ${ }^{38}$ the $\mathrm{UK}^{57}$ and the USA ${ }^{30,58}$ among other countries. Average concentrations are around 15-30 ng/l, although maximum concentrations of $0.3 \mu \mathrm{g} / \mathrm{l}$ have been reported (Table 5.1). In these studies, MX amounts to about $40 \%$ of the total mutagenicity of drinking water.

Table 5.1. Concentration of MX in plant effluent and tap waters

\begin{tabular}{|c|c|}
\hline Location & Conc. (ng/L) \\
\hline UK (Treatment plant) ${ }^{57}$ & $<3-41$ \\
\hline Australia 59 & $<0.5-33$ \\
\hline China ${ }^{51}$ & $3.8-58.4$ \\
\hline Finland ${ }^{12}$ & $<4-67$ \\
\hline Japan ${ }^{54}$ & $<3-9$ \\
\hline $\mathrm{USA}^{58}$ & $2-33$ \\
\hline $\mathrm{USA}^{30}$ & $4.0-79.9$ \\
\hline Spain 38 & $0.1-56$ \\
\hline
\end{tabular}

The concentration of brominated analogues of MX in plant effluent water vary from below the detection limit, to values of $0.7 \mu \mathrm{g} / \mathrm{l}$, depending mostly on the presence of bromine in the chlorination step (Table 5.2).

Table 5.2. Maximum concentration of brominated MX in plant effluent

\begin{tabular}{ccc}
\hline DBP & & Max. Conc. (ng/L) \\
\cline { 1 - 1 } BMX-1 & & 170 \\
BEMX-1 & 720 \\
BMX-2 & 30 \\
BEMX-2 & 810 \\
BMX-3 & 40 \\
BEMX-3 & 410 \\
\hline
\end{tabular}

The halogenated furanones are often stable in the distribution system and in simulated distribution 
system tests. Previous controlled laboratory studies had suggested that halogenated furanones, particularly MX, may not be stable in distribution systems. In at least five instances, MX levels actually increased in concentration from the finished water leaving the water treatment plant to the distribution system point sampled. ${ }^{3}$

In the few early drinking-water occurrence studies, concentrations of MX were generally $60 \mathrm{ng} / \mathrm{L}$ or lower. However, in 2002, Wright et al. reported MX maximum levels of $80 \mathrm{ng} / \mathrm{L}$ in 36 drinking water samples from Massachusetts (USA). ${ }^{30}$ Later, in the U.S. Nationwide Occurrence Study, which specifically focused on waters high in natural organic matter and/or bromide, Weinberg et al. found much higher levels of MX (frequently > $100 \mathrm{ng} / \mathrm{L}$ ) in finished drinking waters across the United States. ${ }^{48,49}$

In drinking-water treatment-plant effluents, a maximum level of $310 \mathrm{ng} / \mathrm{L}$ was observed for MX. Maximum levels of brominated MX analogues included 720, 810 and 410 ng/L for BEMX-1, BEMX-2 and BEMX-3, respectively.

MX levels reached a high of $850 \mathrm{ng} / \mathrm{L}$ in the average distribution system sample from a chlorine dioxide-chlorine-chloramine treatment plant. The highest levels of total halogenated furanones occurred in a water systems that disinfected with chlorine-chloramines $(2380 \mathrm{ng} / \mathrm{L}$ in drinking-water treatmentplant effluent) and at a treatment plant that disinfected with chlorine dioxidechlorine-chloramines (1020 $\mathrm{ng} / \mathrm{L}$ in the distribution system).

In a follow-up of the Nationwide DBP Occurrence Study, thirteen chlorinated and brominated MX analogues in the finished drinking waters from six cities across the United States. ${ }^{7,41}$ B-MX compounds were formed in waters with higher bromide levels, as would be expected. Chlorination formed higher levels than chloramination, but chloramines appeared to stabilize MX analogues in the distribution system. Pretreatment with ozone and granular activated carbon minimized MX formation upon subsequent chlorination or chloramination but pretreatment with chlorine dioxide did not. In chlorinated drinking waters, the concentrations of MX analogues and trihalomethanes and haloacetic acids showed similar patterns, which that all are formed through a common.

Mucochloric acid, along with other halogenated furanones, has been detected in drinking waters (plant effluent) at concentrations up to $1 \mu \mathrm{g} / 1 .{ }^{11,21,49,52}$ The total concentration of MCA in plant effluent from U.S. water treatment plants ranges from below detection limit $(0.02 \mu \mathrm{g} / \mathrm{l})$ up to $0.71 \mu \mathrm{g} / \mathrm{l}$, depending on the location of the plant, the season, the amount of natural organic matter and the chlorination product used. ${ }^{41}$

\subsubsection{Mucohalic acids}

Among the halogenated furanones formed in chlorination is mucochloric acid (MCA, 3,4-dichloro-5hydroxyfuran-2(5H)-one), which, like its analogue mucobromic acid (MBA, 3,4-dibromo-5-hydroxyfuran$2(5 H)$-one), is a direct genotoxin and a potential carcinogen ${ }^{60-63}$ has been identified in drinking water at approximately the same concentration of $\mathrm{MX}^{21,52}$ Although initially it had not been identified in drinking water, MBA was believed to be formed in the chlorination of water with high amounts of bromide, and was finally detected in as recently as $2006 .{ }^{49}$ 


\subsubsection{Reactivity of mucohalic acids}

The first studies of mucohalic acids addressed their structure, which was correctly deduced as soon as the $1880^{\prime}$ s, ${ }^{64-70}$ the nature of the ring-chain equilibrium and their reactivity with electrophiles and nucleophiles. ${ }^{14,15,71-74}$

In the closed-chain halofuranone form, the hydroxyl group has alcohol-like nucleophilic character, and reacts with alcohols in acidic medium affording mucohalic esters (3,4-dichloro-5-alkoxy-2(5)furanone) and also with anhydrides such as acetic or benzyl anhydride, affording mucohalic acetic or benzyl anhydride (3,4-dichloro-5-acetoxy-2(5)-furanone).

Mucohalic acids have two electrophilic sites through which they can react: the aldehyde group and the halogen in $\alpha$ with respect to the ester/acid group.

As regards the aldehyde group, its own electrophilicity reduces its ability to react with nucleophiles in the reaction medium: since the aldehyde open-chain form is minor in organic solvents, the fraction of MXA available for reaction at this site is limited. Thus, little reactivity is observed in solvents other than basic aqueous solution, where the aldehyde reacts with enolates or primary amines. ${ }^{69,73}$ This preference of the furanone form in the tautomeric equilibrium is itself a good proof of the electrophilic character of the aldehyde group. In aqueous conditions the aldehyde could expected to be partially hydrated, which again reduces its reactivity with nucleophiles. For the same reason, the acid group is quite unreactive in comparison to other carboxylic acids.

When substitution a halogen atom is observed it is always the one in $\alpha$ to the carboxylate. For instance, in the reaction of MXA with excess aniline, one equivalent of aniline reacts with the aldehyde group, affording the Schiff base, and then a dianilino derivative is formed, by substitution of the halogen $\alpha$ to the carboxylate. The hydrolysis of MXA also takes place at the same position.

Mucohalic acids are known to react in basic aqueous media to form mucoxyhalic acids (MOXA): the $\alpha$ halogen atom of the carboxyl group undergoes nucleophilic substitution by a hydroxide ion. ${ }^{15,74}$ The direct product of this reaction is the enol tautomer of MOXA $\left(\mathrm{MOXA}_{\mathrm{en}}\right)$, which is in tautomeric equilibrium with the keto tautomer $\left(\mathrm{MOXA}_{\mathrm{ket}}\right)$, as is shown in Scheme 5.4.

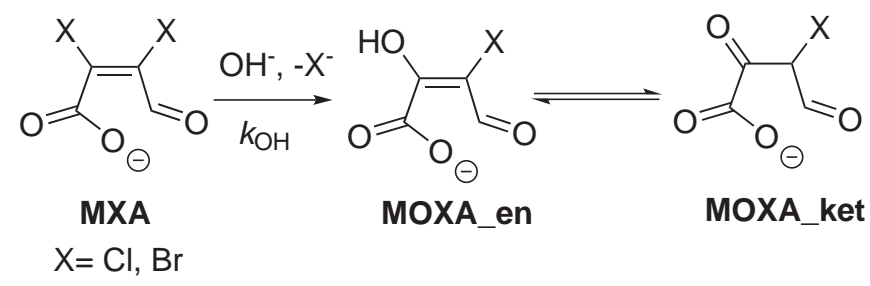

Scheme 5.4. Reaction of MXA with $\mathrm{OH}^{-}$

The reaction of MXA to form MOXA is of ecological importance, since it may represent the first step in a possible abiotic pathway for the degradation of mucohalic acids in the environment. The reaction products, MOXA, have received only modest attention in the literature, and little is known about their 
toxicity, stability, tautomeric equilibria or potential environmental and health effects. For instance, mucoxyhalic acids are known to be unstable in acidic conditions 14,15 and have been suggested to undergo decarboxylation, yielding highly toxic malondialdehydes. ${ }^{75-78}$ These effects could become significant, should the formation of mucoxyhalic acids be favored under environmentally relevant conditions or during water treatment processes.

5.1.2.1.1. Applications in organic synthesis. Mucohalic acids are the only halohydroxyfuranones formed in water chlorination that also find use in organic synthesis. They are highly functionalized, affordable building blocks that have been used in a variety of reactions since the 1960's

They have been reacted with Wittig reagents, ${ }^{79}$ Grignard reagents, ${ }^{80}$ alkyl phosphites, ${ }^{81-84} 1,2-$ ethanedithiol ${ }^{85}$

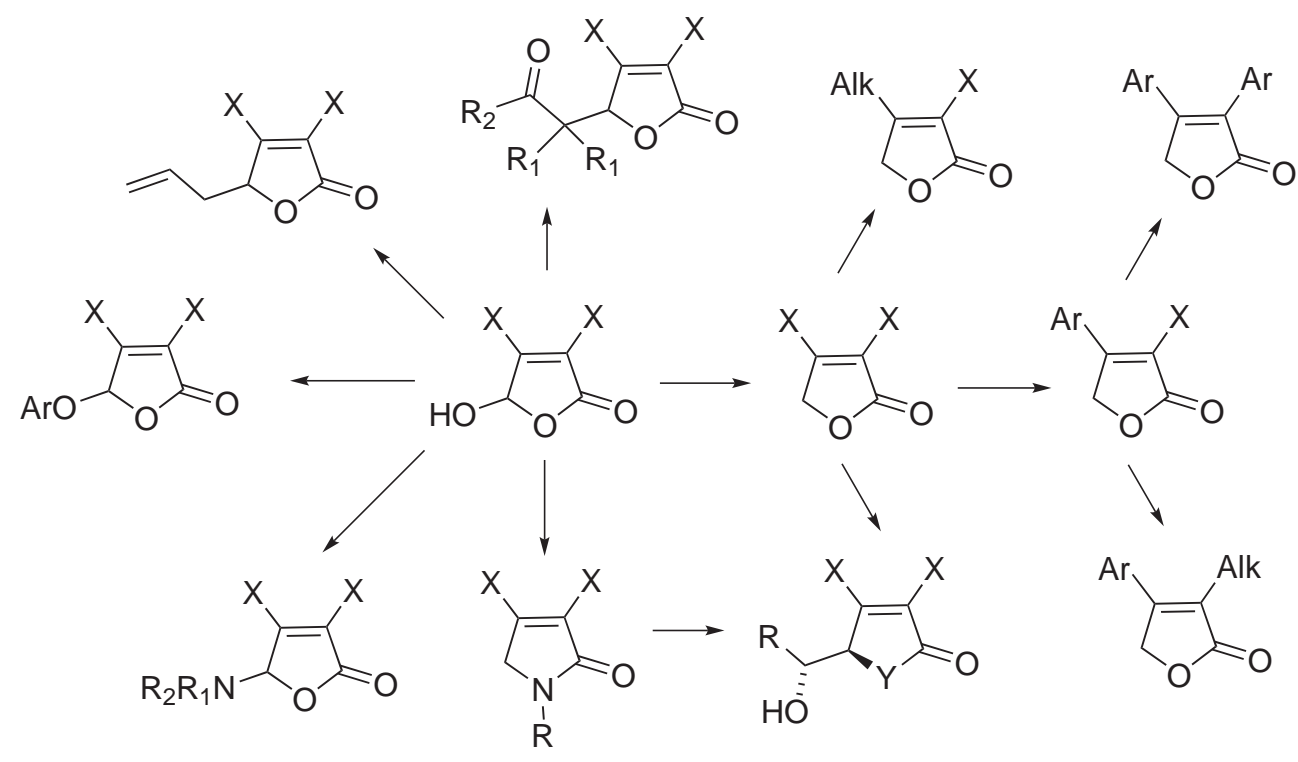

Scheme 5.5. Products synthesized from MXA

In many cases, the use of MXA as building blocks involves reduction as a first step to afford 3,4dihalo-2(5H)-furanone. This has subsequently been transformed into:

- 4-aryl-3-halo-2(5H)-furanones and 3,4-diaryl-2(5H)-furanones either by Stille-type reactions (palladiumcatalyzed reaction with aryl(trialkyl)stannanes), ${ }^{86,87}$ or regioselective Suzuki cross-coupling reaction with alkylboronic acids ${ }^{88}$ in the presence of catalytic amounts of $\mathrm{PdCl} 2(\mathrm{MeCN})(2)$ and $\mathrm{AsPh}_{3}$ and a large molal excess of $\mathrm{Ag}_{2} \mathrm{O}^{89}$ and also $\mathrm{AsPh}_{3}$ and a $\mathrm{Pd}(\mathrm{II})$ or a $\mathrm{Pd}(0)$ compound 90

- 3-Benzyl-4-isopropyl-2(5H)-furanone, which is a precursor to two naturally-occurring cytotoxic (Z)-5-ylidene-2(5H)furanone derivatives ${ }^{91}$

- $\gamma$-butyrolactones and $\gamma$-butyrolactams afford 5-(1'-hydroxy)- $\gamma$-butyrolactone and $\gamma$-butyrolactam subunits by direct vinylogous aldol addition of $\alpha, \beta$-dichloro. ${ }^{92}$ Use of an axially chiral guanidine 
base allows an asymmetric reaction. ${ }^{93}$

Several other transformations of MXA have been addressed, such as: regioselective etherification and amination of MXA-carbonates and acetates in a palladium free reaction; ${ }^{94}$ indium- and tin-mediated Barbier-type allylation to afford $\gamma$-allylic- $\alpha, \beta$ unsaturated gamma-butyrolactones; ${ }^{95}$ successive reaction with amines followed by in situ reduction to afford $N$-aryl (or alkyl)-3,4-dichloro-1,5-dihydro-pyrrol2-ones; ${ }^{96}$ Lewis-acid-catalyzed Mukaiyama aldol reaction; ${ }^{97}$ reaction with $\alpha$ - and $\beta$-amino acid esters, amides and short peptides yielding $\gamma$-lactam-based short peptides ${ }^{98}$ reaction with anilines gives dibromo- and dichloro-pyridazinone; ${ }^{99}$ glycoconjugation using Hunig's base; ${ }^{100}$ Friedel-Crafts hydroxyalkylation ${ }^{101}$...

Lewis-acid catalyzed addition of active methylene compounds to MXA affords Knoevenagel aldol adducts, gamma-substituted gamma-butenolides. ${ }^{102}$ This, in turn, undergo DABCO-mediated highly selective dehalogenation. ${ }^{103}$

Use of Pd cross-coupling and conjugate addition/elimination reactions have allowed production of a compound library including bisaryl maleimides, anilinoaryl maleimides, and bisanilino maleimides and also bisaryl substituted $\alpha, \beta$-unsaturated- $\gamma$-butyrolactams. ${ }^{104}$

MXA have also been applied to the synthesis of antifungal 5-chloro-6-phenylpyridazin-3(2H)-one derivatives, ${ }^{105}$ antibacterial and antiprotozoal 5-(omega-Hydroxyalkylamino) derivatives, ${ }^{106}$

\subsubsection{Genotoxicity of mucohalic acids}

Because they are formed during water chlorination, and especially because of their structural similarities with the strong genotoxin MX, mucohalic acids have attracted much attention in regards to their biological properties. They have been shown to bind covalently to nucleobases, both in the monomeric form and forming part of DNA and also to afford positive results in the Ames test and a number of test systems. Therefore, they have been concluded to be mutagenic and genotoxic, although at much lower levels than other halohydroxyfuranones.

5.1.2.2.1. Biological assays. Mucohalic acids are direct acting mutagens in the Ames assay, ${ }^{21}$ where it induced 3.6 revertants per nmol in E coli TA100 (earlier values of $3.3^{107}$ and $60^{108}$ revertants per nmol have been reported). LaLonde et al. studied the effect of $\mathrm{Cl}$-by- $\mathrm{Br}$ and $\mathrm{H}$-by-OH replacement. The effect of the Cl-by-Br replacement on mutagenicity amounted to a 1.9-fold enhancement in the presence of C-5 $\mathrm{OH}$, but an 11-fold enhancement in the presence of C-5 H. ${ }^{109}$

Positive results were also obtained in the SOS chromotest with E. coli110 and in the differential DNA repair test: ${ }^{60}$ when tested in animal-mediated assays with $E$. coli $\mathrm{K}-12$ cells, genotoxicity was detected in various organs of mice with the most pronounced effect in the gastrointestinal tract.

C. Helma et al. reported a dose dependent-increase of micronuclei in pollen mother cells of Tradescantia when doses up to $100 \mu \mathrm{g}$ MX and $500 \mu \mathrm{g}$ MCA were applied directly to the inflorescences. In 
contrast, exposure of the stems in aqueous solutions containing up to $10 \mathrm{mg} / \mathrm{l} \mathrm{MCA}$ did not cause a positive response. ${ }^{111}$ Toxicity towards rainbow trout hepatocytes has also been observed ${ }^{112}$

MCA also induced chromosomal aberrations and DNA strand breaks in mammalian cell cultures $58,113,114$ (CHO cells, rodent lymphocytes, rat hepatocytes and cultured human cells) and in the $\phi$ X174 plasmid. ${ }^{115}$

MCA was found to be a strong genotoxin, second only to CMCF - and thus, stronger than MX - in the microscale micronucleus assay on L5178Y mouse lymphoma cells and unscheduled DNA synthesis assay on hepatocyte primary culture from Fisher F344 rats. ${ }^{116}$

According to the results in the alkaline comet assay in TK6 cells, both mucochloric and mucobromic acids are clearly genotoxic and lead to high levels of DNA breaks. MBA was seen to be somewhat more effective, but the DNA damage induced by MBA repairs better over time. Furthermore, the two MXA produce high levels of oxidized bases. In contrast, the results from the micronucleus assay, which measures the induction of clastogenic and/or aneugenic effects, are mainly negative for the two compounds in TK6 (MCA is able to increase significantly the frequency of micronuclei in binucleated cells at the concentration tested, $10 \mu \mathrm{M}) .63$

In a recent study, MBA - but not MCA - was able to increase the frequency of mutations in the thymidine kinase $(T k)$ gene in the mouse lymphoma assay. This assay quantifies a wide range of genetic alterations affecting the expression of this gene in L5178Y/Tk ${ }^{+/-}-3.7 .2 \mathrm{C}$ cells. The effect was detected at the highest evaluated dose $(10 \mu \mathrm{M})$, and is most possibly related to cytotoxicity, and not necessarily direct DNA damage. ${ }^{117}$

The bioactivation of alpha-haloacrolyl-containing molecules, which is present in a variety of DBP, has been modeled using alpha-bromo-2-cyclopentenone. The resulting products are consistent with a mechanism in which initial addition of thiols to the double bond is followed by intramolecular displacement of bromide to yield a DNA-alkylating episulfonium ion intermediate, whether this mechanism applies to mucohalic acids is unknown. ${ }^{118}$

The mutagenic spectrum of mucohalic acids consists mainly in GC $\rightarrow$ AT transitions, as measured in the hisG46 allele of Salmonella typhimurium using allele specific oligonucleotide hybridization. This pattern consistent with modification of guanosine positions in DNA. ${ }^{62}$

5.1.2.2. DNA adduct formation. Both mucohalic acids (MXA) are known to bind covalently to the DNA bases guanosine, adenosine and cytosine, both in the form of monomers and forming part of DNA. ${ }^{78}$ These alkylation reactions give rise to the adducts depicted in Schemes 5.6 and 5.7: etheno ${ }^{75}$ $\left(\mathrm{R}=\mathrm{H} ; \epsilon \mathrm{A}, \epsilon \mathrm{G}\right.$ and $\epsilon \mathrm{C}$ ), ethenocarbaldehyde ${ }^{76}(\mathrm{R}=\mathrm{COH} ; \epsilon \mathrm{CA}, \epsilon \mathrm{CG}$ and $\epsilon c \mathrm{C})$, oxalo-etheno ${ }^{119}$ ( $\mathrm{R}=\mathrm{CO}$ $\mathrm{COOH} ; \epsilon O A, \epsilon O G$ and $\epsilon O C)$, and halopropenal ${ }^{77}\left(\mathrm{M}_{X} \mathrm{~A}\right)$ derivatives. Adducts containing two adenosine moieties haven been also observed $(\epsilon \mathrm{CA}, \mathrm{A} \text { and } \epsilon \mathrm{OA}, \mathrm{A})^{120}$ and also regioisomeric $\alpha$ chlorohydrins. ${ }^{121}$

The presence of nucleophiles such as glutathione and N-acetylcysteine reduces the biological effects of mucohalic acids. ${ }^{109,122,123}$ It was concluded that this occurs by reaction with the C-4 halogen atom (the same that undergoes displacement by hydroxide ions in the hydrolysis reaction) 


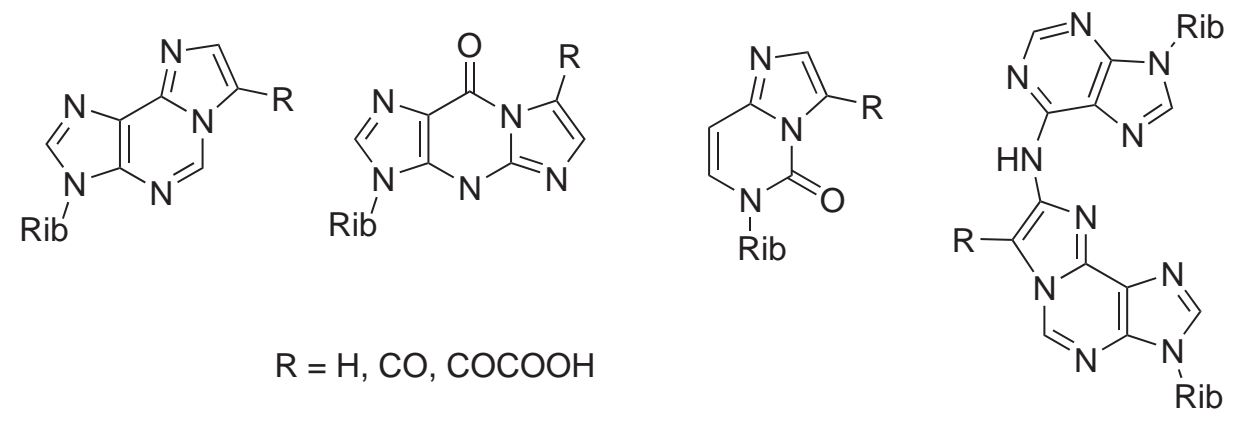

Scheme 5.6. Common nucleotide-MXA adducts<smiles>[X]/C(C=O)=C\Nc1ncnc2c1ncn2[PH]</smiles>

Scheme 5.7. Rare nucleotide-MXA adducts

The product distribution of the nucleotide+MXA reaction depends on the reaction conditions, mostly $\mathrm{pH}$ and temperature: whereas the etheno derivatives are major when the reaction is carried out in harsh conditions $\left(T=90{ }^{\circ} \mathrm{C}\right)$, they are very minor at lower temperatures $\left(T=37^{\circ} \mathrm{C}\right)$. The oxalo-etheno derivatives are observed to be very abundant when the reaction was performed in acidic medium $(\mathrm{pH}$ $=4.6$ ), and so are the ethenocarbaldehyde adducts. The halopropenal derivatives are major when the reaction is carried out in an aqueous solvent and thus, were detected for the first time when the reaction was performed in dimethylformamide. They have been also detected in alkylation reactions carried out in aqueous conditions, albeit at much lower concentrations.

Based on the nature of the products formed (including the use of isotopic tracers), an alkylation mechanism has been proposed for MXA (Scheme 5.8): attack of the exocyclic amine is followed by elimination forming a Schiff base. Then, the halogen atom in $\alpha$ to the carboxylate is substituted by a hydroxyl group, which is followed by cyclization to afford a five-membered cycle. In some conditions this ring-closure reaction is followed by decarboxylation or even loss of the oxalo group. In this pathway, it is unsure whether substitution of chlorine by hydroxide precedes - which would imply that the true alkylating agents are mucoxyhalic acids - or succeeds the formation of the adduct.

\subsubsection{Mutagen X}

Unlike mucohalic acids, MX is only produced for research purposes and has no known uses, and thus the only source of exposure is chlorinated water. The interest in MX arises mostly from its record-high 


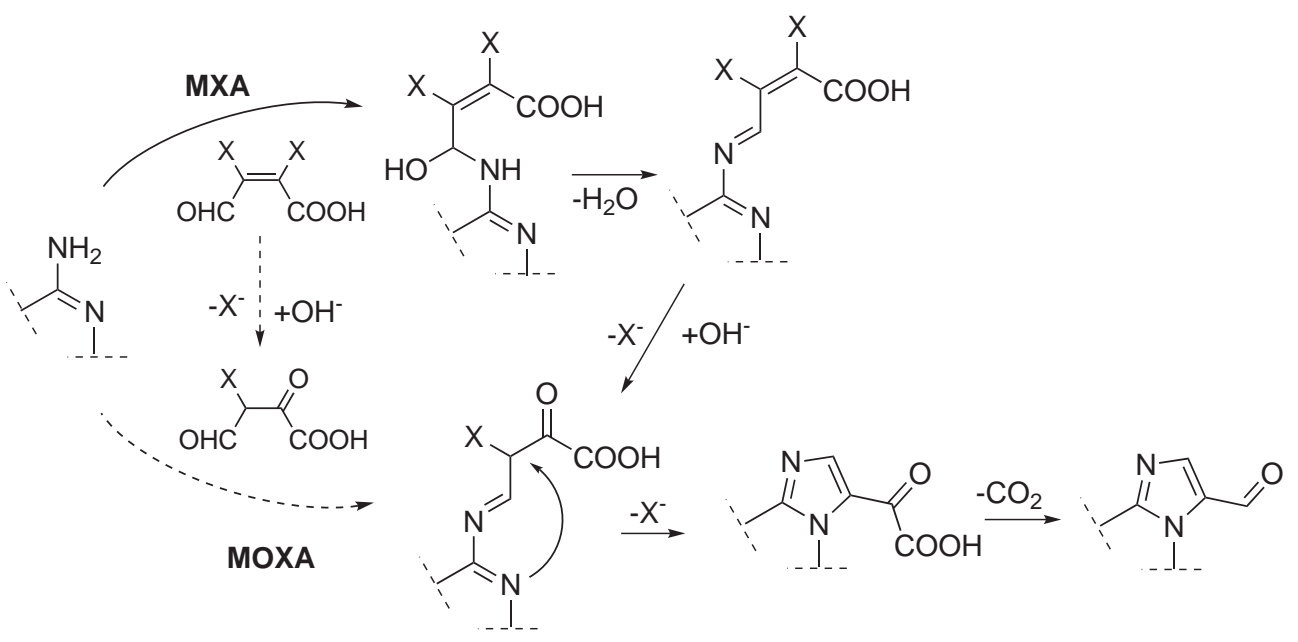

Scheme 5.8. Mechanism of alkylation by MXA proposed in the literature

positive results in standard bacterial mutagenicity tests. Of the several hydroxyhalofuranones formed in water chlorination, MX is the only one to have been tested for carcinogenicity in animal models ${ }^{124}$ and is the only one of them to have been designated as a carcinogen. IARC considered MX as "possibly carcinogenic to humans" and ascribed it to the 2B Group in 2004. ${ }^{9}$

Aside from the ring-chain equlibrium, MX has two open-ring tautomeric forms that exist at physiological pH: (Z)-2-chloro-3-(dichloromethyl)-4-oxobutenoic acid (ZMX) and (E)-2-chloro-3-(dichloromethyl)4-oxobutenoic acid (EMX). Both are potent genotoxicants in a wide variety of genetic assays and endpoints in vitro

The $Z \rightarrow E$ isomerization reaction takes place at basic $\mathrm{pH}$. MX also undergoes hydrolysis in alkaline media: at $\mathrm{pH} 8$ and $23^{\circ} \mathrm{C}$, the half-life of MX is 6 days ${ }^{125}$ (7.4 days at $\mathrm{pH} 7.8^{16}$ ), compared to several years at $\mathrm{pH} 2.8 .^{16}$

Various other halohydroxyfuranones, analogue to MX exist: 3-chloro-4-methyl-5-hydroxy-2(5H)-furanone (MCF) and 3-chloro-4-chloromethyl-5-hydroxy-2(5H)-furanone (CMCF) are of special interest, since they differ from MX only in the presence of one or no chlorine atoms on the methyl group. The set of MX, CMCF and MCF will be referred in this work as "MX and analogues" or MXY.

\subsubsection{Genotoxicity of MXY}

The carcinogenicity of MX has been addressed extensively in a review by McDonald and Komulainen, ${ }^{10}$ and also in reports by the California Environmental Protection Agency ${ }^{124}$ and the IARC, ${ }^{9}$ where it was evaluated as possibly carcinogenic to humans and ascribed to the Group 2B in 2004. According to the World Health Organization (WHO), MX concentrations of $1.8 \mu \mathrm{g} \mathrm{l} \mathrm{l}^{-1}$ are associated with a $10^{-5}$ cancer risk for a $60 \mathrm{~kg}$ adult drinking 21 of water per day. ${ }^{126}$ The genotoxic effects of the weaker pollutants MCF and CMCF have attracted comparatively less attention, and mostly, as a comparison to MX. 
Brominated analogues of MXY with a variety of $\mathrm{Cl} \rightarrow \mathrm{Br}$ substitution are also formed in drinking water treatment, in waters with high concentration of inorganic bromine.

5.1.3.1.1. Tumorigenicity in animal models. The carcinogenic effects of $M X$ on male and female Wistar rats was studied by Komulainen et al., who found that dosage of few $\mathrm{mg} / \mathrm{kg} /$ day produced a significant, dose-dependent increase in the incidence of certain tumors. No toxic effects of MX were observed at those levels. ${ }^{127}$

Combined incidence of thyroid gland follicular carcinoma or adenoma were observed $90 \%$ of the treated animals. Since the mean plasma levels of thyroid hormones were similar for MX-treated and control groups, these tumor were probably not due to a proliferative mechanism resulting from thyroid hormone disruption but to the direct effect of MX.

For males linear dose-related increases were observed for basal cell skin tumors, lung adenoma, liver adenoma (and carcinoma), liver cholangioma, pancreas Langerhans' cell adenoma (and carcinoma), and adrenal gland cortical adenoma. In the female rats MX induced increases in the incidence of mostly the same tumors, together with mammary gland tumors.

No gastric tumors were observed in genetically modified CB57Bl/6Min/+ mice, which develop gastrointestinal tumors at a very young age. ${ }^{128}$ However, gastrointestinal tumors have neither been observed in rats in the two-year study, which could suggest that these organs are not targeted by MX. ${ }^{127}$

Other studies using genetically modified Long-Evas (Ecker) rats have found that MX was unique among several water contaminants in increasing the incidence of renal adenomas, but not gastric tumors. However, none of these were observed using non modified rats. ${ }^{129}$

MX has been observed to act as a tumor initiator in experiments with SENCAR mice in which an initial dose of $0-50 \mathrm{mg} / \mathrm{kg}$ was followed by continued treatment with known tumor promoter $12-\mathrm{O}$ tetradecanoyl-phorbol-13-acetate (TPA). ${ }^{130}$ No effect was observed in the dermal application experiments, but a significant increase in skin tumors occurred in the experiments in which MX was administrated orally. Single dosage without TPA produced no increase in tumors. These experiments suggest that MX is an effective tumor initiator.

MX in drinking water at $0-30 \mathrm{ppm}$ was observed to act as a tumor promoter following initial administration of $N$-methyl- $N^{\prime}$-nitro- $N$-nitrosoguanidine. MX increased the incidence and multiplicity of adenocarcinoma of the glandular stomach and the incidence of atypical hyperplasia of the glandular stomach. ${ }^{131}$ Since none of these were observed in the study of MX carcinogenicity, they are probably attributable to the role of MX as a tumor promoter. No tumor promotion was observed in the case of colon cancer following administration of azoxymethane or $\mathrm{N}$-bis(2hydroxypropyl)nitrosamine. ${ }^{128}$

5.1.3.1.2. Genotoxicity in vitro. MX is a direct-acting mutagen and clastogen, and close to a hundred publications have addressed the DNA-damaging potential of MX. The results of some of these are summarized in Tables 5.3 and 5.4 and include mutations, chromosomal aberrations, sister chromatid ex- 
changes, strand breaks, unscheduled DNA synthesis and cell transformation. Very extensive data can be found in the report by the IARC. ${ }^{9}$

Table 5.3. Genotoxicity of MX in non-mammalian in vitro test systems

\begin{tabular}{|c|c|c|}
\hline Test System & Assay & Rslt. \\
\hline \multirow{2}{*}{ Purified DNA } & Abasic sites/Strand breaks supercoiled PM2 DNA ${ }^{132}$ & + \\
\hline & Strand breaks $\phi X 174^{115}$ & + \\
\hline \multirow{5}{*}{ Bacteria } & Reverse Mutation Salmonella typhimurium strains TA92, TA97, & \\
\hline & 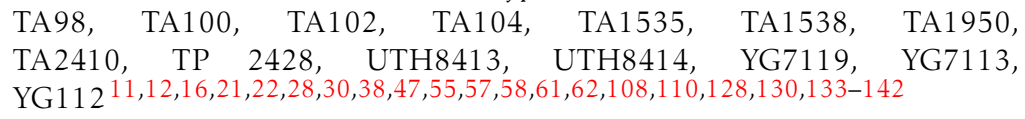 & + \\
\hline & Rev Mut Salmonella typhimurium strain RSJ100143 & - \\
\hline & Forw Mut Salmonella typhimurium strain TM677 138 & + \\
\hline & $\begin{array}{l}\text { Rev Mut Eschernichia coli strains WP2, CC101, CC102, CC103, CC104, } \\
\text { CC105, CC106, CC107, CC108, CC109, CC110, CC111, CL101P, CL102P, } \\
\text { CL103P, CL104P, CL105P, ZA500, ZA4107, ZA4108, ZA4109, ZA2102, } \\
\text { ZA2104, ZA2107, ZA2108, ZA2109, ZA2110, ZA2111, ZA4107, ZA4108, } \\
\text { ZA4110, ZA4111, ZA5102, ZA5104, ZA5108, ZA5109144-147 }\end{array}$ & + \\
\hline Chromotest & E. coli strain PQ37110 & + \\
\hline $\begin{array}{l}\text { Prophage } \lambda \\
\text { induction }\end{array}$ & E. coli system ${ }^{138}$ & + \\
\hline DNA repair & E. coli strain $\mathrm{K}-12^{60}$ & + \\
\hline Yeast & $\begin{array}{l}\text { Deletions, interchromosomal recombination, aneuploidy, Saccharomyces } \\
\text { cerevisiae RS } 112^{148}\end{array}$ & + \\
\hline
\end{tabular}

The results of MX in some in vitro tests are the highest ever recorded for any compounds, for instance, MX produced 5-13 $\times 10^{3}$ revertants per nmol in Salmonella typhimurium strain TA100.

Results in this and other strains suggest that the mutational pattern of MX includes base-pair substitutions and frameshift mutations. The positive results in the absence of S9 activating mixture suggest that MX requires no metabolic activation, and, in fact, Ames-test results are lower in the presence of the enzyme preparation.

MX proved twice as potent as aflatoxin B1 in the 8-azaguanine resistance assay in Salmonella TM6776 and has the greatest potency to induce deletions, interchromosomal recombinarion and aneuploidy of any carcinogen tested in the deletion-events assay in yeast (S. cerevisae strain RS112). ${ }^{148}$ These effects are decreased by the presence of added nucleophiles.

The mutational pattern of MX is of interest, ${ }^{138,139}$ and has received much attention. ${ }^{62,149}$

In S. typhimurium strain TA100, MX induces GC $\rightarrow$ TA transversions, especially in a GGG-CCC hot spot. GC $\rightarrow$ TA transversions have been observed too in TA1535, TA1950 and TP 2428 treated with MX and other halohydroxyfuranones and also in E. coli bacteria. MX also produced frameshift mutations in $S$. typhimurium TA98: two-base deletions in 40-70\% of the mutants and complex deletions (single deletion 
plus contiguous base-pair substitution) in 30-50\%. In Chinese hamster ovary cell GC $\rightarrow$ TA transversions were also detected, together with AT $\rightarrow$ TA transversions, deletions of GC base pairs and larger deletions.

These results are consistent with MX damaging mainly guanosine residues, since the DNA polymerases preferentially insert adenine nucleotides in positions opposite non-instructional lesions, such as abasic sites. ${ }^{150}$ Frameshift mutations arising from deletion within runs of GC are also consistent with the abasic sites at the positions of damaged guanosine nucleotides.

Table 5.4. Genotoxicity of MX in mammalian cells in vitro

\begin{tabular}{|c|c|c|}
\hline Assay & Test System & Result \\
\hline $\begin{array}{l}\text { Fwd. mutation, thymidine ki- } \\
\text { nase locus }\end{array}$ & $\begin{array}{l}\text { Mouse L5178Y lymphoma cells, human B-lympho- } \\
\text { blastoid cells MCL-5, AHH1 151,152 }\end{array}$ & + \\
\hline $\begin{array}{l}\text { Fwd. mutation, HPRT locus or } \\
\mathrm{Na} / \mathrm{K} \text { ATPase locus }\end{array}$ & $\begin{array}{l}\text { Chinese hamster ovary cells, Chinese hamster V79 } \\
\text { cells } 113,149,153-155\end{array}$ & \pm \\
\hline DNA Strand breaks & $\begin{array}{l}\text { Human lymphoblastoid CCRF-CEM, human lympho- } \\
\text { cytes, human peripheral blood mononuclear cells, hu- } \\
\text { man LLC-PK1 renal epithelial cells, HL cells, pig kid- } \\
\text { ney cells, rat hepatocytes, rat testicular cells, CHV79 } \\
\text { cells, CHO cells } 130,155-161\end{array}$ & + \\
\hline Chromosome aberrations & $\begin{array}{l}\text { Chinese hamster ovary cells, mouse } \begin{array}{c}\text { L5178Y } \\
\text { lymphoma cells, rat } \\
\text { cytes } 58,114,130,151,154,160\end{array} \\
\text { peripheral lympho- }\end{array}$ & + \\
\hline Micronuclei & $\begin{array}{l}\text { Mouse L5178Y lymphoma cells, rat peripheral lym- } \\
\text { phocytes } 116,162\end{array}$ & + \\
\hline Sister chromatide exchanges & $\begin{array}{l}\text { Chinese hamster ovary cells, rat peripheral lympho- } \\
\text { cytes } 114,154,160\end{array}$ & + \\
\hline Cell transformation & Mouse C $3 \mathrm{H} 10 \mathrm{~T} 1 / 2$ cells $^{163}$ & + \\
\hline Unscheduled DNA synthesis & Mouse hepatocytes, rat hepatocytes 116,164 & + \\
\hline
\end{tabular}

CMCF has been reported to around five times less mutagenic than MX in S. typhimurium strain TA100 with $579,{ }^{21} 1000^{136}$ and $1200^{165} \mathrm{rev}$ per mol. MCF exerted an effect approximately one hundred times smaller, $10 \mathrm{rev} / \mathrm{nmol}^{19,21}$

In a study of the relationship between the structure and activity of hydroxyhalofuranones, LaLonde et al. concluded that, the halomethyl group was key to the mutagenicity. Cl-by- $\mathrm{H}$ replacement at the chloromethyl group, that is from CMCF to MCF, had a greater effect in reducing mutagenicity (up to a 1:1000 factor), than a similar replacement at C-3 or a hydroxyl-by-hydrogen replacement at C-5. ${ }^{135,136}$

The mutational pattern of CMCF is similar to that of MX, whereas the mutations induced by MCF are somewhat halfway between MX and mucohalic acids...

LaLonde and his colleagues synthesized and determined the mutagenic potency (S. typhimurium strain TA100) of 12 bromine-, chlorine-, and mixed halogen-substituted 4-methyl-2(5H)-furanones. ${ }^{140}$ 
Table 5.5. Relative molar genotoxicity of MX to analogue hydroxyhalofuranones ${ }^{10}$

\begin{tabular}{ccccccc}
\hline Test system & MX & CMCF & MCF & BMX-1 & BMX-2 & BMX-3 \\
\hline Relative concentration & 1.0 & 0.44 & 2.5 & $0-6$ & $0-4$ & $0-9$ \\
$\quad$ S. typhimurium & & & & & & \\
$\quad$ Rev. in TA97 & 1.0 & 0.40 & 0.005 & - & - & - \\
Rev. in TA98 & 1.0 & 0.013 & 0.0004 & - & 2.6 & 0.9 \\
Rev. in TA100 & 1.0 & 0.10 & 0.0014 & 0.4 & 1.5 & 1.3 \\
Mammalian cells (CHO) & & & & & & \\
$\quad$ Comet Assay & 1.0 & 1.1 & 0.025 & - & - & - \\
Sister Chromatide Exchange & 1.0 & 0.17 & 0.005 & - & - & - \\
Chromosomal aberrations & 1.0 & 0.08 & 0.037 & - & - & - \\
\hline
\end{tabular}

The most mutagenic compounds were the trihalo- $\left(\sim 10^{3} \mathrm{rev} / \mathrm{nmol}\right)$, followed by the dihalo-4-methyl5-hydroxy-2(5H)-furanones, $\left(\sim 10^{2} \mathrm{rev} / \mathrm{nmol}\right)$ irrespective of bromine or chlorine substitutions. Trihalides and dihalides lacking the C-5 hydroxyl group expressed lower mutagenic potencies $\left(\sim 10^{2}\right.$ and 10 $\mathrm{rev} / \mathrm{nmol}$, respectively). Replacement of a $\mathrm{C}-5 \mathrm{H}$ by $\mathrm{OH}$ enhanced mutagenicity more for the dihalides than the trihalides, indicating that increasing the C-6 halogen number simultaneously with replacing C-5 $\mathrm{H}$ by $\mathrm{OH}$ results in a nonlinear, additive enhancement.

For fewer than half of the compound pairs compared, changing the C-6 halogen from chlorine to bromine resulted in small increases in mutagenicity, and for the remaining compound pairs, no increase could be discerned. 3-Chloro-4-(dibromomethyl)-5-hydroxy-2(5H)-furanone (BMX-2), which induced a $140 \%$ increase in mutagenicity compared to MX

Some effort has been attempted to correlate mutagenicity of halofuranones in the Ames assay with simple molecular properties like HOMO-LUMO separation, or electron affinity. ${ }^{166}$

In a 12 -week study, MX was given to gpt delta mice at concentrations of 10, 30, or $100 \mathrm{ppm}$. No induction of mutation in the reporter gene (gpt) was detected nor were deletions larger than $1 \mathrm{~kb}$ observed using a phage-insensitive selection system (Spi). However, MX inhibited gap junctions in rat WB cells. ${ }^{167}$

5.1.3.1.3. Genotoxicity in vivo. MX has also observed to be genotoxic in vivo. Although in has afforded negative results in some tests, these have been attributed to different DNA-damage and repair kinetics, and thus MX is understood to be able to exert DNA damage to a wide range of tissues in vivo. These include

- Micronuclei No micronuclei in polychromatic erictrocytes were observed by Meier et al either in Swiss Webster mice trated with $22-90 \mathrm{mg} / \mathrm{km} /$ day oral doses for two days following 48-72 hours following exposure, ${ }^{58}$ or in $\mathrm{B}_{6} \mathrm{C}_{3} \mathrm{~F}_{1}$ mice treated for 14 days. ${ }^{162}$ Similar negative results were observed by Jansson, ${ }^{168}$ Fawell and Horth in CD-1 mice with single doses of $144 \mathrm{mg} / \mathrm{kg}^{57}$ and Tikkanen and Kronberg at 4.4 and $8.8 \mathrm{mg} / \mathrm{kg}$, ${ }^{110}$ Daniel et al. observed significant positive effects at 50.0 and $62.2 \mathrm{mg} / \mathrm{kg}$ in the forestomach and duodenum. ${ }^{169}$ 
- Sister chromatide exchanges Statistically significant periferal blood lymphocytes SCEs were observed in Han:Wistar rats of both sexes treated during 14 to 18 weeks and also in kidney cells. ${ }^{114,170,171}$

- DNA damage No single strand breaks or alkali-labile sites were observed in organs of male Wistar rats which had been given 18, 63, or $125125 \mathrm{mg} / \mathrm{kg}$ MX. ${ }^{113}$ However DNA single strand breaks were observed in the stomach of F344/Du Crj rats (at $10-48 \mathrm{mg} / \mathrm{kg}$ doses). ${ }^{172}$ Also, dose-related increases in replicative DNA synthesis, as well as increases in the activity of ornithine decarboxylase were observed. Assays with E coli strains differing in their DNA-repair capability suggest that the strain with deficient repair system had its viability reduced. ${ }^{60} \mathrm{MX}$ at $100 \mathrm{mg} / \mathrm{kg}$ doses also induced DNA damage as measured by the alkaline comet assay in the liver, kidney, lung, brain and all mucosa of male CD-1 mice. The kinetics of damage and repair varied depending on the target organ. ${ }^{173}$ The same dose induced unscheduled DNA synthesis in Balb/c mice. ${ }^{164}$ Single strand breaks and alkali labile sites were observed in $\mathrm{B}_{6} \mathrm{C}_{3} \mathrm{~F}_{1}$ mice at $40-80 \mathrm{mg} / \mathrm{kg}$ doses, but only in those that had been pre-treated with DNA repair enzyme inhibitors. ${ }^{159}$

As occurs with in vitro models, the effects of CMCF are somewhat weaker, but qualitatively close to those of MX; on the other hand, MCF is a much milder genotoxin.

5.1.3.1.4. DNA adduct formation. The reaction products of nucleotide alkylation by MX (as well as MCF and CMCF) have been studied in several works. In these, the reaction of nucleotides with the hydroxyhalofuranones were carried out in vitro, with no in vivo results having been reported. Adenine and guanine adducts have been detected in the reaction with nucleotides, but only those formed with adenosine have been observed in the reaction with double stranded DNA. ${ }^{78,174}$

Scheme 5.9 shows the adducts detected for MCF, ${ }^{175} \mathrm{CMCF}^{176}$ and MX, ${ }^{174,176}$ and the proposed mechanisms that lead to them, when any, are shown in Scheme 5.10, 5.11 and 5.12 respectively. The proposed general pathway, common to the three MXY, implies attack on the exocyclic amine in a Michael addition to the terminal methylene of the isomerized halofuranone. This is followed by elimination of $\mathrm{HCl}$. Whereas the reaction of MCF is over at this point, CMCF and MX still have chlorine groups that can be substituted, by either hydrolysis or intramolecular nucleophilic attack, involving ring-closure, followed by decarboxylation. In addition, the adduct formed in the reaction of MX with monomeric guanosine ( $\in$ fGuo-dR) suggests that MX can also react by substitution of a chlorine atom in the dichloromethyl group followed by cyclization. Interestingly, no explanation have been given for the formation of either pfA-dR or $\mathrm{M}_{1} \mathrm{~A}-\mathrm{dR}$ in the reaction of MX.

A second alkylation mechanism has been proposed for MCF, in which a different product is formed (MCF-dA-I): attack of the amine onto the carbonyl carbon and ring closure of the furanone. This is similar to the way mucohalic acids modify DNA, however, this reaction has not been observed for the other MXY. An additional alkylation reaction occurring trough a decomposition product has been proposed for MX (enaminal) but since it includes two MX molecules reacting with the same nucleotide it is unlikely to take place in vivo, where the concentrations of MX are extremely low. ${ }^{177}$

Since the mutagenic effects of MX seem to arise from guanosine lesions and no guanosine adducts have been detected in the reaction with double-stranded DNA; stable, covalent adducts with nucleobases 


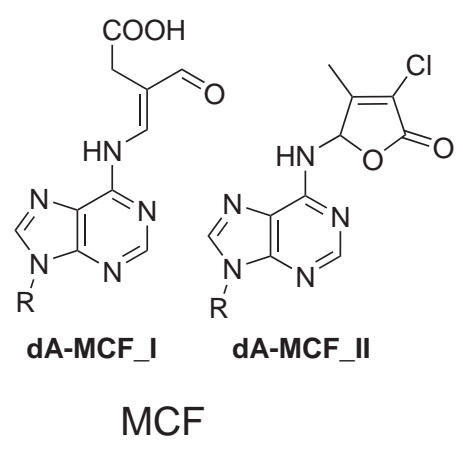

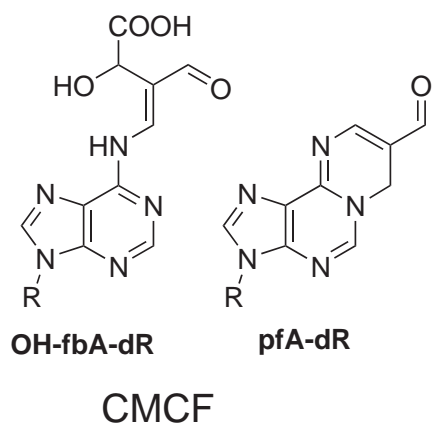<smiles></smiles>

$M_{1}$ A-dR<smiles>O=CC1=CN=C2c3ncn(P)c3N=CN2C1</smiles>
pfA-dR<smiles>O=CC1=C(Cl)N=C2c3ncn(P)c3N=CN2C1</smiles><smiles>O=c1c2ncn(P)c2nc2n1C(C=P)C=N2</smiles>

efGuo-dR

\section{MX}

Scheme 5.9. Nucleotide-MXY adducts

can hardly explain the biological effects of MX, or CMCF, whose biological effects are qualitatively similar.

Based on this mutational pattern and some basic theoretical molecular properties of MX, some authors have proposed that MX abstracts an electron from DNA (guanosine residues being the most easily oxidized), which yields a radical cation. This, in turn, can undergo depurination or other processes that lead to abasic sites or other lesions. This is in good agreement with the mutational pattern of MX, since DNA polymerases usually insert adenine residues opposing non-instructional lesions. Nevertheless, the hypothesis of MX oxidizing DNA lacks experimental support as of 2011. 


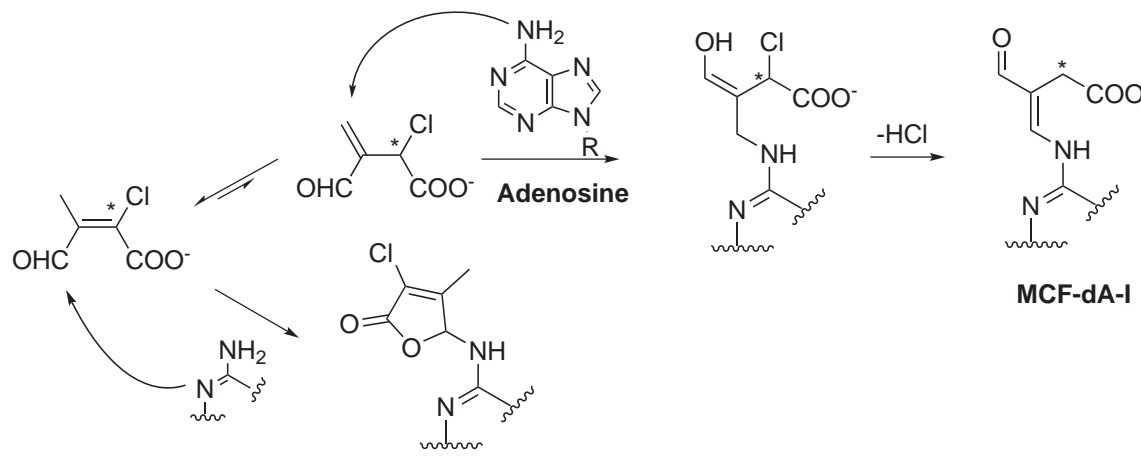

MCF_dA-II

Scheme 5.10. Proposed mechanisms for the formation of nucleotide-MCF adducts
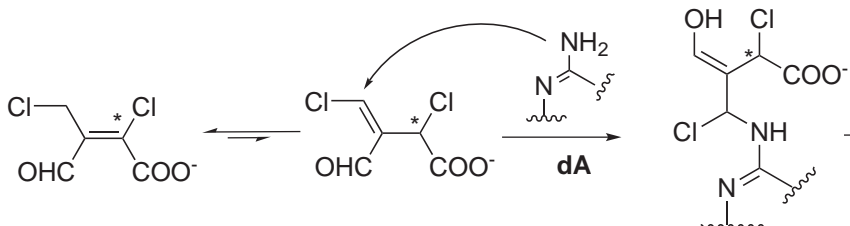

$\stackrel{-\mathrm{HCl}}{\longrightarrow}$

<smiles>CCC(C)N/C(=C\NC)C(=O)O</smiles>

$\mathrm{OHC}$<smiles>[CH]1CCCCC1</smiles>

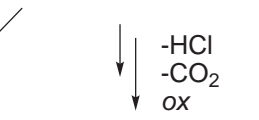

OH-fbaA-dR<smiles>CC1CC2CCN1CC(CC(=O)O)C2</smiles>

pfA-dR<smiles>CC(C)C1=NC=C(C=O)C(C(C)C)N1C(=O)[O-]</smiles>

OOC

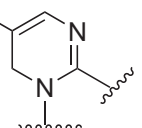

pcA-dR

Scheme 5.11. Proposed mechanisms for the formation of nucleotide-CMCF adducts

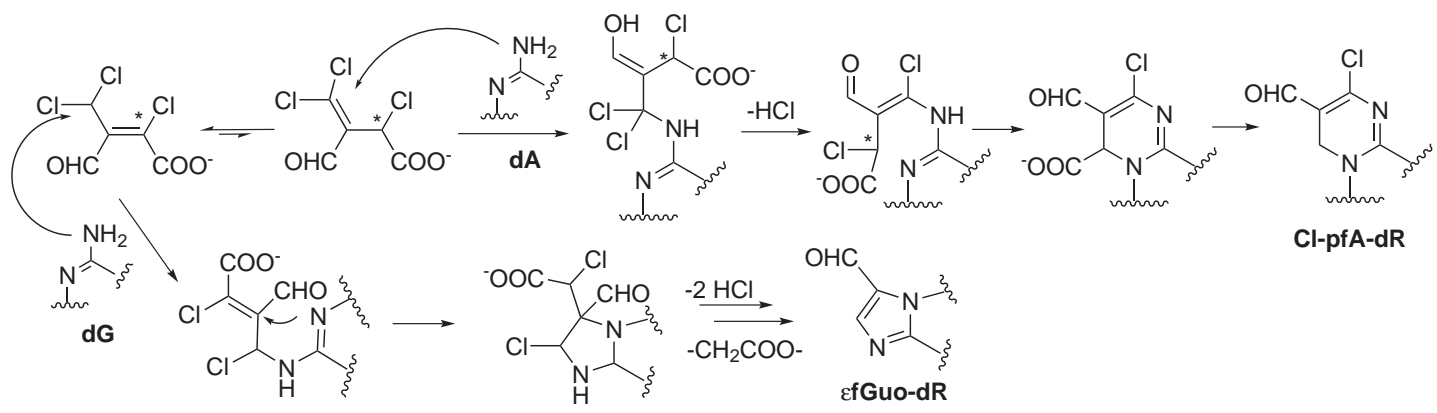

Scheme 5.12. Proposed mechanisms for the formation of nucleotide-MX adducts 


\subsection{Materials \& methods}

\subsubsection{Reactivity of halohydroxyhalofuranones}

\subsubsection{Calculation of hydration and hemiacetalization constants}

The computational calculation of equilibrium constants in solution is very demanding. It may be seen from the thermodynamic definition of $K$ that an error of $5.7 \mathrm{~kJ} \mathrm{~mol}^{-1}$ in $\Delta G^{\ominus}$ results in a deviation of one logarithmic unit in $K$.

$$
\log K=-\frac{\Delta G^{\ominus}}{\ln 10 R T}
$$

As was the case with $\mathrm{p} K_{\mathrm{a}}$ calculations, gas-phase free energies are usually calculated using high accuracy ab initio methods, and solution energies are calculated using Self-Consistent Reaction Field (SCRF) methods. In this work, carbonyl hydration and hemiacetalization constants were calculated from free energy differences between compounds using two different approaches (Scheme 5.13): an absolute and a relative approach.

$\mathrm{R}$ can be a hydrogen atom, in which case, hydration constants are computed, or an alkyl group, so that hemiacetalization constants are computed. $R_{1}$ and $R_{3}$ are alkyl groups, and depending on the nature of the $\mathrm{R}_{2}$ and $\mathrm{R}_{4}$ groups, addition constants were computed for adelhydes (-H), ketones (-Alkyl), esters (-O-Alkyl), thioesters (-S-Alkyl) and amides $\left(-\mathrm{N}-(\mathrm{R})_{2}\right)$.

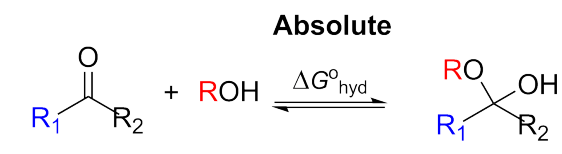

\section{Relative}

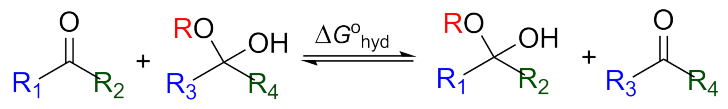

Scheme 5.13. Thermodynamic cycles for the determination of $K_{\text {hyd }}$ values

In the absolute approach the equilibrium constant is computed using equation 5.2

$$
\Delta G^{\ominus}=G_{\mathrm{R}}{ }^{1} \mathrm{C}(\mathrm{OH})(\mathrm{OR}) \mathrm{R}^{2}-G_{\mathrm{ROH}}-G_{\mathrm{R}^{1} \mathrm{COR}^{2}}
$$

In the relative approach the equilibrium constant is computed using equation 5.3

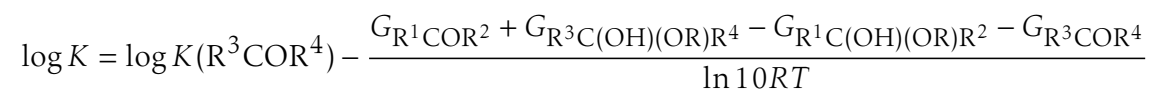


The rationale behind these methods is the same as in the calculation of acid dissociation constants: use of an experimental reference in an homodesmic reaction decreases systematic errors through favorable error cancellation. The method could also be applied to charged molecules, since the charge is present on both sides of the chemical equation and solvent effects are compensated.

The quality of the results in the relative approach depends strongly on the similarity between $\mathrm{R}^{1} \mathrm{COR}^{2}$ and $\mathrm{R}^{3} \mathrm{COR}^{4}$, and thus, we studied how the choice of references can influence the accuracy of the results. Two kinds of reference were used: either a common reference for all compounds (acetaldehyde), independently of their functionalization, or group references for each type of compound, with acetaldehyde for aldehydes, acetone for ketones and methyl acetate for carboxylic acid derivatives. In the case of hemiacetalization constants, since the equilibrium constant for acetone was not available, acetaldehyde and chloroacetone were used as group references. However, the lack of sufficient experimental data hinders the study of how this choice affects the results.

Calculations were performed at the DFT-B3LYP/6-31G++(d,p), MP2/6-31G++(d,p) and CBS-Q levels of theory, using the IEF-PCM method for solvation, with the default parameters of the software (UA0 radii). The Gaussian 03 suite of programs was used for all calculations.

\subsubsection{Equilibria of mucohalic acids}

In acidic aqueous solution, the major species is known to be the cyclic halofuranone, which suggests that in the ring-closure reaction $\left(K_{\mathrm{iso}}\right) \mathrm{MXA}_{\mathrm{cl}}$ is the predominant form. When $\mathrm{pH}$ is increased, $\mathrm{MXA}_{\mathrm{op}}^{\mathrm{O}}$ undergoes deprotonation, forming $\mathrm{MXA}_{\mathrm{op}}^{-}\left(K_{\mathrm{a}}\right)$, and the global equilibrium is shifted to the right. Thus, MXA $\mathrm{op}_{\mathrm{op}}^{\mathrm{O}}$ and its hydrate, $\mathrm{MXA}_{\mathrm{hyd}}^{\mathrm{O}}$, are expected to be minor species. In organic solvents, in which $\mathrm{MXA}_{\mathrm{op}}^{\mathrm{O}}$ cannot undergo dissociation, only the isomerization equilibrium is observed and $\mathrm{MXA}_{\mathrm{cl}}$ is always major.

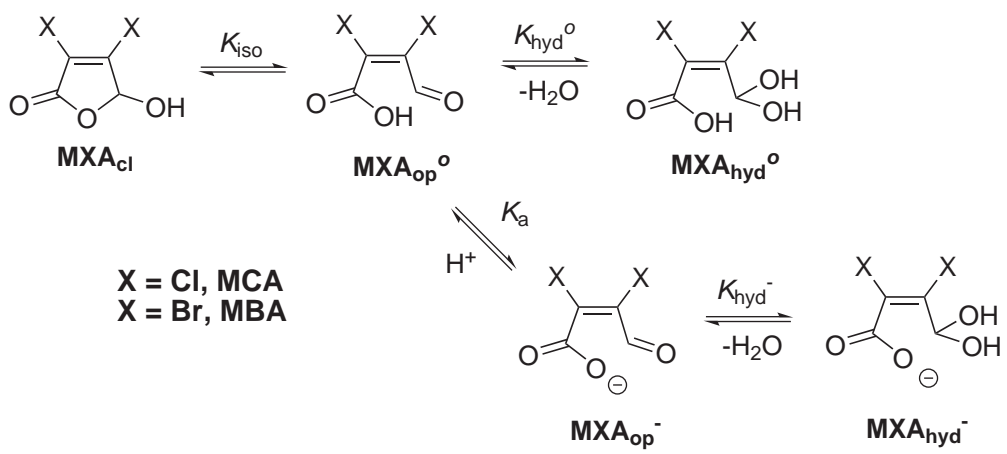

Scheme 5.14. Forms of mucohalic acids in equilibrium in aqueous solution.

\subsection{Experimental study.}

\subsection{Apparent acid dissociation constant. Methodology}


An apparent acid dissociation constant exists between protonated and unprotonated species:

$$
K_{\mathrm{a}}^{\mathrm{app}}=\left[\mathrm{H}^{+}\right] \frac{\left[\mathrm{MXA}_{\mathrm{op}}^{-}\right]+\left[\mathrm{MXA}_{\text {hyd }}^{-}\right]}{\left[\mathrm{MXA}_{\mathrm{cl}}\right]+\left[\mathrm{MXA}_{\mathrm{op}}^{\mathrm{o}}\right]+\left[\mathrm{MXA}_{\text {hyd }}^{\mathrm{o}}\right]}
$$

This can be rewritten in terms of the four microscopic equilibrium constants depicted in Scheme 5.14

$$
\begin{aligned}
K_{\mathrm{a}} & =\frac{\left[\mathrm{MXA}_{\mathrm{op}}^{-}\right]}{\left[\mathrm{MXA}_{\mathrm{op}}^{\mathrm{o}}\right]}\left[\mathrm{H}^{+}\right] \\
K_{\mathrm{iso}} & =\frac{\left[\mathrm{MXA}_{\mathrm{op}}^{\mathrm{o}}\right]}{\left[\mathrm{MXA}_{\mathrm{cl}}\right]} \\
K_{\text {hyd }}^{-} & =\frac{\left[\mathrm{MXA}_{\mathrm{hyd}}^{-}\right]}{\left[\mathrm{MXA}_{\mathrm{op}}^{-}\right]} \\
K_{\text {hyd }}^{o} & =\frac{\left[\mathrm{MXA}_{\text {hyd }}^{\mathrm{o}}\right]}{\left[\mathrm{MXA}_{\mathrm{op}}^{\mathrm{o}}\right]}
\end{aligned}
$$

as

$$
K_{\mathrm{a}}^{\mathrm{app}}=K_{\mathrm{a}} \frac{1+K_{\text {hyd }}^{-}}{1+K_{\text {hyd }}^{o}+1 / K_{\text {iso }}}
$$

Since all the equilibria are relatively fast, the UV-Vis spectra obtained in the spectrophotometric titration of MXA correspond to the equilibrium mixtures and eq. 5.4 can be expressed as eq. 5.10 can be written. $\epsilon_{\text {acid }}$ and $\epsilon_{\text {bas }}$ stand for the combined absortion of all species present in acidic and basic medium, respectively, and $\epsilon$ is the measured absorbance at each $\mathrm{pH}$. Fitting of the spectroscopic data to eq 5.10 affords $K_{\mathrm{a}}^{\text {app }}$.

$$
\mathrm{p} K_{\mathrm{a}}^{\mathrm{app}}=\mathrm{pH}-\log \frac{\epsilon-\epsilon_{\mathrm{bas}}}{\epsilon_{\mathrm{acid}}-\epsilon}
$$

${ }^{1} \mathrm{H}$ NMR spectroscopy is more sensitive than UV-Vis spectrophotometry to faster equilibria. Whereas the ring-chain tautomerization reaction is very rapid, and an average signal corresponding to $\mathrm{MXA}_{\mathrm{op}}^{\mathrm{O}}$, $\mathrm{MXA}_{\mathrm{cl}}$ and $\mathrm{MXA}_{\mathrm{op}}^{-}$is observed; the hydration equilibria are slower, and the signals of the hydrate forms appear as separate, easily resolvable, peaks. Therefore, the equilibrium constant measured by nuclear magnetic resonance (NMR) titration is that of the faster equilibrium between $\mathrm{MXA}_{\mathrm{cl}}$ and $\mathrm{MXA}_{\mathrm{op}}^{-}$, hydrate forms being excluded (eq. 5.11).

$$
\mathrm{p} K_{\mathrm{NMR}}^{\mathrm{app}}=-\log \frac{K_{\mathrm{a}}}{1+1 / K_{\mathrm{iso}}}=\mathrm{pH}-\log \frac{\delta-\delta_{\text {bas }}}{\delta_{\mathrm{acid}}-\delta}
$$




\section{EXPERIMENTAL PROCEDURE}

MCA (99\%) and MBA (99\%) were purchased from Aldrich. UV-Vis spectra measurements were carried out on a Shimadzu UV-2401-PC spectrophotometer equipped with a thermoelectric six-cell holder temperature control system $\left( \pm 0.1^{\circ} \mathrm{C}\right)$. The temperature of the reaction mixtures was kept constant $( \pm$ $0.05{ }^{\circ} \mathrm{C}$ ) with a Lauda Ecoline RE120 thermostat. ${ }^{1} \mathrm{H}$ NMR spectra were recorded with a Brucker 400 $\mathrm{MHz}$ in $\mathrm{D}_{2} \mathrm{O}$. Spectrophotometric titration of the MXA was carried out by adding concentrated $\mathrm{HCl}$ dropwise to $200 \mathrm{~cm}^{3}$ MXA at $\sim 1 \times 10^{-4} \mathrm{M}$, starting at $\mathrm{pH} \sim 7$. Because the total added volume did not exceed $1.0 \mathrm{~cm}^{3}$, the effect of dilution was not taken into account. Ionic strength was fixed at $0.10 \mathrm{M}$ withNaNO${ }_{3}$.

5.2.1.2.1.2. Hydration constant. The experimental determination of the hydration constants of the two mucohalic acids in neutral aqueous solution was performed as suggested in the literature. ${ }^{178,179}$ Briefly, solutions of mucohalic acids $(0.15 \mathrm{M})$ were prepared in $\mathrm{D}_{2} \mathrm{O}$, the $\mathrm{pH}$ values of these were adjusted to approximately 7.0 by the addition of small amounts of concentrated sodium hydroxide in $\mathrm{D}_{2} \mathrm{O}$, and their ${ }^{1} \mathrm{H}$ NMR were recorded at $25{ }^{\circ} \mathrm{C}$ using a Brucker $400 \mathrm{MHz}$ apparatus. By dividing the area of the peak corresponding to the hydrate $\left(\right.$ Area $_{\text {hyd }}$ ) by the area of the main peak ( Area $_{\text {MXA }}$ ), which corresponds to un-hydrated MXA ( $\mathrm{MXA}_{\mathrm{op}}^{-}$), it is possible to calculate the hydration constants.

$$
K_{\text {hyd }}^{\text {MXA }}=K_{\text {hyd }}^{-}=\frac{\left[\text { MXA }_{\text {hyd }}^{-}\right]}{\left[\text {MXA }_{\text {op }}^{-}\right]}=\frac{\text { Area }_{\text {hyd }}}{\text { Area }_{\text {MXA }}}
$$

5.2.1.2.2. Theoretical study. Since the open-chain tautomers of halogenated furanones are strong acids (due to the inductive and conjugative effects of halogen atoms and the double bond, respectively) the species of interest are anionic at the working $\mathrm{pH}$, which hinders the use of the easily available models such as SPARC (SPARC Performs Automated Reasoning in Chemistry) to predict their properties, and $a b$ initio or DFT computational approaches are needed.

5.2.1.2.2.1. Ring-opening constant. Applying the thermodynamic definition of the equilibrium constant to $K_{\text {iso }}$, eq 5.13 is obtained

$$
\mathrm{p} K_{\mathrm{iso}}=-\log K_{\mathrm{iso}}=\frac{\Delta G_{\mathrm{iso}}}{R T \ln 10}=\frac{G_{\mathrm{MXA}_{\mathrm{op}}^{o}}-G_{\mathrm{MXA}_{\mathrm{cl}}}}{R T \ln 10}
$$

5.2.1.2.2.2. Acid dissociation constant. The difficulties associated with the accurate determination of acid dissociation constants have already been described (vide supra). In this case, the relative method was used, so that the $\mathrm{p} K_{\mathrm{a}}$ values of $\mathrm{MXA}_{\mathrm{op}}^{o}$ were computed using equations 5.14 and 5.15. Tricholoroacetate was chosen as a reference $\left(\mathrm{p} K_{\mathrm{a}}(\mathrm{BH})=0.7\right)$. 


$$
\begin{aligned}
\mathrm{p} K_{\mathrm{a}}(\mathrm{AH}) & =-\log K_{\mathrm{a}}(\mathrm{AH})=\mathrm{p} K_{\mathrm{a}}(\mathrm{BH})+\frac{\Delta G_{\mathrm{pK}}^{\ominus}}{R T \ln 10} \\
\Delta G_{\mathrm{pK}}^{\ominus} & =G(\mathrm{BH})+\left(\mathrm{A}^{-}\right)-G\left(\mathrm{~B}^{-}\right)-G(\mathrm{AH})
\end{aligned}
$$

5.2.1.2.2.3. Computational details. All calculations were performed using Gaussian 03. Reaction free energies were obtained at the DFT-B3LYP 6-31++G(d,p), ${ }^{180,181}$ DFT-B3LYP 6-31++G(2df,2pd), MP2 6-31++G(d,p), CBS-QB3, and G3MP2B3 levels of theory and all structures were checked to be true minima (zero imaginary frequencies). The presence of two halogen atoms, containing many electrons, and especially in the case of bromine, hinders the use of larger basis sets or higher levels of theory. The effect of solvation on structure and energy was computed via the SCRF keyword, using the Gaussian 03 default Integral Equation Formalism for the Polarizable Continuum Model (IEF-PCM). ${ }^{182-184}$

\subsubsection{Hydrolysis of mucohalic acids}

5.2.1.3.1. Experimental study. Mucochloric acid (99\%), mucobromic acid (99\%) and $\mathrm{D}_{2} \mathrm{O}$ were purchased from Aldrich. $\mathrm{NaOH}(99 \%)$ was from Panreac. Water was purified using a MiliQ system.

Reactions were carried in $\mathrm{NaOH}$ solutions ( $\mathrm{pH} 11$ - 13.5) that had been previously normalized with potassium hydrogen phthalate. The final concentration of MXA was in the $1 \times 10^{-5}-1 \times 10^{-4} \mathrm{M}$ range and the applied concentration of sodium hydroxide was between 0.01 and $0.50 \mathrm{M}$ for MCA, and 0.005 and $0.10 \mathrm{M}$ for MBA. Reactions were monitored between 15 and $35^{\circ} \mathrm{C}$. The experimental procedure was as follows: $50 \mathrm{ml}$ of MXA $\left(5 \times 10^{-4}-5 \times 10^{-3} \mathrm{M}\right)$ in slightly acidic aqueous solution $(\mathrm{pH} \sim 4.5)$ was delivered with a Hamilton syringe to a Hellma quartz UV-Vis cuvette $\left(10.0 \mathrm{~mm}\right.$ optical length) containing $3 \mathrm{~cm}^{3}$ of the thermostated $\mathrm{NaOH}$ solution. Immediately after the mixture was complete, data acquisition was started. Reaction kinetics were monitored at $\lambda=225$ and $290 \mathrm{~nm}$ for MCA and $\lambda=275 \mathrm{~nm}$ for MBA. UVVis spectra and kinetic measurements were carried out on a Shimadzu UV-2401-PC spectrophotometer equipped with a thermoelectric six-cell holder temperature control system $\left( \pm 0.1^{\circ} \mathrm{C}\right)$. The temperature of the reaction mixtures prior to mixing was kept constant $\left(0.05^{\circ} \mathrm{C}\right)$ with a Lauda Ecoline RE120 thermostat. The UV-Vis, ${ }^{1} \mathrm{H}$ NMR and mass spectra of the reaction products were consistent with those reported for MOXA in the literature.

\subsubsection{Reactivity of MXY}

MX and related furanones exist in aqueous solution as a quite complex mixture (Scheme 5.15). Like other hydroxyhalofuranones, they undergo ring-chain tautomerism, and the formed acid undergoes dissociation in neutral and basic medium. MX in its open-chain forms is also known to undergo base-catalyzed isomerization between the $Z$ and $E$ forms. Also, based on the structures of the DNA adducts, the reaction of MX and CMCF with DNA bases has been suggested to occur through a $\alpha, \beta$-unsaturated MX isomer, 
in which the double bond is in a terminal position, this species being the proposed effective alkylating agent.
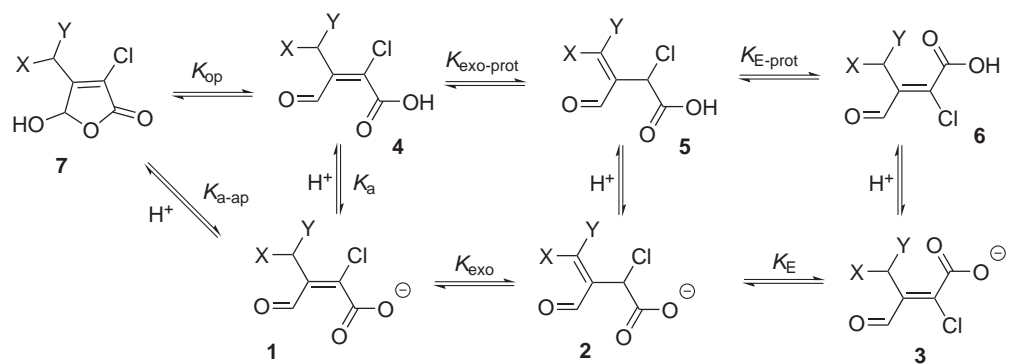

Scheme 5.15. Forms of MX and analogues in equilibrium in aqueous solution.

MX has been proposed to oxididize DNA, and thus we have computed the electron affinities, which are highly related to the one-electron reduction potential, for MXY.

5.2.1.4.1. Equilibrium constants. We have calculated the free energies in solution of compounds 1-7 at different levels of theory, and by using the definition of equilibrium constant we have computed the values for the equilibrium constants of the processes shown in Scheme 5.15.

Calculation of pKa values. The aforementioned approach is likely to introduce systematic errors in $G$, mainly in the solvation energies, since short-range solvent-solute interaction such as hydrogen bonding are not taken into account. If free energy differences are computed between similar species, such as, for instance $1,2,3$ or 4, 5, 6, these systematic deviations tend to cancel out. However, errors are not favorably compensated if the species at both sides of the chemical equation have very different solvation patterns, such as for instance, when one species is charged and the other is not.

As stated above, this is a very common problem in the calculation of $\mathrm{p} K_{\mathrm{a}}$ values, and convenient solutions exist: the use of relative methods, or addition of explicit solvent molecules to accurately determine solvation energies. We have opted for the use of a relative approach, using a homodesmic reaction. Trichloroacetic acid $\left(\mathrm{p} K_{\mathrm{a}}=0.7\right)$ was used as a reference for its structural similarities with open-chain halofuranones.

An apparent acid dissociation constant exists between protonated and unprotonated species:

$$
K_{\mathrm{a}}^{\mathrm{app}}=\left[\mathrm{H}^{+}\right] \frac{[1]+[2]+[3]}{[4]+[5]+[6]+[7]}
$$

And also several acid dissociation and isomerization equilibria exist, some of which are governed by the following equilibrium constants: 


$$
\begin{aligned}
K_{\mathrm{a}} & =\frac{[1]}{[4]}\left[\mathrm{H}^{+}\right] \\
K_{\mathrm{op}} & =\frac{[4]}{[7]} \\
K^{\text {exo-prot }} & =\frac{[5]}{[4]} \\
K^{\text {exo }} & =\frac{[2]}{[1]} \\
K^{\mathrm{E}-\text { prot }} & =\frac{[6]}{[5]} \\
K^{\mathrm{E}} & =\frac{[3]}{[2]}
\end{aligned}
$$

5.2.1.4.2. Electron affinities. Vertical electron affinities were calculated as the free energy difference of the starting molecule and a radical anion with same geometry. Adiabatic electron affinities were obtained using the equilibrium geometry of the radical anion.

5.2.1.4.3. Computational details. Geometries were optimized and free energies were computed using the B3LYP hybrid functional with the $6-31++G(d, p)$ and $6-31++G(2 d f, 2 p d)$ basis sets, at the MP2/6$31++\mathrm{G}(\mathrm{d}, \mathrm{p})$ level and also using compound methods CBS-QB3 and G3MP2B3. To compute electron affinities the B3LYP hybrid functional was used with $6-31 G(d), 6-31++G(d, p)$ and $6-311+G(2 d f, 2 p)$ basis sets. Post-HF and compound methods (CBS-QB3 and G2) were also assayed, but the presence of large spin contamination made the results unreliable.

\subsubsection{Alkylating potential of halohydroxyfuranones}

\subsubsection{Alkylating potential of mucohalic acids}

Reactions were monitored by UV-Vis spectroscopy in a Shimadzu UV2401 PC with a thermoelectric sixcell holder temperature control system $\left( \pm 0.1^{\circ} \mathrm{C}\right)$. The reactions were carried out in an excess of nucleophile, and thus the pseudo-first-order approximation was applied. $\mathrm{pH}$ was kept constant by using buffer solutions: $0.020 \mathrm{M} \mathrm{CH}_{3} \mathrm{COOH} / \mathrm{CH}_{3} \mathrm{COO}^{-}$for $4<\mathrm{pH}<6$ and $0.020 \mathrm{M} \mathrm{H}_{2} \mathrm{PO}_{4}^{-} / \mathrm{HPO}_{42}^{-}$for $6<\mathrm{pH}<8$. Electrospray ionization mass spectra were recorded on a Waters ZQ4000 spectrometer, by direct injection.

5.2.2.1.1. Reaction with aniline. Reactions of the electrophiles with aniline (AN) were carried out in water, and in 7:3 (vol.) water/dioxane $(w / d)$ mixtures. The kinetic species was monitored at $\lambda=320 \mathrm{~nm}$ for both MBA and MCA, where aniline shows almost null absorption. The concentration of MXA was in the $7-50 \mu \mathrm{M}$ range and that of aniline was in the $1-20 \mathrm{mM}$ range. 
5.2.2.1.2. Reaction with NBP. Since the adduct formed in the alkylation of NBP by MXA shows significant absorption in the visible region of the spectrum, a variation of the NBP test was used in which basification of the reaction media to obtain color was not necessary, as occurred in the alkylation of NBP by DIK. Thus, the alkylation reaction was monitored continuously in the reaction medium at $\lambda=485 \mathrm{~nm}$ for MBA and $\lambda=510 \mathrm{~nm}$ for MCA. The MXA concentration was in the $30-200 \mu \mathrm{M}$ and that of NBP in the $5-20 \mathrm{mM}$ range. Because NBP is insoluble in water, reactions were carried out only in aquo-organic medium (7:3 w/d mixtures).

5.2.2.1.3. Computational study of nucleobase alkylation. The reaction of MXA with nucleobases was studied theoretically. Geometries were optimized at the B3LYP/6-31G(d) level, and were characterized as either minima or transition states by harmonic analysis. Solvent effects were included using the IEF-PCM method with default parameters. Calculations were performed using the Gaussian 03 suite of programs.

\subsubsection{Alkylating potential of $M X Y$}

5.2.2.2.1. Reaction pathway. The alkylation mechanism of adenosine and guanosine by MXY was modeled using density functional theory. All minima on the potential energy hypersurface were characterized by harmonic analysis (zero imaginary frequencies for reactants and one for transition states) and the computed frequencies were used to obtain thermodynamic parameters. The polarizable continuum model was used, to take into account the effect of aqueous solvent.

Vertical electron affinities were calculated as the free energy difference of the starting molecule and a radical anion with same geometry. Adiabatic electron affinities were obtained using the equilibrium geometry of the radical anion.

5.2.2.2.2. Computational details. Geometries were optimized at the DFT-B3LYP 6-31G(d) level, and so were electron affinities. In cases in which more precision was needed, the $6-31++G(d, p)$ level of theory was used. Solvent was taken into account using the Integral Equation Formalism Polarizable Continuum Model (IEF-PCM). The Gaussian 03 suite of programs was used for all calculations. 


\subsection{Results and discussion}

\subsubsection{Reactivity of halohydroxyhalofuranones}

The study of the aqueous reactivity of hydroxyhalofuranones comprises their acid-base, hydration and isomerization equilibria, and their hydrolysis and decomposition reactions as well. Since carbonyl compounds are known to undergo hydration, and highly aldehydes ones exist almost uniquely in their gemdiol form, the ability to measure and compute hydration constants for the open-chain forms of halohydroxyfuranones is of paramount importance.

\subsubsection{Calculation of hydration and hemiacetalization constants}

5.3.1.1.1. Hydration constants. The study of the hydration equilibria of aldehydes and other carbonyl compounds began more than a century ago, following the observation that the hydrate form amounts for a significant fraction of the total compound; e.g. less than $0.1 \%$ of formaldehyde is present as the carbonyl compound in aqueous solution. Ketones and other carbonyl compounds also add a water molecule to form gemdiols, although the reaction is usually less favored.

The hydration reaction represents one of the simplest additions to the carbonyl group. Since the formation of a tetrahedral intermediate is a step in the most common hydrolysis mechanisms $\left(\mathrm{A}_{\mathrm{AC}} 2\right.$ and $\mathrm{B}_{\mathrm{AC}} 2$, vide supra), the hydration of carboxylic acid derivatives, such as esters, thioesters, and amides, is of mechanistic importance. Hemiacetalization, as well, is a kinetic step in certain alcoholysis mechanisms.

Much attention has been devoted to the measurement and calculation of hydration rates. ${ }^{185-188} \mathrm{Hy}-$ dration free energies can be used to calculate hydration rate constants in neutral, acidic, and basic media, using multidimensional Marcus theory and the no-barrier theory. ${ }^{189,190}$

Aldehyde hydrates lack the electrophilic carbonyl function, and thus, are unable to react with nucleophiles such as DNA. Therefore the hydration of aldehydes is also significant in their role as alkylating and potentially mutagenic and carcinogenic agents, e.g., 99.997\% of chloral is in the hydrate form, which significantly reduces its potential reactivity as an electrophile, and this in turn influences its genotoxic potential. ${ }^{191-197}$

Because carbohydrates show intramolecular hemiacetal equilibria, and since the reactivity of aldehydes as alkylating agents may be strongly influenced by the formation of cyclic and linear hemiacetals, hemiacetal formation is also of biological importance.

Hydration and hemiacetalization equilibrium constants have been measured with a variety of methods, most commonly UV and NMR spectroscopy, in which ${ }^{1} \mathrm{H},{ }^{13} \mathrm{C},{ }^{17} \mathrm{O}$, and ${ }^{19} \mathrm{~F}$ have been used. ${ }^{178,179,198}$ Since the sensitivity of NMR spectroscopy makes it difficult to measure very displaced equilibria directly, and the use of UV spectroscopy forces the assumption that the molar absorption coefficient is solvent independent (which may imply significant error), ${ }^{199}$ the experimental determination of hydration 
and hemiacetalization equilibrium constants is complex. Indirect methods are often used: linear free energy relationships, equilibrium constant extrapolations, acetalization constants or hemiacetalization constants, calculation of formation and solvation free energies, and so forth.

The functionalization of halohydroxyfuranones is expected to enhance the electrophilicity of their aldehyde group, in the light of the strong inductive and mesomer effects of the halogen atoms, and the unsaturation and carboxylate group, respectively. Thus, hydration of the major and minor open-chain forms could play an important role in their in vivo reactivity and thus, condition their genotoxicity. No first-principle computational methods exist for the calculation of hydration constants, so, in a close parallel to the methodology used in the calculation of acid dissociation constants, a method for the calculation of hydration equilibrium constants has been developed.

Hydration free energies are only available for few compounds, and thus, only a narrow range of choice is available. The molecules studied (Figure 5.1) comprise a wide range of $K_{\text {hyd }}$ values of almost 20 logarithm units. Different types of carbonyl compounds were included: aldehydes, ketones, and carboxylic acid derivatives (esters, thioesters, and amides).

\section{Aldehydes}

ㅇ<smiles>O=CCC(=O)C(Cl)(Cl)CC=O</smiles><smiles>CCCC(=O)CC</smiles><smiles>CCC(Cl)C=O</smiles><smiles>CCC(Br)C=O</smiles><smiles>CC(C)(Cl)C=O</smiles><smiles>CC(C)(C)C=O</smiles><smiles>C/C=C/C=O</smiles>

\section{Esters}<smiles>COC=O</smiles><smiles>COC(=O)C(F)C(F)Cl</smiles><smiles>COC(=O)C(F)(F)F</smiles>

(a) Acting<smiles>CC(=O)CF</smiles><smiles>CC(=O)CCl</smiles><smiles>CC(=O)C(Cl)Cl</smiles><smiles>O=C1CCC1</smiles><smiles>CCC(=O)C(C)=O</smiles><smiles>COC(=O)C(C)=O</smiles>

Thioesters<smiles>CCSC(C)=O</smiles>

Amides<smiles>CC(=O)N(C)C=O</smiles>

Figure 5.1. Carbonyl compounds studied

Experimental $K_{\text {hyd }}$ values in the following order: aldehydes $>$ ketones $>$ (thio)esters $>$ amides. It can also be observed that electron-withdrawing substituents on the $\alpha$ carbons increase the hydration constants, whereas electron-donating groups, such as alkyl substituents have the opposite effect.

Figure 5.2 shows the hydration constants obtained at different levels of theory, using the absolute method described above, plotted against the respective experimental values. Results show that:

i The values at the DFT-B3LYP/6-31++G(d,p) are well correlated, however, the slope shows significant deviation from unity $(\alpha=1.24 \pm 4)$

ii Correlation is slightly worse at the MP2/6-31++G(d,p) level but the slope is significantly improved $(\alpha=1.12 \pm 0.04)$. 
iii Results at the CBS-Q level show excellent correlation, and, more importantly, the value for the slope is improved, to the point of being significantly equal to unity $(\alpha=1.05 \pm 0.03)$
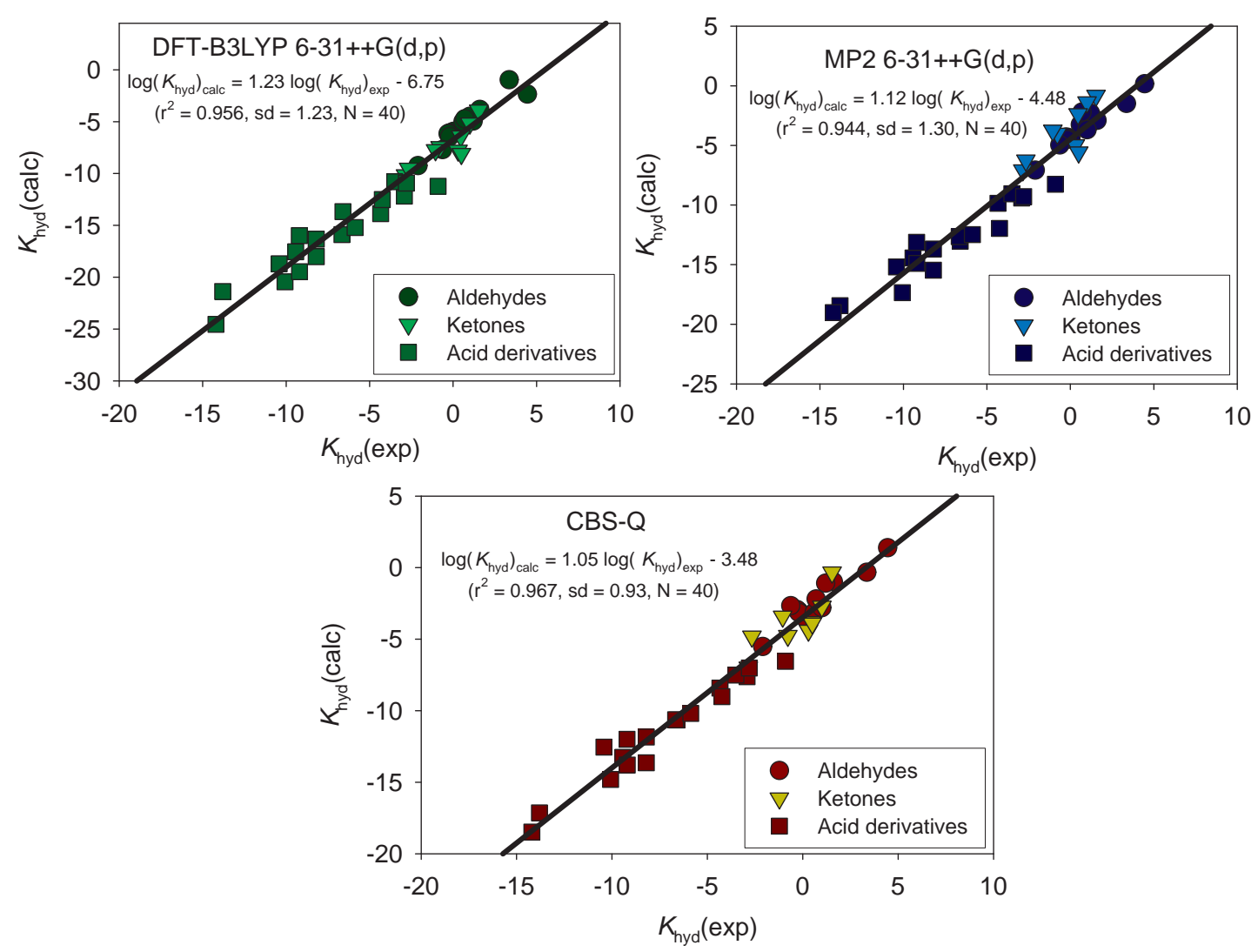

Figure 5.2. Calculated absolute $K_{\mathrm{Hyd}}$

All the calculations are affected by a significant systematic error, which corresponds with the large $y$-intercept obtained at the three levels of theory. This deviation is mostly attributable to errors in the solvation energy, and, as is the case in $\mathrm{p} K_{\mathrm{a}}$ calculations, it can be largely corrected by the use of a relative approach (vide infra). The choice of the reference molecules for the relative method (acetaldehyde, acetone, and methyl acetate) was based on their capacity to account for the whole of the $y$-intercept.

Table 5.6 shows the calculated $K_{\text {hyd }}$ values, obtained using a relative approach. As shown, different experimental references for each group were used: acetaldehyde for aldehydes, acetone for ketones, and methyl acetate for acid derivatives.

As was the case in $\mathrm{p} K_{\mathrm{a}}$ calculations, the use of a relative approach allows the compensation of systematic errors in the solvation energies. However, a minor tendency for calculated values to be larger than experimental ones can be observed (Table 5.6), this arises from the fact that the plots in Figure 5.2 have slopes larger than unity. The use of the complete calibration lines instead of just one single reference 
molecule would further correct this remaining systematic error, at the cost of reducing the applicability of this method to other groups of compounds or to hemiacetalization reactions (vide infra), for which experimental values are scarce.

It is also important to keep in mind that, in certain cases, the literature values are estimations, sometimes obtained by making excessively optimistic assumptions; this can lead to error in the experimental values. For instance, the high deviations observed for $\mathrm{CF}_{3} \mathrm{COCH}_{3}, \mathrm{CH}_{3} \mathrm{OCH}_{2} \mathrm{COOCH}_{3}$, and $\left(\mathrm{CH}_{3}\right)_{2} \mathrm{CHCOOCH}_{3}$ suggest that the experimental values may be affected by significant error.

Mean unsigned errors (mue) for results obtained the relative method with a common reference and with group references are shown in Table 5.7. Mue are in the order CBS-Q < MP2 < DFT-B3LYP; higher level methods give more accurate results and only at the CBS-Q level are mue below $1.0 \log$ units. All three methods afford mues of about $0.5 \mathrm{log}$ units for aldehydes. In the case of ketones, the DFT-B3LYP results are close to MP2, with the mue higher than $1.0 \log$ units. CBS-Q gives mue under $1.0 \log$ units. On considering carboxylic acid derivatives, it can be seen that the DFT-B3LYP results are very inaccurate, with mue over $2.5 \log$ units. The mue for MP2 is also over $1.5 \log$ units, whereas the CBS-Q remains under $1.0 \mathrm{log}$ units. The use of the mean of the three methods gives very good results for aldehydes and ketones, but the high mue of carboxylic acid derivatives using DFT-B3LYP is reflected in the mean values, making the global results inaccurate.

Switching to group references increases the accuracy of the method at the lower levels of theory because, as expected, the higher-level methods need less empirical correction. Thus, this effect is most important for the DFT-B3LYP results, especially with acid derivatives, which is now reflected in the global mue being under 1.0 logarithm units. The MP2 results are almost insensitive to the use of group references and generally the least accurate. The CBS-Q results for carboxylic acid derivatives are in fact worse than those obtained using the common reference, and this translates into an increased global mue. Since the DFT-B3LYP values are largely corrected with the use of group references, the values obtained with the mean of the three methods also improve. Only the results obtained at the CBS-Q level using the common reference show lower mue than these average values.

In sum, the CBS-Q results with the common reference are the most accurate for all types of compounds, outdoing DFT-B3LYP, MP2, and the mean results, with or without group references. Almost as accurate, but much cheaper in terms of CPU time and resources, is DFT-B3LYP 6-31++G(d,p) using group references. The mean values of the three methods are in general highly accurate due to error cancellation but very expensive computationally.

Thus, with the relative approach, the proposed methodology can predict hydration constants for aldehydes within $\pm 0.5 \log$ units on average, using any of the three levels of theory. The calculation of the hydration constants of ketones and carboxylic acid derivatives can be carried out on average within \pm 1.0 logarithm units using a group reference or the higher levels of theory.

5.3.1.1.2. Hemiacetalization constants. Using the same method, (Scheme 5.13), hemiacetalization constants were computed for some of the compounds with both ethanol and methanol as solvents. Since 
Table 5.6. Experimental and calculated relative hydration constants using group references

\begin{tabular}{|c|c|c|c|c|c|}
\hline & \multicolumn{5}{|c|}{$-\log K_{\text {hyd }}$} \\
\hline & \multirow[t]{2}{*}{$\begin{array}{c}\text { DFT-B3LYP } \\
6-31 G++(d, p)\end{array}$} & \multirow{2}{*}{$\begin{array}{c}\text { MP2 } \\
6-31 G++(d, p) \\
\text { Aldehydes }\end{array}$} & \multirow[t]{2}{*}{ CBS-Q } & \multirow[t]{2}{*}{ Average } & Experimental \\
\hline & & & & & \\
\hline $\mathrm{CH}_{2} \mathrm{O}$ & -5.00 & -2.94 & -3.13 & $-3.69 \pm 1.14$ & $-3.36^{200,20}$ \\
\hline $\mathrm{CH}_{3} \mathrm{CHO}$ & REF & REF & REF & REF & $-0.03^{200,202}$ \\
\hline $\mathrm{CH}_{2}^{3} \mathrm{ClCHO}$ & -2.14 & -1.52 & -2.48 & $-2.05 \pm 0.49$ & $-1.6^{200}$ \\
\hline $\mathrm{CCl}_{3} \mathrm{CHO}$ & -3.62 & -4.59 & -4.85 & $-4.35 \pm 0.65$ & $-4.45^{200,203}$ \\
\hline $\mathrm{CH}_{3} \mathrm{CH}_{2} \mathrm{CHO}$ & 0.12 & -0.11 & -0.40 & $-0.13 \pm 0.26$ & $0.20^{200,201}$ \\
\hline $\mathrm{CH}_{3} \mathrm{CH}_{2} \mathrm{CH}_{2} \mathrm{CHO}$ & 0.19 & -0.09 & -0.53 & $-0.14 \pm 0.36$ & $0.30^{200,20}$ \\
\hline$\left(\mathrm{CH}_{3}\right)_{2} \mathrm{CHCHO}$ & 0.48 & 0.09 & -0.33 & $0.08 \pm 0.40$ & $0.21^{200,20}$ \\
\hline $\mathrm{CH}_{3} \mathrm{CH}_{2} \mathrm{CHClCHO}$ & -1.02 & -2.13 & -2.37 & $-1.84 \pm 0.72$ & $-1.2^{205}$ \\
\hline $\mathrm{CH}_{3} \mathrm{CH}_{2}^{2} \mathrm{CHBrCHO}$ & -0.98 & -1.16 & -0.34 & $-0.83 \pm 0.43$ & $-0.6^{205}$ \\
\hline $\mathrm{CH}_{3} \mathrm{CH}_{2} \mathrm{CBr}_{2} \mathrm{CHO}$ & -1.47 & -0.74 & -0.65 & $-0.95 \pm 0.45$ & $-1.0^{205}$ \\
\hline$\left(\mathrm{CH}_{3}\right)_{2} \mathrm{CClCHO}$ & -1.24 & -2.22 & -1.27 & $-1.58 \pm 0.56$ & $-0.70^{205}$ \\
\hline$\left(\mathrm{CH}_{3}\right)_{3} \mathrm{CCHO}$ & 1.74 & 0.55 & -0.79 & $0.50 \pm 1.27$ & $0.63^{200,201}$ \\
\hline $\mathrm{PhCHO}$ & 3.28 & $\begin{array}{l}2.66 \\
\text { Ketones }\end{array}$ & 2.05 & $2.66 \pm 0.61$ & $2.1^{206}$ \\
\hline $\mathrm{CH}_{3} \mathrm{COCH}_{3}$ & REF & REF & REF & REF & $2.85^{189,20}$ \\
\hline $\mathrm{CH}_{2} \mathrm{FCOCH}_{3}$ & 0.13 & -0.22 & 0.64 & $0.18 \pm 0.43$ & $0.78^{204}$ \\
\hline $\mathrm{CF}_{3} \mathrm{COCH}_{3}$ & -4.30 & -3.40 & -3.80 & $-3.53 \pm 0.23$ & $-1.54^{207}$ \\
\hline $\mathrm{CH}_{2} \mathrm{ClCOCH}_{3}$ & 0.43 & -0.48 & -0.71 & $-0.26 \pm 0.60$ & $1.05^{204}$ \\
\hline $\mathrm{CH}_{2}^{2} \mathrm{ClCH}_{2} \mathrm{Cl}^{3}$ & -2.14 & -2.85 & -1.41 & $-2.13 \pm 0.72$ & $-1.0^{178}$ \\
\hline $\mathrm{CHCl}_{2} \mathrm{COCH}_{3}$ & -0.90 & -1.86 & -0.33 & $-1.03 \pm 0.77$ & $-0.46^{178}$ \\
\hline Cyclobutanone & 2.21 & 2.03 & 0.69 & $1.64 \pm 0.83$ & $2.66^{208}$ \\
\hline $\mathrm{CH}_{3} \mathrm{COCOCH}_{3}$ & 0.47 & 0.74 & 0.27 & $0.49 \pm 0.23$ & $-0.3^{205}$ \\
\hline $\mathrm{CH}_{3} \mathrm{COCOOCH}_{3}$ & 0.79 & 1.35 & -0.23 & $-0.64 \pm 0.80$ & $-0.41^{204}$ \\
\hline & & Esters 209 & & & \\
\hline $\mathrm{HCOOCH}_{3}$ & 8.20 & 5.76 & 5.21 & $6.39 \pm 1.59$ & 6.6 \\
\hline $\mathrm{CH}_{3} \mathrm{COOCH}_{3}$ & REF & REF & REF & REF & 8.2 \\
\hline $\mathrm{CH}_{2} \mathrm{ClCOOCH}_{3}$ & 6.08 & 5.39 & 5.20 & $5.56 \pm 0.46$ & 6.66 \\
\hline $\mathrm{CHCl}_{2} \mathrm{COOCH}_{3}$ & 4.06 & 2.59 & 2.98 & $3.21 \pm 0.76$ & 4.34 \\
\hline $\mathrm{CHF}_{2} \mathrm{COOCH}_{3}{ }^{3}$ & 2.36 & 2.14 & 2.19 & $2.23 \pm 0.12$ & 2.92 \\
\hline $\mathrm{CF}_{3} \mathrm{COOCH}_{3}$ & 1.41 & 0.99 & 1.11 & $1.17 \pm 0.22$ & 0.97 \\
\hline $\mathrm{CCl}_{3} \mathrm{COOCH}_{3}$ & 2.70 & 4.70 & 3.58 & $3.66 \pm 1.00$ & 4.24 \\
\hline $\mathrm{CH}_{3} \mathrm{CH}_{2} \mathrm{COOCH}_{3}$ & 7.73 & 7.17 & 7.82 & $7.57 \pm 0.35$ & 9.43 \\
\hline $\mathrm{NCCH}_{2} \mathrm{COOCH}_{3}{ }^{3}$ & 5.40 & 5.22 & 4.75 & $5.12 \pm 0.34$ & 5.87 \\
\hline $\mathrm{CH}_{3} \mathrm{OCH}_{2} \mathrm{COOCH}_{3}$ & 6.18 & 5.87 & 6.56 & $6.20 \pm 0.35$ & 9.21 \\
\hline$\left(\mathrm{CH}_{3}\right)_{2} \mathrm{CHCOOCH}_{3}$ & 8.89 & 7.92 & 7.11 & $7.97 \pm 0.89$ & 10.42 \\
\hline $\mathrm{PhCOOCH}_{3}$ & 10.63 & 10.09 & 10.03 & $9.43 \pm 0.64$ & 10.07 \\
\hline & & Thiosters 210 & & & \\
\hline $\mathrm{HCOSC}_{2} \mathrm{H}_{5}$ & 0.98 & 1.79 & 2.07 & $1.61 \pm 0.57$ & 3.5 \\
\hline $\mathrm{CH}_{3} \operatorname{COSC}_{2} \mathrm{H}_{5}$ & 6.52 & 6.42 & 6.40 & $6.45 \pm 0.06$ & 8.2 \\
\hline $\mathrm{CF}_{3} \mathrm{COSC}_{2} \mathrm{H}_{5}$ & 1.11 & 2.04 & 1.59 & $1.58 \pm 0.46$ & 2.8 \\
\hline & & Amides 210 & & & \\
\hline $\operatorname{HCON}\left(\mathrm{CH}_{3}\right)_{2}$ & 11.57 & 11.18 & 11.70 & $11.48 \pm 0.27$ & 13.8 \\
\hline $\mathrm{CH}_{3} \mathrm{CON}\left(\mathrm{CH}_{3}\right)_{2}$ & 14.73 & 11.76 & 13.04 & $13.18 \pm 1.49$ & 14.2 \\
\hline $\mathrm{CF}_{3} \mathrm{CON}\left(\mathrm{CH}_{3}\right)_{2}$ & 9.66 & 7.63 & 8.36 & $8.55 \pm 1.03$ & 9.2 \\
\hline
\end{tabular}


Table 5.7. Mean unsigned errors in relative $K_{\text {hyd }}$ values

\begin{tabular}{|c|c|c|c|c|c|c|c|}
\hline & \multicolumn{7}{|c|}{ Mean unsigned error } \\
\hline & \multirow{2}{*}{$\frac{\text { Aldehydes }}{\mathrm{GR}^{a}}$} & \multicolumn{2}{|c|}{ Ketones } & \multicolumn{2}{|c|}{ Acid Deriv. } & \multicolumn{2}{|c|}{ Global } \\
\hline & & $\mathrm{CR}$ & GR & $\mathrm{CR}$ & GR & $\mathrm{CR}$ & GR \\
\hline DFT-B3LYP 6-31G++(d,p) & 0.57 & 1.11 & 0.79 & 2.84 & 1.19 & 1.70 & 0.90 \\
\hline MP2 6-31G++(d,p) & 0.41 & 1.32 & 1.23 & 1.58 & 1.38 & 1.15 & 1.08 \\
\hline CBS-Q & 0.56 & 0.93 & 0.83 & 0.92 & 1.32 & 0.80 & 1.00 \\
\hline Mean & 0.33 & 0.75 & 0.94 & 2.40 & 1.23 & 1.23 & 0.86 \\
\hline
\end{tabular}

${ }^{a} \mathrm{CR}$ - common reference; GR - group reference.

experimental hemiacetalization constants are scarce, only some compounds could be included. As is the case with hydration constants, aldehydes have higher $K_{\text {hyd }}$ values than ketones, since electron-withdrawing substituents on the $\alpha$ carbons increase the hemiacetalization constants, whereas electron-donating groups have the opposite effect.

Figure 5.3 shows the plot of hemiacetalization constants computed with the absolute method, against the respective experimental values. Comparison with Figure 5.2 shows that the quality of the results is inferior to those obtained for hydration constants. This is due in part to the scarcity of experimental values. In addition, these empirical benchmarks correspond mostly to alkyl aldehydes, and thus, tend to cluster around the same central value. As before, MP2 results show a slope closer to unity and better linearity.
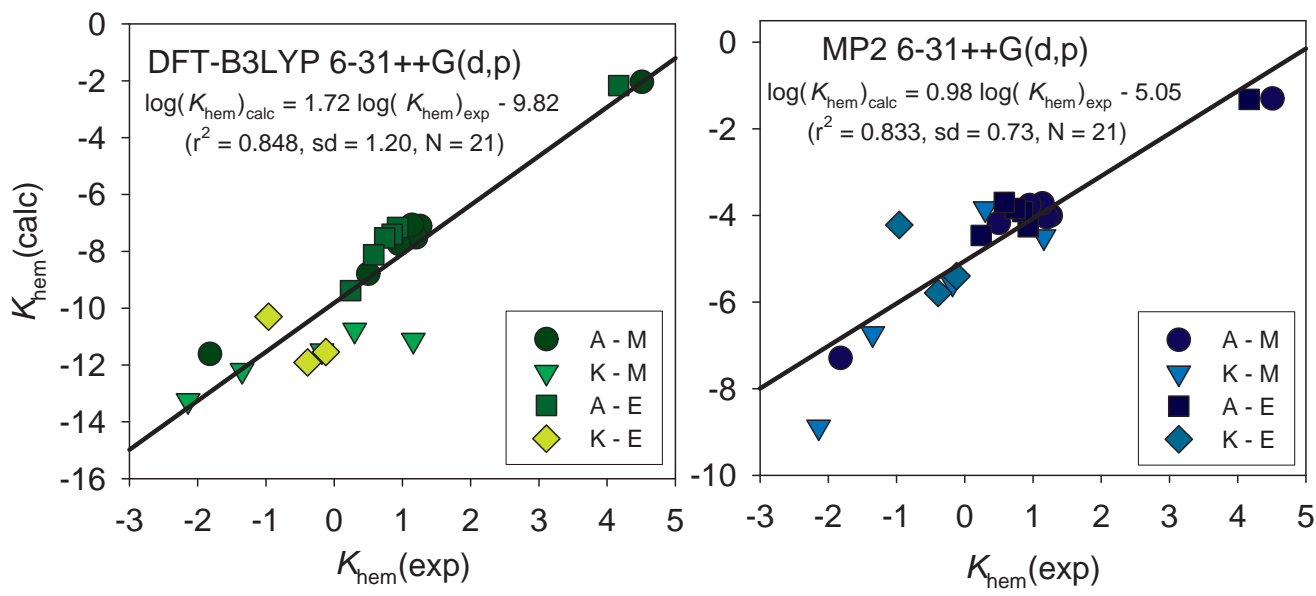

Figure 5.3. Calculated absolute $K_{\mathrm{Hem}}$

The existence of a large y-intercept can be corrected with the use of the relative method. Relative values, calculated using group references, are shown in Table 5.8. 
Table 5.8. Experimental and calculated relative hemiacetalization constants

\begin{tabular}{|c|c|c|c|c|}
\hline & \multicolumn{4}{|c|}{$\log K_{\text {hem }}$} \\
\hline & $\begin{array}{c}\text { DFT-B3LYP } \\
6-31 G++(d, p)\end{array}$ & $\begin{array}{c}\text { MP2 } \\
6-31 G++(d, p)\end{array}$ & Mean & Experimental \\
\hline \multicolumn{5}{|c|}{ In MethanoL 207} \\
\hline \multicolumn{5}{|c|}{ Aldehydes } \\
\hline $\mathrm{CH}_{2} \mathrm{O}$ & 6.32 & 3.96 & 5.14 & 4.51 \\
\hline $\mathrm{CH}_{3}^{2} \mathrm{CHO}$ & REF & REF & REF & $1.26^{208}$ \\
\hline $\mathrm{CH}_{3} \mathrm{CH}_{2} \mathrm{CHO}$ & 0.85 & 1.22 & 1.04 & 1.20 \\
\hline $\mathrm{CH}_{3} \mathrm{CH}_{2}^{2} \mathrm{CH}_{2} \mathrm{CHO}$ & 1.36 & 1.54 & 1.45 & $1.14^{208}$ \\
\hline$\left(\mathrm{CH}_{3}\right)_{2} \mathrm{CHCHO}$ & 0.62 & 1.50 & 1.06 & 0.95 \\
\hline$\left(\mathrm{CH}_{3}\right)_{3} \mathrm{CCHO}$ & -0.43 & 1.09 & 0.33 & 0.50 \\
\hline $\begin{array}{c}\mathrm{CH}_{3} \mathrm{CH}=\mathrm{CHCHO} \\
\text { Ketones }\end{array}$ & \multicolumn{4}{|c|}{ Ketones } \\
\hline $\mathrm{CH}_{3} \mathrm{COCH}_{3}$ & -1.93 & -3.50 & -2.72 & $-2.14^{208}$ \\
\hline $\mathrm{CH}_{2} \mathrm{ClCOCH}_{3}$ & REF & REF & REF & -0.18 \\
\hline $\mathrm{CH}_{2}^{2} \mathrm{ClCOCH}_{2}^{3} \mathrm{Cl}$ & 0.98 & 0.55 & 0.94 & 1.16 \\
\hline $\mathrm{CHCl}_{2} \mathrm{COCH}_{2}^{2} \mathrm{Cl}$ & 0.55 & 0.03 & 0.29 & 0.30 \\
\hline Cyclobutanone & -0.87 & -1.34 & -1.10 & $-1.35^{208}$ \\
\hline \multicolumn{5}{|c|}{ IN ETHANOL 207} \\
\hline \multicolumn{5}{|c|}{ Aldehydes } \\
\hline $\mathrm{CH}_{2} \mathrm{O}$ & 5.92 & 3.87 & 4.90 & 4.17 \\
\hline $\mathrm{CH}_{3}^{2} \mathrm{CHO}$ & REF & REF & REF & 0.93 \\
\hline $\mathrm{CH}_{3}^{3} \mathrm{CH}_{2} \mathrm{CHO}$ & 0.70 & 1.27 & 0.99 & 0.85 \\
\hline $\mathrm{CH}_{3} \mathrm{CH}_{2} \mathrm{CH}_{2} \mathrm{CHO}$ & 0.57 & 1.35 & 0.96 & 0.74 \\
\hline$\left(\mathrm{CH}_{3}\right)_{2} \mathrm{CHCHO}$ & -0.03 & 1.49 & 0.73 & 0.58 \\
\hline$\left(\mathrm{CH}_{3}\right)_{3} \mathrm{CCHO}$ & -1.28 & 0.73 & -0.28 & 0.24 \\
\hline \multicolumn{5}{|l|}{ Ketones } \\
\hline $\mathrm{CH}_{2} \mathrm{ClCOCH}_{3}$ & REF & REF & REF & -0.39 \\
\hline $\mathrm{CH}_{2} \mathrm{ClCOCH}_{2} \mathrm{Cl}$ & 1.22 & 1.18 & 1.20 & 0.96 \\
\hline $\mathrm{CHCl}_{2} \mathrm{COCH}_{2}^{2} \mathrm{Cl}$ & -0.02 & -0.01 & -0.02 & -0.12 \\
\hline
\end{tabular}

Given the scarceness of experimental $K_{\text {hem }}$ values, the proposed methodology cannot be tested thoroughly, but the results do suggest that this approach can be a useful tool in the calculation of hemiacetalization constants. Table 5.9 shows the mean unsigned errors computed for each of the methods, using both a common reference or group references.

Since the lack of appropriate experimental data hampers the study of how the use of group references affects the results, ketones and aldehydes cannot be discussed separately. Nevertheless, it is quite clear that the DFT-B3LYP 6-31++G(d,p) values improve significantly with the use of group references, while those at the MP2 $6-31++G(d, p)$ level of theory are rather insensitive to the change in the reference molecules.

The mues obtained are almost equal for methanol and ethanol, suggesting that the alkyl chain of the alcohol does not affect the accuracy of the method, although it does increase the computational cost. The results suggest that the proposed methodology can predict hemiacetal formation constants within \pm 0.5 logarithm units on average at the MP2 6-31++G(d,p) level of theory using a common reference. 
The results obtained using the much cheaper DFT-B3LYP $6-31++G(d, p)$ level of theory are almost as accurate if group references are used. The use of mean values further improves the results by favorable error cancellation.

Table 5.9. Mean unsigned errors in relative $K_{\text {hem }}$ values

\begin{tabular}{lcccccc}
\hline & \multicolumn{4}{c}{ Mean unsigned error } \\
\cline { 2 - 3 } \cline { 6 - 7 } & \multicolumn{2}{c}{ Common ref. } & & \multicolumn{2}{c}{ Group ref. } \\
\cline { 2 - 3 } \cline { 6 - 7 } & MeOH & EtOH & & MeOH & EtOH \\
\hline DFT-B3LYP 6-31G++(d,p) & 1.57 & 1.57 & & 0.51 & 0.51 \\
MP2 6-31G++(d,p) & 0.40 & 0.34 & & 0.35 & 0.34 \\
Mean & 0.87 & 0.76 & & 0.27 & 0.23 \\
\hline
\end{tabular}




\subsubsection{Equilibria of mucohalic acids}

As noted above, mucohalic acids exist a mixture of several dissociated and undissociated isomers (Scheme 5.16). The knowledge of the respective equilibrium constants (defined above, see eq. 5.4 to 5.11) is of interest, since the reactivity of the different form against biological nucleophiles is expected to be very different.
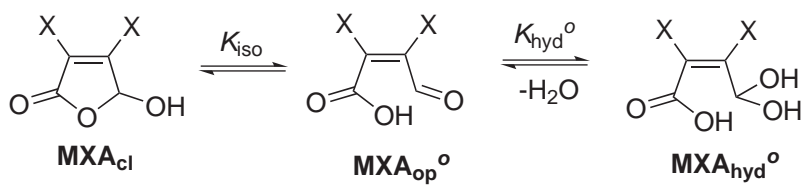

$$
\begin{aligned}
& X=C I, M C A \\
& X=B r, M B A
\end{aligned}
$$$$
\underbrace{K_{a}}_{O_{\odot}} \frac{K_{\text {hyd }}}{-\mathrm{H}_{2} \mathrm{O}}
$$$$
\text { MXAop }^{-}
$$

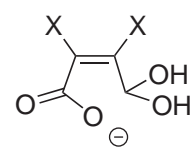

$\mathrm{MXA}_{\text {hyd }} \mathbf{C}^{-}$

Scheme 5.16. Equilibria of MXA in aqueous solution

5.3.1.2.1. Experimental study. We have determined the apparent acid dissociation constant of mucohalic acids, which roughly corresponds to the equilibrium between the major closed-chain furanone and open-chain carboxylate forms. In addition, the hydration constant of MXA was measure by NMR spectroscopy.

5.3.1.2.1.1. Apparent acid dissociation constant. Figure 5.4 shows the spectra of MCA and MBA at various $\mathrm{pH}$. Absorbance values were measured at the wavelengths where $\mathrm{MXA}_{\mathrm{cl}}$ and $\mathrm{MXA}_{\mathrm{op}}^{-}$ showed maximum absorption; i.e. 231.0 and $263.0 \mathrm{~nm}$ for MCA; 245.0 and $276.0 \mathrm{~nm}$ for MBA.

Figure 5.5 shows the excellent fit of the variation in absorbance with $\mathrm{pH}$ to eq 5.10 . The $\mathrm{p} K_{\mathrm{a}}^{\text {app }}$ values shown in Table 5.10 were calculated from the fits at the two wavelengths.

The ${ }^{1} \mathrm{H}$ NMR spectra of MXA is composed of only one major signal (Figure 5.6). Its variation with $\mathrm{pH}$ reveals a displacement from the chemical shift of the dihalohydroxyfuranone, $\mathrm{MXA}_{\mathrm{cl}},\left(\delta_{\mathrm{MCA}}=\right.$ $6.198 \mathrm{ppm}$ and $\left.\delta_{\mathrm{MBA}}=6.198 \mathrm{ppm}\right)$ to that of the aldehyde, $\left(\mathrm{MXA}_{\mathrm{op}}^{-}\right),\left(\delta_{\mathrm{MCA}}=9.696 \mathrm{ppm}\right.$ and $\delta_{\mathrm{MBA}}=$ $9.409 \mathrm{ppm})$. A smaller peak corresponding to the hydrate of the aldehyde group $\mathrm{MXA}_{\text {hyd }}^{-}\left(\delta_{\mathrm{MCA}}=\right.$ $6.110 \mathrm{ppm}$ and $\delta_{\mathrm{MBA}}=5.7155 \mathrm{ppm}$ ) is also observed at high $\mathrm{pH}$, where the linear aldehyde is the most abundant species.

Fitting of said results to eq. 5.11 affords $\mathrm{p} K_{\mathrm{NMR}}^{\mathrm{app}}=4.06 \pm 0.10$ for MCA and $\mathrm{p} K_{\mathrm{NMR}}^{\mathrm{app}}=4.34 \pm 0.10$ for MBA 

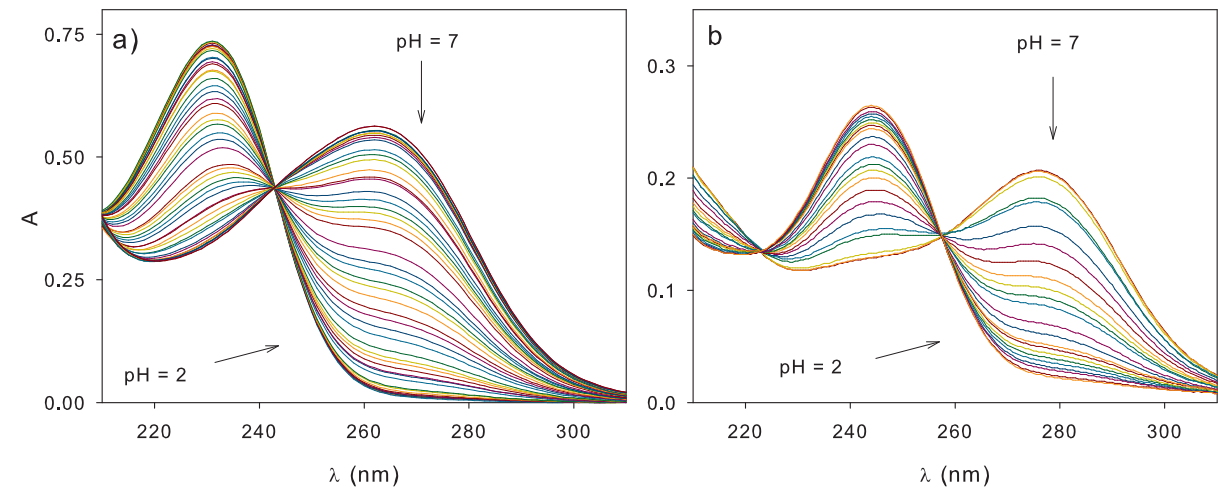

Figure 5.4. Variation in the UV-Vis spectra of $M X A$ with $p H, T=25.0^{\circ} \mathrm{C}$. (a) $X=\mathrm{Cl},[\mathrm{MCA}]=1.0 \times 10^{-4} \mathrm{M}$; (b) $\mathrm{X}=\mathrm{Br}$, $[\mathrm{MBA}]=3.0 \times 10^{-5} \mathrm{M}$;
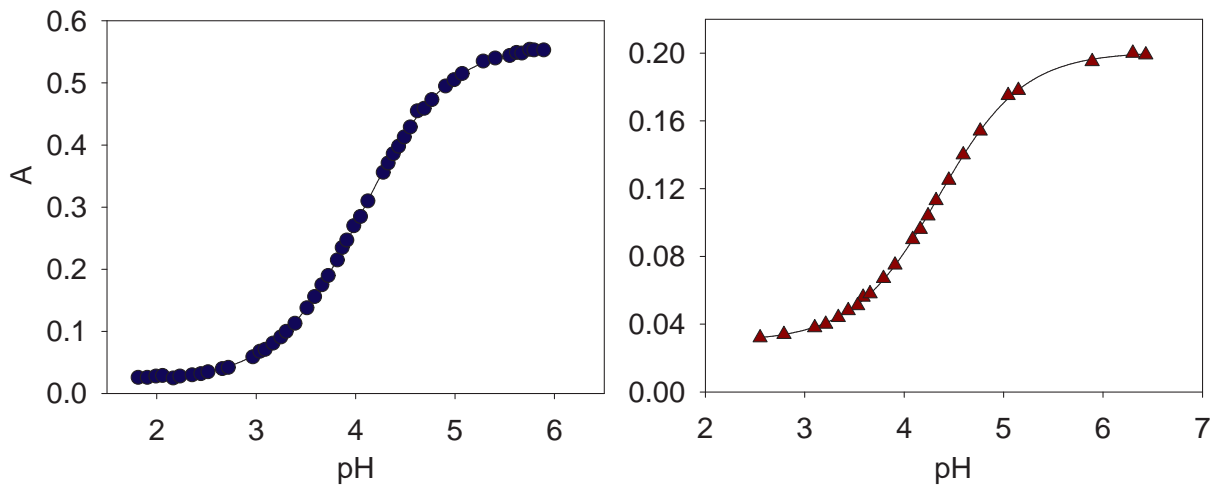

Figure 5.5. Fit of experimental data to eq. $5.10, \mathrm{~T}=25.0^{\circ} \mathrm{C}$. (a) $\mathrm{X}=\mathrm{Cl},[\mathrm{MCA}]=1.0 \times 10^{-4} \mathrm{M}, \lambda=263 \mathrm{~nm}$; (b) $\mathrm{X}=\mathrm{Br}$, $[\mathrm{MBA}]=3.0 \times 10^{-5} \mathrm{M}, \lambda=276 \mathrm{~nm}$;

Inspection of eq. 5.9 reveals that if $K_{\text {iso }} \ll 1$, as is the case (see below for a quantitive discussion), the term $1 / K_{\text {iso }}$ dominates the denominator in both eqs. 5.9 and 5.11 ( $\mathrm{p} K_{\text {hyd }}^{o}$ is estimated to be close to 0 , and thus, negligible).

$$
K_{\mathrm{a}}^{\mathrm{app}}=K_{\mathrm{a}} \frac{1+K_{\text {hyd }}^{-}}{1+K_{\text {hyd }}^{o}+1 / K_{\text {iso }}} \simeq K_{\mathrm{a}} K_{\text {iso }}\left(1+K_{\text {hyd }}^{-}\right) \simeq K_{\mathrm{NMR}}^{\mathrm{app}}\left(1+K_{\text {hyd }}^{-}\right)
$$

$K_{\text {hyd }}^{-}$were determined to be 0.23 for MCA and 0.17 for MBA (vide infra), which, combined with $\mathrm{p} K_{\mathrm{NMR}}^{\mathrm{app}}$ values afford $\mathrm{p} K_{\mathrm{a}}^{\mathrm{app}}=3.97 \pm 0.10$ and $4.27 \pm 0.10$ for MCA and MBA respectively. These are in excellent agreement with those obtained by spectrophotometric measurements.

Fitting $K_{\mathrm{a}}^{\text {app }}$ values to the van't Hoff equation (eq 5.24) affords the reaction enthalpies (17.7 \pm 0.9 and $14.0 \pm 0.5 \mathrm{~kJ} \mathrm{~mol}^{-1}$ for MCA and MBA respectively), which are positive and small. 
Table 5.10. Experimental values for $p K_{a}^{a p p}$ obtained by UV-Vis spectroscopy

\begin{tabular}{ccc}
\hline & \multicolumn{2}{c}{$\mathrm{p} K_{\mathrm{a}}^{\mathrm{app}}$} \\
\cline { 2 - 3 }$T\left({ }^{\circ} \mathrm{C}\right)$ & $\mathrm{MCA}$ & $\mathrm{MBA}$ \\
\hline 15.0 & $4.06 \pm 0.01$ & $4.35 \pm 0.01$ \\
20.0 & $4.02 \pm 0.01$ & $4.31 \pm 0.02$ \\
25.0 & $3.95 \pm 0.02$ & $4.27 \pm 0.02$ \\
30.0 & $3.90 \pm 0.02$ & $4.22 \pm 0.02$ \\
35.0 & $3.86 \pm 0.01$ & $4.19 \pm 0.02$ \\
\hline
\end{tabular}
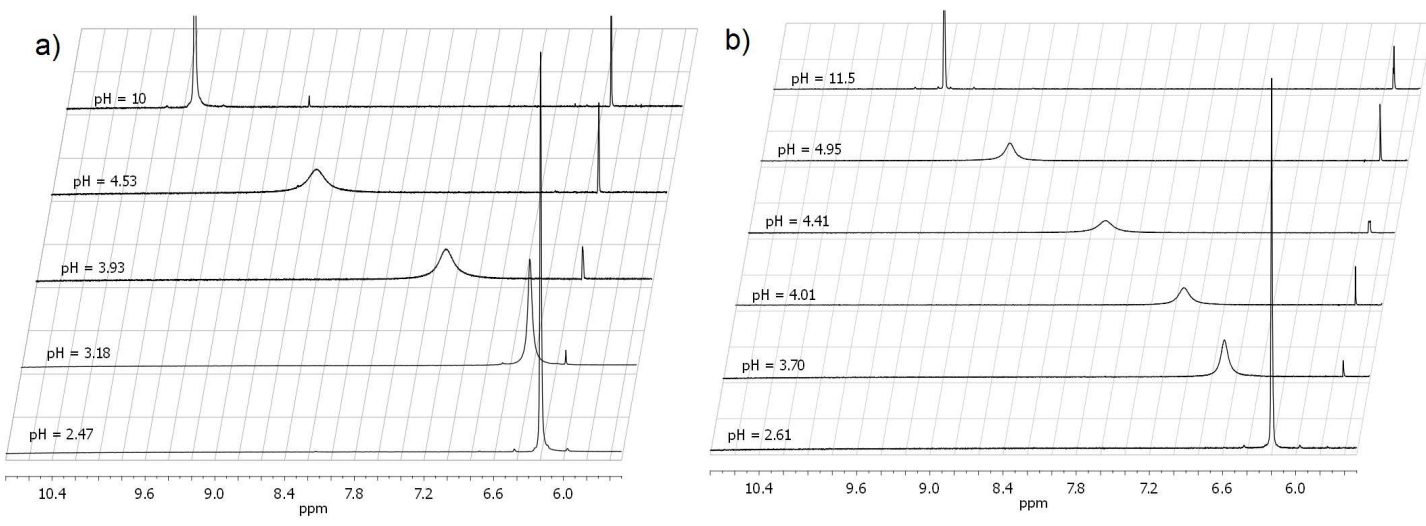

Figure 5.6. Variation in the ${ }^{1} \mathrm{H}$ NMR spectra of $\mathrm{MXA}$ with $\mathrm{pH}, \mathrm{T}=25.0^{\circ} \mathrm{C}$. (a) $\mathrm{X}=\mathrm{Cl},[\mathrm{MCA}]=7.0 \times 10^{-2} \mathrm{M} ;(\mathrm{b}) \mathrm{X}=\mathrm{Br}$, $[\mathrm{MBA}]=7.0 \times 10^{-2} \mathrm{M}$

$$
\frac{\partial \ln K_{\mathrm{a}}^{\mathrm{app}}}{\partial\left(T^{-1}\right)}=\Delta H
$$

The apparent acid dissociation constants of $\mathrm{MXA}_{\mathrm{cl}}$ to form $\mathrm{MXA}_{\mathrm{op}}^{-}$, defined as in eq 5.4, are around $1 \times 10^{-4}$. The values for MCA and MBA are very similar and the greater apparent acidity of MCA is consistent with the higher electronegativity of chlorine.

These values suggest that at the approximately neutral $\mathrm{pH}$ of tap water or most natural waters MXA are present, by about $10^{3}$ to 1 , as $\mathrm{MXA}_{\mathrm{op}}^{-}$rather than $\mathrm{MXA}_{\mathrm{cl}}$. In addition, at physiological $\mathrm{pH}, \mathrm{MXA}$ are mostly in their open-chain dissociated form and can therefore react through the aldehyde group with nucleophilic sites in macromolecules.

The amount of $\mathrm{MXA}_{\mathrm{op}}^{\mathrm{o}}$ present in the equilibrium cannot be determined experimentally, and was therefore addressed using a computational approach.

5.3.1.2.1.2. Hydration constant. Since aldehydes in their hydrate form cannot react with nucleophilic sites in DNA, the hydration of aldehydes is also significant in their role as alkylating and 
potentially mutagenic and carcinogenic agents. For instance, at any given pH, 99.997\% of chloral in aqueous solution is in its hydrate form, which significantly reduces its potential reactivity as an electrophile, and this in turn influence its genotoxic potential. ${ }^{192,195-197}$

The minor signal observed in the ${ }^{1} \mathrm{H}$ NMR spectra of $\mathrm{MXA}\left(\delta_{\mathrm{MCA}}=6.110 \mathrm{ppm}\right.$ and $\left.\delta_{\mathrm{MBA}}=5.7155 \mathrm{ppm}\right)$ is similar to that of gem-diols, hemiacetals, and acetals, which suggests that this peak corresponds to the hydrate of the aldehyde group. The ratio of peak areas between the major and minor signals is time- and concentration-independent.

By introducing the peak areas in eq. 5.12, the values $K_{\text {hyd }}^{\mathrm{MCA}}=0.23 \pm 0.04$ and $K_{\text {hyd }}^{\mathrm{MBA}}=0.17 \pm 0.03$ were obtained.

The hydration constants are somewhat lower than those of other haloaldehydes, such as chloroacetaldehyde, trichloroacetaldehyde, 2- chlorobutyraldehyde or 2-bromobutyraldehyde, which have $K_{\text {hyd }}$ values of $10^{1.6}, 10^{4.45}, 10^{1.2}$ and $10^{0.6}$, respectively. ${ }^{200,205}$ This suggests that the presence of the double bond, together with the conjugation with the carboxylate group, decreases the electrophilicity of the aldehyde group, and hence the hydration constant.

Nevertheless, the amount of hydrate in the equilibrium is significant, since close to $20 \%$ of MXA is present in this form. Because aldehyde hydrates lack the electrophilicity of the free aldehyde group, these values imply a decrease in the alkylating capacity of mucohalic acids.

5.3.1.2.2. Theoretical study. Since the separation of $K_{\mathrm{a}}^{\mathrm{app}}$ into the two main contributions of $K_{\text {iso }}$ and $K_{\mathrm{a}}$ is very interesting but experimentally unattainable, it was approached using computational methods by calculating $K_{\text {iso }}$ and $K_{\mathrm{a}}$ separately. To do so, the optimized energies and geometries of the compounds at several levels of theory using IEF-PCM for solvation were calculated.

5.3.1.2.2.1. Structure of mucohalic acids. $\mathrm{MXA}_{\mathrm{cl}}$ was calculated to have a planar structure at all levels of theory, in both water and organic solvents (DMSO and heptane), as shown in Figure 5.7.
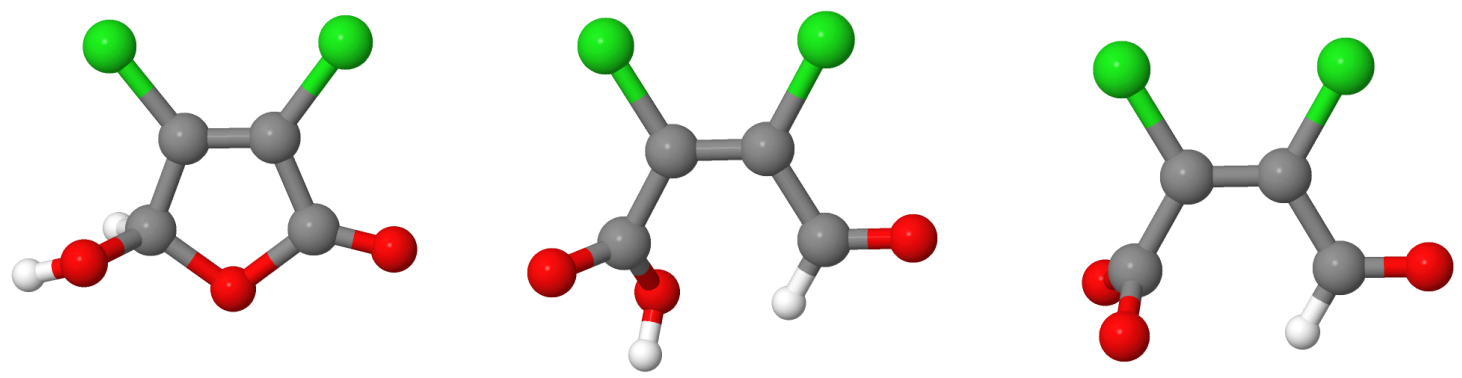

Figure 5.7. Calculated equilibrium geometries for mucohalic acids 
In $\mathrm{MXA}_{\mathrm{op}}^{\mathrm{O}}$ and $\mathrm{MXA}_{\mathrm{op}}^{-}$, the $\mathrm{CHO}-\mathrm{CCl}=\mathrm{CCl}$ atoms lie on a plane due to double bond conjugation, but the carboxylic/carboxylate group is rotated out, because the decrease in $\pi$ conjugation energy is compensated by a decreased repulsion between the aldehydic hydrogen and the carboxylic oxygen. The dihedral angles are shown in Table 5.11 and, with one exception (MP2 for MXA ${ }_{\mathrm{Op}}^{\mathrm{O}}$ ), the values for the same compound are consistent within the different methods in the three solvents studied $\left( \pm 5^{\circ}\right)$.

Table 5.11. Dihedral angle $\mathrm{C}=\mathrm{C}-\mathrm{C}-\mathrm{O}$

\begin{tabular}{cccccc}
\hline & \multicolumn{2}{c}{ MCA } & & \multicolumn{2}{c}{ MBA } \\
\cline { 2 - 3 } \cline { 5 - 6 } Method & MXA op $_{\text {ONXA }}^{-}$ & & MXA op $_{\text {op }}^{\circ}$ & MXA $_{\text {op }}^{-}$ \\
\hline DFT-B3LYP/6-31G++(d,p) & $137.1^{\circ}$ & $83.3^{\circ}$ & & $121.9^{\circ}$ & $90.5^{\circ}$ \\
DFT-B3LYP/6-31G++(2df,2pd) & $132.6^{\circ}$ & $90.3^{\circ}$ & & $114.5^{\circ}$ & $90.5^{\circ}$ \\
MP2/6-31G++(d,p) & $130.6^{\circ}$ & $66.4^{\circ}$ & & $121.1^{\circ}$ & $74.1^{\circ}$ \\
\hline
\end{tabular}

Interestingly, the calculated dihedral angle $(\mathrm{C}=\mathrm{C}-\mathrm{C}-\mathrm{O})$ in $\mathrm{MXA}_{\mathrm{op}}^{-}$depends on the level of theory used. When the correlation energy is not taken into account (DFT-B3LYP), the carboxylate group is almost perpendicular to the plane formed by the rest of the molecule, but when MP2 is used a significant deviation from the right angle is observed.

If the dihedral angle is fixed at certain values, a plot like the one shown in Figure 5.8 is obtained. It may be observed that, as expected since the rest of the molecule is planar, the plots are symmetrical with respect to $90^{\circ}$, and, more importantly, that at the DFT-B3LYP level there is a minimum at $90^{\circ}$ (the plot is therefore $\mathrm{U}$ shaped), whereas at the MP2 level, minima exist at $\sim 65^{\circ}$ and $\sim 115^{\circ}$ (and the plot is $\mathrm{W}$ shaped). This is possibly caused by the negative charge and the resulting lone pair present in $\mathrm{MXA}_{\mathrm{op}}^{-}$, which may increase the need for correction of the correlation energy. Nevertheless, the energy differences over a wide range of angles were small and non-significant for the purposes of the present work.

5.3.1.2.2.2. Ring-opening constant. The experimental results suggest that, both in organic solvents and acidic aqueous media, MXA are mainly present in their cyclic form. ${ }^{14-18}$ Calculation of the free energies in aqueous solution of $\mathrm{MXA}_{\mathrm{cl}}$ and $\mathrm{MXA}_{\mathrm{op}}^{\mathrm{o}}$ at different levels of theory and the use of eq. 5.13 afford the values shown in Table 5.12.

Table 5.12. Calculated $\mathrm{p} K_{\text {iso }}$ of MXA in water

\begin{tabular}{cccccc}
\hline & B3LYP & B3LYP & MP2 & CBS-QB3 & G3MP2B3 \\
& $6-31++\mathrm{G}(\mathrm{d}, \mathrm{p})$ & $6-31++\mathrm{G}(2 \mathrm{df}, 2 \mathrm{pd})$ & $6-31++\mathrm{G}(\mathrm{d}, \mathrm{p})$ & & \\
\hline MCA & 2.42 & 2.42 & 3.48 & 5.13 & 4.57 \\
MBA & 4.14 & 3.29 & 4.29 & 5.73 & - \\
\hline
\end{tabular}

The mean values of $\mathrm{p} K_{\mathrm{iso}}$ considering the different levels of theory are 3.60 for MCA and 3.91 for 


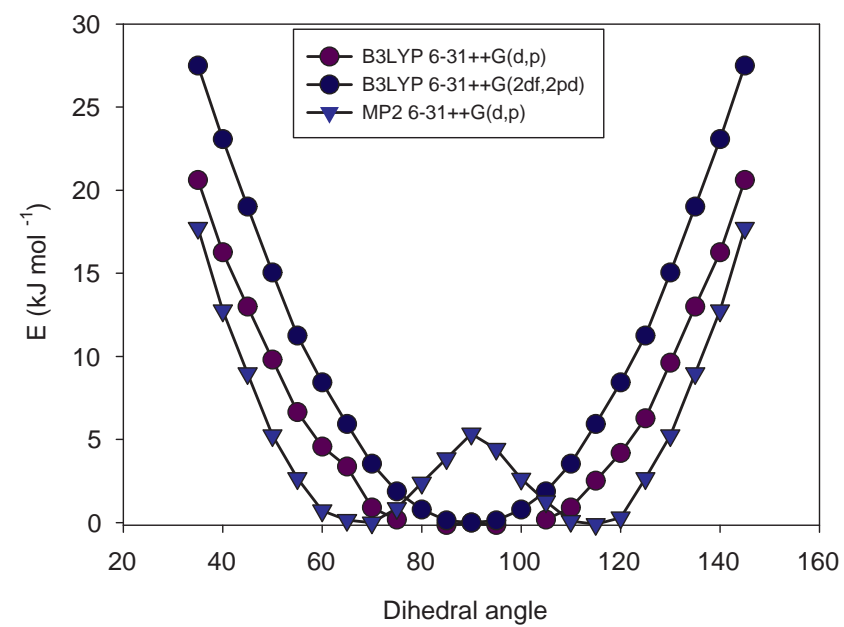

Figure 5.8. Energy of $\mathrm{MXA}_{\mathrm{op}}^{-}$at different levels of theory as a function of the $\mathrm{C}=\mathrm{C}-\mathrm{C}-\mathrm{O}$ dihedral angle

MBA, which shows that the cyclic forms are more stable than the open-chain isomers. Thus, at any given $\mathrm{pH}, \mathrm{MXA}_{\mathrm{op}}^{\mathrm{O}}$ are present only at minor proportions with respect to MXAcl $\left(1\right.$ to $\left.10^{2.5}-10^{5.7}\right)$. Increasing $\mathrm{p} K_{\text {iso }}$ values are observed at the higher levels of theory increases, with more powerful methods affording reaction free energies around $30 \mathrm{~kJ} \mathrm{~mol}^{-1}$.

The $\mathrm{p} K_{\text {iso }}$ values in organic solvents DMSO and heptane (Table 5.13) do not differ much from those in water. This shifted equilibrium and the presence of a small amount of aldehyde should be taken into account when carrying out reactions of MXA in organic solvents.

Table 5.13. Calculated $\mathrm{pK}$ iso of MXA in DMSO and heptane

\begin{tabular}{|c|c|c|c|c|c|c|c|c|c|c|}
\hline & \multicolumn{10}{|c|}{$\mathrm{p} K_{\text {iso }}$} \\
\hline & \multicolumn{2}{|c|}{$\begin{array}{c}\text { DFT-B3LYP } \\
6-31++G(d, p)\end{array}$} & \multicolumn{2}{|c|}{$\begin{array}{c}\text { DFT-B3LYP } \\
6-31++G(2 d f, 2 p d)\end{array}$} & \multicolumn{2}{|c|}{$\begin{array}{c}M P 2 \\
6-31++G(d, p)\end{array}$} & \multicolumn{2}{|c|}{ CBS-QB3 } & \multicolumn{2}{|c|}{ G3MP2B3 } \\
\hline & DMSO & Hep. & DMSO & Hep. & DMSO & Hep. & DMSO & Hep. & DMSO & Hep. \\
\hline MCA & 3.38 & 3.23 & 3.17 & 3.16 & 3.61 & 3.56 & 5.22 & 5.22 & 4.61 & 4.64 \\
\hline MBA & 4.17 & 3.94 & 3.50 & 3.28 & 4.15 & 3.99 & 6.05 & 5.72 & - & - \\
\hline
\end{tabular}

5.3.1.2.2.3. Acid dissociation constant. $\quad \mathrm{MXA}_{\mathrm{op}}^{\mathrm{O}}$ are acidic and dissociate in aqueous solution yielding $\mathrm{MXA}_{\mathrm{op}}^{-}$. Since the higher the acidity, the lower the concentration of highly reactive and potentially toxic MXA $\mathrm{op}_{\mathrm{op}}^{\mathrm{O}}$ knowledge of their $\mathrm{p} K_{\mathrm{a}}$ values is important.

The values calculated for $\mathrm{p} K_{\mathrm{a}}$ using a relative approach (eqs. 5.14 and 5.15) are shown in Table 5.14. 
Table 5.14. Calculated $\mathrm{p} K_{\mathrm{a}}$ and $\mathrm{p} K_{\text {calc }}^{\mathrm{app}}$ of $\mathrm{MXA}$ in water

\begin{tabular}{|c|c|c|c|c|c|c|c|c|c|c|}
\hline & \multicolumn{2}{|c|}{$\begin{array}{l}\text { DFT-B3LYP } \\
6-31++G(d, p)\end{array}$} & \multicolumn{2}{|c|}{$\begin{array}{c}\text { DFT-B3LYP } \\
6-31++G(2 d f, 2 p d)\end{array}$} & \multicolumn{2}{|c|}{$\begin{array}{c}\mathrm{MP} 2 \\
6-31++\mathrm{G}(\mathrm{d}, \mathrm{p}) \\
\end{array}$} & \multicolumn{2}{|c|}{ CBS-QB3 } & \multicolumn{2}{|c|}{ G3MP2B3 } \\
\hline & $\mathrm{p} K_{\mathrm{a}}$ & $\mathrm{p} K_{\text {calc }}^{\mathrm{app}}$ & $\mathrm{p} K_{\mathrm{a}}$ & $\mathrm{p} K_{\text {calc }}^{\text {app }}$ & $\mathrm{p} K_{\mathrm{a}}$ & $\mathrm{p} K_{\text {calc }}^{\text {app }}$ & $\mathrm{p} K_{\mathrm{a}}$ & $\mathrm{p} K_{\text {calc }}^{\text {app }}$ & $\mathrm{p} K_{\mathrm{a}}$ & $\mathrm{p} K_{\text {calc }}^{\text {app }}$ \\
\hline MCA & 1.07 & 3.44 & 0.80 & 3.13 & 1.26 & 4.65 & 0.57 & 5.61 & 1.27 & 5.75 \\
\hline MBA & 1.48 & 5.55 & 1.55 & 4.77 & 1.10 & 5.32 & 0.85 & 6.15 & - & - \\
\hline
\end{tabular}

These results suggest that $\mathrm{MXA}_{\mathrm{op}}^{\mathrm{o}}$ are very strong carboxylic acids, with $\mathrm{p} K_{\mathrm{a}}$ values close to 1 . This is consistent with the inductive effect of the halogen substituents and the electron delocalization in the $\alpha-\beta$ unsaturation; for instance, $\mathrm{p} K_{\mathrm{a}}$ (chloroacetic acid) $=2.85$ and $\mathrm{p} K_{\mathrm{a}}$ (acrylic acid $)=4.25$ as compared to $\mathrm{p} K_{\mathrm{a}}$ (acetic acid $)=4.75$. The fact that the predicted $\mathrm{p} K_{\mathrm{a}}$ values show little variation at the different levels of theory is probably due to favorable error cancellation arising from the use of a relative approach.

The low $\mathrm{p} K_{\mathrm{a}}$ values suggest that $\mathrm{MXA}_{\mathrm{op}}^{\mathrm{o}}$ are present at very low proportions in aqueous solution at neutral or basic $\mathrm{pH}$ (accounting for about 1 in $10^{6}$ at $\mathrm{pH}=7$ ). They also explain why, despite the high $K_{\text {iso }}$ values, MXA are not in their cyclic forms at neutral or basic $\mathrm{pH}$, but rather in an open-chain (dissociated) form.

Since its concentration is always orders of magnitude lower, the minor presence of the open-chain undissociated aldehyde is expected to limit its impact on environment and health.

The computational values for $\mathrm{p} K_{\text {calc }}^{\mathrm{app}}=\mathrm{p} K_{\mathrm{a}}+\mathrm{p} K_{\mathrm{iso}}-\log \left(1+K_{\text {hyd }}^{-}\right)$are also shown in Table 5.14. The calculated values are around 4 in the case of MCA and 5 in the case of MBA (the averages for the three methods are 3.8 and 5.3 for MCA and MBA, respectively). Computationally estimated equilibrium constants in solution are usually affected by errors of not less than \pm 1.0 logarithm units, and $\mathrm{p} K_{\text {calc }}^{\text {app }}$ values in this work arise from the combination of two calculated equilibrium constants. The agreement between the experimental (Table 5.10) and computational (Table 5.14) results supports the accuracy of the computational values $\left(\mathrm{p} K_{\text {iso }}\right.$ and $\mathrm{p} K_{\mathrm{a}}$ ) 


\subsubsection{Hydrolysis of mucohalic acids}

As has been shown (Scheme 5.14), mucohalic acids exist mostly as the aldehyde-carboxylate in neutral and basic medium, as used in this work and thus, $\mathrm{MXA}_{\mathrm{cl}}$ and $\mathrm{MXA}_{\mathrm{op}}^{o}$ play a negligible role in the context of the alkaline hydrolysis of mucohalic acids. The formation of mucoxyhalic acids has been proposed as a possible initial step in the reaction of MXA with DNA bases. ${ }^{76-78,121}$ Whereas currently some evidence has discarded mucoxyhalic acids as intermediates in the alkylation reaction of mucohalic acids, ${ }^{119,120}$ a kinetic study of the formation of MOXA is still lacking<smiles>[X]C(C=O)=C([X])C(=O)[O-]</smiles>

MXA<smiles>[X]C(C=O)=C(O)C(=O)[O-]</smiles>

MOXA_en<smiles>[X]C(C=O)C(=O)C(=O)[O-]</smiles>

MOXA_ket

$$
\mathrm{X}=\mathrm{Cl}, \mathrm{Br}
$$

Scheme 5.17. Proposed hydrolysis mechanism of MXA

5.3.1.3.1. Kinetics of MXA decomposition. The reactions were followed by UV-Vis spectroscopy. In the case of MCA, its disappearance was monitored at $\lambda=255 \mathrm{~nm}$, and the appearance of MOCA at $\lambda=290 \mathrm{~nm}$ (Figure $5.9 \mathrm{a}$ ); equal rate constants were obtained in both cases. In the case of MBA, owing to the low molar absorption of MOBA only the disappearance of MBA was observed $(\lambda=275 \mathrm{~nm}$; Fig. 5.9 b).
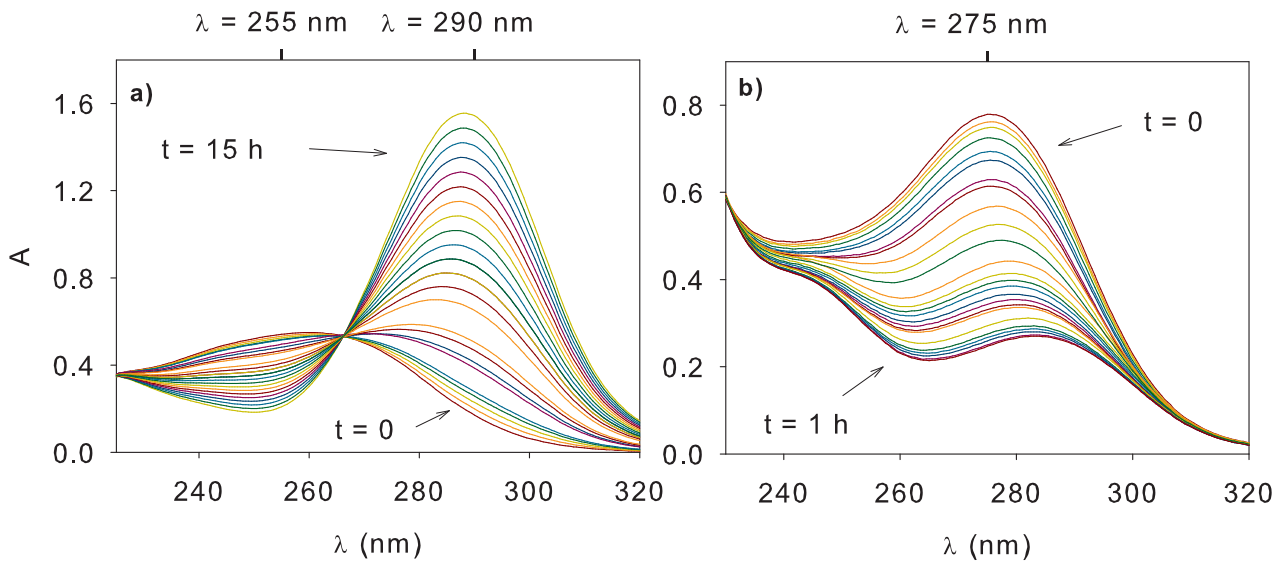

Figure 5.9. Variation with time in the UV-Vis spectra of MXA in the reaction with $\mathrm{OH}^{-}, \mathrm{T}=25.0^{\circ} \mathrm{C}\left(\mathrm{OH}^{-}\right)=3.3 \times 10^{-2} \mathrm{M}$ (a) $\mathrm{X}=\mathrm{Cl}$, $[\mathrm{MCA}]=7.5 \times 10^{-5} \mathrm{M}$; (b) $\mathrm{X}=\mathrm{Br}$, [MBA] $=1.1 \times 10^{-4} \mathrm{M}$

The following rate equation describes the formation of MOXA from MXA as illustrated in Scheme 5.4 . 


$$
\frac{d[\mathrm{MOXA}]}{d t}=k_{\mathrm{OH}}^{\mathrm{MXA}}\left[\mathrm{OH}^{-}\right][\mathrm{MXA}]
$$

Since hydroxide ions were present in large excess, their concentration was considered to remain constant, and thus the pseudo-first-order approximation was used, such that $k_{\mathrm{OH}-\exp }^{\mathrm{MXA}}=k_{\mathrm{OH}}^{\mathrm{MXA}}\left[\mathrm{OH}^{-}\right]$, as in eq. 5.26, and its integrated counterpart, eq. 5.27.

$$
\begin{aligned}
\frac{d[\mathrm{MOXA}]}{d t} & =k_{\mathrm{OH}-\exp }^{\mathrm{MXA}}[\mathrm{MXA}] \\
{[\mathrm{MOXA}] } & =[\mathrm{MXA}]_{O}\left(1-e^{k_{\mathrm{OH}-\exp }^{\mathrm{MXA}}}\right)
\end{aligned}
$$

Since both MXA and MOXA show significant absorption at the wavelengths of measurement, the total absorbance is

$$
\begin{aligned}
A & =l[\mathrm{MXA}]_{o}\left(\epsilon_{\mathrm{MXA}}\left(e^{k_{\mathrm{OH}-\operatorname{\mathrm {MXp}}}^{\mathrm{A}}}\right)+\epsilon_{\mathrm{MOXA}}\left(1-e^{k_{\mathrm{OH}-\operatorname{\mathrm {MXp}}}^{\mathrm{MA}}}\right)\right) \\
& =l[\mathrm{MXA}]_{O}\left(\epsilon_{\mathrm{MXA}}+\left(\epsilon_{\mathrm{MOXA}}-\epsilon_{\mathrm{MXA}}\right)\left(1-e^{k_{\mathrm{OH}-\exp }^{\mathrm{MXA}}}\right)\right) \\
& =A_{O}+\Delta A\left(1-e^{k_{\mathrm{OH}-\exp }^{\mathrm{MX}} t}\right)
\end{aligned}
$$

$A_{O}$ is the initial absorbance and $\triangle A$ is the difference in absorption between MXA and MOXA. Figure 5.10 shows the excellent fit of the results to eq. 5.30
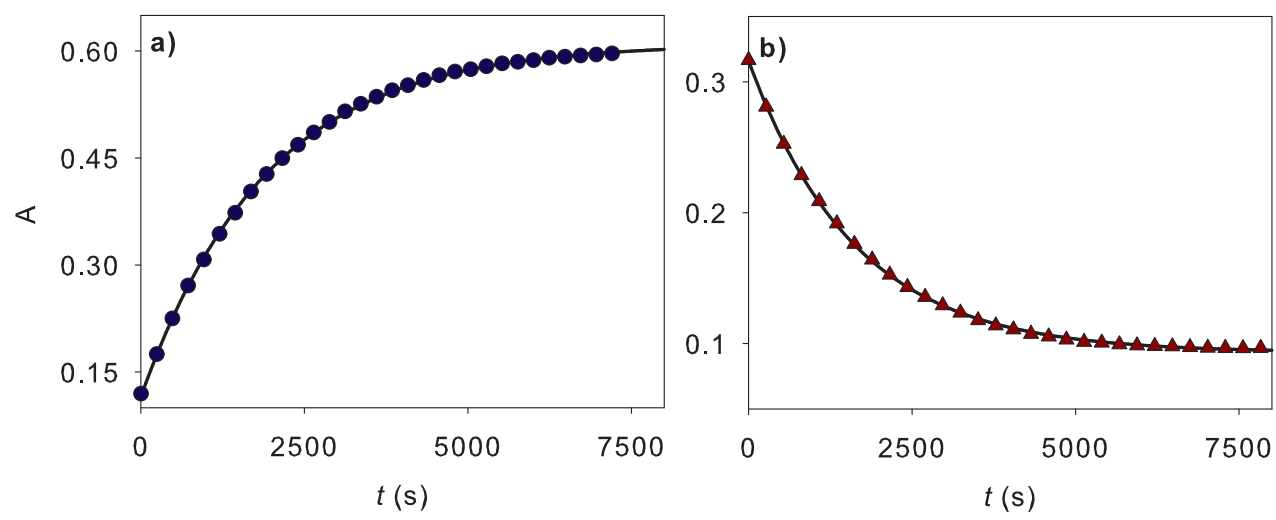

Figure 5.10. Kinetic profiles of the reaction of $\mathrm{MXA}$ with $\mathrm{OH}^{-}, \mathrm{T}=25.0^{\circ} \mathrm{C}(\mathrm{a}) \mathrm{X}=\mathrm{Cl},\left[\mathrm{OH}^{-}\right]=2.6 \times 10^{-2} \mathrm{M}, \lambda=290 \mathrm{~nm}$, $[\mathrm{MCA}]=3 \times 10^{-5} \mathrm{M} ;(\mathrm{b}) \mathrm{X}=\mathrm{Br},\left[\mathrm{OH}^{-}\right]=6.5 \times 10^{-3} \mathrm{M}, \lambda=275 \mathrm{~nm},[\mathrm{MBA}]=4 \times 10^{-5} \mathrm{M}$.

The reaction orders were determined from the slope of the logarithmic plots of log $k_{\mathrm{OH}-\exp }^{\mathrm{MXA}}$ against $\log \left[\mathrm{OH}^{-}\right]$(Figure 5.11). The observed reaction orders with respect to hydroxide ions in the experimental conditions are 1.23 for MCA and 1.82 for MBA. 


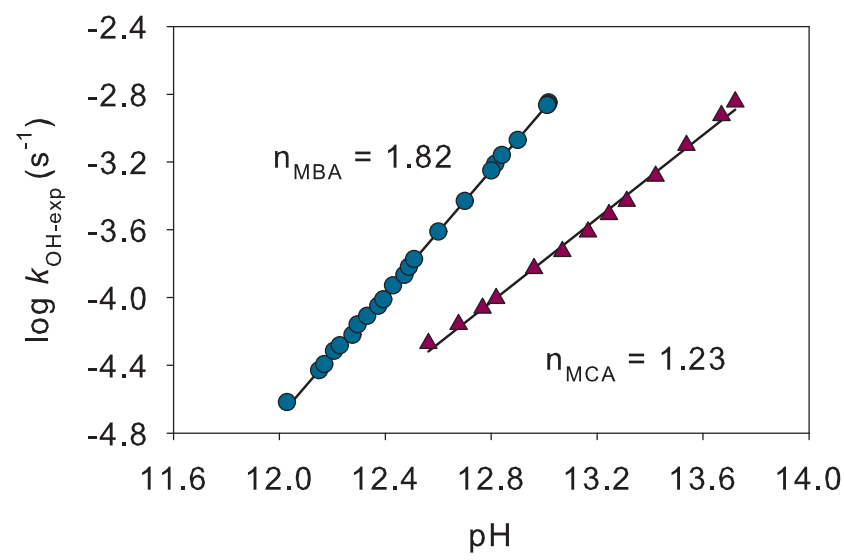

Figure 5.11. Reaction order of MXA hydrolysis with respect to hydroxide. $T=25.0^{\circ} \mathrm{C}$.

These values suggest that the reaction takes place by both first-order and second-order reaction mechanisms, with rate constants $k_{1}^{\mathrm{OH}}$ and $k_{2}^{\mathrm{OH}}$, respectively (eq. 5.31).

$$
k_{\mathrm{OH}-\exp }^{\mathrm{MXA}}=k_{1}^{\mathrm{OH}}\left[\mathrm{OH}^{-}\right]+k_{2}^{\mathrm{OH}}\left[\mathrm{OH}^{-}\right]^{2}
$$

Fig. 5.12 shows the excellent fit of the experimental $k_{\mathrm{OH}-\exp }^{\mathrm{MXA}}$ values to eq. 5.31. The values obtained for $k_{1}^{\mathrm{OH}}$ and $k_{2}^{\mathrm{OH}}$ are shown in Table 5.15.
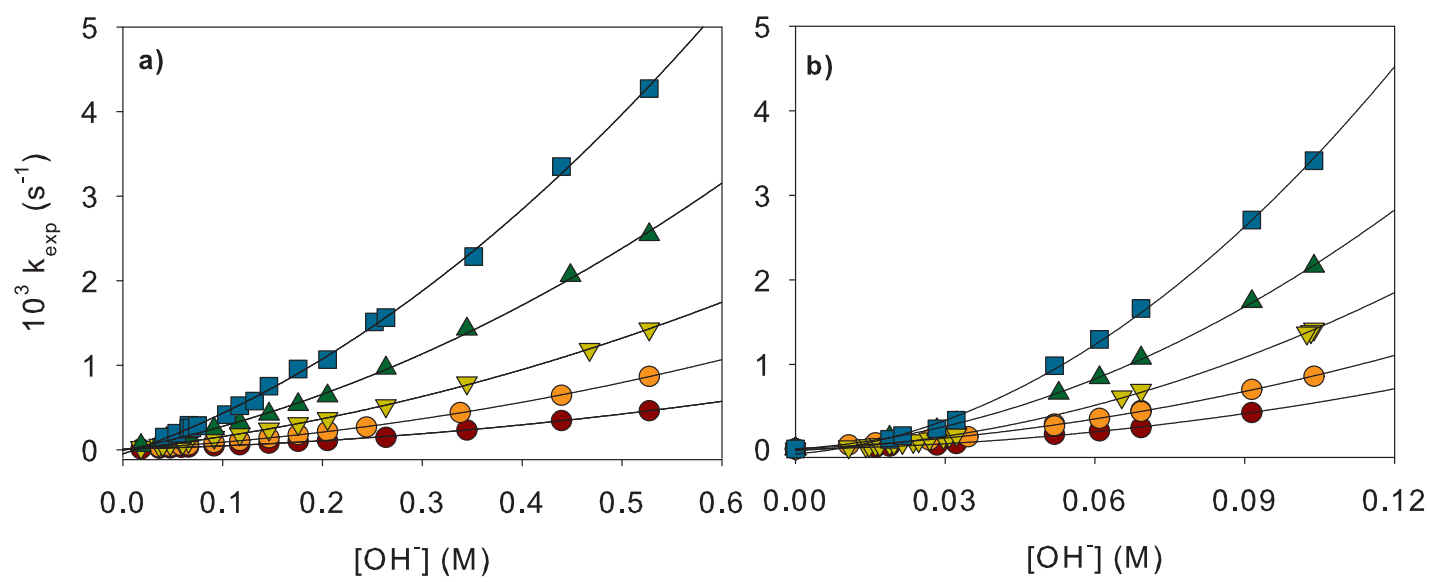

Figure 5.12. Fit of experimental $k_{\mathrm{OH} \text { exp }}^{\mathrm{MX}}$ values to eq. 5.31.

a) $\mathrm{X}=\mathrm{Cl}$ b) $\mathrm{X}=\mathrm{Br}$. T: $15^{\circ} \mathrm{C}(\bullet) ; 20^{\circ} \mathrm{C}(\bullet) ; 25^{\circ} \mathrm{C}(\nabla) ; 30^{\circ} \mathrm{C}(\boldsymbol{\Delta}) ; 35^{\circ} \mathrm{C}(\square)$

Kinetic isotope effect (KIE) experiments afford larger reaction rate constants. KIE $\left(k_{\mathrm{H}} / k_{\mathrm{D}}\right.$ for the firstorder term is 0.87 for MCA and 0.67 for MBA, and thus inverse. This inverse KIE is in keeping with that 
Table 5.15. Rate constants for the reaction of $\mathrm{MXA}$ with $\mathrm{OH}^{-}$

\begin{tabular}{crrrrr}
\hline & \multicolumn{2}{c}{$k_{1}^{\mathrm{OH}}\left(\mathrm{M}^{-1} \mathrm{~s}^{-1}\right)$} & & \multicolumn{2}{c}{$k_{2}^{\mathrm{OH}}\left(\mathrm{M}^{-2} \mathrm{~s}^{-1}\right)$} \\
\cline { 2 - 3 } \cline { 5 - 6 } $\mathrm{T}\left({ }^{\circ} \mathrm{C}\right)$ & \multicolumn{1}{c}{$\mathrm{MCA}$} & \multicolumn{1}{c}{$\mathrm{MBA}$} & & \multicolumn{1}{c}{$\mathrm{MCA}$} & \multicolumn{1}{c}{ MBA } \\
\hline 15.0 & $3.5 \pm 0.1$ & $7.0 \pm 1.1$ & & $9.8 \pm 0.5$ & $3.4 \pm 0.3$ \\
20.0 & $7.0 \pm 0.2$ & $13.4 \pm 1.6$ & & $17.7 \pm 0.4$ & $5.6 \pm 0.2$ \\
25.0 & $13.0 \pm 0.3$ & $20.8 \pm 1.6$ & & $31.1 \pm 0.7$ & $10.6 \pm 0.2$ \\
30.0 & $22.9 \pm 1.0$ & $35.0 \pm 2.1$ & & $49.6 \pm 2.3$ & $15.9 \pm 0.3$ \\
35.0 & $41.2 \pm 1.3$ & $59.6 \pm 1.1$ & & $88.4 \pm 3.2$ & $28.1 \pm 1.2$ \\
\hline
\end{tabular}

observed for reactions in which hydroxide acts as a nucleophile, and is interpreted in terms of $\mathrm{OD}^{-}$being a stronger nucleophile than $\mathrm{OH}^{-}$. KIE for the second order term is also inverse but larger in magnitude ( 0.51 for MCA, 0.53 for MBA). This can be interpreted as two cumulative inverse KIE, one arising from a nucleophilic attack and the other perhaps from a preequilibrium protonation reaction.

The variation in the rate constants (Table 5.15) with temperature allows calculation of the activation energies using the Arrhenius equation; the values are shown in Table 5.16.

The values for $k_{1}^{\mathrm{OH}}$ are somewhat higher for MBA, which together with the lower activation energies is consistent with bromide as the better leaving group. Moreover, $k_{2}^{\mathrm{OH}}$ is almost two hundred times larger in the case of MBA, which can be interpreted in terms of the proposed mechanism (vide infra). In general, the high activation energies are consistent with the attack of a hard charged nucleophile such as the hydroxide ion.

Table 5.16. Activation energies for the hydrolysis of MXA

\begin{tabular}{lcc}
\hline & \multicolumn{2}{c}{$\mathrm{E}_{\mathrm{a}}\left(\mathrm{kJ} \mathrm{mol}^{-1}\right)$} \\
\cline { 2 - 3 } & $k_{1}^{\mathrm{OH}}$ & $k_{2}^{\mathrm{OH}}$ \\
\hline $\mathrm{MCA}$ & $90.0 \pm 1.1$ & $80.1 \pm 1.5$ \\
$\mathrm{MBA}$ & $77.3 \pm 2.2$ & $77.4 \pm 2.4$ \\
\hline
\end{tabular}

These results show that the reaction of mucohalic acids with hydroxide ions to form mucoxyhalic acids becomes kinetically significant only at moderately high concentrations of $\mathrm{OH}^{-}$, and hence the reaction rate when the $\mathrm{pH}$ value is close to neutrality can be considered negligible. Since the extracellular $\mathrm{pH}$ is approximately $7.40^{211}$ and $\mathrm{pH}$ levels inside human cells are about 7.00-7.40212 the formation of MOXA from MXA in vivo is expected to be negligible. This is in agreement with current evidence suggesting that MOXA do not participate in the alkylation reactions of mucohalic acids. ${ }^{19,120}$

MXA do not decompose to MOXA at the common $\mathrm{pH}$ levels of tap water, 6.5-8.5 being the values recommended by the US Environmental Protection Agency in the National Secondary Drinking Water Regulations, ${ }^{213}$ or those of surface waters, since the global median $\mathrm{pH}$ value is 7.7 , most average annual 
pH values are between 6.5 and 8.3, and very rarely exceed 9.0. ${ }^{214}$ Therefore, the amount of time elapsed since water treatment is not expected to modify exposure to MXA or MOXA. A situation worth noting could arise in the case of prechlorination followed by lime softening, where the $\mathrm{pH}$ values are raised to high levels such as those used in this work $(\mathrm{pH}>10)$. At these high concentrations of hydroxide, the formation of mucoxyhalic acids is kinetically significant. Whereas the environmental fate of MOXA is unknown, in the acidification following lime softening, they could undergo decarboxylation and evolve into malondialdehydes, which are known carcinogens.

5.3.1.3.2. Mechanism of MXA decomposition. The quadratic dependence of $k_{\mathrm{OH}-\exp }^{\mathrm{MXA}}$ on hydroxide ions suggests a mechanism involving two successive reactions with hydroxide ions. Such a dependence has often been documented in the hydrolysis of carbonyl compounds such as anilides, ${ }^{215,216}$ arylaminoacrolein, ${ }^{217}$ and acylpyrroles ${ }^{218}$ or sulfonyl compounds such as sultams. ${ }^{219-221}$ The existence of a second-order term is generally interpreted as coming from the reversible addition of the first hydroxide anion to the carbonyl group and the subsequent deprotonation of the hydroxyl group formed by the second $\mathrm{OH}^{-}$, resulting in a dianion, which expels the leaving group.

The second-order term measured in this work is, to our knowledge, a novelty with respect to those described before,since in the reaction of mucohalic acids with hydroxide ions the leaving group is not located on the carbonylic carbon. Therefore, some variation in the accepted hydrolysis mechanism can be proposed, such as two successive attacks of the hydroxide ion on different electrophilic sites, the first one being a fast equilibrium and the second one the actual nucleophilic substitution. Alternatively, a preequilibrium deprotonation, followed by nucleophilic attack is also kinetically equivalent and also plausible.

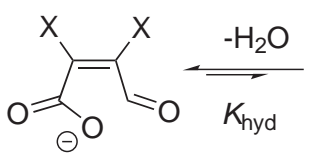

MXA

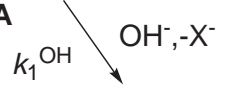<smiles>[X]C(C=C)=C(O)C(=O)[O-]</smiles>

MOXAen<smiles>[X]C(C(=O)[O-])=C([X])C(O)(O)O</smiles>

MXA hyd

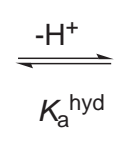

$K_{\mathrm{a}}^{\text {hyd }} \mathrm{O}=\mathrm{K}_{\mathrm{OH}}^{\mathrm{O}}$<smiles>[Y][C@H](O)[C@H]([Y])O</smiles><smiles>[X]C(=C(O)C(=O)O)C(O)(O)C(=O)O</smiles>

Scheme 5.18. Reaction of MXA with $\mathrm{OH}^{-}$

Since the only hydrogen atom in MXA is not expected to dissociate in the working pH interval, and, since aldehydes have high hydration constants, we suggest a possible reaction mechanism that could help to understand the observed results (Scheme 5.18)

In the proposed mechanism, mucohalic acids react directly with hydroxide ions, with rate constant $k_{1}^{\mathrm{OH}}$ in a first-order reaction. At the same time, MXA can add water to form the corresponding hydrate, 
the equilibrium constant for the formation of the gem-diol being $K_{\text {hyd }}$ The hydrate can, in turn, undergo deprotonation $\left(K_{\mathrm{a}}^{\text {hyd }}\right)$; the sum of these two first steps is equivalent to the reversible attack of a hydroxide anion on the carbonyl group. The deprotonated hydrate can subsequently react with $\mathrm{OH}^{-}\left(k_{\text {hyd }} \mathrm{OH}\right)$ The predicted rate equation arising from this mechanismis eq. 5.32, which has both first-order and secondorder terms, as observed experimentally.

$$
\frac{d[\mathrm{MOXA}]}{d t}=\left(k_{1}^{\mathrm{OH}}\left[\mathrm{OH}^{-}\right]+K_{\mathrm{hyd}} K_{\mathrm{a}}^{\mathrm{hyd}} k_{\mathrm{hyd}}^{\mathrm{OH}}\left[\mathrm{OH}^{-}\right]^{2}\right)[\mathrm{MXA}]
$$

Thus, the product $K_{\text {hyd }} K_{\mathrm{a}}^{\text {hyd }} k_{\text {hyd }}^{\mathrm{OH}}$ would equal the experimental second-order rate constant $k_{2}^{\mathrm{OH}}$. Since there are several contributions to $k_{2}^{\mathrm{OH}}$, the large differences observed between MBA and MCA can be explained in terms of a difference in $k_{\text {hyd }}^{\mathrm{OH}}$, (suggesting an inherently higher reactivity of MBA as compared with MCA), in $K_{a}^{\text {hyd }}$ (suggesting that the hydrate of MBA is more acidic than that of MCA), or in $K_{\text {hyd }}$ (suggesting greater electrophilicity of the aldehyde group in MBA) or any combination of the three.

$K_{\text {hyd }}$ values of 0.23 and 0.17 for MCA and MBA have been reported in this work (vide supra) and imply high concentrations of hydrate, which is in keeping with this species playing a role in the proposed mechanism. Since the hydration constants of both compounds are very similar, the large difference in $k_{2}^{\mathrm{OH}}$ between MBA and MCA (one hundred-fold) could be due both to the acidity of the hydrates $K_{\mathrm{a}}^{\text {hyd }}$ and to the inherent reactivity of the deprotonated hydrates $k_{\text {hyd }}^{\mathrm{OH}}$. A higher $k_{\text {hyd }}^{\mathrm{OH}}$ should be expected for MBA since bromide is a better leaving group, and this tendency may be increased by the presence of one extra negative charge in the molecule, as in the deprotonated hydrate.

Whereas the experimental determination of $K_{a}^{\text {hyd }}$ is challenging, standard procedures exist for the computational calculation of acid dissociation constants and have been used along this work several times. ${ }^{222}$ We have estimated the values at the DFT B3LYP $6-31 \mathrm{G}++(\mathrm{d}, \mathrm{p})$ level of theory . The p $K_{\mathrm{a}}^{\text {hyd }}$ values predicted were 23.0 for MCA and 23.6 for MBA. Absolute computational $\mathrm{p} K_{\mathrm{a}}$ values are known to show significant errors systematic errors and, as regards to the present work, only the difference between MCA and MBA is of interest. Since both values are almost equal within the error of the methodology applied (at least $1 \log$ unit), the large difference in $k_{2}^{\mathrm{OH}}$ can only be attributed to bromide as the better leaving group.

5.3.1.3.3. Mucoxyhalic acids. As regards the reaction products, little is known about their reactivity and environmental fate. MOXA are known to be reasonably stable in strongly basic media, but to decompose when the $\mathrm{pH}$ of the solution is lowered. It was concluded that at very high $\mathrm{pH}$ they exist as a double dianion, which also explains the stability of their barium salt. ${ }^{15}$

The instability of MOXA hinders their experimental study. Given the lack of experimental values for comparison, they will only be discussed briefly.

We computed the free energy of dissociated $\mathrm{MOCA}_{\mathrm{en}}$ and $\mathrm{MOCA}_{\mathrm{ke}}$ in aqueous solution at the DFT B3LYP $6-31++G(2 d f, 2 p d)$ level of theory with the Polarizable Continuum Model for solvation. The difference in free energy between these two species is less than $1 \mathrm{~kJ} \mathrm{~mol}^{-1}\left(\Delta G=G_{\text {enol }}-G_{\text {ket }}=-0.3 \mathrm{~kJ}\right.$ 
<smiles>[X]C(C=O)=C(O)C(=O)OC1C(=O)OC(O)C1[X]</smiles>

Scheme 5.19. Equilibria of mucoxyhalic acids

$\mathrm{mol}^{-1}$ ), which suggests an equilibrium constant very close to unity, and hence, that neither of the two is major and both have similar concentrations in the reaction conditions. The open-chain neutral species shows a similar distribution, whereas the closed-chain tautomer favors the keto form by $\sim 20 \mathrm{~kJ} \mathrm{~mol}^{-1}$.

As occurs with MXA, the closed-chain form is more stable than its open counterpart, with $\mathrm{p} K_{\text {iso }} \sim 3$. The $\mathrm{p} K_{\mathrm{a}}$ of the carboxylate group is estimated to be similar to that of mucohalic acids, and thus, MOXA are also strong acids.
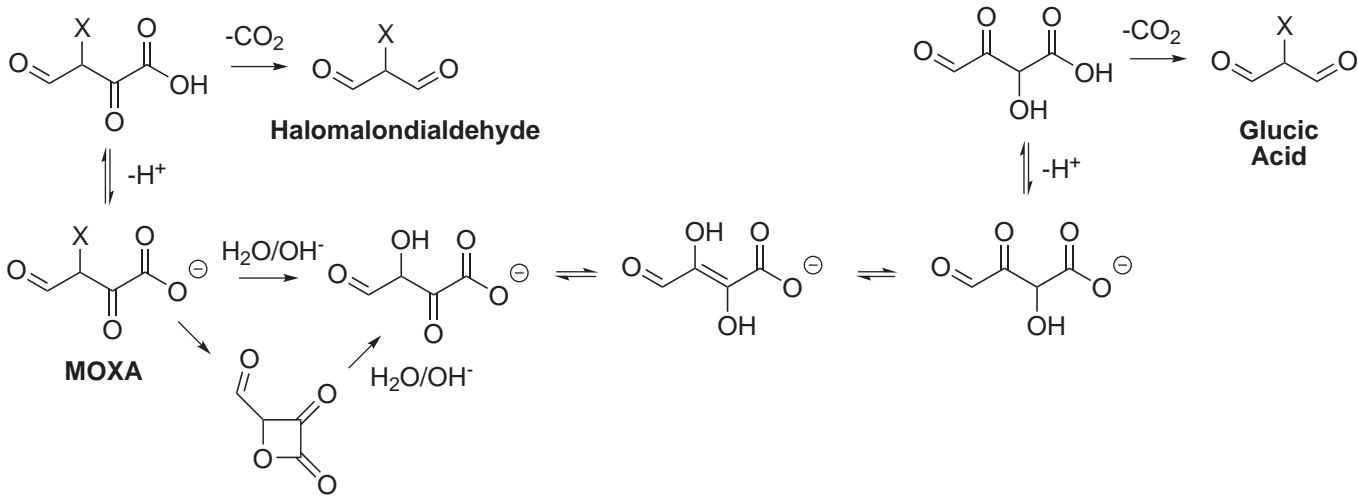

Scheme 5.20. Reactivity of mucoxyhalic acids

As regards their reactivity, MOXA have a very labile halo group in $\alpha$ with respect to two carbonyl moieties, which can undergo hydrolysis, especially in the keto form. Calculated $\Delta^{\ddagger} G^{\ominus}$ values at the DFTB3LYP 6-31G(d) level are 109 and $101 \mathrm{~kJ} \mathrm{~mol}^{-1}$ for MCA and MBA, respectively, for the reaction with water, and 33 and $32 \mathrm{~kJ} \mathrm{~mol}^{-1}$ for the reaction with hydroxide. In addition, a intramolecular ring-closure reaction in which the carboxylate expels the halide and affords a $\beta$-lactone is also possible $\left(\Delta^{\ddagger} G^{\ominus}=70\right.$ and $67 \mathrm{~kJ} \mathrm{~mol}^{-1}$ for MCA and MBA, respectively). The intermediate $\beta$-lactone is expected to hydrolize very rapidly, affording 2,3-hydroxy-4-oxo-but-2-en-oic acid. This reactivity is in keeping with the observation that MOXA are at moderate and low $\mathrm{pH}$. The decarboxylation reaction of the undissociated MOXA, which affords halomalonaldehyde has a high barrier $\left(\Delta^{\ddagger} G^{\ominus}=161\right.$ and $146 \mathrm{~kJ} \mathrm{~mol}^{-1}$ for $\mathrm{X}=\mathrm{Cl}$ and $\mathrm{X}=\mathrm{Br}$, respectively) and can be expected to be unimportant.

The calculated free energies for the different isomers suggest that the acid exists as a mixture of the $\alpha$ - 
and $\beta$-ketoacid mixture, with a minor contribution from the diol. Like most $\beta$-keto acids, the compound undergoes decarboxylation in acidic medium, with a calculated barrier of $\Delta^{\ddagger} G^{\ominus}=115 \mathrm{~kJ} \mathrm{~mol}^{-1}$, affording glucic acid (hydroxymalondialdehyde). 


\subsubsection{Reactivity of MXY}

5.3.1.4.1. Equilibrium constants. How MXY are distributed among all the possible species in aqueous solution (Scheme 5.21) is of interest, especially since the amount of the highly electrophilic exospecies (2 and 5) could be very influential on the ability to react with DNA. Also, comparison with other 3-chloro-4-(methyl)-5-hydroxy-2(5H)-furanones could shed some light onto the origins of the higher genotoxicity of MX. The high number of different species, their low relative concentrations and the rate at which they interconvert make the experimental determination of the equilibrium constants for the reactions in question extremely complex, and thus, we have approached the subject using ab intio and DFT computational methods. The low availability and high hazards of hydroxyhalofuranones also encourage the use of a theoretical approach.
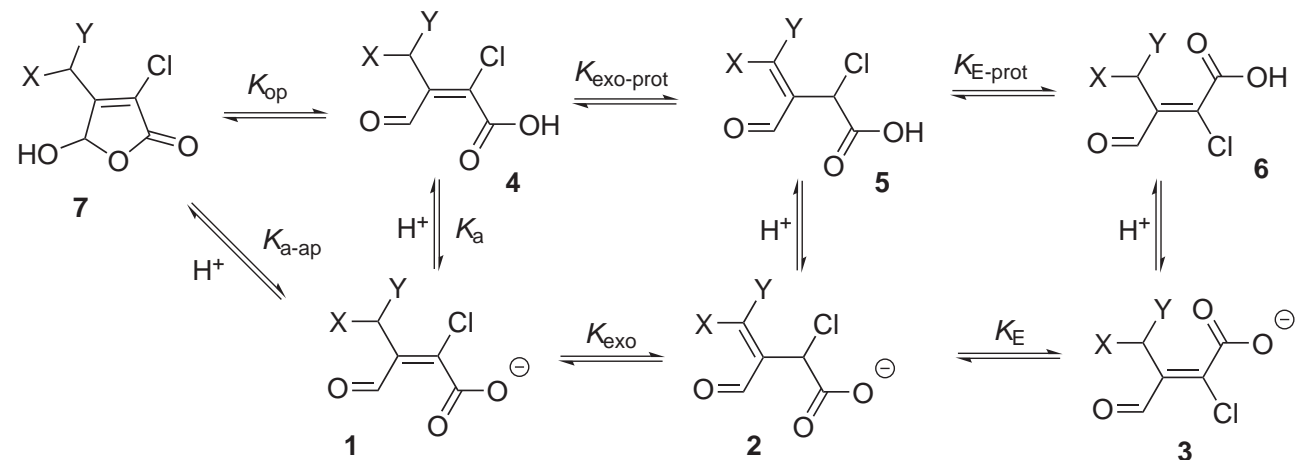

Scheme 5.21. Forms of MX and analogues in equilibrium in aqueous solution.

The computed equilibrium constants for the most significant reactions $\left(K_{\mathrm{a}}, K_{\mathrm{op}}\right.$ and $\left.K_{\mathrm{a}}^{\mathrm{app}}\right)$ are reported in Table 5.17.

Consideration of all negatively charged species and all non-charged species for the calculation for the calculation of $K_{\mathrm{a}}^{\text {app }}$ yields values within \pm 0.01 of those reported in Table 5.17, and thus a simplified definition of $K_{\mathrm{a}}^{\mathrm{app}}$ was used (eq. 5.33)

$$
K_{\mathrm{a}}^{\mathrm{app}}=\left[\mathrm{H}^{+}\right] \frac{[1]+[2]+[3]}{[4]+[5]+[6]+[7] \simeq\left[\mathrm{H}^{+}\right] \frac{[1]}{[7]}}=K_{\mathrm{a}} \times K_{\mathrm{op}}
$$

It is observed that, as occurs with other hydroxyhalofuranones, the cyclic form is far more stable than all the open-chain undissociated counterparts. Since the inductive effect of the $\mathrm{CHX}_{2}$ group modifies simultaneously the nucleophilicity of the carboxylate group and the electrophilicity of the aldehyde group in opposite directions, little variation in $\mathrm{p} K_{\mathrm{op}}$ is observed among MCF, CMCF and MX.

The most stable open-chain form, 4 , has a $\mathrm{p} K_{\mathrm{a}}$ value between approximately 1 and 2 . This suggests that MCF, CMCF and MX are strong acids, and justifies the choice of trichloroacetic acid as a reference for $\mathrm{p} K_{\mathrm{a}}$ calculation. This result is not unexpected, given the inductive effect of the halogen atoms and 
Table 5.17. Constants for some MXY-equilibria

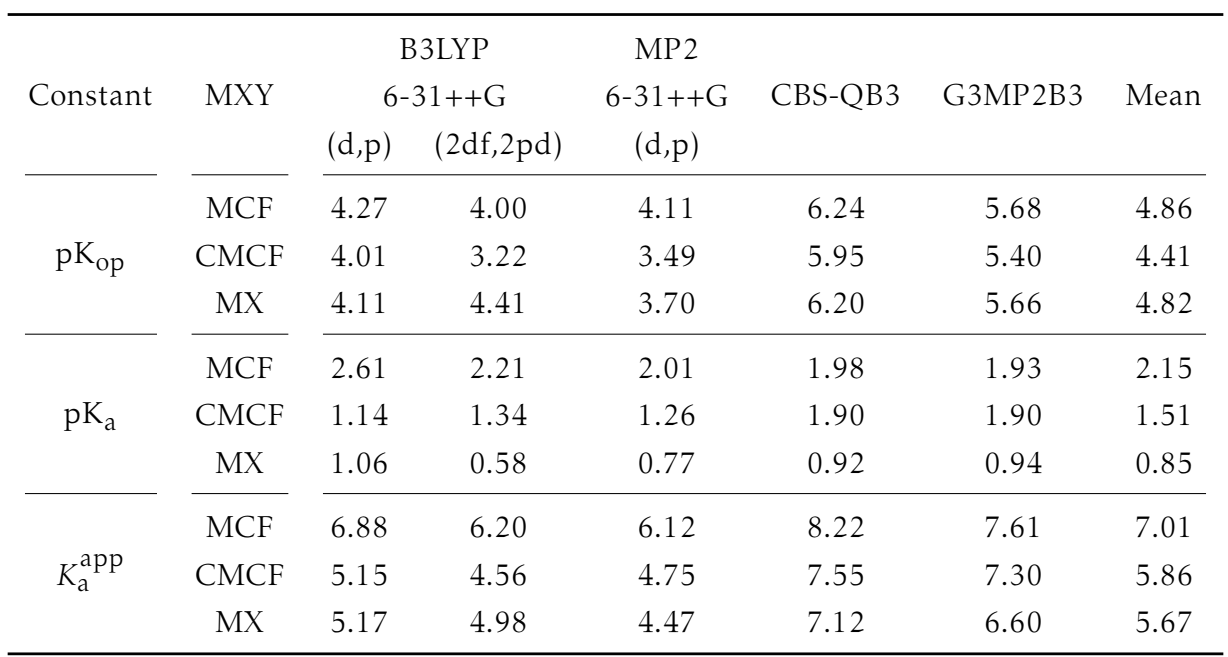

the conjugative effect of the $\alpha, \beta$-insaturation. For the same reasons, the order of decreasing $\mathrm{p} K_{\mathrm{a}}$ is MCF $>\mathrm{CMCF}>\mathrm{MX}$. Previous estimates for the $\mathrm{p} K_{\mathrm{a}}$ of MCF and MX (3.27 and 1.85) ${ }^{138}$ result rather high, especially when compared to $\mathrm{p} K_{\mathrm{a}}$ of chloroacetic acid (2.85), which lacks de conjugative effect of the double bond and the carbonyl group.

Because of this high acidity, halofuranones exist either as the cyclic form in acidic media (7), or as the open-chain anions at higher $\mathrm{pH}$ values (mostly the $Z$-form, 1 ), the intermediate protonated open-chain species being always minor.

Thus, the apparent dissociation constants of 7 to 1 , which are the ones measured experimentally (either by UV-Vis or NMR spectroscopy or by titration), are about $10^{-6}$ for the more halogenated furanones and around $10^{-7}$ for MCF. This value compares favorably with the experimental values of $K_{\mathrm{a}}^{\mathrm{app}}=5.25$ for $\mathrm{MX}^{125,176}$ and $K_{\mathrm{a}}^{\text {app }} \sim 6$ for MCF. ${ }^{175}$

Species Distribution. Since the $\mathrm{pH}$ values of tap and natural waters and the cellular medium are close to neutrality, we have determined the equilibrium concentrations of $1-7$ at $\mathrm{pH}=7.0$. These values are reported as the negative logarithms (p[i]) of the relative concentrations, with respect to the major species (1) in Table 2.

At the $\mathrm{pH}$ levels of the cellular medium or tap water, MXY exist in equilibrium mostly in the dissociated $Z$ form (1), with significant equilibrium concentrations of the $E$ form (3): about $10 \%$ for MCF and CMCF and $1 \%$ for MX. This observation can be understood in terms of the steric bulk of the CXY group: steric hindrance forces the carboxylate moiety to deviate from the more conjugated planar conformation. Since the chlorine atom in CMCF is oriented outwards, similar effects are observed for MCF and CMCF, whereas repulsion is larger in MX. 
Table 5.18. Relative concentration of species $1-7$ expressed as $\mathrm{p}(\mathrm{i})$ at $\mathrm{pH}=7.00$

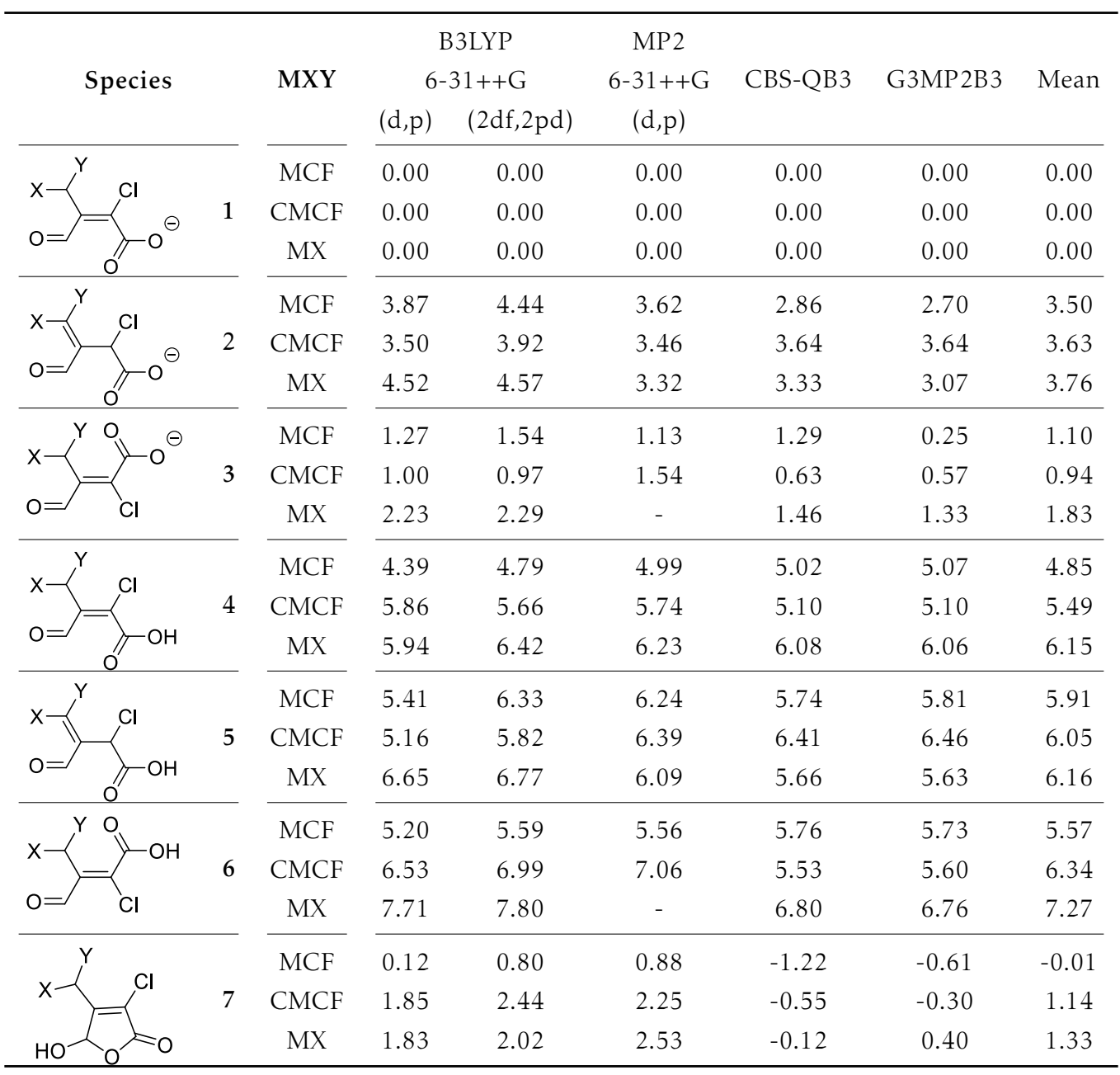

The cyclic form (7) is also a major species, with an equilibrium concentration of about $10 \%$ for $\mathrm{CMCF}$ and MX. In the case of MCF, whose somewhat low acidity displaces the $7 \rightleftharpoons 4 \rightleftharpoons 1+\mathrm{H}^{+}$equilibrium towards the closed-chain form, the equilibrium concentration of $\mathbf{7}$ is higher and amounts to more than $25 \%$ of the total concentration.

For all three halofuranones, the relative equilibrium concentration of the putative alkylating intermediate 2 is very low, of the order of 1 in 10,000. No significant differences, within the accuracy of the method, are observed in the concentration of 2 for MCF, CMCF and MX, which suggests that the extraordinary behavior of MX does not arise from an abnormally high concentration of 2 . Thus, the unmatched genotoxicity of MX could lie in the inherent reactivity of MX-2, rather than its high concentration, or, as has been proposed, be due to a mechanism other than DNA-alkylation. The methylene carbon in 2-CMCF in unsymetrically substituted and, due the increased steric repulsion, the isomer in which the chlorine 
atom opposes the aldehydic oxygen is less stable by about $5 \mathrm{~kJ} \mathrm{~mol}^{-1}$.

All undissociated open-chain forms are present in very minor amounts, with concentrations about six logarithm units below that of major species, however, the exo species is much more significant among the neutral forms than it is among the anions, the concentrations of 4,5 and 6 being very similar. The reasons for this different behavior probably lie in the negative charge being highly stabilized in 1 and 3 by the extended conjugation, as compared to 2. Loss of this conjugation by species 4 and 6 results in a less dramatic destabilization since no charges are present.

Due to its high acidity, MX has the lower concentration of undissociated species of the three compounds studied, which further rules out the high concentration of $\mathbf{5}$ as a source of the high mutagenicity.

Isomer interconversion. The direct interconversion of the $Z$ and $E$ forms by rotation around their double bond is highly unfavorable, due to the loss of conjugation and the presence of bulky substituents. The fact that no $E \rightleftharpoons Z$ reaction is observed at acidic or neutral $\mathrm{pH}$ suggests that the direct reaction does not take place, and only a base-catalyzed pathway is available, i.e., the reaction proceeds as $1 \rightleftharpoons 2 \rightleftharpoons 3$, since the internal rotation barrier for 2 is much lower. This requires the successive deprotonation and protonation of $\mathbf{1}$ (or $\mathbf{3}$ ), and thus, the accessibility of this pathway must be closely related to the ability of 1 (or 3 ) to release a proton.

For this reason, we have computed the second pKa of MCF, CMCF and MX (Table 5.19). Since we lack an appropriate reference to apply the relative method, (see above) absolute $\mathrm{p} K_{\mathrm{a} 2}$ values are affected by high systematic errors which reach up to several logarithm units. Thus, only the differences in acidity $\left(\Delta \mathrm{p} K_{\mathrm{a} 2}\right.$, referred to $\left.\mathrm{MX}\right)$ can be discussed in quantitative terms.

Table 5.19. Difference in $\mathrm{p} K_{\mathrm{a} 2}$ among $\mathrm{MXY}$

\begin{tabular}{|c|c|c|c|c|c|c|}
\hline \multirow[t]{2}{*}{ MXY } & \multicolumn{2}{|c|}{$\begin{array}{c}\text { B3LYP } \\
6-31++G\end{array}$} & \multirow{2}{*}{$\begin{array}{c}\mathrm{MP} 2 \\
6-31++\mathrm{G} \\
(\mathrm{d}, \mathrm{p}) \\
\end{array}$} & \multirow[t]{2}{*}{ CBS-QB3 } & \multirow[t]{2}{*}{ G3MP2B3 } & \multirow[t]{2}{*}{ Mean } \\
\hline & $(\mathrm{d}, \mathrm{p})$ & $(2 \mathrm{df}, 2 \mathrm{pd})$ & & & & \\
\hline $\mathrm{MCF}$ & 2.2 & 3.3 & 3.8 & 2.8 & 2.1 & 2.8 \\
\hline $\mathrm{CMCF}$ & 0.5 & -0.2 & 2.9 & 3.3 & 3.1 & 1.9 \\
\hline
\end{tabular}

As can be seen, MX is about 2-3 log units more acidic than the less halogenated homologues. This translates in the existence of a 100-1000 fold more accessible pathway towards the terminally doublebonded isomer (2) and the E-form in the case of MX. Perhaps in the availability of this pathway allowing more rapid formation of 2 lies one of the causes for then enhanced genotoxicity of MX.

A concerted pathway, in which loss and gain of the protons occur simultaneously is also possible and would also be favored by the increased acidity of MX.

5.3.1.4.2. Electron affinities. It has been hypothesized that MX can extract an electron from DNA, especially from guanosine hot spots for oxidation. This would afford a radical cation, which is likely 
to lead to abasic lesions. This would be in better agreement with the observed mutational pattern of MX than the nucleotide adducts found so far. Also, some evidence for the ability MXY to cause oxidative damage exists. REFERNCIA We have computed the electron affinities of MXY in their open-chain anionic and closed forms ( 1 and 7 ).

Table 5.20. Electron affinities of MXY

\begin{tabular}{|c|c|c|c|c|c|c|}
\hline \multirow{3}{*}{$\begin{array}{l}\text { Basis set } \\
\text { Isomer }\end{array}$} & \multicolumn{6}{|c|}{ Vertical electron affinity $(\mathrm{eV})$} \\
\hline & \multicolumn{2}{|c|}{$6-31 G(d)$} & \multicolumn{2}{|c|}{$6-31++G(d, p)$} & \multicolumn{2}{|c|}{$6-311+G(2 d f, 2 p)$} \\
\hline & 7 & 1 & 7 & 1 & 7 & 1 \\
\hline $\mathrm{MCF}$ & 1.7 & 1.5 & 2.2 & 2.1 & 2.3 & 2.2 \\
\hline CMCF & 2.3 & 1.8 & 2.7 & 2.3 & 2.8 & 2.5 \\
\hline \multirow[t]{2}{*}{ MX } & 2.5 & 2.0 & 2.9 & 2.5 & 3.0 & 2.7 \\
\hline & \multicolumn{6}{|c|}{ Adiabatic electron affinity $(\mathrm{eV})$} \\
\hline Basis set & \multicolumn{2}{|c|}{$6-31 G(d)$} & \multicolumn{2}{|c|}{$6-31++G(d, p)$} & \multicolumn{2}{|c|}{$6-311+G(2 d f, 2 p)$} \\
\hline Isomer & 7 & 1 & 7 & 1 & 7 & 1 \\
\hline MCF & 2.2 & 2.1 & 2.7 & 2.7 & 2.6 & 2.7 \\
\hline $\mathrm{CMCF}$ & 4.1 & 3.9 & 4.6 & 4.3 & 4.5 & 4.3 \\
\hline MX & 4.5 & 4.3 & 4.9 & 4.6 & 4.9 & 4.6 \\
\hline
\end{tabular}

Results suggest that MXY have high electron affinities, and thus are easily reduced, in keeping with previous calculations. ${ }^{109}$ Post-HF and compound methods (CBS-QB3 and G2) were also assayed, but the presence of large spin contamination made the results unreliable.

The radical anion forms of CMCF and MX were observed to be unstable, and expel a chloride anion. This greatly stabilizes the system, and hence the difference between vertical and adiabatic electron affinities.

The ionization potentials of nucleobases and (poly)nucleotides, and the closely related one-electron oxidation potentials, have been determined experimentally. In general, guanine and guanine-containing oligonucleotides show the lowest ionization potential, which is around $5 \mathrm{eV}$ in solution. Several computational works have also addressed the issue, finding good accord between theoretical and empirical values. For comparison with the electron affinities of MXY, we have obtained are, $6.3 \mathrm{eV}$ for adenosine; $6.0 \mathrm{eV}$ for guanosine both at the B3LYP 6-31G(d) and 6-31++G(d,p) levels respectively.

The differences between IP of the nucleotides and $\mathrm{E}_{\mathrm{A}}$ of the MXY are conclusive: the vertical reduction potentials of MXY are too small for the MXY in either their open-chain or closed forms to oxidize nucleotides directly . 


\subsubsection{Alkylating potential of halohydroxyfuranones}

Halohydroxyfuranones, both MXA and MXY have been proven to bond covalently to DNA, affording a variety of adducts.

\subsubsection{Alkylating potential of mucohalic acids}

MXA are known to produce mutagenic lesions in accord with their observed ability to form covalent adducts with guanosine nucleotides. Whereas an alkylation mechanism has been proposed, on the basis of the final products formed, no additional empirical or theoretical evidence has been found. Also, the putative role of mucoxyhalic acids in the DNA-binding reaction of MXA is unclear.

5.3.2.1.1. Reaction with aniline. The changes in the UV-Vis spectrum of the reaction mixture of MXA with excess aniline along time during the reaction are shown in Figure 5.13. The variation in absorbance (A) with time at the wavelength of maximum absorption of the $\mathrm{AD}_{\mathrm{AN}-\mathrm{MXA}}$ adduct, $\lambda=320 \mathrm{~nm}$, (Figure 5.14), complies with a pseudo-first order rate equation (eq. 5.34),
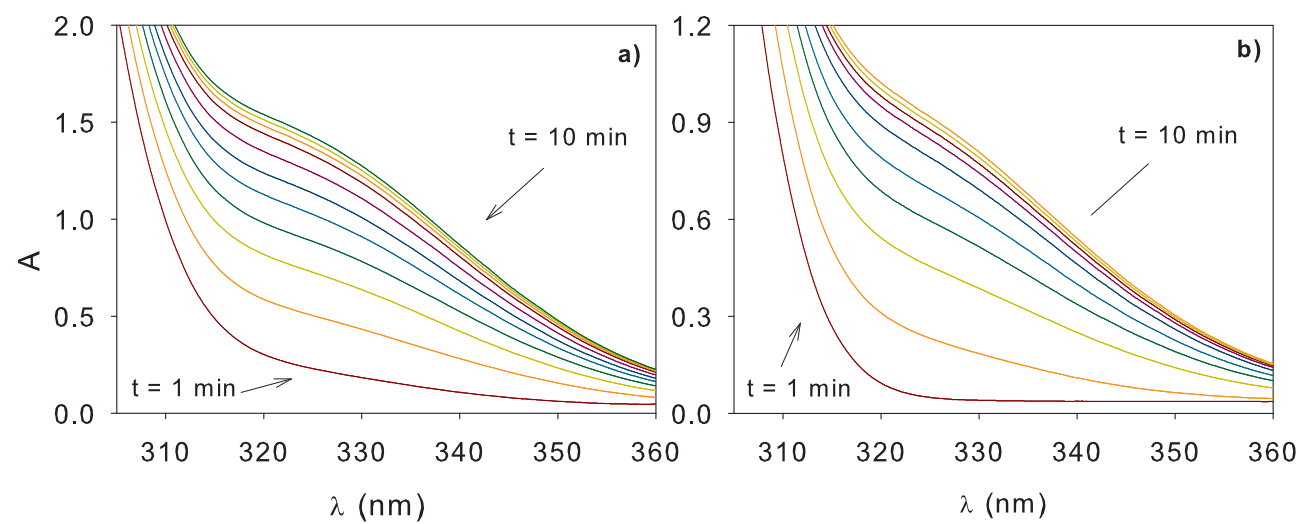

Figure 5.13. Spectrograms showing the formation of the AN-MXA adducts in water, $[A N]=0.010 \mathrm{M}, \mathrm{T}=25.0^{\circ} \mathrm{C}, \mathrm{pH}$ $=7.00$. a) $\left.[\mathrm{MCA}]=5.0 \times 10^{-5} \mathrm{M}, \mathrm{b}\right)[\mathrm{MBA}]=3.0 \times 10^{-5} \mathrm{M}$.

$$
A=\epsilon_{\mathrm{AN}-\mathrm{MXA}} l\left[\mathrm{MXA}_{\mathrm{O}}\right]\left(1-\exp \left(-k_{1 \mathrm{AN}}^{\mathrm{MXA}} t\right)\right)
$$

$\epsilon_{\mathrm{AN}-\mathrm{MXA}}$ is the molar absorption coefficient of the adduct; $l$ is the optic path, and $k_{1 \mathrm{AN}}^{\mathrm{MXA}}$ is the alkylation rate pseudo-constant.

Since $k_{1 \mathrm{AN}}^{\mathrm{MX}}$ is first order in the concentration of aniline,

$$
k_{1 \mathrm{AN}}^{\mathrm{MXA}}=k_{\mathrm{AN}}^{\mathrm{MXA}}[\mathrm{AN}] \mathrm{o}
$$




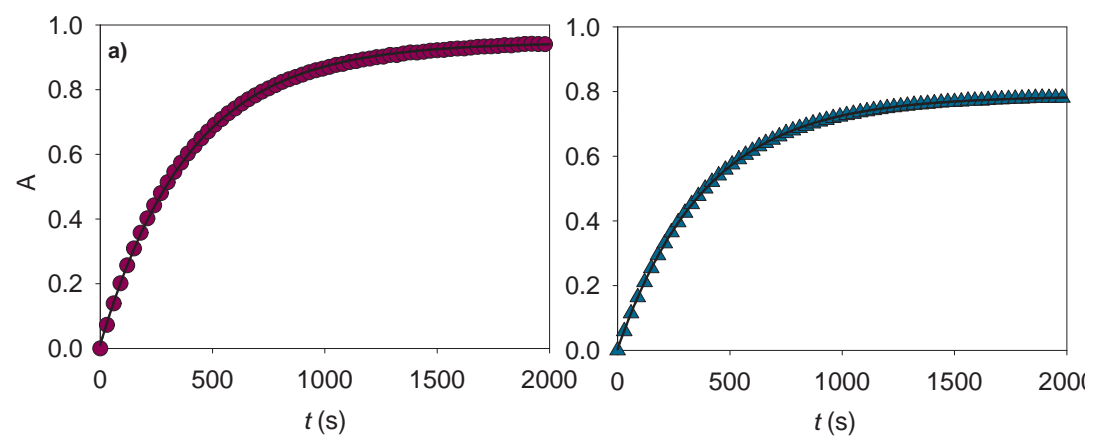

Figure 5.14. Typical kinetic profile of the alkylation reaction of aniline by $M X A$ in water, $T=25.0^{\circ} \mathrm{C}, \mathrm{pH}=7.00$. a) $\left.[\mathrm{MCA}]=3.1 \times 10^{-5} \mathrm{M},[\mathrm{AN}]=0.015 \mathrm{M} ; \mathrm{b}\right)[\mathrm{MBA}]=3.0 \times 10^{-5} \mathrm{M} ;[\mathrm{AN}]=0.010 \mathrm{M}$.

From these results the following experimental rate equation (eq. 5.36) can be derived:

$$
\frac{d\left[\mathrm{AD}_{\mathrm{AN}-\mathrm{MXA}}\right]}{d t}=k_{\mathrm{AN}}^{\mathrm{MXA}}[\mathrm{AN}][\mathrm{MXA}]
$$

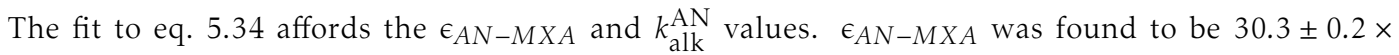
$10^{-3} \mathrm{M}^{-1} \mathrm{~cm}^{-1}$ and $\left(27.3 \pm 0.3 \times 10^{-3} \mathrm{M}^{-1} \mathrm{~cm}^{-1}\right.$ for MCA and MBA respectively at $\lambda=320 \mathrm{~nm}$. The reaction rate was observed to be $\mathrm{pH}$-independent in the $5.5<\mathrm{pH}<8.0$ range. Table 5.21 shows the alkylation rate constants obtained in water and aquo-organic reaction media at different temperatures. The two mucohalic acids react at very similar rates, the reaction with MBA being slightly faster. The decrease in the dielectric constant when switching from pure water to $7: 3(w / d)$ medium elicits approximately a six-fold decrease in the rate constant.

Table 5.21. Rate constants of aniline alkylation by mucohalic acids as a function of temperature and the reaction medium

\begin{tabular}{|c|c|c|c|c|}
\hline \multirow{3}{*}{$\mathrm{T}\left({ }^{\circ} \mathrm{C}\right)$} & \multicolumn{4}{|c|}{$k_{\mathrm{alk}}^{\mathrm{AN}}\left(\mathrm{M}^{-1} \mathrm{~s}^{-1}\right)^{a}$} \\
\hline & \multicolumn{2}{|c|}{ Water } & \multicolumn{2}{|c|}{$7: 3 w / d$} \\
\hline & MCA & MBA & MCA & MBA \\
\hline 25.0 & 0.162 & 0.242 & 0.0304 & 0.0454 \\
\hline 27.5 & 0.190 & 0.273 & 0.0329 & 0.0490 \\
\hline 30.0 & 0.210 & 0.302 & 0.0368 & 0.0529 \\
\hline 32.5 & 0.235 & 0.335 & 0.0392 & 0.0559 \\
\hline 35.0 & 0.275 & 0.370 & 0.0430 & 0.0610 \\
\hline 37.5 & 0.300 & 0.404 & 0.0475 & 0.0640 \\
\hline
\end{tabular}

${ }^{a}$ Values are reproducible to within $5 \%$

The reaction rate for the alkylation of aniline is very fast compared to the rate of formation of mucoxy- 
halic acids (MOXA) from mucohalic acids (vide supra). The hydrolysis half-life of MXA at $25.0^{\circ} \mathrm{C}$ and $\mathrm{pH}$ $=8.0$ is of the order of years, whereas the alkylation half-life in the same conditions ranges from minutes to hours. This confirms that the role of MOXA in the alkylation by MXA is very small or inexistent, and thus the presence of hydroxyl groups in the earlier position of halogen atoms must be due to a reaction with water or hydroxide ions succeeding, and not preceding, the alkylation.

The fit of the $k_{\text {alk }}^{\mathrm{AN}}$ values (Table 5.21) to the Eyring-Wynne-Jones equation, gives the activation parameters for the alkylation reaction 5.22 .

Table 5.22. Activation parameters for the alkylation of aniline by MXA in aqueous and water/dioxane media

\begin{tabular}{|c|c|c|c|c|}
\hline & Medium & $\begin{array}{c}\Delta^{\ddagger} H^{\ominus} \\
\left(\mathrm{kJ} \mathrm{mol}{ }^{-1}\right)\end{array}$ & $\begin{array}{c}-\Delta^{\ddagger} S^{\ominus} \\
\left(\mathrm{J} \mathrm{mol}^{-1} \mathrm{~K}^{-1}\right)\end{array}$ & $\begin{array}{c}\Delta^{\ddagger} G^{\ominus}\left(35^{\circ} \mathrm{C}\right) \\
\left(\mathrm{kJ} \mathrm{mol}{ }^{-1}\right)\end{array}$ \\
\hline \multirow{2}{*}{ MCA } & Water & $35 \pm 1$ & $141 \pm 4$ & $78 \pm 2$ \\
\hline & $7: 3 w / d$ & $25 \pm 1$ & $191 \pm 2$ & $84 \pm 2$ \\
\hline \multirow{2}{*}{ MBA } & Water & $29 \pm 1$ & $159 \pm 2$ & $78 \pm 2$ \\
\hline & $7: 3 w / d$ & $19 \pm 1$ & $207 \pm 4$ & $83 \pm 2$ \\
\hline
\end{tabular}

The low values for the activation enthalpies are consistent with the high reactivity of the aldehyde group of mucohalic acids, whereas the negative activation entropies are consistent with the idea of alkylation being an addition reaction.

The reaction products $\left(\mathrm{AD}_{\mathrm{AN}-\mathrm{MXA}}\right)$ were identified as the 2:1 aniline-MXA adducts by mass spectrometry, with $m / z=301.1$ for MCA and $m / z=345.1$ for MBA (calc. 301.1 and 345.1). This is consistent with the observation that mucohalic acids react with a stoichiometric amount of aniline to form the Schiff base, whereas in an excess of aniline the diadduct is formed by substitution of the halogen atom $\alpha$ to the carboxylic group. ${ }^{69}$ Minor amounts of a secondary product showing monoalkylation and substitution of the halogen in $\alpha$ by a hydroxyl group were also detected $(\mathrm{m} / z=226.1$ for MCA and $m / z=270.0$ for MBA).

This allows us to propose the following reaction mechanism (Scheme 5.22): as shown before, mucohalic acids exist mostly in the open-chain conformation in the reaction conditions. The attack of aniline on the aldehyde group is the limiting step, whereas the subsequent elimination to form the Schiff base and further reaction with aniline or water take place rapidly.

It is known that no substitution of the halogen atom by aniline is observed prior to formation of the Schiff base ${ }^{69}$ and that substitution of $\alpha$-halogens by water to form mucoxyhalic acids is very slow. This, together with our results, suggests that the reactivity of the halogen $\alpha$ to the carboxyl group in the Schiff base is increased with respect to that in mucohalic acids. This enhanced electrophilicity is also consistent with the formation of products with hydroxyl substituents $\alpha$ to the carboxylate group in adducts of MXA with adenosine, guanosine and cytidine.

Cyclic adducts are the main product in the alkylation of cytidine, adenosine and guanosine in water, as shown in Scheme 5.6. They are formed by the attack of an aromatic nitrogen in the nucleobases on the carbon $\beta$ to the carboxylate group after alkylation of the exocyclic amine group. 


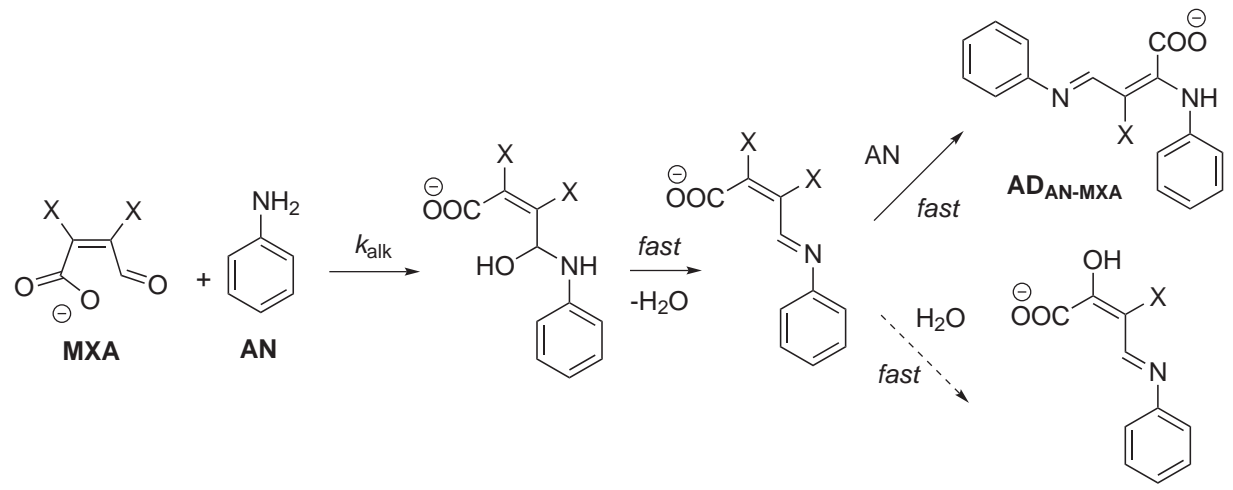

Scheme 5.22. Reaction of MXA with aniline

The fact that the intramolecular reaction takes place at the $\beta$ instead of the more reactive $\alpha$ position is possibly due to a combination of various factors: a) the potential six-ring products obtained by intramolecular attack on the carbon $\alpha$ are disfavored; b) the endocyclic nitrogen atoms are not nucleophilic enough to react with the $s p^{2} \alpha$-carbon, while stronger nucleophilic hydroxide ions do react at that site; and c) substitution in $\alpha$ by water or hydroxide is relatively fast and takes place before cyclization in $\alpha$ can occur. Since the position $\beta$ to the carboxylate shows almost no reactivity prior to alkylation - MXA undergo substitution by hydroxide only in the $\alpha$ position - it may be concluded that the electrophilicity of this carbon must also be enhanced before cyclization. Substitution of chlorine in $\alpha$ by a hydroxyl group yields an enol, whose corresponding keto form would show a strongly increased reactivity on the $\beta$ carbon, since it would acquire a more electrophilic $s p^{3}$ hybridization and yield a favored five-membered ring.

5.3.2.1.2. Reaction with NBP. As described above, the alkylation reaction of NBP by mucohalic acids was followed spectrophotometrically, at at $\lambda=510 \mathrm{~nm}$ in the case of MCA and $\lambda=485 \mathrm{~nm}$ for MBA, where the adducts show maximum absorption (Figure 5.15). The kinetic profiles of typical reactions are shown in Figure 5.16 and show that the adduct is unstable, decomposing quite rapidly in the reaction medium. This suggests a bi-exponential (consecutive reaction) mechanism.

However, closer scrutiny reveals the existence of an induction period. This feature has important mechanistic implications since it implies the existence of an additional step. Thus, the need to take into account an extra reaction is imposed. When this third step is considered (Scheme 5.23), the rate equations (5.37-5.39) are obtained. If $k_{\mathrm{NBP}}^{\mathrm{MXA}}$ is the alkyaltion rate constant, $k_{1 \mathrm{NBP}}^{\mathrm{MXA}}$ is the alkylation pseudo-constant, $k_{\mathrm{conv}}^{\mathrm{AD}}$ is the rate constant for the conversion of $\mathrm{AD} 2 \mathrm{MXA}$ into $\mathrm{AD} 2 \mathrm{MXA}$, and $k_{\mathrm{dec}}^{\mathrm{AD}_{2}}$ is the rate constant for the decomposition of $\mathrm{AD} 2 \mathrm{MXA}$, we have 

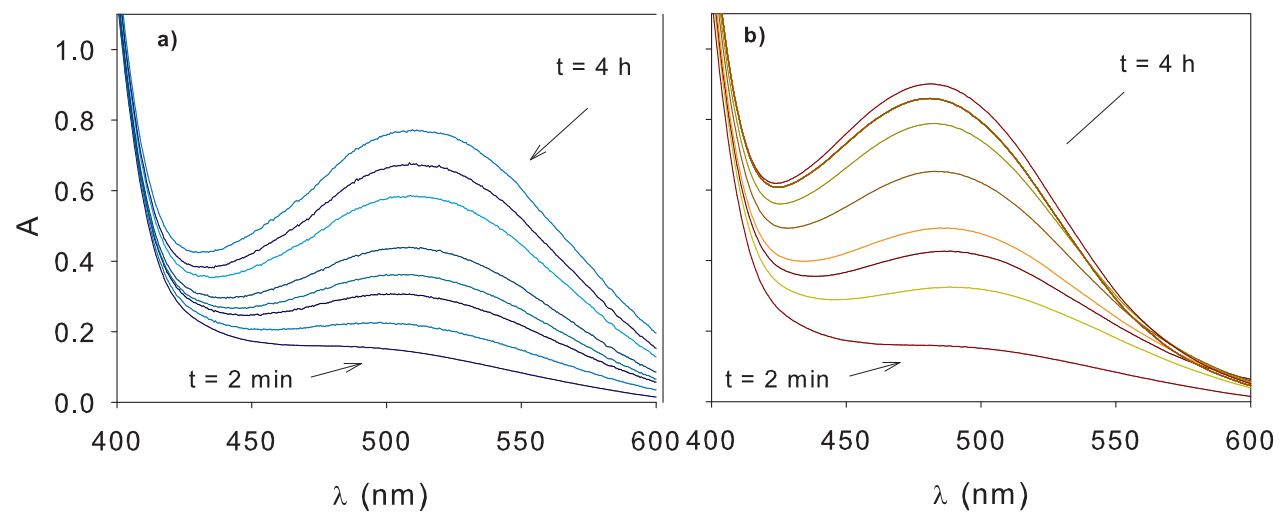

Figure 5.15. Spectrograms showing the formation of the NBP-MXA adducts in 7:3 $\mathrm{w} / \mathrm{d}$, [NBP] $=0.010 \mathrm{M}, \mathrm{T}=25.0^{\circ} \mathrm{C}$, $\mathrm{pH}=7.00$. a) $\left.[\mathrm{MCA}]=2.5 \times 10^{-5} \mathrm{M}, \mathrm{b}\right)[\mathrm{MBA}]=2.5 \times 10^{-5} \mathrm{M}$

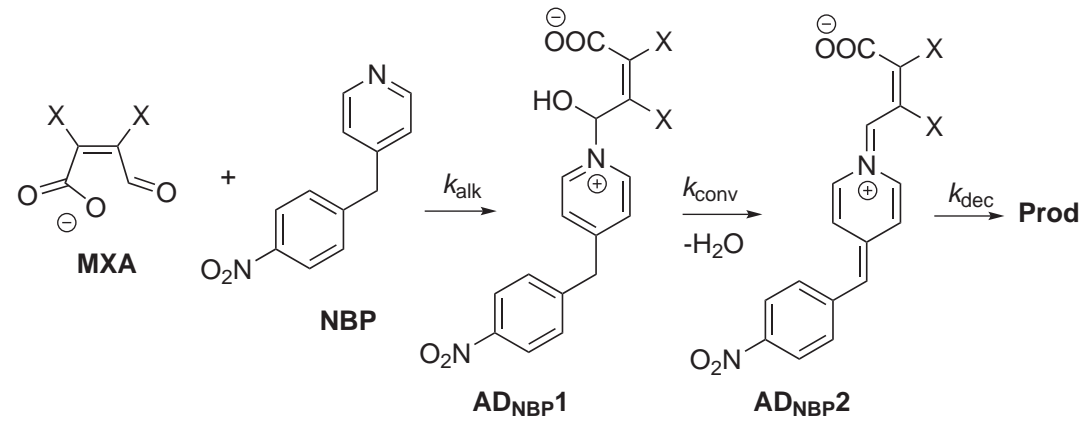

Scheme 5.23. Reaction of MXA with NBP

$$
\begin{aligned}
& \frac{d[\mathrm{MXA}]}{d t}=-k_{\mathrm{NBP}}^{\mathrm{MXA}}[\mathrm{NBP}][\mathrm{MXA}]=-k_{1 \mathrm{NBP}}^{\mathrm{MXA}}[\mathrm{MXA}] \\
& \frac{d\left[\mathrm{AD} 1_{\mathrm{NBP}}^{\mathrm{MXA}}\right]}{d t}=k_{1 \mathrm{NBP}}^{\mathrm{MXA}}[\mathrm{MXA}]-k_{\mathrm{conv}}^{\mathrm{AD}_{1}}\left[\mathrm{AD} 1_{\mathrm{NBP}}^{\mathrm{MXA}}\right] \\
& \frac{d\left[\mathrm{AD}_{\mathrm{NBP}}^{\mathrm{MXA}}\right]}{d t}=k_{\mathrm{conv}}^{\mathrm{AD}_{1}}\left[\mathrm{AD} 1_{\mathrm{NBP}}^{\mathrm{MXA}}\right]-k_{\mathrm{dec}}^{\mathrm{AD}_{2}}\left[\mathrm{AD} 2_{\mathrm{NBP}}^{\mathrm{MXA}}\right]
\end{aligned}
$$

The rate equations (5.37-5.39) can be integrated to yield: 


$$
\begin{aligned}
& {\left[\mathrm{AD} 2_{\mathrm{NBP}}^{\mathrm{MXA}}\right]=\frac{[\mathrm{MXA}]_{\mathrm{o}} k_{1 \mathrm{NBP}}^{\mathrm{MXA}} k_{\mathrm{conv}}^{\mathrm{AD}_{1}}}{\left(k_{1 \mathrm{NBP}}^{\mathrm{MXA}}+k_{\mathrm{conv}}^{\mathrm{AD}}\right)\left(k_{1 \mathrm{NBP}}^{\mathrm{MXA}}+k_{\mathrm{dec}}^{\mathrm{AD}_{2}}\right)\left(k_{\mathrm{dec}}^{\mathrm{AD}_{2}}-k_{\mathrm{conv}}^{\mathrm{AD}_{1}}\right)} \times} \\
& \times\left(\left(-k_{1 \mathrm{NBP}}^{\mathrm{MXA}}-k_{\mathrm{conv}}^{\mathrm{AD}}\right) e^{-k_{\mathrm{dec}}^{\mathrm{AD}_{2}} t}+\left(k_{\mathrm{conv}}^{\mathrm{AD}_{1}}-k_{\mathrm{dec}}^{\mathrm{AD}_{2}}\right) e^{-k_{1 \mathrm{NBP}}^{\mathrm{MXA}} t}+\left(k_{1 \mathrm{NBP}}^{\mathrm{MXA}}+k_{\mathrm{dec}}^{\mathrm{AD}_{2}}\right) e^{-k_{\mathrm{conv}} \mathrm{AD}_{1} t}\right)
\end{aligned}
$$

The quality of the fit of the experimental data to eq. 5.40 is shown in Figure 5.16. The fit to a biexponential mechanism, including only two successive reactions is also pictured. The quality of this simplified fit is insufficient since an important deviation from the experimental points exist, especially at the beginning of the reaction. This confirms the need to include a third step in the mechanism.

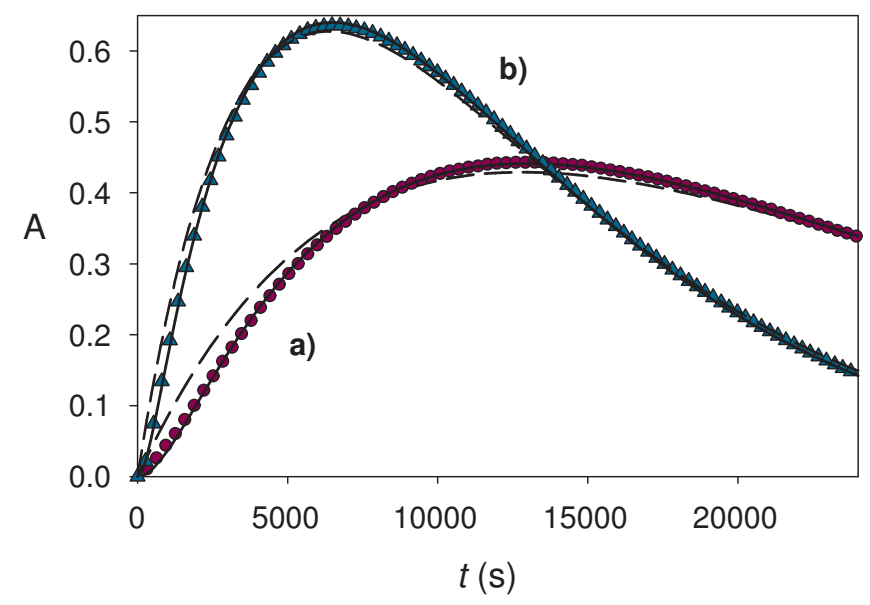

Figure 5.16. Typical kinetic profile of the alkylation reaction NBP aniline by $M X A$ in $7: 3 \mathrm{~W} / \mathrm{d}, \mathrm{T}=25.0^{\circ} \mathrm{C}, \mathrm{pH}=7.00$, $[\mathrm{NBP}]=0.015 \mathrm{M} \mathrm{a})[\mathrm{MCA}]=2.0 \times 10^{-5} \mathrm{M}$; ; b) $[\mathrm{MBA}]=2.0 \times 10^{-5} \mathrm{M}$. Dashed line (- - ): fit to a tworeaction mechanism; Solid line (-): fit to a three-reaction mechanism; Symbols: Experimental, (•) MCA, ( $\mathbf{\Lambda})$ MBA

Fitting the experimental data to eq. 5.40 affords values for the three rate constants and the molar absorption coefficient of the adducts. $\mathrm{AD} 1_{\mathrm{NBP}}^{\mathrm{MXA}}$ has null absorption and the wavelength of measurement, whereas $\mathrm{AD} 2 \mathrm{NXA}$ shows $\epsilon_{\mathrm{MCA}-\mathrm{NBP}}=25.6 \pm 0.4 \times 10^{3} \mathrm{M}^{-1} \mathrm{~cm}^{-1}$ at $\lambda=510 \mathrm{~nm}$ and $\epsilon_{\mathrm{MBA}-\mathrm{NBP}}=30.1 \pm$ $1.4 \times 10^{3} \mathrm{M}^{-1}$ at $\lambda=510 \mathrm{~nm}$.

5.3.2.1.2.1. Adduct formation. The experimental rate constant $k_{1 \mathrm{NBP}}^{\mathrm{MXA}}$ is first order with respect to the concentration of NBP, confirming that is, indeed, the alkylation rate constant. Values for the second-order rate constant $k_{\mathrm{NBP}}^{\mathrm{MXA}}$ are reported in 5.23. No dependence on $\mathrm{pH}$ was observed in the working range $(5.5<\mathrm{pH}<8.0)$.

Mucochloric acid shows higher rate constans, in keeping with the higher electronegativity of chlorine and also with the greater steric repulsion of bromine atoms. The values are between five- and ten-fold 
Table 5.23. Rate constants of NBP alkylation by mucohalic acids as a function of temperature, in 7:3 w/d at pH= 7.00

\begin{tabular}{|c|c|c|}
\hline \multirow{2}{*}{$\mathrm{T}\left({ }^{\circ} \mathrm{C}\right)$} & \multicolumn{2}{|c|}{$k_{\mathrm{NBP}}^{\mathrm{MXA}}\left(\mathrm{M}^{-1} \mathrm{~s}^{-1}\right)$} \\
\hline & MCA & MBA \\
\hline 25.0 & $8.0 \pm 0.2$ & $4.7 \pm 0.1$ \\
\hline 27.5 & $9.1 \pm 0.2$ & $5.6 \pm 0.1$ \\
\hline 30.0 & $10.3 \pm 0.5$ & $6.5 \pm 0.2$ \\
\hline 32.5 & $11.6 \pm 0.5$ & $7.5 \pm 0.2$ \\
\hline 35.0 & $12.6 \pm 0.4$ & $8.6 \pm 0.1$ \\
\hline 37.5 & $14.0 \pm 0.3$ & $10.0 \pm 0.2$ \\
\hline
\end{tabular}

lower than those observed for the alkylation of aniline, which is in keeping with the observation that mucohalic acids react primarily at the exocyclic amino groups of DNA bases rather than at the $s p^{2}$ nitrogen atoms in the ring. As a matter of fact, no nucleobase adducts of MXA with thymine, which lacks exocyclic nitrogen atoms, have been detected. However, the reactivity of MXA towards endocyclic nitrogen atoms is not completely negligible; a minor alkylation of aromatic nitrogen has also been observed in the reaction of MXA and guanosine, ${ }^{121}$ for whose endocyclic nitrogen atoms NBP is a nucleophilicity mode.

Table 5.24 reports the activation parameters for the alkylation of NBP.

Table 5.24. Activation parameters for the alkylation of NBP by MXA in 7:3 water/dioxane media

\begin{tabular}{|c|c|c|c|}
\hline & $\begin{array}{c}\Delta^{\ddagger} H^{\ominus} \\
\left(\mathrm{kJ} \mathrm{mol}{ }^{-1}\right)\end{array}$ & $\begin{array}{c}-\Delta^{\ddagger} S^{\ominus} \\
\left(\mathrm{J} \mathrm{mol}^{-1} \mathrm{~K}^{-1}\right)\end{array}$ & $\begin{array}{c}\Delta^{\ddagger} G^{\ominus}\left(35^{\circ} \mathrm{C}\right) \\
\left(\mathrm{kJ} \mathrm{mol}{ }^{-1}\right)\end{array}$ \\
\hline MCA & $32 \pm 1$ & $177 \pm 4$ & $86 \pm 2$ \\
\hline MBA & $43 \pm 1$ & $144 \pm 2$ & $88 \pm 2$ \\
\hline
\end{tabular}

The activation parameters parallel those obtained with aniline: low activation enthalpies, consistent with the electrophilicity of the aldehyde, and high negative activation entropies, consistent with an addition mechanism. The values of the activation free energies are slightly higher than the values obtained for the alkylation of aniline in water/dioxane medium. This is consistent with the lower reactivity of aromatic nitrogen atoms with mucohalic acids.

Comparison of the activation parameters with those obtained for the alkylation of NBP by other alkylating agents such as diketene $36\left(\Delta^{\ddagger} G^{\ominus}=71 \pm 2 \mathrm{~kJ} \mathrm{~mol}{ }^{-1}\right), \beta$-propiolactone $35\left(\Delta^{\ddagger} G^{\ominus}=87 \pm 2\right.$ $\left.\mathrm{kJ} \mathrm{mol}{ }^{-1}\right)$, potassium sorbate $42\left(\Delta^{\ddagger} G^{\ominus}=99 \pm 6 \mathrm{~kJ} \mathrm{~mol}^{-1}\right)$ or acrylamide $43\left(\Delta^{\ddagger} G^{\ominus}=104 \pm 2 \mathrm{~kJ} \mathrm{~mol}^{-1}\right)$ in the same reaction conditions suggests that mucohalic acids are strong alkylating agents when attacking endocyclic nitrogen atoms, and even more so when reacting with exocyclic amino groups, as is suggested by the higher rate constants obtained in the reaction with aniline. 
5.3.2.1.2.2. Adduct conversion. NBP has been shown to be a reasonable nucleophilicity model of guanosine N-7 and other nitrogen atoms in the cycle of nucleobases. However, its structure is very different from that of nucleotides and hence the subsequent transformations undergone by the MXA-NBP adduct after alkylation are hardly extrapolable to DNA. Therefore, they will only be discussed briefly. The values for $k_{\text {conv }}^{\mathrm{AD}_{1}}$ are shown in 5.25. These values are $\mathrm{pH}$-independent in the $5.5<\mathrm{pH}<8.0$ range. Since water elimination reactions occur through a base-catalyzed pathway, the possibility of general base catalysis by the phosphate buffer cannot be ruled out. It can be seen that the values for MBA are almost four-fold higher than those of MCA.

Table 5.25. Rate constants of NBP-MXA adduct conversion as a function of temperature, in 7:3 $\mathrm{w} / \mathrm{d}$ at $\mathrm{pH}=7.00$

\begin{tabular}{|c|c|c|}
\hline \multirow{2}{*}{$\mathrm{T}\left({ }^{\circ} \mathrm{C}\right)$} & \multicolumn{2}{|c|}{$k_{\text {conv }}^{\mathrm{AD}_{1}}\left(\mathrm{M}^{-1} \mathrm{~s}^{-1}\right)$} \\
\hline & MCA & MBA \\
\hline 25.0 & 0.12 & 0.45 \\
\hline 27.5 & 0.18 & 0.71 \\
\hline 30.0 & 0.24 & 1.10 \\
\hline 32.5 & 0.36 & 1.54 \\
\hline 35.0 & 0.52 & 2.03 \\
\hline 37.5 & 0.74 & 3.20 \\
\hline
\end{tabular}

The UV-Vis spectrum of $\mathrm{AD} 2 \mathrm{MXA}$ (Figure 5.15) deserves some attention: it shows absorption in the visible part of the spectrum, without the need for basification; the wavelengths of maximum absorption are significantly blue-shifted when compared to NBP adducts with other alkylating agents and the molar absorption coefficients are high as compared to those of other NBP adducts, which are about $5 \times 10^{3} \mathrm{M}^{-1} \mathrm{~cm}^{-1}$. This suggests that $\mathrm{AD} 2{ }_{\mathrm{NBP}}^{\mathrm{MXA}}$ is the highly conjugated elimination product shown in Scheme 5.23 .

5.3.2.1.3. Computational study of nucleobase alkylation. As exposed above, several works have addressed the nature of the MXA-nucleoside adducts in vitro, both those formed in the reaction of mucohalic acids with isolated nucleotides, and also in the reaction with double stranded DNA. Whereas several alkylation mechanisms have been proposed recursively, on the basis of the adduct structure, including using of isotopic tracers, no works have actually addressed the alkylation reaction path.

Whereas the model nucleophiles used so far in this work give reactions that can be followed more or less cleanly, the actual reaction of nucleotides and MXA is complex, since the reactives - which are quite unsoluble - have several possible reaction sites, close to a dozen different species are involved, and none of them has properties that allow easy kinetic study of the reaction. For those reasons, the study of the actual nucleotide alkylation path by MXA was undertaken using computational methods.

In order to bring together the kinetic evidence obtained with the model substrates, and the products identified by Kronberg et al. in the reaction of MXA and nucleotides, we have modeled the proposed 
reaction mechanism of nucleobase alkylation by MXA in silico (Scheme 5.24).

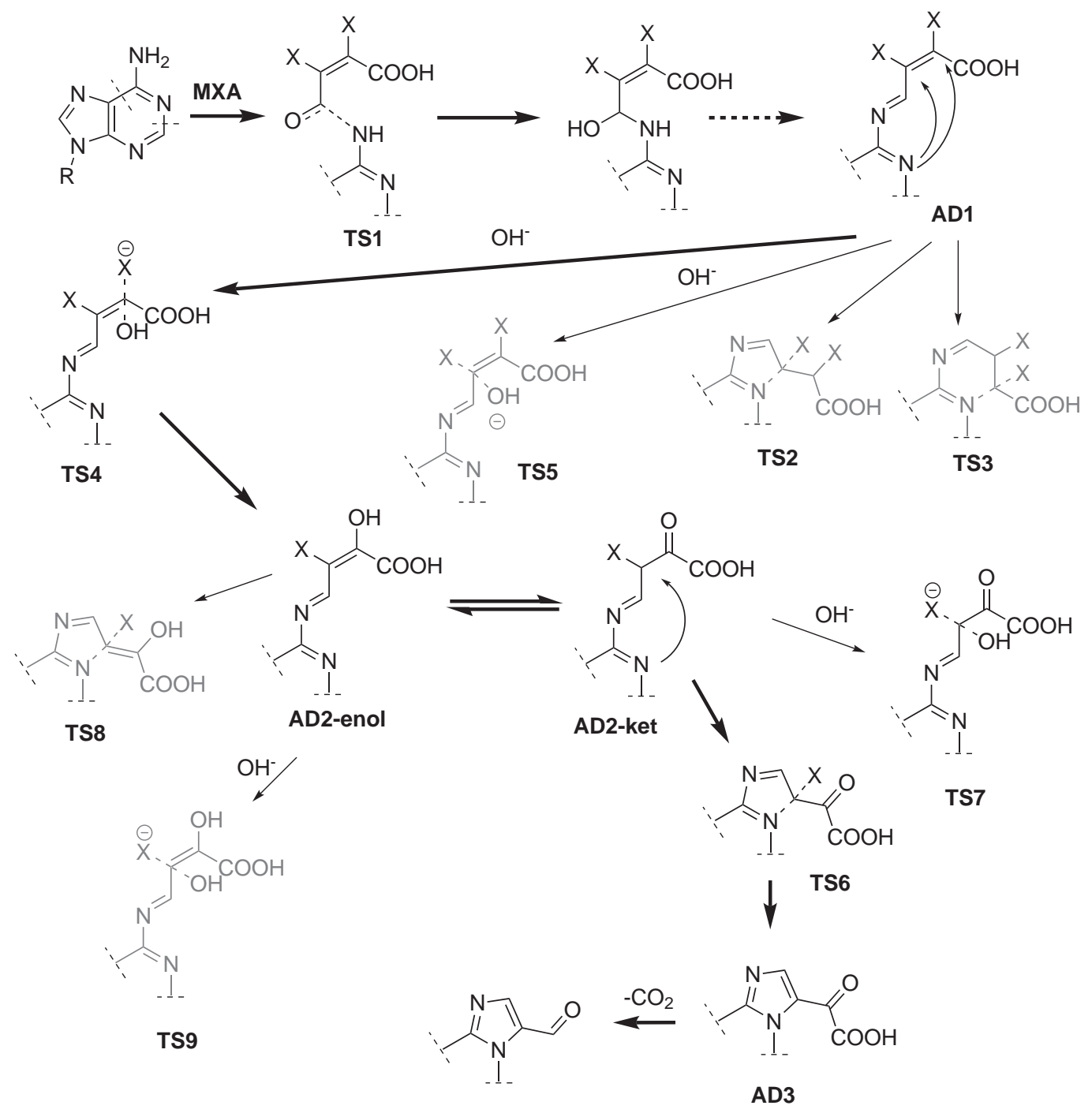

Scheme 5.24. Reaction of MXA with nucleotides

As shown in Scheme 5.24, transition states were computed for the following reactions: the addition to the aldehyde group (TS1); the putative ring closure reactions of AD1, affording both five- or six-membered rings (TS2 and TS3); substitution of the $\alpha$ and $\beta$ halogen atoms in AD1 by hydroxide (TS4 and TS5); the ring-closure reaction of AD2 in both its keto and enol forms (TS6 and TS8); the hydrolysis of AD2 in the (TS7 and TS9) and the decarboxylation of AD3. Of these, several reactions could be possible, at least in principle, but do not lead to the observed products (TS2, TS3 and TS5).

The energy barriers for the reactions as well as energy differences between different possible isomers are reported in Table 5.26 and 5.27. Guanosine adducts can exist both as keto and enol tautomers. The 
results reported correspond to the lower in energy, which is usually the keto form for minima and the enol form in species where the endocyclic nitrogen atom acts as a nucleophile.

Table 5.26. Relative energies along the alkylation path of nucleotides by MXA - I

\begin{tabular}{|c|c|c|c|c|c|c|c|c|}
\hline \multirow{4}{*}{ Species } & \multicolumn{8}{|c|}{ Relative energy $\left(\mathrm{kJ} \mathrm{mol}^{-1}\right)$} \\
\hline & \multicolumn{4}{|c|}{ Adenosine } & \multicolumn{4}{|c|}{ Guanosine } \\
\hline & \multicolumn{2}{|c|}{ MCA } & \multicolumn{2}{|c|}{ MBA } & \multicolumn{2}{|c|}{ MCA } & \multicolumn{2}{|c|}{ MBA } \\
\hline & $\Delta H^{\ominus}$ & $\Delta G^{\ominus}$ & $\Delta H^{\ominus}$ & $\Delta G^{\ominus}$ & $\Delta H^{\ominus}$ & $\Delta G^{\ominus}$ & $\Delta H^{\ominus}$ & $\Delta G^{\ominus}$ \\
\hline $\mathrm{TS} 1^{a}$ & 74.4 & 89.3 & 71.3 & 84.5 & 64.3 & 78.7 & 62.8 & 77.3 \\
\hline $\mathrm{TS} 2^{b}$ & 158.8 & 168.3 & 142.4 & 149.1 & 217.1 & 232.6 & 239.0 & 243.0 \\
\hline TS3 & 119.9 & 130.9 & 112.8 & 122.1 & 193.1 & 200.9 & 185.2 & 191.8 \\
\hline TS4 & 33.4 & 42.4 & 30.7 & 38.5 & 34.4 & 36.1 & 28.3 & 46.0 \\
\hline TS5 & 95.2 & 105.4 & 93.5 & 106.3 & 102.4 & 126.1 & 100.2 & 120.7 \\
\hline
\end{tabular}

The first inspection of the results suggests that reaction follows the path marked with boldface arrows in Scheme 5.24. The addition reaction of the amine at the carbonyl group has the highest barrier in the pathway, and thus this is expected to be the rate-limiting step for the complete alkylation pathway. However, the free energy barriers are very similar for some of the steps, especially in the case of guanosine, and hence the indetermination in the calculated energies is compatible with more than one step being rate-limiting, rather than a single specific reaction being a bottleneck.

Of the three nucleobases studied, guanosine shows the lowest alkylation barrier (Table 5.23), which is quite similar to that of aniline (Table 5.24), the calculated activation free energies for adenosine being about $10-15 \mathrm{~kJ} \mathrm{~mol}^{-1}$ higher. The initial barrier for the alkylation of cytidine is much higher than those of the purine bases $\left(\Delta^{\ddagger} H^{\ominus}=95.6 \mathrm{~kJ} \mathrm{~mol}^{-1}, \Delta^{\ddagger} G^{\ominus}=99.8 \mathrm{~kJ} \mathrm{~mol}^{-1}\right)$, and thus no further steps in the alkylation path were computed for this compound.

Computational calculation of the energy barrier for the elimination step is complex, since this kind of reactions are susceptible to both general base and acid catalysis by number of species that are present in both experimental conditions and in vivo, especially the phosphate buffer. Experimental evidence from the reaction of aniline suggests that this elimination reaction is very fast, and thus that it has little influence on the global reaction rate. For these reasons, the elimination step in the reaction pathway was not computed.

AD1 can undergo a variety of reactions that cleave the halogen-carbon bonds: substitution by hydroxide (or water) or by endocyclic nitrogen atoms, which yields cyclic products. Of these reactions, the one corresponding to the hydrolysis of the halogen atom $\alpha$ to the carboxylate group (TS4) is by far the most favored. This is consistent with the final reaction products and the proposed reaction pathway 
(Schemes 5.6 and 5.7). It also confirms that hydrolysis follows the formation of the adduct, but precludes cyclization, as was the case with aniline.

We also computed the barriers for the non-catalyzed hydrolysis reaction, with water as a nucleophile. The barrier is comparatively higher than that for hydroxide ions $\left(\Delta^{\ddagger} H^{\ominus}=82.1 \mathrm{~kJ} \mathrm{~mol}^{-1}, \Delta^{\ddagger} G^{\ominus}=89.7\right.$ $\mathrm{kJ} \mathrm{mol}^{-1}$ for adenosine-MCA), but the reaction is still faster than the intramolecular alternatives or the hydrolysis at the $\beta$-position. Thus, the alkylation pathway proceeds through either TS4 or its neutral counterpart, possibly depending on the reaction conditions.

In the reaction of MXA with aniline, further alkylation of a second aniline moiety is observed, instead of hydrolysis. This occurs because aniline is in quite high concentration, and also because its amino group is a stronger nucleophile than the $\mathrm{N}-1$ atom in adenosine or guanosine.

Table 5.27. Relative energies along the alkylation path of nucleotides by MXA - II

\begin{tabular}{|c|c|c|c|c|c|c|c|c|}
\hline \multirow{4}{*}{ Species } & \multicolumn{8}{|c|}{ Relative energy ${ }^{a}\left(\mathrm{~kJ} \mathrm{~mol}^{-1}\right)$} \\
\hline & \multicolumn{4}{|c|}{ Adenosine } & \multicolumn{4}{|c|}{ Guanosine } \\
\hline & \multicolumn{2}{|c|}{ MCA } & \multicolumn{2}{|c|}{ MBA } & \multicolumn{2}{|c|}{ MCA } & \multicolumn{2}{|c|}{ MBA } \\
\hline & $\Delta H^{\ominus}$ & $\Delta G^{\ominus}$ & $\Delta H^{\ominus}$ & $\Delta G^{\ominus}$ & $\Delta H^{\ominus}$ & $\Delta G^{\ominus}$ & $\Delta H^{\ominus}$ & $\Delta G^{\ominus}$ \\
\hline $\mathrm{AD} 2_{\mathrm{en}}$ & 0 & 0 & 0 & 0 & 0 & 0 & 0 & 0 \\
\hline TS8 & 187.6 & 189.5 & 182.7 & 185.7 & 194.3 & 192.6 & 189.7 & 191.4 \\
\hline TS9 & 172.8 & 177.7 & 164.7 & 170.4 & 182.5 & 186.9 & 175.5 & 179.4 \\
\hline $\mathrm{AD} 2_{\text {ket }}$ & 20.1 & 12.5 & 14.0 & 8.2 & 14.2 & 11.0 & 6.8 & 4.5 \\
\hline TS6 & 75.1 & 77.5 & 69.7 & 69.7 & 77.0 & 83.0 & 75.5 & 79.5 \\
\hline TS7 & 29.2 & 34.8 & 20.9 & 29.3 & 28.5 & 33.7 & 26.5 & 31.5 \\
\hline
\end{tabular}

The results in Table 5.27 show that the enol form of AD2 is slightly more stable than the keto form. This is possibly a consequence of both the formation of a hydrogen bond between the enol hydrogen and the carboxylate oxygen, and the extended conjugation that connects the aromatic system and the carboxylate unit. Reactivity, however, is favored in the keto form. The $s p^{2}$ carbon in the enol form is less reactive in electrophilic reactions, such as hydrolysis or displacement by cyclic nitrogen atoms, as suggested by the high activation free energies for TS8 and TS9, as compared to TS6 and TS7.

The activation free energy for the ring-closure reaction (TS6) is quite low - the activation entropy being close to null, as expected for unimolecular reactions - and hence the reaction is expected to proceed rapidly. The activation free energies for the competing hydrolysis are also very low, which suggests that hydrolysis by hydroxide ions is very fast. However, the concentration of hydroxide ions in neutral medium is very low $\left(10^{-7} \mathrm{M}\right)$. Since the effect of reducing the concentration of hydroxide from the reference standard state of $1 \mathrm{M}$ to $10^{-7} \mathrm{M}$ is approximately equivalent to an increase of $40 \mathrm{~kJ} \mathrm{~mol}^{-1}$ in the activation free energy, both ring-closure and base-catalyzed hydrolysis could be expected to occur similar at similar rates at neutral $\mathrm{pH}$. As was the case with AD1, the hydrolysis of AD2 by the more abundant, 
and less nucleophilic, water molecules has a higher energy barrier (adenosine: $\Delta^{\ddagger} H^{\ominus}=59.7 \mathrm{~kJ} \mathrm{~mol}^{-1}$, $\Delta^{\ddagger} G^{\ominus}=81.3 \mathrm{~kJ} \mathrm{~mol}^{-1}$ for MCA; $\Delta^{\ddagger} H^{\ominus}=52.6 \mathrm{~kJ} \mathrm{~mol}^{-1}, \Delta^{\ddagger} G^{\ominus}=79.8 \mathrm{~kJ} \mathrm{~mol}^{-1}$ for MBA) and is somewhat higher than that observed for the formation of the cycle. Thus, it may be concluded that hydrolysis by both hydroxide and water might pose some competition to intramolecular cyclization. However, the formation of nucleotide-adducts showing substitution of the second halogen atom by a hydroxyl group has not been observed experimentally.

When the experimental alkylation reactions of nucleotides with MXA are carried out at high temperatures $\left(\sim 90^{\circ} \mathrm{C}\right)$, decarboxylated and even de-oxalo products are encountered. However, these are hardly detectable when alkylation reactions are carried out at temperatures closer to biological conditions. Since they lack a keto group to assist the reaction, no transition states were found for the decarboxylation of MXA or AD1, which is consistent with the fact that no decarboxylated products have been observed in the alkylation reaction, prior to the formation of AD2. The decarboxylation reactions of the protonated MOXA, AD2 are somewhat unfavored with free energies of activation about 150-160 kJ mol-1 (MOCA: $\Delta^{\ddagger} G^{\ominus}=161 \mathrm{~kJ} \mathrm{~mol}^{-1}$; MOBA: $\Delta^{\ddagger} H^{\ominus}=146 \mathrm{~kJ} \mathrm{~mol}^{-1}, \Delta^{\ddagger} G^{\ominus}=156 \mathrm{~kJ} \mathrm{~mol}^{-1}$; adenosine-AD2: $\Delta^{\ddagger} H^{\ominus}=$ $161 \mathrm{~kJ} \mathrm{~mol}^{-1}, \Delta^{\ddagger} G^{\ominus}=156 \mathrm{~kJ} \mathrm{~mol}^{-1}$ for MCA; $\Delta^{\ddagger} H^{\ominus}=162 \mathrm{~kJ} \mathrm{~mol}^{-1}, \Delta^{\ddagger} G^{\ominus}=158 \mathrm{~kJ} \mathrm{~mol}^{-1}$ for MBA). The decarboxylation of AD3 is more favored, consistently with the increased amount of decarboxylated AD3 adduct observed in the experiments (adenosine-AD3: $\Delta^{\ddagger} H^{\ominus}=113 \mathrm{~kJ} \mathrm{~mol}^{-1}, \Delta^{\ddagger} G^{\ominus}=116 \mathrm{~kJ} \mathrm{~mol}^{-1}$ ). These reactions show high activation enthalpies and positive activation entropies (consistent with a unimolecular cleavage that affords two product molecules) which imply a large increase in the decarboxylation rate at higher temperatures; this is in keeping with the observation of decarboxylated products in these conditions.

The mutational pattern of MXA - which consists mainly of GC $\rightarrow$ AT transitions, has been explained by the formation of guanosine - and possibly to a lesser extent, cytidine-etheno derivatives. ${ }^{62}$ Our results are in good agreement with these conclusions: guanosine shows the lower theoretical free energy barrier for alkylation and thus it is expected to be the main target of mucohalic acids. The formed adducts have shown no tendency to lead to abasic sites and it is difficult to envision such bulky tricyclic adducts mispairing with large purine residues be them A or G. Of the two pyrimidine nucleobases, only pairing with thymidine leads to a net mutation, and thus, $G C \rightarrow G^{*} C \rightarrow G^{*} T \rightarrow A T$ ( $G^{*}$ being the modified guanosine) would be the expected sequence of base pairs, which is in keeping with the results reported in the literature.

\subsubsection{Alkylating potential of $M X Y$}

5.3.2.2.1. Adduct formation. Certain evidence suggests that the mode of action of MX is very different to that of other halohydroxyfuranones, and thus that the formation of covalent DNA adducts is not the cause of the extremely high mutagenicity observed by MX, but rather some unknown mechanism.

For instance, the mutational pattern of $\mathrm{MX}$, consisting mainly in GC $\rightarrow$ TA transversions, is different to that of other halofuranones like MCF (but not CMCF) or mucohalic acids, which are known to alkylate adenine residues. In addition, only adenosine adducts have been observed in the reaction of MX with purified double-stranded DNA. No guanosine adducts, which would be more consistent with the observed 

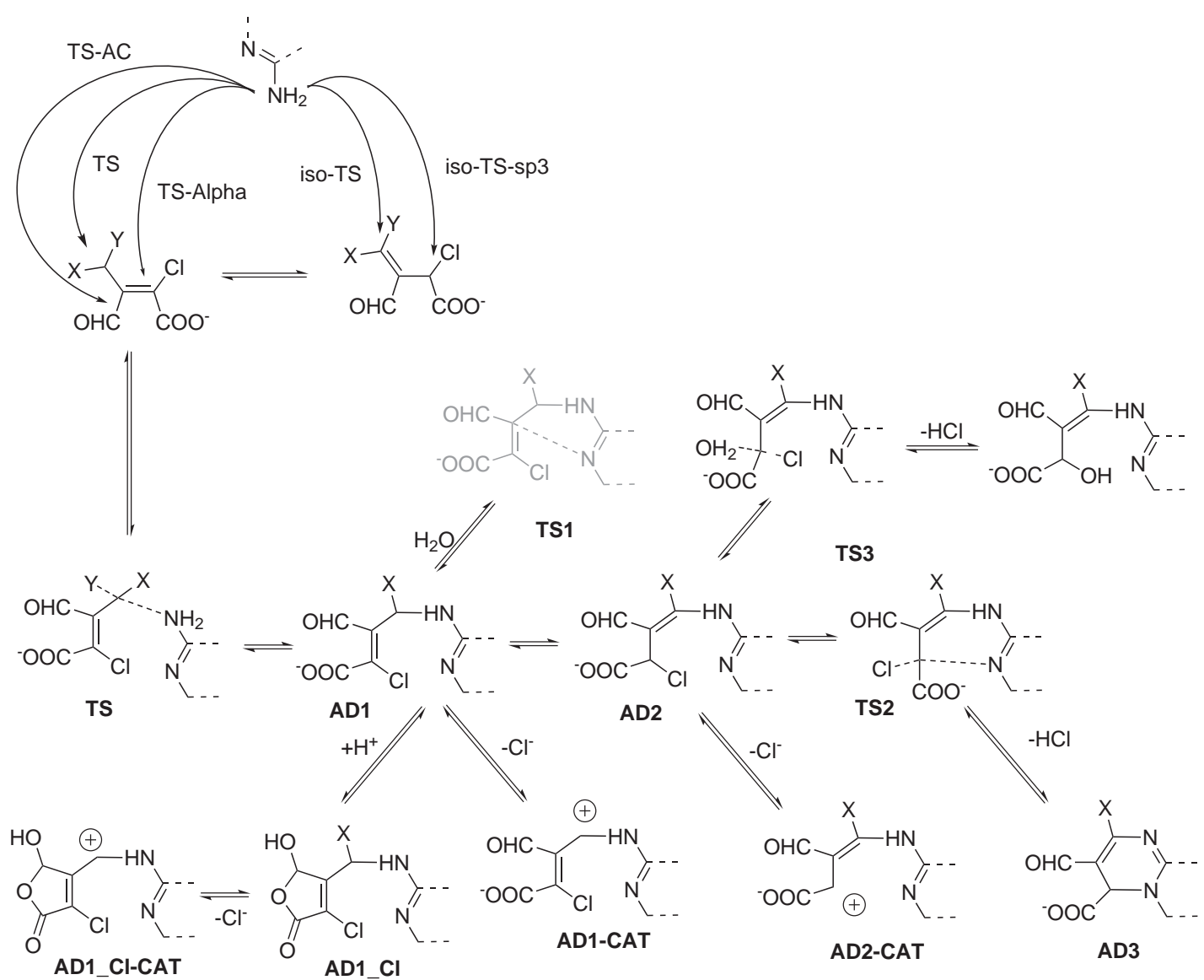

Scheme 5.25. Alkylation of nucleotides by $M X$ and analogues. $X, Y=H, C l$

mutational pattern of MX, were detected. This suggests that lesions in guanine bases may occur by some mechanism other than formation of stable DNA adducts with guanine.

Whereas the presence of high concentrations of terminally double-bonded isomers can be dismissed as the source of the exceptional behavior of MX, the inherent reactivity of these electrophiles could still be related to the particular biological effects of MX.

In order to gain understanding about how the adducts reported in the literature are formed (Scheme 5.9), and to assess whether MX in any of its isomeric forms shows exceptional reactivity as an alkylating agent, we have modeled the following alkylation pathways (Scheme 5.25).

We have determined the energy barrier for the direct displacement of a halogen atom on the methyl group by the exocyclic amino group in adenosine and guanosine (TS). Since MCF lacks any substitution on the methyl group, this pathway is only available for CMCF and MX. The formation of the Schiff base (TS-AC), the Michael addition of the nucleophile on the terminal double bond in the iso form of the halofuranones (iso-TS) and the substitution of the chlorine atom $\alpha$ to the carboxylate group, both 
in the major form (TS-Alpha) and in the terminally double-bonded isomer (iso-TS-sp3) have also been considered.

Table 5.28 shows the computed enthalpy and free energy differences for the significant steps in the proposed alkylation mechanism. It has also been considered that guanosine can exist both as keto and enol isomers; values for the lower-energy isomers (the keto form) are reported.

Table 5.28. Calculated energy barriers for the alkylation of nucleotides by MXY

\begin{tabular}{|c|c|c|c|c|c|c|c|}
\hline \multirow[b]{3}{*}{ Nucleotide } & \multirow[b]{3}{*}{ Attack } & \multicolumn{6}{|c|}{ Reaction barrier $\left(\mathrm{kJ} \mathrm{mol}^{-1}\right)$} \\
\hline & & \multicolumn{2}{|c|}{ MCF } & \multicolumn{2}{|c|}{ CMCF } & \multicolumn{2}{|c|}{ MX } \\
\hline & & $\Delta H$ & $\Delta G$ & $\Delta H$ & $\Delta G$ & $\Delta H$ & $\Delta G$ \\
\hline \multirow{6}{*}{$\mathrm{dA}$} & TS-Z & - & - & 92.2 & 96.5 & 88.9 & 95.7 \\
\hline & TS-E & - & - & 57.8 & 75.6 & 90.2 & 98.1 \\
\hline & TS-AC & 118 & 135.5 & 108 & 131.3 & 99.7 & 126.6 \\
\hline & iso-TS & 72.5 & 87.1 & 105.5 & 107.4 & 107.2 & 135.9 \\
\hline & iso-TS-sp3 & 98.4 & 99.5 & 108 & 109.2 & 107.9 & 109.7 \\
\hline & TS-Alpha & - & - & 98.4 & 99.5 & 88.7 & 110.3 \\
\hline Nucleotide & Attack & $\Delta H$ & $\Delta G$ & $\Delta H$ & $\Delta G$ & $\Delta H$ & $\Delta G$ \\
\hline \multirow{6}{*}{$\mathrm{dG}$} & TS-Z & - & - & 105.1 & 111.3 & 115.0 & 117.4 \\
\hline & TS-E & - & - & 72.0 & 81.5 & 90.4 & 96.0 \\
\hline & TS-AC & & & & & 100.6 & 129.9 \\
\hline & iso-TS & 79.5 & 86.6 & 90.8 & 101.5 & 119.1 & 123.7 \\
\hline & iso-TS-sp3 & 106.3 & 110.3 & 90.8 & 101.5 & 114.4 & 117.0 \\
\hline & TS-Alpha & - & - & 114.2 & 120.4 & 103.7 & 118.1 \\
\hline
\end{tabular}

It can be seen from the lower energy barriers that, in the case of CMCF and MX, the most favorable alkylation pathway proceeds through the $\mathrm{Z}$ and $\mathrm{E}$ forms, and not the terminally double bonded isomer, contrarily to what has been proposed in the literature. Both reactions, however, lead to the same products. Interestingly, the barrier for the alkylation by the $Z$ form is a few $\mathrm{kJ} \mathrm{mol}^{-1}$ lower, in keeping with the observation that the $Z$ form is more mutagenic. ${ }^{137}$

Additionally, our results show that in the unfavored nucleophilic attack onto the exo double bond in 2 , it is the chlorine atom $\alpha$ to the carboxyl group that acts as a leaving group, as observed experimentally for MCF. This confirms that the alkylation of both adenosine and guanosine by CMCF and MX proceed most likely through $\mathbf{1}$. The reaction with adenosine is faster, in keeping with the fact that no guanosineadducts have been detected in the reaction of MX with double stranded DNA.

The favored pathway for CMCF and MX does not exist for MCF, and hence the nucleophilic attack on MCF takes place at the terminal double bond of 2, or at the carbonyl group, in keeping with the products observed. 
These results are coherent with the products of the nucleotide-MXY reactions and suggest that MXY are moderately strong alkylating agents. Nevertheless, they are weaker (in their $E$ form) than similar compounds, e.g. mucohalic acids, or other genotoxic compounds which however afford weaker results in biological assays. ${ }^{223-232}$

More importantly, little difference is observed in the alkylation reaction by MX and CMCF: the activation free energy for the rate-limiting step is similar for both compounds, and the subsequent reactions have comparable barriers as shown in Table 5.29.

Table 5.29. Energy barriers for further reactions of the nucleotide-MXY adducts

\begin{tabular}{|c|c|c|c|c|c|c|c|}
\hline \multicolumn{4}{|c|}{$\mathrm{dA}$} & \multicolumn{4}{|c|}{$\mathrm{dG}$} \\
\hline \multirow{3}{*}{ Species } & \multicolumn{3}{|c|}{$\Delta G\left(\mathrm{~kJ} \mathrm{~mol}^{-1}\right)$} & \multicolumn{4}{|c|}{$\Delta G\left(\mathrm{~kJ} \mathrm{~mol}^{-1}\right)$} \\
\hline & \multirow{2}{*}{$\frac{\mathrm{CMCF}}{\mathrm{Op}-\mathrm{H}}$} & \multicolumn{2}{|c|}{ MX } & \multirow{2}{*}{ Species } & \multirow{2}{*}{$\frac{\mathrm{CMCF}}{\mathrm{Op}-\mathrm{H}}$} & \multicolumn{2}{|c|}{ MX } \\
\hline & & $\mathrm{Op}-\mathrm{H}$ & $\mathrm{Cl}$ & & & $\mathrm{Op}-\mathrm{H}$ & $\mathrm{Cl}$ \\
\hline AD1 & 38.6 & 0 & 0 & AD1-ket & 58.7 & 0 & 0 \\
\hline \multirow[t]{3}{*}{ AD1-CAT } & - & -13.2 & 14.2 & AD1-ket-CAT & - & -16.8 & 31.3 \\
\hline & & & & AD1-en & 93.6 & 11.8 & 15.6 \\
\hline & & & & AD1-en-CAT & - & -24.4 & 10.3 \\
\hline AD2 & 0 & 1.5 & - & AD2-ket & 0 & 31.2 & - \\
\hline \multirow[t]{3}{*}{ AD2-CAT } & 16.4 & 44.1 & - & AD2-ket-CAT & 20.6 & -7.9 & - \\
\hline & & & & AD2-en & 42.5 & 40.8 & - \\
\hline & & & & AD2-en-CAT & -23.3 & -10.4 & - \\
\hline \multirow[t]{2}{*}{ TS-HID } & 86.3 & 59.7 & - & TS-HID-ket & 40.7 & & - \\
\hline & & & & TS-HID-en & 68.9 & & - \\
\hline \multirow[t]{2}{*}{ TS2 } & 85.7 & 81.3 & - & TS2-ket & 164 & 163 & - \\
\hline & & & & TS2-en & 152 & 157 & - \\
\hline AD3 & -47.5 & -53.1 & - & AD3 & -54.2 & -60.7 & - \\
\hline TS-deCARB & 120 & 109 & - & TS-deCARB & 118 & 112 & - \\
\hline
\end{tabular}

The initial adduct formed by MX and CMCF undergoes rapid loss of a proton yielding AD1, which can isomerize to AD2.

Several ring-closure reactions involving nucleophilic attack by the endocyclic nitrogen atom are possible, in principle: (i) Ring closure of AD1 to afford a five-membered cycle (TS1). (ii) Ring closure of AD2 (TS2), which is much more favored, since the product is a more stable six-membered ring (AD3). (iii) Ring closure of AD1 with loss of the chlorine atom in the allylic position, which is especially difficult since a strained four-membered ring as a product.

Experimental evidence exists for the reactions occurring through TS2 and TS3: products arising from the reaction of AD2-CMCF have been observed experimentally (pfA-dR and OH-fbA-dR).

Reaction products including a five-membered ring have also been reported for guanosine-MX, but not for guanosine-CMCF or adenosine-MXY. This is partially consistent with the calculated energies: in 
the case of $\mathrm{CMCF}, \mathrm{AD} 1$ isomerizes to the more stable $\mathrm{AD} 2$ and then reacts, but in the case of MX, a major amount of AD1, able to undergo cyclization is present in the equilibrium, especially in the case of guanosine. However, in this work no TS1-like transition states could be found for any of the halofuranones, and thus, the products are expected to arise from another intermediate (see below). As regards TS2, energy barriers are moderate for adenosine nucleotides, and quite large for guanosine; as with TS1, the formation of endocyclic products is perhaps better explained by the reaction of other intermediates.

As regards the hydrolysis reaction (TS2), the energy barriers are quite low, in agreement with the lability of the carbon-chlorine bonds in these adducts, and the nucleophilicity of the hydroxide ions. As said before, the low concentration of $\mathrm{OH}^{-}$in physiological conditions is equivalent to an additional increase in $40 \mathrm{~kJ} \mathrm{~mol}^{-1}$ in activation free energy. Even when accounting for the low concentration of hydroxide ions in vivo, the barriers for the experimentally-observed ring-closure reactions is still much higher than that for the experimentally-unobserved hydrolysis. This evidence can be reconciled if additional aspects of the reactivity of these MXY-nucleotide adducts is considered.

The only large difference observed to this point between MX and CMCF is the relative stability of AD1 and AD2: AD2 is clearly favored by CMCF, and in the case of MX, AD1 is preferred by a smaller margin. Whereas this observation is consistent with nucleotide adducts, it still does not account for the ability of MX to damage guanosine positions. Therefore, additional reactions must be considered.

5.3.2.2.2. Unimolecular cleavage. Since adenosine adducts of MX lacking any chlorine atoms have been observed experimentally (pfA-dR) and the $\mathrm{C}-\mathrm{Cl}$ bond length in AD1, but not AD2, is exceptionally long ( $205 \mathrm{pm}$ and $\sim 195 \mathrm{pm}$, respectively, compared to a common value around 170-195 pm), the unimolecular cleavage of the adducts has been considered. This reaction affords chloride ion and a cationic adduct that could be expected to be a highly reactive electrophile.

Calculations show that the $\mathrm{S}_{\mathrm{N}} 1$-formation of the cation is quite favored: it has low positive reaction free energies, and is even exoergonic in some cases (Table 5.29). Some differences between CMCF and MX are observed: most importantly, that CMCF can only yield the AD2-like cation. Also, the energies differences reveal that MX is somewhat more prone to heterolytic cleavage.

These carbocationic adducts may react in several different ways: trapping nucleophiles such as water or nucleophilic nitrogen positions, or undergoing elimination.

In fact, the polycyclic adducts observed in nucleotide-alkylation experiments (Scheme 5.9) may be formed by reaction of the cations, rather than by $\mathrm{S}_{\mathrm{N}} 2$ displacement of $\mathrm{AD} 1$ and $\mathrm{AD} 2$. Here, it was found that he ring-closure barrier for the cations is much lower than the bimolecular counterpart for the uncleavaged adduct: $\Delta H=96.6 \mathrm{~kJ} \mathrm{~mol}^{-1}, \Delta G=112.0 \mathrm{~kJ} \mathrm{~mol}^{-1}$ and $\Delta H=74.7 \mathrm{~kJ} \mathrm{~mol}^{-1}, \Delta G=79.9 \mathrm{~kJ}$ $\mathrm{mol}^{-1}$ for the TS1- and TS2-like ring closure of MX-Guo-AD1 and MX-Guo-AD2. This contrasts with the inability to locate a TS1 transition state and also with the barrier for bimolecular TS2, about $100 \mathrm{~kJ}$ $\mathrm{mol}^{-1}$ higher.

Therefore, this unimolecular cleavage reaction is in accord with the formation of polycyclic adducts, and accounts for the existence of a five-membered adduct, whose formation could not be explained in 
terms of bimolecular reactions. Also, it helps understand the formation of adducts showing substitution of chlorine by hydroxide, since the barrier for the $\mathrm{S}_{\mathrm{N}} 2$ reaction is very high, considering the high nucleophilicity of hydroxide ions and their low concentration in vivo.

However, the matter of guanosine residues being damaged is not accounted for by these covalent adducts, either cationic or neutral. An oxidation scheme would indeed explain the damage to guanosine -the most easily oxidized base. Nevertheless, the calculated vertical electron affinities of MXY suggest that this cannot be a direct reaction. Thus, we have addressed the ability of the nucleotide-MXY adducts and their cations to undergo one-electron reduction.

5.3.2.2.3. Reduction. Like their parent halofuranones, nucleotide-MXY adducts are easily reduced (Table 5.30). Results at the DFT-B3LYP/6-31G(d) level of theory suggest that MXY-nucleotide adducts have high adiabatic electron affinities, which is due to the loss of chloride, as occurred in the parent compounds. Vertical electron affinities are moderate and, in general, insufficient to oxidize the bases.

Table 5.30. Electron affinities in aqueous solution for the MXY-Nucleotide adducts

\begin{tabular}{|c|c|c|c|c|c|c|c|c|c|}
\hline & \multirow{4}{*}{ Species } & \multicolumn{8}{|c|}{$\mathrm{E}_{\mathrm{A}}$ DFT-B3LYP/6-31G(d) (eV) } \\
\hline & & \multicolumn{4}{|c|}{ Vertical } & \multicolumn{4}{|c|}{ Adiabatic } \\
\hline & & \multicolumn{2}{|c|}{$\mathrm{CMCF}$} & \multicolumn{2}{|c|}{ MX } & \multicolumn{2}{|c|}{$\mathrm{CMCF}$} & \multicolumn{2}{|c|}{ MX } \\
\hline & & $\mathrm{Op}-\mathrm{H}$ & $\mathrm{Cl}$ & Op-H & $\mathrm{Cl}$ & Op-H & $\mathrm{Cl}$ & $\mathrm{Op}-\mathrm{H}$ & $\mathrm{Cl}$ \\
\hline \multirow{4}{*}{$\mathrm{dA}$} & $\mathrm{AD} 1$ & 1.5 & & 2.0 & 2.2 & 2.2 & & 4.4 & 4.7 \\
\hline & AD2 & 2.3 & - & 1.9 & - & 3.9 & - & 4.3 & - \\
\hline & AD1-CAT & - & - & 3.9 & 4.6 & - & - & 4.2 & 4.9 \\
\hline & AD2-CAT & 3.6 & - & 3.4 & - & 3.9 & - & 4.8 & - \\
\hline \multirow{8}{*}{$\mathrm{dG}$} & AD1-ket & 1.1 & & 1.7 & 2.5 & ? & & 4.3 & 4.7 \\
\hline & AD1-en & 1.1 & & 2.9 & 2.5 & $?$ & & 4.2 & 4.6 \\
\hline & AD2-ket & 2.0 & - & 2.1 & - & 3.8 & - & 4.1 & - \\
\hline & AD2-en & 1.6 & - & 1.8 & - & 4.0 & - & 3.7 & - \\
\hline & AD1-ket-CAT & - & - & 3.7 & 4.7 & - & - & 4.1 & 5.0 \\
\hline & AD1-en-CAT & - & - & 3.5 & 4.4 & - & - & 3.9 & 4.7 \\
\hline & AD2-ket-CAT & 4.0 & - & 3.6 & - & 4.0 & - & 4.0 & - \\
\hline & AD2-en-CAT & 3.3 & - & 3.4 & - & 3.8 & - & 3.6 & - \\
\hline
\end{tabular}

Nevertheless, one exception exists: the cations arising from the carbon-chlorine cleavage of the adducts. These molecules, having already expelled the chloride anion, are in a favored disposition to accept an electron, especially those from the closed-chain furanone adduct.

Since the electron affinities depend strongly on the basis set, we have improved the results for the most interesting species, i.e. the cations, using the $6-31++G(d, p)$ basis set. In a similar effect to that 
observed for the parent MXY, this superior basis set afford larger electron affinities, by about 0.25-0.50 $\mathrm{eV}$. The larger $6-311+\mathrm{G}(2 \mathrm{df}, 2 \mathrm{p})$ basis set affords very similar results.

Table 5.31. Electron affinities in aqueous solution for the closed-chain MX-Nucleotide cationic adducts

\begin{tabular}{|c|c|c|c|c|c|}
\hline & \multirow{3}{*}{ Species } & \multicolumn{4}{|c|}{$\mathrm{E}_{\mathrm{A}}$ DFT-B3LYP $(\mathrm{eV})$} \\
\hline & & \multicolumn{2}{|c|}{$6-31++G(d, p)$} & \multicolumn{2}{|c|}{$6-311+G(2 d f, 2 p)$} \\
\hline \multirow{5}{*}{$\mathrm{dA}$} & & Op-H & $\mathrm{Cl}$ & Op-H & $\mathrm{Cl}$ \\
\hline & AD1-CAT & 4.1 & 4.9 & 4.1 & 4.9 \\
\hline & AD2-CAT & 4.0 & - & 4.0 & - \\
\hline & AD1-ket-CAT & 4.1 & 5.0 & 4.2 & 4.9 \\
\hline & AD1-en-CAT & 3.9 & 4.7 & 3.9 & 4.7 \\
\hline \multirow{2}{*}{$\mathrm{dG}$} & AD2-ket-CAT & 4.1 & - & 4.1 & - \\
\hline & AD2-en-CAT & 3.8 & - & 3.9 & - \\
\hline
\end{tabular}

The electron affinities of the cations are quite high, and the top values overlap with the experimental ionization potential of guanosine. Whereas the precise determination of redox properties would require higher levels of theory and, more importantly, modelization of the adjacent bases, our results suggest that MXY and especially MX-nucleotide adducts, could indeed oxidize guanosine residues.

The $\pi-\pi$ stacking of the bases in DNA, and the orbital overlap would allow facile electron transfer from the guanine nucleotides to the nucleotide-MXY cationic adducts, affording two radicals, whose further reactivity could produce a variety of DNA lesions, including formation of 8-oxoguanin, abasic sites...

Radicals are known to be able to migrate along the DNA polymer, which would be consistent with the lesions taking place in mutational hot-spots for oxidation, somewhat independently of the position of the alkylated nucleotide. 


\subsection{Conclusions}

1. The use of a relative approach allows accurate prediction of hydration equilibrium constants of the carbonyl group in aldehydes, ketones and esters, even at low levels of theory.

2. Mucoxyhalic acids exist in aqueous solution as an apparent acid-base equilibrium between the closed-ring furanone form and the open-chain dissociated carboxylate. The global equilibrium constant for this reaction are $\mathrm{p} K_{\mathrm{a}}^{\mathrm{app}}=3.95 \pm 0.02$ and $4.27 \pm 0.02$.

3. MXA are strong acids that dissociate readily, with $\mathrm{p} K_{\mathrm{a}}$ values close to unity. As regards the undissociated forms, the closed-chain tautomer is favored by $\sim 30 \mathrm{~kJ} \mathrm{~mol}^{-1}\left(10^{4}\right.$ to 1$)$.

4. MXA are hydrolyzed to MOXA at negligible rates, except in highly alkaline conditions, such as those used for lime softening. This discards any role for MOXA in the in vivo alkylation of DNA.

5. MXA react preferentially with the exocyclic amino groups of DNA nucleotides, in a rate-limiting formation of a Schiff-base derivative. Subsequent reactions of nucleotide-MXA adducts include hydrolysis, isomerization and cyclization or hydrolysis. The adducts formed and the preference for the more nucleophilic guanosine is in keeping with the mutational spectrum of MXA.

6. MXY exist in solution as a complex equilibrium mixture composed mostly by closed-chain and dissociated $E$ and $Z$ forms. Undissociated and terminal-methylene forms are minor. The product distribution hardly explains the biological effects of MX.

7. One electron reduction potentials of MXY suggest that these compounds are easily reduced, but the reaction is no exergonic enough to promote the direct oxidation of DNA-nucleotides.

8. MX and CMCF alkylate DNA through the $\mathrm{S}_{\mathrm{N}} 2$ displacement of chlorine, especially in the $E$ form. MCF reacts through its carbonyl moiety and the terminal-methylene isoform.

9. The DNA adducts of MXY cannot explain the record-high mutagenicity of MX. However, the electron affinity of some cationic adducts are sufficiently high to support the hypothesis of MX inflecting oxidative DNA damage. 


\section{References}

[1] Villanueva, C. M.; Cantor, K. P.; Cordier, S.; Jaakkola, J. J.; King, W. D.; Lynch, C. F.; Porru, S.; Kogevinas, M. Epidemiology 2004, 15, 357-367.

[2] Rahman, M.; Driscoll, T.; Cowie, C.; Armstrong, B. Int. J. Epidemiol. 2010, 39, 733-745.

[3] Richardson, S. D.; Plewa, M. J.; Wagner, E. D.; Schoeny, R.; DeMarini, D. M. Mutat. Res. 2007, 636, 178-242.

[4] Richardson, S. D. Anal. Chem. 2003, 75, 2831-2857.

[5] Richardson, S. D.; Ternes, T. A. Anal. Chem. 2005, 77, 3807-3838.

[6] Richardson, S. D. Anal. Chem. 2007, 79, 4295-4323.

[7] Richardson, S. D. Anal. Chem. 2009, 81, 4645-4677.

[8] Richardson, S. D.; Ternes, T. A. Anal. Chem. 2011, 83, 4614-4648.

[9] IARC, In Monographs on the Evaluation of Carcinogenic Risks to Humans.; Lyon,, Ed.; IARC Monograph 84, IARC: Lyon, France, 2004.

[10] McDonald, T. A.; Komulainen, H. Environ. Carcin. Eco. R. 2005, 23, 163-214.

[11] Kronberg, L.; Holmbom, B.; Reunanen, M.; Tikkanen, L. Environ. Sci. Technol. 1988, 22, 1097-1103.

[12] Kronberg, L.; Vartiainen, T. Mutat. Res. 1988, 206, 177-182.

[13] Kanniganti, R.; Johnson, J. D.; Ball, L. M.; Charles, M. J. Environ. Sci. Technol. 1992, 26, 1998-2004.

[14] Mowry, D. T. J. Am. Chem. Soc. 1950, 72, 2535-2537.

[15] Wasserman, H. H.; Precopio, F. M. J. Am. Chem. Soc. 1952, 74, 326-328.

[16] Vartianinen, T.; Heiskanen, K.; Lötjönen, S. Fresen. J. Anal. Chem. 1991, 340, 230-233.

[17] Valente, E. J.; Fuller, J. F.; Ball, J. D. Acta Crystallogr., Sect. B: Struct. Sci. 1998, 54 ( Pt 2), 162-173.

[18] Franzen, R.; Tanabe, K.; Morita, M. Chemosphere 1999, 38, 973-980.

[19] Holmbom, B.; R.H., V.; R.D., M.; A., W. Tappi J. 1981, 64, 172-174.

[20] Holmbom, B. IARC Sci Publ 1990, 333340.

[21] Kronberg, L.; Franzen, R. Environ. Sci. Technol. 1993, 27, 1181-1818.

[22] Hemming, J.; Holmbom, B.; Reunanen, M.; Kronberg, L. Chemosphere 1986, 15, 549-556.

[23] Backlund, P.; Kronberg, L.; Tikkanen, L. Chemosphere 1988, 17, 1329-1336.

[24] Langvik, V.-A.; Holmbom, B.; Tikkanen, L. Chemosphere 1991, 23, 873 - 880.

[25] Backlund, P. Environ. Int. 1994, 20, 113-120.

[26] Backlund, P.; Wondergem, E.; Voogd, K.; De Jong, A. Chemosphere 1989, 18, 1903-1911.

[27] Backlund, P.; Wondergem, E.; Voogd, K.; De Jong, A. Sci. Total Environ. 1989, 84, 273-282. 
[28] Langvik, V.-A.; Hormi, O.; Tikkanen, L.; Holmbom, B. Chemosphere 1991, 22, 547 - 555.

[29] Zhuo, C.; Chengyong, Y.; Junhe, L.; Huixian, Z.; Jinqi, Z. Chemosphere 2001, 45, 379 - 385.

[30] Wright, J. M.; Schwartz, J.; Vartiainen, T.; Mäki-Paakkanen, J.; Altshul, L.; Harrington, J. J.; Dockery, D. W. Environ. Health Perspect. 2002, 110, 157164.

[31] LaLonde, R. T.; Perakyla, H.; Hayes, M. P. J. Org. Chem. 1990, 55, 2847-2855.

[32] Richardson, S. D. Anal. Chem. 2002, 74, 2719-2741.

[33] Richardson, S. D. Anal. Chem. 2004, 76, 3337-3363.

[34] Richardson, S. D. Anal. Chem. 2006, 78, 4021-4045.

[35] Richardson, S. D. Anal. Chem. 2008, 80, 4373-4402.

[36] Richardson, S. D. Anal. Chem. 2010, 82, 4742-4774.

[37] Charles, M. J.; Chen, G.; Kanniganti, R.; Marbury, G. Environ. Sci. Technol. 1992, 26, 1030-1035.

[38] Romero, J.; Ventura, F.; Caixach, J.; Rivera, J.; Guerrero, R. Bull. Environ. Contam. Toxicol. 1997, 59, $715-722$.

[39] Zwiener, C.; Kronberg, L. Fresen. J. Anal. Chem. 2001, 371, 591597.

[40] Onstad, G. D.; Weinberg, H. S. Anal. Chim. Acta 2005, 534, 281-292.

[41] Onstad, G. D.; Weinberg, H. S.; Krasner, S. S. Environ. Sci. Technol. 2008, 42, 3341-3348.

[42] Nawrocki, J.; Andrzejewski, P.; Kronberg, L.; Jeleń, H. J. Chromatogr. A 1997, 790, 242247.

[43] Nawrocki, J.; Andrzejewski, P.; Jeleń, H.; Wasowicz, E. Water Res. 2001, 35, 18911896.

[44] Kubwabo, C.; Stewart, B.; Gauthier, S. A.; Gauthier, B. R. Anal. Chim. Acta 2009, 649, 222-229.

[45] Rezemini, A.; Vaz, J.; Carvalho, L. J. Braz. Chem. Soc. 2008, 19, 922-928.

[46] Kronberg, L.; Christman, R.; Singh, R.; Ball, L. Environ. Sci. Technol. 1991, 25, 99-104.

[47] Suzuki, N.; Nakanishi, J. Chemosphere 1995, 30, 1557 - 1564.

[48] Weinberg, H.; Krasner, S.; Richardson, S.; Jr., A. T. The occurrence of disinfection by-products (DBPs) of health concern in drinking water: results of a nationwide DBP occurrence study EPA/600/R02/068; 2002.

[49] Krasner, S. S.; Weinberg, H. S.; Richardson, S. D.; Pastor, S. J.; Chinn, R.; Sclimenti, M. J.; Onstad, G. D.; Thruston, A. D. J. Environ. Sci. Technol. 2006, 40, 7175-7185.

[50] Andrews, R.; Daignault, S.; Laverdure, C.; Williams, D.; Huck, P. Environ. Technol. 1990, 11, 685-694.

[51] Zou, X.; Xu, X.; Zhang, J.; Zhu, Z. Chemosphere 1995, 30, 22192225.

[52] Smeds, A.; Vartianinen, J., T Mäki-Paakkanen; Kronberg, L. Environ. Sci. Technol. 1997, 31, 10331039.

[53] Rantakokko, P.; Yritys, M.; Vartiainen, T. J. Chromatogr. A 2004, 1028, 179-188.

[54] Suzuki, N.; Nakanishi, J. Chemosphere 1990, 21, 387-392. 
[55] Kinae, N.; Sugiyama, C.; Nasuda, M. Y.; Goto, K.; Tokumoto, K.; Furugori, M.; Shimoi, K. Water Sci. Technol. 1992, 25, 333-340.

[56] Egorov, A. I.; Tereschenko, A. A.; Altshul, L. M.; Vartiainen, T.; Samsonov, D.; LaBrecque, B.; MäkiPaakkanen, J.; Drizhd, N. L.; Ford, T. E. Int. J. Hyg. Environ. Health 2003, 206, 539-551.

[57] Fawell, J.; Horth, H. In Assessment and identification of genotoxic compounds in water. In: Genetic Toxicology of Complex Mixtures; Press, N., Ed.; 1990; p 197-214.

[58] Meier, J. R.; Knohl, R. B.; Coleman, W. E.; Ringhand, H. P.; Munch, J. W.; Kaylor, W. H.; Streicher, R. P.; Kopfler, F. C. Mutat. Res. 1987, 189, 363-373.

[59] Simpson, K., K.L. \& Hayes Water Res. 1998, 32, 1522-1528.

[60] Fekadu, K.; Parzefall, W.; Kronberg, L.; Franzen, R.; Schulte-Hermann, R.; Knasmuller, S. Environ. Mol. Mutagen. 1994, 24, 317-324.

[61] Jansson, K.; Hyttinen, J.; Niittykoski, M.; Mäki-Paakkanen, J. Environ. Mol. Mutagen. 1995, 25, $284-287$.

[62] Knasmuller, S.; Zohrer, E.; Kronberg, L.; Kundi, M.; Franzen, R.; SchulteHermann, R. Chem. Res. Toxicol. 1996, 9, 374-381.

[63] Liviac, D.; Creus, A.; Marcos, R. Environ. Mol. Mutagen. 2009, 50, 413-420.

[64] Hill,; Palmer, Am. Chem. J. 1887, 9, 147.

[65] Hill, Am. Chem. J. 1881, 3, 165.

[66] Hill,; Cornelison, Am. Chem. J. 1894, 16, 188.

[67] Hill,; Widtsoe,; Dunlap, Am. Chem. J. 1897, 19, 627.

[68] Simonis, H. Ber. Dtsch. Chem. Ges. 1899, 32, 2084.

[69] Simonis, H. Ber. Dtsch. Chem. Ges. 1901, 34, 509-519.

[70] Simonis, H. Ber. Dtsch. Chem. Ges. 1905, 38, 3981.

[71] Wasserman, H. H.; Precopio, F. M.; Liu, T. C. J. Am. Chem. Soc. 1952, 74, 4093-4095.

[72] Wasserman, H. H.; Precopio, F. M.; Liu, T. C. J. Am. Chem. Soc. 1953, 75, 2527-2528.

[73] Mowry, D. T. J. Am. Chem. Soc. 1953, 75, 1909-1910.

[74] Wasserman, H. H.; Precopio, F. M. J. Am. Chem. Soc. 1954, 76, 1242-1243.

[75] Kronberg, L.; Sjoholm, R.; Karlsson, S. Chem. Res. Toxicol. 1992, 5, 852-5.

[76] Kronberg, L.; Karlsson, S.; Sjoholm, R. Chem. Res. Toxicol. 1993, 6, 495-9.

[77] Kronberg, L.; Asplund, D.; Mäki, J.; Sjöholm, R. Chem. Res. Toxicol. 1996, 9, 1257-1263.

[78] LeCurieux, F.; Munter, T.; Kronberg, L. Chem. Res. Toxicol. 1997, 10, 1180-1185.

[79] Al-Hakim, A. H.; Haines, A. H. Tetrahedron Lett. 1982, 23, 5295 - 5298.

[80] Beska, E.; Rapos, P. J. Chem. Soc., Perkin Trans. 1 1976, 2470-2471.

[81] Ratts, K.; Phillips, W. J. Org. Chem. 1974, 39, 3300-3301. 
[82] Polezhaeva, N.; Sakhibullina, V.; Elshina, E.; Kostyunina, T.; Arbuzov, B. Zh. Obshch. Khim. 1993, $63,2422-2425$.

[83] Polezhaeva, N.; Loginova, I.; Ovechkina, E.; Galkin, V.; Sakhibullina, V.; Cherkasov, R. Zh. Obshch. Khim. 1996, 66, 927-929.

[84] Polezhaeva, N.; Loginova, I.; Ovechkina, E.; Galkin, V.; Sakhibullina, V.; Cherkasov, R. Zh. Obshch. Khim. 1997, 67, 1076-1079.

[85] Kurbangalieva, A. R.; Lodochnikova, O. A.; Devyatova, N. F.; Berdnikov, E. A.; Gnezdilov, O. I.; Litvinov, I. A.; Chmutova, G. A. Tetrahedron 2010, 66, 9945-9953.

[86] Rossi, R.; Bellina, F.; Raugei, E. Synlett 2000, 1749-1752.

[87] Bellina, F.; Anselmi, C.; Martina, F.; Rossi, R. Eur. J. Org. Chem. 2003, 2290-2302.

[88] Zhang, J.; Blazecka, P. G.; Belmont, D.; Davidson, J. G. Org. Lett. 2002, 4, 4559-4561.

[89] Bellina, F.; Anselmi, C.; Rossi, R. Tetrahedron Lett. 2001, 42, 3851-3854.

[90] Bellina, F.; Anselmi, C.; Viel, S.; Mannina, L.; Rossi, R. Tetrahedron 2001, 57, 9997-10007.

[91] Bellina, F.; Rossi, R. Synthesis-stuttgart 2002, 2729-2732.

[92] Das Sarma, K.; Zhang, J.; Curran, T. T. J. Org. Chem. 2007, 72, 3311-3318.

[93] Ube, H.; Shimada, N.; Terada, M. Angew. Chem., Int. Ed. 2010, 49, 1858-1861.

[94] Blazecka, P. G.; Belmont, D.; Curran, T.; Pflum, D.; Zhang, J. Org. Lett. 2003, 5, 5015-5017.

[95] Zhang, J.; Blazecka, P. G.; Berven, H.; Belmont, D. Tetrahedron Lett. 2003, 44, 5579-5582.

[96] Zhang, J.; Blazecka, P. G.; Davidson, J. G. Org. Lett. 2003, 5, 553-556.

[97] Angell, P.; Zhang, J.; Belmont, D.; Curran, T.; Davidson, J. G. Tetrahedron Lett. 2005, 46, $2029-2032$.

[98] Das Sarma, K.; Zhang, J.; Huang, Y.; Davidson, J. G. Eur. J. Org. Chem. 2006, 3730-3737.

[99] Zhang, J.; Morton, H. E.; Ji, J. G. Tetrahedron Lett. 2006, 47, 8733-8735.

[100] Gondela, E.; Walczak, K. Z. Molecules 2011, 16, 1011-1020.

[101] Zhang, J.; Blazecka, P. G.; Curran, T. T. Tetrahedron Lett. 2007, 48, 2611-2615.

[102] Zhang, J.; Das Sarma, K.; Curran, T. T.; Belmont, D. T.; Davidson, J. G. J. Org. Chem. 2005, 70, 5890-5895.

[103] Zhang, J.; Das Sarma, K.; Curran, T. T. Tetrahedron Lett. 2005, 46, 6433-6436.

[104] Awuah, E.; Capretta, A. J. Org. Chem. 2011, 76, 3122-3130.

[105] Wu, J.; Song, B.; Chen, H.; Bhadury, P.; Hu, D. Molecules 2009, 14, 3676-3687.

[106] Gondela, E.; Walczak, K. Z. Eur. J. Med. Chem. 2010, 45, 3993-3997.

[107] Smeds, A.; Holmbom, B.; Tikkanen, L. Nord. Pulp Paper Res.J. 1990, 5, 142.

[108] Ishiguro, Y.; Santodonato, J.; Neal, M. W. Environ. Mol. Mutagen. 1988, 11, 225-234.

[109] LaLonde, R. T.; Leo, H. R. Chem. Res. Toxicol. 1994, 7, 779783. 
[110] Tikkanen, L.; Kronberg, L. Mutat. Res. 1990, 240, 109116.

[111] Helma, C.; Kronberg, L.; Ma, T.-H.; KnasmÃ¹/4ller, S. Mutat. Res. Lett. 1995, 346, 181-186.

[112] Isomaa, B.; Holmstrom, T. H.; Lilius, H.; Franzen, R.; Kronberg, L. Toxicology 1995, 100, 69-77.

[113] Brunborg, G.; Holme, J. A.; Søderlund, E. J.; Hongslo, J. K.; Vartiainen, T.; Lötjönen, S.; Becher, G. Mutat. Res. 1991, 260, 5564.

[114] Jansson, K.; Maki-Paakkanen, J.; Vaittinen, S.-L.; Vartiainen, T.; Komulainen, H.; Tuomisto, J. Mutat. Res. 1993, 229, 25-28.

[115] LaLonde, R. T.; Ramdayal, F. Chem. Res. Toxicol. 1997, 10, 205-210.

[116] Le Curieux, F.; Nesslany, F.; Munter, T.; Kronberg, L.; Marzin, D. Mutagenesis 1999, 14, 457-462.

[117] Liviac, D.; Creus, A.; Marcos, R. J. Hazard. Mater. 2011, 190, 1045-1052.

[118] Fekry, M. I.; Price, N. E.; Zang, H.; Huang, C. F.; Harmata, M.; Brown, P.; Daniels, J. S.; Gates, K. S. Chem. Res. Toxicol. 2011, 24, 217-228.

[119] Mäki, J.; Sjöholm, R.; Kronberg, L. J. Chem. Soc., Perkin Trans. 1 1999, 2923-2928.

[120] Asplund, D.; Kronberg, L.; Sjöholm, R.; Munter, T. Chem. Res. Toxicol. 1995, 8, 841-846.

[121] Klika, K. D.; Mäki, J.; Sjöholm, R.; Kronberg, L. Arkivoc 2006, 65-74.

[122] LaLonde, R. T.; Xie, S. Chem. Res. Toxicol. 1992, 5, 618-624.

[123] LaLonde, R. T.; Xie, S. Chem. Res. Toxicol. 1993, 6, 445-451.

[124] McDonald, T. A. Evidence on the carcinogenicity of MX (3-chloro-4-(dichloromethyl)-5-hydroxy-2(5h)furanone); 2000.

[125] Kronberg, L.; Christman, R. F. Sci. Total Environ. 1989, 81-2, 219-230.

[126] WHO, MX in Drinking-water. Background document for development of WHO Guidelines for Drinkingwater Quality; 2004.

[127] Komulainen, H.; Kosma, V.-M.; Vaittinen, S.-L.; Vartiainen, T.; Kaliste-Korhonen, E.; Latjanen, S.; Tuominen, R. K.; Tuomisto, J. J. Natl. Cancer Inst. 1997, 89, 848-856.

[128] Steffensen, I. L.; Paulsen, J. E.; Engeset, D.; Kronberg, L.; Alexander, J. Pharmacol. Toxicol. 1999, 85, $56-64$.

[129] Hooth, M. J.; McDorman, K. S.; Hester, S. D.; George, M. H.; Brooks, L. R.; Swank, A. E.; Wolf, D. C. Toxicol. Sci. 2002, 69, 322331.

[130] Meier, J. R.; DeAngelo, A. B.; Daniel, F. B.; Doerger, J.; Chang, L.; Kopfler, F. C.; Robinson, M.; Ringhand, H. P. In Genotoxic and carcinogenic properties of chlorinated furanones: important by-products of water chlorination; Waters, M. e. a., Ed.; Genetic Toxicology of Complex Mixtures; Plenum Press, New York, 1990; pp 185-195.

[131] Nishikawa, A.; Furukawa, F.; Lee, I. S.; Kasahara, K.; Tanakamaru, Z.; Nakamura, H.; Miyauchi, M.; Kinae, N.; Hirose, M. Cancer Res. 1999, 59, 20452049.

[132] Hyttinen, J. M. T.; Jansson, K. Mutat. Res. Lett. 1995, 348, 183-186.

[133] Ishiguro, Y.; Lalonde, R. T.; Dence, C. W.; Santodonato, J. Environ. Toxicol. Chem. 1987, 6, 935-946. 
[134] Backlund, P.; Smeds, A.; Holmbom, B. Chemosphere 1990, 21, 771-779.

[135] LaLonde, R. T.; Cook, G. P.; Perakyla, H.; Bu, L. Chem. Res. Toxicol. 1991, 4, 540-545.

[136] LaLonde, R. T.; Cook, G. P.; Perakyla, H.; Dence, C. W. Chem. Res. Toxicol. 1991, 4, 35-40.

[137] LaLonde, R. T.; Cook, G. P.; Perakyla, H.; Dence, C. W.; Babish, J. G. Environ. Mol. Mutagen. 1991, 17,4048 .

[138] DeMarini, D. M.; AbuShakra, A.; Felton, C. F.; Patterson, K. S.; Shelton, M. L. Environ. Mol. Mutagen. $1995,26,270-285$.

[139] Hyttinen, J. M.; Niittykoski, M.; Jansson, K. Mutagenesis 1995, 10, 321-323.

[140] LaLonde, R. T.; Bu, L.; Henwood, A.; Fiumano, J.; Zhang, L. Chem. Res. Toxicol. 1997, 10, 14271436.

[141] Franzén, R.; Goto, S.; Tanabe, K.; Morita, M. Mutat. Res. 1998, 417, 31-37.

[142] Shaughnessy, D. T.; Ohe, T.; Landi, S.; Warren, S. H.; Richard, A. M.; Munter, T.; Franzen, R.; Kronberg, L.; DeMarini, D. Environ. Mol. Mutagen. 2000, 35, 106-113.

[143] Kargalioglu, Y.; McMillan, B. J.; Minear, R. A.; Plewa, M. J. Teratog. Carcinog. Mutagen. 2002, 22, 113128.

[144] Watanabe, M.; Kobayashi, H.; Ohta, T. Mutat. Res. 1994, 312, 131138.

[145] Watanabe-Akanuma, M.; Ohta, T. Mutat. Res. 1994, 311, 295304.

[146] Watanabe-Akanuma, M.; Shimoi, K.; Kinae, N.; Ohta, T. Mutat. Res. 1997, 377, 225229.

[147] Ohta, T.; Tokishita, S.-i.; Tsunoi, R.; Ohmae, S.; Yamagata, H. Mutagenesis 2002, 17, 313316.

[148] Egorov, A. I.; Howlett, N. G.; Schiestl, R. H. Mutat. Res.-Genet. Tox. 2004, 563, 159-169.

[149] Hyttinen, J. M.; Myöhänen, S.; Jansson, K. Carcinogenesis 1996, 17, 11791181.

[150] Strauss, B. S. Bioessays 1991, 13, 7984.

[151] Harrington-Brock, K.; Doerr, C. L.; Moore, M. M. Mutat. Res. 1995, 348, 105110.

[152] Woodruff, N. W.; Durant, J. L.; Donhoffner, L. L.; Penman, B. W.; Crespi, C. L. Mutat. Res. 2001, $495,157168$.

[153] Jansson, K.; Hyttinen, J. M. Mutat. Res. 1994, 322, 129-132.

[154] Mäki-Paakkanen, J.; Jansson, K.; Vartiainen, T. Mutat. Res. 1994, 310, 117123.

[155] Matsumura, H.; Watanabe, M.; Matsumoto, K.; Ohta, T. J. Toxicol. Environ. Health 1994, 43, 65-72.

[156] Chang, L. W.; Daniel, F. B.; DeAngelo, A. B. Teratog. Carcinog. Mutagen. 1991, 11, 103114.

[157] Nunn, J. W.; Chipman, J. K. Mutat. Res. 1994, 341, 133140.

[158] Marsteinstredet, U.; Brunborg, G.; Bjørås, M.; Søderlund, E.; Seeberg, E.; Kronberg, L.; Holme, J. A. Mutat. Res. 1997, 390, 171178.

[159] Holme, J. A.; Haddeland, U.; Haug, K.; Brunborg, G. Mutat. Res. 1999, 441, 145153. 
[160] Mäki, J.; Laaksonen, M.; Munter, T.; Kronberg, L.; Komulainen, H. Environ. Mol. Mutagen. 2001, $38,297-305$.

[161] Plewa, M. J.; Kargalioglu, Y.; Vankerk, D.; Minear, R. A.; Wagner, E. D. Environ. Mol. Mutagen. 2002, 40, 134142.

[162] Meier, J. R.; Monarca, S.; Patterson, K. S.; Villarini, M.; Daniel, F. B.; Moretti, M.; Pasquini, R. Toxicology 1996, 110, 5970.

[163] Laaksonen, M.; Mäki-Paakkanen, J.; Komulainen, H. Arch. Toxicol. 2001, 75, 613617.

[164] Nunn, J. W.; Davies, J. E.; Chipman, J. K. Mutat. Res. 1997, 373, 67-73.

[165] Council, N. R. Drinking Water and Wealth. Disinfectants and Disinfectant By-products; National Academy Press: Washington, DC., 1997; Vol. 7.

[166] LaLonde, R. T.; Leo, H.; Perakyla, H.; Dence, C. W.; Farrell, R. P. Chem. Res. Toxicol. 1992, 5, 392400.

[167] Nishikawa, A.; Sai, K.; Okazaki, K.; Son, H.-Y.; Kanki, K.; Nakajima, M.; Kinae, N.; Nohmi, T.; Trosko, J. E.; Inoue, T.; Hirose, M. Environ. Mol. Mutagen. 2006, 47, 4855.

[168] Jansson, K. Environ. Mol. Mutagen. 1998, 32, 185187.

[169] Daniel, F. B.; Olson, G. R.; Stober, J. A. Environ. Mol. Mutagen. 1991, 17, 32-39.

[170] Mäki-Paakkanen, J.; Jansson, K. Mutat. Res. 1995, 343, 151156.

[171] Vaittinen, S. L.; Komulainen, H.; Kosma, V. M.; Julkunen, A.; Mäki-Paakkanen, J.; Jansson, K.; Vartiainen, T.; Tuomisto, J. Food Chem. Toxicol. 1995, 33, 10271037.

[172] Furihata, C.; Yamashita, M.; Kinae, N.; Matsushima, T. Water Sci. Technol. 1992, 26, 341-345.

[173] Sasaki, Y. F.; Nishidate, E.; Izumiyama, F.; Watanabe-Akanuma, M.; Kinae, N.; Matsusaka, N.; Tsuda, S. Mutat. Res. 1997, 393, 4753.

[174] Munter, T.; Le Curieux, F.; Sjöholm, R.; Kronberg, L. Chem. Res. Toxicol. 1998, 11, 226-233.

[175] Munter, T.; Kronberg, L.; Sjoholm, R. Chem. Res. Toxicol. 1996, 9, 703-708.

[176] Munter, T.; Le Curieux, F.; Sjöholm, R.; Kronberg, L. Chem. Res. Toxicol. 1999, 12, 46-52.

[177] Franzen, R.; Tanabe, K.; Morita, M. Chemosphere 1998, 36, 2803-2808.

[178] Greenzaid, P.; Luz, Z.; Samuel, D. J. Am. Chem. Soc. 1967, 89, 749-756.

[179] Hooper, D. L. J. Chem. Soc. B 1967, 169-170.

[180] Becke, A. D. Physical Review A 1988, 38, 3098.

[181] Lee, C.; Yang, W.; Parr, R. G. Phys. Rev. B 1988, 37, 785.

[182] Cancès, M. T.; Mennucci, B.; Tomasi, J. J. Chem. Phys. 1997, 107, 3032.

[183] Mennucci, B.; Tomasi, J. J. Chem. Phys. 1997, 106, 5151.

[184] Cossi, M.; Barone, V.; Mennucci, B.; Tomasi, J. Chem. Phys. Lett. 1998, 286, 253.

[185] Ahrens, M. L.; Maass, G.; Schuster, P.; Winkler, H. J. Am. Chem. Soc. 1970, 92, 6134-6139. 
[186] Andres, G. O.; Pierini, A. B.; de Rossi, R. H. J. Org. Chem. 2006, 71, 7650-7656.

[187] Wolfe, S.; Kim, C. K.; Yang, K.; Weinberg, N.; Shi, Z. J. Am. Chem. Soc. 1995, 117, 4240-4260.

[188] Wolfe, S.; Shi, Z.; Yang, K. Y.; Ro, S.; Weinberg, N.; Kim, C. K. Can. J. Chem. 1998, 76, 114-124.

[189] Guthrie, J. P. J. Am. Chem. Soc. 2000, 122, 5529-5538.

[190] Guthrie, J. P.; Pitchko, V. J. Am. Chem. Soc. 2000, 122, 5520-5528.

[191] Keller, D. A.; Heck, H. d. Toxicol. Lett. 1988, 42, 183-191.

[192] Daniel, F. B.; DeAngelo, A. B.; Stober, J. A.; Olson, G. R.; Page, N. P. Fund. Appl. Toxicol. 1992, 19, 159-168.

[193] Kauffman, R. E. et al. Pediatrics 1993, 92, 471-473.

[194] Steinberg, A. D. Pediatrics 1993, 92, 442-446.

[195] Salmon, A. G.; Kizer, K. W.; Zeise, L.; Jackson, R. J.; Smith, M. T. J. Toxicol. Clin. Toxicol. 1995, 33, $115-121$.

[196] Seng, J. E.; Agrawal, N.; Horsley, E. T. M.; Leakey, T. I.; Scherer, E. M.; Xia, S. J.; Allaben, W. T.; Leakey, J. E. A. Toxicol. Appl. Pharmacol. 2003, 193, 281-292.

[197] Haselkorn, T.; Whittemore, A. S.; Udaltsova, N.; Friedman, G. D. Drug Safety 2006, 29, 67-77.

[198] Greenzaid, P.; Luz, Z.; Samuel, D. J. Am. Chem. Soc. 1967, 89, 756-759.

[199] Greenzaid, P.; Rappoport, Z.; Samuel, D. J. Chem. Soc., Far. Trans. 1967, 63, 2131-2139.

[200] Bell, R. P. Adv. Phys. Org. Chem. 1966, 4, 1-29.

[201] Le Henaff, P. B. Soc. Chim. Fr. 1968, 11, 4687-4700.

[202] Kurz, J. J. Am. Chem. Soc. 1967, 89, 3524-3528.

[203] Gruen, L. C.; McTigue, P. T. Journal of the Chemical Society, Chemical Communications 1963, 52175223.

[204] Buschmann, H.-J.; Dutkiewicz, E.; Knoche, W. Ber. Dtsch. Chem. Ges. 1982, 86, 129-134.

[205] Hilal, S. H.; Bornander, L. L.; Carreira, L. A. QSAR Comb. Sci. 2005, 24, 631-638.

[206] Guthrie, J. P. Can. J. Chem. 1978, 56, 962-973.

[207] Guthrie, J. P. Can. J. Chem. 1975, 53, 898-906.

[208] Wiberg, K. B.; Morgan, K. M.; Maltz, H. J. Am. Chem. Soc. 1994, 116, 11067-11077.

[209] Guthrie, J. P.; Cullimore, P. A. Can. J. Chem. 1980, 58, 1281-1294.

[210] Guthrie, J. P. J. Am. Chem. Soc. 1978, 100, 5892-5904.

[211] Ross, A.; Boron, W. Physiology Reviews 1981, 2, 296-434.

[212] Bright, G. R.; Fisher, G. W.; Rogowska, J.; Taylor, D. L. J. Cell Biol. 1987, 104, 1019 - 1044.

[213] USEPA, US Code of Federal Regulations. Title 40, Volume 19, Chapter I, Part 143.1-4. 2002. 
[214] GEMStat, United Nations Global Environment Monitoring System Water Programme, Global Water Quality Data and Statistics. 2010; http://www.gemstat.org.

[215] Pollack, R. M.; Bender, M. L. J. Am. Chem. Soc. 1970, 92, 7190-7194.

[216] Biechler, S. S.; Taft, R. W. J. Am. Chem. Soc. 1957, 79, 4927-4935.

[217] Ono, M.; Todoriki, R.; Mitake, A.; Yukawa, M.; Goto, Y.; Tamura, S. Chem. Pharm. Bull. 1989, 37, 2902-2908.

[218] Menger, F. M.; Donohue, J. A. J. Am. Chem. Soc. 1973, 95, 432-437.

[219] King, J. F.; GIll, M. S.; Klassen, D. F. Pure Appl. Chem. 1996, 68, 825-830.

[220] Baxter, N. J.; Rigoreau, L. J. M.; Laws, A. P.; Page, M. I. J. Am. Chem. Soc. 2000, 122, 3375-3385.

[221] Page, M. I. Acc. Chem. Res. 2004, 37, 297-303.

[222] Ho, J. M.; Coote, M. L. Theor. Chem. Acc. 2010, 125, 3-21.

[223] Manso, J. A.; Pérez-Prior, M. T.; García-Santos, M. P.; Calle, E.; Casado, J. Chem. Res. Toxicol. 2005, $18,1161-1166$.

[224] Pérez-Prior, M. T.; Manso, J. A.; García-Santos, M. D.; Calle, E.; Casado, J. J. Agric. Food Chem. 2005, 53, 10244-10247.

[225] Fernández-Rodríguez, E.; Manso, J. A.; Pérez-Prior, M. T.; García-Santos, M. D. P.; Calle, E.; Casado, J. Int. J. Chem. Kinet. 2007, 39, 591-594.

[226] Manso, J. A.; Pérez-Prior, M. T.; García-Santos, M. P.; Calle, E.; Casado, J. J. Phys. Org. Chem. 2008, 21, 932-938.

[227] Pérez-Prior, M. T.; Manso, J. A.; García-Santos, M. P.; Calle, E.; Casado, J. J. Solution Chem. 2008, 37, 459-466.

[228] Manso, J. A.; Pérez-Prior, M. T.; Gómez-Bombarelli, R.; González-Pérez, M.; Céspedes, I. F.; GarcíaSantos, M. P.; Calle, E.; Casado, J. J. Phys. Org. Chem. 2009, 22, 386-389.

[229] Pérez-Prior, M. T.; Gómez-Bombarelli, R.; González-Pérez, M.; Manso, J. A.; García-Santos, M. P.; Calle, E.; Casado, J. Chem. Res. Toxicol. 2009, 22, 1320-1324.

[230] Céspedes, I. F.; Manso, J. A.; Pérez-Prior, M. T.; Gómez-Bombarelli, R.; González-Pérez, M.; Calle, E.; Casado, J. J. Phys. Org. Chem. 2010, 23, 171-175.

[231] Pérez-Prior, M. T.; Gómez-Bombarelli, R.; González-Pérez, M.; Manso, J. A.; García-Santos, M. P.; Calle, E.; Casado, J. J. Org. Chem. 2010, 75, 1444-1449.

[232] González-Pérez, M.; Gómez-Bombarelli, R.; Pérez-Prior, M. T.; Manso, J. A.; CespedesCamacho, I. F.; Calle, E.; Casado, J. Org. Biomol. Chem. 2011, 9, 7016-7022. 


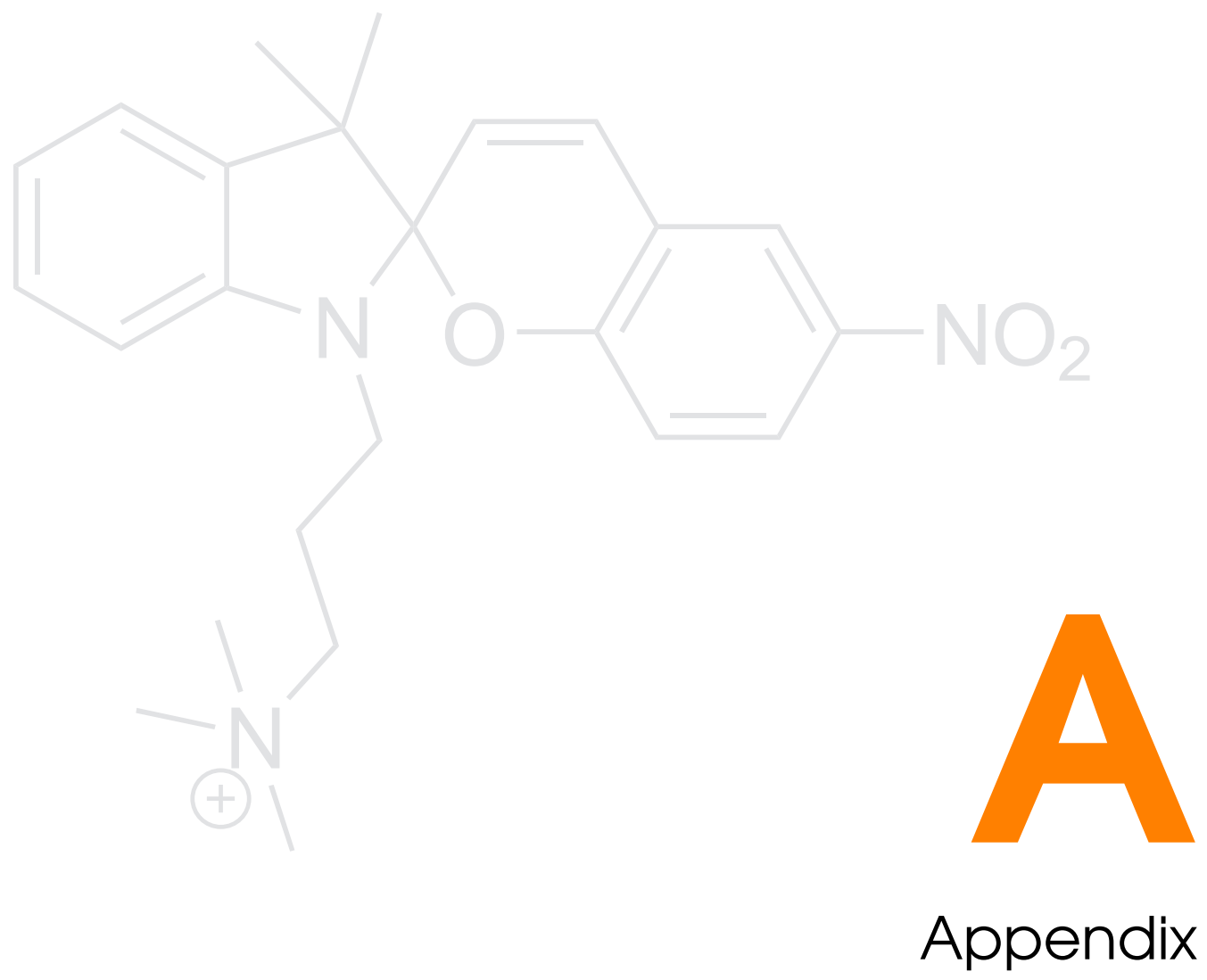





\section{Contents}

A.1 DNA-Intercalating photochromic spiropyran . . . . . . . . . A5

A.1.1 Methodology and computational details . . . . . . . . . . . A5

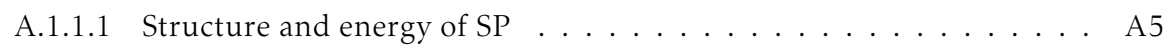

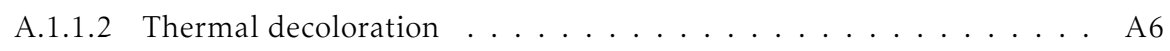

A.1.1.3 Intercalation of $\mathrm{SP} \ldots \ldots \ldots \ldots$ A6

A.1.2 Results and discussion . . . . . . . . . . . . A6

A.1.2.1 Structure and energy of $\mathrm{SP} \ldots \ldots \ldots \ldots$ A6

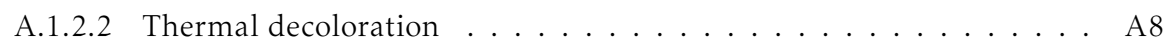

A.1.2.3 Optical transitions . . . . . . . . . . . . . . . A9

A.1.2.4 Intercalation and optical properties . . . . . . . . . . A10

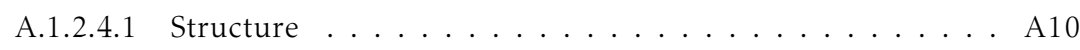

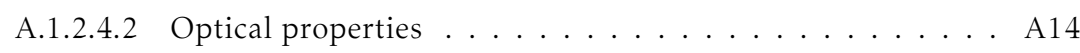

A.1.3 Conclusions .............................. A16

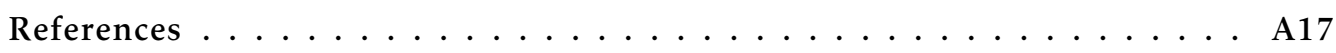





\section{A.1. DNA-Intercalating photochromic spiropyran}

Among the many potential applications of photochromes, their use as reversible photoswitches of biological processes results particularly promising. ${ }^{1}$ In recent years, photochromic units have been proposed as a plausible way to prepare optobioelectronic devices based on macromolecules such as DNA or proteins. Whereas the approaches so far usually include the covalent modification of the macromolecule, and thus greatly limit the in vivo applicability, ${ }^{2,3}$ Andersson et al. have recently reported the reversible photoswitched DNA-binding of a photochromic spiropyran (SP, A.1). ${ }^{4}$ Some later works have also addressed other DNA-binding photochromes such as tetraaryl-pyridinium derivatives, ${ }^{5}$ a molecular glue, ${ }^{6}$ acylated aminonaphthyridine and an azaquinolone, ${ }^{7}$ other spiropyran, ${ }^{8}$ methylated azobenzenes ${ }^{9}$ and chromene derivatives containing a charged side-chain.

The ability to control the binding of ligands to DNA using external stimuli is of great pharmaceutical interest, and would potentially allow control of both DNA replication and transcription. In order to give some details at the molecular level of the interaction first reported by Andersson et al.; ${ }^{4}$ we have undertaken a computational study of the spiropyran, especially as regards its DNA-intercalating ability

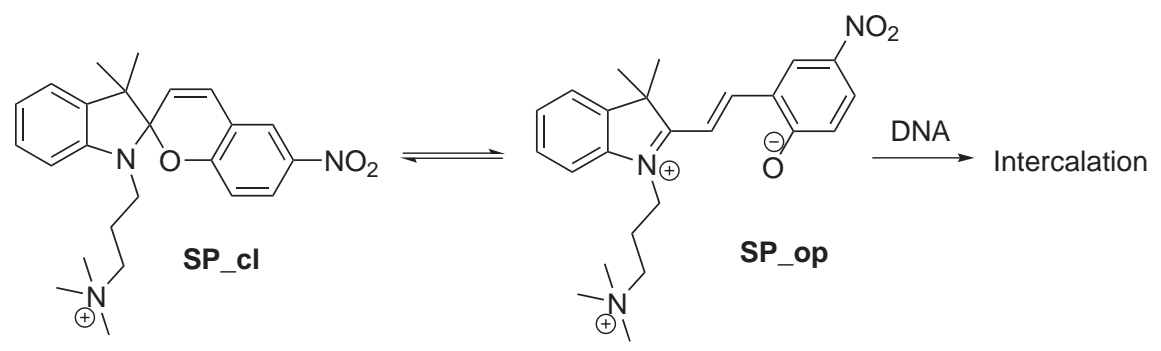

Scheme A.1. Photochromic spiropyran with DNA-intercalating ability

\section{A.1.1. Methodology and computational details}

\section{A.1.1.1. Structure and energy of SP}

The equilibrium geometries and energies of the different conformers of SP were determined using density functional theory. The B3LYP, BPE and MWPB1K functionals with the 6-31G(d), 6-31G+(d), 6$31 \mathrm{G}++(\mathrm{d}, \mathrm{p})$ basis sets were used. When necessary, solvation (in both water and chloroform) was taken into account implicitly using the Integral Equation Formalism Polarizable Continuum Model (IEF-PCM). All minima on the potential energy hypersurface were characterized by harmonic analysis. Transition states connecting the different isomers were also modeled at the same level of theory.

Electronic transition spectra were calculated using time dependent density functional theory (TDDFT) with the B3LYP hybrid functional. 


\section{A.1.1.2. Thermal decoloration}

The thermal decoloration and isomer-interconversion pathways were studied at the DFT-B3LYP/6-31G(d) level of theory using the PCM model for aqueous solvation.

\section{A.1.1.3. Intercalation of SP}

The interaction of SP with two oligonucleotides $[\operatorname{tri}(\mathrm{dA}-\mathrm{dT})]_{2}$ and $[\operatorname{tri}(\mathrm{dG}-\mathrm{dC})]_{2}$ was studied. The intercalation of SP in DNA was modeled using a two-layer quantum mechanics/quantum mechanics (QM/QM) approach. The high-level layer (HL) contained the photochromic molecule and the two base pairs that make up the intercalation site. The HL was modeled at the DFT-B3LYP 6-31G(d) and DFT-MPWB1K 6-31G(d) levels. The low-level layer (LL) contained the remaining base pairs and the phosphate-sugar backbone. The LL was modeled at the semiempirical PM3 level of theory. The total energy of the system can be computed as:

$$
E=E_{\mathrm{LL}}^{\mathrm{PM} 3}+E_{\mathrm{HL}}^{\mathrm{DFT}}-E_{\mathrm{HL}}^{\mathrm{PM} 3}
$$

In order to prevent the unphysical elongation of the double helix structure that is usually observed in gas-phase calculations and to model the effect of additional nucleotides, the positions of the top and bottom ribose rings were frozen. This model has some obvious limitations due to border effects; as a consequence, the object of discussion will be solely the central region of the system where the intercalation takes place. The effect of intercalation upon the structure of the oligonucleotide is also beyond the reach of this work, since it would call for a more precise model for the LL and a much larger LL region. The electronic transitions for the SP+DNA system were computed also at a QM/QM two-layer system, using TD-DFT-B3LYP 6-31G(d)/PM3 and the equilibrium geometries previously determined. A total of ten excited states were studied (nstates $=10$ ) The Gaussian 03 suite of programs was used for all calculations.

\section{A.1.2. Results and discussion}

\section{A.1.2.1. Structure and energy of SP}

Spyropyrans in their open forms can exist as a variety of conformers that are usually identified by the $\mathrm{C}$ (for cis) or $\mathrm{T}$ (for trans) initials depending on the configuration $(Z$ or $E$ ) of the three rigid conjugated bonds that connect the two cyclic regions, beginning the count at the indolin unit. Generally the conformers showing a trans-configuration in the central bond are more stable, and thus, only the conformers CTC, CTT, TTC and TTT were considered in this work. CL stands for the closed form.

Table A.1 shows the relative energies of the open-form isomers and the closed form, referred to the most stable isomer. A first inspection shows that the choice of functional and basis set has a large effect, 
Table A.1. Relative energies for the isomers of $\mathbf{S P}$ in gas phase and solution

\begin{tabular}{|c|c|c|c|c|c|}
\hline \multirow{3}{*}{ Solvent } & \multirow{3}{*}{ Isomer } & \multicolumn{4}{|c|}{ Relative free energy $\left(\mathrm{kJ} \mathrm{mol}^{-1}\right)$} \\
\hline & & \multicolumn{3}{|c|}{ B3LYP/6-31 } & \multirow{2}{*}{$\frac{\text { MPWB1K }}{6-31 G(d)}$} \\
\hline & & $\mathrm{G}(\mathrm{d})$ & $+\mathrm{G}(\mathrm{d})$ & $++G(d, p)$ & \\
\hline \multirow{5}{*}{ Water } & $\mathrm{CL}$ & 3.4 & 7.8 & 11.2 & 0 \\
\hline & CTC & 21.3 & 9.6 & 10.0 & 62.9 \\
\hline & CTT & 5.0 & 4.3 & 5.0 & 38.8 \\
\hline & TTC & 0 & 0 & 0 & 32.6 \\
\hline & TTT & 13.3 & 2.0 & 2.7 & 73.9 \\
\hline \multirow{5}{*}{ Chloroform } & $\mathrm{CL}$ & 4.9 & 8.6 & 9.4 & 13.2 \\
\hline & CTC & 39.7 & 32.4 & 29.6 & 54.7 \\
\hline & CTT & 8.4 & 9.1 & 9.3 & 4.3 \\
\hline & TTC & 0 & 0 & 0 & 0 \\
\hline & TTT & 26.5 & 17.6 & 18.8 & 35.3 \\
\hline \multirow{5}{*}{ Gas } & $\mathrm{CL}$ & 15 & 18.8 & 0 & 12.8 \\
\hline & СТC & 50.9 & 49.2 & 71.7 & 49.6 \\
\hline & CTT & 4.9 & 5.4 & 17.9 & 3.6 \\
\hline & TTC & 0 & 0 & 14 & 0 \\
\hline & TTT & 31.8 & 31.6 & 53.9 & 32.7 \\
\hline
\end{tabular}

especially in the energy differences between the closed form and the open-chain forms as a whole. Energy differences among the open isomers show less variation. It can be observed that the most stable open conformer in solution is TTC, by $2-5 \mathrm{~kJ} \mathrm{~mol}^{-1}$.

More importantly, results show that in solution, some open-chain conformers are more stable than the closed form, contrarily to what occurs for most spiropyranic photochromes. The thermal equilibrium between the open and closed forms has been reported to be close to $50 \%$ in water, ${ }^{4}$ and thus, an energy difference close to null should be expected between the open and closed forms. The observed difference can be attributed, in part, to errors in the solvation energy: the solvation energies for SP are relatively large due to the presence of a charged substituent, and even small errors in the solvation energies can easily reach the magnitude of a few $\mathrm{kJ} \mathrm{mol}^{-1}$. Thus, within the reach of the method, our results are in agreement with the experimental values. As can be seen, the solvation in either chloroform or water shows only moderate influence on the relative energies of the conformers.

Since the only structural difference between SP and other well studied spyropyrans is the presence of a charged substitutent on the indolin nitrogen, the same calculation was applied to a spyropyran with a methyl substituent in the place of the trimethylpropylammonium chain, in order to confirm the accuracy of the method. The results (Table A.2) are in keeping with experimental measurements and show the closed form as the most stable isomer. 
Table A.2. Relative free energies of the isomers of a SP-analogue lacking the charged substituent

\begin{tabular}{cccc}
\hline \multirow{2}{*}{ Isomer } & & \multicolumn{2}{c}{ Free energy $\left(\mathrm{kJ} \mathrm{mol}^{-1}\right)$} \\
\cline { 1 - 1 } $\mathrm{Cl}$ & & B3LYP/6-31G(d) & MPWB1K/6-31G(d) \\
CTC & & 7.5 & 0.0 \\
$\mathrm{CTT}$ & & 10.1 & 32.9 \\
$\mathrm{TTC}$ & & 3.4 & 35.9 \\
TTT & 5.4 & 23.8 \\
\hline
\end{tabular}

Results with the MPWB1K functional are less satisfactory, since they predict a very displaced thermal equilibrium, however, the differences among the open-chain isomers are similar to those using the B3LYP hybrid functional.
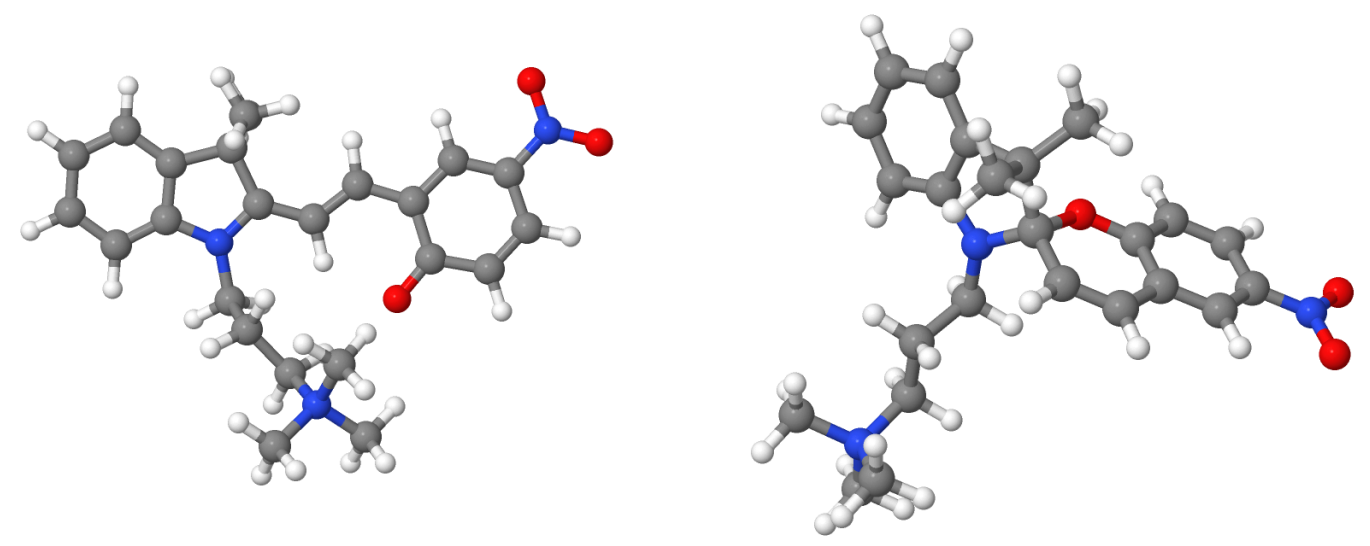

Figure A.1. Calculated geometries for the closed form and most abundant open form of SP in aqueous solution

From the structural point of view, and as would be expected, all open-chain SP forms are highly planar. Some interaction between the charged group and the $\mathrm{C}=\mathrm{O}$ (in CTT and TTC) or the $\mathrm{NO}_{2}$ (in CTC and TTT) groups is observed in the gas-phase geometries. However, only the interaction of with the oxo with CTT and TTC oxygen in maintained in solution (Figure A.1).

\section{A.1.2.2. Thermal decoloration}

Given the rather high time constant reported for the decoloration of SP-CL in water $\left(124 \mathrm{~min}\right.$ at $\left.23^{\circ} \mathrm{C}\right)$ and the presence of a bulky substituent on the indolin nitrogen, whose influence is unclear, we have 
studied the thermal closing of SP and the interconversion of the different conformers using DFT methods. Depending on the geometry of the transition state for the ring-opening reaction, two reaction pathways are available (Scheme A.2), one of which is effectively blocked by the two methyl groups present.

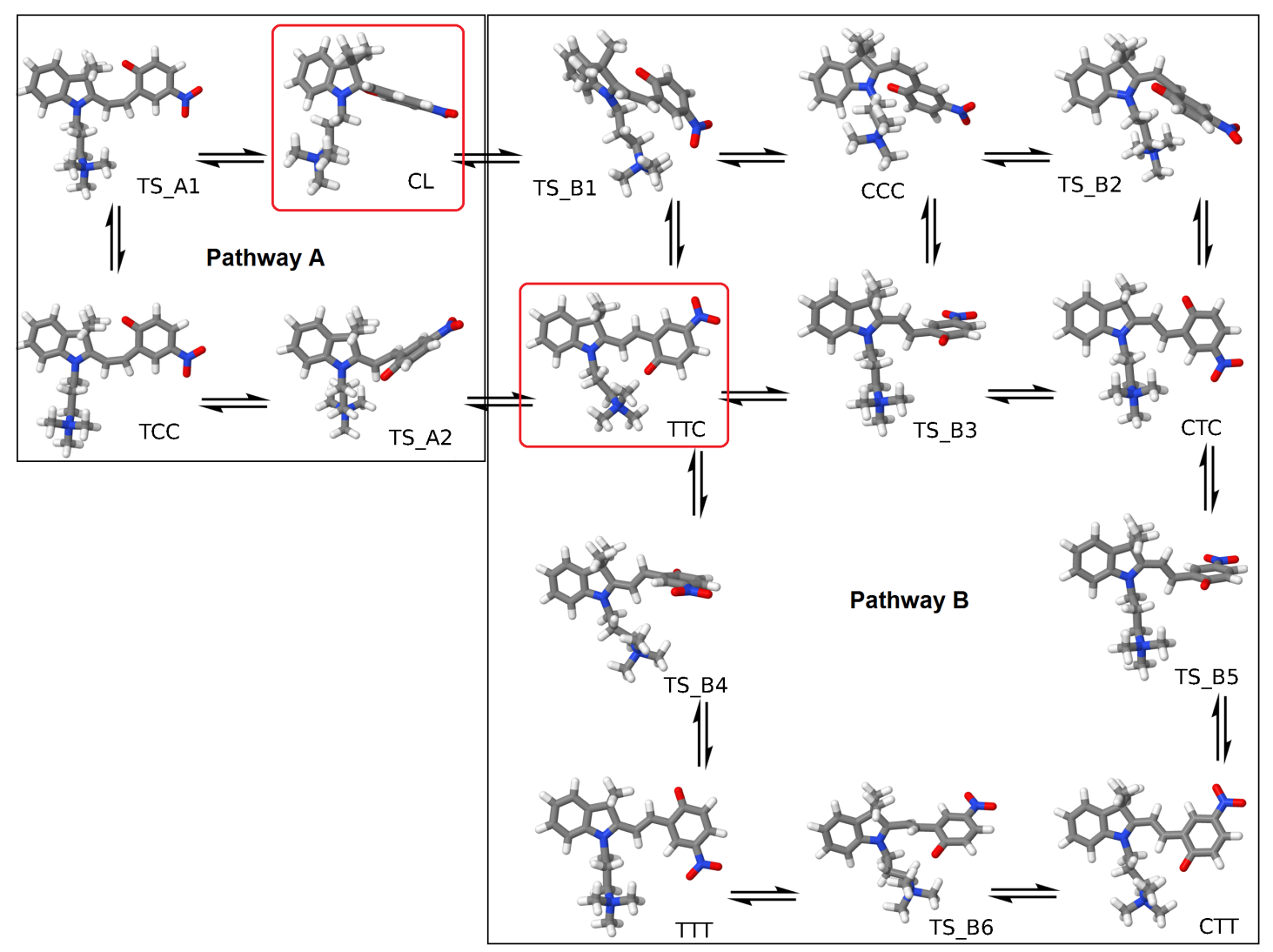

Scheme A.2. Thermal decoloration and isomerization pathways

The calculated relative energies for both the pathway B and the cis-trans interconversion are reported in Table A.3. Results are in agreement with previously computed thermal barriers for other spyropyrans with similar substitution patterns and suggest that the presence of the two methyl groups blocks one of the thermal opening/closing pathways whereas the trimethylpropylammonium substituent produces scarcely more hindrance on the other reaction pathway than a methyl group. CITAR REVIEW

\section{A.1.2.3. Optical transitions}

Since the applications of photochromes are necessarily related to their ability to absorb UV-Vis radiation, the UV-Vis absorption spectra of SP were calculated, for comparison with experimental results, and also to address the effect of DNA-binding (see below). 
Table A.3. Relative energies of SP-isomers and transition states in aqueous solution at the DFT-B3LYP/6-31G(d) level of theory $\left(\mathrm{kJ} \mathrm{mol}^{-1}\right)$

\begin{tabular}{lccccc}
\hline Species & TS-A1 & CL & TS-B1 & CCC & TS-B2 \\
Energy & $\sim 200$ & 5 & 80 & 40 & 130 \\
Species & TCC & TS-A2 & TTC & TS-B3 & CTC \\
Energy & 160 & & 0 & 85 & 21 \\
Species & & & TS-B4 & & TS-B5 \\
Energy & & & 140 & & 125 \\
Species & & & TTT & TS-B6 & CTT \\
Energy & & & 13 & 100 & 5 \\
\hline
\end{tabular}

The optical transitions of SP in the CL and CTC, CTT, TTC and TTT conformations have been computed in solution and in the gas phase from both B3LYP 6-31G(d) and MPWB1K 6-31G(d) geometries using TD-B3LYP 6-31G(d) and, since B3LYP 6-31+G(d) energies proved to be somewhat superior, TDB3LYP $6-31+G(d)$ calculations on B3LYP $6-31+G(d)$ geometries were also performed (Table A.4) .

Very little difference exists between the $6-31 G(d)$ and $6-31+G(d)$ results, despite the increased computational cost, and thus, the more affordable ones were used for later calculations.

The experimental values for the wavelengths of maximum absorption are $348 \mathrm{~nm}$ for the closed form and $512 \mathrm{~nm}$ for the open form. B3LYP results are in excellent agreement with these measurements, particularly when considering that the more abundant minor species may also contribute in part to the observed UV-Vis spectrum. MPWB1K geometries afford fairly blue-shifted transition wavelengths, which are in somewhat worse agreement with experimental values. Figure A.2 reports the theoretical UV-Vis spectra for the closed isomer and the most common open chain form (TTC). Results are in excellent agreement with the experimental values reported by. ${ }^{4}$

\section{A.1.2.4. Intercalation and optical properties}

We have calculated the equilibrium geometries of the SP-DNA complexes using density functional theory. Two functionals have been used: B3LYP which is very often used for many classes of compounds and reactions and MPWB1K, which has shown to produce improved results in processes governed by $\pi-\pi$ interaction and dispersion forces. The electronic transitions of the HL section (SP + surrounding nucleotides) have also been computed, using the much superior B3LYP functional. The PM3 semi-empirical QM method has been used for the modelling of the LL section. PM3 is known to be able to reproduce hydrogen bonding.

A.1.2.4.1. Structure. The computed structures show a clean insertion of the photochrome into the intercalation site comprising the two central base pairs. Significant distortion to the central part of the 
Table A.4. Wavelength of maximum absorption $(\mathrm{nm})$ for the SP-isomers in different solvents at different levels of theory

\begin{tabular}{|c|c|c|c|c|}
\hline \multirow{6}{*}{ Solvent } & \multirow{6}{*}{ Isomer } & \multicolumn{3}{|c|}{ Geometry } \\
\hline & & B3LYP & B3LYP & MPWB1K \\
\hline & & $6-31 G(d)$ & $6-31+G(d)$ & $6-31 G(d)$ \\
\hline & & \multicolumn{3}{|c|}{ TD-DFT } \\
\hline & & B3LYP & B3LYP & B3LYP \\
\hline & & $6-31 G(d)$ & $6-31+G(d)$ & $6-31 G(d)$ \\
\hline \multirow{5}{*}{ Gas-Phase } & CL & 331.8 & 347.6 & 340.2 \\
\hline & СТС & 524 & 532.0 & 488 \\
\hline & CTT & 515 & 522489 & \\
\hline & TTC & 503 & 506 & 479 \\
\hline & TTT & 553 & 566 & 510 \\
\hline \multirow{5}{*}{ Water } & $\mathrm{CL}$ & 323 & 343 & 349 \\
\hline & СТС & 517 & 514 & 488 \\
\hline & СTT & 525 & 531 & 499 \\
\hline & TTC & 506 & 507 & 482 \\
\hline & TTT & 536 & 539 & 506 \\
\hline \multirow{5}{*}{ Chloroform } & CL & 353 & 359 & - \\
\hline & СТС & 519 & 529 & - \\
\hline & CTT & 522 & 531 & - \\
\hline & TTC & 506 & 510 & - \\
\hline & TTT & 534 & 545 & - \\
\hline
\end{tabular}

oligonucleotide is observed; so that the spacing between the base pairs surrounding the spyropyran is about $6 \AA$. However, Watson-Crick hydrogen bonding between all pairs is conserved.

All the open-form isomers show some structural changes in their intercalation geometries, mainly a loss of coplanarity between the two rings, which acquire somewhat parallel orientations. Interestingly, TTC and CTT intercalate mostly on the side of the base pair, whereas TTT and CTC have more central positions. This is due to the general shape of the isomers: in TTC and CTT are more rectangular, whereas and TTT and CTC are more T-shaped.

The presence of the two bulky substituents on the indolin moiety limits the degree of intercalation of SP into the site. Thus, the two methyl substituents play opposite roles: as stated above, they block a thermal decoloration pathway, effectively increasing the elapsed time until thermal equilibrium is reached, and, at the same time, limit how far SP can slide into the intercalation site. As expected from the perpendicular rings, SP-CL shows the least extent of intercalation, due to the steric repulsion.

An important structural feature is the interaction between the ammonium group and the negativelycharged phosphate backbone. 

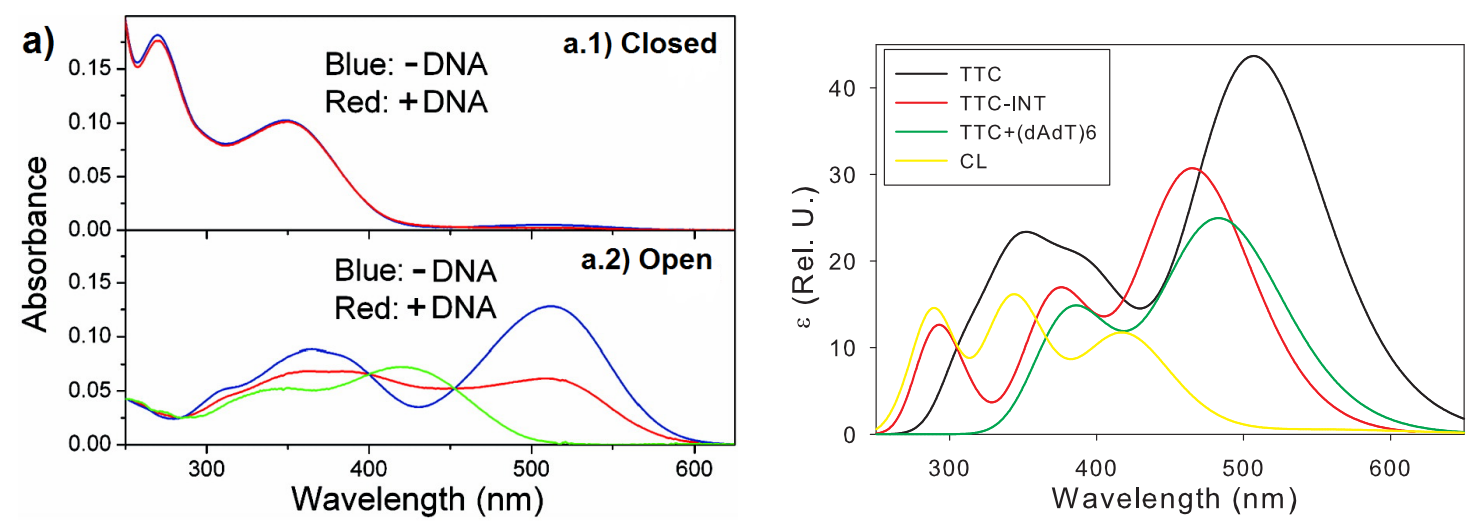

Figure A.2. a) Reported UV-Vis spectra of the open and closed forms of SP, in the absence and presence of DNA. Reproduced from. ${ }^{4}$ b) Calculated UV-Vis spectra of TTC; isolated TTC in its intercalation geometry; TTC bound to $(\text { tri }(d A-d T))_{2} ; C L$

Table A.5 summarize the gas-phase interaction energies between SP in the different conformations and the oligonucleotides. Also shown are the interaction energies between SP and the base pairs contained in the HL part, thus excluding the electrostatic interaction of the charged groups. The counterpoise correction to the Basis Set Superposition Error (BSSE) is included in the results.

Table A.5. SP-oligonucleotide interaction energy

\begin{tabular}{|c|c|c|c|c|c|c|c|}
\hline \multirow{3}{*}{ Oligo } & \multirow{3}{*}{ Isomer } & \multicolumn{6}{|c|}{ Interaction Energy $\left(\mathrm{kJ} \mathrm{mol}^{-1}\right)$} \\
\hline & & \multicolumn{3}{|c|}{ MPWB1K-31G(d)/PM3 } & \multicolumn{3}{|c|}{ B3LYP-31G(d)/PM3 } \\
\hline & & $\mathrm{HL}+\mathrm{LL}$ & Rel. HL +LL & $\mathrm{HL}$ & $\mathrm{HL}+\mathrm{LL}$ & Rel. HL +LL & $\mathrm{HL}$ \\
\hline \multirow{5}{*}[\operatorname{tri}(\mathrm{dA}-\mathrm{dT})]{$_{2}$} & CL & -1085.2 & 98.2 & -42.4 & -1095.1 & 70.7 & -27.4 \\
\hline & СТC & -1151.2 & 32.1 & -11.5 & -1165.8 & 0 & 13.1 \\
\hline & CTT & -1183.4 & 0 & -65.1 & -1103.7 & 62.1 & 1.6 \\
\hline & TTC & -1153.4 & 30.0 & -42.0 & -1139.6 & 26.2 & 21.9 \\
\hline & TTT & -1119.7 & 63.7 & -48.9 & -1051.2 & 114.6 & 23.3 \\
\hline \multirow{5}{*}[\operatorname{tri}(\mathrm{dA}-\mathrm{dT})]{$_{2}$} & $\mathrm{CL}$ & -1103.8 & 106.1 & -62.4 & -1104 & 71.8 & -48.1 \\
\hline & CTC & -1209.9 & 0 & -114.3 & -1175.8 & 0 & -51.3 \\
\hline & CTT & -1152.1 & 57.8 & -94.5 & -1148.3 & 27.5 & -41.7 \\
\hline & TTC & -1085.2 & 124.7 & -78.1 & -1068.5 & 107.3 & -16.4 \\
\hline & TTT & -1186.1 & 23.9 & -134.2 & -1149.7 & 26.1 & -62.1 \\
\hline
\end{tabular}

The very high binding energies obtained are due to the gas-phase electrostatic interaction of the ammonium and phosphate groups. The HL interaction energies between the planar region of SP and the nucleosides are of some tens $\mathrm{kJ} \mathrm{mol}^{-1}$, which is consistent with the usual values in intercalation and other 
processes governed by dispersion forces or $\pi-\pi$ stacking.

We can also see the clear superiority of the MPWB1K functional - which predicts much more favorable SP-DNA interactions - over the B3LYP functional, to the point that the latter predicts repulsive intercalation energies in some cases.

Some sequence specificity among the different open-form isomers is observed: AT favors the CTT conformation, whereas GC favors the TTT conformation. This specificity, if well-tuned, has the potential for practical applications.

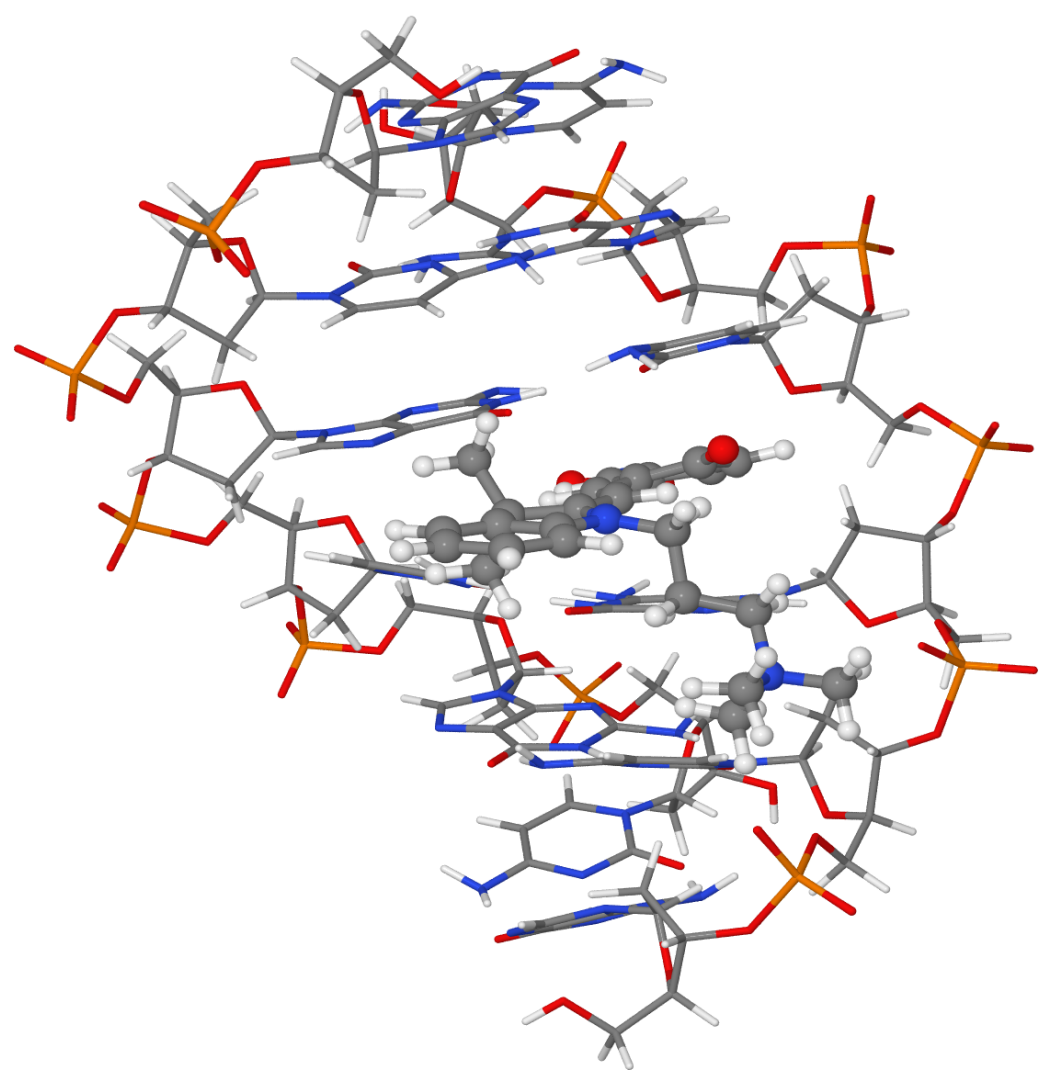

Figure A.3. SP TTC-(tri(dA-dT) $)_{2}$ intercalation complex

For both oligonucleotides, the intercalation energy of the closed form is lower than that of the open chain isomers, which is in good agreement with experimental results that show null variation in the $\mathrm{UV}$-vis spectrum of SP-CL when in the presence of DNA.

Since only a portion of the photochrome is intercalated, the extent of intercalation and thus, the interaction energy, could be increased by (i) expanding the intercalating region of SP by adding a fused cycle to the nitrobenzene region (ii) modifying the two methyl groups so that they allow further inclusion 
of the nitrobenzene moiety, although this would have negative effects on the thermal decoloration rate, since a new low-energy thermal opening pathway would be available.

The role of the trimethylpropylammonium chain also opens an interesting horizon for the synthesis of other potential photo switched DNA-binding photochromes: the length of the spacer chain, the nature of the charged group, the presence of additional charged groups to have extra interactions with other phosphate moities... are all parameters which could be optimized. Since other photochromes, such as diarylethenes, show very little thermal decoloration, addition of such positively-charged chains could also allow the preparation of DNA-binding photochromes of other classes. The extent of intercalation could also be modulated depending on the size and planarity of the probe.

Water solvent is expected to have a modest influence on the HL intercalation energy. However, the magnitude of the electrostatic interaction between the charged groups is expected to be highly dependent on the aqueous environment. Should solvent effects be included, the scale of this interaction would be significantly reduced.

We have computed the interaction energy between the propylammonium group and the phosphateribose chain at the DFT B3LYP/6-31G(d) level of theory. In the gas phase, the attractive interaction is about $600 \mathrm{~kJ} \mathrm{~mol}^{-1}$ more intense than in solution (-679 vs $-43 \mathrm{~kJ} \mathrm{~mol}^{-1}$ ), which confirms that the magnitude of the interaction between the ammonium and phosphate groups decreases significantly in aqueous solution.

A.1.2.4.2. Optical properties. In order to investigate the effect of intercalation upon the optical transitions of the photochrome, TD-DFT calculations were performed, the results of which are shown in Table A. 6 as $\lambda_{\text {DNA }}$.

Two concurrent processes affect the electronic transitions: geometrical changes in SP and that of the inclusion itself, including the orbital overlap with the nucleotides. To detach the geometric effect from that of polarization and $\pi-\pi$ interaction, the electronic transitions were also calculated for the isolated photochromes in their intercalation geometries ( $\lambda_{\text {SP-Int }}$ ) Table A.6). These two separate effects are reported as $\Delta \lambda_{\mathrm{GEOM}}$ and $\Delta \lambda_{\mathrm{INT}}$ respectively (eq A.2 and A.3)

$$
\begin{aligned}
\Delta \lambda_{\mathrm{GEOM}} & =\lambda_{\mathrm{SP}-\mathrm{Int}}-\lambda_{\mathrm{SP}} \\
\Delta \lambda_{\mathrm{INT}} & =\lambda_{\mathrm{DNA}}-\lambda_{\mathrm{SP}-\mathrm{Int}}
\end{aligned}
$$

Results show that the changes in geometry, chiefly the loss of coplanarity between the two cycles, results in an important blue-shift in the UV-Vis spectra and a decrease in absorptivity (A.2, red line). On the other hand, intercalation produces some red-shift and a decrease in the transition strength, and thus, the molar absorption coefficients (A.2, blue line). The hypsochromic shift due to the geometrical changes is generally larger than the batochromic shift due to the interaction with the bases, which results in transitions appearing at shorter wavelengths for all isomers. 
Table A.6. UV-Vis transitions of the SP-oligonucleotide HL complex, TD-DFT B3LYP/6-31G(d)

\begin{tabular}{|c|c|c|c|c|c|c|}
\hline \multirow{2}{*}{ Oligonucleotide } & \multirow{2}{*}{ Isomer } & \multicolumn{5}{|c|}{ MPWB1K-31G(d)/PM3 Geometry } \\
\hline & & $\lambda_{\text {SP-Int }}$ & $\lambda_{\mathrm{DNA}}$ & $\Delta \lambda_{\mathrm{GEOM}}$ & $\Delta \lambda_{\mathrm{INT}}$ & $\Delta \lambda_{\mathrm{TOT}}$ \\
\hline \multirow{5}{*}[\operatorname{tri}(\mathrm{dA}-\mathrm{dT})]{$_{2}$} & $\mathrm{CL}$ & 312 & 323 & -28 & 10 & -18 \\
\hline & СТC & 470 & 521 & -18 & 51 & 33 \\
\hline & CTT & 495 & 510 & 6 & 15 & 21 \\
\hline & TTC & 465 & 475 & -14 & 10 & -4 \\
\hline & TTT & 471 & 489 & -39 & 18 & -21 \\
\hline \multirow{5}{*}[\operatorname{tri}(\mathrm{dG}-\mathrm{dC})]{$_{2}$} & $\mathrm{CL}$ & 349 & 363 & 8 & 15 & 23 \\
\hline & СТC & 470 & 506 & -17 & 35 & 18 \\
\hline & CTT & 486 & 497 & -3 & 11 & 8 \\
\hline & TTC & 471 & 467 & -8 & -40 & -12 \\
\hline & TTT & 480 & 498 & -30 & 17 & -12 \\
\hline \multirow{2}{*}{ Oligonucleotide } & Isomer & \multicolumn{5}{|c|}{ B3LYP 6-31G(d)/PM3 Geometry } \\
\hline & & $\lambda_{\text {SP-Int }}$ & $\lambda_{\mathrm{DNA}}$ & $\Delta \lambda_{\mathrm{GEOM}}$ & $\Delta \lambda_{\mathrm{INT}}$ & $\Delta \lambda_{\mathrm{TOT}}$ \\
\hline \multirow{5}{*}[\operatorname{tri}(\mathrm{dA}-\mathrm{dT})]{$_{2}$} & $\mathrm{CL}$ & 346 & 348 & 14 & 1.7 & 16 \\
\hline & СТC & 488 & 504 & -36 & 16 & -20 \\
\hline & CTT & 507 & 511 & -8 & 4 & -4 \\
\hline & TTC & 479 & 484 & -24 & 5 & -19 \\
\hline & TTT & 485 & 506 & -68 & 21 & -47 \\
\hline \multirow{5}{*}[\operatorname{tri}(\mathrm{dG}-\mathrm{dC})]{$_{2}$} & $\mathrm{CL}$ & 375 & 367 & 44 & -9 & 35 \\
\hline & СТC & 493 & 512 & -31 & -19 & -11 \\
\hline & CTT & 505 & 501 & -10 & -3 & -14 \\
\hline & TTC & 486 & 472 & -17 & -14 & -31 \\
\hline & TTT & 487 & 507 & -65 & 20 & -46 \\
\hline
\end{tabular}

The closed-chain isomer is, in part, an exception; since the two rings are perpendicular and the extent of intercalation is limited by steric repulsion, intercalation induces small geometrical distortions and has little effect on the UV-Vis spectrum.

Little variation in both the size and position of the absorption band are observed in the spectrum of the closed form in the presence of DNA. This is in keeping with our results, which suggest that the interaction between SP-CL and DNA is unfavored. 


\section{A.1.3. Conclusions}

1. The DNA-binding properties of a photochromic spiropyran have been modeled in silico.

2. Intercalation energies are in good agreement with the observed experimental behavior. Results using the MPWB1K functional are superior to those obtained with the B3LYP hybrid functional.

3. The optical properties of the DNA-SP complex are also in accord with the experimental UV-Vis spectra. 


\section{References}

[1] Willner, I. Acc. Chem. Res. 1997, 30, 347-356.

[2] Asanuma, H.; Shirasuka, K.; Yoshida, T.; Takarada, T.; Liang, X.; Komiyama, M. Chem. Lett. 2001, 108-109.

[3] Liu, M.; Asanuma, H.; Komiyama, M. J. Am. Chem. Soc. 2006, 128, 1009-1015.

[4] Andersson, J.; Li, S.; Lincoln, P.; Andreasson, J. J. Am. Chem. Soc. 2008, 130, 11836-11837.

[5] Di Pietro, M. L.; Puntoriero, F.; Tuyeras, F.; Ochsenbein, P.; Laine, P. P.; Campagna, S. Chem. Comm. 2010, 46, 5169-5171.

[6] Dohno, C.; nosuke Uno, S.; Sakai, S.; Oku, M.; Nakatani, K. Bioorg. Medicinal Chem. 2009, 17, 2536 2543.

[7] Dohno, C.; Yamamoto, T.; Nakatani, K. Eur. J. Org. Chem. 2009, 4051-4058.

[8] Hammarson, M.; Andersson, J.; Li, S.; Lincoln, P.; Andreasson, J. Chem. Comm. 2010, 46, 7130-7132.

[9] Nishioka, H.; Liang, X.; Asanuma, H. Chem.-Eur. J. 2010, 16, 2054-2062. 

Resumen en español 



\section{Publication list}

1. Gómez-Bombarelli, R.; González-Pérez, M.; Teresa Pérez-Prior, M.; Manso, J. A.; Calle, E.; Casado, J., Chemical reactivity and biological activity of diketene. Chem. Res. Toxicol. 2008, 21, (10), 1964-1969.

2. Gómez-Bombarelli, R.; González-Pérez, M.; Pérez-Prior, M. T.; Calle, E.; Casado, J., Computational calculation of equilibrium constants: Addition to carbonyl compounds. J. Phys. Chem. A 2009, 113, (42), 11423-11428.

3. Gómez-Bombarelli, R.; González-Pérez, M.; Pérez-Prior, M. T.; Calle, E.; Casado, J., Computational study of the acid dissociation of esters and lactones. A case study of diketene. J. Org. Chem. 2009, 74, (14), 4943-4948.

4. Gómez-Bombarelli, R.; González-Pérez, M.; Pérez-Prior, M. T.; Manso, J. A.; Calle, E.; Casado, J., Kinetic study of the neutral and base hydrolysis of diketene. J. Phys. Org. Chem. 2009, 22, (5), 438-442.

5. Gómez-Bombarelli, R.; Palma, B. B.; Martins, C.; Kranendonk, M.; Rodrigues, A. S.; Calle, E.; Rueff, J.; Casado, J., Alkylating potential of oxetanes. Chem. Res. Toxicol. 2010, 23, (7), 1275-1281.

6. Gómez-Bombarelli, R.; González-Pérez, M.; Arenas-Valgañón, J.; Céspedes-Camacho, I. F.; Calle, E.; Casado, J., DNA-damaging disinfection byproducts: Alkylation mechanism of mutagenic mucohalic acids. Environ. Sci. Technol. 2011, 45, (20), 9009-9016.

7. Gómez-Bombarelli, R.; González-Pérez, M.; Calle, E.; Casado, J., Reactivity of mucohalic acids in water. Water Res. 2011, 45, (2), 714-720.

8. Gómez-Bombarelli, R.; González-Pérez, M.; Pérez-Prior, M. T.; Calle, E.; Casado, J., Genotoxic halofuranones in water: Isomerization and acidity of mucohalic acids. J. Phys. Org. Chem. 2011 $10.1002 /$ poc. 1857. 